\title{
Cyclization reactions of oxyallyl cation. A method for cyclopentane ring formation
}

\author{
Bojan Vulovic, ${ }^{\mathrm{a}}$ Milena Trmcic, ${ }^{\mathrm{b}}$ Radomir Matovic, ${ }^{\mathrm{c}}$ Radomir N. Saicic ${ }^{*}, \mathrm{a}, \mathrm{d}$ \\ ${ }^{a}$ Faculty of Chemistry, University of Belgrade, Studentski trg 16, P. O. Box 51, 11158 Belgrade \\ 118, Serbia; rsaicic@chem.bg.ac.rs \\ ${ }^{\mathrm{b}}$ Innovation Centre of the Faculty of Chemistry, University of Belgrade \\ ${ }^{\mathrm{c}}$ ICTM Center for Chemistry, Njegoseva 12, Belgrade \\ ${ }^{\mathrm{d}}$ Serbian Academy of Sciences and Arts, Knez Mihailova 35, Belgrade
}

Supporting Information 


\section{Table of Contents}

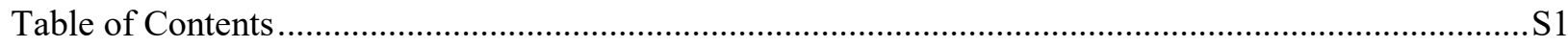

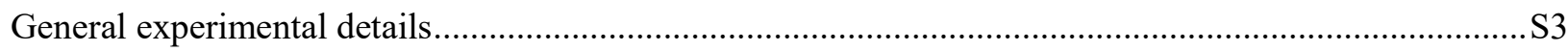

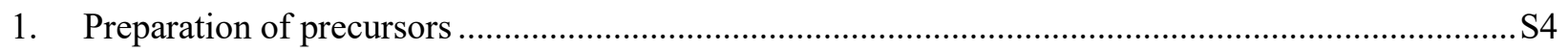

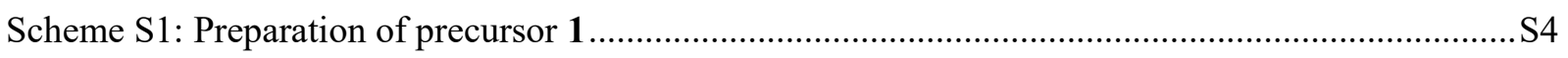

General procedure 1. (G.P.1.): mesylation of hydroxy ketones .....................................................S9

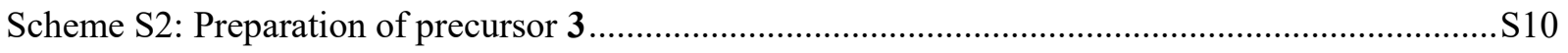

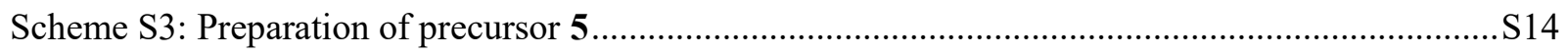

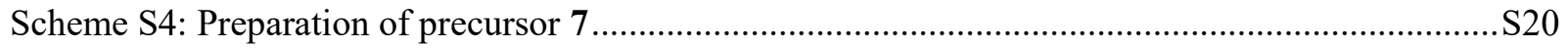

General procedure 2. (G.P.2.) - cross-acyloin condensation .......................................................S2 1

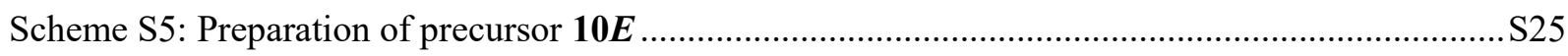

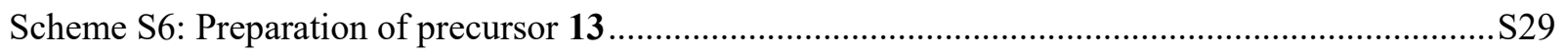

2,6,11,15-Tetramethyl-9-oxohexadeca-2,14-dien-8-yl methanesulfonate (16) .................................S32

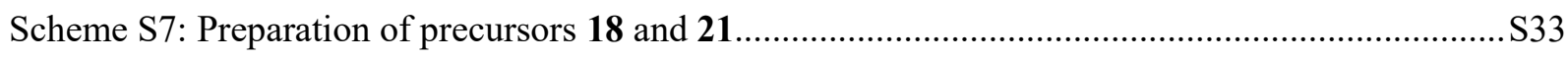

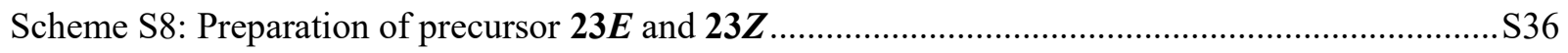

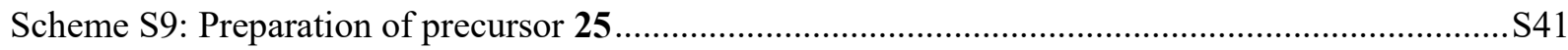

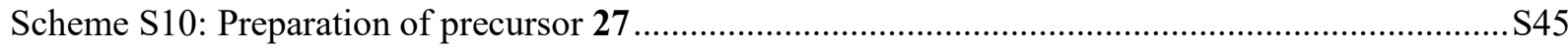

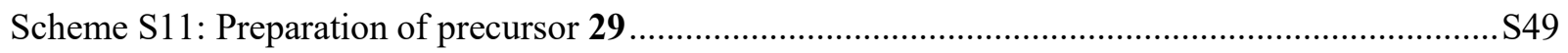

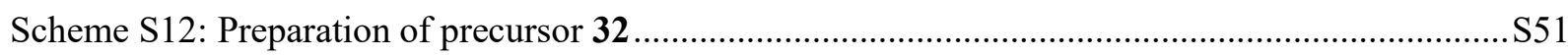

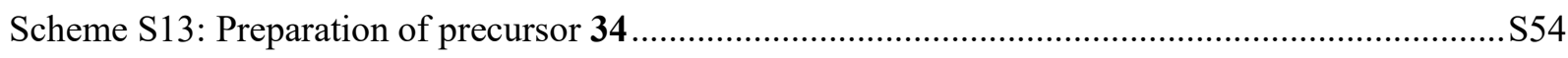

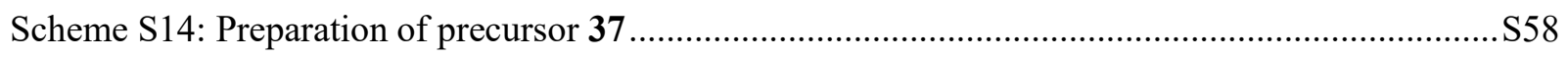

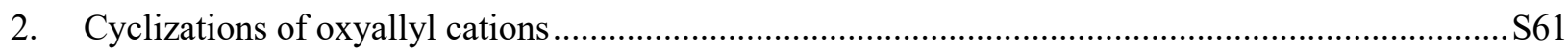

1-(2-Ethyl-5-methylcyclopentyl)ethan-1-one (2) (General procedure for the cyclization- Method A) S61

1-(2-Ethyl-5-methylcyclopentyl)ethan-1-one (2) (General procedure for the cyclization- Method C) S62

Distribution of products 2a:2b:2c over time (conditions: Method C) .................................................. 63

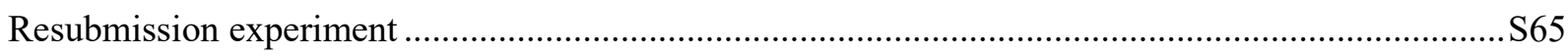

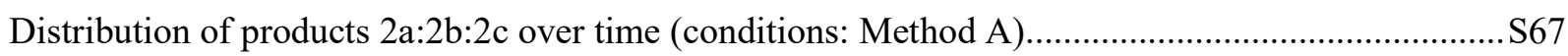

Distribution of products 2a:2b:2c over time (conditions: Method A) ................................................. 68

Distribution of products $2 \mathrm{a}: 2 \mathrm{~b}: 2 \mathrm{c}$ over time in the presence of menthone as an "in situ" internal

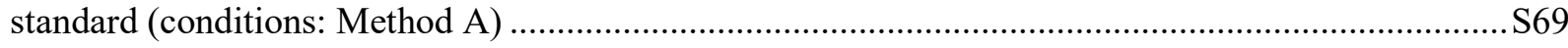

1-(2-(Prop-1-en-2-yl)spiro[4.5]decan-1-yl)ethan-1-one (4) ……....................................................... S72

(E)-1-(3-(Pent-1-en-1-yl)spiro[4.5]decan-2-yl)ethan-1-one (6)....................................................S72 
1-(2-Methyl-5-(prop-1-en-2-yl)cyclopentyl)-2-phenylethan-1-one (8) and (Z)-3-benzylidene-1,1,4trimethylhexahydro-1 $H$-cyclopenta $[c]$ furan (9). S73

1-(2-Methyl-5-(prop-1-en-2-yl)cyclopentyl)-2-phenylethan-1-one (8) and (Z)-3-benzylidene-1,1,4trimethylhexahydro-1H-cyclopenta[c]furan (9) (General procedure for the cyclization - Method B).S74 1-((1R,2S,5R)-2-Methyl-5-(prop-1-en-2-yl)cyclopentyl)-2-phenylethanone $\left(\mathbf{8}^{*}\right)$ and $(3 \mathrm{a} R, 4 S, 6 \mathrm{a} S, Z)-3$ benzylidene-1,1,4-trimethylhexahydro-1 $H$-cyclopenta[c] furan $\left(9^{*}\right)$.

1-(2-Methyl-5-vinylcyclopentyl)-2-phenylethan-1-one (11) and (Z)-3-benzylidene-1,4dimethylhexahydro- $1 H$-cyclopenta[c] furan (12) S76

1-(2-Methyl-5-styrylcyclopentyl)-2-phenylethan-1-one (14) and 1-benzyl-3-benzylidene-4methylhexahydro-1H-cyclopenta[c]furan (15) S77

4,8-Dimethyl-1-(2-methyl-5-(prop-1-en-2-yl)cyclopentyl)non-7-en-1-one (17). . $\mathrm{S} 78$

2-Phenyl-1-(4-(prop-1-en-2-yl)tetrahydrofuran-3-yl)ethan-1-one (19) and (Z)-3-benzylidene-1,1dimethyltetrahydro- $1 H, 3 H$-furo[3,4-c] furan (20) S79

4-((3-Methylbut-2-en-1-yl)oxy)-1-(4-(prop-1-en-2-yl)tetrahydrofuran-3-yl)butan-1-one (22) .80

trans-(E)-4-(but-2-en-1-yloxy)-1-(4-vinyltetrahydrofuran-3-yl)butan-1-one (24trans) and cis-(E)-4(but-2-en-1-yloxy)-1-(4-vinyltetrahydrofuran-3-yl)butan-1-one (24cis)..... S81

1,1-Dimethyloctahydroindeno[7,1-bc]furan-2a(1H)-ol (26) S82

(E)-2,3,3a,5,6,7,8,10a-Octahydrocyclopenta[9] annulen-4(1H)-one (28) . $\mathrm{S} 83$

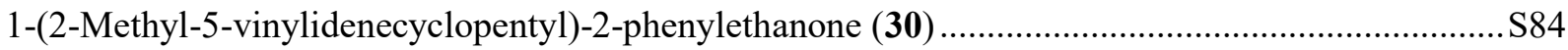

1-(5-Methyl-2-vinylcyclopent-1-enyl)-2-phenylethanone (31) .......................................................S84

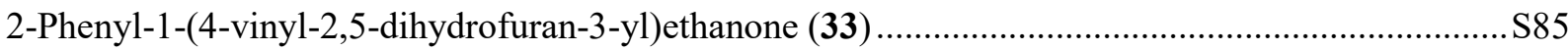

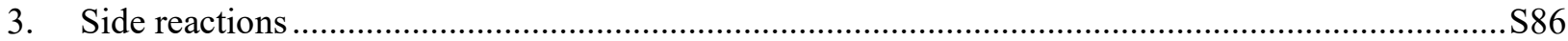

2,2,2-Trifluoroethyl (E)-3-(2-(hept-2-en-1-yl)phenyl)propanoate (35) ............................................S86

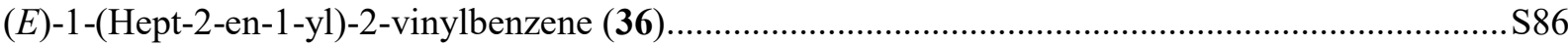

(E)-4-(5-Acetyl-3-((benzyloxy)methyl)tetrahydrofuran-3-yl)but-2-en-1-yl acetate (38) ...................S87

4. Copies of NMR spectra S89 


\section{General experimental details}

All chromatographic separations ${ }^{1}$ were performed on Silica, 10-18, 60A , ICN Biomedicals and Merck Silica gel $60(0.063-0.200 \mathrm{~mm})(70-230$ mesh ASTM). Standard techniques were used for the purification of reagents and solvents. ${ }^{2}$ Petroleum-ether refers to the fraction boiling at 70-72 ${ }^{\circ} \mathrm{C}$. For all reactions that required heating, oil bath was used as the heat source. NMR spectra were recorded on a Varian Gemini 200, $\left({ }^{1} \mathrm{H}\right.$ NMR at $200 \mathrm{MHz},{ }^{13} \mathrm{C}$ NMR at $50 \mathrm{MHz}$, and on a Bruker Avance III $500\left({ }^{1} \mathrm{H}\right.$ NMR at $500 \mathrm{MHz},{ }^{13} \mathrm{C}$ NMR at $\left.125 \mathrm{MHz}\right)$. Chemical shifts are expressed in ppm $(\delta)$ using tetramethylsilane as internal standard, coupling constants $(J)$ are in Hz. Gas chromatography (GC) and gas chromatography coupled to low resolution mass spectrometry (GCMS) analysis were performed on two different instruments. Agilent Technologies 7890A instrument, using HP-5MSI capillary column (length: $30 \mathrm{~m}$; diam. $0.25 \mathrm{~mm}$; film: $0.25 \mu$ ) and using $\mathrm{He}$ gas as carrier. GC was equipped with an FID detector. GC-MS was performed on Agilent Technologies 5975C Inert XL EI/CI MSD quadrupole detector. Shimadzu GCMS-QP2010 Ultra instrument, a comprehensive two dimensional gas chromatograph - quadrupole mass spectrometer (GC $\times$ GC-qMS) with ZX2 thermal modulation system, using RtxR-1 (first column: RESTEK, CrossbondR 100\% dimethyl polysiloxane, $30 \mathrm{~m}, 0.25 \mathrm{~mm} \mathrm{ID}, \mathrm{df}=0.25 \mu \mathrm{m}$ ) and a BPX50 (SGE Analytical Science, $1 \mathrm{~m}, 0.1 \mathrm{~mm} \mathrm{ID}, \mathrm{df}=0.1 \mu \mathrm{m}$ ) columns (columns were connected through the $\mathrm{GC} \times \mathrm{GC}$ modulator as the first and second capillary columns, and samples were analysed without modulation). IR spectra were recorded on a Nicolet $6700 \mathrm{FT}$ instrument, and are expressed in $\mathrm{cm}^{-}$ ${ }^{1}$. Mass spectra were obtained on Agilent technologies 6210 TOF LC/MS instrument (LC: series 1200) and LTQ Orbitrap XL hybrid FTMS (Thermo Scientific). Melting points were determined on a Kofler hot-stage apparatus and are uncorrected.

\footnotetext{
${ }^{1}$ For description of the technique of dry-flash chromatography, see: a) Harwood, L. M. Aldrichimica Acta 1985, 18 , 25; b) Vogel's Textbook of Practical Organic Chemistry, Longman Scientific\&Technical, $5^{\text {th }}$ edition, London, 1989, p. 220 ; c) A recent account which includes some improvements of the separation technique: Pedersen, D. S.;

Rosenbohm, C. Synthesis 2001, 2431.

${ }^{2}$ Perrin, D. D.; Armarego, W. L. F. Purification of Laboratory Chemicals, 3rd edition, Pergamon Press, 1988.
} 


\section{Preparation of precursors}

Scheme S1: Preparation of precursor 1<smiles>CC(C)=CCCC(C)C=O</smiles>

$( \pm)$-melonal

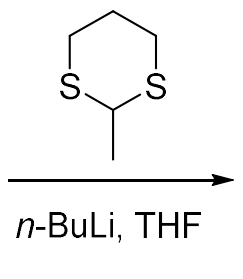<smiles>CC(C)=CCC[C@H](C)[C@H](O)C1(C)SCCCS1</smiles>

S1-1<smiles>CC(C)=CCC[C@@H](C)[C@H](O)C1(C)SCCCS1</smiles>

S1-2

two isomers separated by column cromatography<smiles>CC(C)=CCC[C@H](C)[C@H](O)C1(C)SCCCS1</smiles>

S1-1

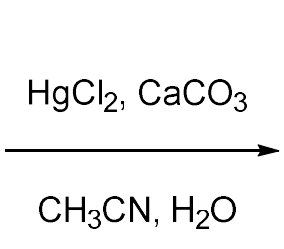

$\mathrm{CH}_{3} \mathrm{CN}, \mathrm{H}_{2} \mathrm{O}$<smiles>CC(C)=CCC[C@@H](C)[C@H](O)C1(C)SCCCS1</smiles>

S1-2
$\mathrm{HgCl}_{2}, \mathrm{CaCO}_{3}$

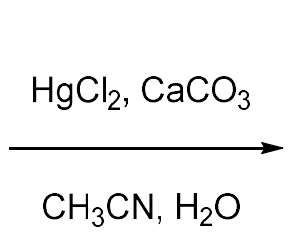

S1-4<smiles>CC(=O)[C@H](O)[C@@H](C)CCC=C(C)C</smiles>

S1-3

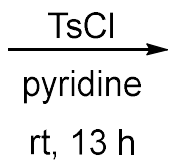

$\mathrm{rt}, 13 \mathrm{~h}$

1 syn<smiles>CC(=O)C([OH2+])[C@H](C)CCC=C(C)C</smiles>

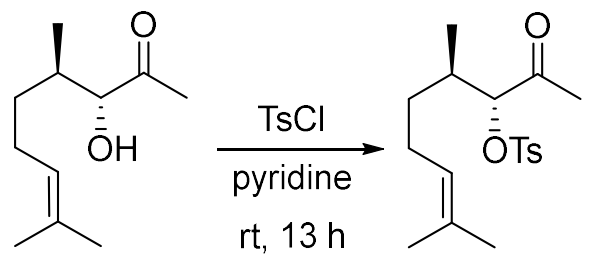

1 anti<smiles>CC(C)=CCCC(C)C=O</smiles>

( \pm )-melonal<smiles>C=COCC</smiles>

$\underset{\mathrm{HCl}}{\stackrel{\mathrm{MeOH}}{\longrightarrow}}$<smiles>CO[14CH2]CCC(C)C(O)C(C)=O</smiles>

$S 1-3+S 1-4$<smiles>CCN(CC)C(C)CCCC(C)C(OC)C(C)=O</smiles>

1 syn+anti 


\section{2,6-Dimethyl-1-(2-methyl-1,3-dithian-2-yl)hept-5-en-1-ol, syn and anti (S1-1 and S1-2) ${ }^{3}$}

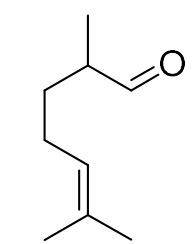

$( \pm)$-melonal

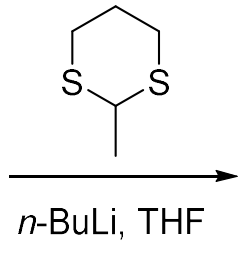

S1-1

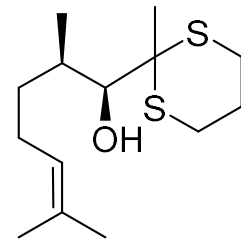

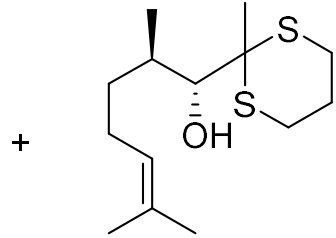

S1-2

To a solution of 1,3-dithiane $\mathrm{e}^{3}(0.4 \mathrm{~mL}, 3.34 \mathrm{mmol}, 1 \mathrm{eq})$ in THF $(6.7 \mathrm{~mL})$ was added $n$-BuLi in hexane $(2.15 \mathrm{M}, 1.8 \mathrm{~mL}, 3.87 \mathrm{mmol}, 1.16 \mathrm{eq})$, with stirring at $-78{ }^{\circ} \mathrm{C}$, under an argon atmosphere. The mixture was allowed to warm to $0{ }^{\circ} \mathrm{C}$ within $1 \mathrm{~h}$, cooled down to $-78{ }^{\circ} \mathrm{C}$ again, and 2,6dimethyl-5-heptenal $(0.7 \mathrm{~mL}, 4.25 \mathrm{mmol}, 1.27 \mathrm{eq})$ was added dropwise. The reaction mixture was allowed to warm to room temperature within $3 \mathrm{~h}$ and then quenched by the addition of water. The aqueous layer was extracted with $\mathrm{CH}_{2} \mathrm{Cl}_{2}$. The combined organic extract was washed with water, $\mathrm{KOH}(7 \%)$ and water, dried over anh. $\mathrm{MgSO}_{4}$, and the solvents were removed under reduced pressure. The crude product was purified by dry flash chromatography (petroleum-ether/ethyl acetate $=95 / 5)$ followed by column chromatography (petroleum-ether/ethyl acetate $=95 / 5)$ to afford $503 \mathrm{mg}$ (55\%) of 2,6-dimethyl-1-(2-methyl-1,3-dithian-2-yl)hept-5-en-1-ol (S1-1) and 216 mg (23\%) of 2,6-dimethyl-1-(2-methyl-1,3-dithian-2-yl)hept-5-en-1-ol (S1-2), both as pale yellow oils.

S1-1:

${ }^{1} \mathbf{H}$ NMR $\left(500 \mathrm{MHz}, \mathrm{CDCl}_{3}\right) \delta 5.17-5.11(\mathrm{~m}, 1 \mathrm{H}), 3.89(\mathrm{t}, J=1.8 \mathrm{~Hz}, 1 \mathrm{H}), 3.01-2.92(\mathrm{~m}, 2 \mathrm{H})$, $2.69-2.62(\mathrm{~m}, 2 \mathrm{H}), 2.56(\mathrm{dd}, J=2.1,1.1 \mathrm{~Hz}, 1 \mathrm{H}), 2.24-2.15(\mathrm{~m}, 1 \mathrm{H}), 2.13-1.98(\mathrm{~m}, 3 \mathrm{H}), 1.92$ $-1.82(\mathrm{~m}, 1 \mathrm{H}), 1.69(\mathrm{~d}, J=1.0 \mathrm{~Hz}, 3 \mathrm{H}), 1.62(\mathrm{~s}, 3 \mathrm{H}), 1.56-1.48(\mathrm{~m}, 1 \mathrm{H}), 1.47(\mathrm{~s}, 3 \mathrm{H}), 1.46-$ $1.39(\mathrm{~m}, 1 \mathrm{H}), 0.96(\mathrm{~d}, J=6.8 \mathrm{~Hz}, 3 \mathrm{H})$.

${ }^{13} \mathrm{C}$ NMR (125 MHz, $\left.\mathrm{CDCl}_{3}\right) \delta 131.5,124.5,73.5,55.0,38.1,32.5,26.3,25.9,25.8,25.7,24.4$, 23.0, 17.1, 14.6.

IR (ATR) $v_{\max }: 3480,2967,2916,2856,1448,1381,1252$.

HRMS (ESI) calcd. for $\mathrm{C}_{14} \mathrm{H}_{27} \mathrm{OS}_{2}^{+}[\mathrm{M}+\mathrm{H}]^{+}: 275.1498$, found: 275.1493.

\section{S1-2:}

${ }^{1} \mathbf{H}$ NMR $\left(500 \mathrm{MHz}, \mathrm{CDCl}_{3}\right) \delta 5.16-5.09(\mathrm{~m}, 1 \mathrm{H}), 3.81(\mathrm{t}, J=2.1 \mathrm{~Hz}, 1 \mathrm{H}), 3.01-2.92(\mathrm{~m}, 2 \mathrm{H})$, $2.68-2.59(\mathrm{~m}, 2 \mathrm{H}), 2.61(\mathrm{dd}, J=1.9,0.9 \mathrm{~Hz}, 1 \mathrm{H}), 2.13-2.03(\mathrm{~m}, 3 \mathrm{H}), 1.97-1.79(\mathrm{~m}, 2 \mathrm{H}), 1.68$ $(\mathrm{d}, J=2.1,3 \mathrm{H}), 1.67-1.61(\mathrm{~m}, 1 \mathrm{H}), 1.61(\mathrm{~s}, 3 \mathrm{H}), 1.45(\mathrm{~s}, 3 \mathrm{H}), 1.28-1.19(\mathrm{~m}, 1 \mathrm{H}), 1.14(\mathrm{~d}, J=$ $6.9 \mathrm{~Hz}, 3 \mathrm{H})$.

${ }^{13} \mathrm{C}$ NMR $\left(125 \mathrm{MHz}, \mathrm{CDCl}_{3}\right) \delta 131.4,124.8,75.4,55.0,32.8,32.7,26.4,26.1,25.8,25.7,24.4$, 22.6, 21.0, 17.6.

IR (ATR) $v_{\max }: 3484,2961,2914,1447,1377,1271$.

HRMS (ESI) calcd. for $\mathrm{C}_{14} \mathrm{H}_{27} \mathrm{OS}_{2}{ }^{+}[\mathrm{M}+\mathrm{H}]^{+}: 275.1498$, found: 275.1493 .

${ }^{3}$ Dickschat, J. S., Wickel, S., Bolten, C. J., Nawrath, T., Schulz, S., Wittmann, C., Eur. J. Org. Chem. 2010, 26872695. 
3-Hydroxy-4,8-dimethylnon-7-en-2-one (S1-3) ${ }^{4}$

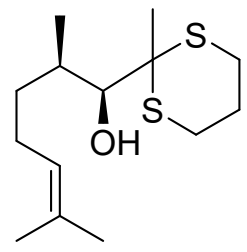

S1-1

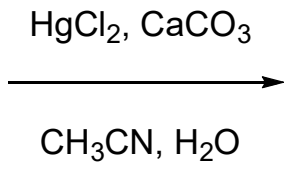

To a solution of S1-1 (163 mg, $0.59 \mathrm{mmol}, 1 \mathrm{eq})$ in acetonitrile/water (4:1, $12 \mathrm{~mL})$ were added mercury(II) chloride (478 mg, $1.76 \mathrm{mmol}, 3 \mathrm{eq}$ ) and calcium carbonate (239 $\mathrm{mg}, 2.39 \mathrm{mmol}, 4$ eq). The reaction mixture was heated to $65^{\circ} \mathrm{C}$ for $45 \mathrm{~min}$, then cooled to rt. After addition of diethyl ether $(20 \mathrm{~mL})$, reaction mixture was filtered through Celite. The filtrate was washed with water and brine, dried over anh. $\mathrm{MgSO}_{4}$ and concentrated in vacuo. Dry flash chromatography (petroleum-ether/ethyl acetate $=925 / 75)$ afforded $76 \mathrm{mg}(70 \%)$ of compound $\mathbf{S 1 - 3}$ as a colorless oil.

${ }^{1}$ H NMR $\left(500 \mathrm{MHz}, \mathrm{CDCl}_{3}\right) \delta 5.17-5.10(\mathrm{~m}, 1 \mathrm{H}), 4.19(\mathrm{dd}, J=4.8,2.2 \mathrm{~Hz}, 1 \mathrm{H}), 3.37(\mathrm{~d}, J=4.8$ $\mathrm{Hz}, 1 \mathrm{H}), 2.18(\mathrm{~s}, 3 \mathrm{H}), 2.15-2.04(\mathrm{~m}, 2 \mathrm{H}), 2.03-1.95(\mathrm{~m}, 1 \mathrm{H}), 1.71(\mathrm{~d}, J=1.0 \mathrm{~Hz}, 3 \mathrm{H}), 1.65-$ $1.57(\mathrm{~m}, 1 \mathrm{H}), 1.63(\mathrm{~s}, 3 \mathrm{H}), 1.47-1.37(\mathrm{~m}, 1 \mathrm{H}), 0.70(\mathrm{~d}, J=6.8 \mathrm{~Hz}, 3 \mathrm{H})$.

${ }^{13}$ C NMR $\left(125 \mathrm{MHz}, \mathrm{CDCl}_{3}\right) \delta 210.1,131.9,124.0,79.2,35.1,33.8,25.7,25.6,25.2,17.7,12.7$. IR (ATR) $v_{\max }:$ 3480, 2961, 2925, 2856, 1712, 1458, 1379, 1265.

HRMS (ESI) calcd. for $\mathrm{C}_{11} \mathrm{H}_{24} \mathrm{O}_{2} \mathrm{~N}^{+}\left[\mathrm{M}+\mathrm{NH}_{4}\right]^{+}:$202.1802, found: 202.1801 .

\section{4,8-Dimethyl-2-oxonon-7-en-3-yl 4-methylbenzenesulfonate (1 syn)}

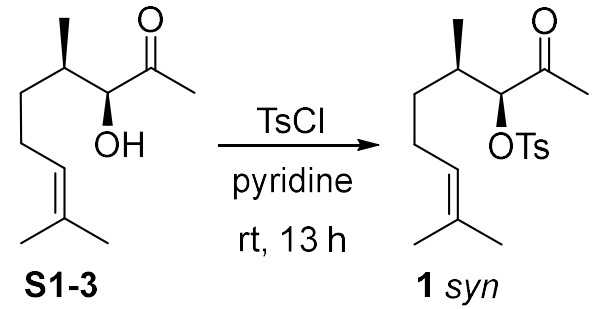

Tosyl chloride (168 mg, $0.88 \mathrm{mmol}, 4 \mathrm{eq})$ was added to a solution of S1-3 (41.6 mg, $0.22 \mathrm{mmol}$, $1 \mathrm{eq})$ in pyridine $(1.1 \mathrm{~mL})$ and the reaction mixture was stirred at room temperature for $13 \mathrm{~h}$. The reaction mixture was then diluted with $\mathrm{CH}_{2} \mathrm{Cl}_{2}$, washed with sat. $\mathrm{CuSO}_{4}$ solution, water, brine, dried over anh. $\mathrm{MgSO}_{4}$ and concentrated in vacuo. Purification by column chromatography (petroleum-ether/ethyl acetate $=9 / 1)$ afforded $60 \mathrm{mg}(82 \%)$ of compound $1 \boldsymbol{s y n}$ as a colorless oil.

${ }^{4}$ Love, B.; Jones, E. J. Org. Chem. 1999, 3755-3756. 
${ }^{1} \mathbf{H}$ NMR $\left(500 \mathrm{MHz}, \mathrm{CDCl}_{3}\right) \delta 7.84-7.79(\mathrm{~m}, 2 \mathrm{H}), 7.39-7.35(\mathrm{~m}, 2 \mathrm{H}), 4.93-4.87(\mathrm{~m}, 1 \mathrm{H})$, $4.62(\mathrm{~d}, J=3.9 \mathrm{~Hz}, 1 \mathrm{H}), 2.47$ (s, 3H), $2.20(\mathrm{~s}, 3 \mathrm{H}), 2.03-1.95(\mathrm{~m}, 1 \mathrm{H}), 1.91(\mathrm{dt}, J=14.7,7.2 \mathrm{~Hz}$, $1 \mathrm{H}), 1.79$ (dt, $J=14.8,7.4 \mathrm{~Hz}, 1 \mathrm{H}), 1.67$ (d, $J=1.1 \mathrm{~Hz}, 3 \mathrm{H}), 1.56$ (s, 3H), $1.24-1.15(\mathrm{~m}, 1 \mathrm{H})$, $1.15-1.06(\mathrm{~m}, 1 \mathrm{H}), 0.85(\mathrm{~d}, J=6.9 \mathrm{~Hz}, 3 \mathrm{H})$.

${ }^{13}$ C NMR $\left(125 \mathrm{MHz}, \mathrm{CDCl}_{3}\right) \delta 205.8,145.2,133.0,132.1,129.8,128.0,123.4,87.2,35.1,32.5$, 27.1, 25.6, 24.9, 21.6, 17.6, 14.0 .

IR (ATR) $v_{\max }: 2969,2927,1720,1597,1452,1370$.

HRMS (ESI) calcd. for $\mathrm{C}_{18} \mathrm{H}_{30} \mathrm{O}_{4} \mathrm{SN}^{+}\left[\mathrm{M}+\mathrm{NH}_{4}\right]^{+}: 356.1890$, found: 356.1889 .

\section{3-Hydroxy-4,8-dimethylnon-7-en-2-one (S1-4) ${ }^{4}$}

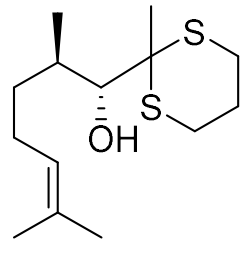

S1-2

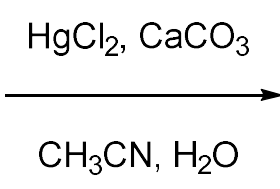

$\mathrm{CH}_{3} \mathrm{CN}, \mathrm{H}_{2} \mathrm{O}$

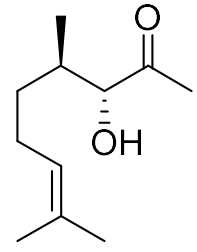

S1-4

To a solution of $\mathbf{S 1 - 2}(35 \mathrm{mg}, 0.127 \mathrm{mmol}, 1 \mathrm{eq})$ in acetonitrile/water $(4: 1,2.6 \mathrm{~mL})$ were added mercury(II) chloride (104 mg, $0.38 \mathrm{mmol}, 3 \mathrm{eq}$ ) and calcium carbonate $(51 \mathrm{mg}, 0.51 \mathrm{mmol}, 4 \mathrm{eq})$. The reaction mixture was heated to $65^{\circ} \mathrm{C}$ for $45 \mathrm{~min}$, then cooled to rt. After addition of diethyl ether $(5 \mathrm{~mL})$, reaction mixture was filtered through Celite. The filtrate was washed with water and brine, dried over anh. $\mathrm{MgSO}_{4}$ and concentrated in vacuo. Dry flash chromatography (petroleumether/ethyl acetate $=9 / 1)$ afforded $11.8 \mathrm{mg}(50 \%)$ of compound $\mathbf{S 1 - 4}$, as a colorless oil.

${ }^{1}$ H NMR $\left(500 \mathrm{MHz}, \mathrm{CDCl}_{3}\right) \delta 5.07-5.00(\mathrm{~m}, 1 \mathrm{H}), 4.09(\mathrm{dd}, J=4.7,2.5 \mathrm{~Hz}, 1 \mathrm{H}), 3.40(\mathrm{~d}, J=4.7$ $\mathrm{Hz}, 1 \mathrm{H}), 2.19$ (s, 3H), $2.08-1.96(\mathrm{~m}, 2 \mathrm{H}), 1.94-1.84(\mathrm{~m}, 1 \mathrm{H}), 1.68(\mathrm{~d}, J=0.7 \mathrm{~Hz}, 3 \mathrm{H}), 1.59$ (s, $3 \mathrm{H}), 1.29-1.20(\mathrm{~m}, 1 \mathrm{H}), 1.12(\mathrm{~d}, J=6.9 \mathrm{~Hz}, 3 \mathrm{H}), 1.07-0.98(\mathrm{~m}, 1 \mathrm{H})$.

${ }^{13}$ C NMR $\left(125 \mathrm{MHz}, \mathrm{CDCl}_{3}\right) \delta 209.9,132.2,123.9,81.8,35.5,29.7,25.7,25.6,25.4,17.7,16.9$. IR (ATR) $v_{\max }: 3480,3054,2968,2928,1710,1453,1358,1265$.

HRMS (ESI) calcd. for $\mathrm{C}_{11} \mathrm{H}_{24} \mathrm{O}_{2} \mathrm{~N}^{+}\left[\mathrm{M}+\mathrm{NH}_{4}\right]^{+}: 202.1802$, found: 202.1800 . 


\section{4,8-Dimethyl-2-oxonon-7-en-3-yl 4-methylbenzenesulfonate (1 anti)}

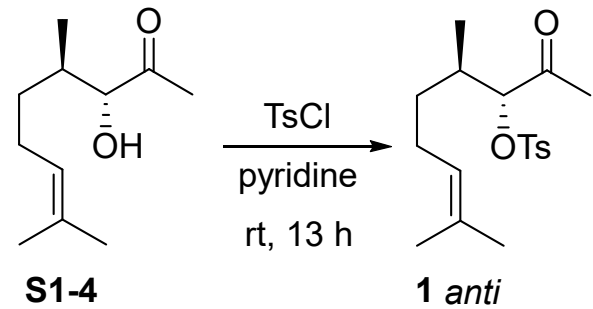

Tosyl chloride ( $65 \mathrm{mg}, 0.34 \mathrm{mmol}, 4 \mathrm{eq})$ was added to a solution of $\mathbf{S 1 - 4}(15.8 \mathrm{mg}, 0.085 \mathrm{mmol}$, $1 \mathrm{eq})$ in pyridine $(0.4 \mathrm{~mL})$ and the reaction mixture was stirred at room temperature for $13 \mathrm{~h}$. The reaction mixture was then diluted with $\mathrm{CH}_{2} \mathrm{Cl}_{2}$, washed with sat. $\mathrm{CuSO}_{4}$ solution, water, brine, dried over anh. $\mathrm{MgSO}_{4}$ and concentrated in vacuo. Purification by column chromatography (petroleum-ether/ethyl acetate $=95 / 5)$ afforded $16.2 \mathrm{mg}(56 \%)$ of $\mathbf{1}$ anti, as a colorless oil.

${ }^{1} \mathbf{H}$ NMR $\left(500 \mathrm{MHz}, \mathrm{CDCl}_{3}\right) \delta 7.82-7.78(\mathrm{~m}, 2 \mathrm{H}), 7.37-7.33(\mathrm{~m}, 2 \mathrm{H}), 4.97-4.91(\mathrm{~m}, 1 \mathrm{H})$, $4.48(\mathrm{~d}, J=5.3 \mathrm{~Hz}, 1 \mathrm{H}), 2.46(\mathrm{~s}, 3 \mathrm{H}), 2.18(\mathrm{~s}, 3 \mathrm{H}), 2.01-1.78(\mathrm{~m}, 3 \mathrm{H}), 1.65(\mathrm{~d}, J=0.8 \mathrm{~Hz}, 3 \mathrm{H})$, $1.55(\mathrm{~s}, 3 \mathrm{H}), 1.38-1.28(\mathrm{~m}, 1 \mathrm{H}), 1.20-1.10(\mathrm{~m}, 1 \mathrm{H}), 0.80(\mathrm{~d}, J=6.9 \mathrm{~Hz}, 3 \mathrm{H})$.

${ }^{13} \mathbf{C}$ NMR $\left(125 \mathrm{MHz}, \mathrm{CDCl}_{3}\right) \delta 205.6,145.3,133.0,132.2,129.9,128.0,123.4,88.5,35.2,31.1$, 27.1, 25.6, 24.9, 21.7, 17.6, 15.2.

IR (ATR) $v_{\max }: 2970,2927,1721,1598,1455,1369$.

HRMS (ESI) calcd. for $\mathrm{C}_{18} \mathrm{H}_{26} \mathrm{O}_{4} \mathrm{SK}^{+}[\mathrm{M}+\mathrm{K}]^{+}: 377.1183$, found: 377.1184 .

For a gram scale preparation of S1-3 and S1-4 mixture, a shorter and higher yielding procedure was used:

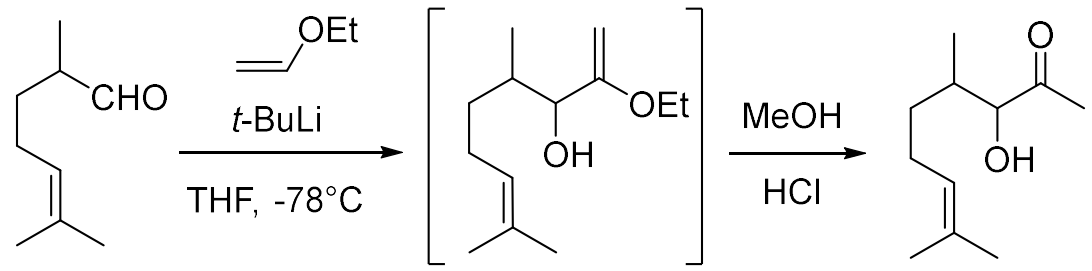

( \pm -melonal

$\mathrm{S} 1-3+\mathrm{S} 1-4$

To a solution of methyl vinyl ether $(10.98 \mathrm{~mL}, 0.114 \mathrm{M}, 8 \mathrm{eq})$, in THF $(130 \mathrm{~mL})$, at $-78{ }^{\circ} \mathrm{C}, t$ BuLi (1.6 M, $35 \mathrm{~mL}, 4$ eq) was added dropwise over a period of 15 minutes, under an argon atmosphere. A yellow precipitate appeared and the mixture was stirred for additional 15 minutes at $-78^{\circ} \mathrm{C}$. The yellow suspension was then warmed to $0{ }^{\circ} \mathrm{C}$ and stirred for 20 minutes to give a colorless solution. The reaction mixture was cooled to $-78{ }^{\circ} \mathrm{C}$ and 2,6-dimethylhept-5-enal $(2 \mathrm{~g}$, $0.0143 \mathrm{M})$ in THF $(5 \mathrm{~mL})$ was added dropwise. The reaction mixture was stirred at $-78{ }^{\circ} \mathrm{C}$ for $1 \mathrm{~h}$, then warmed up to room temperature. The reaction was quenched with water and the mixture was 
extracted with $\mathrm{Et}_{2} \mathrm{O}(2 \times 25 \mathrm{~mL})$. The combined organic extract was washed with $\mathrm{H}_{2} \mathrm{O}$ and brine, dried over anh. $\mathrm{MgSO}_{4}$, filtered and concentrated. The residue was stirred with aqueous methanolic $\mathrm{HCl}(0.05 \mathrm{M}, 45 \mathrm{~mL})$ for $20 \mathrm{~min}$. The reaction mixture was concentrated in vacuo, diluted with water, extracted with $\mathrm{Et}_{2} \mathrm{O}(2 \times 30 \mathrm{~mL})$, dried over anh. $\mathrm{MgSO}_{4}$ and concentrated under reduced pressure. Purification by column chromatography (petroleum-ether/ethyl acetate $=95 / 5$ ) afforded $2.1 \mathrm{~g}(77 \%)$ 3-hydroxy-4,8-dimethylnon-7-en-2-one (S1-3 + S1-4), as a colorless oil.

\section{General procedure 1. (G.P.1.): mesylation of hydroxy ketones} 4,8-Dimethyl-2-oxonon-7-en-3-yl methanesulfonate (1 syn + anti)

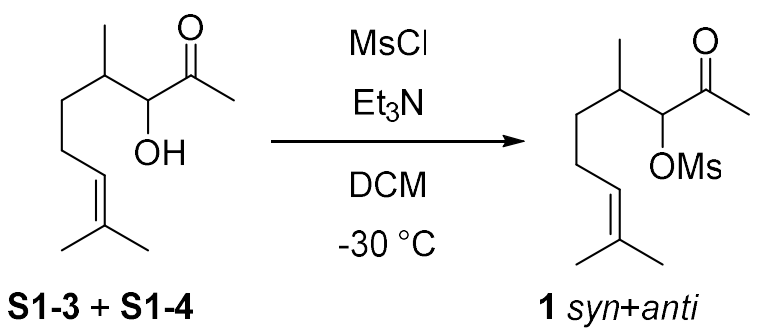

Mesyl chloride (0.044 mmol, $3.4 \mu \mathrm{L}, 2 \mathrm{eq})$ was added to a solution of S1-3 + S1-4 (4.1 mg, 0.022 mmol, 1 eq), triethylamine $(9 \mu \mathrm{L}, 0.067 \mathrm{mmol}, 3 \mathrm{eq})$ and a few crystals of DMAP in $\mathrm{CH}_{2} \mathrm{Cl}_{2}(0.17$ $\mathrm{mL})$ at $-30{ }^{\circ} \mathrm{C}$ and the reaction was completed almost immediately. The reaction mixture was concentrated in vacuo and the residue was purified by column chromatography (petroleumether/ethyl acetate $=8 / 2)$ to afford $3.6 \mathrm{mg}(62 \%)$ of $\mathbf{1} \mathbf{s y n}+\boldsymbol{a n t i}$ as a colorless oil (mixture of isomers in a relative ratio $1.5: 1$, as determined by $\left.{ }^{1} \mathrm{H} \mathrm{NMR}\right)$.

Spectral data for a mixture of isomers, where $\mathrm{H}$ : $\mathrm{H}^{\prime}$ are isomers in the ratio 1.5:1

${ }^{1} \mathbf{H}$ NMR $\left(500 \mathrm{MHz}, \mathrm{CDCl}_{3}\right) 5.12-5.06(\mathrm{~m}, 1 \mathrm{H}), 5.12-5.06\left(\mathrm{~m}, 1 \mathrm{H}^{\prime}\right), 4.96(\mathrm{~d}, J=3.1 \mathrm{~Hz}, 1 \mathrm{H})$, $4.82\left(\mathrm{~d}, J=4.1 \mathrm{~Hz}, 1 \mathrm{H}^{\prime}\right), 3.14(\mathrm{~s}, 3 \mathrm{H}), 3.12\left(\mathrm{~s}, 3 \mathrm{H}^{\prime}\right), 2.24\left(\mathrm{~s}, 3 \mathrm{H}^{\prime}\right), 2.22(\mathrm{~s}, 3 \mathrm{H}), 2.20-2.12(\mathrm{~m}$, $\left.1 \mathrm{H}^{+}+\mathrm{H}^{\prime}\right), 2.12-2.02\left(\mathrm{~m}, 2 \mathrm{H}+1 \mathrm{H}^{\prime}\right), 1.97-1.88\left(\mathrm{~m}, 1 \mathrm{H}^{\prime}\right), 1.70(\mathrm{~d}, J=1.0 \mathrm{~Hz}, 3 \mathrm{H}), 1.68(\mathrm{~d}, J=$ $\left.0.9 \mathrm{~Hz}, 3 \mathrm{H}^{\prime}\right), 1.63(\mathrm{~s}, 3 \mathrm{H}), 1.60\left(\mathrm{~s}, 3 \mathrm{H}^{\prime}\right), 1.58-1.49(\mathrm{~m}, 1 \mathrm{H}), 1.44-1.24\left(\mathrm{~m}, 1 \mathrm{H}+2 \mathrm{H}^{\prime}\right), 1.07$ (d, $\left.J=6.9 \mathrm{~Hz}, 3 \mathrm{H}^{\prime}\right), 0.90(\mathrm{~d}, J=6.9 \mathrm{~Hz}, 3 \mathrm{H})$.

${ }^{13} \mathbf{C}$ NMR (125 MHz, $\left.\mathrm{CDCl}_{3}\right) \delta$ 204.2, 204.2, 132.6, 123.3, 123.2, 88.5, 86.9, 38.9, 38.7, 34.6, $34.4,33.0,30.7,27.0,26.8,25.7(2 \times \mathrm{C}), 25.2,25.1,17.7(2 \times \mathrm{C}), 15.9,13.7$.

IR (ATR) $v_{\max }: 2970,2934,1728,1456,1358$.

HRMS (ESI) calcd. for $\mathrm{C}_{11} \mathrm{H}_{22} \mathrm{O}_{4} \mathrm{SNa}^{+}[\mathrm{M}+\mathrm{Na}]^{+}: 285.1131$, found: 285.1123 . 


\section{Scheme S2: Preparation of precursor 3}
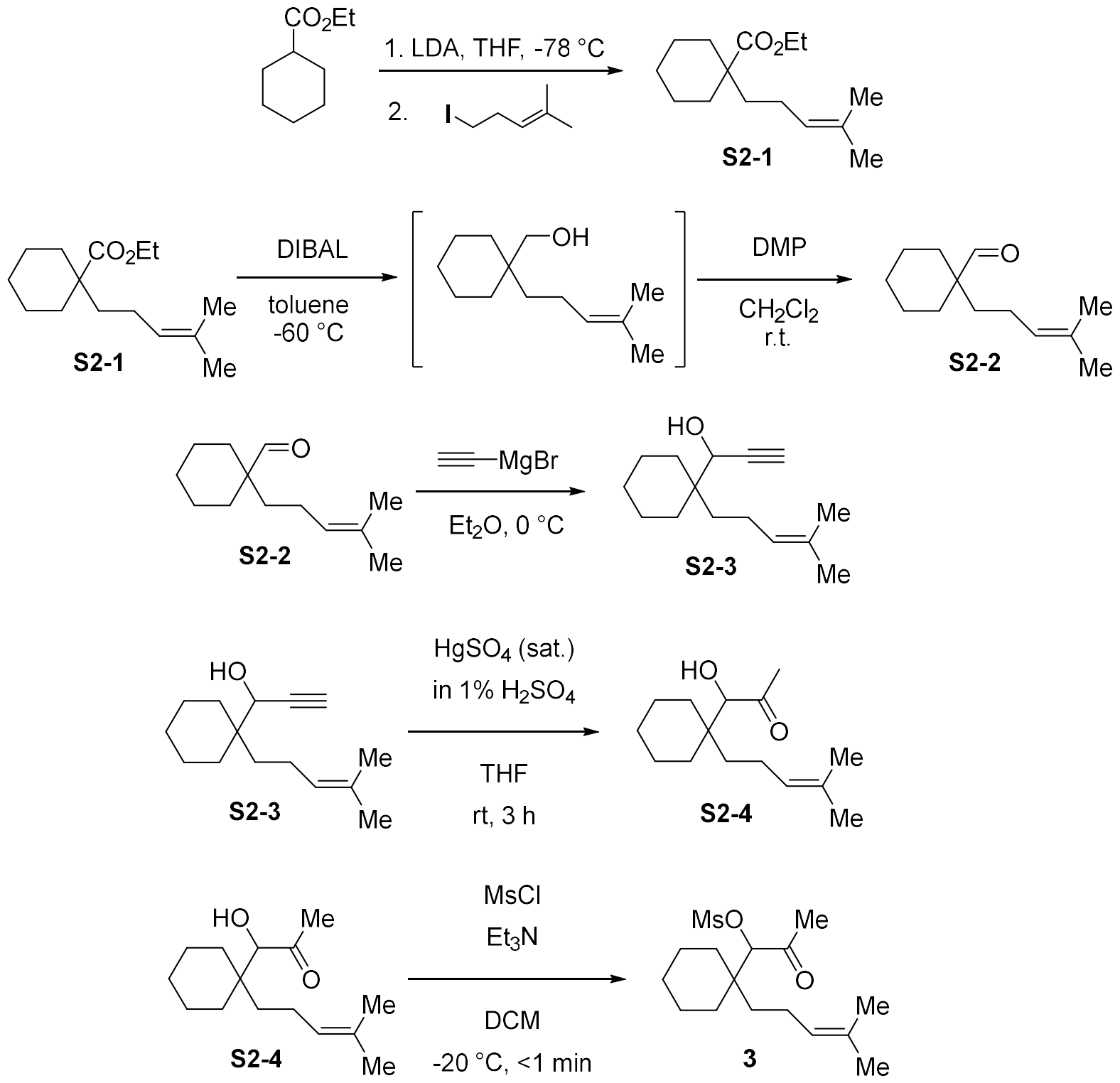

Ethyl 1-(4-methylpent-3-en-1-yl)cyclohexane-1-carboxylate (S2-1)

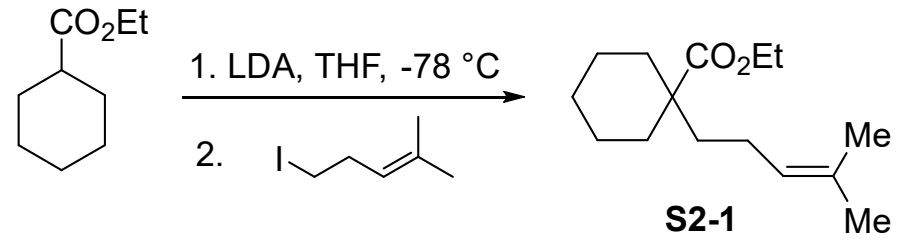

To a cold $\left(0{ }^{\circ} \mathrm{C}\right)$ solution of diisopropylamine $(1.80 \mathrm{~g} ; 17.8 \mathrm{mmol} ; 1.1 \mathrm{eq})$ in dry THF $(15.0 \mathrm{~mL})$, butyllithium was added (14.85 $\mathrm{mL}$ of the $1.2 \mathrm{M}$ solution in hexane; $17.8 \mathrm{mmol}, 1.1 \mathrm{eq})$, under an 
argon atmosphere. The solution was stirred for $10 \mathrm{~min}$ at that temperature, cooled to $-78{ }^{\circ} \mathrm{C}$, and then ethyl cyclohexanecarboxylate $(2.50 \mathrm{~g} ; 16.0 \mathrm{mmol})$ was added dropwise, over $15 \mathrm{~min}$. After the addition was complete, the reaction mixture was stirred for 1 hour at $-78^{\circ} \mathrm{C}$, when a solution of 5-iodo-2-methylpent-2-ene (3.70 g; $17.8 \mathrm{mmol}$; $1.1 \mathrm{eq})$ in DMSO (3.0 mL) was added dropwise. The reaction mixture was stirred at $-78{ }^{\circ} \mathrm{C}$ for $30 \mathrm{~min}$, warmed to room temperature and stirred overnight. The reaction mixture was quenched with $1.5 \mathrm{M} \mathrm{HCl}$ and the organic phase was washed with water and brine, dried over anh. $\mathrm{MgSO}_{4}$, filtered and concentrated under reduced pressure. The crude product was purified by dry-flash chromatography $\left(\mathrm{SiO}_{2}\right.$, eluent petroleum-ether/ethyl acetate $=975 / 25)$ to provide $3.0 \mathrm{~g}(78 \%)$ of ethyl 1-(4-methylpent-3-enyl)cyclohexanecarboxylate (S2-1), as a colorless oil. NMR spectra consistent with literature. ${ }^{5}$

${ }^{1} \mathbf{H}$ NMR $\left(500 \mathrm{MHz}, \mathrm{CDCl}_{3}\right) \delta 5.05-5.02(\mathrm{~m}, 1 \mathrm{H}), 4.13(\mathrm{q}, J=7.0 \mathrm{~Hz}, 2 \mathrm{H}), 2.06(\mathrm{bd}, J=13.0$ $\mathrm{Hz}, 2 \mathrm{H}), 1.88-1.83(\mathrm{~m}, 2 \mathrm{H}), 1.65(\mathrm{~s}, 3 \mathrm{H}), 1.58-1.51(\mathrm{~m}, 3 \mathrm{H}), 1.56(\mathrm{~s}, 3 \mathrm{H}), 1.49-1.46(\mathrm{~m}$, $2 \mathrm{H}), 1.38-1.28(\mathrm{~m}, 2 \mathrm{H}), 1.25(\mathrm{t}, J=7.0 \mathrm{~Hz}, 3 \mathrm{H}), 1.23-1.16(\mathrm{~m}, 3 \mathrm{H})$.

${ }^{13} \mathrm{C}$ NMR $\left(126 \mathrm{MHz}, \mathrm{CDCl}_{3}\right) \delta 176.9,131.7,124.3,60.0,46.8,40.6,34.2$ (2 x C), 26.1, 25.8, 23.4 ( 2 x C), 22.9, 17.6, 14.5 .

IR (ATR) $v_{\max } 2930,2857,1696,1453,1244,1199 \mathrm{~cm}^{-1}$.

HRMS (ESI) calcd. for $\mathrm{C}_{15} \mathrm{H}_{27} \mathrm{O}_{2}^{+}[\mathrm{M}+\mathrm{H}]^{+}:$239.2006, found: 239.2006.

\section{1-(4-Methylpent-3-en-1-yl)cyclohexane-1-carbaldehyde (S2-2)}

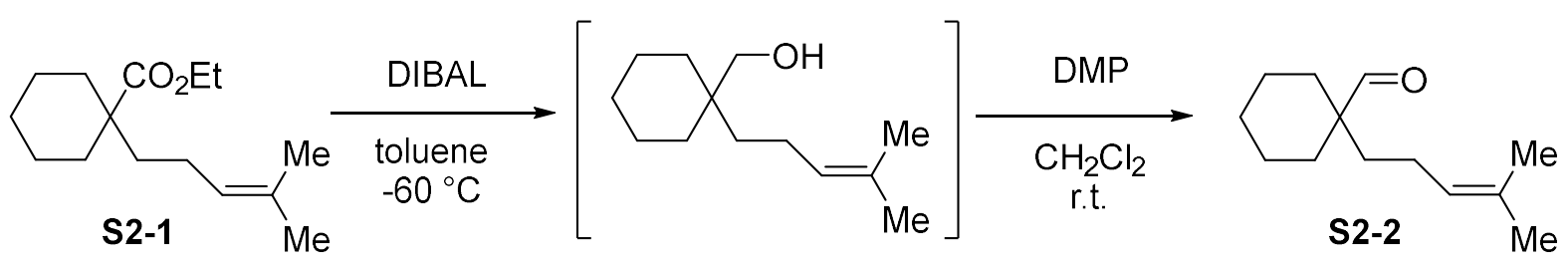

Solution of ethyl 1-(4-methylpent-3-en-1-yl)cyclohexane-1-carboxylate S2-1 (300 mg, 1.26 $\mathrm{mmol})$ in toluene $(10 \mathrm{~mL})$ was cooled to $-60^{\circ} \mathrm{C}$ and a $1.0 \mathrm{M}$ solution of DIBAL-H in toluene $(2.52$ $\mathrm{mL} ; 2.52 \mathrm{mmol}$ ) was added. The solution was stirred for $30 \mathrm{~min}$ at $-78{ }^{\circ} \mathrm{C}$ and then warmed to the room temperature. The reaction was diluted with EtOAc $(50 \mathrm{~mL})$ and quenched by successive addition of $\mathrm{MeOH}(2 \mathrm{~mL})$ and a saturated aqueous solution of potassium sodium tartrate. The mixture was stirred for $30 \mathrm{~min}$, the organic layer was separated, filtered through a short pad of silica gel and concentrated in vacuo. The residue (used immediately in the next step, without further purification) was dissolved in $\mathrm{CH}_{2} \mathrm{Cl}_{2}(25 \mathrm{~mL})$ and Dess-Martin periodinane $(1.06 \mathrm{~g}, 2.52$ mmol) was added. The reaction mixture was stirred at room temperature for $30 \mathrm{~min}$, after which time it was quenched with $20 \%$ aq. $\mathrm{Na}_{2} \mathrm{~S}_{2} \mathrm{O}_{3}(10 \mathrm{~mL})$ and a saturated aqueous solution $\mathrm{NaHCO}_{3}(8$ $\mathrm{mL})$. The mixture was stirred until the aqueous layer was clear. The aqueous layer was extracted

\footnotetext{
${ }^{5}$ Compound S2-1 was prepared according to the procedure: M. Trajkovic, Z. Ferjancic, R. N. Saicic, F. Bihelovic.
} Chem. Eur. J. 2019, 25, 4340-4344 
with $\mathrm{CH}_{2} \mathrm{Cl}_{2}(3 \times 10 \mathrm{~mL})$. The combined organic extract was dried over anh. $\mathrm{MgSO}_{4}$ and concentrated. The residue was purified by dry-flash chromatography $\left(\mathrm{SiO}_{2}\right.$; eluent: petroleumether/ethyl acetate $=95: 5)$ to give $162 \mathrm{mg},(66 \%)$ of 1-(4-methylpent-3-en-1-yl)cyclohexane-1carbaldehyde (S2-2), as colorless oil.

${ }^{1} \mathrm{H}$ NMR $\left(500 \mathrm{MHz}, \mathrm{CDCl}_{3}\right) \delta 9.42(\mathrm{~s}, 1 \mathrm{H}), 5.02(\mathrm{t}, J=7.1 \mathrm{~Hz}, 1 \mathrm{H}), 1.91-1.81(\mathrm{~m}, 4 \mathrm{H}), 1.65(\mathrm{~s}$, $3 \mathrm{H}), 1.61-1.50(\mathrm{~m}, 3 \mathrm{H}), 1.55(\mathrm{~s}, 3 \mathrm{H}), 1.47-1.41(\mathrm{~m}, 2 \mathrm{H}), 1.34-1.26(\mathrm{~m}, 6 \mathrm{H})$.

$9.44(\mathrm{~s}, 1 \mathrm{H}), 5.09-4.99(\mathrm{~m}, 1 \mathrm{H}), 1.94-1.84(\mathrm{~m}, 4 \mathrm{H}), 1.67(\mathrm{~s}, 3 \mathrm{H}), 1.62-1.53(\mathrm{~m}, 3 \mathrm{H}), 1.57(\mathrm{~s}$, $3 \mathrm{H}), 1.49-1.44(\mathrm{~m}, 2 \mathrm{H}), 1.34-1.26(\mathrm{~m}, 5 \mathrm{H})$.

${ }^{13}$ C NMR $\left(125 \mathrm{MHz}, \mathrm{CDCl}_{3}\right) \delta 207.2,132.3,124.0,49.8,36.7,31.1(2 \mathrm{x} \mathrm{C}), 26.0,25.8,22.7(2 \mathrm{x}$ C), 22.4, 17.7 .

IR (ATR) $v_{\max } 3391,2932,2858,1699,1452,1380,1260,1131,1072,901$.

\section{1-(1-(4-Methylpent-3-en-1-yl)cyclohexyl)prop-2-yn-1-ol (S2-3)}

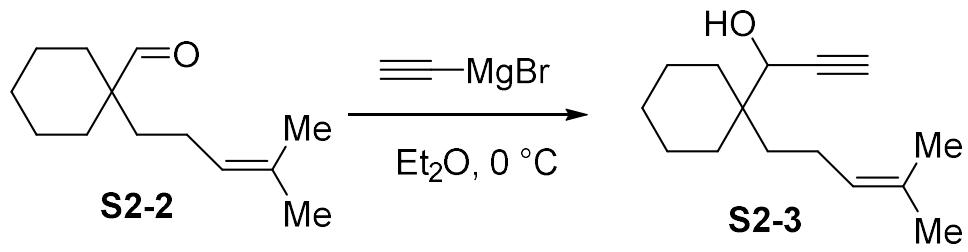

A solution of ethynylmagnesium bromide ( $0.5 \mathrm{M}$ in THF, $4 \mathrm{~mL}, 2 \mathrm{mmol})$ was added to a solution of aldehyde S2-2 $(221 \mathrm{mg}, 1.0 \mathrm{mmol})$ in $\mathrm{Et}_{2} \mathrm{O}(15 \mathrm{~mL})$, at $0{ }^{\circ} \mathrm{C}$, and then warmed to room temperature, under an argon atmosphere. After $20 \mathrm{~min}, 2 \%$ aqueous solution of $\mathrm{HCl}$ was added, and the mixture was extracted with EtOAc $(2 \times 25 \mathrm{~mL})$. The combined organic extract was washed with $\mathrm{H}_{2} \mathrm{O}$ and brine, dried over anh. $\mathrm{MgSO}_{4}$, filtered and concentrated. The residue was purified by dry-flash chromatography $\left(\mathrm{SiO}_{2}\right.$; eluent: petroleum-ether/ethyl acetate $\left.=9: 1\right)$ to give 1-(1-(4methylpent-3-en-1-yl)cyclohexyl)prop-2-yn-1-ol (S2-3; $155 \mathrm{mg}, 62 \%)$, as colorless oil.

${ }^{1} \mathbf{H}$ NMR $\left(500 \mathrm{MHz}, \mathrm{CDCl}_{3}\right) \delta 5.17-5.10(\mathrm{~m}, 1 \mathrm{H}), 4.32(\mathrm{~d}, J=4.1 \mathrm{~Hz}, 1 \mathrm{H}), 2.47(\mathrm{~d}, J=2.2 \mathrm{~Hz}$, $1 \mathrm{H}), 1.95\left(\mathrm{dd}, J^{l}=16.2, J^{2}=8.0 \mathrm{~Hz}, 2 \mathrm{H}\right), 1.69(\mathrm{~s}, 3 \mathrm{H}), 1.62(\mathrm{~s}, 3 \mathrm{H}), 1.58-1.41(\mathrm{~m}, 12 \mathrm{H}), 1.29$ $-1.24(\mathrm{~m}, 1 \mathrm{H})$.

$5.17-5.10(\mathrm{~m}, 1 \mathrm{H}), 4.32(\mathrm{~d}, J=2.3 \mathrm{~Hz}, 1 \mathrm{H}), 2.47$ (d, $J=2.3, \mathrm{~Hz}, 1 \mathrm{H}), 1.99-1.91(\mathrm{~m}, 2 \mathrm{H}), 1.69$ (s, 3H), $1.62(\mathrm{~s}, 3 \mathrm{H}), 1.57-1.41(\mathrm{~m}, 10 \mathrm{H}), 1.35-1.20(\mathrm{~m}, 2 \mathrm{H})$.

${ }^{13} \mathrm{C}$ NMR $\left(125 \mathrm{MHz}, \mathrm{CDCl}_{3}\right) \delta 131.6,125.1,83.6,74.6,68.4,40.1,32.3,30.8,30.1,26.2,25.8$, 22.0, 21.5, 21.4, 17.7 .

IR (ATR) $v_{\max }$ 3424, 3307, 2929, 2860, 1670, 1454, 1039.

HRMS (ESI) calcd. for $\mathrm{C}_{15} \mathrm{H}_{25} \mathrm{O}^{+}[\mathrm{M}+\mathrm{H}]^{+}:$221.1900, found: 221.1903 . 


\section{1-Hydroxy-1-(1-(4-methylpent-3-en-1-yl)cyclohexyl)propan-2-one (S2-4)}

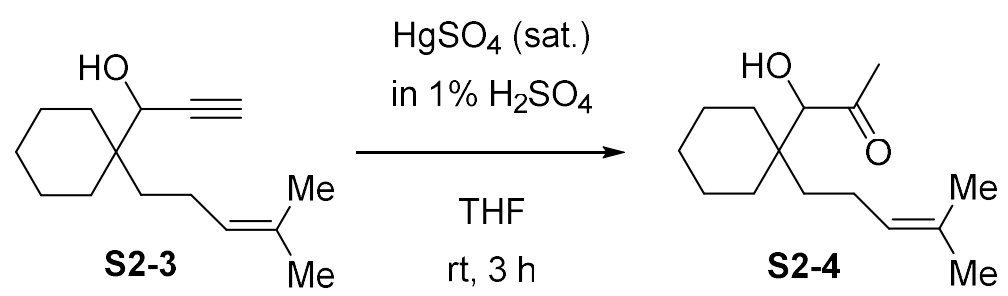

Propargylic alcohol S2-3 (200 mg, $0.91 \mathrm{mmol})$ was added to a solution containing of THF (1.8 $\mathrm{ml})$, water, $(0.2 \mathrm{ml})$ and $\mathrm{HgSO}_{4}\left(2.0 \mathrm{ml}\right.$ of a saturated solution in $\left.1 \% \mathrm{H}_{2} \mathrm{SO}_{4}\right)$. The reaction mixture was stirred at room temperature for $3 \mathrm{~h}$. The progress of the reaction was monitored by TLC. On completion of the reaction, the mixture was diluted with water, neutralized with saturated aqueous solution of $\mathrm{NaHCO}_{3}$, and extracted with diethyl ether. The extracts were dried over anh. $\mathrm{MgSO}_{4}$ and concentrated under reduced pressure. The residue was purified by dry-flash chromatography (eluent: petroleum-ether/ethyl acetate $=9 / 1)$ to give 1-hydroxy-1-(1-(4-methylpent-3-en-1yl)cyclohexyl)propan-2-one (S2-4; $126 \mathrm{mg}, 58 \%$ ), as colorless oil.

${ }^{1} \mathbf{H}$ NMR $\left(500 \mathrm{MHz}, \mathrm{CDCl}_{3}\right) \delta 5.06\left(\mathrm{dddd}, J^{1}=7.1, J^{2}=5.9, J^{3}=2.7, J^{4}=1.4 \mathrm{~Hz}, 1 \mathrm{H}\right), 4.15(\mathrm{~d}$, $J=5.3 \mathrm{~Hz}, 1 \mathrm{H}), 3.19(\mathrm{~d}, J=6.0 \mathrm{~Hz}, 1 \mathrm{H}), 2.26(\mathrm{~s}, 3 \mathrm{H}), 1.99-1.84(\mathrm{~m}, 2 \mathrm{H}), 1.66(\mathrm{~d}, J=0.7 \mathrm{~Hz}$, $3 \mathrm{H}), 1.59(\mathrm{~s}, 3 \mathrm{H}), 1.57-1.37(\mathrm{~m}, 10 \mathrm{H}), 1.29\left(\mathrm{ddd}, J^{1}=14.4, J^{2}=12.0, J^{3}=5.2 \mathrm{~Hz}, 2 \mathrm{H}\right)$.

${ }^{13} \mathrm{C}$ NMR $\left(125 \mathrm{MHz}, \mathrm{CDCl}_{3}\right) \delta 211.9,131.6,124.7,81.6,41.1,32.8,31.93,31.8,29.8$ 26.1, 25.8, $22.2,21.5,21.5,17.7$.

IR (ATR) $v_{\max }: 3470,2928,1701,1610,1455,1354,1072$.

HRMS (ESI) calcd. for $\mathrm{C}_{15} \mathrm{H}_{27} \mathrm{O}_{2}^{+}[\mathrm{M}+\mathrm{H}]^{+}:$239.2006, found: 239.2008

\section{1-(1-(4-Methylpent-3-en-1-yl)ciclohexyl)-2-oxopropyl methanesulfonate (3)}

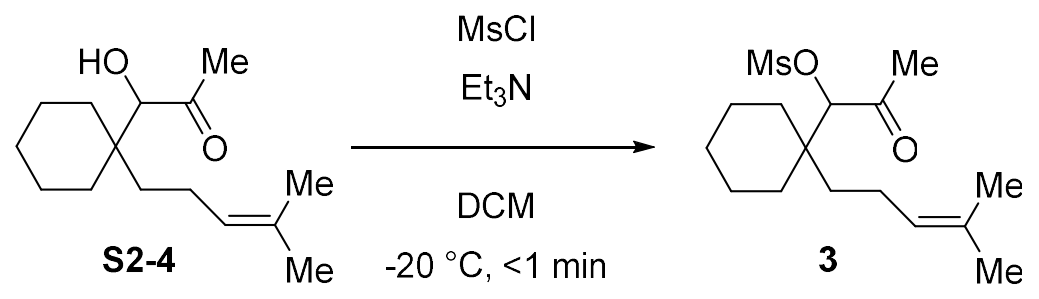

Mesyl chloride $(66.5 \mu \mathrm{L}, 0.86 \mathrm{mmol})$ was added to a solution of hydroxy ketone $\mathbf{S 2 - 4}$ (102 $\mathrm{mg}$, $0.43 \mathrm{mmol})$, triethylamine $(180 \mu \mathrm{L}, 1.29 \mathrm{mmol})$ and a few crystals of DMAP in $\mathrm{CH}_{2} \mathrm{Cl}_{2}(3 \mathrm{~mL})$ at $-30{ }^{\circ} \mathrm{C}$ and the reaction was completed almost immediately. The reaction mixture was diluted with $\mathrm{CH}_{2} \mathrm{Cl}_{2}(20 \mathrm{~mL})$, filtered through a pad of silica gel and concentrated in vacuo. Since mesylate 3 
was prone to decomposition, the crude product was used immediately in the cyclization step, without further purification.

\section{Scheme S3: Preparation of precursor 5}
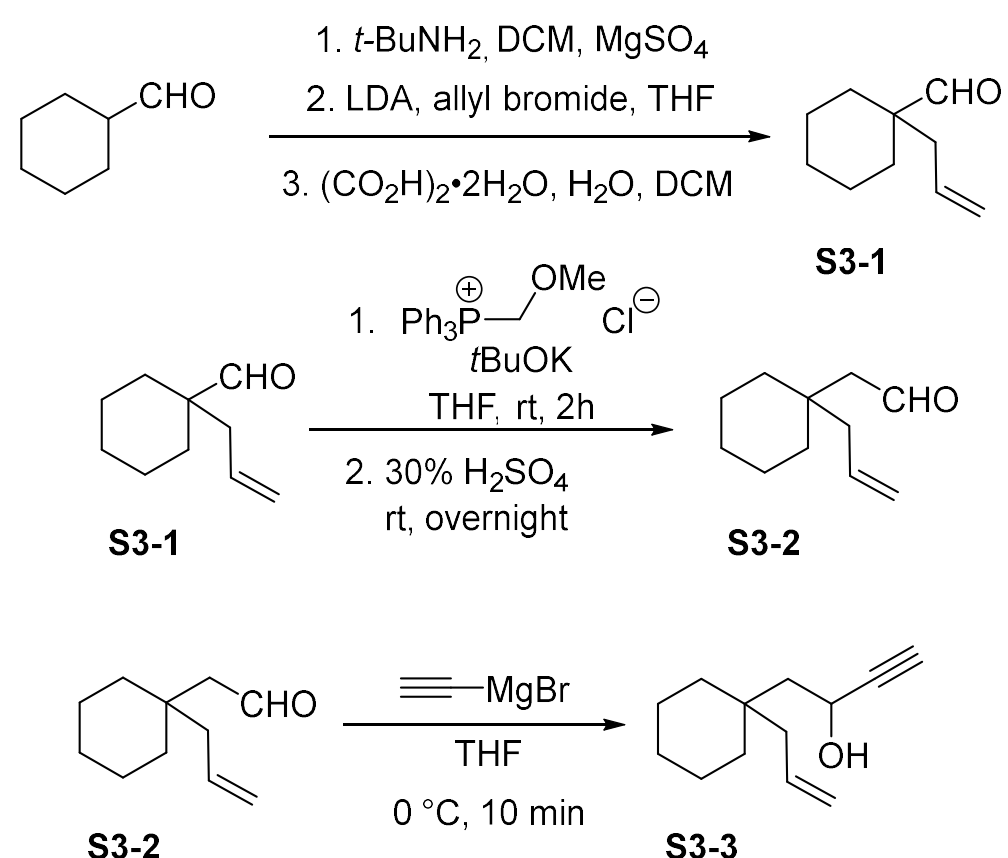

S3-2<smiles>C=CCC1(CC(O)C(C)=O)CCCCC1</smiles>

S3-3

$\mathrm{rt}$, overnight

S3-4<smiles>C=CCC1(CC(O)C(C)=O)CCCCC1(CC=C)CC1(CC)CCCCC1</smiles>

S3-4 $-20^{\circ} \mathrm{C}, 15 \mathrm{~min}$

S3-5<smiles>C=CCCCCCCCC(OC)C(C)=O</smiles>

S3-5

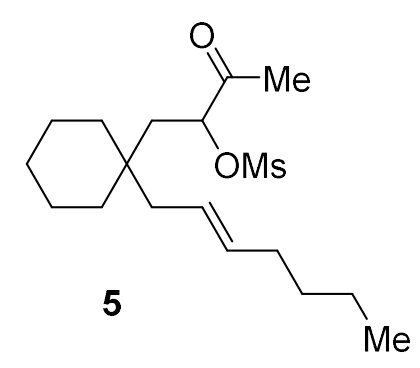




\section{1-Allylcyclohexanecarbaldehyde (S3-1) ${ }^{6}$}

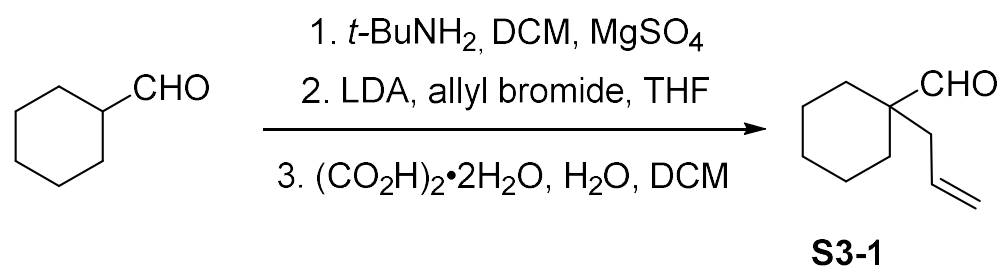

A suspension of anh. $\mathrm{MgSO}_{4}(10 \mathrm{~g}, 83 \mathrm{mmol})$, cyclohexane carbaldehyde $(6 \mathrm{~mL}, 50 \mathrm{mmol})$ and $t$ $\mathrm{BuNH}_{2}(5.6 \mathrm{~mL}, 53 \mathrm{mmol})$ in dry $\mathrm{CH}_{2} \mathrm{Cl}_{2}(50 \mathrm{~mL})$ was refluxed for $1 \mathrm{~h}$, then cooled to room temperature, filtered under reduced pressure, and concentrated at rotavap to give $7.1 \mathrm{~g}(86 \%)$ of the crude aldimine that was used immediately in the next step.

To an ice cold solution of diisopropylamine $(8.1 \mathrm{~mL}, 58 \mathrm{mmol})$ in THF $(60 \mathrm{~mL})$ under argon, $n$ BuLi (35 mL, $1.49 \mathrm{M}, 52 \mathrm{mmol}$ ) was added dropwise, and after $15 \mathrm{~min}$ of stirring at that temperature a solution of crude aldimine $(7.1 \mathrm{~g}, 42 \mathrm{mmol})$ in THF $(10 \mathrm{~mL})$ was added. After 3.5 $\mathrm{h}$ of stirring at $0{ }^{\circ} \mathrm{C}$, a solution of allyl bromide $(4.6 \mathrm{~mL}, 53 \mathrm{mmol})$ in THF $(10 \mathrm{~mL})$ was added, and stirring continued overnight, allowing the reaction mixture to warm up to room temperature. After quenching with $\mathrm{H}_{2} \mathrm{O}(10 \mathrm{~mL})$, reaction mixture was poured in $\mathrm{H}_{2} \mathrm{O}(240 \mathrm{~mL})$ and extracted with $\mathrm{Et}_{2} \mathrm{O}(3 \times 120 \mathrm{~mL})$. The combined organic extract was dried over $\mathrm{K}_{2} \mathrm{CO}_{3}$, filtered and concentrated at rotavap to give $8.8 \mathrm{~g}$ of the crude allylated aldimine.

A solution of crude allylated aldimine $(8.8 \mathrm{~g})$ and $(\mathrm{COOH})_{2} \cdot \mathrm{H}_{2} \mathrm{O}(6.3 \mathrm{~g})$ in $\mathrm{CH}_{2} \mathrm{Cl}_{2}(100 \mathrm{~mL})$ and $\mathrm{H}_{2} \mathrm{O}(60 \mathrm{~mL})$ was vigorously stirred under reflux for $1 \mathrm{~h}$. The organic layer was separated, the water layer was extracted with $\mathrm{CH}_{2} \mathrm{Cl}_{2}(2 \times 20 \mathrm{~mL})$, and the combined organic extract was dried over anh. $\mathrm{MgSO}_{4}$ and concentrated in vacuo. The residue was distilled under reduced pressure to give $3.7 \mathrm{~g}$ (50\% overall) of 1-allylcyclohexanecarbaldehyde (S3-1) as a clear colorless liquid (bp. 90-100 $\left.{ }^{\circ} \mathrm{C} / 7 \mathrm{~mm} \mathrm{Hg}\right)$. NMR spectra consistent with literature. ${ }^{7}$

${ }^{1} \mathbf{H}$ NMR $\left(200 \mathrm{MHz} \mathrm{CDCl}_{3}\right) \delta 9.43(\mathrm{~s}, 1 \mathrm{H}), 5.80-5.50(\mathrm{~m}, 1 \mathrm{H}), 5.12-4.90(\mathrm{~m}, 2 \mathrm{H}), 2.16\left(\mathrm{dt}, J^{l}\right.$ $\left.=7.4, J^{2}=1.0 \mathrm{~Hz}, 2 \mathrm{H}\right), 1.94-1.76(\mathrm{~m}, 2 \mathrm{H}), 1.66-1.43(\mathrm{~m}, 2 \mathrm{H}), 1.41-1.14(\mathrm{~m}, 6 \mathrm{H})$.

${ }^{13} \mathrm{C}$ NMR $\left(50 \mathrm{MHz}, \mathrm{CDCl}_{3}\right) \delta 207.1,132.1,118.4,49.7,40.9,30.8,25.7,22.5$.

\footnotetext{
${ }^{6}$ (a) According to the published procedure: De Kimpe, N; De Smaele, D; Hofkens, A; Dejaegher, Y; Kesteleyn, B. Tetrahedron, 1997, 53, 10803-10816. (b) A published procedure that did not work in our hands: Ibrahem, I; Cordova, A. Angew. Chem. Int. Ed. 2006, 45, 1952-1956.

${ }^{7}$ Cozzi, P. G.; Benfatti, F.; Capdevila, M. G.; Mignogna, A. Chem Comm.. 2008, 3317-3318.
} 


\section{2-(1-Allylcyclohexyl)acetaldehyde (S3-2) ${ }^{8}$}

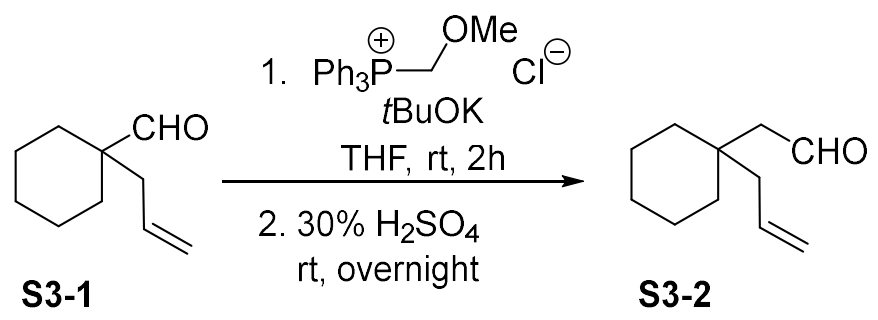

A solution of $t$-BuOK (344 mg, $3.1 \mathrm{mmol})$ in THF (5 mL) was added dropwise, with stirring, to an ice-cold suspension of (methoxymethyl)triphenylphosphonium chloride (1.05 g, $3.1 \mathrm{mmol})$ in dry THF ( $4 \mathrm{~mL})$, under an argon atmosphere. The resulting cherry-red solution was stirred at $0{ }^{\circ} \mathrm{C}$ for $1 \mathrm{~h}$, when a solution of 1-allylcyclohexanecarbaldehyde S3-1 (310 mg, $2.0 \mathrm{mmol})$ in dry THF $(2 \mathrm{~mL})$ was added at $0{ }^{\circ} \mathrm{C}$, and stirring was continued at room temperature for $2 \mathrm{~h}$. Water $(2.3 \mathrm{~mL})$ was added, the reaction mixture was concentrated under reduced pressure to approximately $5 \mathrm{~mL}$, when $30 \% \mathrm{H}_{2} \mathrm{SO}_{4}(1 \mathrm{~mL})$ was added and the mixture was stirred overnight at room temperature. Saturated $\mathrm{NaHCO}_{3}$ solution $(2.8 \mathrm{~mL})$ was added to reaction mixture, followed by extraction with diethyl ether $(3 \times 5 \mathrm{~mL})$. The combined organic extract was washed with water $(5 \mathrm{~mL})$, dried over anh. $\mathrm{MgSO}_{4}$ and carefully concentrated at rotavap (ca. $20 \mathrm{~mm} \mathrm{Hg} / 34{ }^{\circ} \mathrm{C}$ bath temperature). The residue was purified by column chromatography (petroleum-ether/ethyl acetate $=95 / 5$ ) to afford $262 \mathrm{mg}$ (77\%) of 2-(1-allylcyclohexyl)acetaldehyde (S3-2) as a clear liquid. ${ }^{9}$

${ }^{1} \mathbf{H}$ NMR $\left(200 \mathrm{MHz}, \mathrm{CDCl}_{3}\right) \delta 9.86\left(\mathrm{td}, J^{1}=3.1, J^{2}=0.6 \mathrm{~Hz}, 1 \mathrm{H}\right), 5.97-5.66(\mathrm{~m}, 1 \mathrm{H}), 5.22-$ $4.95(\mathrm{~m}, 2 \mathrm{H}), 2.34(\mathrm{~d}, J=3.1 \mathrm{~Hz}, 2 \mathrm{H}), 2.22(\mathrm{~d}, J=7.5 \mathrm{~Hz}, 2 \mathrm{H}), 1.46(\mathrm{~s}, 10 \mathrm{H})$.

${ }^{13} \mathrm{C}$ NMR $\left(50 \mathrm{MHz}, \mathrm{CDCl}_{3}\right) \delta 203.9,134.1,118.4,50.7,42.7,36.9,36.0,26.1,21.6$.

\section{1-(1-Allylcyclohexyl)but-3-yn-2-ol (S3-3)}

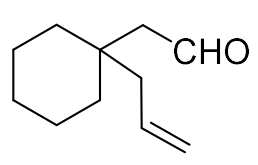

S3-2

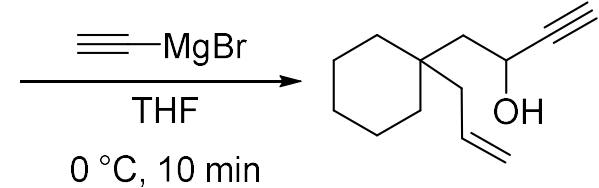

S3-3

To an ice-cold solution of 2-(1-allylcyclohexyl)acetaldehyde S3-2 (262 mg, $1.57 \mathrm{mmol}$ ) in THF $(10 \mathrm{~mL})$, ethynylmagnesium bromide solution $(7 \mathrm{~mL}, 0.5 \mathrm{M}$ in $\mathrm{THF}, 3.5 \mathrm{mmol})$ was added dropwise via syringe, under an argon atmosphere. After $10 \mathrm{~min}$ of stirring TLC indicated complete consumption of the starting material. The reaction was quenched by addition of $\mathrm{NH}_{4} \mathrm{Cl}_{\text {(sat.) }}(42$

\footnotetext{
${ }^{8}$ Following the procedure from: Clavette, C; Rocan, J-F. V; Beauchemin, A. M. Angew. Chem. Int. Ed. 2013, 52, $12705-12708$.

${ }^{9}$ Known compound: Bai, Y.; Davis, D. C.; Dai, M. Angew. Chem. Int. Ed. 2014, 53, 6519-6522.
} 
$\mathrm{mL})$ and extracted with EtOAc $(3 \times 30 \mathrm{~mL})$. Combined organic extract was washed with brine $(30$ $\mathrm{mL}$ ), dried over anh. $\mathrm{MgSO}_{4}$ and concentrated under reduced pressure. The residue was purified by column chromatography (petroleum-ether/ethyl acetate $=8 / 1)$ to afford $249 \mathrm{mg}(82 \%)$ of 1 - $(1$ allylcyclohexyl)but-3-yn-2-ol (S3-3), as a pale yellow oil.

${ }^{1} \mathbf{H}$ NMR $\left(200 \mathrm{MHz}, \mathrm{CDCl}_{3}\right) \delta 5.83\left(\mathrm{ddt}, J^{1}=15.0, J^{2}=11.6, J^{3}=7.4 \mathrm{~Hz}, 1 \mathrm{H}\right), 5.12-4.96(\mathrm{~m}$, $2 \mathrm{H}), 4.51\left(\mathrm{qd}, J^{l}=6.3, J^{2}=2.1 \mathrm{~Hz}, 1 \mathrm{H}\right), 2.48(\mathrm{~d}, J=2.1 \mathrm{~Hz}, 1 \mathrm{H}), 2.17(\mathrm{~d}, J=7.4 \mathrm{~Hz}, 2 \mathrm{H}), 1.92-$ $1.83(\mathrm{~m}, 1 \mathrm{H}), 1.77\left(\mathrm{dd}, J^{1}=6.4, J^{2}=1.6 \mathrm{~Hz}, 2 \mathrm{H}\right), 1.51-1.32(\mathrm{~m}, 10 \mathrm{H})$.

${ }^{13} \mathrm{C}$ NMR $\left(50 \mathrm{MHz}, \mathrm{CDCl}_{3}\right) \delta 135.1,117.5,86.5,72.9,72.8,59.2,45.3,42.0,36.1,35.7,35.4$, 26.3, 21.7 .

IR (ATR) $v_{\max }: 3306,2927,2856,1639,1455,1006,914$.

\section{4-(1-Allylcyclohexyl)-3-hydroxybutan-2-one (S3-4)}

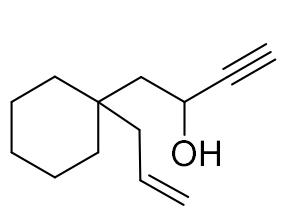

S3-3

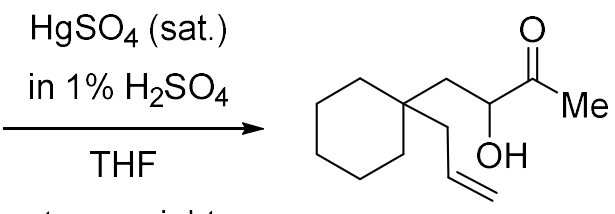

S3-4

Water (11.6 mL) was added to a solution of 1-(1-allylcyclohexyl)but-3-yn-2-ol S3-3 (246 mg, 1.28 mmol) in THF (27.6 mL), followed by dropwise addition of saturated $\mathrm{HgSO}_{4}$ solution in $1 \% \mathrm{H}_{2} \mathrm{SO}_{4}$ $(2.77 \mathrm{~mL})$. After $16 \mathrm{~h}$ of stirring at room temperature, the reaction mixture was poured into brine $(130 \mathrm{~mL})$ and extracted with $\mathrm{Et}_{2} \mathrm{O}(3 \times 40 \mathrm{~mL})$. The combined organic extract was dried over anh. $\mathrm{MgSO}_{4}$ and concentrated at rotavap. The residue was purified by column chromatography (petroleum-ether/ethyl acetate $=6 / 1$ ) to afford $213 \mathrm{mg}(79 \%)$ of hydroxy ketone S3-4, as a colorless oil.

${ }^{1} \mathbf{H}$ NMR $\left(500 \mathrm{MHz}, \mathrm{CDCl}_{3}\right) \delta 5.92-5.80(\mathrm{~m}, 1 \mathrm{H}), 5.13-5.03(\mathrm{~m}, 2 \mathrm{H}), 4.27\left(\mathrm{ddd}, J^{1}=9.9, J^{2}=\right.$ $\left.5.3, J^{3}=1.7 \mathrm{~Hz}, 1 \mathrm{H}\right), 3.40(\mathrm{~d}, J=5.3 \mathrm{~Hz}, 1 \mathrm{H}), 2.32\left(\mathrm{dd}, J^{1}=14.1, J^{2}=6.6 \mathrm{~Hz}, 1 \mathrm{H}\right), 2.24-2.15$ $(\mathrm{m}, 1 \mathrm{H}), 2.19(\mathrm{~s}, 3 \mathrm{H}) 1.65\left(\mathrm{dd}, J^{1}=14.7, J^{2}=1.7 \mathrm{~Hz}, 1 \mathrm{H}\right), 1.51-1.31(\mathrm{~m}, 11 \mathrm{H})$.

${ }^{13} \mathrm{C}$ NMR $\left(126 \mathrm{MHz}, \mathrm{CDCl}_{3}\right) \delta 210.8,135.2,117.5,74.8,41.8,41.2,36.1,36.0(2 \times \mathrm{C}), 26.3,25.3$, $21.8(2 \times \mathrm{C})$.

IR (ATR) $v_{\max }: 3471,2927,2857,1714,1639,1454,1358,1097$. 


\section{1-(1-Allylcyclohexyl)-3-oxobutan-2-yl methanesulfonate (S3-5)}<smiles>C=CCC1(CC(O)C(C)=O)CCCCC1</smiles>

S3-4

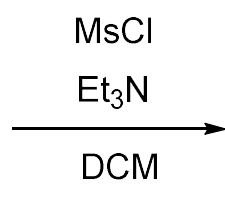

$-20^{\circ} \mathrm{C}, 15 \mathrm{~min}$<smiles>C=CCC1(CC(C(C)=O)C(C)=O)CCCCC1</smiles>

S3-5

According to G.P.1: $\mathrm{MsCl}(25 \mu \mathrm{L}, 0.323 \mathrm{mmol})$ was added to a cold $\left(-20{ }^{\circ} \mathrm{C}\right)$ solution of 4-(1allylcyclohexyl)-3-hydroxybutan-2-one S3-4 (38 mg, $0.120 \mathrm{mmol}$ ), DMAP (1 mg, $0.008 \mathrm{mmol})$ and $\mathrm{Et}_{3} \mathrm{~N}(70 \mu \mathrm{L}, 0.502 \mathrm{mmol})$. After $10 \mathrm{~min}$ TLC indicated complete conversion. $\mathrm{SiO}_{2}(200 \mathrm{mg})$ was added to the reaction mixture and the volatiles were removed under reduced pressure. The residue was purified by column chromatography (petroleum-ether/ethyl acetate $=9 / 1$ ) to afford $36.8 \mathrm{mg}$ (71\%) of 1-(1-allylcyclohexyl)-3-oxobutan-2-yl methanesulfonate (S3-5), as colorless oil.

${ }^{1} \mathbf{H}$ NMR $\left(500 \mathrm{MHz}, \mathrm{CDCl}_{3}\right) \delta 5.88-5.74(\mathrm{~m}, 1 \mathrm{H}), 5.21-5.03(\mathrm{~m}, 3 \mathrm{H}), 3.12(\mathrm{~s}, 3 \mathrm{H}), 2.26\left(\mathrm{dd}, J^{1}\right.$ $\left.=14.4, J^{2}=6.7 \mathrm{~Hz}, 1 \mathrm{H}\right), 2.22(\mathrm{~s}, 3 \mathrm{H}), 2.17\left(\mathrm{dd}, J^{1}=14.9, J^{2}=8.7 \mathrm{~Hz}, 1 \mathrm{H}\right), 1.77\left(\mathrm{dd}, J^{l}=5.6, J^{2}\right.$ $=9.6 \mathrm{~Hz}, 1 \mathrm{H}), 1.59\left(\mathrm{dd}, J^{l}=15.8, J^{2}=1.7 \mathrm{~Hz}, 2 \mathrm{H}\right), 1.52-1.33(\mathrm{~m}, 10 \mathrm{H})$.

${ }^{13} \mathrm{C}$ NMR $\left(126 \mathrm{MHz}, \mathrm{CDCl}_{3}\right) \delta 204.4,134.1,118.4,81.4,41.4,39.4,37.6,35.8(2 \times \mathrm{C}), 35.7,26.1$, 25.9, $21.6(2 \times \mathrm{C})$.

IR (ATR) $v_{\max }: 2928,2857,1733,1638,1352,1175,951,914,882,795$.

HRMS (ESI) calcd. for $\mathrm{C}_{14} \mathrm{H}_{28} \mathrm{O}_{4} \mathrm{SN}^{+}[\mathrm{M}+\mathrm{NH} 4]^{+}$: 306.1734; found: 306.1727 .

\section{(E)-1-(1-(Hept-2-enyl)cyclohexyl)-3-oxobutan-2-yl methanesulfonate (5)}

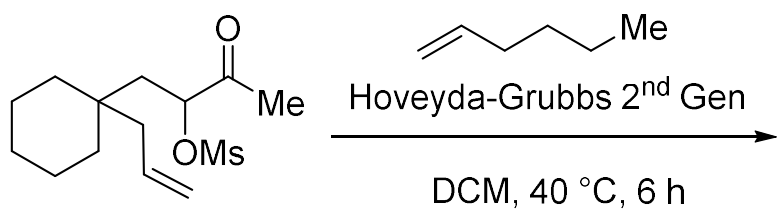

S3-5

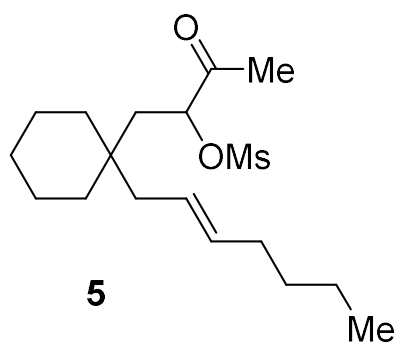

A solution of 1-(1-allylcyclohexyl)-3-oxobutan-2-yl methanesulfonate $\mathbf{S 3 - 5}$ (28.6 mg, $0.1 \mathrm{mmol}$ ), 1-hexene $(25 \mu \mathrm{L}, 0.2 \mathrm{mmol})$ and and Hoveyda-Grubbs $2^{\text {nd }}$ generation catalyst $(2.0 \mathrm{mg}, 0.003$ $\mathrm{mmol})$ in dry $\mathrm{CH}_{2} \mathrm{Cl}_{2}(1 \mathrm{~mL})$ was stirred overnight at room temperature under an argon atmosphere. TLC showed slow reaction, and more Hoveyda-Grubbs $2^{\text {nd }}$ generation catalyst $(2.0$ $\mathrm{mg}, 0.033 \mathrm{mmol})$ and 1 -hexene $(25 \mu \mathrm{L}, 0.2 \mathrm{mmol})$ were added, and the reaction mixture was heated to reflux. After $3 \mathrm{~h}$ of reflux, one more portion of Hoveyda-Grubbs $2^{\text {nd }}$ generation catalyst $(2.0$ $\mathrm{mg}, 0.033 \mathrm{mmol})$ and 1-hexene $(25 \mu \mathrm{L}, 0.2 \mathrm{mmol})$ was added and the reflux was continued for 3 
h more. $\mathrm{SiO}_{2}(120 \mathrm{mg})$ was added to the reaction mixture and volatiles were removed under reduced pressure. The residue was purified by column chromatography (petroleum-ether/ethyl acetate $=9 / 1)$ to afford $18.5 \mathrm{mg}(54 \%)$ of $(E)-1$-(1-(hept-2-enyl)cyclohexyl)-3-oxobutan-2-yl methanesulfonate (5), as a yellow oil. Mixture of $E$ and $Z$ isomers in a relative ratio 6.7:1, according to ${ }^{1} \mathrm{H}$ NMR spectrum integration of [-CHOMs-] signals at $5.11\left(\mathrm{dd}, J^{l}=9.5, J^{2}=2.0\right.$ $\mathrm{Hz}, 1 \mathrm{H}_{\text {major }}$ ) and $5.07\left(\mathrm{dd}, J^{l}=9.6, J^{2}=2.0 \mathrm{~Hz}, 1 \mathrm{H}_{\text {minor }}\right.$.

NMR data for the major $(E)$-isomer

${ }^{1} \mathbf{H}$ NMR $\left(500 \mathrm{MHz}, \mathrm{CDCl}_{3}\right) \delta 5.53-5.46(\mathrm{~m}, 1 \mathrm{H}), 5.41-5.33(\mathrm{~m}, 1 \mathrm{H}), 5.11\left(\mathrm{dd}, J^{l}=9.5, J^{2}=\right.$ $2.0 \mathrm{~Hz}, 1 \mathrm{H}), 3.11(\mathrm{~s}, 3 \mathrm{H}), 2.20(\mathrm{~s}, 3 \mathrm{H}), 2.17\left(\mathrm{dd}, J^{l}=14.6, J^{2}=6.7 \mathrm{~Hz}, 1 \mathrm{H}\right), 2.08\left(\mathrm{dd}, J^{l}=14.5\right.$, $\left.J^{2}=7.8 \mathrm{~Hz}, 1 \mathrm{H}\right), 2.01(\mathrm{q}, J=6.6 \mathrm{~Hz}, 2 \mathrm{H}), 1.75\left(\mathrm{dd}, J^{l}=15.5, J^{2}=9.6 \mathrm{~Hz}, 1 \mathrm{H}\right), 1.57\left(\mathrm{dd}, J^{l}=\right.$ $\left.15.5, J^{2}=2.0 \mathrm{~Hz}, 1 \mathrm{H}\right), 1.52-1.27(\mathrm{~m}, 14 \mathrm{H}), 0.88(\mathrm{t}, J=7.1 \mathrm{~Hz}, 3 \mathrm{H})$.

${ }^{13} \mathbf{C}$ NMR $\left(126 \mathrm{MHz}, \mathrm{CDCl}_{3}\right) \delta 204.4,134.6,125.0,81.6,40.0,39.3,37.6$ 35.9, 35.8, 35.6, 32.6, $31.8,26.1,25.8,22.3,21.7,21.6,14.0$.

IR (ATR) $v_{\text {max }}: 2928,2857,1733,1456,1355,1177,972,944$.

HRMS (ESI) calcd. for $\mathrm{C}_{18} \mathrm{H}_{36} \mathrm{O}_{4} \mathrm{SN}^{+}\left[\mathrm{M}+\mathrm{NH}_{4}\right]^{+}: 362.2360$; found: 362.2358 . 


\section{Scheme S4: Preparation of precursor 7}

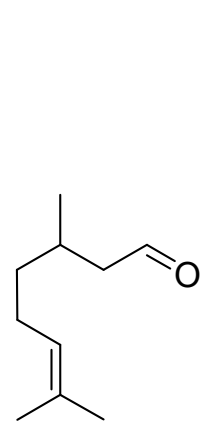

$( \pm)$-citronellal

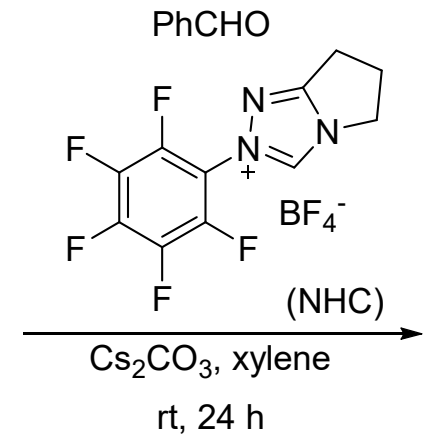

$\mathrm{rt}, 24 \mathrm{~h}$<smiles>CC(C)=CCCC(C)CC(=O)C(O)c1ccccc1</smiles>

S4-1

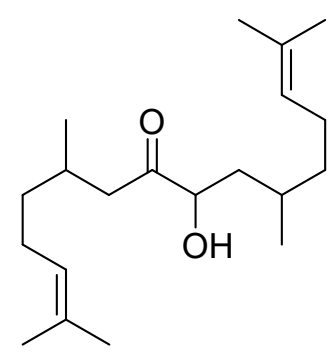

S4-2<smiles>CC(C)=CCCC(C)CC(=O)C(O)c1ccccc1</smiles>

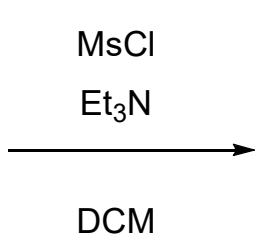<smiles>COC(C(=O)CC(C)CCC=C(C)C)c1ccccc1</smiles>

S4-1

7<smiles>CC(C)=CCC[C@H](C)CC=O</smiles><smiles>N#CC1CCCCC1</smiles>

rt, $24 \mathrm{~h}$

(S)-(-)-citronellal<smiles>CC(C)=CCC[C@@H](C)CC(=O)C(O)c1ccccc1</smiles>

S4-1*<smiles>CC(C)=CCC[C@@H](C)CC(=O)C(O)c1ccccc1</smiles><smiles>CCCCCCN(C)CC</smiles>

S4-1*<smiles>COC(C(=O)C[C@@H](C)CCC=C(C)C)c1ccccc1</smiles>

$7^{*}$ 
General procedure 2. (G.P.2.) - cross-acyloin condensation

1-Hydroxy-4,8-dimethyl-1-phenylnon-7-en-2-one (S4-1) tetramethylhexadeca-2,14-dien-8-one (S4-2) ${ }^{10}$

and 9-hydroxy-2,6,11,15-

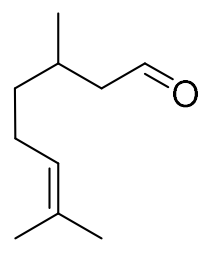

( \pm -citronellal

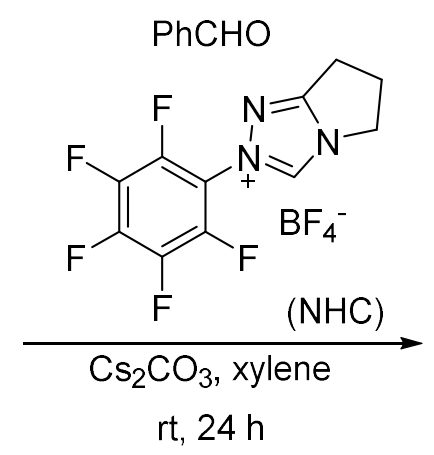

$\mathrm{rt}, 24 \mathrm{~h}$

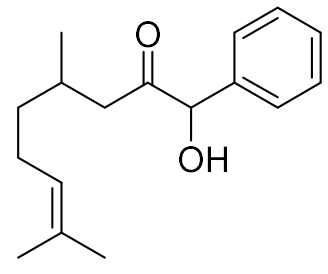

S4-1

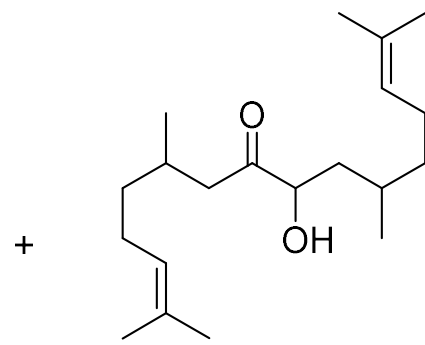

S4-2

A suspension of anhydrous $\mathrm{Cs}_{2} \mathrm{CO}_{3}(16 \mathrm{mg}, 0.049 \mathrm{mmol}, 0.1 \mathrm{eq}$ ), benzaldehyde (50 $\mu \mathrm{L}, 0.49$ mmol, $1 \mathrm{eq}), 3,7$-dimethyloct-6-enal $(0.322 \mathrm{~mL}, 2.45 \mathrm{mmol}, 5 \mathrm{eq})$ and $N$-heterocyclic carbene precatalyst NHC (17.8 mg, $0.049 \mathrm{mmol}, 0.1 \mathrm{eq})$ in dry xylene $(1 \mathrm{~mL})$ was stirred at room temperature for $24 \mathrm{~h}$. The reaction mixture was then quenched with distilled water and extracted with ethyl acetate. The combined organic extract was washed with brine, dried over anh. $\mathrm{MgSO}_{4}$ and concentrated in vacuo. Dry flash chromatography of the residue (petroleum-ether/ethyl acetate $=95 / 5$ ), followed by column chromatography (benzene/ethyl acetate $=95 / 5$ ) afforded $40.4 \mathrm{mg}$ (33\%) of hydroxy ketone $\mathbf{S 4 - 1}$, as a colorless oil (mixture of two diastereoisomers in a relative ratio 1.5:1, as determined by ${ }^{1} \mathrm{H}$ NMR), and $64.0 \mathrm{mg}$ (16\%) of citronellal dimer $\mathbf{S 4 - 2}$ as a colorless oil (mixture of diastereoisomers, ratio not determined).

\section{S4-1}

Spectral data for a mixture of isomers, where $\mathrm{H}$ and $\mathrm{H}^{\prime}$ correspond to the isomers in a ratio 1.5:1

${ }^{1} \mathbf{H}$ NMR $\left(500 \mathrm{MHz}, \mathrm{CDCl}_{3}\right) \delta 7.40-7.28\left(\mathrm{~m}, 5 \mathrm{H}+5 \mathrm{H}^{\prime}\right), 5.08-5.01(\mathrm{~m}, 1 \mathrm{H}+2 \mathrm{H}$ '), $4.97-4.91$ (m, 1H), 4.39 (d, $J=4.3 \mathrm{~Hz}, 1 \mathrm{H}$ ), 4.39 (d, $\left.J=4.5 \mathrm{~Hz}, 1 \mathrm{H}^{\prime}\right), 2.37$ (dd, $\left.J=16.3,5.4 \mathrm{~Hz}, 1 \mathrm{H}^{\prime}\right), 2.26$ (dd, $J=16.4,6.0 \mathrm{~Hz}, 1 \mathrm{H}), 2.20$ (dd, $J=16.4,7.7 \mathrm{~Hz}, 1 \mathrm{H}), 2.13$ (dd, $\left.J=16.3,8.3 \mathrm{~Hz}, 1 \mathrm{H}^{\prime}\right), 2.05$ $-1.75\left(\mathrm{~m}, 3 \mathrm{H}+3 \mathrm{H}^{\prime}\right), 1.67$ (d, $\left.J=1.0 \mathrm{~Hz}, 3 \mathrm{H}^{\prime}\right), 1.67$ (d, $\left.J=1 \mathrm{~Hz}, 3 \mathrm{H}\right), 1.57$ (s, 3H'), $1.52(\mathrm{~s}, 3 \mathrm{H})$, $1.28-1.20\left(\mathrm{~m}, 1 \mathrm{H}^{\prime}\right), 1.19-1.10\left(\mathrm{~m}, 1 \mathrm{H}+1 \mathrm{H}^{\prime}\right), 1.08-0.99(\mathrm{~m}, 1 \mathrm{H}), 0.86(\mathrm{~d}, J=6.7 \mathrm{~Hz}, 3 \mathrm{H}), 0.69$ (d, $\left.J=6.6 \mathrm{~Hz}, 3 \mathrm{H}^{\prime}\right)$.

${ }^{13}$ C NMR $\left(125 \mathrm{MHz}, \mathrm{CDCl}_{3}\right) \delta 209.3,209.2,138.0,137.9,128.9,128.6,127.5,124.0,80.2,79.8$, 45.1, 45.0, 36.8, 36.6, 29.0, 28.9, 25.6, 25.4, 19.6, 19.4, 17.6, 17.6.

IR (ATR) $v_{\max }: 3462,2963,2921,2854,1714,1454,1379$.

HRMS (ESI) calcd. for $\mathrm{C}_{17} \mathrm{H}_{24} \mathrm{O}_{2} \mathrm{Na}^{+}[\mathrm{M}+\mathrm{Na}]^{+}: 283.1668$, found: 283.1679 .

${ }^{10}$ Jin, M. Y., Kim, S. M., Mao, H., Ryu, D. H., Song C. E., Yang, J. W., Org. Biomol. Chem., 2014, 12, 1547-1550. 


\section{S4-2}

Spectral data for a mixture of isomers

${ }^{1} \mathbf{H}$ NMR $\left(500 \mathrm{MHz}, \mathrm{CDCl}_{3}\right) 5.15-5.05(\mathrm{~m}, 2 \mathrm{H}), 4.20-4.11(\mathrm{~m}, 1 \mathrm{H}), 3.46-3.39(\mathrm{~m}, 1 \mathrm{H}), 2.48$ $-2.39(\mathrm{~m}, 1 \mathrm{H}), 2.33-2.22(\mathrm{~m}, 1 \mathrm{H}), 2.13-1.90(\mathrm{~m}, 5 \mathrm{H}), 1.86-1.76(\mathrm{~m}, 1 \mathrm{H}), 1.72-1.66(\mathrm{~m}$, $6 \mathrm{H}), 1.63-1.58(\mathrm{~m}, 6 \mathrm{H}), 1.46-1.39(\mathrm{~m}, 1 \mathrm{H}), 1.36-1.12(\mathrm{~m}, 5 \mathrm{H}), 1.02-0.94(\mathrm{~m}, 3 \mathrm{H}), 0.93-$ $0.89(\mathrm{~m}, 3 \mathrm{H})$.

${ }^{13} \mathrm{C}$ NMR $\left(125 \mathrm{MHz}, \mathrm{CDCl}_{3}\right) \delta 212.7(2 \times \mathrm{C}), 212.6,131.7,131.4,124.6,124.5,124.1(2 \times \mathrm{C}), 75.7$, $75.3,75.1,74.8,45.3(2 \times \mathrm{C}), 45.2,41.3,41.2,41.1,41.0,38.0,36.9,35.7,29.5,29.1,29.0(2 \times \mathrm{C})$, $28.9,25.7,25.5,25.4(2 \times \mathrm{C}), 25.3,20.4,19.9,19.7,18.5,17.7$.

IR (ATR) $v_{\max }: 3481,2962,2922,2854,1709,1454,1378$.

HRMS (ESI) calcd. for $\mathrm{C}_{20} \mathrm{H}_{36} \mathrm{O}_{2} \mathrm{Na}^{+}[\mathrm{M}+\mathrm{Na}]^{+}: 331.2607$, found: 331.2604 .

\section{4,8-Dimethyl-2-oxo-1-phenylnon-7-enyl methanesulfonate (7)}

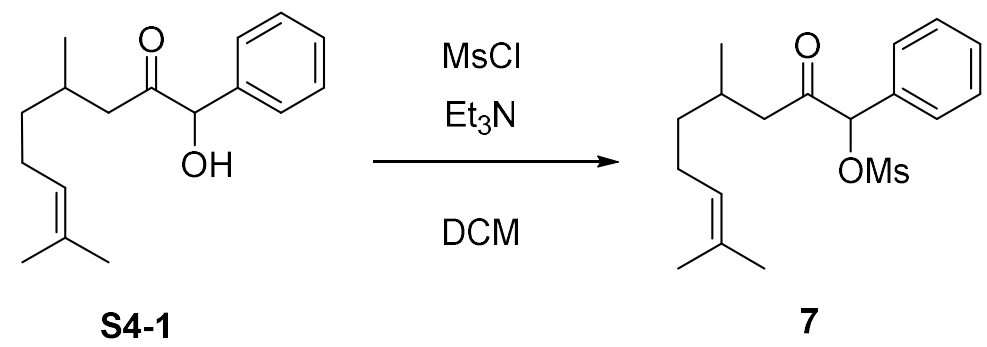

Prepared according G.P.1, using S4-1 (8.3 mg, $0.032 \mathrm{mmol}, 1 \mathrm{eq})$, mesyl chloride (7.4 $\mu \mathrm{L}, 0.095$ mmol, $3 \mathrm{eq})$, triethylamine $(22 \mu \mathrm{L}, 0.159 \mathrm{mmol}, 5 \mathrm{eq})$, a few crystals of DMAP, and $\mathrm{CH}_{2} \mathrm{Cl}_{2}(0.2$ $\mathrm{mL})$. Column chromatography (petroleum-ether/ethyl acetate $=85 / 15)$ afforded $8.7 \mathrm{mg}(81 \%)$ of mesylate 7 , as a colorless oil (mixture of isomers in a relative ratio $1.5: 1$, as determined by ${ }^{1} \mathrm{H}$ NMR).

Spectral data for a mixture of isomers, where $\mathrm{H}$ and $\mathrm{H}^{\prime}$ correspond to the isomers in a ratio $\mathrm{H} / \mathrm{H}^{\prime}=$ 1.5: 1

${ }^{1} \mathbf{H}$ NMR $\left(500 \mathrm{MHz}, \mathrm{CDCl}_{3}\right) \delta 7.45-7.37$ (m, 5H+5H'), $5.90\left(\mathrm{~s}, 1 \mathrm{H}^{\prime}\right), 5.88(\mathrm{~s}, 1 \mathrm{H}), 5.06-4.96$ (m, 1H+1H'), $3.04\left(\mathrm{~s}, 3 \mathrm{H}+3 \mathrm{H}^{\prime}\right), 2.46\left(\mathrm{dd}, J^{l}=17.0,5.2 \mathrm{~Hz}, 1 \mathrm{H}^{\prime}\right), 2.38(\mathrm{dd}, J=17.0,5.8 \mathrm{~Hz}, 1 \mathrm{H})$, 2.30 (dd, $J=17.0,7.7 \mathrm{~Hz}, 1 \mathrm{H}), 2.23$ (dd, $\left.J=17.0,8.4 \mathrm{~Hz}, 1 \mathrm{H}^{\prime}\right), 2.07-1.80$ (m, 3H+3H'), 1.67 $\left(\mathrm{d}, J=1.0 \mathrm{~Hz}, 3 \mathrm{H}^{\prime}\right), 1.65(\mathrm{~d}, J=1.0 \mathrm{~Hz}, 3 \mathrm{H}), 1.56\left(\mathrm{~s}, 3 \mathrm{H}^{\prime}\right), 1.54(\mathrm{~s}, 3 \mathrm{H}), 1.29-1.04\left(\mathrm{~m}, 2 \mathrm{H}+2 \mathrm{H}^{\prime}\right)$, $0.85(\mathrm{~d}, J=6.7 \mathrm{~Hz}, 3 \mathrm{H}), 0.76\left(\mathrm{~d}, J=6.6 \mathrm{~Hz}, 3 \mathrm{H}^{\prime}\right)$.

${ }^{13} \mathrm{C}$ NMR (125 MHz, $\left.\mathrm{CDCl}_{3}\right) \delta$ 202.8, 202.6, 132.2, 132.1, 131.7, 131.6, 130.1, 130.0, 129.3, 128.2, 128.1, 124.0, 85.7, 85.5, 45.9, 39.4, 36.7, 36.5, 28.4, 28.3, 25.7, 25.3, 25.2, 19.5, 19.4, 17.6. IR (ATR) $v_{\max }: 2958,2917,2852,1732,1455,1367$.

HRMS (ESI) calcd. for $\mathrm{C}_{18} \mathrm{H}_{26} \mathrm{O}_{4} \mathrm{SK}[\mathrm{M}+\mathrm{K}]^{+}: 377.1183$, found: 377.1174 . 

dimethyl-1-phenylnon-7-en-2-one (S4-1*)

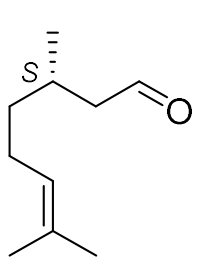

(S)-(-)-citronellal

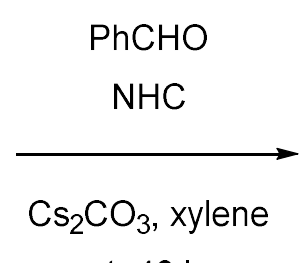

$\mathrm{rt}, 40 \mathrm{~h}$<smiles>CC(C)=CCCCC(=O)C(O)c1ccccc1</smiles>

S4-1*

Prepared by G.P.2, using (S)-3,7-dimethyloct-6-enal (1.01 g, $6.5 \mathrm{mmol}, 10$ eq), benzaldehyde (66 $\mu \mathrm{L}, 0.65 \mathrm{mmol}, 1 \mathrm{eq}), \mathbf{N H C}$ (23.6 mg, $0.065 \mathrm{mmol}, 0.1 \mathrm{eq}$ ), cesium carbonate (21 mg, $0.065 \mathrm{mmol}$, $0.1 \mathrm{eq})$ and xylene $(1.3 \mathrm{~mL})$, during $40 \mathrm{~h}$. Purification by dry-flash chromatography (petroleumether/ethyl acetate $=95 / 5$ ) and two consecutive column chromatographies (benzene/ethyl acetate $=95 / 5$ and petroleum-ether/ethyl acetate $=9 / 1)$ afforded $76.3 \mathrm{mg}(45 \%)$ of a mixture of isomers S4-1* (ratio 1:1 as determined by ${ }^{1} \mathrm{H}$ NMR), as a colorless oil.

Spectral data for a mixture of isomers in a ratio $\mathrm{H}: \mathrm{H}^{\prime}=2: 1$

${ }^{1} \mathbf{H}$ NMR $\left(500 \mathrm{MHz}, \mathrm{CDCl}_{3}\right) \delta 7.40-7.28\left(\mathrm{~m}, 5 \mathrm{H}+5 \mathrm{H}^{\prime}\right), 5.08-5.01\left(\mathrm{~m}, 1 \mathrm{H}+2 \mathrm{H}^{\prime}\right), 4.97-4.91$ (m, 1H), 4.39 (d, $J=4.3 \mathrm{~Hz}, 1 \mathrm{H}), 4.39$ (d, $\left.J=4.5 \mathrm{~Hz}, 1 \mathrm{H}^{\prime}\right), 2.37$ (dd, $\left.J=16.3,5.4 \mathrm{~Hz}, 1 \mathrm{H}^{\prime}\right), 2.26$ $(\mathrm{dd}, J=16.4,6.0 \mathrm{~Hz}, 1 \mathrm{H}), 2.20$ (dd, $J=16.4,7.7 \mathrm{~Hz}, 1 \mathrm{H}), 2.13$ (dd, $\left.J=16.3,8.3 \mathrm{~Hz}, 1 \mathrm{H}^{\prime}\right), 2.05$ $-1.75\left(\mathrm{~m}, 3 \mathrm{H}+3 \mathrm{H}^{\prime}\right), 1.67\left(\mathrm{~d}, J=1.0 \mathrm{~Hz}, 3 \mathrm{H}^{\prime}\right), 1.67$ (d, J=1 Hz, 3H), 1.57 (s, 3H'), 1.52 (s, 3H), $1.28-1.20\left(\mathrm{~m}, 1 \mathrm{H}^{\prime}\right), 1.19-1.10\left(\mathrm{~m}, 1 \mathrm{H}+1 \mathrm{H}^{\prime}\right), 1.08-0.99(\mathrm{~m}, 1 \mathrm{H}), 0.86(\mathrm{~d}, J=6.7 \mathrm{~Hz}, 3 \mathrm{H}), 0.69$ (d, $\left.J=6.6 \mathrm{~Hz}, 3 \mathrm{H}^{\prime}\right)$.

${ }^{13} \mathrm{C}$ NMR $\left(125 \mathrm{MHz}, \mathrm{CDCl}_{3}\right) \delta 209.3,209.2,138.0,131.6,128.9,128.7,127.5,124.0,80.2,79.8$, 45.1, 45.0, 36.8, 36.6, 29.0, 28.9, 25.7, 25.6, 25.4, 25.2, 19.6, 19.4, 17.6, 17.6.

IR (ATR) v $v_{\max }:$ 3460, 3062, 3030, 2963, 2919, 2729, 1712, 1600, 1493, 1453, 1378, 1287.

HRMS (ESI) calcd. for $\mathrm{C}_{17} \mathrm{H}_{24} \mathrm{O}_{2} \mathrm{Na}^{+}[\mathrm{M}+\mathrm{Na}]^{+}:$283.1668, found: 283.1679 . 
$(1 S, 4 S)-4,8$-Dimethyl-2-oxo-1-phenylnon-7-enyl methanesulfonate and $(1 R, 4 S)-4,8-$ dimethyl-2-oxo-1-phenylnon-7-enyl methanesulfonate $(7 *)$

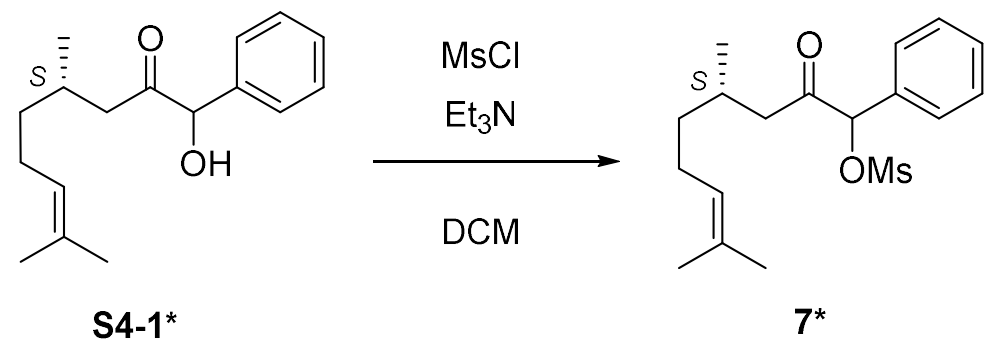

Prepared by G.P.1, using S4-1* (57 mg, 0.22 mmol, 1 eq), mesyl chloride (34 $\mu \mathrm{L}, 0.44$ mmol, 2 eq), triethylamine ( $92 \mu \mathrm{L}, 0.66 \mathrm{mmol}, 3 \mathrm{eq})$, DMAP (2.7 mg. $0.022 \mathrm{mmol}, 0.1 \mathrm{eq})$ and $\mathrm{CH}_{2} \mathrm{Cl}_{2}(1.1$ $\mathrm{mL}$ ). Column chromatography (petroleum-ether/acetone $=85 / 15)$ afforded $65.9 \mathrm{mg}(88 \%)$ of $7 *$, as a colorless oil (mixture of two isomers in a relative ratio 1:1).

Spectral data for mixture, where $\mathrm{H}$ and $\mathrm{H}^{\prime}$ correspond to isomers in a relative ratio 1:1

${ }^{1} \mathbf{H}$ NMR $\left(500 \mathrm{MHz}, \mathrm{CDCl}_{3}\right) \delta 7.45-7.37$ (m, 5H+5H'), 5.90 (s, 1H'), $5.88(\mathrm{~s}, 1 \mathrm{H}), 5.05-4.96$ (m, 1H+1H'), 3.04 (s, 3H+3H'), 2.46 (dd, $\left.J=16.9,5.2 \mathrm{~Hz}, 1 \mathrm{H}^{\prime}\right), 2.38$ (dd, $\left.J=17.0,5.8 \mathrm{~Hz}, 1 \mathrm{H}\right)$, 2.30 (dd, $J=17.0,7.7 \mathrm{~Hz}, 1 \mathrm{H}), 2.23$ (dd, $\left.J=17.0,8.4 \mathrm{~Hz}, 1 \mathrm{H}^{\prime}\right), 2.06-1.80$ (m, 3H+3H'), 1.66 $\left(\mathrm{d}, J=0.7 \mathrm{~Hz}, 3 \mathrm{H}^{\prime}\right), 1.65(\mathrm{~s}, 3 \mathrm{H}), 1.56\left(\mathrm{~s}, 3 \mathrm{H}^{\prime}\right), 1.54(\mathrm{~s}, 3 \mathrm{H}), 1.29-1.04\left(\mathrm{~m}, 2 \mathrm{H}+2 \mathrm{H}^{\prime}\right), 0.85$ (d, $J$ $=6.7 \mathrm{~Hz}, 3 \mathrm{H}), 0.76\left(\mathrm{~d}, J=6.6 \mathrm{~Hz}, 3 \mathrm{H}^{\prime}\right)$.

${ }^{13} \mathrm{C}$ NMR $\left(125 \mathrm{MHz}, \mathrm{CDCl}_{3}\right) \delta 202.8,202.6,132.3,132.2,131.7,131.6,130.1,130.1,129.3$, $128.2,128.1,124.1,85.7,85.5,45.9,39.5,39.5,36.7,36.6,28.5,28.3,25.7,25.4,25.3,19.6,19.4$, 17.6 .

IR (ATR) $v_{\max }:$ 3032, 2963, 2921, 1732, 1495, 1454, 1409, 1361.

HRMS (ESI) calcd. for $\mathrm{C}_{18} \mathrm{H}_{26} \mathrm{O}_{4} \mathrm{SNa}^{+}[\mathrm{M}+\mathrm{Na}]^{+}: 361.1444$, found: 361.1432 . 
Scheme S5: Preparation of precursor $10 E$
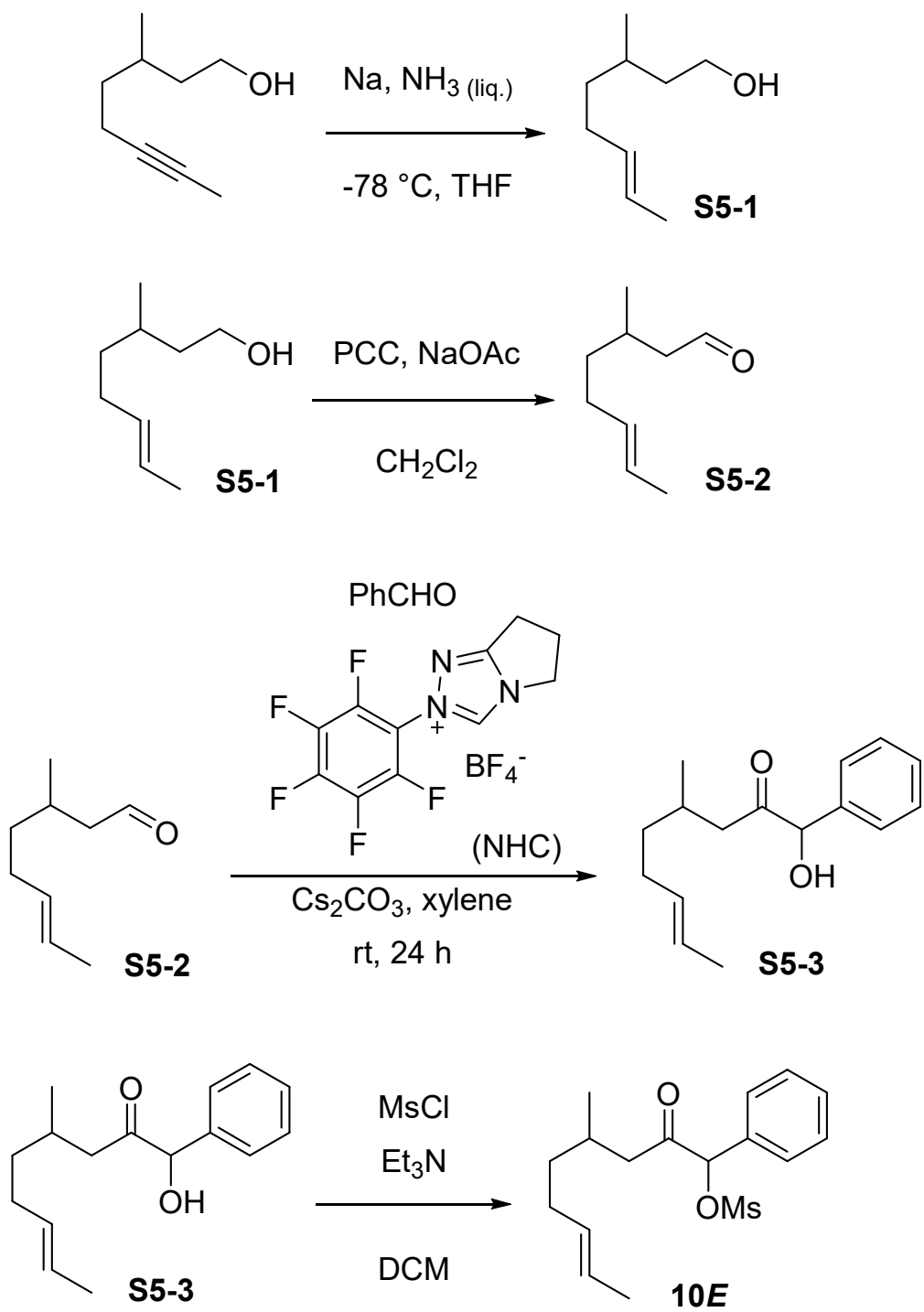

(E)-3-Methyloct-6-en-1-ol (S5-1)

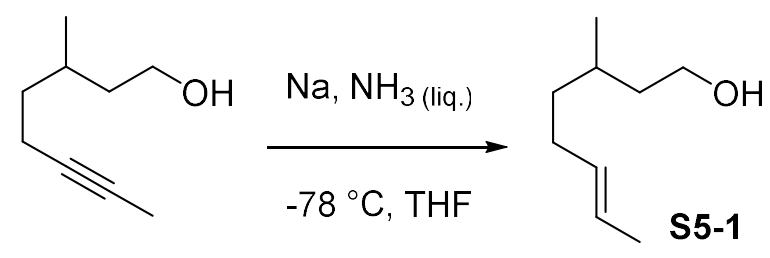

Sodium (5.3 g, $0.23 \mathrm{~mol}, 10.7 \mathrm{eq})$ was dissolved in liquid ammonia $(350 \mathrm{~mL})$ at $-78{ }^{\circ} \mathrm{C}$ and 3methyloct-6-yn-1-ol ${ }^{11}(3 \mathrm{~g}, 0.0214 \mathrm{~mol}, 1 \mathrm{eq})$ in THF $(10 \mathrm{~mL})$ was added to the resulting blue

${ }^{11}$ Gao, P., Xu, P. F.,Zhai, H., J. Org. Chem. 2009, 74, 2592-2593. 
solution. The reaction mixture was stirred at $-78{ }^{\circ} \mathrm{C}$ for $6 \mathrm{~h}$, then warmed to room temperature and stirred until all ammonia has evaporated. The residual solid was carefully quenched with sat. $\mathrm{NH}_{4} \mathrm{Cl}$ solution, extracted with ethyl acetate, washed with water, dried over anh. $\mathrm{MgSO}_{4}$, and concentrated in vacuo. Purification of the residue by dry-flash chromatography (petroleumether/ethyl acetate $=8 / 2$ ), followed by column chromatography (the same eluent) afforded $704 \mathrm{mg}$ $(23 \%)$ of alkene S5-1, as a colorless oil.

${ }^{1} \mathbf{H}$ NMR $\left(500 \mathrm{MHz}, \mathrm{CDCl}_{3}\right) 5.46-5.36(\mathrm{~m}, 2 \mathrm{H}), 3.71-3.60(\mathrm{~m}, 2 \mathrm{H}), 2.07-1.90(\mathrm{~m}, 3 \mathrm{H}), 1.66$ $-1.53(\mathrm{~m}, 5 \mathrm{H}), 1.42-1.33(\mathrm{~m}, 2 \mathrm{H}), 1.24-1.15(\mathrm{~m}, 1 \mathrm{H}), 0.89(\mathrm{~d}, J=6.6 \mathrm{~Hz}, 3 \mathrm{H})$.

${ }^{13}$ C NMR $\left(125 \mathrm{MHz}, \mathrm{CDCl}_{3}\right) \delta 131.4,124.6,60.9,39.7,36.9,29.9,28.9,19.4,17.8$.

IR (ATR) $v_{\max }: 3348,3020,2924,1730,1455,1376,1176$.

HRMS (ESI) calcd. for $\mathrm{C}_{9} \mathrm{H}_{19} \mathrm{O}^{+}[\mathrm{M}+\mathrm{H}]^{+}:$143.1430, found: 143.1425 .

\section{(E)-3-Methyloct-6-enal (S5-2)}

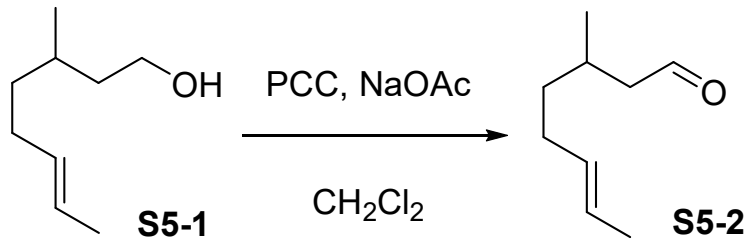

Alcohol S5-1 (608 mg, $4.27 \mathrm{mmol}, 1 \mathrm{eq}$ ) was added to the suspension of anhydrous sodium acetate (111 mg, $1.345 \mathrm{mmol}, 0.315 \mathrm{eq})$ and PCC (1.47 g, $6.84 \mathrm{mmol}, 1.6 \mathrm{eq})$ in dry $\mathrm{CH}_{2} \mathrm{Cl}_{2}(6 \mathrm{~mL})$, and the mixture was stirred for $1.5 \mathrm{~h}$. Diethyl ether $(70 \mathrm{~mL})$ was added to the reaction mixture and the suspension was filtered through Celite. The resulting filtrate was washed with sat. $\mathrm{NaHCO}_{3}$ solution, brine, dried over anh. $\mathrm{MgSO}_{4}$ and concentrated in vacuo. Purification of the residue by dry flash chromatography (petroleum-ether/ethyl acetate $=975 / 25$ ), followed by column chromatography (with the same eluent) afforded $315 \mathrm{mg}$ (53\%) of aldehyde S5-2, as a volatile colorless liquid.

${ }^{1}$ H NMR $\left(500 \mathrm{MHz}, \mathrm{CDCl}_{3}\right) 9.76-9.74(\mathrm{~m}, 1 \mathrm{H}), 5.48-5.35(\mathrm{~m}, 2 \mathrm{H}), 2.40\left(\mathrm{ddd}, J^{1}=16.1, J^{2}=\right.$ $\left.5.6, J^{3}=2.0 \mathrm{~Hz}, 1 \mathrm{H}\right), 2.23\left(\mathrm{ddd}, J^{l}=16.1, J^{2}=7.9, J^{3}=2.6 \mathrm{~Hz}, 1 \mathrm{H}\right), 2.12-1.93(\mathrm{~m}, 3 \mathrm{H}), 1.66-$ $1.62(\mathrm{~m}, 3 \mathrm{H}), 1.43-1.32(\mathrm{~m}, 1 \mathrm{H}), 1.33-1.25(\mathrm{~m}, 1 \mathrm{H}), 0.96(\mathrm{~d}, J=6.7 \mathrm{~Hz}, 3 \mathrm{H})$.

${ }^{13}$ C NMR $\left(125 \mathrm{MHz}, \mathrm{CDCl}_{3}\right) \delta 203.6,131.5,125.9,51.6,37.4,30.6,28.3,20.5,18.6$.

IR (ATR) $v_{\max }: 3020,2959,2880,2853,2715,1726,1456,1410,1380$.

HRMS (ESI) calcd. for $\mathrm{C}_{16} \mathrm{H}_{26} \mathrm{O}_{3}{ }^{+}[\mathrm{M}+\mathrm{Na}]^{+}:$289.1774, found: 289.1774 . 


\section{(E)-1-Hydroxy-4-methyl-1-phenylnon-7-en-2-one (S5-3)}

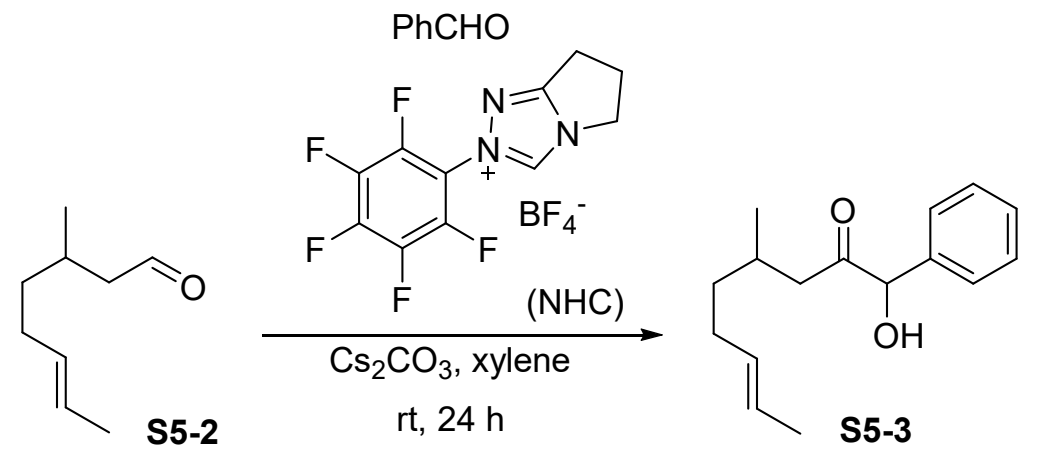

Prepared by G.P.2 using S5-2 (250 mg, $1.78 \mathrm{mmol}, 4$ eq), benzaldehyde (45 $\mu \mathrm{L}, 0.445 \mathrm{mmol}, 1$ eq), NHC (23 mg, $0.063 \mathrm{mmol}, 0.14 \mathrm{eq})$, cesium carbonate $(21.5 \mathrm{mg}, 0.066 \mathrm{mmol}, 0.15 \mathrm{eq})$ and xylene $(1 \mathrm{~mL})$ during $64 \mathrm{~h}$; dry-flash chromatography (petroleum-ether/ethyl acetate $=95 / 5$ ) followed by column chromatography (petroleum-ether/ethyl acetate 9/1) afforded $36.5 \mathrm{mg}(33 \%)$ of hydroxy ketone S5-3, as a colorless oil (mixture of isomers in a relative ratio 1.1:1, as determined by ${ }^{1} \mathrm{H}$ NMR),

Spectral data for a mixture of isomers in a ratio of $\mathrm{H}: \mathrm{H}^{\prime}=1.1: 1$

${ }^{1} \mathbf{H}$ NMR $\left(500 \mathrm{MHz}, \mathrm{CDCl}_{3}\right) \delta 7.39-7.28\left(\mathrm{~m}, 5 \mathrm{H}+5 \mathrm{H}^{\prime}\right), 5.43-5.23\left(\mathrm{~m}, 2 \mathrm{H}+2 \mathrm{H}^{\prime}\right), 5.05(\mathrm{~d}, J=$ $4.4 \mathrm{~Hz}, 1 \mathrm{H}), 5.02$ (d, $\left.J=4.3 \mathrm{~Hz}, 1 \mathrm{H}^{\prime}\right), 4.41$ (d, $\left.J=4.3 \mathrm{~Hz}, 1 \mathrm{H}\right), 4.40$ (d, J=4.5 Hz, 1H'), 2.36 (dd, $\left.J=16.3,5.5 \mathrm{~Hz}, 1 \mathrm{H}^{\prime}\right), 2.26(\mathrm{dd}, J=16.4,6.0 \mathrm{~Hz}, 1 \mathrm{H}), 2.19(\mathrm{dd}, J=16.4,7.7 \mathrm{~Hz}, 1 \mathrm{H}), 2.12(\mathrm{dd}, J$ $\left.=16.3,8.3 \mathrm{~Hz}, 1 \mathrm{H}^{\prime}\right), 2.05-1.75\left(\mathrm{~m}, 3 \mathrm{H}+3 \mathrm{H}^{\prime}\right), 1.65-1.61\left(\mathrm{~m}, 3 \mathrm{H}^{\prime}\right), 1.61-1.58(\mathrm{~m}, 3 \mathrm{H}), 1.30-$ $1.22\left(\mathrm{~m}, 1 \mathrm{H}^{\prime}\right), 1.22-1.12\left(\mathrm{~m}, 1 \mathrm{H}^{\prime}+1 \mathrm{H}\right), 1.09-1.00(\mathrm{~m}, 1 \mathrm{H}), 0.85(\mathrm{~d}, J=6.7 \mathrm{~Hz}, 3 \mathrm{H}), 0.68(\mathrm{~d}, J$ $\left.=6.6 \mathrm{~Hz}, 3 \mathrm{H}^{\prime}\right)$.

${ }^{13} \mathrm{C}$ NMR $\left(125 \mathrm{MHz}, \mathrm{CDCl}_{3}\right) \delta 209.5,209.4,138.2(2 \times \mathrm{C}), 131.0(2 \times \mathrm{C}), 129.2,128.9,127.7,125.3$, $125.2,80.5,80.0,45.3,45.2,36.8,36.5,29.9,29.0,19.8,19.6,18.1(2 \times \mathrm{C})$.

IR (ATR) $v_{\max }: 3464,3063,3026,2928,2853,1717$, 1600, 1494, 1455, 1362.

HRMS (ESI) calcd. for $\mathrm{C}_{16} \mathrm{H}_{23} \mathrm{O}_{2}^{+}[\mathrm{M}+\mathrm{H}]^{+}: 247.1693$, found: 247.1687 .

\section{(E)-4-Methyl-2-oxo-1-phenylnon-7-enyl methanesulfonate (10E)}

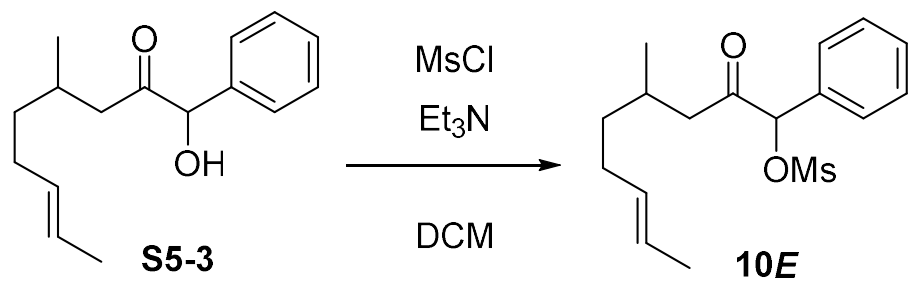


Prepared by G.P.1, using S5-3 (31.6 mg, 0.128 mmol, 1 eq), mesyl chloride (20 $\mu \mathrm{L}, 0.256$ mmol, 2 eq), triethylamine (53 $\mu \mathrm{L}, 0.384 \mathrm{mmol}, 3 \mathrm{eq})$, DMAP (1.64 mg, $0.0128 \mathrm{mmol}, 0.1 \mathrm{eq})$ and $\mathrm{CH}_{2} \mathrm{Cl}_{2}$ $(1 \mathrm{~mL})$. Dry flash chromatography (petroleum-ether/ethyl acetate $=85 / 15)$ afforded $36.7 \mathrm{mg}(88 \%)$ of mesylate $\mathbf{1 0 E}$ as a colorless oil (mixture of isomers, in a relative ratio 1.1:1, as determined by ${ }^{1} \mathrm{H}$ NMR).

Spectral data for a mixture of isomers where $\mathrm{H}$ and $\mathrm{H}^{\prime}$ correspond to the isomers in a ratio of $\mathrm{H}$ : $\mathrm{H}^{\prime}=1.1: 1$

${ }^{1} \mathbf{H}$ NMR $\left(500 \mathrm{MHz}, \mathrm{CDCl}_{3}\right) \delta 7.44-7.37\left(\mathrm{~m}, 5 \mathrm{H}+5 \mathrm{H}^{\prime}\right), 5.90\left(\mathrm{~s}, 1 \mathrm{H}^{\prime}\right), 5.87(\mathrm{~s}, 1 \mathrm{H}), 5.42-5.26$ (m, $\left.2 \mathrm{H}+2 \mathrm{H}^{\prime}\right), 3.04\left(\mathrm{~s}, 3 \mathrm{H}+3 \mathrm{H}^{\prime}\right), 2.45$ (dd, $\left.J=16.9,5.2 \mathrm{~Hz}, 1 \mathrm{H}^{\prime}\right), 2.38$ (dd, $\left.J=17.0,5.9 \mathrm{~Hz}, 1 \mathrm{H}\right)$, $2.30(\mathrm{dd}, J=17.0,7.7 \mathrm{~Hz}, 1 \mathrm{H}), 2.22\left(\mathrm{dd}, J=16.9,8.3 \mathrm{~Hz}, 1 \mathrm{H}^{\prime}\right), 2.06-1.80\left(\mathrm{~m}, 3 \mathrm{H}+3 \mathrm{H}^{\prime}\right), 1.64-$ $1.59\left(\mathrm{~m}, 3 \mathrm{H}^{\prime}+3 \mathrm{H}\right), 1.31-1.05\left(\mathrm{~m}, 2 \mathrm{H}^{\prime}+2 \mathrm{H}\right), 0.86-0.81(\mathrm{~m}, 3 \mathrm{H}), 0.75\left(\mathrm{~d}, J=6.6 \mathrm{~Hz}, 3 \mathrm{H}^{\prime}\right)$.

${ }^{13} \mathbf{C ~ N M R}\left(125 \mathrm{MHz}, \mathrm{CDCl}_{3}\right) \delta$ 202.3, 202.1, 131.8, 131.7, 130.4, $129.7(2 \times \mathrm{C}), 128.9,127.8$, 124.7, 124.6, 85.3, 85.0, 45.4, $39.1(2 \times \mathrm{C}), 36.0,35.8,29.4,29.3,27.9,27.8,19.1,18.9,17.5$.

IR (ATR) $v_{\max }:$ 3025, 2931, 2853, 1732, 1600, 1495, 1455, 1361.

HRMS (ESI) calcd. for $\mathrm{C}_{17} \mathrm{H}_{24} \mathrm{O}_{4} \mathrm{SK}^{+}[\mathrm{M}+\mathrm{K}]^{+}: 363.1027$, found: 363.1021 . 


\section{Scheme S6: Preparation of precursor 13}
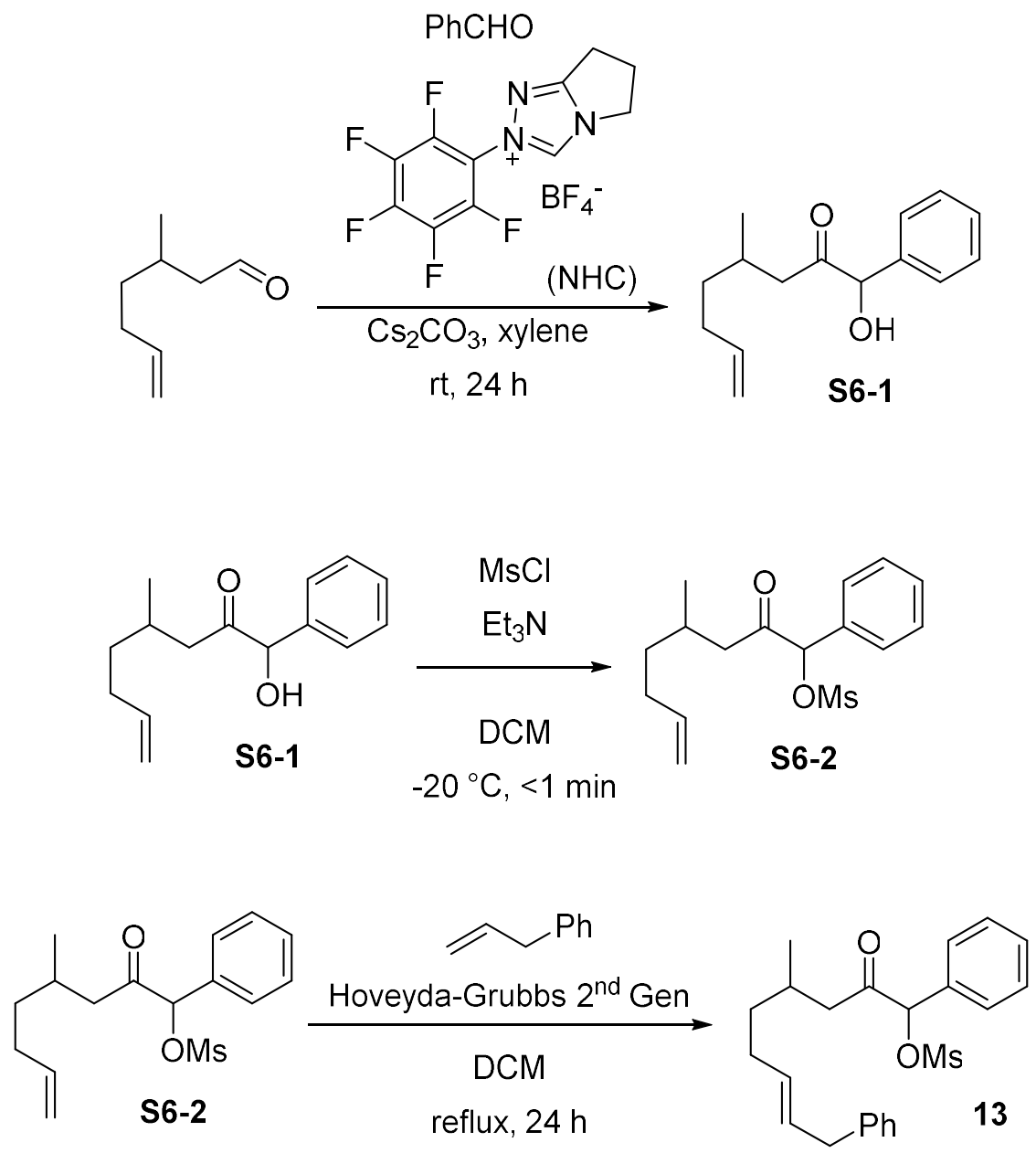

1-Hydroxy-4-methyl-1-phenyloct-7-en-2-one (S6-1)<smiles>C=CCCC(C)CC=O</smiles> 
Prepared by G.P.2, using 3-methylhept-6-enal ${ }^{12}(640 \mathrm{mg}, 5 \mathrm{mmol}, 5 \mathrm{eq})$, benzaldehyde (103.5 $\mu \mathrm{L}$, $1 \mathrm{mmol}, 1 \mathrm{eq})$, NHC (37 mg, $0.1 \mathrm{mmol}, 0.1 \mathrm{eq})$, cesium carbonate (33 mg, $0.1 \mathrm{mmol}, 0.1 \mathrm{eq})$ and xylene $(2 \mathrm{~mL})$, during $40 \mathrm{~h}$. Purification by dry-flash chromatography (petroleum-ether/ethyl acetate $=99 / 1)$ followed by column chromatography (petroleum-ether/ethyl acetate $=9 / 1$ ) afforded $93.3 \mathrm{mg}(40 \%)$ of S6-1 (a mixture of isomers in ratio $\mathrm{H}: \mathrm{H}^{\prime}=1.5: 1$, as determined by ${ }^{1} \mathrm{H} \mathrm{NMR}$ ), as a colorless oil.

Spectral data for mixture of isomers in a ratio of $\mathrm{H}^{\prime} \mathrm{H}^{\prime}=1.5: 1$

${ }^{1} \mathbf{H}$ NMR $\left(500 \mathrm{MHz}, \mathrm{CDCl}_{3}\right) \delta 7.41-7.27\left(\mathrm{~m}, 5 \mathrm{H}+5 \mathrm{H}^{\prime}\right), 5.79-5.62\left(\mathrm{~m}, 1 \mathrm{H}+1 \mathrm{H}^{\prime}\right), 5.06\left(\mathrm{~s}, 1 \mathrm{H}^{\prime}\right)$, $5.03(\mathrm{~s}, 1 \mathrm{H}), 5.00-4.86\left(\mathrm{~m}, 2 \mathrm{H}+2 \mathrm{H}^{\prime}\right), 4.39\left(\mathrm{~s}, 1 \mathrm{H}+1 \mathrm{H}^{\prime}\right), 2.37\left(\mathrm{dd}, J^{l}=16.4, J^{2}=5.5 \mathrm{~Hz}, 1 \mathrm{H}^{\prime}\right)$, $2.28\left(\mathrm{dd}, J^{l}=16.5, J^{2}=6.2 \mathrm{~Hz}, 1 \mathrm{H}\right), 2.21\left(\mathrm{dd}, J^{1}=16.5, J^{2}=7.5 \mathrm{~Hz}, 1 \mathrm{H}\right), 2.14\left(\mathrm{dd}, J^{l}=16.4, J^{2}\right.$ $\left.=8.2 \mathrm{~Hz}, 1 \mathrm{H}^{\prime}\right), 2.06-1.81\left(\mathrm{~m}, 3 \mathrm{H}+3 \mathrm{H}^{\prime}\right), 1.36-1.26\left(\mathrm{~m}, 1 \mathrm{H}^{\prime}\right), 1.25-1.16\left(\mathrm{~m}, 1 \mathrm{H}+1 \mathrm{H}^{\prime}\right), 1.12-$ $1.03(\mathrm{~m}, 1 \mathrm{H}), 0.86(\mathrm{~d}, J=6.7 \mathrm{~Hz}, 3 \mathrm{H}), 0.70\left(\mathrm{~d}, J=6.7 \mathrm{~Hz}, 3 \mathrm{H}^{\prime}\right)$.

${ }^{13} \mathbf{C}$ NMR (125 MHz, $\left.\mathrm{CDCl}_{3}\right) \delta 209.1,209.0,138.3,137.9,128.9,128.6,127.4,114.6,114.5,80.2$, 79.8, 45.0, 44.9, 35.8, 35.5, 31.0, 30.8, 28.7, 28.6, 19.5, 19.3.

IR (ATR) $v_{\max }:$ 3457, 3068, 3031, 2961, 2925, 1712, 1640, 1601, 1493, 14541371.

HRMS (ESI) calcd. for $\mathrm{C}_{15} \mathrm{H}_{24} \mathrm{O}_{2} \mathrm{~N}\left[\mathrm{M}+\mathrm{NH}_{4}\right]^{+}: 250.1802$, found: 250.1799 .

\section{4-Methyl-2-oxo-1-phenyloct-7-enyl methanesulfonate (S6-2)}

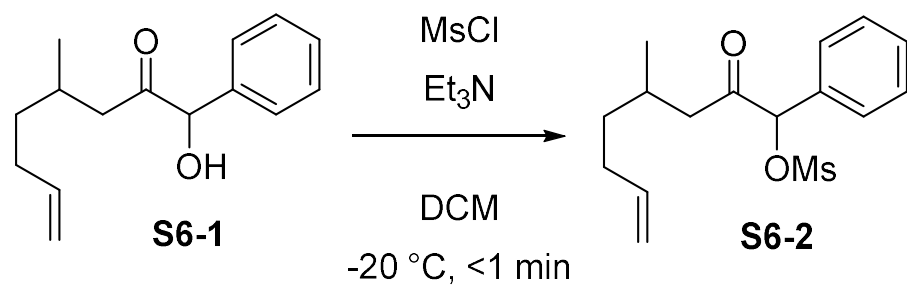

Prepared by G.P.1, using S6-1 (60.4 mg, $0.26 \mathrm{mmol}, 1 \mathrm{eq})$, mesyl chloride (40 $\mu \mathrm{L}, 0.52 \mathrm{mmol}, 2$ eq), triethylamine (109 $\mu \mathrm{L}, 0.78 \mathrm{mmol}, 3 \mathrm{eq})$, DMAP (3 mg, $0.026 \mathrm{mmol}, 0.1 \mathrm{eq})$ and $\mathrm{CH}_{2} \mathrm{Cl}_{2}(1.7$ $\mathrm{mL}$ ). Purification by dry-flash chromatography (petroleum-ether/ethyl acetate $=9 / 1$ ) afforded 69.7 $\mathrm{mg}(86 \%)$ of mesylate S6-2, as a colorless oil (mixture of isomers in a relative ratio $1: 1$, as determined by ${ }^{1} \mathrm{H}$ NMR).

Spectral data for the mixture of isomers in a ratio of $\mathrm{H}^{\prime} \mathrm{H}^{\prime}=1: 1$

${ }^{1} \mathbf{H}$ NMR $\left(500 \mathrm{MHz}, \mathrm{CDCl}_{3}\right) \delta 7.44-7.37$ (m, 5H+5H'), $5.89(\mathrm{~s}, 1 \mathrm{H}), 5.87$ (s, 1H'), $5.78-5.64$

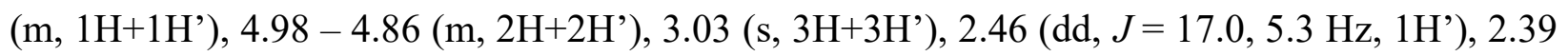

\footnotetext{
${ }^{12}$ Hagiwara, H., Katsumi, T., Vijayendra, P. K., Hoshi, T., Suzuki, T., Ando, M., J. Org. Chem. 2000, 65, 7231-
} 7234. 
(dd, $J=17.1,6.0 \mathrm{~Hz}, 1 \mathrm{H}), 2.31(\mathrm{dd}, J=17.1,7.5 \mathrm{~Hz}, 1 \mathrm{H}), 2.24$ (dd, $\left.J=17.0,8.2 \mathrm{~Hz}, 1 \mathrm{H}^{\prime}\right), 2.08$ $-1.86\left(\mathrm{~m}, 3 \mathrm{H}+3 \mathrm{H}^{\prime}\right), 1.35-1.07\left(\mathrm{~m}, 2 \mathrm{H}+2 \mathrm{H}^{\prime}\right), 0.84(\mathrm{~d}, J=6.7 \mathrm{~Hz}, 3 \mathrm{H}), 0.76\left(\mathrm{~d}, J=6.6 \mathrm{~Hz}, 3 \mathrm{H}^{\prime}\right)$. ${ }^{13}$ C NMR $\left(125 \mathrm{MHz}, \mathrm{CDCl}_{3}\right) \delta 204.2,204.0,139.9,133.7(2 \times \mathrm{C}), 131.6,130.9,129.7(2 \times \mathrm{C}), 116.2$, 116.1, 87.2, 87.0, 47.3, 41.0, 37.3, 37.1, 32.6, 32.5, 29.8, 29.7, 21.0, 20.9.

IR (ATR) $v_{\max }:$ 3070, 3033, 2961, 2931, 2852, 1733, 1640, 1495, 1456, 1362.

HRMS (ESI) calcd. for $\mathrm{C}_{16} \mathrm{H}_{22} \mathrm{O}_{4} \mathrm{SNa}^{+}[\mathrm{M}+\mathrm{Na}]^{+}: 349.0870$, found: 349.0873 .

\section{(E)-4-Methyl-2-oxo-1,9-diphenylnon-7-enyl methanesulfonate (13)}

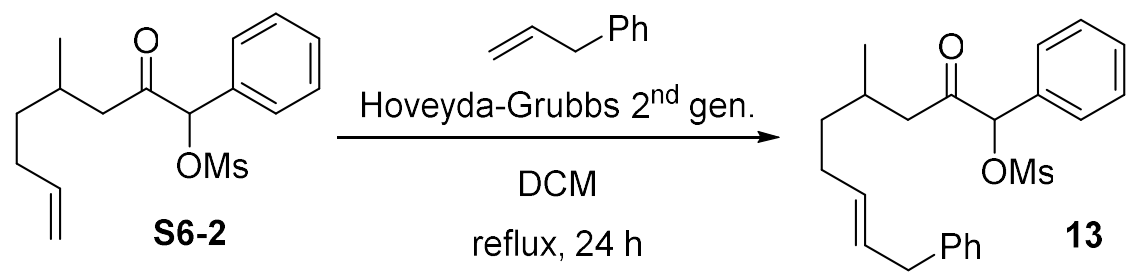

Allyl benzene ( $236 \mu \mathrm{L}, 1.78 \mathrm{mmol}, 20 \mathrm{eq})$ was added to the solution of $\mathbf{S 6 - 2}(27.6 \mathrm{mg}, 0.089$ mmol, $1 \mathrm{eq})$ in $\mathrm{CH}_{2} \mathrm{Cl}_{2}(0.7 \mathrm{~mL})$, followed by the addition of Hoveyda-Grubbs $2^{\text {nd }}$ generation catalyst $(16.8 \mathrm{mg}, 0.027 \mathrm{mmol}, 0.3 \mathrm{eq})$. The reaction mixture was stirred at room temperature for $16 \mathrm{~h}$ then at $40{ }^{\circ} \mathrm{C}$ for $24 \mathrm{~h}$. The reaction mixture was concentrated in vacuo and the residue was purified by column chromatography (petroleum-ether/ethyl acetate $=85 / 15$ ) followed by a second column chromatography with silica gel impregnated with $\mathrm{AgNO}_{3}{ }^{13}$ (benzene/ethyl acetate $=98 / 2$ ) to afford $22.3 \mathrm{mg}(63 \%)$ of mesylate $\mathbf{1 3}$, as a colorless oil (mixture of isomers in a relative ratio $1: 1$, as determined by ${ }^{1} \mathrm{H}$ NMR).

Spectral data for the mixture of isomers in a ratio of $\mathrm{H}: \mathrm{H}^{\prime}=1: 1$

${ }^{1} \mathbf{H}$ NMR $\left(500 \mathrm{MHz}, \mathrm{CDCl}_{3}\right) \delta 7.45-7.35\left(\mathrm{~m}, 5 \mathrm{H}+5 \mathrm{H}^{\prime}\right), 7.31-7.26\left(\mathrm{~m}, 2 \mathrm{H}^{2} 2 \mathrm{H}^{\prime}\right), 7.21-7.14$ (m, 3H+3H'), 5.89 (s, 1H), 5.87 (s, 1H'), $5.57-5.36\left(\mathrm{~m}, 2 \mathrm{H}+2 \mathrm{H}^{\prime}\right), 3.30$ (t, $\left.J=7.2 \mathrm{~Hz}, 2 \mathrm{H}+2 \mathrm{H}^{\prime}\right)$, 3.04 (s, 3H), 3.03 (s, 3H'), 2.46 (dd, $\left.J=17.0,5.3 \mathrm{~Hz}, 1 \mathrm{H}^{\prime}\right), 2.38$ (dd, $\left.J=17.0,6.0 \mathrm{~Hz}, 1 \mathrm{H}\right), 2.30$ (dd, $J=17.0,7.5 \mathrm{~Hz}, 1 \mathrm{H}), 2.23$ (dd, $\left.J=16.9,8.3 \mathrm{~Hz}, 1 \mathrm{H}^{\prime}\right), 2.14-1.82\left(\mathrm{~m}, 3 \mathrm{H}+3 \mathrm{H}^{\prime}\right), 1.38-1.07$ $\left(\mathrm{m}, 2 \mathrm{H}^{+}+\mathrm{H}^{\prime}\right), 0.85(\mathrm{~d}, J=6.7 \mathrm{~Hz}, 3 \mathrm{H}), 0.76\left(\mathrm{~d}, J=6.6 \mathrm{~Hz}, 3 \mathrm{H}^{\prime}\right)$.

${ }^{13}$ C NMR $\left(125 \mathrm{MHz}, \mathrm{CDCl}_{3}\right) \delta 202.6,202.5,140.9,132.1,131.2,130.1,129.3,129.2,129.1$, $128.4,128.3,128.2,128.1,125.9,85.7,85.4,45.8,39.4,39.0,36.3,36.1,29.8,29.6,28.3,28.2$, 19.5, 19.3.

IR (ATR) $v_{\max }: 3062,3028,2959,2929,2849,1733,1601,1494,1455,1362$.

HRMS (ESI) calcd. for $\mathrm{C}_{23} \mathrm{H}_{28} \mathrm{O}_{4} \mathrm{SK}[\mathrm{M}+\mathrm{K}]^{+}:$439.1334, found: 439.1336 .

\footnotetext{
${ }^{13}$ For a review on chromatography on silver nitrate impregnated silica, see: Williams, C. M.; Mander, L. N. Tetrahedron 2001, 55, 425-447, and references therein.
} 
2,6,11,15-Tetramethyl-9-oxohexadeca-2,14-dien-8-yl methanesulfonate (16)

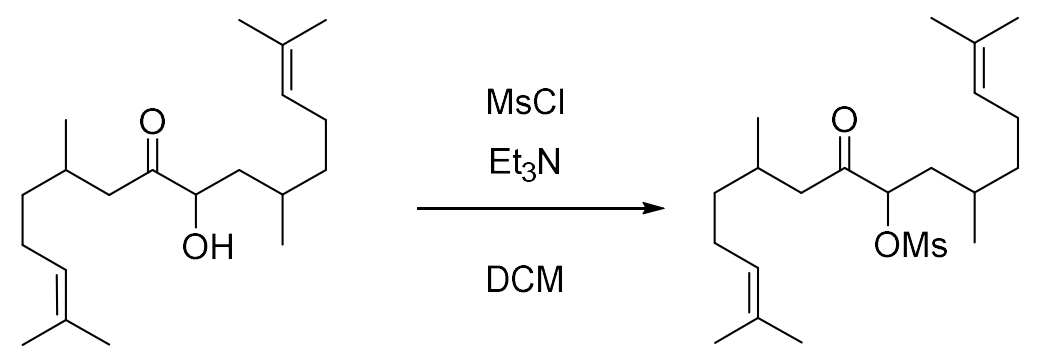

S4-2

16

Prepared by G.P.1, using hydroxy ketone S4-2 (10 mg, $0.03 \mathrm{mmol}, 1 \mathrm{eq})$, mesyl chloride (5 $\mu \mathrm{L}$, $0.06 \mathrm{mmol}, 2 \mathrm{eq})$, triethylamine $(13.5 \mu \mathrm{L}, 0.097 \mathrm{mmol}, 3 \mathrm{eq})$, a few crystals of DMAP and $\mathrm{CH}_{2} \mathrm{Cl}_{2}$ $(0.2 \mathrm{~mL})$. Purification by column chromatography (petroleum-ether/ethyl acetate $=9 / 1)$ afforded $12 \mathrm{mg}(96 \%)$ of mesylate $\mathbf{1 6}$, as a colorless oil (mixture of four isomers, ratio not determined).

Spectral data for a mixture of isomers

${ }^{1} \mathbf{H}$ NMR (500 MHz, $\left.\mathrm{CDCl}_{3}\right) 5.12-5.05(\mathrm{~m}, 2 \mathrm{H}), 5.04-4.95(\mathrm{~m}, 1 \mathrm{H}), 3.14-3.08(\mathrm{~m}, 3 \mathrm{H}), 2.55$ $-2.44(\mathrm{~m}, 1 \mathrm{H}), 2.39-2.30(\mathrm{~m}, 1 \mathrm{H}), 2.12-1.90(\mathrm{~m}, 5 \mathrm{H}), 1.82-1.58(\mathrm{~m}, 14 \mathrm{H}), 1.54-1.45(\mathrm{~m}$, $1 \mathrm{H}), 1.38-1.14(\mathrm{~m}, 4 \mathrm{H}), 1.02-0.96(\mathrm{~m}, 3 \mathrm{H}), 0.94-0.89(\mathrm{~m}, 3 \mathrm{H})$.

${ }^{13}$ C NMR $\left(125 \mathrm{MHz}, \mathrm{CDCl}_{3}\right) \delta$ 206.1, $206.0(2 \times \mathrm{C}), 205.9,131.8,131.7,131.6,124.0,82.8,82.6$, $82.5,82.4,45.7,45.6(2 \times \mathrm{C}), 45.6,39.0,38.9,38.9,38.1,37.9,37.4,36.7(2 \times \mathrm{C}), 35.5(2 \times \mathrm{C}), 28.8$ $(2 \times \mathrm{C}), 28.6,28.2,28.1,25.6,25.4,25.3,25.0,19.8,19.6,19.6,18.4,17.6$.

IR (ATR) $v_{\max }: 2963,2920,2855,1727,1455,1358$.

HRMS (ESI) calcd. for $\mathrm{C}_{21} \mathrm{H}_{42} \mathrm{O}_{4} \mathrm{SN}\left[\mathrm{M}+\mathrm{NH}_{4}\right]^{+}:$404.2829, found: 404.2824. 


\section{Scheme S7: Preparation of precursors 18 and 21}
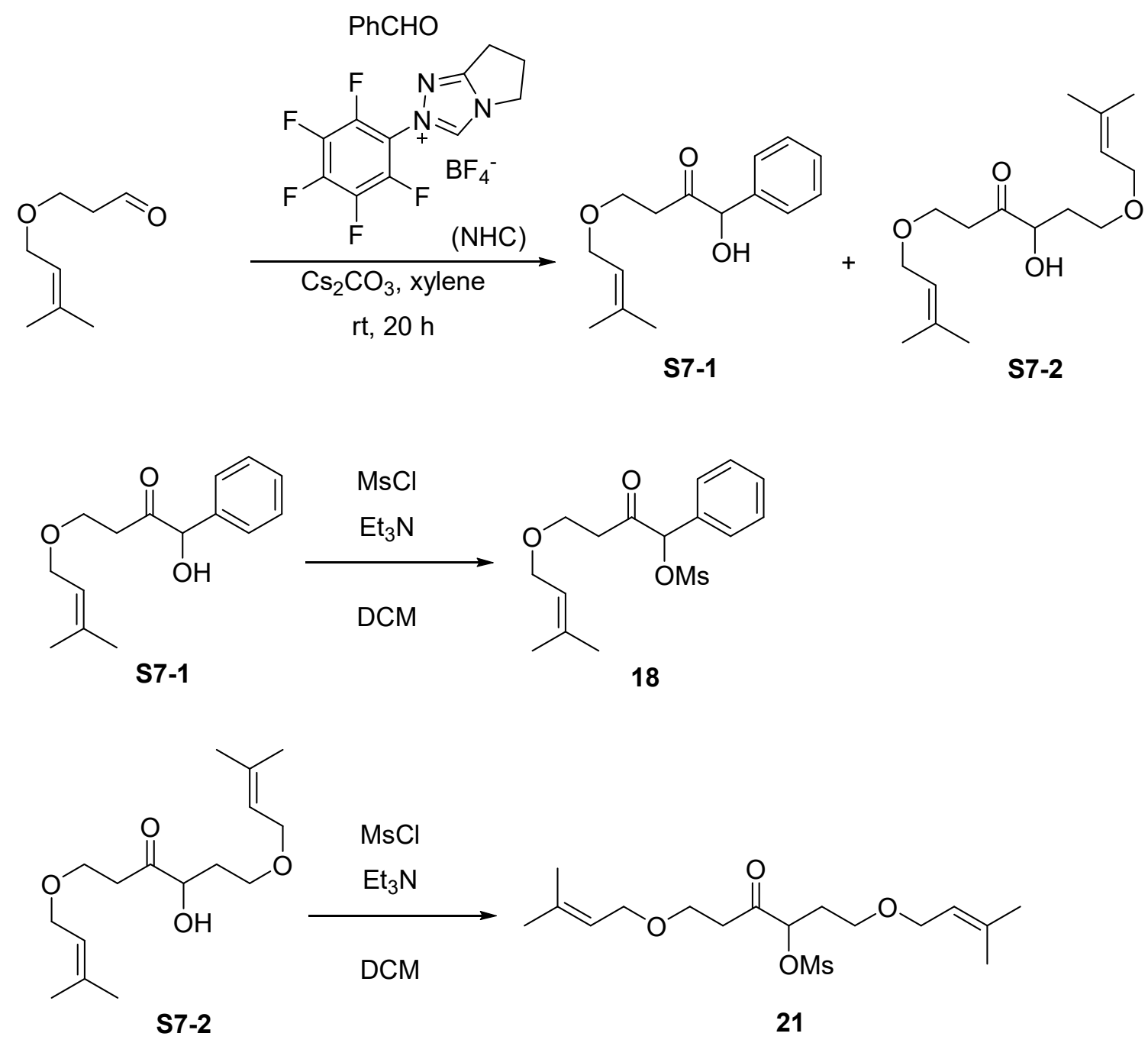

1-Hydroxy-4-(3-methylbut-2-enyloxy)-1-phenylbutan-2-one (S7-1) and 4-hydroxy-1,6-bis(3methylbut-2-enyloxy)hexan-3-one (S7-2)

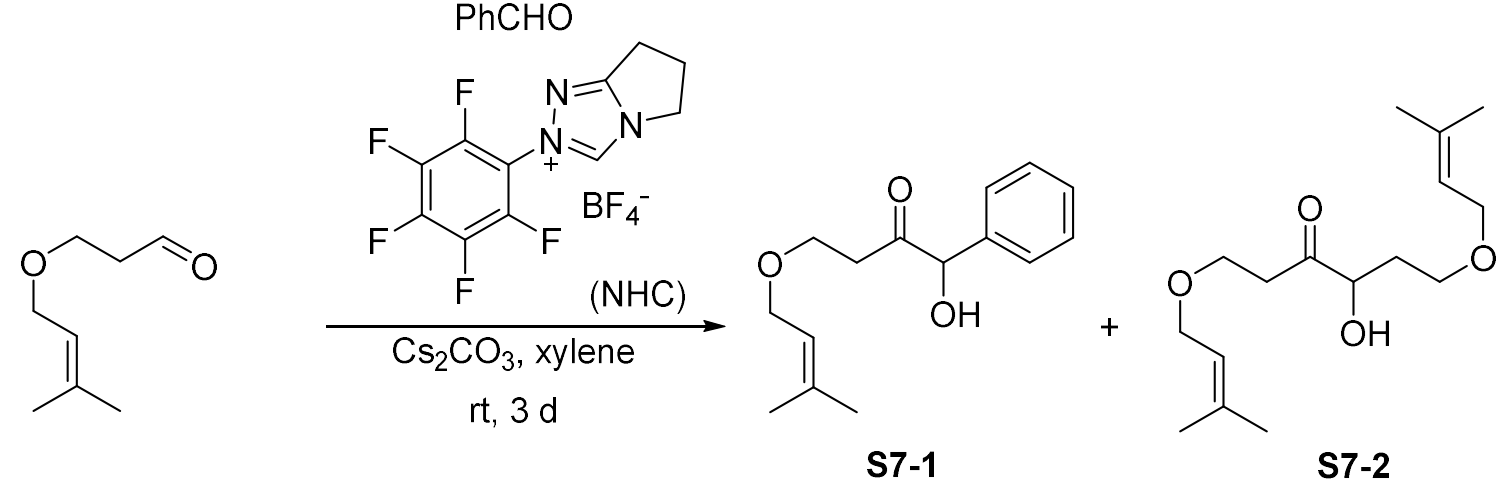


Prepared by G.P.2, using 3-(3-methylbut-2-enyloxy)propanal ${ }^{14}$ (250 mg, $\left.1.76 \mathrm{mmol}, 5 \mathrm{eq}\right)$, benzaldehyde ( $36 \mu \mathrm{L}, 0.35 \mathrm{mmol}, 1 \mathrm{eq})$, NHC (12.8 $\mathrm{mg}, 0.035 \mathrm{mmol}, 0.1 \mathrm{eq})$, cesium carbonate (11.5 mg, $0.035 \mathrm{mmol}, 0.1 \mathrm{eq})$ and xylene $(0.75 \mathrm{~mL})$, at room temperature, during $20 \mathrm{~h}$. Purification by dry-flash chromatography (petroleum-ether/ethyl acetate $=9 / 1$ ), followed by column chromatography (petroleum-ether/ethyl acetate $=9 / 1)$ afforded $63.2 \mathrm{mg}(72 \%)$ of hydroxy ketone S7-1 and 112.1 mg (45\%) of hydroxy ketone S7-2, both as colorless oils.

\section{S7-1}

${ }^{1} \mathbf{H}$ NMR $\left(500 \mathrm{MHz}, \mathrm{CDCl}_{3}\right) \delta 7.40-7.30(\mathrm{~m}, 5 \mathrm{H}), 5.29-5.23(\mathrm{~m}, 1 \mathrm{H}), 5.12(\mathrm{~s}, 1 \mathrm{H}), 4.34(\mathrm{~s}$, $1 \mathrm{H}), 3.89(\mathrm{~d}, J=6.9 \mathrm{~Hz}, 2 \mathrm{H}), 3.69-3.62(\mathrm{~m}, 1 \mathrm{H}), 3.59-3.52(\mathrm{~m}, 1 \mathrm{H}), 2.74-2.66(\mathrm{~m}, 1 \mathrm{H}), 2.51$ $\left(\mathrm{dt}, J^{1}=16.5, J^{2}=6.0 \mathrm{~Hz}, 1 \mathrm{H}\right), 1.73(\mathrm{~s}, 3 \mathrm{H}), 1.64(\mathrm{~s}, 3 \mathrm{H})$.

${ }^{13}$ C NMR (125 MHz, $\left.\mathrm{CDCl}_{3}\right) \delta 208.0,137.7,137.4,128.9,128.6,127.4,120.5,80.1,67.5,64.6$, 38.4, 25.7, 17.9 .

IR (ATR) v $v_{\max }:$ 3457, 2971, 2914, 2867, 1717, 1681, 1599, 1492, 1450, 1378.

HRMS (ESI) calcd. for $\mathrm{C}_{15} \mathrm{H}_{20} \mathrm{O}_{3} \mathrm{Na}^{+}[\mathrm{M}+\mathrm{Na}]^{+}: 271.1305$, found: 271.1307 .

\section{S7-2}

${ }^{1} \mathbf{H}$ NMR $\left(500 \mathrm{MHz}, \mathrm{CDCl}_{3}\right) \delta 5.34-5.24(\mathrm{~m}, 2 \mathrm{H}), 4.26\left(\mathrm{dt}, J^{l}=6.8, J^{2}=4.1 \mathrm{~Hz}, 1 \mathrm{H}\right), 3.95(\mathrm{~d}, J$ $=6.9 \mathrm{~Hz}, 2 \mathrm{H}), 3.91-3.87(\mathrm{~m}, 2 \mathrm{H}), 3.79(\mathrm{~d}, J=4.2 \mathrm{~Hz}, 1 \mathrm{H}), 3.75-3.67(\mathrm{~m}, 2 \mathrm{H}), 3.58-3.51(\mathrm{~m}$, 2H), $2.86\left(\mathrm{dt}, J^{l}=16.4, J^{2}=6.1 \mathrm{~Hz}, 1 \mathrm{H}\right), 2.75\left(\mathrm{dt}, J^{l}=16.4, J^{2}=6.6 \mathrm{~Hz}, 1 \mathrm{H}\right), 2.13-2.03(\mathrm{~m}$, $1 \mathrm{H}), 1.97-1.89(\mathrm{~m}, 1 \mathrm{H}), 1.74(\mathrm{~s}, 6 \mathrm{H}), 1.66(\mathrm{~s}, 3 \mathrm{H}), 1.65(\mathrm{~s}, 3 \mathrm{H})$.

${ }^{13}$ C NMR (125 MHz, $\left.\mathrm{CDCl}_{3}\right) \delta 210.5,136.8,136.5,120.4(2 \times \mathrm{C}), 74.5,67.1(2 \times \mathrm{C}), 65.1,64.5$, $38.1,33.0,25.3,25.3,17.6,17.5$.

IR (ATR) $v_{\max }:$ 3464, 2971, 2915, 2866, 1716, 1676, 1450, 1378, 1327.

HRMS (ESI) calcd. for $\mathrm{C}_{16} \mathrm{H}_{28} \mathrm{O}_{4} \mathrm{~K}^{+}[\mathrm{M}+\mathrm{K}]^{+}: 323.1619$, found: 323.1624 .

\section{4-(3-Methylbut-2-enyloxy)-2-oxo-1-phenylbutyl methanesulfonate (18)}

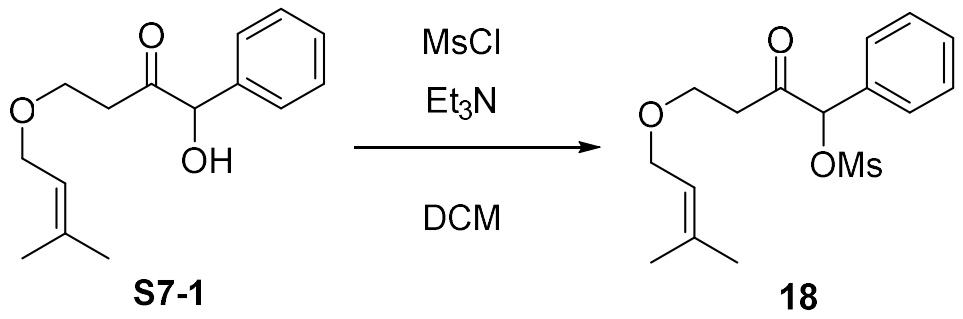

Prepared by G.P.1, using S7-1 (44.8 mg, $0.18 \mathrm{mmol}, 1 \mathrm{eq})$, mesyl chloride ( $28 \mu \mathrm{L}, 0.36 \mathrm{mmol}, 2$ eq), triethylamine $(75 \mu \mathrm{L}, 0.54 \mathrm{mmol}, 3 \mathrm{eq})$, DMAP $(2 \mathrm{mg}, 0.018 \mathrm{mmol}, 0.1 \mathrm{eq})$ and $\mathrm{CH}_{2} \mathrm{Cl}_{2}(1$ $\mathrm{mL}$ ). Purification by dry-flash chromatography (petroleum-ether/ethyl acetate $=85 / 15$ ) afforded $43 \mathrm{mg}(73 \%)$ of mesylate 18, as a colorless oil.

\footnotetext{
${ }^{14}$ Comito, R. J., Finelli, F. G., MacMillan, D. W. C., J. Am. Chem. Soc., 2013, 135 (25), 9358-9361.
} 
${ }^{1} \mathbf{H}$ NMR $\left(500 \mathrm{MHz}, \mathrm{CDCl}_{3}\right) \delta 7.43-7.37(\mathrm{~m}, 5 \mathrm{H}), 5.98(\mathrm{~s}, 1 \mathrm{H}), 5.28-5.23(\mathrm{~m}, 1 \mathrm{H}), 3.89(\mathrm{~d}, J$ $=6.9 \mathrm{~Hz}, 2 \mathrm{H}), 3.67-3.58(\mathrm{~m}, 2 \mathrm{H}), 3.00(\mathrm{~s}, 3 \mathrm{H}), 2.75\left(\mathrm{dt}, J^{l}=16.8, J^{2}=6.6 \mathrm{~Hz}, 1 \mathrm{H}\right), 2.63\left(\mathrm{dt}, J^{1}\right.$ $\left.=16.8, J^{2}=6.1 \mathrm{~Hz}, 1 \mathrm{H}\right), 1.72(\mathrm{~d}, J=0.7 \mathrm{~Hz}, 3 \mathrm{H}), 1.63(\mathrm{~s}, 3 \mathrm{H})$.

${ }^{13} \mathbf{C}$ NMR $\left(125 \mathrm{MHz}, \mathrm{CDCl}_{3}\right) \delta 201.6,137.4,132.3,130.2,129.5,128.5,120.8,85.7,67.7,64.5$, 39.5, 39.4, 25.9, 18.1 .

IR (ATR) $v_{\max }:$ 3031, 2970, 2919, 2864, 1734, 1674, 1495, 1453, 1360.

HRMS (ESI) calcd. for $\mathrm{C}_{16} \mathrm{H}_{22} \mathrm{O}_{5} \mathrm{SNa}^{+}[\mathrm{M}+\mathrm{Na}]^{+}: 349.1080$, found: 349.1083 .

\section{1,6-Bis(3-methylbut-2-enyloxy)-4-oxohexan-3-yl methanesulfonate (21)}

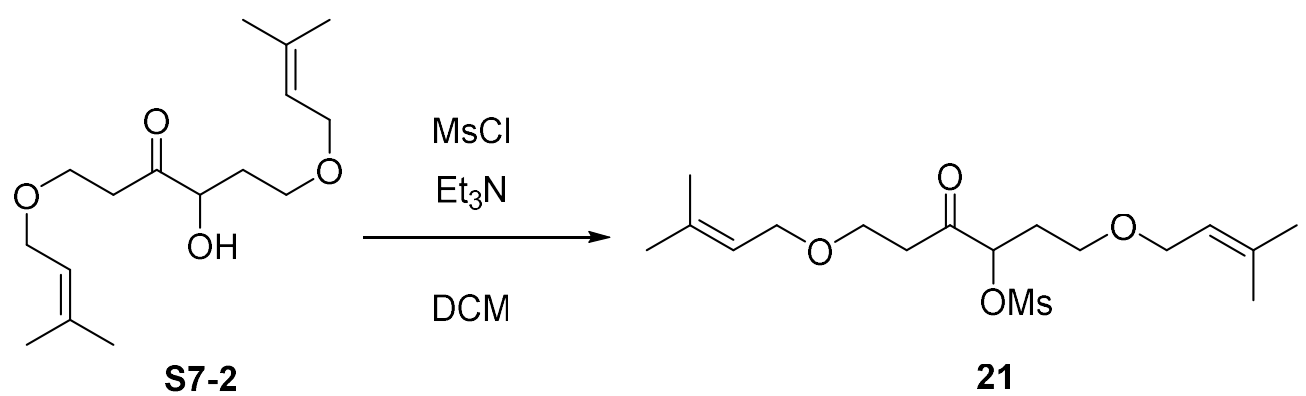

Prepared by G.P.1, using S7-2 ( $40.6 \mathrm{mg}, 0.14 \mathrm{mmol}, 1 \mathrm{eq}$ ), mesyl chloride ( $22 \mu \mathrm{L}, 0.285 \mathrm{mmol}, 2$ eq), triethylamine ( $60 \mu \mathrm{L}, 0.43 \mathrm{mmol}, 3 \mathrm{eq})$, DMAP (1.7 mg, $0.014 \mathrm{mmol}, 0.1 \mathrm{eq})$ and $\mathrm{CH}_{2} \mathrm{Cl}_{2}$ $(0.75 \mathrm{~mL})$. Purification by dry-flash chromatography (petroleum-ether/ethyl acetate $=85 / 15$ ) afforded $32.4 \mathrm{mg}(63 \%)$ of mesylate $\mathbf{2 1}$, as a colorless oil.

${ }^{1} \mathbf{H}$ NMR $\left(500 \mathrm{MHz}, \mathrm{CDCl}_{3}\right) \delta 5.29-5.22(\mathrm{~m}, 2 \mathrm{H}), 5.10\left(\mathrm{dd}, J^{l}=7.9, J^{2}=4.1 \mathrm{~Hz}, 1 \mathrm{H}\right), 3.91(\mathrm{t}, J$ $=7.1 \mathrm{~Hz}, 4 \mathrm{H}), 3.68-3.61(\mathrm{~m}, 2 \mathrm{H}), 3.53-3.45(\mathrm{~m}, 2 \mathrm{H}), 3.06(\mathrm{~s}, 3 \mathrm{H}), 2.84-2.72(\mathrm{~m}, 2 \mathrm{H}), 2.18-$ $2.10(\mathrm{~m}, 1 \mathrm{H}), 2.05-1.97(\mathrm{~m}, 1 \mathrm{H}), 1.70(2 \times 3 \mathrm{H}), 1.62(\mathrm{~s}, 6 \mathrm{H})$.

${ }^{13}$ C NMR $\left(125 \mathrm{MHz}, \mathrm{CDCl}_{3}\right) \delta 204.7,137.3,120.9,120.8,81.7,67.7,67.6,64.6,64.3,39.2,38.7$, $31.8,25.9,18.1$.

IR (ATR) $v_{\max }: 3018,2970,2916,2865,1729,1675,1448,1417,1360$.

HRMS (ESI) calcd. for $\mathrm{C}_{17} \mathrm{H}_{30} \mathrm{O}_{6} \mathrm{SK}^{+}[\mathrm{M}+\mathrm{K}]^{+}$: 401.1395, found: 401.1394 . 
Scheme S8: Preparation of precursor $23 E$ and $23 Z$
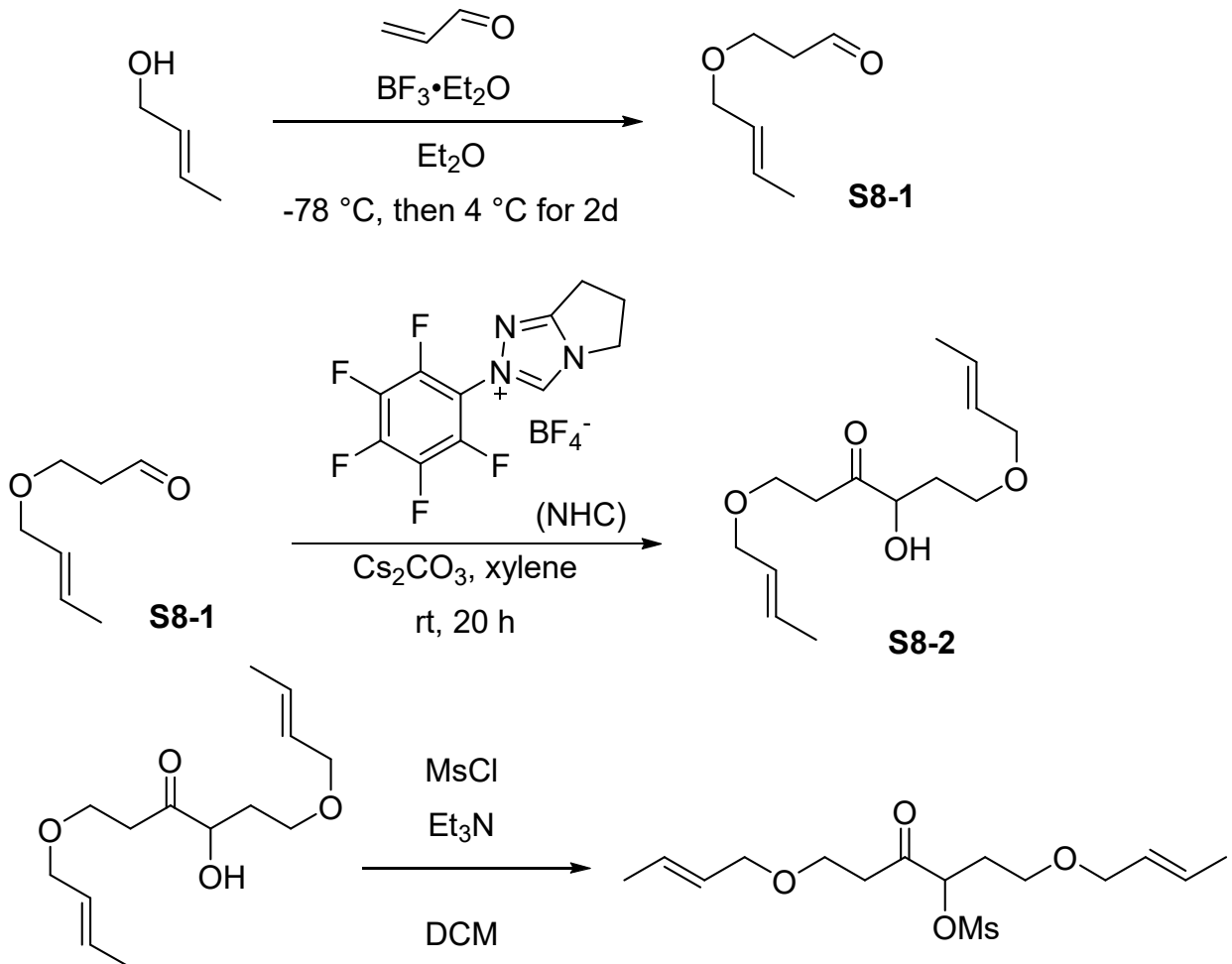

S8-2

$23 E$
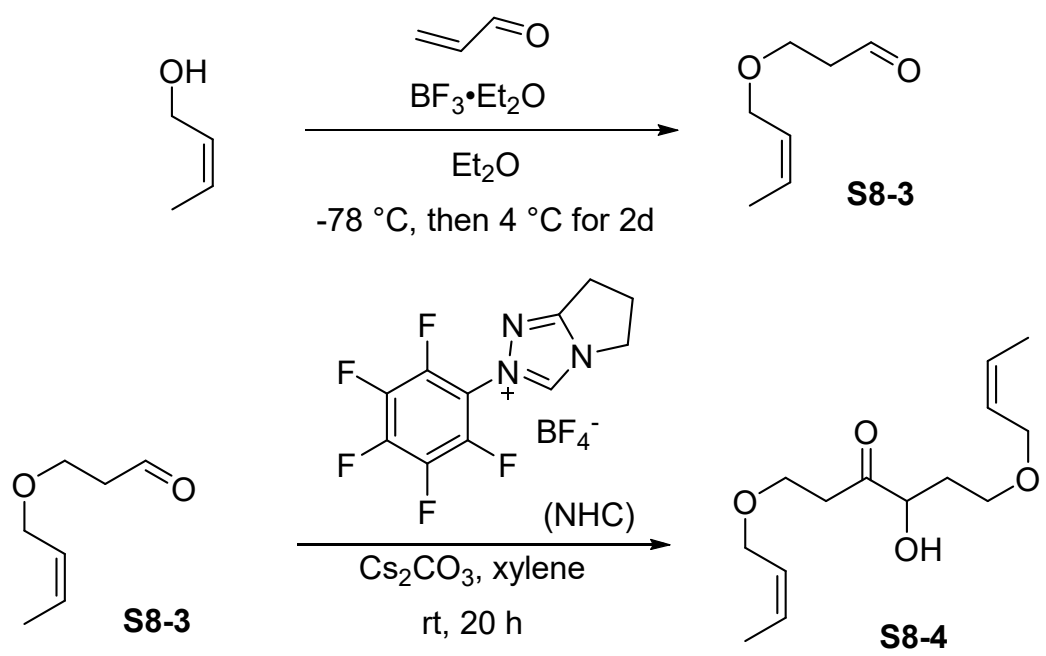

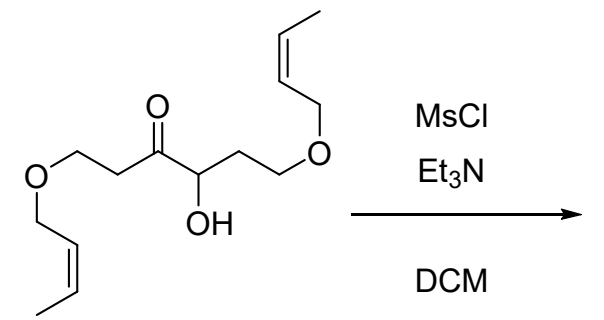

S8-4<smiles>C/C=C\COCCC(=O)C(CCOC/C=C\C)OC</smiles>

$23 Z$ 


\section{(E)-3-(but-2-en-1-yloxy)propanal (S8-1)}

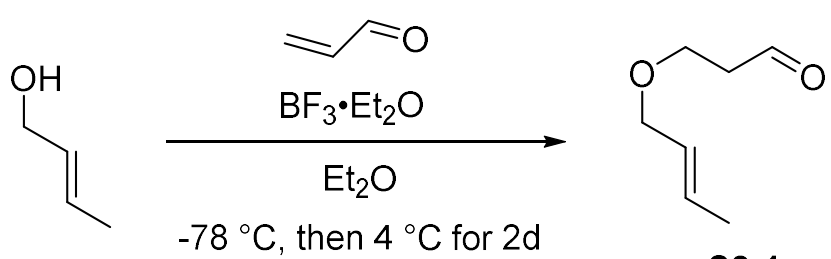

S8-1

(E)-crotyl alcohol $(2.5 \mathrm{~g}, 35 \mathrm{mmol}, 1 \mathrm{eq})$ was dissolved in diethyl ether $(15.1 \mathrm{~mL})$ and $\mathrm{BF}_{3} \cdot \mathrm{Et}_{2} \mathrm{O}$ $(0.53 \mathrm{~mL}, 4.2 \mathrm{mmol}, 0.12 \mathrm{eq})$ was added to the reaction mixture at $-78^{\circ} \mathrm{C}$ followed by the addition of acrolein $(8.8 \mathrm{~mL}, 140 \mathrm{mmol}, 4 \mathrm{eq})$, over the period of $30 \mathrm{~min}$. After stirring for 3 days at $4{ }^{\circ} \mathrm{C}$, the reaction was quenched with pyridine $(0.8 \mathrm{~mL})$, filtered through Celite and washed with diethyl ether. After concentration in vacuo, the residue was purified by dry flash chromatography (petroleum ether/ethyl acetate $9 / 1)$ to afford $0.99 \mathrm{~g}$ (22\%) of aldehyde S8-1, as a colorless oil.

${ }^{1} \mathbf{H}$ NMR $\left(500 \mathrm{MHz}, \mathrm{CDCl}_{3}\right) \delta 9.78(\mathrm{t}, J=1.8 \mathrm{~Hz}, 1 \mathrm{H}), 5.76-5.64(\mathrm{~m}, 1 \mathrm{H}), 5.61-5.49(\mathrm{~m}, 1 \mathrm{H})$, $3.91\left(\mathrm{dd}, J^{1}=6.3, J^{2}=0.9 \mathrm{~Hz}, 2 \mathrm{H}\right), 3.764\left(\mathrm{dt}, J^{1}=6.1, J^{2}=1.9 \mathrm{~Hz}, 2 \mathrm{H}\right), 2.69-2.63(\mathrm{~m}, 2 \mathrm{H}), 1.72$ $-1.68(\mathrm{~m}, 3 \mathrm{H})$.

${ }^{13}$ C NMR $\left(125 \mathrm{MHz}, \mathrm{CDCl}_{3}\right) \delta 201.3,130.0,127.3,72.0,63.6,44.0,17.8$.

\section{1,6-bis(((E)-but-2-en-1-yl)oxy)-4-hydroxyhexan-3-one (S8-2)}

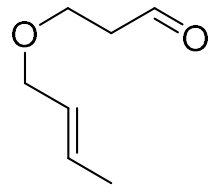

S8-1

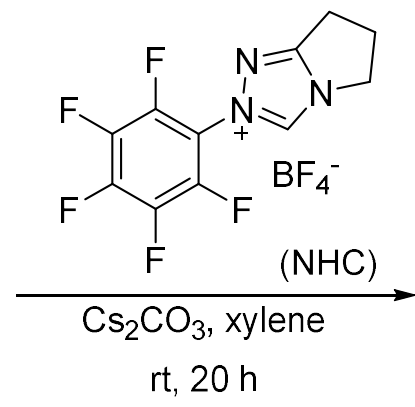

$\mathrm{rt}, 20 \mathrm{~h}$

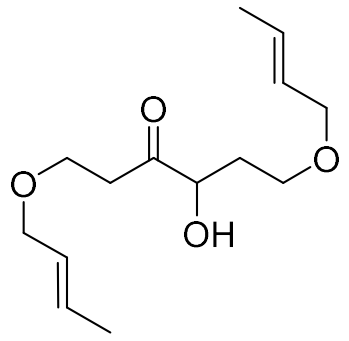

S8-2

Prepared by G.P.2. Using aldehyde S8-1 (50 mg, $0.39 \mathrm{mmol}, 1 \mathrm{eq})$, NHC (8 mg, $0.036 \mathrm{mmol}, 0.06$ eq), cesium carbonate $(12 \mathrm{mg}, 0.036 \mathrm{mmol}, 0.06 \mathrm{eq})$ and xylene $(0.8 \mathrm{~mL})$, during $27 \mathrm{~h}$. Dry flash chromatography (petroleum ether/ethyl acetate $=8 / 2$ ) afforded $20.3 \mathrm{mg}(40.6 \%)$ of hydroxy ketone S8-2, as a colorless oil.

${ }^{1}$ H NMR $\left(500 \mathrm{MHz}, \mathrm{CDCl}_{3}\right) \delta 5.68\left(\mathrm{ddt}, J^{1}=19.3, J^{2}=12.8, J^{3}=6.4 \mathrm{~Hz}, 2 \mathrm{H}\right), 5.59-5.46(\mathrm{~m}$, $2 \mathrm{H}), 4.26\left(\mathrm{dd}, J^{1}=6.3, J^{2}=4.2 \mathrm{~Hz}, 1 \mathrm{H}\right), 3.88(\mathrm{~d}, J=6.2 \mathrm{~Hz}, 2 \mathrm{H}), 3.82(\mathrm{~d}, J=5.7 \mathrm{~Hz}, 2 \mathrm{H}), 3.75-$ $3.63(\mathrm{~m}, 2 \mathrm{H}), 3.56-3.49(\mathrm{~m}, 2 \mathrm{H}), 2.85\left(\mathrm{dt}, J^{1}=16.3, J^{2}=6.1 \mathrm{~Hz}, 1 \mathrm{H}\right), 2.74\left(\mathrm{dt}, J^{1}=16.4, J^{2}=\right.$ $6.6 \mathrm{~Hz}, 1 \mathrm{H}), 2.13-2.04(\mathrm{~m}, 1 \mathrm{H}), 1.98-1.88(\mathrm{~m}, 1 \mathrm{H}), 1.70(\mathrm{~s}, 3 \mathrm{H}), 1.69(\mathrm{~s}, 3 \mathrm{H})$. 
${ }^{13} \mathbf{C}$ NMR $\left(125 \mathrm{MHz}, \mathrm{CDCl}_{3}\right) \delta 210.8,129.7,129.5,127.4,127.3,74.9,71.8,65.4,64.9,38.4$, 33.4, 17.7 .

IR (ATR) $v_{\max }: 3470,3014,2921,2862,1715,1447,1363,1250,1189,1104$.

HRMS (ESI) calcd. for $\mathrm{C}_{14} \mathrm{H}_{25} \mathrm{O}_{4}^{+}[\mathrm{M}+\mathrm{H}]^{+}: 257.1747$, found: 257.1742 .

\section{1,6-bis(((E)-but-2-en-1-yl)oxy)-4- oxohexan-3-yl methanesulfonate (23E)}

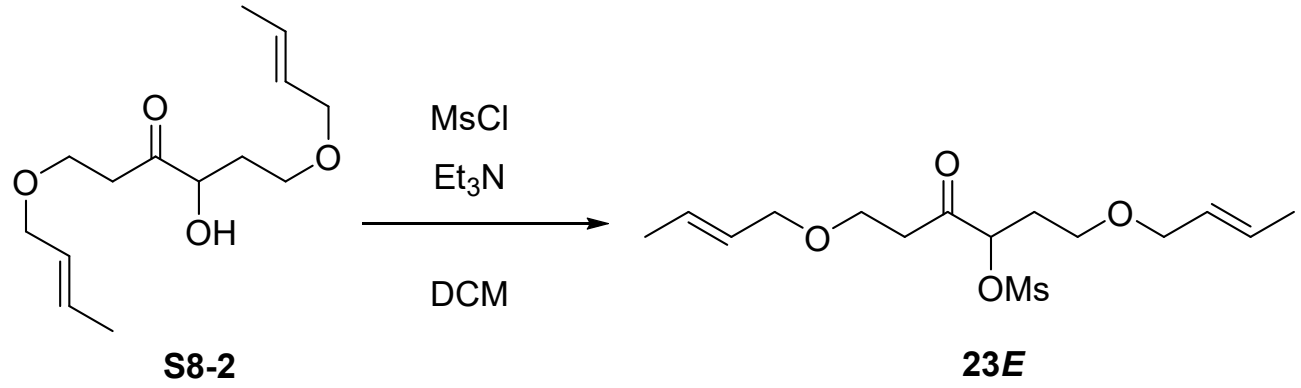

Prepared by G.P.1. Using S8-2 (67.1 mg, $0.26 \mathrm{mmol}, 1 \mathrm{eq}$ ), mesyl chloride ( $41 \mu \mathrm{L}, 0.52 \mathrm{mmol}, 2$ eq), triethylamine ( $109 \mu \mathrm{L}, 0.78 \mathrm{mmol}, 2 \mathrm{eq})$, DMAP ( $3 \mathrm{mg}, 0.029 \mathrm{mmol}, 0.1 \mathrm{eq})$ and $\mathrm{CH}_{2} \mathrm{Cl}_{2}(1.2$ $\mathrm{mL}$ ). Dry flash chromatography (petroleum ether/ethyl acetate 2/1) afforded $67.9 \mathrm{mg}(78 \%)$ of mesylate $\mathbf{2 3} \boldsymbol{E}$, as a colorless oil.

${ }^{1}$ H NMR $\left(500 \mathrm{MHz}, \mathrm{CDCl}_{3}\right) \delta 5.73\left(\mathrm{dq}, J^{I}=12.9, J^{2}=6.4 \mathrm{~Hz}, 2 \mathrm{H}\right), 5.61-5.52(\mathrm{~m}, 2 \mathrm{H}), 5.18(\mathrm{dd}$, $\left.J^{l}=7.8, J^{2}=4.1 \mathrm{~Hz}, 1 \mathrm{H}\right), 3.91(\mathrm{t}, J=7.3 \mathrm{~Hz}, 4 \mathrm{H}), 3.71\left(\mathrm{td}, J^{l}=12.9, J^{2}=6.4 \mathrm{~Hz}, 2 \mathrm{H}\right), 3.60-$ $3.51(\mathrm{~m}, 2 \mathrm{H}), 3.13(\mathrm{~s}, 3 \mathrm{H}), 2.92-2.79(\mathrm{~m}, 2 \mathrm{H}), 2.26-2.17(\mathrm{~m}, 1 \mathrm{H}), 2.14-2.04(\mathrm{~m}, 1 \mathrm{H}), 1.77-$ $1.76-1.71(\mathrm{~m}, 6 \mathrm{H})$.

${ }^{13}$ C NMR $\left(125 \mathrm{MHz}, \mathrm{CDCl}_{3}\right) \delta 204.7,129.9,129.8,127.4,127.3,81.6,72.0,71.8,64.5,64.2$, $39.1,38.7,31.7,17.8$.

IR (ATR) $v_{\max }: 3479$, 3016, 2937, 2862, 1729, 1673, 1448, 1354, 1242, 1176, 1103.

HRMS (ESI) calcd. for $\mathrm{C}_{15} \mathrm{H}_{27} \mathrm{O}_{6} \mathrm{~S}^{+}[\mathrm{M}+\mathrm{H}]^{+}: 335.1523$, found: 335.1517 . 


\section{(Z)-3-(but-2-en-1-yloxy)propanal (S8-3)}

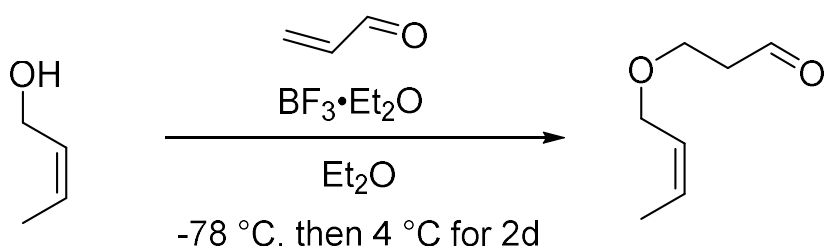

S8-3

(Z)-but-2-en-1-ol ${ }^{15}(1.37 \mathrm{~g}, 19 \mathrm{mmol}, 1 \mathrm{eq})$ was dissolved in diethyl ether $(15 \mathrm{~mL})$ and $\mathrm{BF}_{3} \cdot \mathrm{Et}_{2} \mathrm{O}$ $(0.29 \mathrm{~mL}, 2.3 \mathrm{mmol}, 0.12 \mathrm{eq})$ was added to the reaction mixture at $-78^{\circ} \mathrm{C}$ followed by the addition of acrolein ( $4.8 \mathrm{~mL}, 76.4 \mathrm{mmol}, 4 \mathrm{eq}$ ), over the period of $30 \mathrm{~min}$. After stirring for 2 days at $4{ }^{\circ} \mathrm{C}$, the reaction was quenched with pyridine $(0.6 \mathrm{~mL})$, filtered through Celite and washed with diethyl ether. After concentration in vacuo, the residue was purified by dry flash chromatography (petroleum ether/ethyl acetate 9/1) to afford $0.927 \mathrm{~g} \mathrm{(38 \% )} \mathrm{of} \mathrm{aldehyde} \mathrm{S8-3,} \mathrm{as} \mathrm{a} \mathrm{colorless} \mathrm{oil.}$

${ }^{1} \mathbf{H}$ NMR $\left(500 \mathrm{MHz}, \mathrm{CDCl}_{3}\right) \delta 9.79(\mathrm{t}, J=1.6 \mathrm{~Hz}, 1 \mathrm{H}), 5.74-5.60(\mathrm{~m}, 1 \mathrm{H}), 5.59-5.49(\mathrm{~m}, 1 \mathrm{H})$, $4.05(\mathrm{~d}, J=6.6 \mathrm{~Hz}, 2 \mathrm{H}), 3.76(\mathrm{t}, J=6.1 \mathrm{~Hz}, 2 \mathrm{H}), 2.67\left(\mathrm{td}, J^{l}=6.1, J^{2}=1.7 \mathrm{~Hz}, 2 \mathrm{H}\right), 1.66\left(\mathrm{dd}, J^{l}\right.$ $\left.=6.9, J^{2}=0.6 \mathrm{~Hz}, 3 \mathrm{H}\right)$.

${ }^{13} \mathrm{C}$ NMR $\left(125 \mathrm{MHz}, \mathrm{CDCl}_{3}\right) \delta 201.4,128.5,126.5,66.5,63.8,44.1,13.3$.

IR (ATR) $v_{\max }$ : 3440, 3023, 2928, 2865, 2731, 2159, 1725, 1655, 1454, 1376, 1338, 1238, 1103.

HRMS (ESI) calcd. for $\mathrm{C}_{7} \mathrm{H}_{13} \mathrm{O}_{2}^{+}[\mathrm{M}+\mathrm{H}]^{+}:$: 29.0910 , found 129.0907 .

\section{1,6-bis(((Z)-but-2-en-1-yl)oxy)-4-hydroxyhexan-3-one (S8-4)}

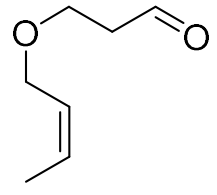

S8-3

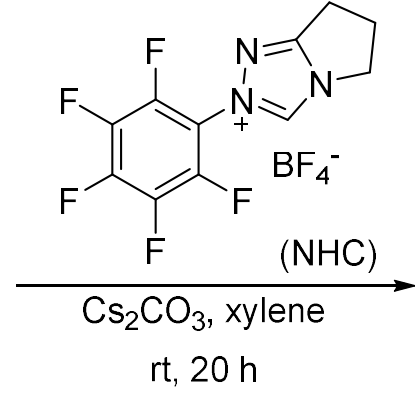

$\mathrm{rt}, 20 \mathrm{~h}$

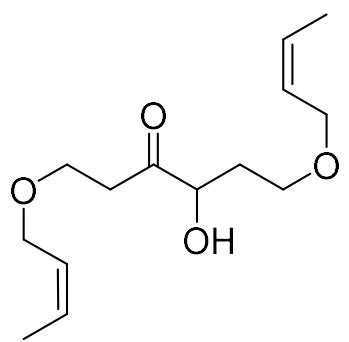

S8-4

Prepared by G.P.2. Using aldehyde S8-3 (300 mg, $2.34 \mathrm{mmol}, 1$ eq), NHC ( $25 \mathrm{mg}, 0.07 \mathrm{mmol}$, $0.03 \mathrm{eq}$ ), cesium carbonate $(23 \mathrm{mg}, 0.07 \mathrm{mmol}, 0.03 \mathrm{eq})$ and xylene $(5 \mathrm{~mL})$, during $27 \mathrm{~h}$. Dry flesh chromatography (petroleum ether/ethyl acetate $=7 / 3$ ) afforded $160.9 \mathrm{mg}(40 \%)$ of hydroxy ketone S8-4 as a colorless oil.

${ }^{15}$ (Z)-but-2-en-1-ol was prepared according to procedure from Balduzzi, S., Brook, M. A., McGlinchey, M. J. Organometallics, 2005, 24, 2617-2627. 
${ }^{1} \mathbf{H}$ NMR $\left(500 \mathrm{MHz}, \mathrm{CDCl}_{3}\right) \delta 5.71-5.58(\mathrm{~m}, 2 \mathrm{H}), 5.56-5.43(\mathrm{~m}, 2 \mathrm{H}), 4.29-4.23(\mathrm{~m}, 1 \mathrm{H}), 4.02$ $(\mathrm{d}, J=6.6 \mathrm{~Hz}, 2 \mathrm{H}), 3.98-3.93(\mathrm{~m}, 2 \mathrm{H}), 3.82-3.65(\mathrm{~m}, 3 \mathrm{H}), 3.55(\mathrm{t}, J=5.7 \mathrm{~Hz}, 2 \mathrm{H}), 2.85\left(\mathrm{dt}, J^{l}=\right.$ $\left.16.4, J^{2}=6.0 \mathrm{~Hz}, 1 \mathrm{H}\right), 2.75\left(\mathrm{dt}, J^{l}=16.4, J^{2}=6.6 \mathrm{~Hz}, 1 \mathrm{H}\right), 2.09\left(\mathrm{dt}, J^{l}=9.3, J^{2}=4.9 \mathrm{~Hz}, 1 \mathrm{H}\right)$, $1.94\left(\mathrm{dt}, J^{l}=12.7, J^{2}=6.3 \mathrm{~Hz}, 1 \mathrm{H}\right), 1.64(\mathrm{~d}, J=5.3 \mathrm{~Hz}, 3 \mathrm{H}), 1.63(\mathrm{~d}, J=5.9 \mathrm{~Hz}, 3 \mathrm{H})$.

${ }^{13} \mathrm{C}$ NMR $\left(125 \mathrm{MHz}, \mathrm{CDCl}_{3}\right) \delta 210.9,128.2,128.0,126.7(2 \times \mathrm{C}), 75.0,66.5(2 \times \mathrm{C}), 65.7,65.2$, 38.6, 33.6, $13.3(2 \times \mathrm{C})$.

IR (ATR) $v_{\max }: 3469,3023,2922,2866,1714,1660,1377,1339,1276,1104$.

HRMS (ESI) calcd. for $\mathrm{C}_{14} \mathrm{H}_{25} \mathrm{O}_{4}{ }^{+}[\mathrm{M}+\mathrm{H}]^{+}: 257.1747$, found: 257.1742 .

\section{1,6-bis(((Z)-but-2-en-1-yl)oxy)-4- oxohexan-3-yl methanesulfonate (23Z)}

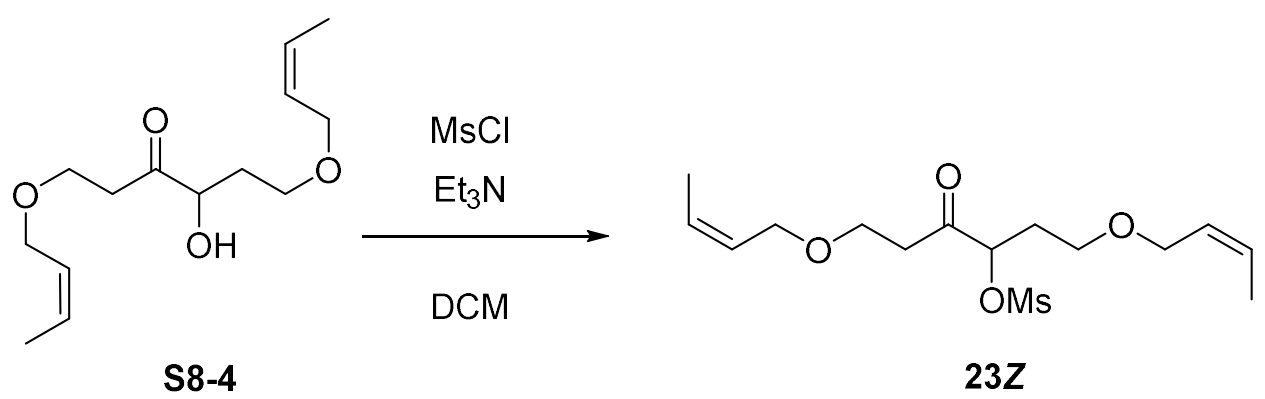

Prepared by G.P.1. Using S8-4 ( $74.3 \mathrm{mg}, 0.29 \mathrm{mmol}, 1 \mathrm{eq})$, mesyl chloride ( $45 \mu \mathrm{L}, 0.58 \mathrm{mmol}, 2$ eq), triethylamine ( $81 \mu \mathrm{L}, 0.58 \mathrm{mmol}, 2 \mathrm{eq})$, DMAP ( $3.5 \mathrm{mg}, 0.029 \mathrm{mmol}, 0.1 \mathrm{eq})$ and $\mathrm{CH}_{2} \mathrm{Cl}_{2}(1.3$ $\mathrm{mL}$ ). Dry flash chromatography (petroleum ether/ethyl acetate 7/3) afforded $72.1 \mathrm{mg}(74 \%)$ of mesylate $\mathbf{2 3 Z}$, as a colorless oil.

${ }^{1} \mathbf{H}$ NMR $\left(500 \mathrm{MHz}, \mathrm{CDCl}_{3}\right) \delta 5.70-5.61(\mathrm{~m}, 2 \mathrm{H}), 5.55-5.46(\mathrm{~m}, 2 \mathrm{H}), 5.16\left(\mathrm{dd}, J^{l}=7.8, J^{2}=\right.$ $4.1 \mathrm{~Hz}, 1 \mathrm{H}), 4.02(\mathrm{t}, J=7.1 \mathrm{~Hz}, 4 \mathrm{H}), 3.71\left(\mathrm{dt}, J^{l}=6.3, J^{2}=1.2 \mathrm{~Hz}, 2 \mathrm{H}\right), 3.60-3.50(\mathrm{~m}, 2 \mathrm{H}), 3.10$ (s, 3H), $2.88-2.76(\mathrm{~m}, 2 \mathrm{H}), 2.23-2.15(\mathrm{~m}, 1 \mathrm{H}), 2.11-2.02(\mathrm{~m}, 1 \mathrm{H}), 1.66(\mathrm{~s}, 3 \mathrm{H}), 1.64(\mathrm{~s}, 3 \mathrm{H})$. ${ }^{13} \mathbf{C}$ NMR $\left(125 \mathrm{MHz}, \mathrm{CDCl}_{3}\right) \delta 204.6,128.3,128.2,126.7,126.5,81.7,66.6,66.4,64.8,64.5$, $39.2,38.7,31.8,13.3(2 \times \mathrm{C})$.

IR (ATR) $v_{\max }: 3023,2933,2868,1729,1359,1359,1177,1103$.

HRMS (ESI) calcd. for $\mathrm{C}_{15} \mathrm{H}_{27} \mathrm{O}_{6} \mathrm{~S}^{+}[\mathrm{M}+\mathrm{H}]^{+}: 335.1523$, found: 335.1516 . 


\section{Scheme S9: Preparation of precursor 25}

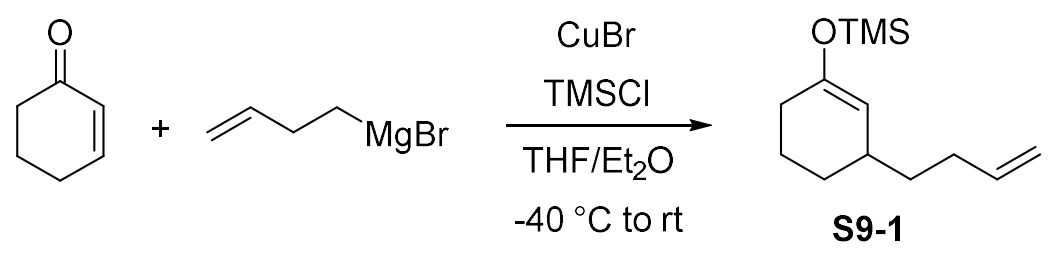<smiles>C=CCCC1C=C(O[Na])CCC1</smiles>

S9-1<smiles>C=CCCC1CCCC(=O)C1O</smiles>

S9-2<smiles>CC(C)=CCCC1CCCC(=O)C1O</smiles>

S9-3
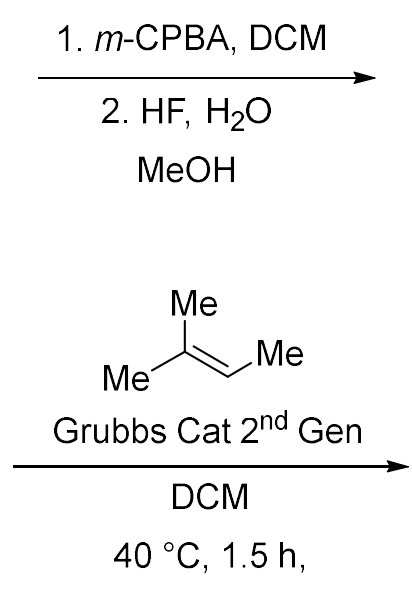<smiles>C=CCCC1CCCC(=O)C1O</smiles>

S9-2<smiles>CC(C)=CCCC1CCCC(=O)C1O</smiles>

S9-3<smiles>CCN(C)CC</smiles>

25

\section{((3-(But-3-en-1-yl)cyclohex-1-en-1-yl)oxy)trimethylsilane (S9-1)}<smiles>C=CCCN(Br)Br</smiles>

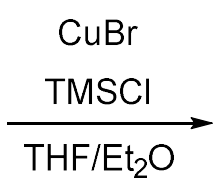

$-40^{\circ} \mathrm{C}$ to rt<smiles>C=CCCC1C=C(OS(C)(=O)=O)CCC1</smiles>

S9-1

A solution of Grignard reagent [freshly prepared from $\mathrm{Mg}(0.36 \mathrm{~g}, 14.8 \mathrm{mmol})$ and 4-bromobut1-ene $(1.3 \mathrm{~mL}, 12.8 \mathrm{mmol})$ in dry $\left.\mathrm{Et}_{2} \mathrm{O}(10.4 \mathrm{~mL})\right]$ was added dropwise to a cold $\left(-40{ }^{\circ} \mathrm{C}\right)$ suspension of dry $\mathrm{CuBr}(0.75 \mathrm{~g}, 5.23 \mathrm{mmol})$ in dry THF $(10.4 \mathrm{~mL})$ under an argon atmosphere, with stirring. At this temperature, a solution of 2-cyclohexen-1-one (1 mL, $10.3 \mathrm{mmol})$ and TMSCl (2.65 mL, $20.9 \mathrm{mmol})$ in $\mathrm{Et}_{2} \mathrm{O}(5.2 \mathrm{~mL})$ was added dropwise over $45 \mathrm{~min}$. The reaction mixture was kept at $-40{ }^{\circ} \mathrm{C}$ for additional $15 \mathrm{~min}$, then allowed to warm up to $0{ }^{\circ} \mathrm{C}$ spontaneously, when $\mathrm{Et}_{3} \mathrm{~N}(3.12 \mathrm{~mL}, 22.4 \mathrm{mmol})$ was added. The reaction mixture was poured into cold $\left(0^{\circ} \mathrm{C}\right)$ solution 
of $\mathrm{NH}_{4} \mathrm{Cl}(3.12 \mathrm{~g})$ in water $(20 \mathrm{~mL})$, the layers were separated and the aqueous layer was extracted with $\mathrm{Et}_{2} \mathrm{O}(2 \times 20 \mathrm{~mL})$. The combined organic extract was washed with saturated $\mathrm{NH}_{4} \mathrm{Cl}(10 \mathrm{~mL})$ solution ( $\mathrm{pH}$ of water layer after washing was around 7), dried over anh. $\mathrm{MgSO}_{4}$ and concentrated at rotavap. The residue was purified by short path distillation under reduced pressure to afford 1.1 g (47\%) of ((3-(but-3-en-1-yl)cyclohex-1-en-1-yl)oxy)trimethylsilane (S9-1) $\left(100-110{ }^{\circ} \mathrm{C} / 1 \mathrm{~mm}\right.$ $\mathrm{Hg}$ ), as colorless liquid that was used immediately in the next step.

\section{3-(But-3-en-1-yl)-2-hydroxycyclohexan-1-one (S9-2)}

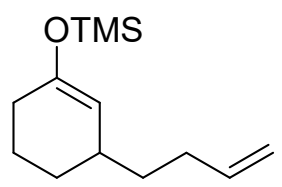

S9-1

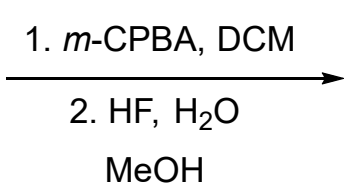

$\mathrm{MeOH}$

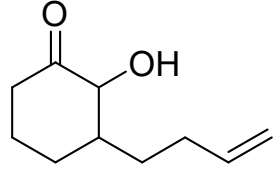

S9-2

A solution of $m$-CPBA (560 mg, 70-75\% (contains water), $2.35 \mathrm{mmol}$ ) in dichloromethane $(3.8$ $\mathrm{mL})$ was added dropwise to a cold $\left(0^{\circ} \mathrm{C}\right)$ suspension of silylenol ether $\mathbf{S 9 - 1}$ (434.3 mg, $1.94 \mathrm{mmol}$; from the previous step) and $\mathrm{NaHCO}_{3}(200 \mathrm{mg}, 2.38 \mathrm{mmol})$ in dichloromethane $(9.5 \mathrm{~mL})$. After stirring for $2 \mathrm{~h}$ at room temperature, the reaction mixture was filtered through a pad of Celite and concentrated in vacuo. The residue was dissolved in methanol $(5 \mathrm{~mL})$ and 50\% $\mathrm{HF}(0.5 \mathrm{~mL}, 14.36$ mmol) was added. After 15 min of stirring, reaction mixture was poured into a saturated $\mathrm{NaHCO}_{3}$ solution in water $(25 \mathrm{~mL})$ and extracted with ethyl acetate $(3 \times 40 \mathrm{~mL})$. The ccombined organic extract was dried over anh. $\mathrm{MgSO}_{4}$ and concentrated at rotavap. Purification of the residue by column chromatography (petroleum-ether/ethyl acetate $=7 / 1$, then toluene/ethyl acetate $=94 / 6$ ) afforded $75.6 \mathrm{mg}$ (23\%) of hydroxy ketone (S9-2), as colorless oil.

${ }^{1} \mathbf{H}$ NMR $\left(500 \mathrm{MHz}, \mathrm{CDCl}_{3}\right) \delta 5.79\left(\mathrm{ddt}, J^{l}=16.9, J^{2}=10.2, J^{3}=6.5 \mathrm{~Hz}, 1 \mathrm{H}\right), 5.01\left(\mathrm{ddd}, J^{l}=\right.$ $\left.17.1, J^{2}=3.4, J^{3}=1.6 \mathrm{~Hz}, 1 \mathrm{H}\right), 4.94\left(\mathrm{ddt}, J^{1}=10.2, J^{2}=2.1, J^{3}=1.2 \mathrm{~Hz}, 1 \mathrm{H}\right), 3.79(\mathrm{dd}, J=11.0$, $1.3 \mathrm{~Hz}, 1 \mathrm{H}), 2.57-2.50(\mathrm{~m}, 1 \mathrm{H}), 2.39-2.30(\mathrm{~m}, 1 \mathrm{H}), 2.25-2.16(\mathrm{~m}, 1 \mathrm{H}), 2.11-1.90(\mathrm{~m}, 4 \mathrm{H})$, $1.60-1.50(\mathrm{~m}, 2 \mathrm{H}), 1.45-1.36(\mathrm{~m}, 2 \mathrm{H})$.

${ }^{13}$ C NMR $\left(126 \mathrm{MHz}, \mathrm{CDCl}_{3}\right) \delta 211.3,138.6,114.8,79.3,47.5,39.3,32.3,30.8,28.8,26.1$.

IR (ATR) $v_{\max }: 3441,3074,2930,2860,1717,1430,1287,1259,1107$.

HRMS (ESI) calcd. for $\mathrm{C}_{10} \mathrm{H}_{15} \mathrm{O}^{+}\left[\mathrm{M}+\mathrm{H}-\mathrm{H}_{2} \mathrm{O}\right]^{+}$: 151.1174; found: 151.1122 . 
2-Hydroxy-3-(4-methylpent-3-en-1-yl)cyclohexan-1-one (S9-3) ${ }^{16}$

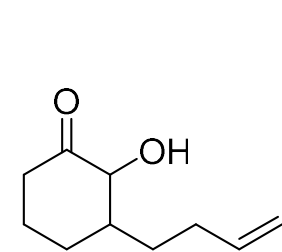

S9-2

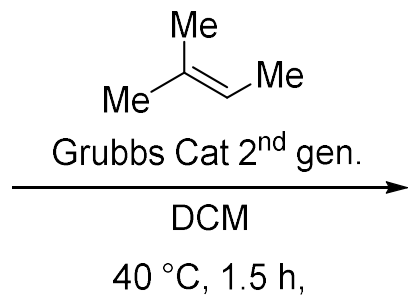

$40{ }^{\circ} \mathrm{C}, 1.5 \mathrm{~h}$,

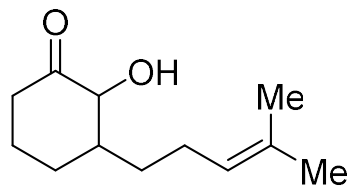

S9-3

Grubbs $2^{\text {nd }}$ generation catalyst $(13.0 \mathrm{mg}, 0.015 \mathrm{mmol})$ was added to a solution of compound S9-2 (49.4 mg, $0.294 \mathrm{mmol}$ ) and 2-methyl-2-butene $(8 \mathrm{~mL})$ in dry $\mathrm{CH}_{2} \mathrm{Cl}_{2}(8 \mathrm{~mL})$, and the reaction mixture was stirred for $1.5 \mathrm{~h}$ at $40{ }^{\circ} \mathrm{C}$, under an argon atmosphere. $\mathrm{SiO}_{2}(260 \mathrm{mg})$ was added to the reaction mixture and the volatiles were removed under reduced pressure. The residue was purified by column chromatography (petroleum-ether/ethyl acetate $=7 / 1)$ to afford $31.7 \mathrm{mg}(55 \%)$ of 2-hydroxy-3-(4-methylpent-3-en-1-yl)cyclohexan-1-one (S9-3) as a very viscous, light yellow oil.

${ }^{1}$ H NMR $\left(500 \mathrm{MHz}, \mathrm{CDCl}_{3}\right) \delta 5.11(\mathrm{t}, J=7.0 \mathrm{~Hz}, 1 \mathrm{H}), 3.79(\mathrm{~d}, J=10.7 \mathrm{~Hz}, 1 \mathrm{H}), 3.63(\mathrm{~s}, 1 \mathrm{H})$, $2.55\left(\mathrm{ddt}, J^{l}=13.7, J^{2}=4.3, J^{3}=2.1 \mathrm{~Hz}, 1 \mathrm{H}\right), 2.35\left(\mathrm{tdd}, J^{1}=13.9, J^{2}=6.5, J^{3}=1.2 \mathrm{~Hz}, 1 \mathrm{H}\right), 2.15$ $-2.05(\mathrm{~m}, 2 \mathrm{H}), 2.03-1.87(\mathrm{~m}, 3 \mathrm{H}), 1.67$ (s, 3H), $1.64-1.50(\mathrm{~m}, 2 \mathrm{H}), 1.60(\mathrm{~s}, 3 \mathrm{H}), 1.45-1.32$ $(\mathrm{m}, 2 \mathrm{H})$.

${ }^{13}$ C NMR $\left(126 \mathrm{MHz} \mathrm{CDCl}_{3}\right) \delta 211.4,131.8,124.3,79.4,47.8,39.4,33.29,29.0,26.2,25.8,25.2$, 17.8 .

IR (ATR) $v_{\max }: 3479,2928,2861,1715,1449,1381,1260,1098$.

HRMS (ESI) calcd. for $\mathrm{C}_{12} \mathrm{H}_{24} \mathrm{NO}_{2}{ }^{+}\left[\mathrm{M}+\mathrm{NH}_{4}\right]^{+}:$214.1802; found: 214.1803.

\section{2-(4-Methylpent-3-en-1-yl)-6-oxocyclohexyl methanesulfonate (25)}<smiles>CC(C)=CCCC1CCCC(=O)C1O</smiles>

S9-3

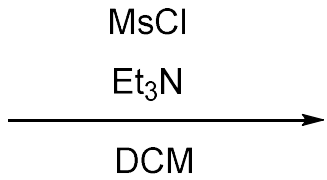

$-20{ }^{\circ} \mathrm{C}, 45 \mathrm{~min}$

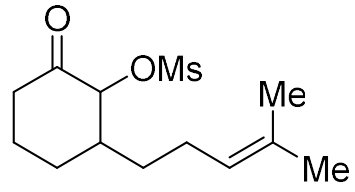

25

According to G.P.1. $\mathrm{MsCl}(20 \mu \mathrm{L}, 0.258 \mathrm{mmol})$ was added to a cold $\left(-20{ }^{\circ} \mathrm{C}\right)$ solution of hydroxy ketone S9-3 (27.3 mg, $0.139 \mathrm{mmol}$ ), DMAP (1.7 mg, $0.014 \mathrm{mmol})$ and $\mathrm{Et}_{3} \mathrm{~N}$ (60 $\left.\mu \mathrm{L}, 0.430 \mathrm{mmol}\right)$. After 45 min TLC indicated complete conversion, when $\mathrm{SiO}_{2}(150 \mathrm{mg})$ was added to the reaction mixture and the volatiles were removed under reduced pressure. The residue was purified by

${ }^{16}$ Following modified published protocol: Chatterjee A. K.; Sanders, D. P.; Grubbs, R. H. Org Lett. 2002, 4, 19391942. 
column chromatography (petroleum-ether/ethyl acetate $=2 / 1)$ to afford $34.4 \mathrm{mg}(90 \%)$ of 2-(4methylpent-3-en-1-yl)-6-oxocyclohexyl methanesulfonate (25), as a clear oil.

${ }^{1} \mathrm{H}$ NMR $\left(500 \mathrm{MHz}, \mathrm{CDCl}_{3}\right) \delta 5.10-5.02(\mathrm{~m}, 1 \mathrm{H}), 4.79(\mathrm{~d}, J=11.2 \mathrm{~Hz}, 1 \mathrm{H}), 3.21(\mathrm{~s}, 3 \mathrm{H}), 2.59$ $-2.48(\mathrm{~m}, 1 \mathrm{H}), 2.36\left(\mathrm{tdd}, J^{1}=13.9, J^{2}=6.1, J^{3}=0.8 \mathrm{~Hz}, 1 \mathrm{H}\right), 2.09\left(\mathrm{dddd}, J^{1}=22.5, J^{2}=13.2, J^{3}\right.$ $\left.=6.0, J^{4}=3.1 \mathrm{~Hz}, 3 \mathrm{H}\right), 1.97\left(\mathrm{dt}, J^{l}=22.3, J^{2}=7.8 \mathrm{~Hz}, 1 \mathrm{H}\right), 1.92-1.84(\mathrm{~m}, 1 \mathrm{H}), 1.84-1.76(\mathrm{~m}$, $1 \mathrm{H}), 1.67(\mathrm{~d}, J=0.9 \mathrm{~Hz}, 3 \mathrm{H}), 1.63-1.56(\mathrm{~m}, 1 \mathrm{H}), 1.59(\mathrm{~s}, 3 \mathrm{H}), 1.52-1.42(\mathrm{~m}, 1 \mathrm{H}), 1.42-1.33$ $(\mathrm{m}, 1 \mathrm{H})$.

${ }^{13}$ C NMR (126 MHz, $\left.\mathrm{CDCl}_{3}\right) \delta 204.1,132.4,123.6,87.0,44.3,40.7,39.6,32.5,29.4,25.8,25.3$, 24.7, 17.8 .

IR (ATR) $v_{\max }: 2938,2865,1743,1450,1352,1175,1018,971,921$.

HRMS (ESI) calcd. for $\mathrm{C}_{13} \mathrm{H}_{26} \mathrm{NO}_{4} \mathrm{~S}^{+}\left[\mathrm{M}+\mathrm{NH}_{4}\right]^{+}:$292.1577; found: 292.1580 . 


\section{Scheme S10: Preparation of precursor 27}
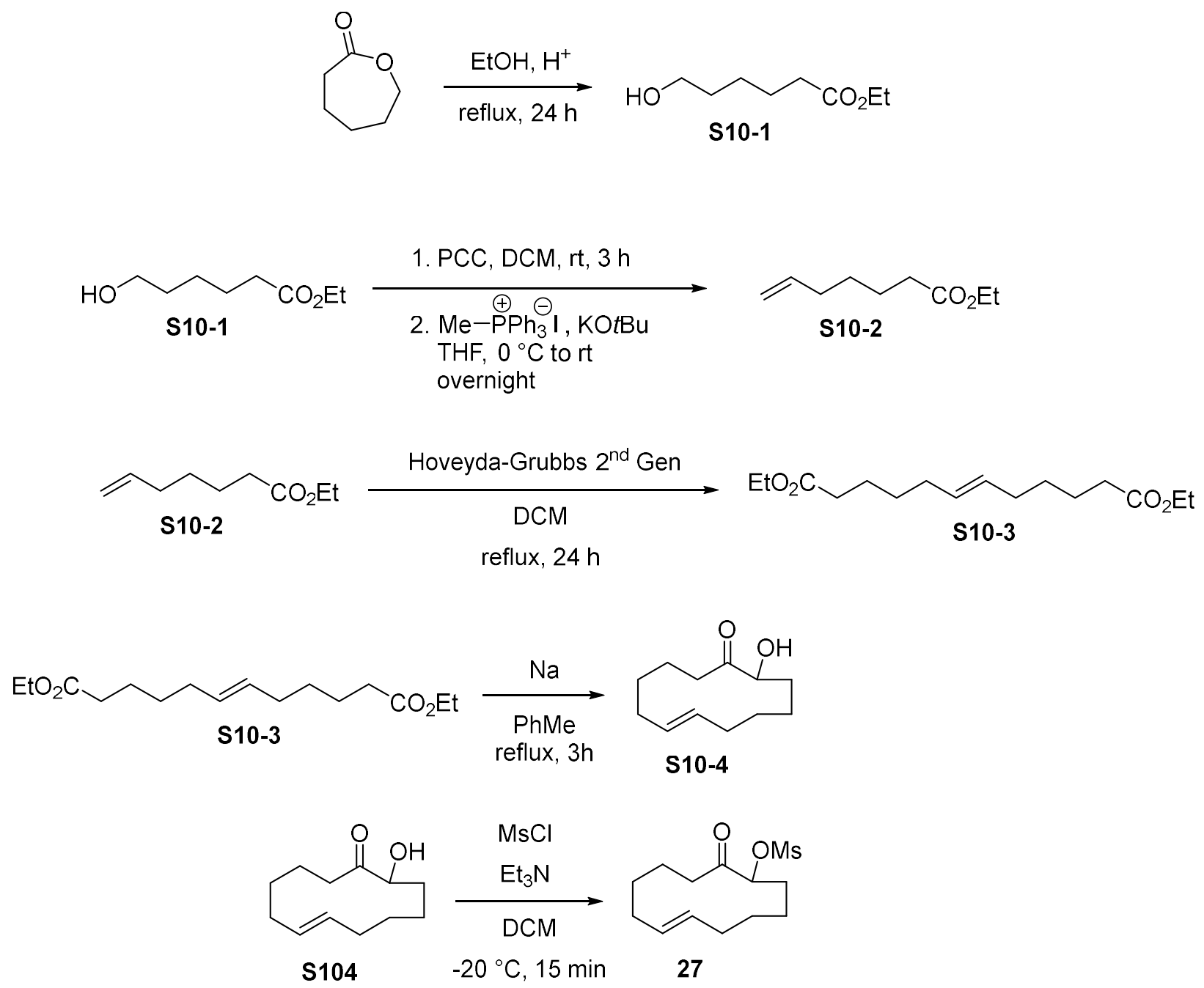

Ethyl 6-hydroxyhexanoate (S10-1) ${ }^{17}$

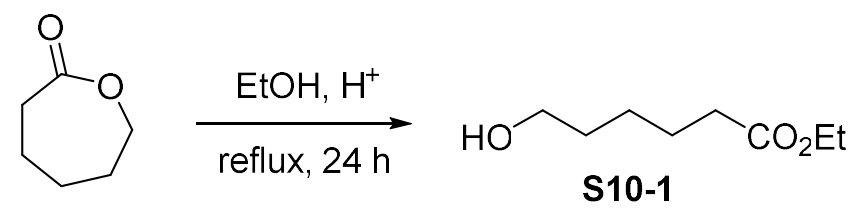

A solution of $\varepsilon$-caprolactone $(11 \mathrm{~mL}, 11.4 \mathrm{~g}, 100 \mathrm{mmol})$ and conc. $\mathrm{H}_{2} \mathrm{SO}_{4}(0.5 \mathrm{~mL})$ in absolute ethanol $(200 \mathrm{~mL})$ was refluxed for $24 \mathrm{~h}$ in the apparatus equipped with drying tube. After cooling to the room temperature, $\mathrm{NaHCO}_{3}(3.5 \mathrm{~g})$ was added with stirring, and the mixture was filtered

\footnotetext{
${ }^{17}$ Following previously reported procedure for $\delta$-valerolactone opening: Cook, C., Liron, F., Guinchard, X., Roulland, E. J. Org. Chem. 2012, 77, 6728.
} 
under reduced pressure. Ethanol was removed in vacuo, water $(70 \mathrm{~mL})$ was added and the product extracted with ethyl acetate $(3 \times 50 \mathrm{~mL})$. The combined organic extract was dried over anh. $\mathrm{MgSO}_{4}$, fitered and concentrated at rotavap. The crude product was distilled under reduced pressure to afford $13.0 \mathrm{~g}$ (81\%) of ethyl-6-hydroxyhexanoate (S10-1), as clear colorless liquid (bp. 101-102 ${ }^{\circ} \mathrm{C} / 1 \mathrm{~mm} \mathrm{Hg}$ ). NMR spectra consistent with literature. ${ }^{18}$

${ }^{1} \mathbf{H}$ NMR $\left(200 \mathrm{MHz}^{\mathrm{CDCl}} 3\right) \delta 4.10(\mathrm{q}, J=7.1 \mathrm{~Hz}, 2 \mathrm{H}), 3.62(\mathrm{t}, J=6.4 \mathrm{~Hz}, 2 \mathrm{H}), 2.29(\mathrm{t}, J=7.4$ $\mathrm{Hz}, 2 \mathrm{H}), 1.88(\mathrm{~s}, 1 \mathrm{H}), 1.74-1.47(\mathrm{~m}, 4 \mathrm{H}), 1.47-1.31(\mathrm{~m}, 2 \mathrm{H}), 1.23(\mathrm{t}, J=7.1 \mathrm{~Hz}, 2 \mathrm{H})$.

${ }^{13}$ C NMR $\left(50 \mathrm{MHz}, \mathrm{CDCl}_{3}\right) \delta 174.0,62.6,60.4,34.3,32.4,25.3,24.7,14.3$.

\section{Ethyl hept-6-enoate (S10-2)}

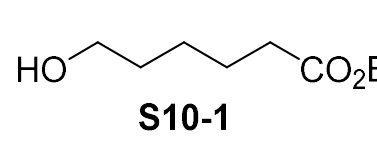

1. PCC DCM, rt $3 \mathrm{~h}$
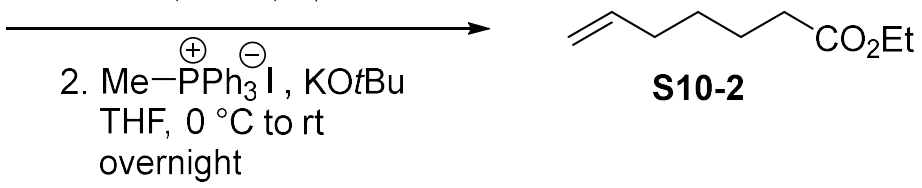

overnight

A solution of ethyl-6-hydroxyhexanoate S10-1 (1.60 g, $10 \mathrm{mmol})$ in dry dichloromethane (2 $\mathrm{mL})$ was added dropwise (over $5 \mathrm{~min}$ ) to a suspension of PCC (3.25 g, $15 \mathrm{mmol}$ ) in dry dichloromethane $(20 \mathrm{~mL})$, with stirring, under an argon atmosphere. After $3 \mathrm{~h}$, the reaction mixture was diluted with dry $\mathrm{Et}_{2} \mathrm{O}(20 \mathrm{~mL})$ and filtered through a pad of silica $(5 \mathrm{~g})$ under reduced pressure. Black residue in the flask was washed thoroughly with $\mathrm{Et}_{2} \mathrm{O}(3 \times 10 \mathrm{~mL})$ and filtered each time. The combined filtrate was carefully concentrated at rotavap, to give a crude aldehyde as yellow liquid $(1.57 \mathrm{~g}$, $99 \%$ ). The crude product was used immediately in the next step, without further purification. ${ }^{19}$

$\mathrm{KO}$-Bu $(1.4 \mathrm{~g}, 12.2 \mathrm{mmol})$ was added in portions to a cold $\left(0^{\circ} \mathrm{C}\right)$ suspension of $\mathrm{PPh}_{3} \mathrm{MeI}(5.1 \mathrm{~g}$, $12.5 \mathrm{mmol})$ in THF $(50 \mathrm{~mL})$, with stirring, under an argon atmosphere. The suspension turned yellow, and after 45 minutes of stirring at $0{ }^{\circ} \mathrm{C}$, a solution of aldehyde $(1.57 \mathrm{~g}, 10 \mathrm{mmol})$ in dry THF ( $5 \mathrm{~mL}$ ) was added dropwise, via syringe, over 5 minutes. Stirring was continued overnight, allowing reaction mixture to spontaneously warm to the room temperature. The reaction was quenched by the addition of $\mathrm{NH}_{4} \mathrm{Cl}_{\text {(sat.) }}(70 \mathrm{~mL})$, and the product was extracted with $\mathrm{Et}_{2} \mathrm{O}(3 \times 25$ $\mathrm{mL})$. The combined organic extract was washed with brine $(50 \mathrm{~mL})$, dried over anh. $\mathrm{MgSO}_{4}$, filtered and concentrated at rotavap. The residue was purified by dry-flash chromatography (petroleum-ether/ethyl acetate $=10 / 1)$ to afford $1.00 \mathrm{~g}(64 \%$ over two steps $)$ of ethyl hept-6-enoate S10-2, as colorless oil. NMR spectra consistent with literature. ${ }^{20}$

\footnotetext{
18 Terent'ev, A. O., Platonov, M. M.,Kutkin, A. V. Central European Journal of Chemistry, 2006, 4, $207-215$.

${ }^{19}$ The crude product could be distilled under reduced pressure $\left(108{ }^{\circ} \mathrm{C} / 8 \mathrm{~mm} \mathrm{Hg}\right)$ to give $1.0 \mathrm{~g}(64 \%)$ of aldehyde. However, this distillation only reduces the amount of aldehyde (due to its volatility), and does not improve the yield in the subsequent Wittig reaction, or the overall yield of alkene formation.

${ }^{20}$ Phapale, V. B., Bunuel, E., Garcia-Iglesias, M., Cardenas, D. J. Angew. Chem. Int. Ed. 2007, 46, 8790-8795.
} 
${ }^{1} \mathbf{H}$ NMR $\left(500 \mathrm{MHz}, \mathrm{CDCl}_{3}\right) \delta 5.78\left(\mathrm{ddt}, J^{l}=16.9, J^{2}=10.2, J^{3}=6.7 \mathrm{~Hz}, 1 \mathrm{H}\right), 5.06-4.89(\mathrm{~m}$, $2 \mathrm{H}), 4.11(\mathrm{q}, J=7.1 \mathrm{~Hz}, 2 \mathrm{H}), 2.29\left(\mathrm{t}, J^{l}=7.5 \mathrm{~Hz}, 2 \mathrm{H}\right), 2.06(\mathrm{q}, J=7.1 \mathrm{~Hz}, 2 \mathrm{H}), 1.63\left(\mathrm{dt}, J^{l}=\right.$ $\left.15.4, J^{2}=7.6 \mathrm{~Hz}, 2 \mathrm{H}\right), 1.48-1.34(\mathrm{~m}, 2 \mathrm{H}), 1.24(\mathrm{t}, J=7.1 \mathrm{~Hz}, 3 \mathrm{H})$.

${ }^{13} \mathrm{C}$ NMR $\left(126 \mathrm{MHz}, \mathrm{CDCl}_{3}\right) \delta 173.8,138.6,114.8,60.3,34.3,33.5,28.5,24.6,14.4$.

\section{(E)-Diethyl dodec-6-enedioate (S10-3)}

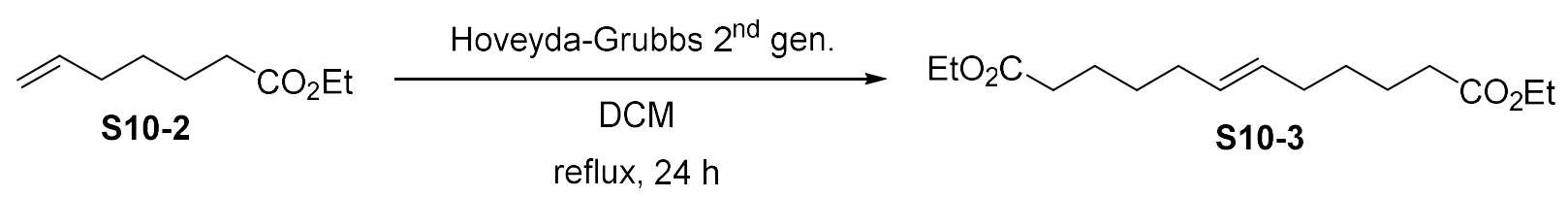

A solution of ethyl hept-6-enoate $\mathbf{S 1 0 - 2}(1.00 \mathrm{~g}, 6.4 \mathrm{mmol})$ and Hoveyda-Grubbs $2^{\text {nd }}$ generation catalyst $(21.0 \mathrm{mg}, 0.033 \mathrm{mmol})$ in dry dichloromethane $(52 \mathrm{~mL})$ was refluxed under an argon atmosphere, for $24 \mathrm{~h}$, when TLC showed complete consumption of starting material. Reaction mixture was cooled to room temperature, silica ( $3 \mathrm{~g})$ was added and the solvent is removed at rotavap. The residue was purified by dry-flash chromatography (petroleum-ether/ethyl acetate $=$ 6/1) to afford (E)-diethyl dodec-6-enedioate (S10-3), as a clear liquid (530 mg, 58\%). Mixture of $E / Z$ isomers in a relative ratio $10 / 1$, as determined by ${ }^{1} \mathrm{H}$ NMR.

Spectral data for the major isomer:

${ }^{1} \mathbf{H}$ NMR $\left(500 \mathrm{MHz}, \mathrm{CDCl}_{3}\right) \delta 5.42-5.30(\mathrm{~m}, 2 \mathrm{H}), 4.10(\mathrm{q}, J=7.1 \mathrm{~Hz}, 4 \mathrm{H}), 2.26(\mathrm{t}, J=7.5 \mathrm{~Hz}$, $4 \mathrm{H}), 2.06-1.92(\mathrm{~m}, 4 \mathrm{H}), 1.63\left(\mathrm{ddt}, J^{l}=30.7, J^{2}=15.4, J^{3}=7.6 \mathrm{~Hz}, 4 \mathrm{H}\right), 1.40-1.31(\mathrm{~m}, 4 \mathrm{H})$, $1.23(\mathrm{t}, J=7.1 \mathrm{~Hz}, 6 \mathrm{H})$.

${ }^{13}$ C NMR $\left(126 \mathrm{MHz}, \mathrm{CDCl}_{3}\right) \delta 173.9,130.3,60.2,34.3,32.2,29.1,24.6,14.3$.

IR (ATR) $v_{\text {max }}$ 2981, 2934, 2858, 1736, 1458, 1372, 1346, 1180, 1100, 1032, 970.

HRMS (ESI) calcd. for $\mathrm{C}_{16} \mathrm{H}_{28} \mathrm{NaO}_{4}{ }^{+}[\mathrm{M}+\mathrm{Na}]^{+}: 307.1880$; found: 307.1878 .

\section{(E)-12-Hydroxycyclododec-6-enone (S10-4)}

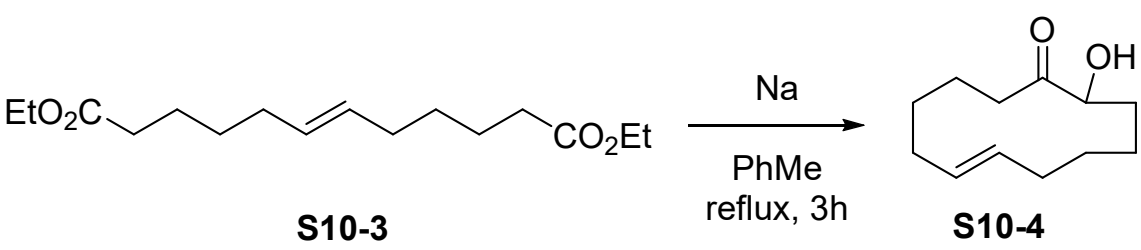

A solution of (E)-diethyl dodec-6-enedioate $\mathbf{S 1 0 - 3}(155 \mathrm{mg}, 0.545 \mathrm{mmol})$ in dry toluene $(0.4 \mathrm{~mL})$ was slowly added dropwise (over $2.5 \mathrm{~h}$ period) to a suspension of sodium ( $77 \mathrm{mg}, 3.35 \mathrm{mmol}$ ) in dry toluene $(3.6 \mathrm{~mL})$, heated to reflux $\left(130^{\circ} \mathrm{C}\right.$ oil bath), with vigorous stirring, under an argon atmosphere. After additional $30 \mathrm{~min}$ of stirring and heating, reaction mixture was cooled to $0{ }^{\circ} \mathrm{C}$, quenched by addition of $\mathrm{AcOH}(0.5 \mathrm{~mL})$, and water $(5 \mathrm{~mL})$ was added to dissolve generated salts. 
The reaction mixture was extracted with toluene $(2 \times 5 \mathrm{~mL})$, the combined organic extract was washed with brine $(4 \mathrm{~mL})$, dried over anh. $\mathrm{MgSO}_{4}$, filtered and concentrated under reduced pressure. The residue was purified by column chromatography (petroleum-ether/ethyl acetate $=$ 5/1) to afford $21 \mathrm{mg}(20 \%)$ of $(E)$-12-hydroxycyclododec-6-enone (S10-4), as colorless oil.

Spectral data for the major isomer:

${ }^{1}$ H NMR $\left(500 \mathrm{MHz}, \mathrm{CDCl}_{3}\right) \delta 5.47-5.38(\mathrm{~m}, 1 \mathrm{H}), 5.22-5.15(\mathrm{~m}, 1 \mathrm{H}), 4.36-4.25(\mathrm{~m}, 1 \mathrm{H}), 3.60$ $(\mathrm{d}, J=4.4 \mathrm{~Hz}, 1 \mathrm{H}), 2.90\left(\mathrm{ddd}, J^{l}=17.6, J^{2}=9.9, J^{3}=4.4 \mathrm{~Hz}, 1 \mathrm{H}\right), 2.22-2.06(\mathrm{~m}, 4 \mathrm{H}), 2.05-$ $1.81(\mathrm{~m}, 3 \mathrm{H}), 1.76-1.60(\mathrm{~m}, 2 \mathrm{H}), 1.59-1.41(\mathrm{~m}, 3 \mathrm{H}), 1.38-1.25(\mathrm{~m}, 2 \mathrm{H}), 0.82-0.70(\mathrm{~m}, 1 \mathrm{H})$. ${ }^{13} \mathbf{C}$ NMR $\left(126 \mathrm{MHz}, \mathrm{CDCl}_{3}\right) \delta 213.3,132.0,130.8,76.4,36.8,33.8,30.2,30.0,26.0,24.7,22.1$, 20.0 .

IR (ATR) $v_{\max }: 3478,2928,2855,1710,1453,1402,1366,1100,982$.

HRMS (ESI) calcd. for $\mathrm{C}_{12} \mathrm{H}_{20} \mathrm{NaO}_{2}{ }^{+}[\mathrm{M}+\mathrm{Na}]^{+}: 219.1356$; found: 219.1359 .

\section{(E)-12-Oxocyclododec-6-en-1-yl methanesulfonate (27)}

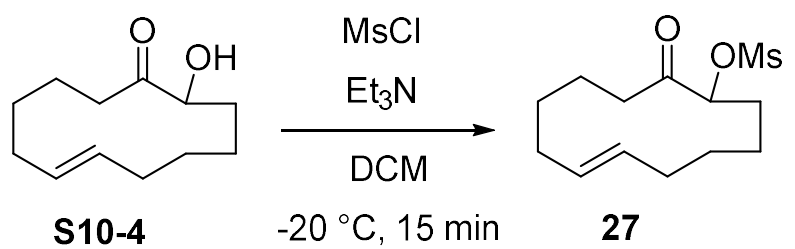

According to G.P.1. $\mathrm{MsCl}(33 \mu \mathrm{L}, 0.426 \mathrm{mmol})$ was added to a cold $\left(-20^{\circ} \mathrm{C}\right)$ solution of $(E)-12-$ hydroxycyclododec-6-enone S10-4 (41 mg, $0.209 \mathrm{mmol})$, DMAP (1 mg, $0.008 \mathrm{mmol})$ and $\mathrm{Et}_{3} \mathrm{~N}$ $(0.10 \mathrm{~mL}, 0.715 \mathrm{mmol})$. After $10 \mathrm{~min}$ TLC indicated complete conversion. $\mathrm{SiO}_{2}(300 \mathrm{mg})$ was added to the reaction mixture and the volatiles were removed under reduced pressure. The residue was purified by column chromatography (petroleum-ether/ethyl acetate $=5 / 1$ ) to afford $40 \mathrm{mg}$ (70\%) of (E)-12-oxocyclododec-6-en-1-yl methanesulfonate (27), as colorless oil.

Spectral data for the major isomer:

${ }^{1}$ H NMR $\left(500 \mathrm{MHz}, \mathrm{CDCl}_{3}\right) \delta 5.51-5.41(\mathrm{~m}, 1 \mathrm{H}), 5.29-5.20(\mathrm{~m}, 1 \mathrm{H}), 5.12\left(\mathrm{dd}, J^{1}=5.6, J^{2}=4.3\right.$ $\mathrm{Hz}, 1 \mathrm{H}), 3.12(\mathrm{~s}, 3 \mathrm{H}), 2.77-2.66(\mathrm{~m}, 1 \mathrm{H}), 2.41-2.31(\mathrm{~m}, 1 \mathrm{H}), 2.18-2.00(\mathrm{~m}, 5 \mathrm{H}), 1.95-1.84$ (m, 1H), $1.81-1.72(\mathrm{~m}, 1 \mathrm{H}), 1.65-1.36(\mathrm{~m}, 6 \mathrm{H}), 1.19-1.08(\mathrm{~m}, 1 \mathrm{H})$.

${ }^{13}$ C NMR $\left(126 \mathrm{MHz}, \mathrm{CDCl}_{3}\right) \delta 205.8,132.6,130.6,84.2,39.3,37.52,33.3,30.0,28.8,26.3,24.5$, 22.1, 20.6.

IR (ATR) $v_{\max }: 3026,2934,2857,1730,1453,1357,1175,978,954$.

HRMS (ESI) calcd. for $\mathrm{C}_{13} \mathrm{H}_{22} \mathrm{NaO}_{4} \mathrm{~S}^{+}[\mathrm{M}+\mathrm{Na}]^{+}:$297.1136; found: 297.1143 . 


\section{Scheme S11: Preparation of precursor 29}
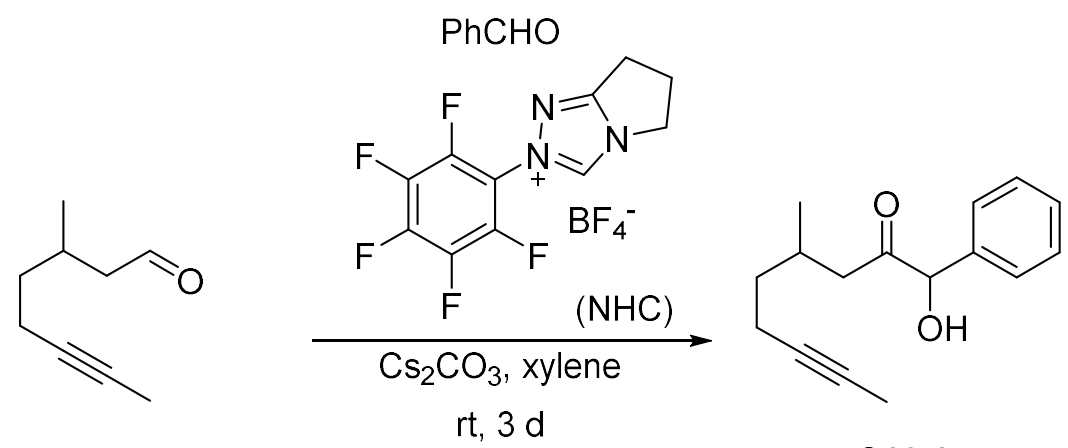

S11-1<smiles>CC#CCCC(C)CC(=O)C(O)c1ccccc1</smiles>

S11-1

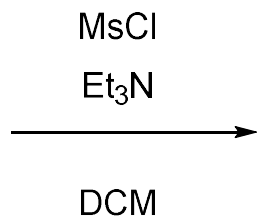<smiles>CC#CCCC(C)CC(=O)C(OC)c1ccccc1</smiles>

29

\section{1-Hydroxy-4-methyl-1-phenylnon-7-yn-2-one (S11-1)}
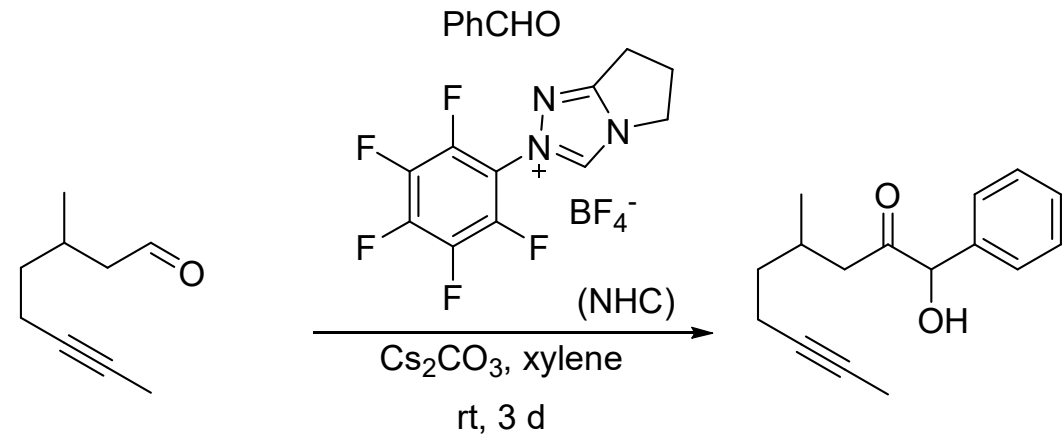

S11-1

Prepared by G.P.2, using 3-methyloct-6-ynal ${ }^{21}(540 \mathrm{mg}, 3.9 \mathrm{mmol}, 5 \mathrm{eq})$, benzaldehyde ( $80 \mu \mathrm{L}$, $0.78 \mathrm{mmol}, 1 \mathrm{eq})$, NHC (28 mg, $0.078 \mathrm{mmol}, 0.1 \mathrm{eq})$, cesium carbonate $(25.4 \mathrm{mg}, 0.078 \mathrm{mmol}$, $0.1 \mathrm{eq})$ and xylene $(1.6 \mathrm{~mL})$, during 3 days. Compared to work up in G.P.2, additional washing of the reaction mixture with sat. $\mathrm{NaHCO}_{3}$ was applied. Purification by dry-flash chromatography (petroleum-ether/ethyl acetate $=925 / 75$ ) followed by column chromatography (petroleum-

${ }^{21}$ Gao, P., Xu, P. F.,Zhai, H., J. Org. Chem. 2009, 74, 2592-2593. 
ether/ethyl acetate $=85 / 15)$ afforded $56.2 \mathrm{mg}(30 \%)$ of hydroxy ketone S11-1, as a colorless oil (mixture of isomers in a relative ratio of $\mathrm{H} / \mathrm{H}^{\prime}=1.1: 1$, as determined by ${ }^{1} \mathrm{H} \mathrm{NMR}$ ).

Spectral data for mixture of isomers in a ratio $\mathrm{H}: \mathrm{H}^{\prime}=1.1: 1$

${ }^{1} \mathbf{H}$ NMR $\left(500 \mathrm{MHz}, \mathrm{CDCl}_{3}\right) \delta 7.41-7.29$ (m, 5H+5H'), 5.08 (s, 1H'), 5.04 (s, 1H), 4.35 (bs, $1 \mathrm{H}), 2.45-2.37\left(\mathrm{~m}, 1 \mathrm{H}^{\prime}\right), 2.30-2.19(\mathrm{~m}, 2 \mathrm{H}), 2.30-2.19(\mathrm{~m}, 2 \mathrm{H}), 2.17-1.95\left(\mathrm{~m}, 3 \mathrm{H}+4 \mathrm{H}^{\prime}\right)$, $1.77\left(\mathrm{t}, J=2.5 \mathrm{~Hz}, 3 \mathrm{H}^{\prime}\right), 1.73(\mathrm{t}, J=2.5 \mathrm{~Hz}, 3 \mathrm{H}), 1.45-1.28\left(\mathrm{~m}, 1 \mathrm{H}+2 \mathrm{H}^{\prime}\right), 1.27-1.15(\mathrm{~m}, 1 \mathrm{H})$, $0.86(\mathrm{~d}, J=6.7 \mathrm{~Hz}, 3 \mathrm{H}), 0.71\left(\mathrm{~d}, J=6.3 \mathrm{~Hz}, 3 \mathrm{H}^{\prime}\right)$.

${ }^{13}$ C NMR (125 MHz, $\left.\mathrm{CDCl}_{3}\right) \delta 209.0,208.9,137.9,129.0,128.9,128.7,127.4,80.2,79.7,78.4$, 75.9, 75.8, 44.8, 44.5, 35.7, 35.5, 28.5, 28.3, 19.3, 19.0, 16.3, 16.2, $3.4(2 \times \mathrm{C})$.

IR (ATR) $v_{\max }: 3463,3062,3031,2958,2922,2872,1713,1493,1454,1372$.

HRMS (ESI) calcd. for $\mathrm{C}_{16} \mathrm{H}_{20} \mathrm{O}_{2} \mathrm{Na}^{+}[\mathrm{M}+\mathrm{Na}]^{+}:$267.1355, found: 267.1349 .

\section{4-Methyl-2-oxo-1-phenylnon-7-ynyl methanesulfonate (29)}

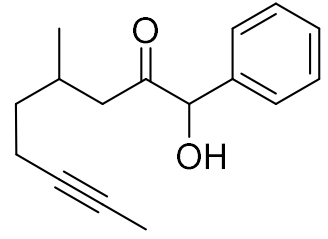

S11-1

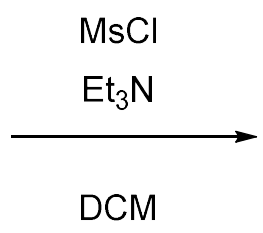

DCM

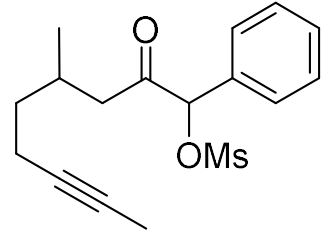

29

Prepared by G.P.1 using S11-1 (89.6 mg, 0.367 mmol, 1 eq), mesyl chloride ( $57 \mu \mathrm{L}, 0.73 \mathrm{mmol}$, 2 eq), triethylamine (153 $\mu \mathrm{L}, 1.1 \mathrm{mmol}, 3 \mathrm{eq})$, DMAP (4.4 mg, $0.037 \mathrm{mmol}, 0.1 \mathrm{eq})$ and $\mathrm{CH}_{2} \mathrm{Cl}_{2}$ $(4 \mathrm{~mL})$. Dry-flash chromatography (petroleum-ether/ethyl acetate $=85 / 15$ ) afforded $99.3 \mathrm{mg}$ (84\%) of compound 29, as a colorless oil (mixture of isomers in a relative ratio 1.5:1, as determined by ${ }^{1} \mathrm{H}$ NMR).

Spectral data for mixture of isomers in a ratio $\mathrm{H}^{\prime} \mathrm{H}^{\prime}=1.2: 1$

${ }^{1} \mathbf{H}$ NMR (500 MHz, $\left.\mathrm{CDCl}_{3}\right) \delta 7.47-7.37\left(\mathrm{~m}, 5 \mathrm{H}+5 \mathrm{H}^{\prime}\right), 5.91\left(\mathrm{~s}, 1 \mathrm{H}^{\prime}\right), 5.88(\mathrm{~s}, 1 \mathrm{H}), 3.04(\mathrm{~s}$, $\left.3 \mathrm{H}+3 \mathrm{H}^{\prime}\right), 2.49\left(\mathrm{dd}, J=16.7,4.7 \mathrm{~Hz}, 1 \mathrm{H}^{\prime}\right), 2.41(\mathrm{dd}, J=17.1,5.6 \mathrm{~Hz}, 1 \mathrm{H}), 2.32(\mathrm{dd}, J=17.1,7.8$ $\mathrm{Hz}, 1 \mathrm{H}), 2.23\left(\mathrm{dd}, J=16.7,8.5 \mathrm{~Hz}, 1 \mathrm{H}^{\prime}\right), 2.19-1.98\left(\mathrm{~m}, 3 \mathrm{H}+3 \mathrm{H}^{\prime}\right), 1.76\left(\mathrm{t}, J=2.6 \mathrm{~Hz}, 3 \mathrm{H}^{\prime}\right), 1.74$ $(\mathrm{t}, J=2.5 \mathrm{~Hz}, 3 \mathrm{H}), 1.45-1.36\left(\mathrm{~m}, 1 \mathrm{H}^{2} 1 \mathrm{H}^{\prime}\right), 1.36-1.20\left(\mathrm{~m}, 1 \mathrm{H}+1 \mathrm{H}^{\prime}\right), 0.84(\mathrm{~d}, J=6.7 \mathrm{~Hz}, 3 \mathrm{H})$, $0.77\left(\mathrm{~d}, J=6.6 \mathrm{~Hz}, 3 \mathrm{H}^{\prime}\right)$.

${ }^{13}$ C NMR $\left(125 \mathrm{MHz}, \mathrm{CDCl}_{3}\right) \delta 202.5,202.3,132.2,132.1,130.1,129.3,128.2,128.1,85.7,85.3$, 78.4, 75.9, 75.8, 45.5, 45.4, 39.5, 39.4, 35.6, 35.5, 28.0, 27.9, 19.1, 19.0, 16.3, 16.2, $3.4(2 \times \mathrm{C})$.

IR (ATR) $v_{\max }: 3032,2925,2852,1733,1495,1455,1361$.

HRMS (ESI) calcd. for $\mathrm{C}_{17} \mathrm{H}_{26} \mathrm{O}_{4} \mathrm{SN}\left[\mathrm{M}+\mathrm{NH}_{4}\right]^{+}: 340.1577$, found: 340.1567 


\section{Scheme S12: Preparation of precursor 32}

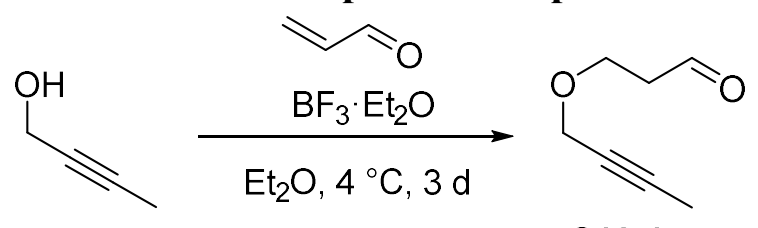

S12-1

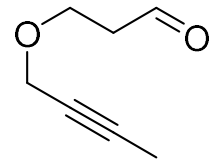

S12-1

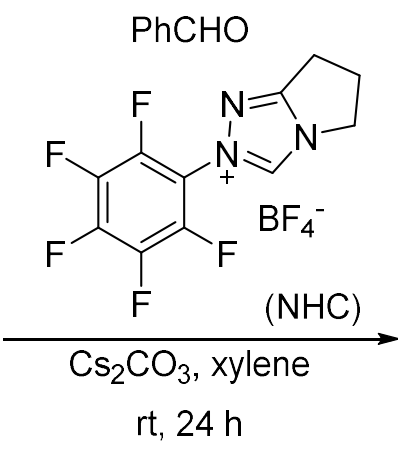

rt, $24 \mathrm{~h}$<smiles>CC#CCOCCC(=O)C(O)c1ccccc1</smiles>

S12-2<smiles>CC#CCOCCC(=O)C(O)c1ccccc1</smiles>

S12-2

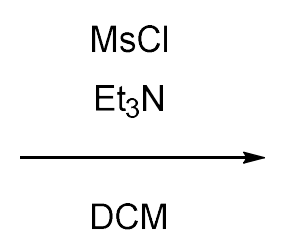

$-20^{\circ} \mathrm{C},<1 \mathrm{~min}$<smiles>CC#CCOCCC(=O)C(OC)c1ccccc1</smiles>

32

\section{3-(But-2-ynyloxy)propanal (S12-1) $)^{22}$}

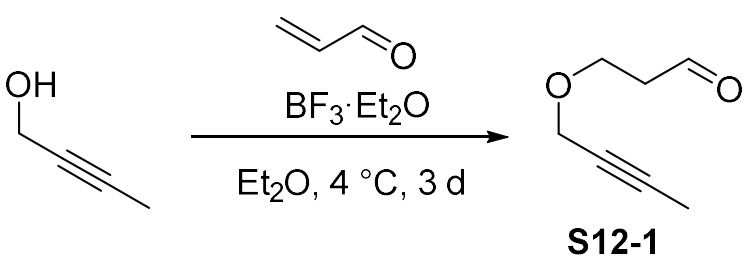

$\mathrm{BF}_{3} \cdot \mathrm{Et}_{2} \mathrm{O}(0.6 \mathrm{~mL}, 4.7 \mathrm{mmol}, 0.12 \mathrm{eq})$ was added to a cold $\left(-78^{\circ} \mathrm{C}\right)$ solution of 2-butin-1-ol (2.6 $\mathrm{mL}, 34.8 \mathrm{mmol}, 1 \mathrm{eq})$ in diethyl ether $(15 \mathrm{~mL})$, followed by the addition of acrolein $(10.2 \mathrm{~mL}$, $162.5 \mathrm{mmol}, 4 \mathrm{eq})$, over the period of $30 \mathrm{~min}$. After stirring for 3 days at $4{ }^{\circ} \mathrm{C}$, the reaction mixture was quenched with pyridine $(0.8 \mathrm{~mL})$, filtered through Celite and washed with diethyl ether. After concentration in vacuo, the residue was purified by dry-flash chromatography (petroleumether/ethyl acetate $=925 / 75$ ) and column chromatography (petroleum-ether/ethyl acetate $=75 / 25$ ) to afford $1.15 \mathrm{~g}(26 \%)$ of aldehyde $\mathbf{S 1 2 - 1}$, as a colorless oil.

${ }^{22}$ Prepared according to the procedure: Carless, H. A. J., Swan, D. I., Haywood, D. J., Tetrahedron, 1993. 49, 1665. 
${ }^{1} \mathbf{H}$ NMR $\left(500 \mathrm{MHz}, \mathrm{CDCl}_{3}\right) \delta 9.73(\mathrm{t}, J=1.8 \mathrm{~Hz}, 1 \mathrm{H}), 4.06\left(\mathrm{dd}, J^{l}=4.6, J^{2}=2.3 \mathrm{~Hz}, 2 \mathrm{H}\right), 3.78$ (t, $J=6.1 \mathrm{~Hz}, 2 \mathrm{H}) 2.63\left(\mathrm{td}, J^{1}=6.1, J^{2}=1.8 \mathrm{~Hz}, 2 \mathrm{H}\right), 1.80(\mathrm{t}, J=2.3 \mathrm{~Hz}, 3 \mathrm{H})$.

${ }^{13} \mathbf{C}$ NMR $\left(125 \mathrm{MHz}, \mathrm{CDCl}_{3}\right) \delta 200.8,82.6,74.6,63.2,58.7,43.5,3.4$.

IR (ATR) $v_{\max }: 2859,2735,1724,1445,1394,1358,1273$.

HRMS (ESI) calcd. for $\mathrm{C}_{7} \mathrm{H}_{10} \mathrm{O}_{2} \mathrm{Na}^{+}[\mathrm{M}+\mathrm{Na}]^{+}:$149.0573, found 149.0570 .

\section{4-(But-2-ynyloxy)-1-hydroxy-1-phenylbutan-2-one (S12-2)}

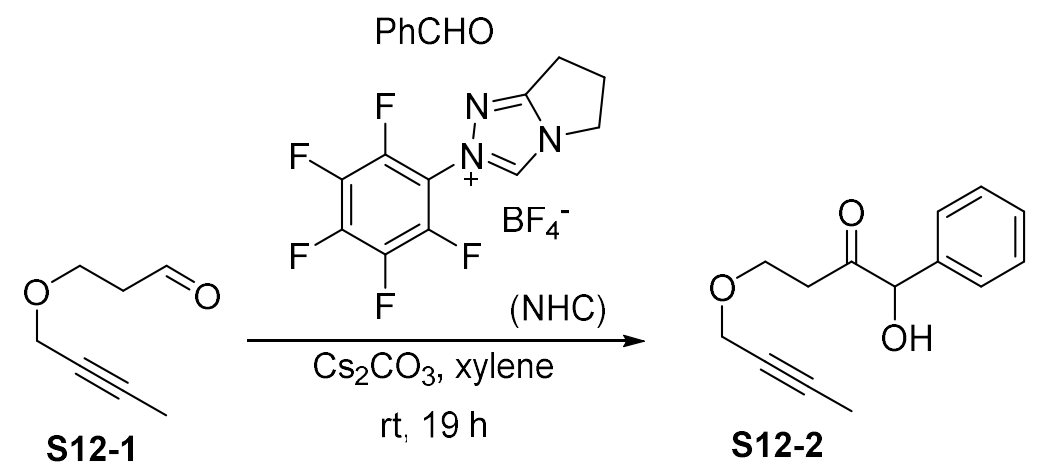

Prepared by G.P.2, using aldehyde S12-1 (312 mg, $2.47 \mathrm{mmol}, 5$ eq), benzaldehyde (50 $\mu \mathrm{L}, 0.49$ mmol, 1 eq), NHC (17.8 mg, $0.049 \mathrm{mmol}, 0.1 \mathrm{eq})$, cesium carbonate (16 mg, $0.049 \mathrm{mmol}, 0.1 \mathrm{eq})$ and xylene $(1 \mathrm{~mL})$, during $19 \mathrm{~h}$. Two consecutive column chromatographies (petroleumether/ethyl acetate $=75 / 25$ and petroleum-ether/acetone $=8 / 2)$ afforded $74 \mathrm{mg}(65 \%)$ of hydroxy ketone $\mathbf{S 1 2 - 2}$, as a colorless oil.

${ }^{1}$ H NMR $\left(500 \mathrm{MHz}, \mathrm{CDCl}_{3}\right) \delta 7.40-7.30(\mathrm{~m}, 5 \mathrm{H}), 5.13(\mathrm{~d}, J=4.1 \mathrm{~Hz}, 1 \mathrm{H}), 4.28(\mathrm{~d}, J=4.1 \mathrm{~Hz}$, $1 \mathrm{H}), 4.06\left(\mathrm{ddd}, J^{l}=15.3, J^{2}=4.7, J^{3}=2.3 \mathrm{~Hz}, 1 \mathrm{H}\right), 4.01\left(\mathrm{ddd}, J^{1}=15.3, J^{2}=4.7, J^{3}=2.3 \mathrm{~Hz}\right.$, $1 \mathrm{H}), 3.76-3.70(\mathrm{~m}, 1 \mathrm{H}), 3.68-3.63(\mathrm{~m}, 1 \mathrm{H}), 2.73\left(\mathrm{ddd}, J^{l}=16.7, J^{2}=7.2, J^{3}=6.3 \mathrm{~Hz}, 1 \mathrm{H}\right)$, $2.52\left(\mathrm{dt}, J^{l}=16.7, J^{2}=5.9 \mathrm{~Hz}, 1 \mathrm{H}\right), 1.84(\mathrm{t}, J=2.3 \mathrm{~Hz}, 3 \mathrm{H})$.

${ }^{13} \mathbf{C}$ NMR $\left(125 \mathrm{MHz}, \mathrm{CDCl}_{3}\right) \delta 207.7,137.6,129.0,128.7,127.5,82.8,80.1,74.6,64.4,58.8$, 38.2, 3.5.

IR (ATR) $v_{\max }: 3455,3062,3031,2917,2872,1718,1601,1493,1452,1360$.

HRMS (ESI) calcd. for $\mathrm{C}_{14} \mathrm{H}_{17} \mathrm{O}_{3}{ }^{+}[\mathrm{M}+\mathrm{H}]^{+}: 233.1172$, found: 233.1171 . 


\section{4-(But-2-ynyloxy)-2-oxo-1-phenylbutyl methanesulfonate (32)}

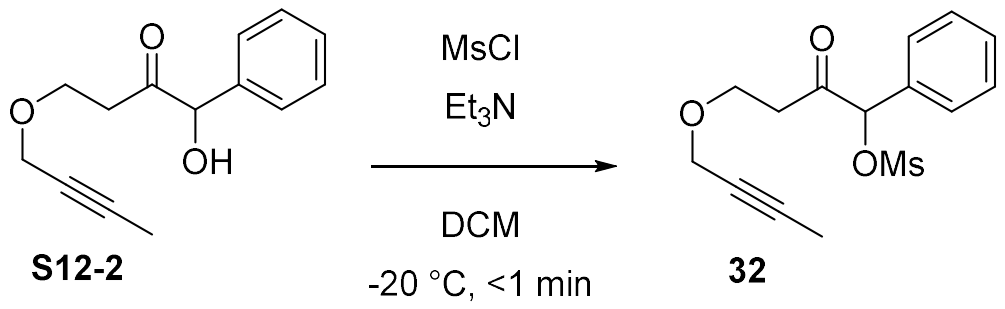

Prepared by G.P.1, using S12-2 (15 mg, $0.065 \mathrm{mmol}, 1 \mathrm{eq})$, mesyl chloride (10 $\mu \mathrm{L}, 0.123 \mathrm{mmol}$, $2 \mathrm{eq}$ ), triethylamine ( $27 \mu \mathrm{L}, 0.194 \mathrm{mmol}, 3 \mathrm{eq})$, DMAP $(0.8 \mathrm{mg}, 0.006 \mathrm{mmol}, 0.1 \mathrm{eq})$ and $\mathrm{CH}_{2} \mathrm{Cl}_{2}$ $(0.5 \mathrm{~mL})$. Column chromatography (petroleum-ether/acetone $=8 / 2)$ afforded $16.8 \mathrm{mg}(88 \%)$ of mesylate 32, as a colorless oil.

${ }^{1} \mathbf{H}$ NMR $\left(500 \mathrm{MHz}, \mathrm{CDCl}_{3}\right) \delta 7.46-7.38(\mathrm{~m}, 5 \mathrm{H}), 5.98(\mathrm{~s}, 1 \mathrm{H}), 4.09-3.99(\mathrm{~m}, 2 \mathrm{H}), 3.76-3.68$ $(\mathrm{m}, 2 \mathrm{H}), 3.02(\mathrm{~s}, 3 \mathrm{H}), 2.82-2.74(\mathrm{~m}, 1 \mathrm{H}), 2.71-2.64(\mathrm{~m}, 1 \mathrm{H}), 1.84(\mathrm{t}, J=2.3 \mathrm{~Hz}, 3 \mathrm{H})$.

${ }^{13}$ C NMR (125 MHz, $\left.\mathrm{CDCl}_{3}\right) \delta 201.2,132.1,130.1,129.4,128.3,85.5,82.9,74.6,64.1,58.9$, 39.3, 39.0, 3.6.

IR (ATR) $v_{\max }: 3442,3031,2939,2875,1735,1496,1454,1361$.

HRMS (ESI) calcd. for $\mathrm{C}_{15} \mathrm{H}_{18} \mathrm{O}_{5} \mathrm{SK}^{+}[\mathrm{M}+\mathrm{K}]^{+}: 349.0506$, found: 349.0495 . 


\section{Scheme S13: Preparation of precursor 34}<smiles>C#CC(O)c1ccccc1CC=C</smiles>

S13-1<smiles>C=CCc1ccccc1C(O)C#CC(=O)O[Na]</smiles><smiles>C=CCCCCCCCC=CCc1ccccc1C(O)C(C)=O</smiles><smiles>CCCC/C=C/Cc1ccccc1C(OC)C(C)=O</smiles> 


\section{1-(2-Allylphenyl)prop-2-yn-1-ol (S13-1)}

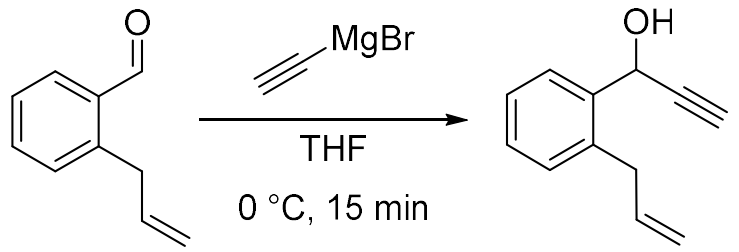

S13-1

Ethynylmagnesium bromide ( $15 \mathrm{~mL}, 0.5 \mathrm{M}$ solution in THF, $7.5 \mathrm{mmol}$ ) was added dropwise to a cold $\left(0^{\circ} \mathrm{C}\right)$ solution of $o$-allylbenzaldehyde ${ }^{23}(450 \mathrm{mg}, 3.1 \mathrm{mmol})$ in dry THF $(25 \mathrm{~mL})$, under an argon atmosphere. After $15 \mathrm{~min}$ TLC indicated complete conversion; the reaction was quenched by the addition of $\mathrm{NH}_{4} \mathrm{Cl}$ (sat.) $(110 \mathrm{~mL})$ and extracted with EtOAc $(3 \times 40 \mathrm{~mL})$. The combined organic extract was dried over anh. $\mathrm{MgSO}_{4}$, filtered and concentrated at rotavap. The residue was purified by dry-flash chromatography (petroleum-ether/ethyl acetate $=8 / 1$ ) to afford $343.6 \mathrm{mg}$ (65\%) of 1-(2-allylphenyl)prop-2-yn-1-ol (S13-1), as a colorless oil. NMR spectra in agreement with the previously reported. ${ }^{24}$

${ }^{1}$ H NMR $\left(500 \mathrm{MHz}, \mathrm{CDCl}_{3}\right) \delta 7.74-7.68(\mathrm{~m}, 1 \mathrm{H}), 7.31-7.24(\mathrm{~m}, 2 \mathrm{H}), 7.22-7.18(\mathrm{~m}, 1 \mathrm{H}), 6.00$ $\left(\mathrm{ddt}, J^{1}=17.0, J^{2}=10.1, J^{3}=6.2 \mathrm{~Hz}, 1 \mathrm{H}\right), 5.64\left(\mathrm{dd}, J^{1}=5.7, J^{2}=2.2 \mathrm{~Hz}, 1 \mathrm{H}\right), 5.09\left(\mathrm{ddd}, J^{1}=\right.$ $\left.10.1, J^{2}=3.2, J^{3}=1.6 \mathrm{~Hz}, 1 \mathrm{H}\right), 5.02\left(\mathrm{dq}, J^{1}=17.1, J^{2}=1.7 \mathrm{~Hz}, 1 \mathrm{H}\right), 3.63-3.47(\mathrm{~m}, 2 \mathrm{H}), 2.63(\mathrm{~d}$, $J=2.3 \mathrm{~Hz}, 1 \mathrm{H}), 2.38(\mathrm{~d}, J=5.8 \mathrm{~Hz}, 1 \mathrm{H})$.

${ }^{13} \mathrm{C}$ NMR $\left(126 \mathrm{MHz}, \mathrm{CDCl}_{3}\right) \delta 138.0,137.6,137.3,130.4,128.9,127.1,127.0,116.3,83.6,74.9$, $61.9,36.7$.

\section{1-(2-Allylphenyl)-1-hydroxypropan-2-one (S13-2)}

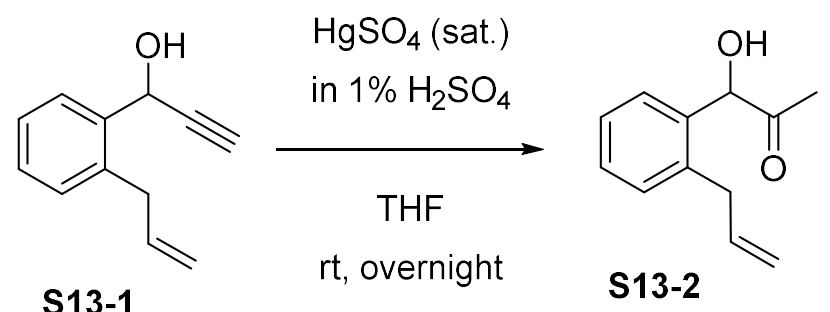

Water (5.5 mL) was added to a solution of 11-(2-allylphenyl)prop-2-yn-1-ol S13-1 (86.4 mg, 0.502 mmol) in THF (13 mL), followed by dropwise addition of saturated $\mathrm{HgSO}_{4}$ solution in $1 \% \mathrm{H}_{2} \mathrm{SO}_{4}$ $(1.30 \mathrm{~mL})$. After $16 \mathrm{~h}$ of stirring at room temperature, the reaction mixture was poured into brine $(60 \mathrm{~mL})$ and extracted with $\mathrm{Et}_{2} \mathrm{O}(3 \times 20 \mathrm{~mL})$. The combined organic extract was dried over anh.

${ }^{23} \mathrm{o}$-allylbenzaldehyde was synthesized as previously reported in Org. Lett. 2014, 16, 5032.

24 J. Org. Chem. 2003, 68, 6238. 
$\mathrm{MgSO}_{4}$ and concentrated at rotavap. The residue was purified by column chromatography (petroleum-ether/ethyl acetate $=4 / 1)$ to afford $76.5 \mathrm{mg}$ (80\%) of 1-(2-allylphenyl)-1hydroxypropan-2-one (S13-2), as a colorless oil.

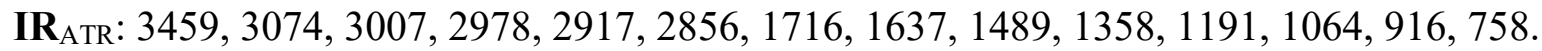

${ }^{1}$ H NMR $\left(500 \mathrm{MHz}, \mathrm{CDCl}_{3}\right) \delta$ 7.31-7.22 (m, 3H), $7.15-7.06(\mathrm{~m}, 1 \mathrm{H}), 6.07-5.94(\mathrm{~m}, 1 \mathrm{H}), 5.29$ $(\mathrm{d}, J=3.8 \mathrm{~Hz}, 1 \mathrm{H}), 5.12\left(\mathrm{dq}, J^{l}=10.1, J^{2}=1.6 \mathrm{~Hz}, 1 \mathrm{H}\right), 5.04\left(\mathrm{dq}, J^{l}=17.1, J^{2}=1.7 \mathrm{~Hz}, 1 \mathrm{H}\right)$, $4.15(\mathrm{~d}, J=3.9 \mathrm{~Hz}, 1 \mathrm{H}), 3.54$ (qdt, $\left.J^{l}=16.1, J^{2}=5.9, J^{3}=1.5 \mathrm{~Hz}, 2 \mathrm{H}\right), 2.04(\mathrm{~s}, 3 \mathrm{H})$.

${ }^{13} \mathbf{C}$ NMR $\left(126 \mathrm{MHz}, \mathrm{CDCl}_{3}\right) \delta 207.9,138.5,137.1,136.1,130.9,129.0,128.35,127.4,116.5$, $77.2,37.0,25.8$.

\section{(E)-1-(2-(Hept-2-en-1-yl)phenyl)-1-hydroxypropan-2-one (S13-3)}
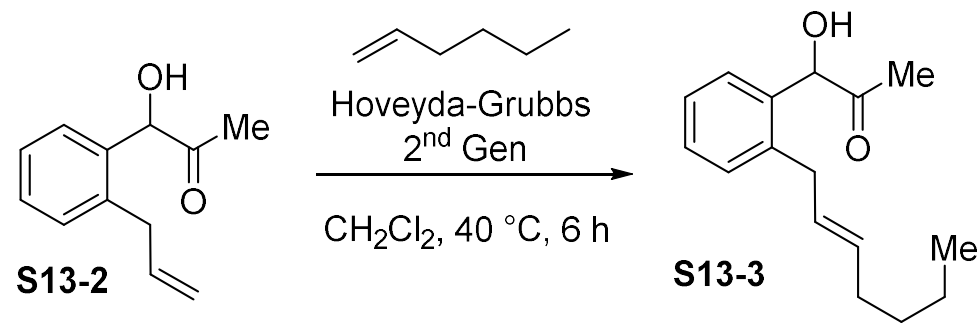

A solution of 1-(2-allylphenyl)-1-hydroxypropan-2-one S13-2 (19.1 mg, $0.1 \mathrm{mmol})$, 1-hexene (38 $\mu \mathrm{L}, 0.3 \mathrm{mmol})$ and Hoveyda-Grubbs $2^{\text {nd }}$ generation catalyst $(1.5 \mathrm{mg}, 0.002 \mathrm{mmol})$ in dry $\mathrm{CH}_{2} \mathrm{Cl}_{2}$ $(1 \mathrm{~mL})$ was stirred overnight at room temperature under argon atmosphere. TLC showed slow reaction, and more Hoveyda-Grubbs $2^{\text {nd }}$ generation catalyst $(1.0 \mathrm{mg}, 0.0015 \mathrm{mmol})$ and 1-hexene $(26 \mu \mathrm{L}, 0.2 \mathrm{mmol})$ were added and reaction mixture was heated to reflux. After $4 \mathrm{~h}$ of reflux one more portion of Hoveyda-Grubbs $2^{\text {nd }}$ generation catalyst $(1.0 \mathrm{mg}, 0.0015 \mathrm{mmol})$ and 1-hexene (38 $\mu \mathrm{L}, 0.3 \mathrm{mmol})$ was added and reflux was continued overnight. $\mathrm{SiO}_{2}(80 \mathrm{mg})$ was added to the reaction mixture and the volatiles were removed under reduced pressure. The residue was purified by column chromatography (petroleum-ether/ethyl acetate $=9 / 1)$ to afford $9.0 \mathrm{mg}(38 \%)$ of $(E)$ 1-(2-(hept-2-en-1-yl)phenyl)-1-hydroxypropan-2-one (S13-3), as a yellow oil. Mixture of $E$ and $Z$ isomers in a ratio $4: 1$.

${ }^{1} \mathbf{H}$ NMR $\left(500 \mathrm{MHz}, \mathrm{CDCl}_{3}\right) \delta 7.34-7.17(\mathrm{~m}, 3 \mathrm{H}), 7.08(\mathrm{~d}, J=7.4 \mathrm{~Hz}, 1 \mathrm{H}), 5.65-5.42(\mathrm{~m}, 2 \mathrm{H})$, $5.37-5.27(\mathrm{~m}, 1 \mathrm{H}), 4.27-4.09(\mathrm{~m}, 1 \mathrm{H}), 3.48\left(\mathrm{qd}, J^{l}=15.7, J^{2}=5.8 \mathrm{~Hz}, 2 \mathrm{H}\right), 2.10-1.94(\mathrm{~m}$, $5 \mathrm{H}), 1.41-1.24(\mathrm{~m}, 4 \mathrm{H}), 0.96-0.86(\mathrm{~m}, 3 \mathrm{H})$.

${ }^{13} \mathbf{C}$ NMR $\left(126 \mathrm{MHz}, \mathrm{CDCl}_{3}\right) \delta 208.0,139.6,136.1,132.8,130.8,129.0,128.42,128.2,127.2$, 77.1, 36.0, 32.4, 31.7, 25.8, 22.4, 14.1 .

IR (ATR) $v_{\max }: 3462,3064,3022,2958,2927,2857$, 1717, 1601, 1452, 1358, 1066, 971, 757.

HRMS (ESI) calcd. for $\mathrm{C}_{16} \mathrm{H}_{22} \mathrm{NaO}_{2}^{+}[\mathrm{M}+\mathrm{Na}]^{+}:$269.1517; found: 269.1516 . 


\section{(E)-1-(2-(Hept-2-en-1-yl)phenyl)-2-oxopropyl methanesulfonate (34)}

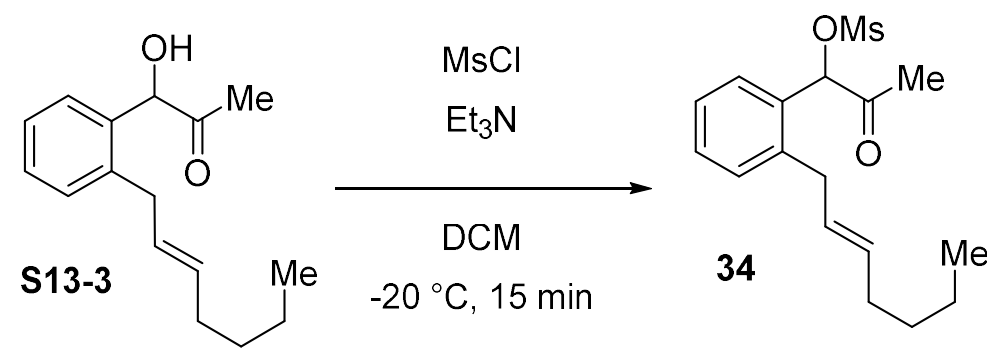

According to G.P.1. $\mathrm{MsCl}(10 \mu \mathrm{L}, 0.129 \mathrm{mmol})$ was added to a cold $\left(-20^{\circ} \mathrm{C}\right)$ solution of hydroxy ketone S13-3 (16 mg, $0.065 \mathrm{mmol})$, DMAP (1 mg, $0.008 \mathrm{mmol})$ and $\mathrm{Et}_{3} \mathrm{~N}(30 \mu \mathrm{L}, 0.215 \mathrm{mmol})$. After 10 min TLC indicated complete conversion and $\mathrm{SiO}_{2}(100 \mathrm{mg})$ was added to the reaction mixture and volatiles were removed under reduced pressure. Residue was purified by column chromatography (petroleum-ether/ethyl acetate $=4 / 1)$ to afford $17.3 \mathrm{mg}(82 \%)$ of $(E)$-1-(2-(hept2-en-1-yl)phenyl)-2-oxopropyl methanesulfonate (34), as a clear oil. Mixture of $E$ and $Z$ isomers in a ratio $6.7: 1$.

Spectral data for a mixture of $Z / E$ isomers:

${ }^{1} \mathbf{H}$ NMR (500 MHz, $\left.\mathrm{CDCl}_{3}\right) \delta 7.42-7.22$ (m, 4H, minor+major), 6.24 (s, 1H, major), 6.18 (s, $1 \mathrm{H}$, minor), $5.63-5.48(\mathrm{~m}, 2 \mathrm{H}$, minor+major), $3.57-3.44(\mathrm{~m}, 2 \mathrm{H}$, minor+major), 3.04 (s, $3 \mathrm{H}$, major), 3.03 (s, 3H, minor), 2.16 (s, 3H, minor), 2.13 (s, 3H, major), $2.06-1.99(\mathrm{~m}, 2 \mathrm{H}$, minor+major), $1.41-1.25$ (m, 4H, minor+major), 0.92 ( $\mathrm{t}, J=7.1 \mathrm{~Hz}, 3 \mathrm{H}$, minor), 0.88 (t, $J=7.1$ $\mathrm{Hz}, 3 \mathrm{H}$, major).

${ }^{13} \mathbf{C}$ NMR $\left(126 \mathrm{MHz}, \mathrm{CDCl}_{3}\right) \delta 201.4,140.4,133.4,131.1,130.7,130.5,128.9,127.7,127.3,82.8$, 39.6, 36.1, 32.3, 31.6, 26.7, 22.4, 14.0.

IR (ATR) $v_{\text {max }}: 3025,2958,2929,2859,1737,1601,1453,1359,1176,950,842,761,529$.

HRMS (ESI) calcd. for $\mathrm{C}_{17} \mathrm{H}_{24} \mathrm{KO}_{4} \mathrm{~S}^{+}[\mathrm{M}+\mathrm{K}]^{+}$: 363.1032; found: 363.1034 . 


\section{Scheme S14: Preparation of precursor 37}
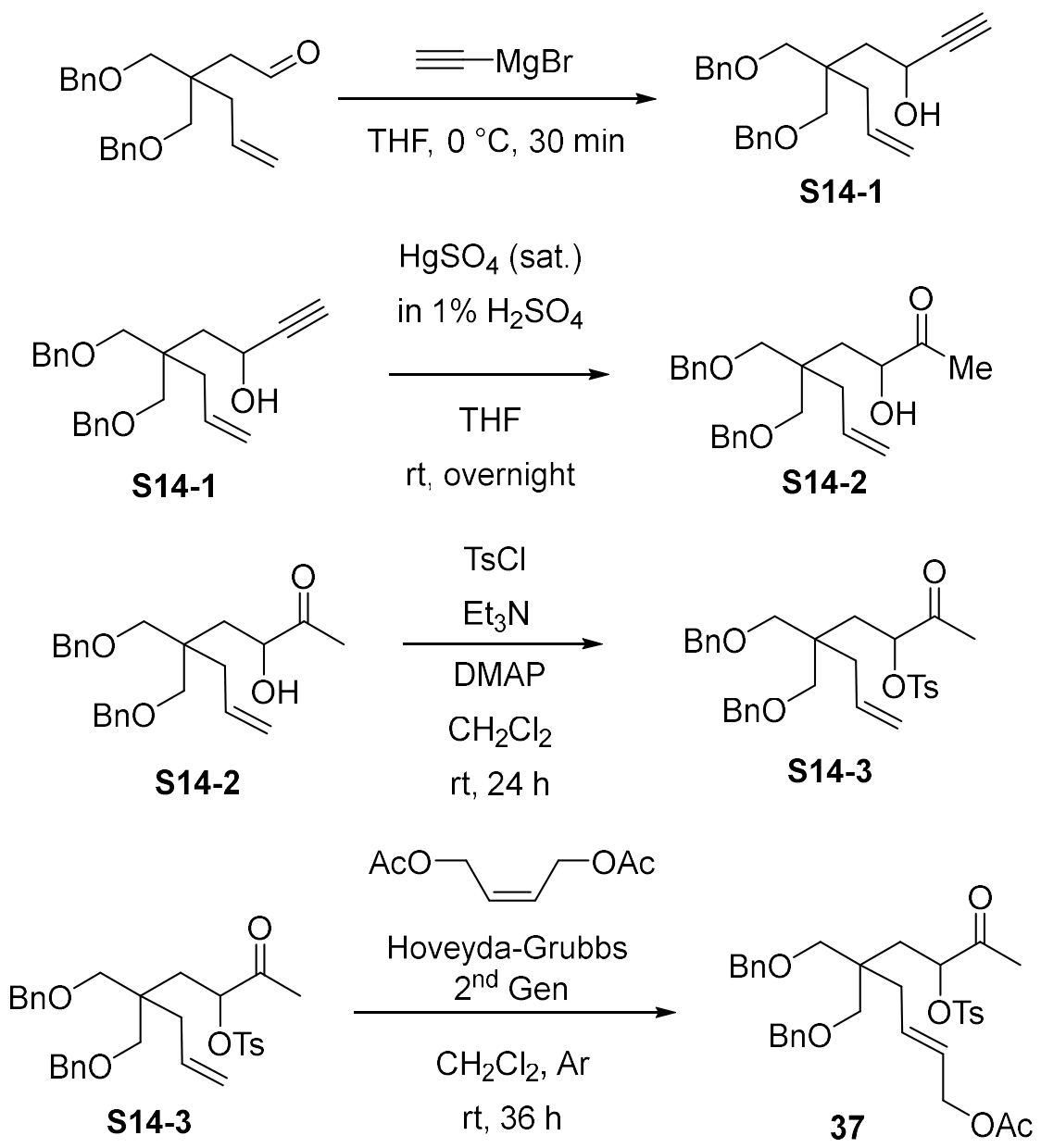

\section{5,5-Bis((benzyloxy)methyl)oct-7-en-1-yn-3-ol (S14-1)}
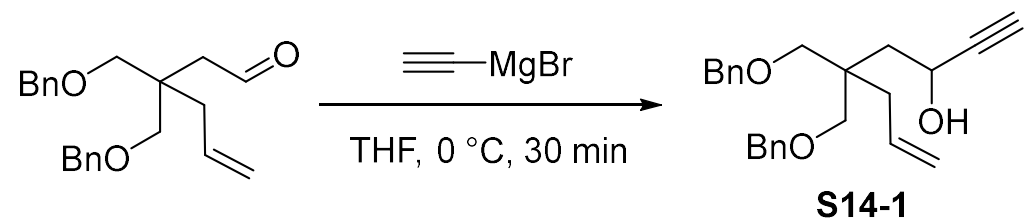

To an ice-cold solution of 3,3-bis((benzyloxy)methyl)hex-5-enal ${ }^{25}$ (31 $\left.\mathrm{mg}, 0.092 \mathrm{mmol}\right)$ in THF $(0.6 \mathrm{~mL})$, ethynylmagnesium bromide solution $(0.4 \mathrm{~mL}, 0.5 \mathrm{M}$ in THF, $0.2 \mathrm{mmol})$ was added dropwise, via syringe, under an argon atmosphere. After 10 min of stirring TLC indicated complete consumption of starting material. The reaction was quenched by the addition of $\mathrm{NH}_{4} \mathrm{Cl}(3 \mathrm{~mL})$ and extracted with EtOAc $(3 \times 3 \mathrm{~mL})$. The combined organic extract was washed with brine $(3 \mathrm{~mL})$,

${ }^{25}$ Makabe, M.; Sato, Y.; Mori, M. J. Org. Chem, 2004, 69, 6238-6243. 
dried over anh. $\mathrm{MgSO}_{4}$ and concentrated under reduced pressure. The residue was purified by column chromatography (petroleum-ether/ethyl acetate $=9 / 1)$ to afford $23.8 \mathrm{mg}(72 \%)$ of propargylic alcohol S14-1, as a pale yellow oil.

${ }^{1}$ H NMR (200 MHz, CDCl $) \delta 7.42-7.20(\mathrm{~m}, 10 \mathrm{H}), 5.86-5.59(\mathrm{~m}, 1 \mathrm{H}), 5.16-4.97(\mathrm{~m}, 2 \mathrm{H})$, $4.61-4.51(\mathrm{~m}, 1 \mathrm{H}), 4.48(\mathrm{~d}, J=3.3 \mathrm{~Hz}, 4 \mathrm{H}), 4.35(\mathrm{~d}, J=3.4 \mathrm{~Hz}, 1 \mathrm{H}), 3.44(\mathrm{~s}, 2 \mathrm{H}), 3.41-3.27$ $(\mathrm{m}, 2 \mathrm{H}), 2.38(\mathrm{~d}, J=2.2 \mathrm{~Hz}, 1 \mathrm{H}), 2.22(\mathrm{~d}, J=7.4 \mathrm{~Hz}, 2 \mathrm{H}), 1.91(\mathrm{~d}, J=0.9 \mathrm{~Hz}, 1 \mathrm{H}), 1.88(\mathrm{~d}, J=$ $5.7 \mathrm{~Hz}, 1 \mathrm{H})$.

${ }^{13} \mathbf{C}$ NMR (50 MHz, $\left.\mathbf{C D C l}_{3}\right) \delta 137.8,133.6,128.6,127.9,127.9,118.7,85.8,74.0,73.7,72.8$, 71.6, 58.8, 43.5, 41.6, 37.9 .

IR (ATR) $v_{\max }: 3386,3293,3066,3030,2910,2862,1638,1451,1365,1095,1026,918,739,699$, 653.

HRMS (ESI) calcd. for $\mathrm{C}_{24} \mathrm{H}_{28} \mathrm{NaO}_{3}{ }^{+}[\mathrm{M}+\mathrm{Na}]^{+}$: 387.1936; found: 387.1943 .

\section{5,5-Bis((benzyloxy)methyl)-3-hydroxyoct-7-en-2-one (S14-2)}

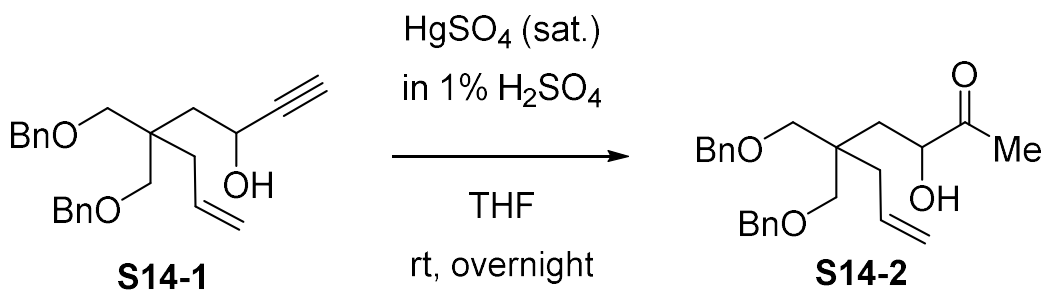

Water $(1.84 \mathrm{~mL})$ was added to a solution of propargylic alcohol S14-1 $(73.0 \mathrm{mg}, 0.20 \mathrm{mmol})$ in THF $(4.3 \mathrm{~mL})$, followed by dropwise addition of saturated solution of $\mathrm{HgSO}_{4}$ in $1 \% \mathrm{H}_{2} \mathrm{SO}_{4}(0.45$ $\mathrm{mL}$ ). After $16 \mathrm{~h}$ of stirring at room temperature, the reaction mixture was poured into brine (18 $\mathrm{mL})$ and extracted with $\mathrm{Et}_{2} \mathrm{O}(3 \times 6 \mathrm{~mL})$. The combined organic extract was dried over anh. $\mathrm{MgSO}_{4}$ and concentrated at rotavap. The residue was purified by column chromatography (petroleumether/ethyl acetate $=7 / 1)$ to afford $60 \mathrm{mg}(78 \%)$ of hydroxy ketone $\mathbf{S 1 4 - 2}$, as a colorless oil.

${ }^{1} \mathbf{H}$ NMR $\left(500 \mathrm{MHz}, \mathrm{CDCl}_{3}\right) \delta 7.36-7.26(\mathrm{~m}, 10 \mathrm{H}), 5.86-5.67(\mathrm{~m}, 1 \mathrm{H}), 5.14-4.97(\mathrm{~m}, 2 \mathrm{H})$, $4.49(\mathrm{~d}, J=8.2 \mathrm{~Hz}, 2 \mathrm{H}), 4.22\left(\mathrm{ddd}, J^{l}=10.1, J^{2}=3.9, J^{3}=1.7 \mathrm{~Hz}, 1 \mathrm{H}\right), 4.18(\mathrm{~d}, J=4.0 \mathrm{~Hz}, 1 \mathrm{H})$, $3.50-3.35(\mathrm{~m}, 4 \mathrm{H}), 2.33-2.20(\mathrm{~m}, 2 \mathrm{H}), 2.15(\mathrm{~s}, 3 \mathrm{H}), 1.88\left(\mathrm{dd}, J^{l}=14.7, J^{2}=1.8 \mathrm{~Hz}, 1 \mathrm{H}\right), 1.46$ (dd, $\left.J^{l}=14.7, J^{2}=10.2 \mathrm{~Hz}, 1 \mathrm{H}\right)$.

${ }^{13} \mathbf{C}$ NMR $\left(126 \mathrm{MHz}, \mathrm{CDCl}_{3}\right) \delta 211.3,138.2,133.9,128.5,127.8,127.7,127.7,118.5,74.5$, 73.6, 73.56, 73.5, 73.0, 41.8, 37.8, 37.6, 25.6.

IR (ATR) $v_{\max }: 3462,3066,3030,2911,2861,1714,1639,1452,1360,1098,739,700$.

HRMS (ESI) calcd. for $\mathrm{C}_{24} \mathrm{H}_{30} \mathrm{NaO}_{4}^{+}[\mathrm{M}+\mathrm{Na}]^{+}$: 405.2042; found: 405.2044. 


\section{5,5-Bis((benzyloxy)methyl)-2-oxooct-7-en-3-yl 4-methylbenzenesulfonate (S14-3)}

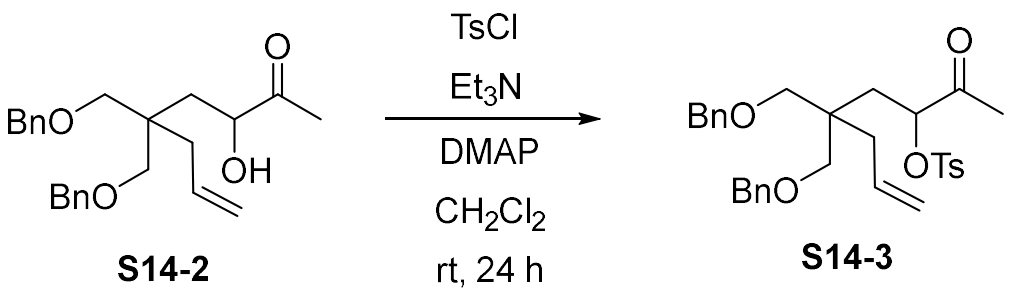

Tosyl chloride ( $107.0 \mathrm{mg}, 0.767 \mathrm{mmol}, 3.76$ equiv.) was added to a solution of hydroxy ketone S14-2 (57 mg, $0.149 \mathrm{mmol})$, DMAP (1.0 mg, $0.008 \mathrm{mmol})$ and $\mathrm{Et}_{3} \mathrm{~N}(0.107 \mathrm{~mL}, 0.767 \mathrm{mmol}$, 5.15 equiv.) in dry $\mathrm{CH}_{2} \mathrm{Cl}_{2}(1.4 \mathrm{~mL})$, under an argon atmosphere, at room temperature. After 24 $\mathrm{h}$ of stirring, the orange reaction mixture was diluted with $\mathrm{CH}_{2} \mathrm{Cl}_{2}(1 \mathrm{~mL})$, silica $(500 \mathrm{mg})$ was added and the volatiles were removed by rotavap. Purification of the residue by column chromatography (petroleum-ether/ethyl acetate $=8 / 1)$ afforded $66.0 \mathrm{mg}(82 \%)$ of tosyloxy ketone S14-3, as a very viscous colorless oil.

${ }^{1} \mathbf{H}$ NMR $\left(200 \mathrm{MHz}, \mathrm{CDCl}_{3}\right) \delta 7.72(\mathrm{~d}, J=8.4 \mathrm{~Hz}, 2 \mathrm{H}), 7.39-7.19(\mathrm{~m}, 12 \mathrm{H}), 5.77-5.51(\mathrm{~m}$, $1 \mathrm{H}), 5.07-4.89(\mathrm{~m}, 3 \mathrm{H}), 4.43-4.31(\mathrm{~m}, 4 \mathrm{H}), 3.30-3.14(\mathrm{~m}, 4 \mathrm{H}), 2.41(\mathrm{~s}, 3 \mathrm{H}), 2.11-2.01(\mathrm{~m}$, 2H), $2.07(\mathrm{~s}, 3 \mathrm{H}), 1.77(\mathrm{~d}, J=6.1 \mathrm{~Hz}, 1 \mathrm{H})$.

${ }^{13} \mathbf{C}$ NMR $\left(50 \mathrm{MHz}, \mathrm{CDCl}_{3}\right) \delta 204.1,145.4,138.6,138.5,133.5,130.0,128.5,128.3,127.7$, $118.7,81.7,73.2,72.5,71.9,41.6,37.2,33.6,25.4,21.8$.

IR (ATR) $v_{\max }$ : 3065, 3031, 3006, 2976, 2917, 2862, 2799, 1724, 1639, 1598, 1452, 1368, 1177 , 1098, 952, 916, 888768, 742, 700 .

HRMS (ESI) calcd. for $\mathrm{C}_{31} \mathrm{H}_{36} \mathrm{NaO}_{6} \mathrm{~S}^{+}[\mathrm{M}+\mathrm{Na}]^{+}$: 559.2130; found: 559.2153 .

(E)-5,5-Bis((benzyloxy)methyl)-8-oxo-7-(tosyloxy)non-2-en-1-yl acetate (37)

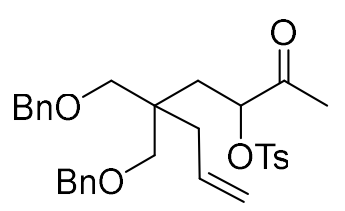

S14-3

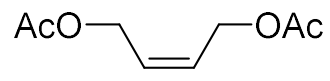

Hoveyda-Grubbs

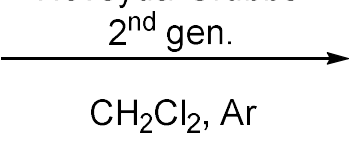

rt. $36 \mathrm{~h}$

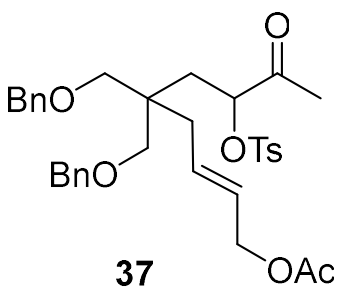

37

A solution of compound S14-3 (60.0 mg, $0.112 \mathrm{mmol}$ ), cis-1,4-diacetoxy-2-butene (40 $\mu \mathrm{L}, 0.250$ $\mathrm{mmol})$ and Hoveyda-Grubbs $2^{\text {nd }}$ generation catalyst $(4.5 \mathrm{mg}, 0.007 \mathrm{mmol})$ in dry $\mathrm{CH}_{2} \mathrm{Cl}_{2}(1 \mathrm{~mL})$ was stirred overnight at room temperature, under an argon atmosphere. TLC showed slow reaction, and more Hoveyda-Grubbs $2^{\text {nd }}$ generation catalyst $(4.5 \mathrm{mg}, 0.007 \mathrm{mmol})$ and cis-1,4-diacetoxy-2butene $(40 \mu \mathrm{L}, 0.250 \mathrm{mmol})$ were added. After $6 \mathrm{~h}$ of stirring at room temperature one more portion 
of Hoveyda-Grubbs $2^{\text {nd }}$ generation catalyst $(2.0 \mathrm{mg}, 0.003 \mathrm{mmol})$ was added and stirring was continued overnight. $\mathrm{SiO}_{2}(400 \mathrm{mg})$ was added to the reaction mixture and the volatiles were removed under reduced pressure. The residue was purified by column chromatography (petroleum-ether/ethyl acetate $=4 / 1)$ to afford $44.8 \mathrm{mg}(66 \%)$ of allylic acetate 37 , as a pale yellow, viscous oil.

${ }^{1} \mathbf{H}$ NMR $\left(500 \mathrm{MHz}, \mathrm{CDCl}_{3}\right) \delta 7.72(\mathrm{~d}, J=8.3 \mathrm{~Hz}, 2 \mathrm{H}), 7.36-7.24(\mathrm{~m}, 12 \mathrm{H}), 5.61-5.54(\mathrm{~m}$, $1 \mathrm{H}), 5.49\left(\mathrm{dt}, J^{1}=15.3, J^{2}=6.1 \mathrm{~Hz}, 1 \mathrm{H}\right), 5.00(\mathrm{t}, J=6.1 \mathrm{~Hz}, 1 \mathrm{H}), 4.43(\mathrm{~d}, J=6.0 \mathrm{~Hz}, 2 \mathrm{H}), 4.40-$ $4.32(\mathrm{~m}, 4 \mathrm{H}), 3.20\left(\mathrm{ddd}, J^{1}=12.8, J^{2}=12.2, J^{3}=6.5 \mathrm{~Hz}, 4 \mathrm{H}\right), 2.42(\mathrm{~s}, 3 \mathrm{H}), 2.08-2.02(\mathrm{~m}, 2 \mathrm{H})$, $2.06(\mathrm{~s}, 3 \mathrm{H}), 2.04(\mathrm{~s}, 3 \mathrm{H}), 1.76(\mathrm{~d}, J=6.1 \mathrm{~Hz}, 2 \mathrm{H})$.

${ }^{13} \mathrm{C}$ NMR $\left(126 \mathrm{MHz}, \mathrm{CDCl}_{3}\right) \delta 203.9,170.9,145.4,138.4,133.3,130.6,130.0,128.5,128.3$, $128.0,127.7,81.6,73.3,72.3,71.8,65.0,41.8,35.6,33.6,25.4,21.8,21.1$.

IR (ATR) $v_{\max }: 3087,3062,3030,2862,1738,1598,1451,1367,1234,1177,1096,1024,953$, $743,699$.

HRMS (ESI) calcd. for $\mathrm{C}_{34} \mathrm{H}_{40} \mathrm{NaO}_{8} \mathrm{~S}^{+}[\mathrm{M}+\mathrm{Na}]^{+}:$631.2342; found: 631.2340 .

\section{Cyclizations of oxyallyl cations}

1-(2-Ethyl-5-methylcyclopentyl)ethan-1-one (2) (General procedure for the cyclization Method A)

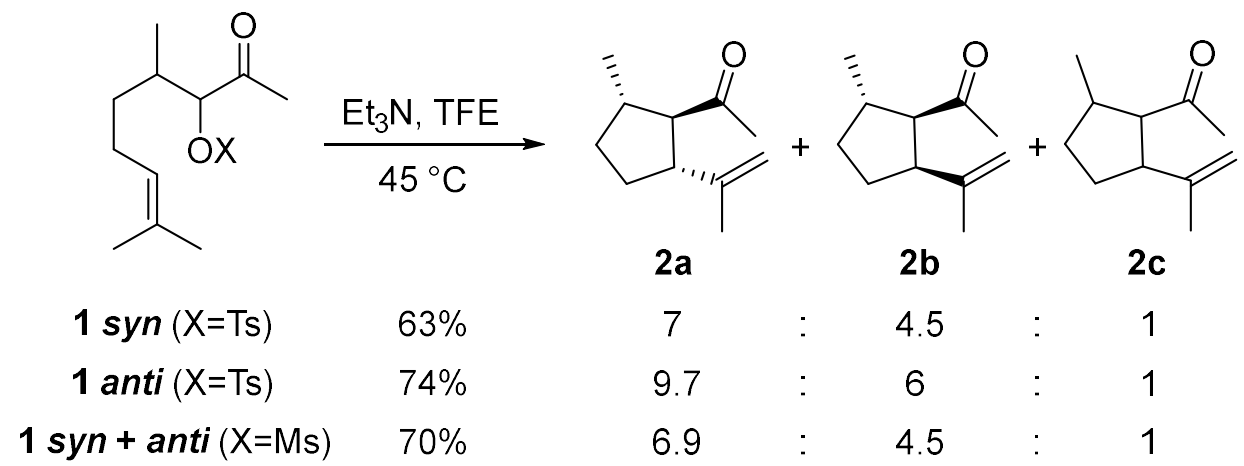

Triethylamine $(61 \mu \mathrm{L}, 0.438 \mathrm{mmol}, 1.6 \mathrm{eq})$ was added to the solution of 1 syn $(93.7 \mathrm{mg}, 0.277$ $\mathrm{mmol}, 1 \mathrm{eq})$ in 2,2,2-trifluoroethanol $(0.85 \mathrm{~mL})$ and the reaction mixture was stirred at $45^{\circ} \mathrm{C}$ for $41 \mathrm{~h}$, under an argon atmosphere. ${ }^{26}$ The reaction mixture was carefully concentrated in vacuo and the residue was purified by column chromatography (pentane/diethyl ether $=95 / 5$ ) to afford 28.9

\footnotetext{
${ }^{26}$ Previously reported procedure was modified: Vander Wal, M. N., Dilger, A. K., MacMillan, D. W. C., Chem. Sci., 2013, 4, 3075.
} 
mg $(63 \%)$ of volatile compound 2 , as a colorless oil (mixture of isomers in a relative ratio 7:4.5:1, as determined by ${ }^{1} \mathrm{H}$ NMR and GC/MS).

According to the General procedure - Method A. Starting from 1 anti (47.2 $\mathrm{mg}, 0.139 \mathrm{mmol})$, triethylamine $(30 \mu \mathrm{L}, 0.219 \mathrm{mmol})$ and 2,2,2-trifluoroethanol $(0.4 \mathrm{~mL}), 2(17.0 \mathrm{mg}, 74 \%)$ was obtained, as mixture of isomers in a relative ratio 9.7:6:1, as determined by ${ }^{1} \mathrm{H}$ NMR and GC/MS.

According to the General procedure - Method A. Starting from 1 anti + syn $(13.0 \mathrm{mg}, 0.050$ $\mathrm{mmol})$, triethylamine $(11 \mu \mathrm{L}, 0.078 \mathrm{mmol})$ and 2,2,2-trifluoroethanol $(0.15 \mathrm{~mL}), 2(5.8 \mathrm{mg}, 70 \%)$ was obtained, as mixture of isomers in a relative ratio 6.9:4.5:1, as determined by ${ }^{1} \mathrm{H}$ NMR.

Spectral data for mixture, spectra given where $\mathrm{H}: \mathrm{H}^{\prime}: \mathrm{H}^{\prime}$ ' correspond to the isomers in a ratio 5.5: 3.5: 1 .

${ }^{1} \mathbf{H}$ NMR (500 MHz, $\left.\mathrm{CDCl}_{3}\right) \delta 4.78-4.66\left(\mathrm{~m}, 2 \mathrm{H}+2 \mathrm{H}\right.$ ' $+2 \mathrm{H}^{\prime}$ ') $), 3.05-2.94(\mathrm{~m}, 1 \mathrm{H}$ '+2H' '), 2.79 $(\mathrm{dd}, J=18.2,9.0 \mathrm{~Hz}, 1 \mathrm{H}), 2.67$ (dd, $\left.J=8.0,8.0 \mathrm{~Hz}, 1 \mathrm{H}^{\prime}\right), 2.56-2.46$ (m, 1H' '), 2.46- 2.36 (m, 1H'), 2.41 (t, $J=9.6 \mathrm{~Hz}, 1 \mathrm{H}), 2.27-2.16(\mathrm{~m}, 1 \mathrm{H}), 2.15\left(\mathrm{~s}, 3 \mathrm{H}^{\prime \prime}\right), 2.12(\mathrm{~s}, 3 \mathrm{H}), 2.09$ (s, 3H'), $2.05-1.97\left(\mathrm{~m}, 1 \mathrm{H}^{\prime}\right), 1.96-1.82\left(\mathrm{~m}, 2 \mathrm{H}+1 \mathrm{H}^{\prime}+2 \mathrm{H}^{\prime}\right), 1.79-1.74\left(\mathrm{~m}, 1 \mathrm{H}^{\prime}\right), 1.72(\mathrm{~s}, 3 \mathrm{H}), 1.69$ (s, $3 \mathrm{H}^{\prime}$ '), 1.69 (s, 3H'), $1.66-1.56(\mathrm{~m}, 1 \mathrm{H}), 1.52-1.28\left(\mathrm{~m}, 1 \mathrm{H}+2 \mathrm{H}^{\prime}\right), 1.27-1.15\left(\mathrm{~m}, 1 \mathrm{H}^{\prime}\right), 1.04(\mathrm{~d}$, $J=6.7 \mathrm{~Hz}, 3 \mathrm{H}), 1.01\left(\mathrm{~d}, J=6.7 \mathrm{~Hz}, 3 \mathrm{H}^{\prime}\right), 0.86\left(\mathrm{~d}, J=7.1 \mathrm{~Hz}, 3 \mathrm{H}^{\prime}{ }^{\prime}\right)$.

${ }^{13} \mathbf{C}$ NMR $\left(125 \mathrm{MHz}, \mathrm{CDCl}_{3}\right) \delta 210.4,210.3,210.1,147.3,146.6,145.9,111.9,110.5,109.2,64.6$, $64.2,59.7,51.8,50.0,46.5,38.6,36.9,35.7,34.1,33.6,33.4,31.4,31.0,30.2,30.1,30.0,29.8$, $21.8,20.9,19.8,19.8,16.8$.

IR (ATR) $v_{\max }:$ 3078, 2955, 2869, 1708, 1644, 1456, 1374, 1356.

HRMS (ESI) calcd. for $\mathrm{C}_{11} \mathrm{H}_{19} \mathrm{O}^{+}[\mathrm{M}+\mathrm{H}]^{+}:$167.1430, found: 167.1423.

Inferior yield was observed when the reaction was performed on a larger scale, due to side reactions with trifluoroethanol. Therefore, the cyclization was performed in toluene on a gram scale (4.5 mmol), under modified conditions (see Method C).

1-(2-Ethyl-5-methylcyclopentyl)ethan-1-one (2) (General procedure for the cyclization Method C)

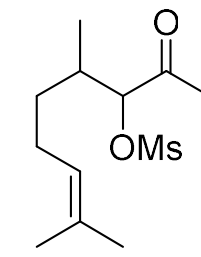

1 syn + anti

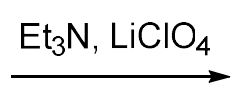

$\mathrm{PhMe}, 60^{\circ} \mathrm{C}$

$55 \%$

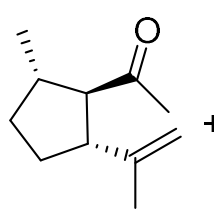

2a

6.9
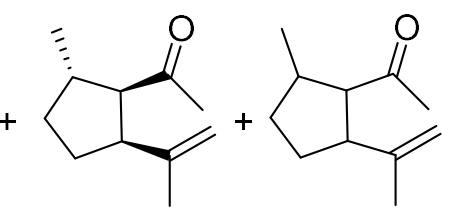

$\mathbf{2 b}$
4.5 2c

1 
A suspension of 1 syn $+\boldsymbol{a n t i}(1.18 \mathrm{~g}, 4.5 \mathrm{mmol})$, trimethylamine $(1.25 \mathrm{~mL}, 9 \mathrm{mmol})$ and lithium perchlorate $(957.5 \mathrm{mg}, 9 \mathrm{mmol})$ in toluene $(16 \mathrm{~mL})$ was stirred at $60^{\circ} \mathrm{C}$ for $7 \mathrm{~h}$ (the reaction was performed under air, reaction vessel was capped). $\mathrm{SiO}_{2}(3 \mathrm{~g})$ was added, the solvent was removed at rotavap and the resulting slurry was put as a cake at the top of a $\mathrm{SiO}_{2}$ column $(8 \mathrm{~g})$ and purified by column chromatography (pentane/diethyl ether $=95 / 5)$ to afford volatile product $2(411 \mathrm{mg}$, $55 \%$ ), as a colorless oil (mixture of isomers in a relative ratio 6.9:4.5:1).

Correlations (NOESY) for major diastereomer 2a:
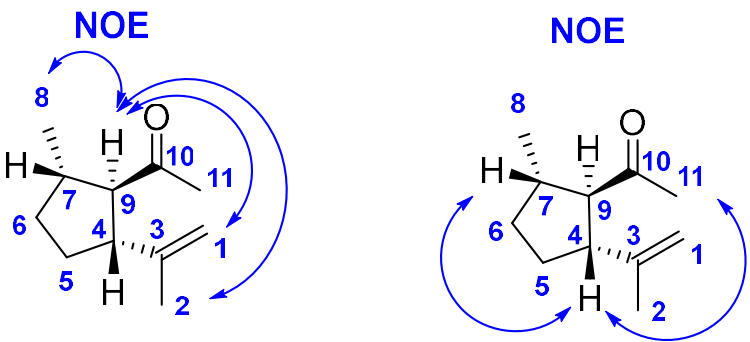

$\mathrm{H}-9 / \mathrm{H}-1$

$\mathrm{H}-9 / \mathrm{Me}-2$

$\mathrm{H}-4 / \mathrm{H}-7$

$\mathrm{H}-9 / \mathrm{Me}-8$

$\mathrm{H}-4 / \mathrm{Me}-11$

In order to determine whether the product distribution ( $2 \mathbf{2}: \mathbf{2 b}: \mathbf{2 c})$ is constant over time, cyclization reaction of $\mathbf{1}$ reaction progress was monitored by GC/MS. This was repeated several times.

\section{Distribution of products 2a:2b:2c over time (conditions: Method C)}<smiles>COC(C(C)=O)C(C)CCC=C(C)C</smiles>

1 syn+anti

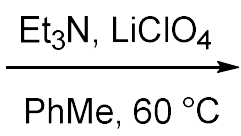

$\mathrm{PhMe}, 60^{\circ} \mathrm{C}$

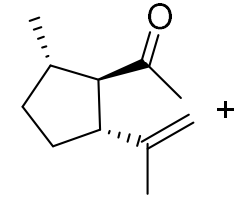

$2 a$

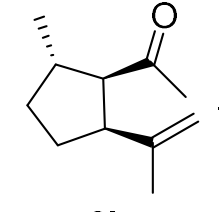

2b

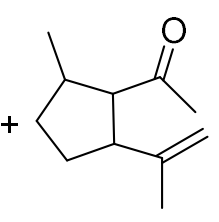

2c

A suspension of $1 \mathrm{syn}+$ anti $(30.0 \mathrm{mg}, 0.114 \mathrm{mmol})$, triethylamine ( $32 \mu \mathrm{L}, 0.229 \mathrm{mmol})$ and lithium perchlorate $(24.3 \mathrm{mg}, 0.229 \mathrm{mmol})$ in toluene $(0.40 \mathrm{~mL})$ was stirred at $60{ }^{\circ} \mathrm{C}$. At certain points of time, reaction mixture aliquots $(40 \mu \mathrm{L})$ were taken, diluted using dichloromethane $(1.00 \mathrm{~mL})$ and analyzed directly by GC-MS. 
Table S1: Product 2 distribution over time

\begin{tabular}{|c|c|}
\hline time (h) & $\begin{array}{c}\text { relative ratio } \\
\mathbf{2 a}: \mathbf{2 b}: \mathbf{2 c}\end{array}$ \\
\hline 1 & $61.1: 33.7: 5.1$ \\
\hline 3 & $60.9: 34.0: 5.1$ \\
\hline 5 & $59.8: 35.2: 4.9$ \\
\hline
\end{tabular}

\section{GC/MS traces of reaction mixture at different reaction times:}

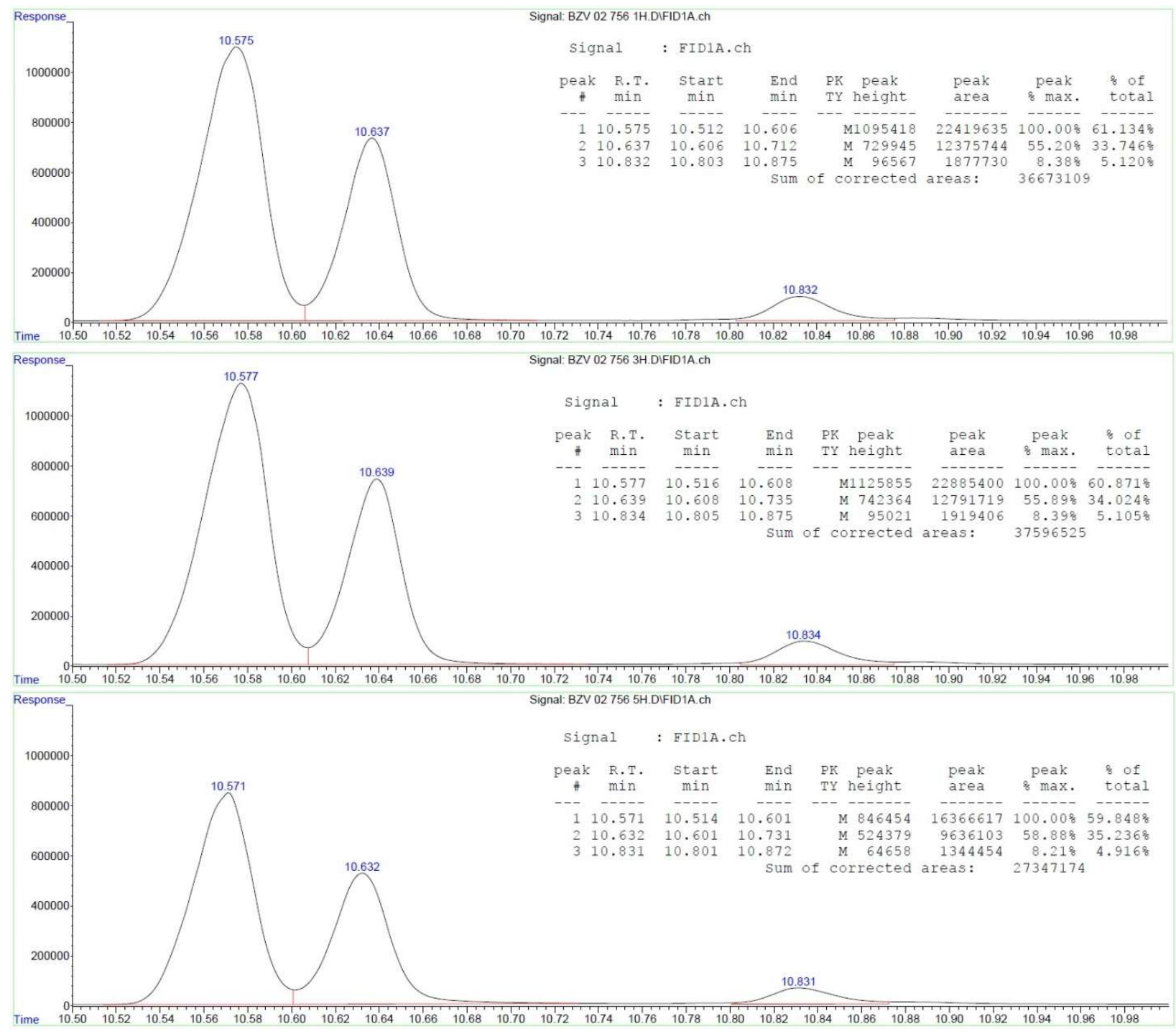




\section{Resubmission experiment}

Isolated product $\mathbf{2}$ (mixture of three diastereoisomers $\mathbf{2 a}: \mathbf{2 b}: \mathbf{2} \mathbf{c}$ in a relative ratio 61:34:5) was resubmitted to the same cyclization reaction conditions:<smiles>C=C(C)[C@H]1CC[C@H](C)[C@H]1C(C)=O</smiles>

$2 a$<smiles>C=C(C)[C@H]1CC[C@@H](C)[C@@H]1C(C)=O</smiles>

$2 b$<smiles>C=C(C)C1CC[CH+]C1C</smiles>

$2 \mathrm{c}$

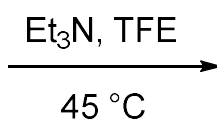<smiles>CC1=CC(=O)[C@@H]2[C@@H](C)CC[C@H]12</smiles>

$2 a$<smiles>C=C(C)[C@H]1CC[C@@H](C)[C@@H]1C(C)=O</smiles>

$2 b$<smiles>CC1=CC(=O)C2C(C)C[CH+]C12</smiles>

2c

Triethylamine ( $9 \mu \mathrm{L}, 0.065 \mathrm{mmol})$ was added to the solution of $\mathbf{2 a}+\mathbf{2} \mathbf{b}+\mathbf{2} \mathbf{c}(13.7 \mathrm{mg}, 0.082 \mathrm{mmol})$ in 2,2,2-trifluoroethanol $(0.125 \mathrm{~mL})$ under argon atmosphere and the reaction mixture was stirred at $45^{\circ} \mathrm{C}$ for $7 \mathrm{~h}$, then overnight at room temperature. Reaction mixture aliquot $(10 \mu \mathrm{L})$ was taken, diluted using dichloromethane $(1.00 \mathrm{~mL})$ and analyzed directly by GC-MS. Another reaction mixture aliquot $(10 \mu \mathrm{L})$ was taken, diluted with $\mathrm{CDCl}_{3}(0.5 \mathrm{~mL})$ and ${ }^{1} \mathrm{H}$ NMR was taken. Results showed that relative ratio $\mathbf{2 a}: \mathbf{2 b}: \mathbf{2 c}$ before and after the treatment with triethylamine in 2,2,2trifluoroethanol at $45{ }^{\circ} \mathrm{C}$ remained identical. Results are depicted in Table S2.

\begin{tabular}{|c|c|c|}
\hline \multicolumn{3}{|c|}{ Table S2: relative ratio 2a:2b:2c } \\
\hline & before & after \\
\hline by ${ }^{1} \mathrm{H} N M R$ & $61: 34: 5$ & $61: 34: 5$ \\
\hline by GC/MS & $58.9: 36.0: 5.0$ & $59.9: 34.9: 5.2$ \\
\hline
\end{tabular}

GC/MS trace of starting material:

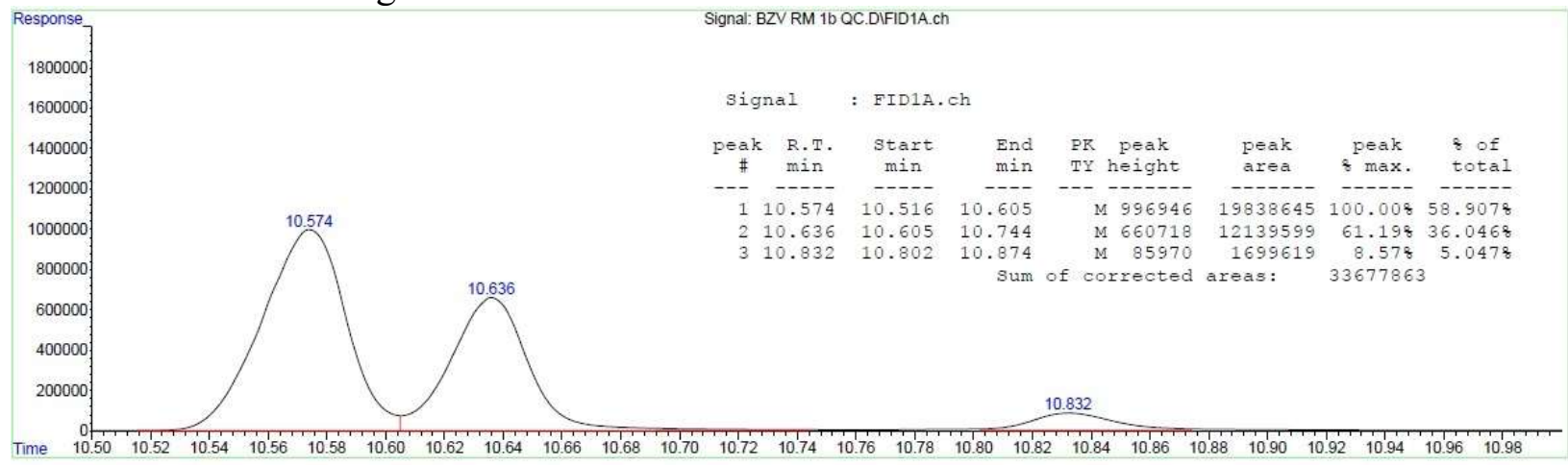

GC/MS trace of reaction mixture:

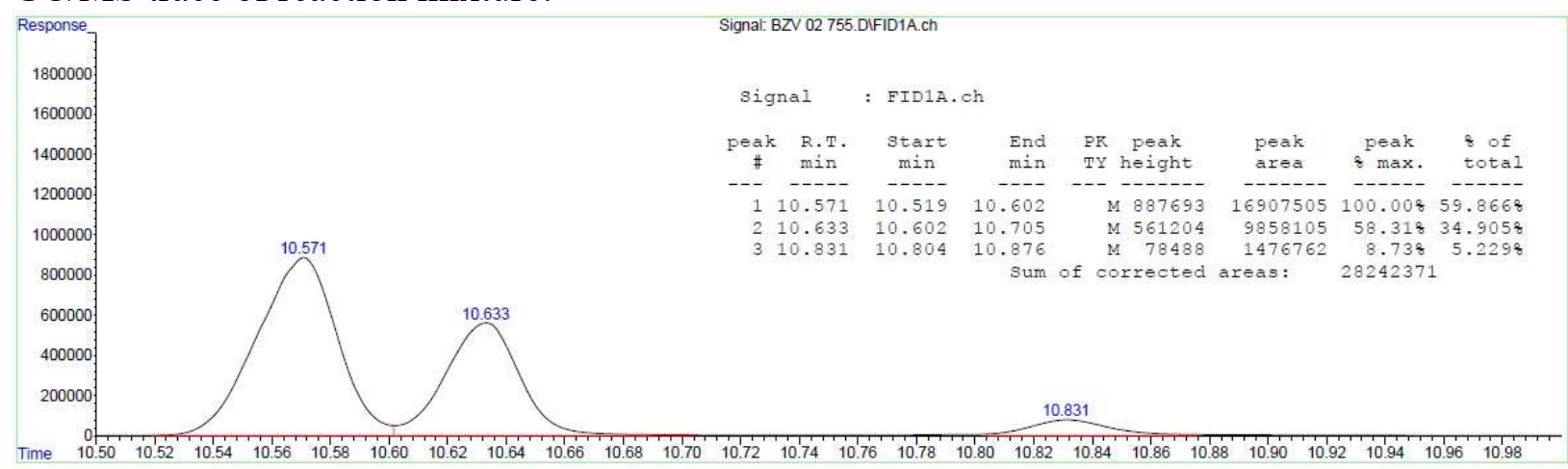



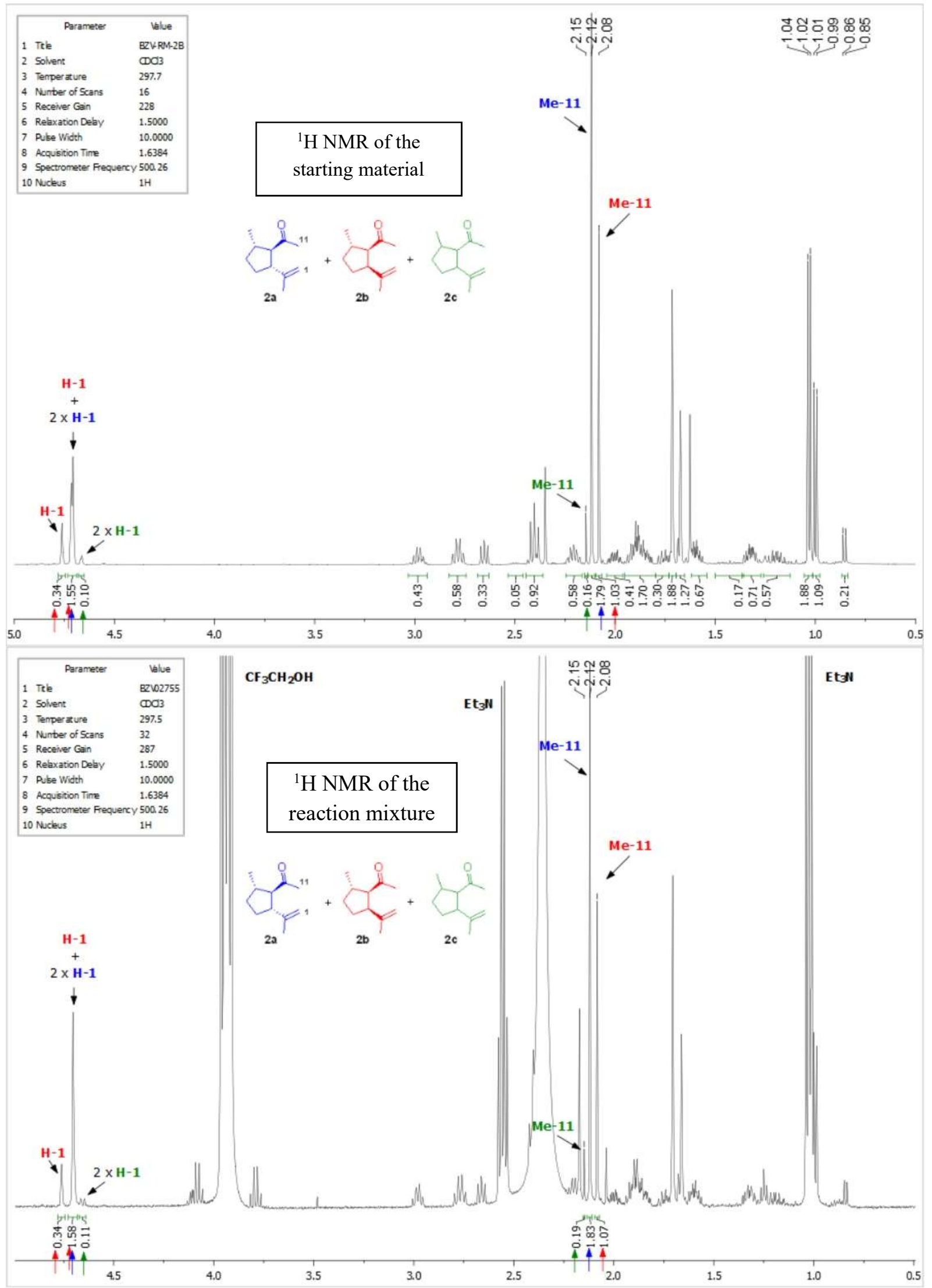
Distribution of products 2a:2b:2c over time (conditions: Method A)<smiles>COC(C(C)=O)C(C)CCC=C(C)C</smiles>

1 syn+anti<smiles>CCN(CC)CC</smiles><smiles>CC1=CC(=O)[C@H]2[C@@H](C)CC[C@@H]12</smiles>

a<smiles>CC1=CC(=O)[C@H]2C(C)CC[C@H]12</smiles>

2b<smiles>CC1=CC(=O)C2C(C)C[CH+]C12</smiles>

2c

Triethylamine $(71.4 \mu \mathrm{L}, 0.51 \mathrm{mmol}, 1.6 \mathrm{eq})$ was added to the solution of 1 syn + anti $(84.0 \mathrm{mg}$, $0.320 \mathrm{mmol}, 1 \mathrm{eq})$ in 2,2,2-trifluoroethanol $(0.1 \mathrm{~mL})$ under argon atmosphere and the reaction mixture was stirred at $45^{\circ} \mathrm{C}$. At certain points of time, reaction mixture aliquots $(30 \mu \mathrm{L})$ were taken, diluted using dichloromethane $(1.00 \mathrm{~mL})$ and analyzed directly by GC-MS. Results are depicted in Table S3.

Table S3: Product 2 distribution over time

\begin{tabular}{|c|c|}
\hline $\begin{array}{c}\text { time } \\
(\mathrm{h})\end{array}$ & $\begin{array}{c}\text { relative ratio } \\
\mathbf{2 a}: \mathbf{2 b}: \mathbf{2 c}\end{array}$ \\
\hline 2 & $57.0: 35.5: 7.5$ \\
\hline 4 & $57.1: 36.5: 6.4$ \\
\hline 7 & $56.3: 37.5: 6.2$ \\
\hline
\end{tabular}

GC/MS traces of reaction mixture at different reaction times:
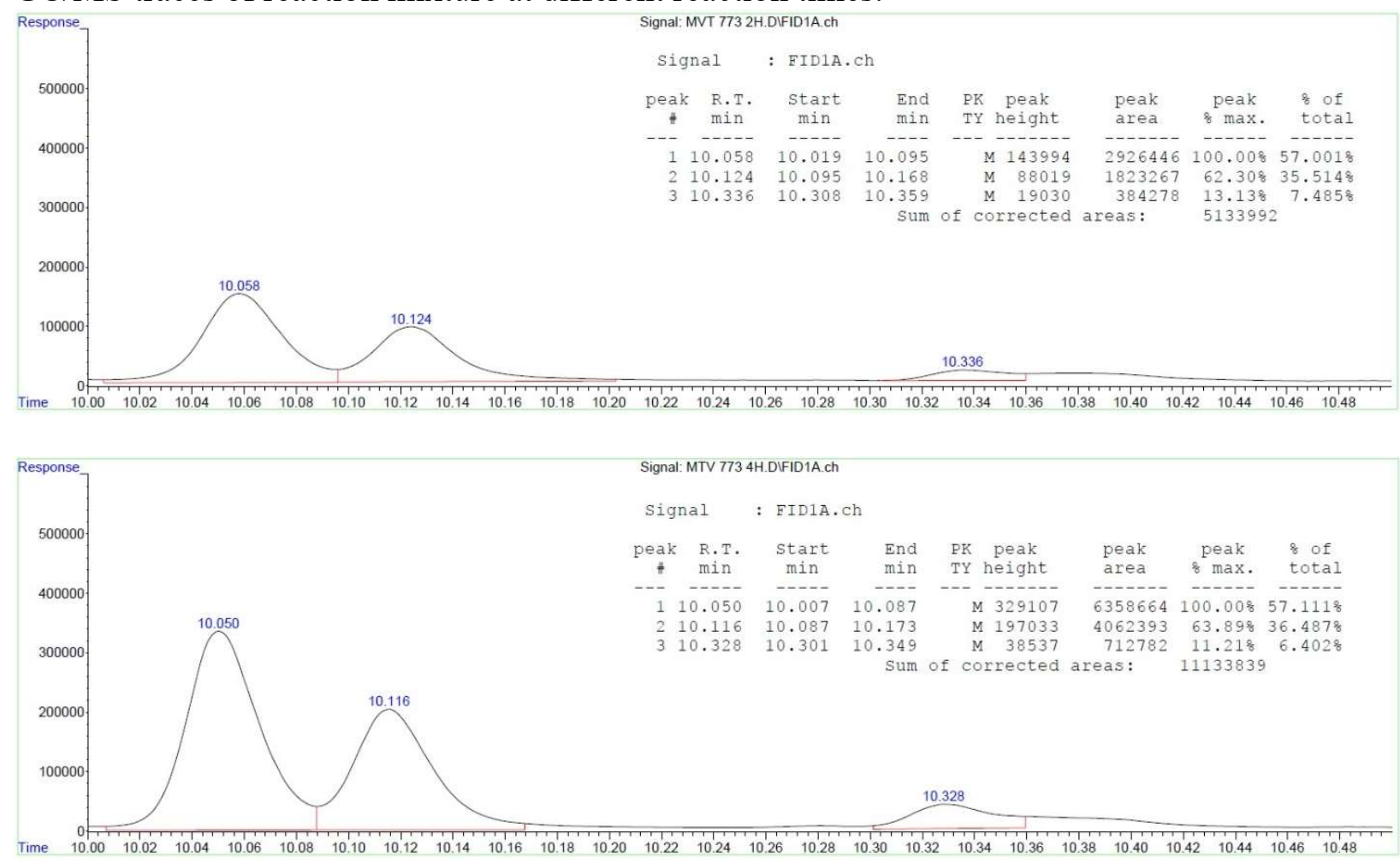


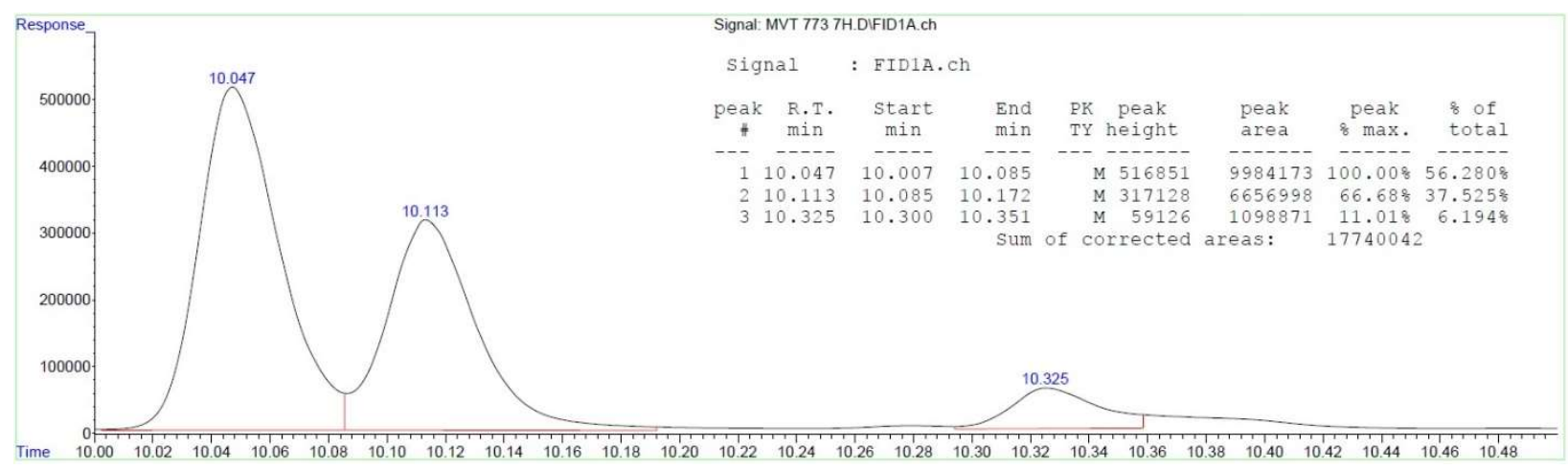

Distribution of products 2a:2b:2c over time (conditions: Method A)<smiles>COC(C(C)=O)C(C)CCC=C(C)C</smiles>

1 syn+anti

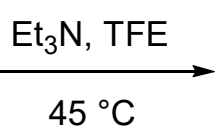

$2 a$<smiles>CC1=[C+]C(=O)C2C1CC[C@@H]2C</smiles>

a<smiles>CC1=CC(=O)[C@H]2C(C)CC[C@@H]12</smiles>

2b<smiles>CC1=CC(=O)C2C(C)C[CH+]C12</smiles>

2c

Triethylamine $(18.7 \mu \mathrm{L}, 0.134 \mathrm{mmol}, 1.6 \mathrm{eq})$ was added to the solution of $1 \mathbf{s y n}+\boldsymbol{a n t i}(22.0 \mathrm{mg}$, $0.084 \mathrm{mmol}, 1 \mathrm{eq})$ in 2,2,2-trifluoroethanol $(0.26 \mathrm{~mL})$ under argon atmosphere and the reaction mixture was stirred at $45^{\circ} \mathrm{C}$. At certain points of time, reaction mixture aliquots $(30 \mu \mathrm{L})$ were taken, diluted using dichloromethane $(1.00 \mathrm{~mL})$ and analyzed directly by GC-MS. Results are depicted in Table S4.

Table S4: Product 2 distribution over time

\begin{tabular}{|c|c|}
\hline $\begin{array}{c}\text { time } \\
(\mathrm{h})\end{array}$ & $\begin{array}{c}\text { relative ratio } \\
\mathbf{2 a}: \mathbf{2 b}: \mathbf{2 c}\end{array}$ \\
\hline 2 & $53.8: 39.2: 7.0$ \\
\hline 4 & $54.8: 38.6: 6.6$ \\
\hline
\end{tabular}

GC/MS traces of reaction mixture at different reaction times:

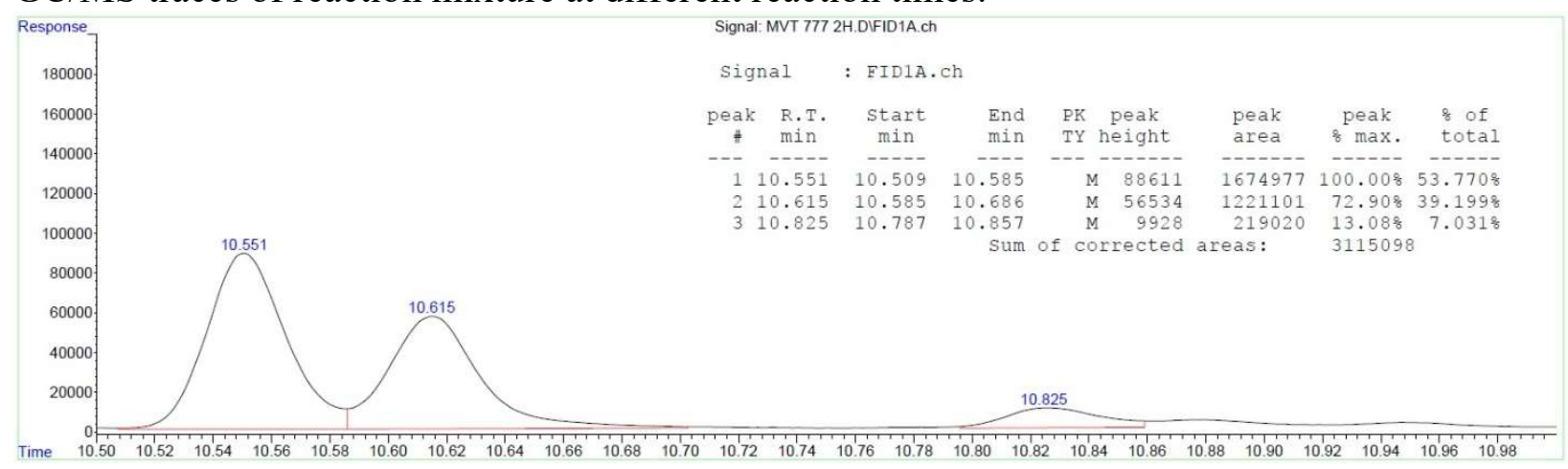




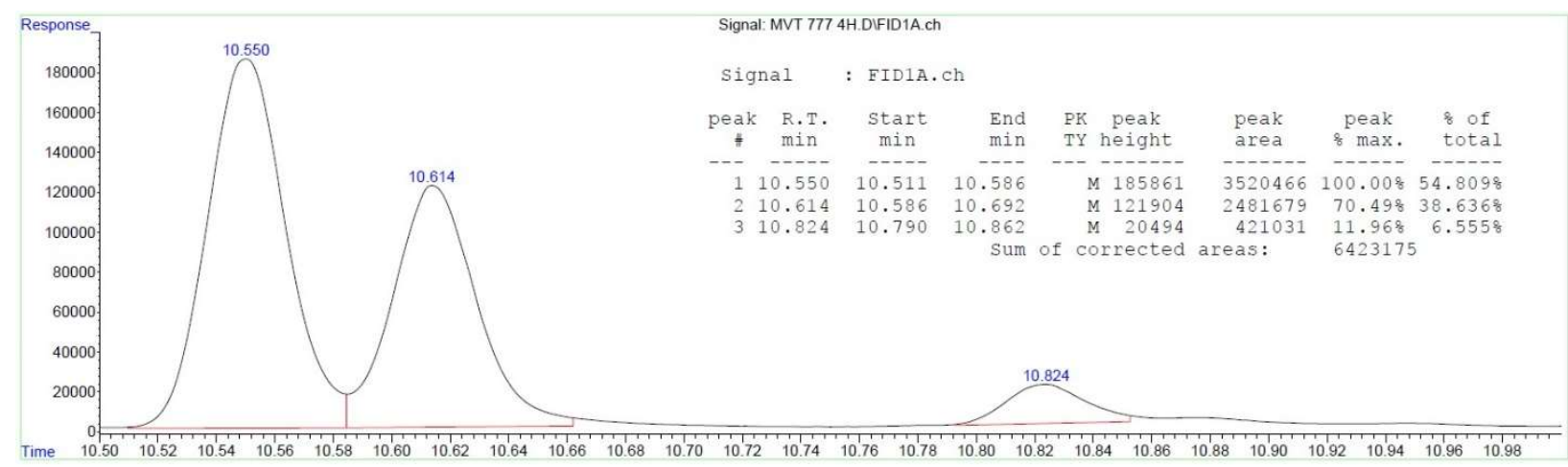

Distribution of products $2 \mathrm{a}: 2 \mathrm{~b}: 2 \mathrm{c}$ over time in the presence of menthone as an "in situ" internal standard (conditions: Method A)<smiles>COC(C(C)=O)C(C)CCC=C(C)C</smiles>

1 syn+anti

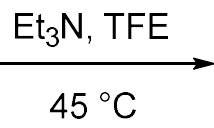

menthone<smiles>C=C1C(=O)[C@H]2[C@@H](C)CC[C@H]12</smiles>

$2 a$<smiles>CC1=CC(=O)[C@@H]2C1CC[C@@H]2C</smiles>

2b<smiles>CC1=CC(=O)C2C(C)[CH+]CC12</smiles>

2c

Triethylamine $(71.4 \mu \mathrm{L}, 0.512 \mathrm{mmol}, 1.6 \mathrm{eq})$ was added to the solution of $1 \mathbf{s y n}+\boldsymbol{a n t i}(84.0 \mathrm{mg}$, $0.320 \mathrm{mmol}, 1 \mathrm{eq})$ and (-)-menthone $(27.5 \mathrm{mg}, 0.160 \mathrm{mmol})$ as internal standard in 2,2,2trifluoroethanol $(0.98 \mathrm{~mL})$ under argon atmosphere and the reaction mixture was stirred at $45^{\circ} \mathrm{C}$. At certain points of time, reaction mixture aliquots $(30 \mu \mathrm{L})$ were taken, diluted using dichloromethane $(1.00 \mathrm{~mL})$ and analyzed directly by GC-MS.

Table S2: Product 2 distribution over time

\begin{tabular}{|c|c|}
\hline time (h) & $\begin{array}{c}\text { relative ratio } \\
\mathbf{2 a}: \mathbf{2 b}: \mathbf{2 c}\end{array}$ \\
\hline 3 & $66.2: 33.7:----$ \\
\hline 7 & $58.3: 32.9: 8.7$ \\
\hline 23 & $58.9: 33.2: 7.8$ \\
\hline 27 & $58.6: 32.6: 8.8$ \\
\hline 31 & $57.1: 33.9: 9.0$ \\
\hline 48 & $57.4: 33.3: 9.3$ \\
\hline
\end{tabular}


GC/MS trace of internal standard (menthone, mixture of isomers):

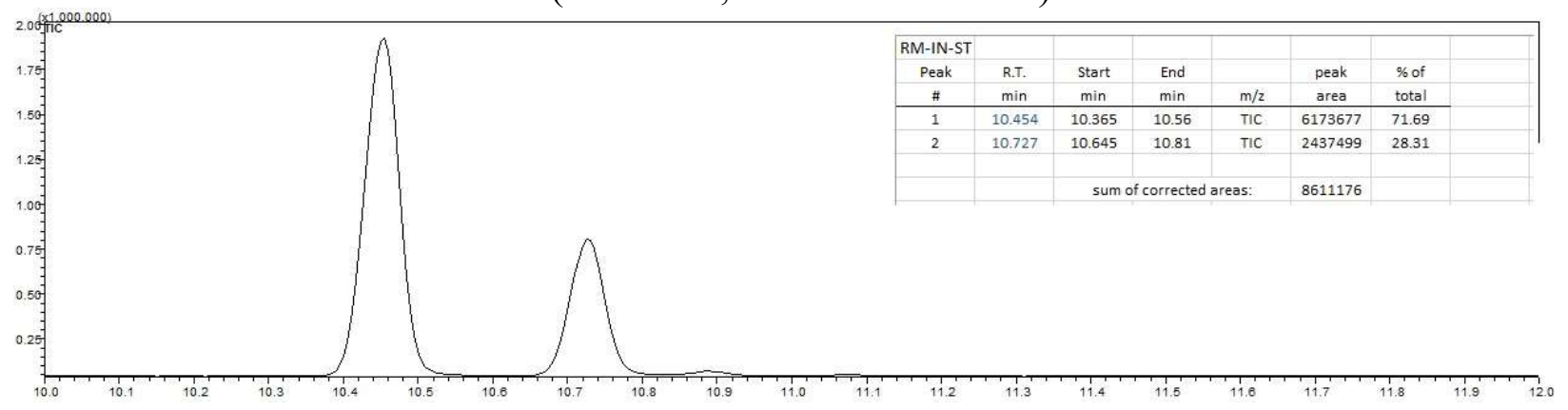

GC/MS trace of the reaction mixture $(3 \mathrm{~h}):^{*}$

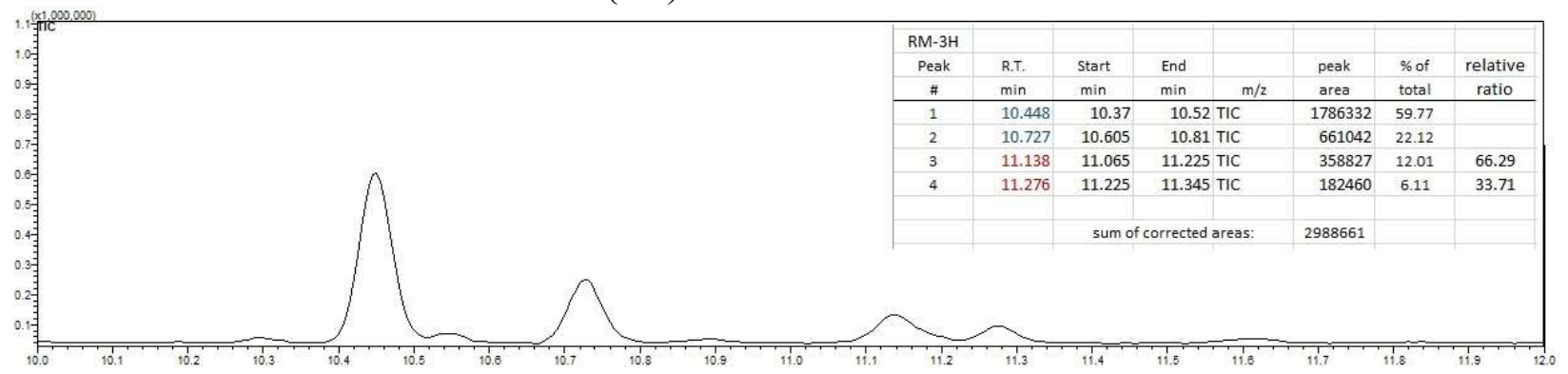

*concentration of $\mathbf{2 c}$ was too low to integrate

GC/MS trace of the reaction mixture $(7 \mathrm{~h})$ :

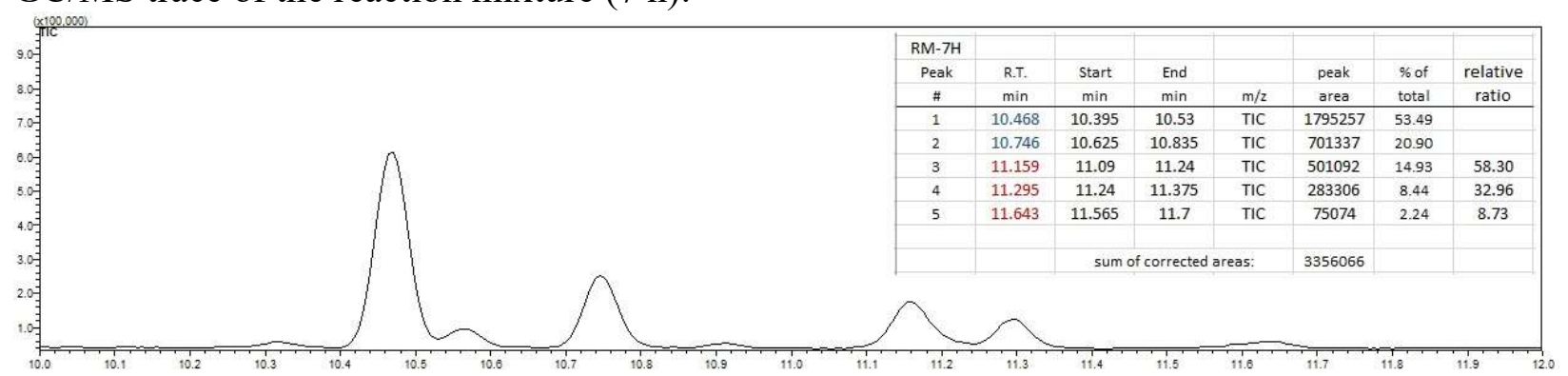

GC/MS trace of the reaction mixture $(23 \mathrm{~h})$ :

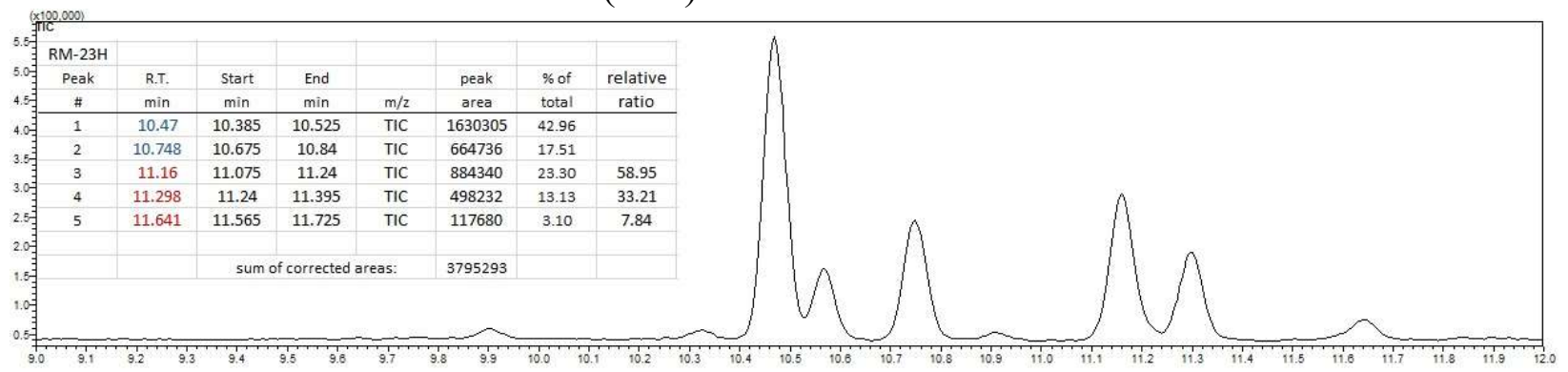

GC/MS trace of the reaction mixture $(27 \mathrm{~h})$ : 


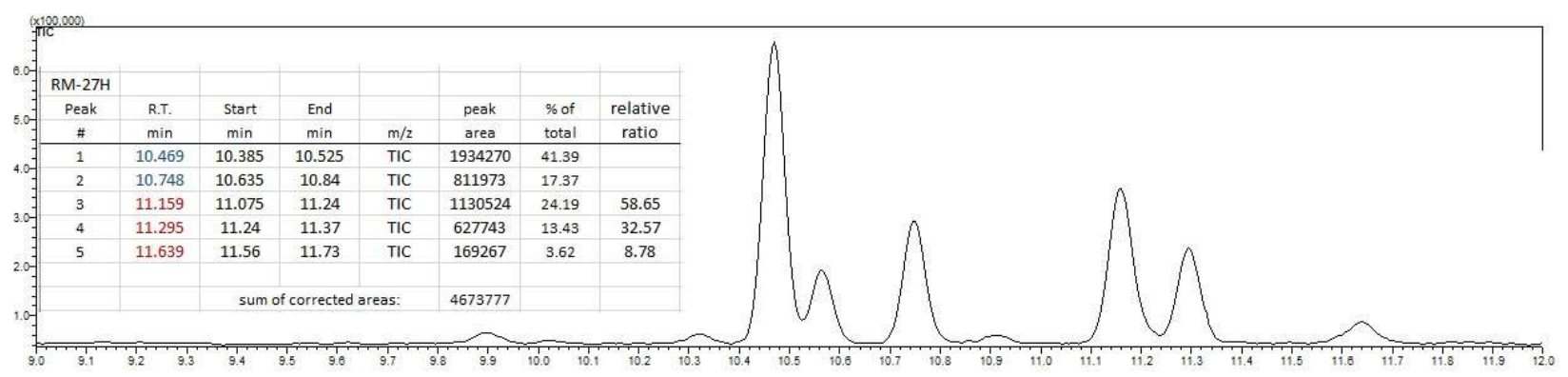

GC/MS trace of the reaction mixture $(31 \mathrm{~h})$ :

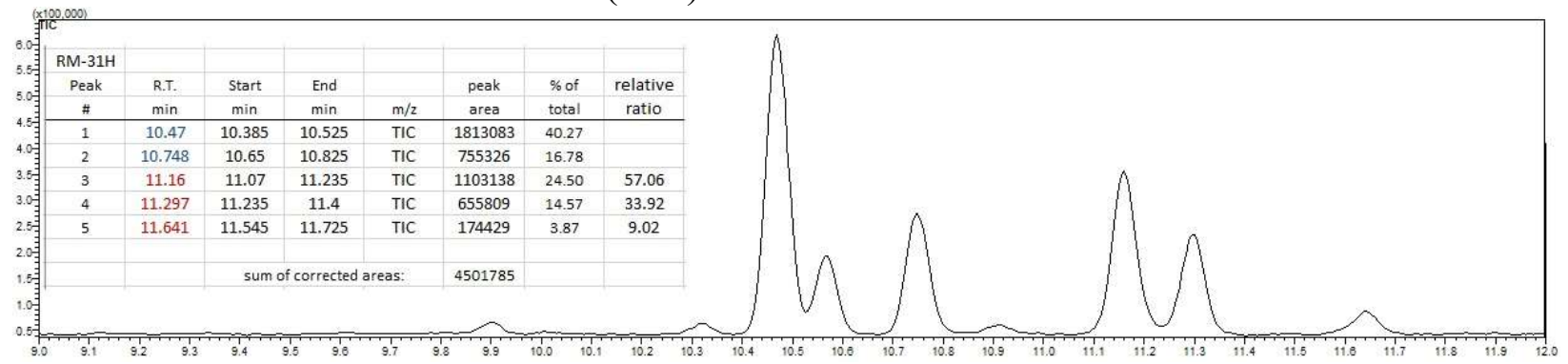

GC/MS trace of the reaction mixture ( $48 \mathrm{~h})$ :

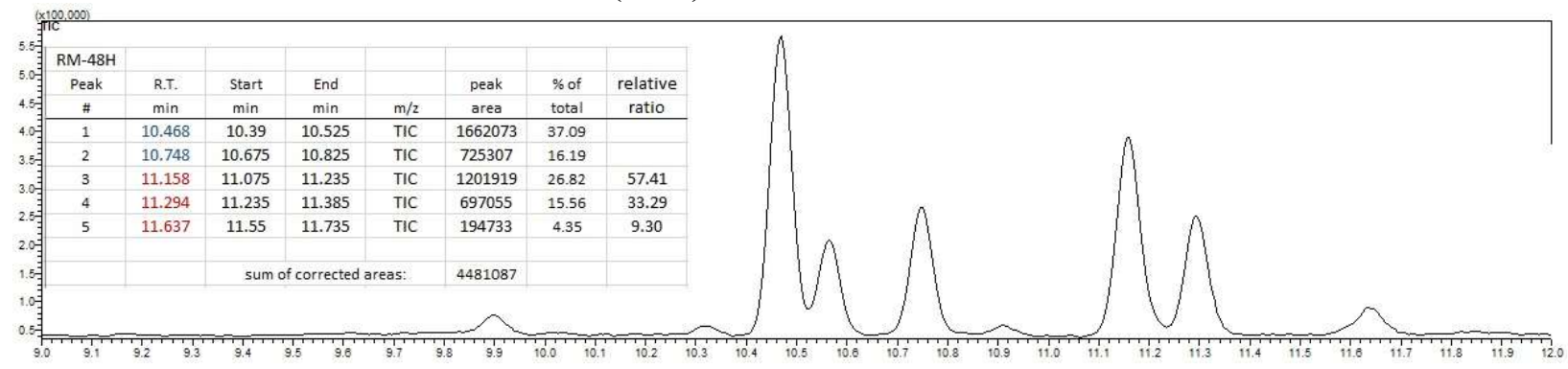

GC/MS trace of product 2 (isolated):

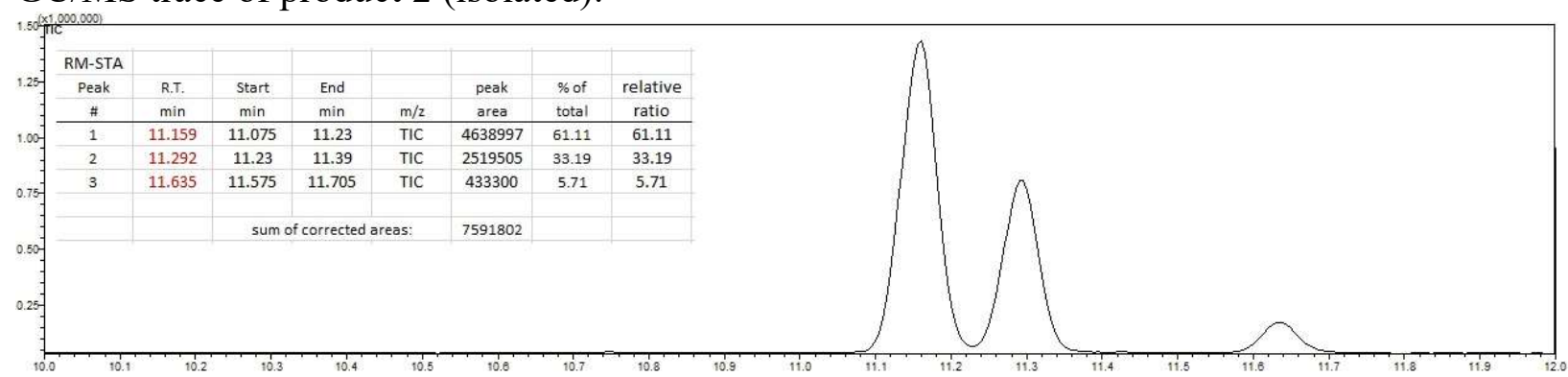




\section{1-(2-(Prop-1-en-2-yl)spiro[4.5]decan-1-yl)ethan-1-one (4)}
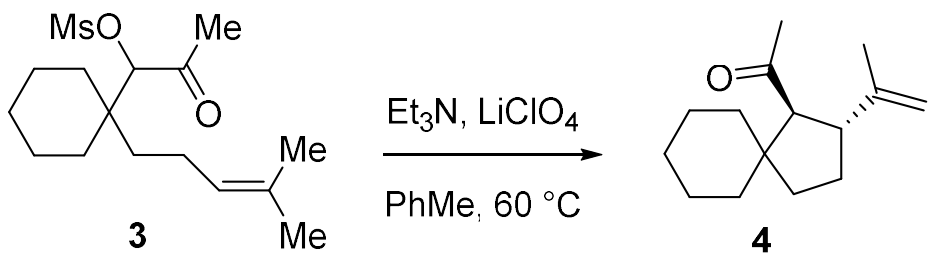

According to the General procedure - Method C. A suspension of crude mesylate $3(0.43 \mathrm{mmol})$ from previous step, triethylamine $(119 \mu \mathrm{L}, 0.854 \mathrm{mmol})$ and lithium perchlorate $(90.8 \mathrm{mg}, 0.854$ mmol) in toluene $(3 \mathrm{~mL})$ was stirred at $60{ }^{\circ} \mathrm{C}$ for $12 \mathrm{~h}$. The reaction mixture was purified by column chromatography (petroleum-ether/ ethyl acetate $=9 / 1)$ to afford $63 \mathrm{mg}(67 \%)$ of 1-(2-(prop-1-en2-yl)spiro[4.5]decan-1-yl)ethan-1-one 4, as a colorless oil (single diastereoisomer).

${ }^{1} \mathbf{H}$ NMR $\left(500 \mathrm{MHz}, \mathrm{CDCl}_{3}\right) \delta 4.68-4.66(\mathrm{~m}, 1 \mathrm{H}), 4.66-4.64(\mathrm{~m}, 1 \mathrm{H}), 2.97\left(\mathrm{td}, J^{1}=10.9, J^{2}=\right.$ $7.6 \mathrm{~Hz}, 1 \mathrm{H}), 2.55(\mathrm{~d}, J=11.0 \mathrm{~Hz}, 1 \mathrm{H}), 2.15(\mathrm{~s}, 3 \mathrm{H}), 1.87\left(\mathrm{dtd}, J^{1}=12.3, J^{2}=7.4, J^{3}=3.7 \mathrm{~Hz}, 1 \mathrm{H}\right)$, $1.71-1.66(\mathrm{~m}, 1 \mathrm{H}), 1.68(\mathrm{~s}, 3 \mathrm{H}), 1.62-1.54(\mathrm{~m}, 6 \mathrm{H}), 1.48-1.37(\mathrm{~m}, 4 \mathrm{H}), 1.33-1.28(\mathrm{~m}, 1 \mathrm{H})$, $1.12\left(\mathrm{tt}, J^{1}=12.7, J^{2}=3.7 \mathrm{~Hz}, 1 \mathrm{H}\right), 1.03\left(\mathrm{td}, J^{1}=12.6, J^{2}=3.6 \mathrm{~Hz}, 1 \mathrm{H}\right)$.

${ }^{13}$ C NMR $\left(125 \mathrm{MHz}, \mathrm{CDCl}_{3}\right) \delta 211.1,147.1,109.7,67.6,49.1,46.8,39.3,35.8,34.7,32.9,29.7$, 26.2, 24.1, 22.8, 21.0.

IR (ATR) $v_{\max }: 2928,2857,1707,1644,1449,1355,1162,889$.

HRMS (ESI) calcd. for $\mathrm{C}_{15} \mathrm{H}_{25} \mathrm{O}^{+}[\mathrm{M}+\mathrm{H}]^{+}: 221.1900$, found: 221.1902 .

Correlations (NOESY) for compound 4:

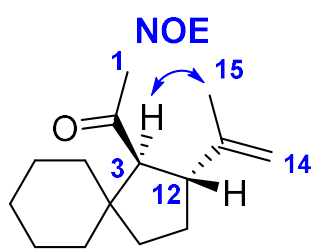

$\mathrm{H}-3 / \mathrm{Me}-15$

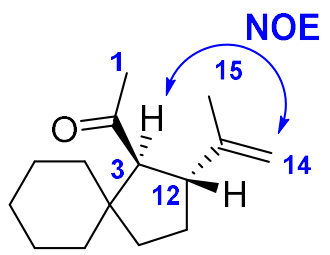

$\mathrm{H}-3 / \mathrm{H}-14$

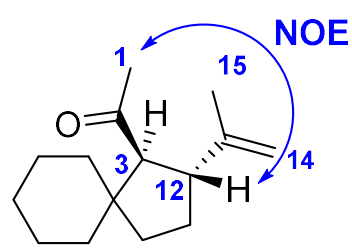

$\mathrm{H}-12 / \mathrm{Me}-1$

(E)-1-(3-(Pent-1-en-1-yl)spiro[4.5]decan-2-yl)ethan-1-one (6)

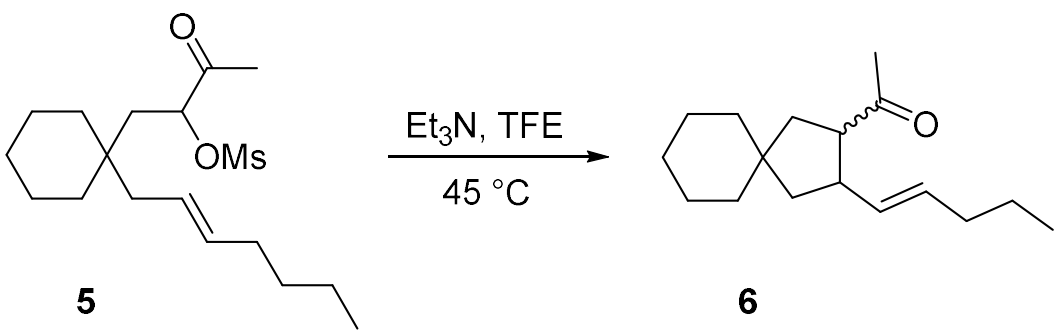


According to the General procedure - Method A. Triethylamine $(20 \mu \mathrm{L}, 0.143 \mathrm{mmol})$ was added to a solution of mesylate $5(31.0 \mathrm{mg}, 0.090 \mathrm{mmol})$ in 2,2,2-trifluoroethanol $(0.42 \mathrm{~mL})$, and the reaction mixture was stirred at $45^{\circ} \mathrm{C}$ for $24 \mathrm{~h}$, under an argon atmosphere. The reaction mixture was concentrated in vacuo and the residue was purified by column chromatography (petroleumether/ethyl acetate $=9 / 1$, then petroleum-ether/ethyl acetate $=975 / 25$, and finally benzene/ethyl acetate $=49 / 1)$ to afford $9.3 \mathrm{mg}(43 \%)$ of compound $\mathbf{6}$, as colorless oil. Obtained as a mixture of trans and cis isomers in a relative ratio 2.56:1, according to ${ }^{1} \mathrm{H}$ NMR spectrum integration of [$\mathrm{COCH}_{3}$ ] signals at $2.10(\mathrm{~s}, 3 \mathrm{H}$, minor) and 2.05 (s, 3H, major).

Spectral data for the mixture of diastereoisomers:

${ }^{1} \mathbf{H}$ NMR (400 MHz, $\left.\mathrm{CDCl}_{3}\right) \delta 5.49-5.16$ (m, 2H, minor+major), $3.39-3.26$ (m, 1H, minor), $3.20-3.07$ (m, 1H, minor+major), $3.03-2.89$ (m, 1H, major), 2.74-2.64 (m, 1H, major), 2.10 (s, $3 \mathrm{H}$, minor), 2.05 (s, 3H, major), $1.97-1.88(\mathrm{~m}, 2 \mathrm{H}$, minor+major), $1.83-1.69$ (m, 2H, minor+major), 1.63 (m, 1H, minor+major), 1.47-1.22 (m, 13H, minor+major), $0.94-0.83$ (m, 3H, minor+major).

${ }^{13} \mathrm{C}$ NMR (101 MHz, $\mathrm{CDCl}_{3}$ ) $\delta 211.0$ (minor), 210.6 (major), 133.2 (minor), 131.3 (major), 131.2 (major), 130.0 (minor), 58.3, 55.6, 55.29, 46.00, 45.6, 44.8, 42.1, 41.9, 40.0, 39.0, 38.9, 38.8, 38.0, $34.6,31.4,31.1,30.0,29.6,26.4,26.3,24.3,23.8,23.6,23.4,23.0,22.8,14.0$ (minor), 13.8 (major).

IR $($ ATR $) \vee 2928,2857,1707,1644,1449,1355,1162,889$.

HRMS (ESI) calcd. for $\mathrm{C}_{17} \mathrm{H}_{29} \mathrm{O}^{+}[\mathrm{M}+\mathrm{H}]^{+}:$249.2213; found: 249.2209.

1-(2-Methyl-5-(prop-1-en-2-yl)cyclopentyl)-2-phenylethan-1-one (8) and (Z)-3-benzylidene1,1,4-trimethylhexahydro-1 $H$-cyclopenta[c]furan (9)
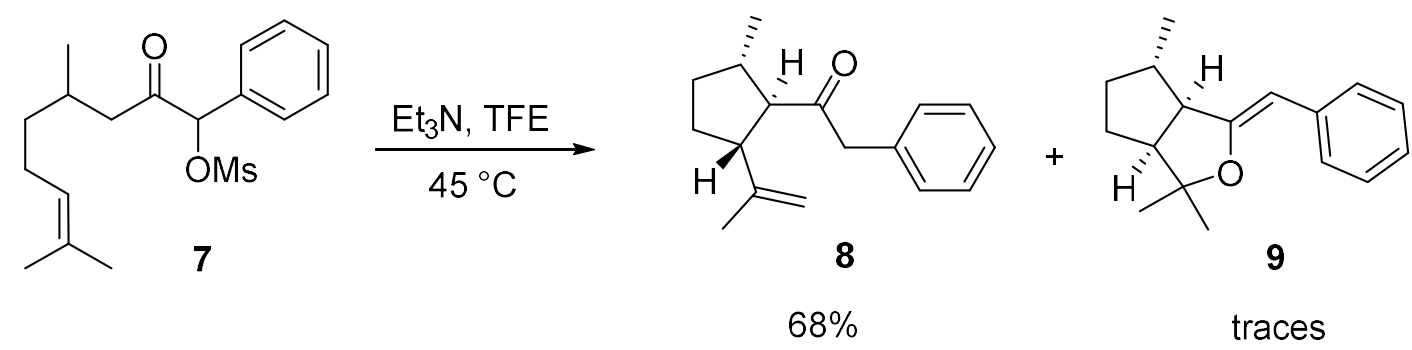

According to the General procedure - Method A. Using 7 ( $8 \mathrm{mg}, 0.024 \mathrm{mmol}, 1 \mathrm{eq})$, triethylamine $(5.3 \mu \mathrm{L}, 0.038 \mathrm{mmol}, 1.6 \mathrm{eq})$ and trifluoroethanol $(0.1 \mathrm{~mL})$, during $1.5 \mathrm{~h}$. Purification by column chromatography (benzene/ethyl acetate $=99 / 1$ ) afforded $3.9 \mathrm{mg}(68 \%)$ of compound $\mathbf{8}$, as a colorless oil. Only traces of $\mathbf{9}$ were formed under these conditions. 
1-(2-Methyl-5-(prop-1-en-2-yl)cyclopentyl)-2-phenylethan-1-one (8) and (Z)-3-benzylidene1,1,4-trimethylhexahydro-1H-cyclopenta[c]furan (9) (General procedure for the cyclization Method B)
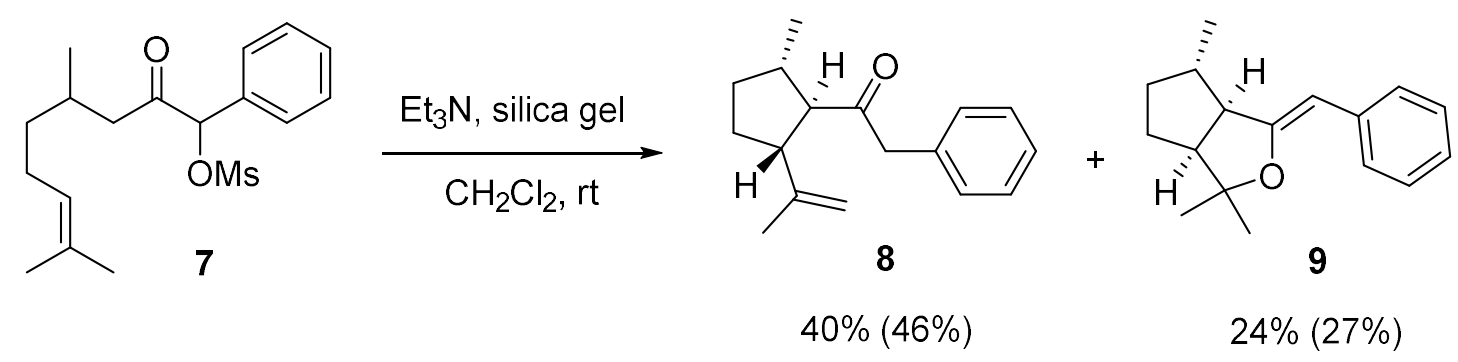

Silica gel $(20 \mathrm{mg})$ was added to the solution of compound 7 (7.6 $\mathrm{mg}, 0.0225 \mathrm{mmol}, 1 \mathrm{eq})$ and triethylamine $(5 \mu \mathrm{L}, 0.036 \mathrm{mmol}, 1.6 \mathrm{eq})$ in $\mathrm{CH}_{2} \mathrm{Cl}_{2}(0.1 \mathrm{~mL})$ and the reaction mixture was stirred at room temperature for 2 days. Purification of the crude reaction mixture by column chromatography (benzene/ethyl acetate $=99 / 1)$ afforded $2.2 \mathrm{mg}(40 \%, 46 \%$ based on the recovered starting material) ) of compound 8 and $1.3 \mathrm{mg}(24 \%, 27 \%$ based on the recovered starting material) of compound $\mathbf{9}$, as colorless oils.

Spectral data for $\mathbf{8}$ :

${ }^{1} \mathbf{H}$ NMR $\left(500 \mathrm{MHz}, \mathrm{CDCl}_{3}\right) \delta 7.34-7.28(\mathrm{~m}, 2 \mathrm{H}), 7.27-7.22(\mathrm{~m}, 1 \mathrm{H}), 7.17-7.13(\mathrm{~m}, 2 \mathrm{H})$, $4.73-4.69(\mathrm{~m}, 2 \mathrm{H}), 3.71(\mathrm{~d}, J=15.4 \mathrm{~Hz}, 1 \mathrm{H}), 3.65(\mathrm{~d}, J=15.4 \mathrm{~Hz}, 1 \mathrm{H}), 2.88-2.80(\mathrm{~m}, 1 \mathrm{H})$, $2.55-2.50(\mathrm{~m}, 1 \mathrm{H}), 2.30-2.20(\mathrm{~m}, 1 \mathrm{H}), 1.93-1.82(\mathrm{~m}, 2 \mathrm{H}), 1.65(\mathrm{~s}, 3 \mathrm{H}), 1.63-1.56(\mathrm{~m}, 1 \mathrm{H})$, $1.34-1.24(\mathrm{~m}, 1 \mathrm{H}), 0.91(\mathrm{~d}, J=6.7 \mathrm{~Hz}, 3 \mathrm{H})$.

${ }^{13} \mathbf{C}$ NMR $\left(125 \mathrm{MHz}, \mathrm{CDCl}_{3}\right) \delta 210.4,146.6,133.9,129.7,128.5,126.9,110.9,62.6,52.4,50.7$, 38.8, 33.6, 30.2, 19.7, 19.5 .

IR (ATR) $v_{\max }: 3066,3029,2954,2867,1706,1643,1495,1454,1363$.

HRMS (ESI) calcd. for $\mathrm{C}_{17} \mathrm{H}_{23} \mathrm{O}^{+}[\mathrm{M}+\mathrm{H}]^{+}:$243.1743, found: 243.1740 .

Correlations (NOESY) of $\mathbf{8}$ :

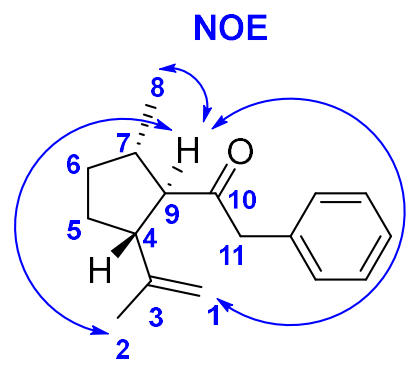

Me-8/H-9

Me-2/H-9

H-9/H-1 
Spectral data for $\mathbf{9}$ :

${ }^{1}$ H NMR $\left(500 \mathrm{MHz}, \mathrm{CDCl}_{3}\right) \delta 7.56-7.53(\mathrm{~m}, 2 \mathrm{H}), 7.26-7.22(\mathrm{~m}, 2 \mathrm{H}), 7.06-7.01(\mathrm{~m}, 1 \mathrm{H}), \quad 5.14$ (d, $J=1.4,1 \mathrm{H}), 3.03(\mathrm{dd}, J=8.1,4.2, \mathrm{~Hz}, 1 \mathrm{H}), 2.41(\mathrm{dd}, J=17.1,8.8,1 \mathrm{H}), 2.23-2.13(\mathrm{~m}, 1 \mathrm{H})$, $1.96-1.89(\mathrm{~m}, 1 \mathrm{H}), 1.75-1.61(\mathrm{~m}, 1 \mathrm{H}), 1.59-1.51(\mathrm{~m}, 1 \mathrm{H}), 1.43(\mathrm{~s}, 3 \mathrm{H}), 1.31(\mathrm{~s}, 3 \mathrm{H}), 1.22-$ $1.16(\mathrm{~m}, 1 \mathrm{H}), 1.16(\mathrm{~d}, J=6.9,1 \mathrm{H})$.

${ }^{13} \mathbf{C}$ NMR $\left(125 \mathrm{MHz}, \mathrm{CDCl}_{3}\right) \delta 162.4,137.7,128.1,127.0,124.2,96.6,86.6,56.8,51.4,42.6$, $36.0,29.8,27.7,23.9,21.4$.

IR (ATR) $v_{\max }: 3062,3030,2962,2868,1728,1600,1493,1452,1373$.

HRMS (ESI) calcd. for $\mathrm{C}_{17} \mathrm{H}_{22} \mathrm{O}^{+}[\mathrm{M}+\mathrm{H}]^{+}: 243.1743$, found: 243. 1733 .

1-((1R,2S,5R)-2-Methyl-5-(prop-1-en-2-yl)cyclopentyl)-2-phenylethanone $\left(8^{*}\right)$ and (3aR,4S,6aS,Z)-3-benzylidene-1,1,4-trimethylhexahydro-1 $H$-cyclopenta[c]furan (9*)
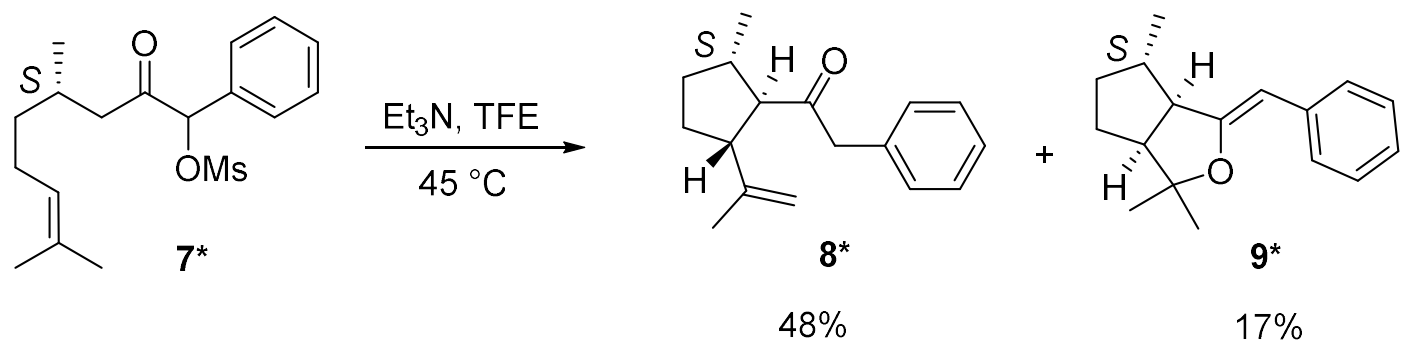

According to the General procedure - Method A. Using 7* (36.7 mg, $0.108 \mathrm{mmol}, 1 \mathrm{eq})$, triethylamine $(24 \mu \mathrm{L}, 0.173 \mathrm{mmol}, 1.6 \mathrm{eq})$ and trifluoroethanol $(0.36 \mathrm{~mL})$, during $4 \mathrm{~h}$. Purification by column chromatography (petroleum-ether/diisopropyl ether $=95 / 5)$ afforded $13.5 \mathrm{mg}(48 \%)$ of compound $\mathbf{8}^{*}$ and $4.8 \mathrm{mg}(17 \%)$ of compound $\mathbf{9}^{*}$, both as colorless oils.

Spectral data for $\mathbf{8}^{*}$ :

$[\alpha]_{\mathrm{D}}^{20}-44.9\left(c 1.27, \mathrm{CDCl}_{3}\right)$.

${ }^{1}$ H NMR $\left(500 \mathrm{MHz}, \mathrm{CDCl}_{3}\right) \delta 7.34-7.29(\mathrm{~m}, 2 \mathrm{H}), 7.28-7.23(\mathrm{~m}, 1 \mathrm{H}), 7.18-7.15(\mathrm{~m}, 2 \mathrm{H}), 4.74$ $-4.71(\mathrm{~m}, 2 \mathrm{H}), 3.72(\mathrm{~d}, J=15.4 \mathrm{~Hz}, 1 \mathrm{H}), 3.66(\mathrm{~d}, J=15.4 \mathrm{~Hz}, 1 \mathrm{H}), 2.85(\mathrm{dd}, J=18.1,8.9 \mathrm{~Hz}$, $1 \mathrm{H}), 2.57-2.51(\mathrm{~m}, 1 \mathrm{H}), 2.32-2.21(\mathrm{~m}, 1 \mathrm{H}), 1.94-1.83(\mathrm{~m}, 2 \mathrm{H}), 1.67-1.66(\mathrm{~m}, 3 \mathrm{H}), 1.65-$ $1.56(\mathrm{~m}, 1 \mathrm{H}), 1.35-1.25(\mathrm{~m}, 1 \mathrm{H}), 0.92(\mathrm{~d}, J=6.7 \mathrm{~Hz}, 3 \mathrm{H})$.

${ }^{13} \mathbf{C}$ NMR $\left(125 \mathrm{MHz}, \mathrm{CDCl}_{3}\right) \delta 210.4,146.6,133.9,129.7,128.5,126.9,110.9,62.6,52.5,50.7$, $38.8,33.6,30.3,19.7,19.5$.

IR (ATR) $v_{\max }: 2924,2854,1710,1495,1458,1375$.

HRMS (ESI) calcd. for $\mathrm{C}_{17} \mathrm{H}_{23} \mathrm{O}^{+}\left[\mathrm{M}+\mathrm{H}^{+}\right]$: 243.1743, found: 243.1740 . 
Correlations (NOESY) for $\mathbf{8}^{*}$ :

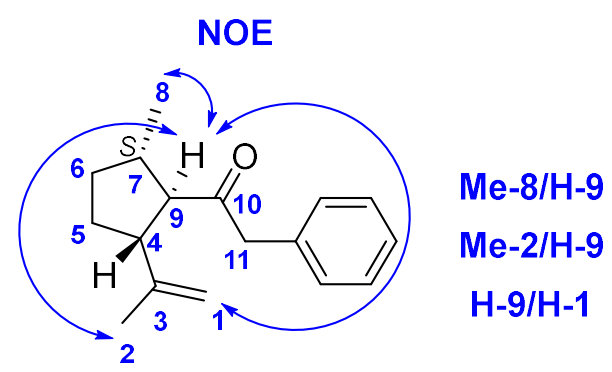

Spectral data for $9 *$ :

$[\alpha]_{\mathrm{D}}^{20}-101.1\left(\mathrm{c} 0.26, \mathrm{C}_{6} \mathrm{H}_{6}\right)$.

${ }^{1}$ H NMR $\left(500 \mathrm{MHz}, \mathrm{C}_{6} \mathrm{D}_{6}\right) \delta 7.85-7.82(\mathrm{~m}, 2 \mathrm{H}), 7.30-7.24(\mathrm{~m}, 1 \mathrm{H}), 7.04-6.99(\mathrm{~m}, 2 \mathrm{H}), 5.28$ $(\mathrm{d}, J=1.4,1 \mathrm{H}), 3.75(\mathrm{ddd}, J=8.6,4.3,1.1, \mathrm{~Hz}, 1 \mathrm{H}), 2.08-1.98(\mathrm{~m}, 1 \mathrm{H}), 1.88(\mathrm{dd}, J=17.1,8.6$, $1 \mathrm{H}), 1.71-1.65(\mathrm{~m}, 1 \mathrm{H}), 1.34-1.28(\mathrm{~m}, 2 \mathrm{H}), 1.17(\mathrm{~s}, 3 \mathrm{H}), 1.05(\mathrm{~s}, 3 \mathrm{H}), 0.95(\mathrm{~d}, J=6.9,3 \mathrm{H})$, $0.93-0.88(\mathrm{~m}, 1 \mathrm{H})$.

${ }^{13}$ C NMR (126 MHz, $\left.\mathrm{C}_{6} \mathrm{D}_{6}\right) \delta 162.2,138.4,128.5,128.4,124.8,97.9,86.4,56.9,51.4,42.8,36.1$, 29.8, 27.8, 23.8, 21.4.

IR (ATR) $v_{\max }: 3053,3020,2955,2864,1662,1599,1493,1449,1365$.

HRMS (ESI) calcd. for $\mathrm{C}_{17} \mathrm{H}_{23} \mathrm{O}^{+}[\mathrm{M}+\mathrm{H}]^{+}:$243.1743, found: 243.1736.

Note: NMR of 9 was taken in $\mathrm{C}_{6} \mathrm{D}_{6}$ because it gradually decomposes in $\mathrm{CDCl}_{3}$ (probably due to acidity of chloroform). Generally, benzylidenetetrahydrofuranes were sensitive to acidic conditions, and very difficult to purify on silica and isolate in pure state.

1-(2-Methyl-5-vinylcyclopentyl)-2-phenylethan-1-one (11) and (Z)-3-benzylidene-1,4dimethylhexahydro- $1 H$-cyclopenta[c]furan (12)

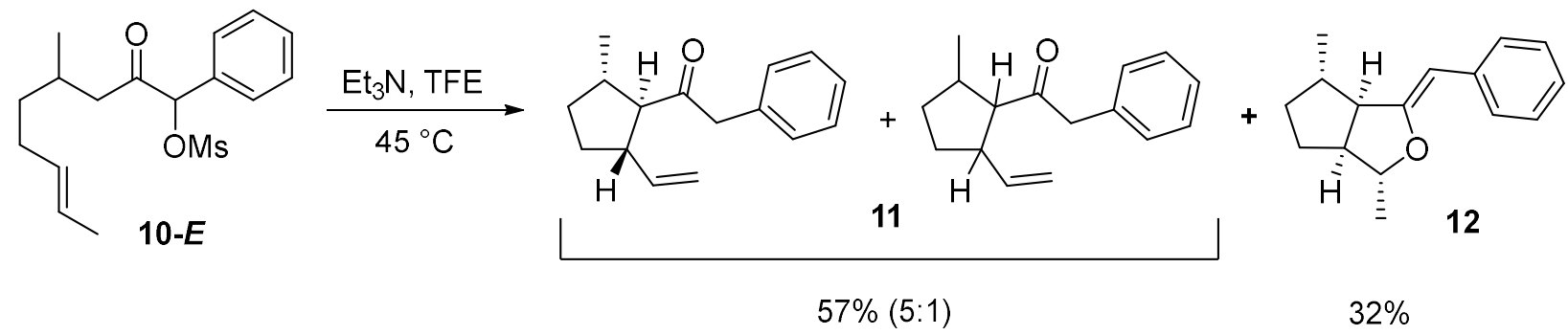

According to the General procedure - Method A. Using mesyloxy ketone 10-E (9.7 mg, 0.03 mmol, $1 \mathrm{eq})$, triethylamine $(6.5 \mu \mathrm{L}, 0.046 \mathrm{mmol}, 1.6 \mathrm{eq})$ and trifluoroethanol $(0.1 \mathrm{~mL})$, during 3 h. Purification by column chromatography (petroleum-ether/diisopropyl ether $=95 / 5$ ) afforded 3.9 $\mathrm{mg}(57 \%)$ of compound 11 as a colorless oil (mixture of isomers in a relative ratio 5:1, as determined by $\left.{ }^{1} \mathrm{H} \mathrm{NMR}\right)$ and $2.2 \mathrm{mg}(32 \%)$ of compound 12, as a colorless oil. 
Spectral data for 11, mixture of isomers in the ratio $\mathrm{H}: \mathrm{H}^{\prime}=5: 1$

${ }^{1} \mathbf{H}$ NMR $\left(500 \mathrm{MHz}, \mathrm{CDCl}_{3}\right) \delta 7.33-7.28\left(\mathrm{~m}, 2 \mathrm{H}+2 \mathrm{H}^{\prime}\right), 7.26-7.22\left(\mathrm{~m}, 1 \mathrm{H}+1 \mathrm{H}^{\prime}\right), 7.17-7.13$ $\left(\mathrm{m}, 2 \mathrm{H}+2 \mathrm{H}^{\prime}\right), 5.74-5.62\left(\mathrm{~m}, 1 \mathrm{H}+1 \mathrm{H}^{\prime}\right), 5.04-5.62\left(\mathrm{~m}, 2 \mathrm{H}+2 \mathrm{H}^{\prime}\right), 3.72(\mathrm{~d}, J=15.4 \mathrm{~Hz}, 1 \mathrm{H}), 3.66$ $(\mathrm{d}, J=15.4 \mathrm{~Hz}, 1 \mathrm{H}), 3.68\left(\mathrm{~d}, J=15.7 \mathrm{~Hz}, 1 \mathrm{H}^{\prime}\right), 3.59$ (d, $\left.J=15.5 \mathrm{~Hz}, 1 \mathrm{H}^{\prime}\right), 2.98-2.88$ (m, 1H'), $2.77-2.67\left(\mathrm{~m}, 1 \mathrm{H}+1 \mathrm{H}^{\prime}\right), 2.44-2.34\left(\mathrm{~m}, 1 \mathrm{H}+1 \mathrm{H}^{\prime}\right), 2.32-2.22(\mathrm{~m}, 1 \mathrm{H}), 1.97-1.84\left(\mathrm{~m}, 2 \mathrm{H}^{2}+\mathrm{H}^{\prime}\right)$, $1.57-1.47\left(\mathrm{~m}, 1 \mathrm{H}^{+}+\mathrm{H}^{\prime}\right), 1.34-1.24(\mathrm{~m}, 1 \mathrm{H}), 1.19-1.11\left(\mathrm{~m}, 1 \mathrm{H}^{\prime}\right), 0.88(\mathrm{~d}, J=6.6 \mathrm{~Hz}, 3 \mathrm{H})$, $0.85\left(\mathrm{~d}, J=6.6 \mathrm{~Hz}, 3 \mathrm{H}^{\prime}\right)$.

${ }^{13} \mathrm{C}$ NMR $\left(125 \mathrm{MHz}, \mathrm{CDCl}_{3}\right) \delta 210.2,208.9,141.4,139.8,129.7,128.5,126.9,126.8,114.8$, $114.3,64.9,62.4,51.6,51.1,49.7,47.4,38.6,33.5,33.3,32.6,31.8,19.7,19.6$.

IR (ATR) $v_{\max }:$ 3066, 3030, 2954, 2869, 1708, 1640, 1495, 1455, 1369.

HRMS (ESI) calcd. for $\mathrm{C}_{17} \mathrm{H}_{26} \mathrm{ON}^{+}\left[\mathrm{M}+\mathrm{NH}_{4}\right]^{+}$: 246.1852, found: 246.1845 .

Spectral data for 12:

${ }^{1}$ H NMR $\left(500 \mathrm{MHz}, \mathrm{C}_{6} \mathrm{D}_{6}\right) \delta 7.89-7.86(\mathrm{~m}, 2 \mathrm{H}), 7.33-7.28(\mathrm{~m}, 2 \mathrm{H}), 7.08-7.04(\mathrm{~m}, 1 \mathrm{H}), 5.31$ $(\mathrm{d}, J=0.6 \mathrm{~Hz}, 1 \mathrm{H}), 3.95(\mathrm{p}, J=6.1 \mathrm{~Hz}, 1 \mathrm{H}), 2.56-2.51(\mathrm{~m}, 1 \mathrm{H}), 1.99-1.86(\mathrm{~m}, 2 \mathrm{H}), 1.71-1.62$ $(\mathrm{m}, 1 \mathrm{H}), 1.60-1.51(\mathrm{~m}, 1 \mathrm{H}), 1.15-1.00(\mathrm{~m}, 2 \mathrm{H}), 1.06(\mathrm{~d}, J=6.2 \mathrm{~Hz}, 3 \mathrm{H}), 0.92(\mathrm{~d}, J=6.8,3 \mathrm{H})$.

${ }^{13} \mathrm{C}$ NMR (125 MHz, $\left.\mathrm{CDCl}_{3}\right) \delta 161.8,138.6,128.9,128.7,128.3,125.2,98.1,86.2,56.9,49.9$, 42.0, 35.7, 29.4, 21.6, 20.1.

IR (ATR) $v_{\max }: 3057,3021,2954,2869,1756,1667,1600,1493,1451,1372,1314$.

HRMS (ESI) calcd. for $\mathrm{C}_{16} \mathrm{H}_{21} \mathrm{O}^{+}[\mathrm{M}+\mathrm{H}]^{+}:$:229.1587, found: 229.1577.

1-(2-Methyl-5-styrylcyclopentyl)-2-phenylethan-1-one (14) and 1-benzyl-3-benzylidene-4methylhexahydro-1H-cyclopenta[c]furan (15)

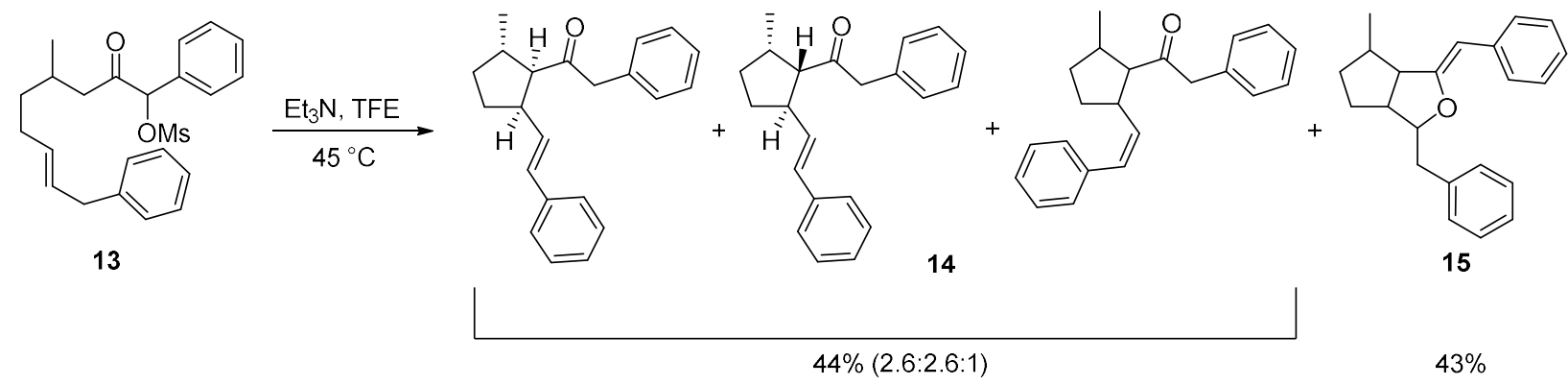

According to the General procedure - Method A. Using mesyloxy ketone 13 (8.3 mg, $0.02 \mathrm{mmol}$, $1 \mathrm{eq})$, triethylamine $(4.6 \mu \mathrm{L}, 0.033 \mathrm{mmol}, 1.6 \mathrm{eq})$ and trifluoroethanol $(0.1 \mathrm{~mL})$, during $4 \mathrm{~h}$. Purification by column chromatography (petroleum-ether/toluene $=1 / 2$ ) afforded $2.8 \mathrm{mg}(44 \%)$ of compound 14, as a colorless oil (mixture of 4 isomers) and $2.7 \mathrm{mg}$ (43\%) of 15, a colorless oil (mixture of 4 isomers, in a relative ratio: 6:1:1:1).

Spectral data for 14, mixture of four isomers: $E$-a, $E$-b, $Z$-a, $Z$-b with the ratio $E: Z=5.25: 1$, where $\mathrm{H}_{\mathrm{Ea}}$ and $\mathrm{H}_{\mathrm{Eb}}$ are $E$ isomers in the ratio of $1: 1$ and $\mathrm{H}_{\mathrm{Za}}$ and $\mathrm{H}_{\mathrm{Zb}}$ are $Z$ isomers in the ratio of 1:1. 
${ }^{1} \mathbf{H}$ NMR $\left(500 \mathrm{MHz}, \mathrm{CDCl}_{3}\right) \delta 7.40-6.99\left(\mathrm{~m}, 10 \mathrm{H}_{\mathrm{Ea}+\mathrm{Eb}+\mathrm{Za}+\mathrm{Zb}}\right), 6.46\left(\mathrm{~d}, J=6.6 \mathrm{~Hz}, 1 \mathrm{H}_{\mathrm{Za}}\right), 6.44$ $\left(\mathrm{d}, J=6.5 \mathrm{~Hz}, 1 \mathrm{H}_{\mathrm{zb}}\right), 6.37\left(\mathrm{~d}, J=15.7 \mathrm{~Hz}, 1 \mathrm{H}_{\mathrm{Ea}}\right), 6.31\left(\mathrm{~d}, J=15.7 \mathrm{~Hz}, 1 \mathrm{H}_{\mathrm{Eb}}\right), 6.08-5.99(\mathrm{~m}$, $\left.1 \mathrm{H}_{\mathrm{Ea}+\mathrm{Eb}}\right), 5.60-5.54\left(\mathrm{~m}, 1 \mathrm{H}_{\mathrm{Za}+Z \mathrm{~b}}\right), 3.73-3.46\left(\mathrm{~m}, 2 \mathrm{H}_{\mathrm{Ea}+\mathrm{Eb}+\mathrm{Za}+\mathrm{Zb}}\right), 3.35-3.26\left(\mathrm{~m}, 1 \mathrm{H}_{\mathrm{Za}+Z \mathrm{~b}}\right), 3.14$ $-3.04\left(\mathrm{~m}, 1 \mathrm{H}_{\mathrm{Ea}}\right), 2.95-2.87\left(\mathrm{~m}, 1 \mathrm{H}_{\mathrm{Eb}}\right), 2.81\left(\mathrm{t}, J=8.9 \mathrm{~Hz}, 1 \mathrm{H}_{\mathrm{Ea}}\right), 2.68\left(\mathrm{t}, J=8.9 \mathrm{~Hz}, 1 \mathrm{~Hz}_{\mathrm{Za}}\right), 2.56$ $-2.45\left(\mathrm{~m}, 2 \mathrm{H}_{\mathrm{Ea}+\mathrm{Eb}}\right), 2.41-2.27\left(\mathrm{~m}, 2 \mathrm{H}_{\mathrm{Eb}+Z \mathrm{~b}}\right), 2.06-1.89\left(\mathrm{~m}, 2 \mathrm{H}_{\mathrm{Ea}+\mathrm{Eb}+Z \mathrm{a}+Z \mathrm{~b}}\right), 1.69-1.56(\mathrm{~m}$,

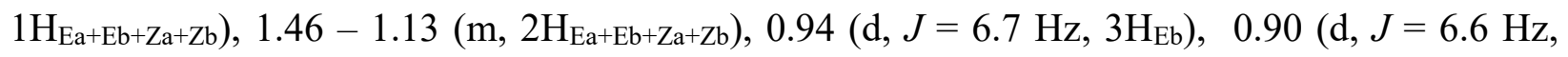
$\left.3 \mathrm{H}_{\mathrm{Ea}}\right), 0.91-0.88\left(\mathrm{~m}, 3 \mathrm{H}_{\mathrm{Zb}}\right), 0.87\left(\mathrm{~d}, J=6.5 \mathrm{~Hz}, 3 \mathrm{~Hz}_{\mathrm{za}}\right)$.

${ }^{13} \mathbf{C}$ NMR $\left(125 \mathrm{MHz}, \mathrm{CDCl}_{3}\right) \delta 210.3,209.1,137.2,137.1,135.9,133.9,133.7,133.6,133.0$, $131.5,130.2,129.7,129.7,129.6,128.7,128.6,128.5,128.3,127.2,127.2,126.9,126.8,126.3$, 126.1, 66.2, 66.1, 62.5, 62.6, 62.3, 51.8, 51.4, 49.1, 46.7, 38.9, 33.4, 33.3, 32.1, 19.9, 19.7.

IR (ATR) $v_{\max }: 3082,3060,3027,2952,2867,1945,1875,1802,1708,1600,1493,1452,1406$, 1372.

HRMS (ESI) calcd. for $\mathrm{C}_{22} \mathrm{H}_{28} \mathrm{ON}^{+}\left[\mathrm{M}+\mathrm{NH}_{4}\right]^{+}: 322.2165$, found: 322.2154 .

Spectral data for $\mathbf{1 5}$, major isomer:

${ }^{1} \mathbf{H}$ NMR $\left(500 \mathrm{MHz}, \mathrm{CDCl}_{3}\right) \delta 7.54-7.50(\mathrm{~m}, 2 \mathrm{H}), 7.36-7.20(\mathrm{~m}, 7 \mathrm{H}), 7.08-7.04(\mathrm{~m}, 1 \mathrm{H}), 5.18$ (s, 1H), 4.37 (dt, $J=7.1,5.8 \mathrm{~Hz}, 1 \mathrm{H}), 3.00$ (dd, $J=13.7,7.3 \mathrm{~Hz}, 1 \mathrm{H}), 2.87$ (dd, $J=13.7,5.8 \mathrm{~Hz}$, $1 \mathrm{H}), 2.79-2.74(\mathrm{~m}, 1 \mathrm{H}), 2.62-2.54(\mathrm{~m}, 1 \mathrm{H}), 2.11-2.03(\mathrm{~m}, 1 \mathrm{H}), 1.91-1.82(\mathrm{~m}, 2 \mathrm{H}), 1.38-$ $1.26(\mathrm{~m}, 2 \mathrm{H}), 1.09(\mathrm{~d}, J=6.8,3 \mathrm{H})$.

${ }^{13}$ C NMR $\left(125 \mathrm{MHz}, \mathrm{CDCl}_{3}\right) \delta 161.3,137.8,137.2,129.5,129.0,128.3,128.2,128.1,127.3$ $(2 \times \mathrm{C}), 127.2,126.4,125.1,124.4,96.9,90.5,57.1,52.2,47.2,42.1,41.8,35.4,29.6,20.4,19.6$. IR (ATR) v $v_{\max }$ 3084, 3061, 3027, 2955, 2931, 2867, 1753, 1699, 1600, 1494, 1453, 1410, 1375. HRMS (ESI) calcd. for $\mathrm{C}_{22} \mathrm{H}_{25} \mathrm{O}^{+}[\mathrm{M}+\mathrm{H}]^{+}: 305.1900$, found: 305.1890 .

\section{4,8-Dimethyl-1-(2-methyl-5-(prop-1-en-2-yl)cyclopentyl)non-7-en-1-one (17)}

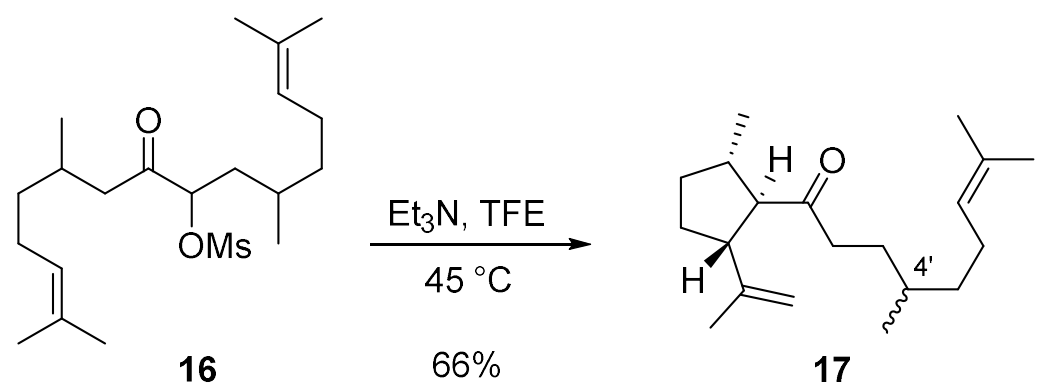

According to the General procedure - Method A. Using mesyloxy ketone 16 ( $8.8 \mathrm{mg}, 0.023 \mathrm{mmol}$, $1 \mathrm{eq})$, triethylamine $(5 \mu \mathrm{L}, 0.036 \mathrm{mmol}, 1.6 \mathrm{eq})$ and trifluoroethanol $(0.1 \mathrm{~mL})$, during $4 \mathrm{~h}$. Purifications by column chromatography (petroleum-ether/toluene $=3 / 1)$ afforded $4.4 \mathrm{mg}(66 \%)$ of $\mathbf{1 7}$, as a colorless oil (mixture of isomers at $\mathrm{C}-4$ ' in a relative ratio $1: 1$, as determined by ${ }^{1} \mathrm{H}$ NMR). 
Spectral data for the mixture of isomers 17

${ }^{1} \mathbf{H}$ NMR $\left(500 \mathrm{MHz}, \mathrm{CDCl}_{3}\right) \delta 5.11-5.05(\mathrm{~m}, 1 \mathrm{H}), 4.75-4.64(\mathrm{~m}, 2 \mathrm{H}), 2.83-2.76(\mathrm{~m}, 1 \mathrm{H})$, $2.47-2.30(\mathrm{~m}, 3 \mathrm{H}), 2.26-2.16(\mathrm{~m}, 1 \mathrm{H}), 2.04-1.82(\mathrm{~m}, 4 \mathrm{H}), 1.71(\mathrm{~s}, 3 \mathrm{H}), 1.68(\mathrm{~s}, 3 \mathrm{H}), 1.63-$ $1.56(\mathrm{~m}, 2 \mathrm{H}), 1.60(\mathrm{~s}, 3 \mathrm{H}), 1.43-1.24(\mathrm{~m}, 4 \mathrm{H})$, ), $1.18-1.09(\mathrm{~m}, 1 \mathrm{H}), 1.02$ (apparent dd, $J=6.7$, $0.9 \mathrm{~Hz}, 3 \mathrm{H},), 0.86(\mathrm{~d}, J=6.0 \mathrm{~Hz}, 3 \mathrm{H})$.

${ }^{13}$ C NMR (125 MHz, $\left.\mathrm{CDCl}_{3}\right) \delta$ 213.9, 147.0, 131.4, 125.0, 110.8, 64.2, 64.1, 52.3, 52.2, 41.2, 39.0, 37.1, 37.1, 33.8, 32.3, 30.5, 30.4, 25.9, 25.7, 20.2, 20.0, 19.6, 17.9.

IR (ATR) $v_{\max }:$ 2955, 2924, 2869, 1708, 1645, 1455, 1376.

HRMS (ESI) calcd. for $\mathrm{C}_{20} \mathrm{H}_{35} \mathrm{O}[\mathrm{M}+\mathrm{H}]^{+}:$291.2682, found: 291.2676.

2-Phenyl-1-(4-(prop-1-en-2-yl)tetrahydrofuran-3-yl)ethan-1-one (19) and (Z)-3benzylidene-1,1-dimethyltetrahydro- $1 \mathrm{H}, 3 \mathrm{H}$-furo[3,4-c]furan (20)

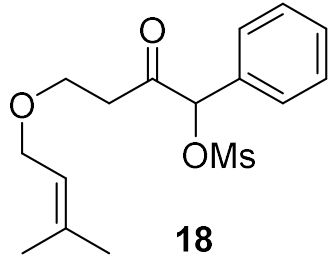

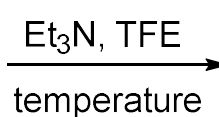

$23^{\circ} \mathrm{C}$

$50{ }^{\circ} \mathrm{C}$

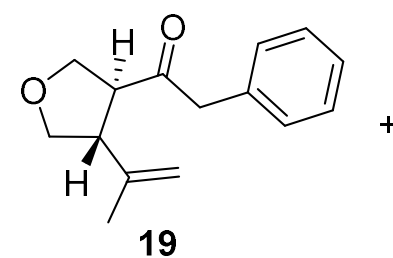

$47 \%$

$50 \%$

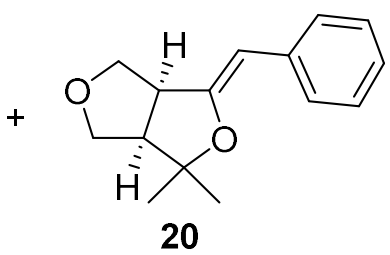

$5 \%$

According to the General procedure - Method A. Using mesyloxy ketone 18 (22.5 mg, 0.069 mmol, $1 \mathrm{eq})$, triethylamine $(11 \mu \mathrm{L}, 0.11 \mathrm{mmol}, 1.6 \mathrm{eq})$ and trifluoroethanol $(0.22 \mathrm{~mL})$ at room temperature, during $40 \mathrm{~min}$. Purification by two consecutive column chromatographies (petroleum-ether/ ethyl acetate $=85 / 15$, followed by benzene/ ethyl acetate $=98 / 2$ ) afforded 7.5 $\mathrm{mg}(47 \%)$ of compound 19 and $0.8 \mathrm{mg}(5 \%)$ of compound $\mathbf{2 0}$, both as colorless oils.

At $50{ }^{\circ} \mathrm{C}$, reaction afforded compound 19 in $50 \%$ yield and only traces of compound 20 were formed.

Spectral data for 19:

${ }^{1} \mathbf{H}$ NMR $\left(500 \mathrm{MHz}, \mathrm{CDCl}_{3}\right) \delta 7.36-7.30(\mathrm{~m}, 2 \mathrm{H}), 7.30-7.25(\mathrm{~m}, 1 \mathrm{H}), 7.19-7.15(\mathrm{~m}, 2 \mathrm{H})$, $4.84-4.80(\mathrm{~m}, 2 \mathrm{H}), 4.00-4.95(\mathrm{~m}, 1 \mathrm{H}), 3.92-3.84(\mathrm{~m}, 2 \mathrm{H}), 3.75(\mathrm{~d}, J=15.2 \mathrm{~Hz}, 1 \mathrm{H}), 3.71$ $(\mathrm{d}, J=15.2 \mathrm{~Hz}, 1 \mathrm{H}), 3.63(\mathrm{dd}, J=8.6,7.3 \mathrm{~Hz}, 1 \mathrm{H}), 3.26(\mathrm{dd}, J=15.1,7.5 \mathrm{~Hz}, 1 \mathrm{H}), 3.13(\mathrm{dd}, J=$ $15.1,7.5 \mathrm{~Hz}, 1 \mathrm{H}), 1.69(\mathrm{~s}, 3 \mathrm{H})$.

${ }^{13} \mathrm{C}$ NMR (125 MHz, $\left.\mathrm{CDCl}_{3}\right) \delta 206.9,143.3,133.4,129.5,128.8,127.2,112.7,72.7,70.4,54.5$, $50.6,50.2,19.9$.

IR (ATR) $v_{\max }:$ 3030, 2956, 2921, 2853, 1714, 1646, 1603, 1495, 1456, 1375.

HRMS (ESI) calcd. for $\mathrm{C}_{15} \mathrm{H}_{22} \mathrm{O}_{2} \mathrm{~N}^{+}\left[\mathrm{M}+\mathrm{NH}_{4}\right]^{+}: 248.1645$, found: 248.1641 . 
Spectral data for 20:

${ }^{1} \mathbf{H}$ NMR $\left(500 \mathrm{MHz}, \mathrm{CDCl}_{3}\right) \delta 7.56-7.51(\mathrm{~m}, 2 \mathrm{H}), 7.30-7.23(\mathrm{~m}, 2 \mathrm{H}), 7.10-7.05(\mathrm{~m}, 1 \mathrm{H})$, $5.15(\mathrm{~s}, 1 \mathrm{H}), 4.08-4.03(\mathrm{~m}, 1 \mathrm{H}), 3.99(\mathrm{dd}, J=8.6,3.0 \mathrm{~Hz}, 1 \mathrm{H}), 3.85(\mathrm{dd}, J=9.3,6.0 \mathrm{~Hz}, 1 \mathrm{H})$, $3.78(\mathrm{dd}, J=9.2,7.5 \mathrm{~Hz}, 1 \mathrm{H}), 3.76-3.70(\mathrm{~m}, 1 \mathrm{H}), 2.70-2.64(\mathrm{~m}, 1 \mathrm{H}), 1.46(\mathrm{~s}, 3 \mathrm{H}), 1.40$ (s, $3 \mathrm{H})$.

${ }^{13} \mathbf{C}$ NMR $\left(125 \mathrm{MHz}, \mathrm{CDCl}_{3}\right) \delta 159.4,128.1,127.2,124.8,98.6,86.6,75.3,69.5,51.6,50.3,30.2$, 23.6 .

IR (ATR) $v_{\max }: 3084,3059,3024,2973,2931,2858,1757,1721,1665,1600,1494,1451,1369$. HRMS (ESI) calcd. for $\mathrm{C}_{15} \mathrm{H}_{22} \mathrm{O}_{2} \mathrm{~N}^{+}\left[\mathrm{M}+\mathrm{NH}_{4}\right]^{+}: 248.1645$, found: 248.1641 .

\section{4-((3-Methylbut-2-en-1-yl)oxy)-1-(4-(prop-1-en-2-yl)tetrahydrofuran-3-yl)butan-1-one (22)}
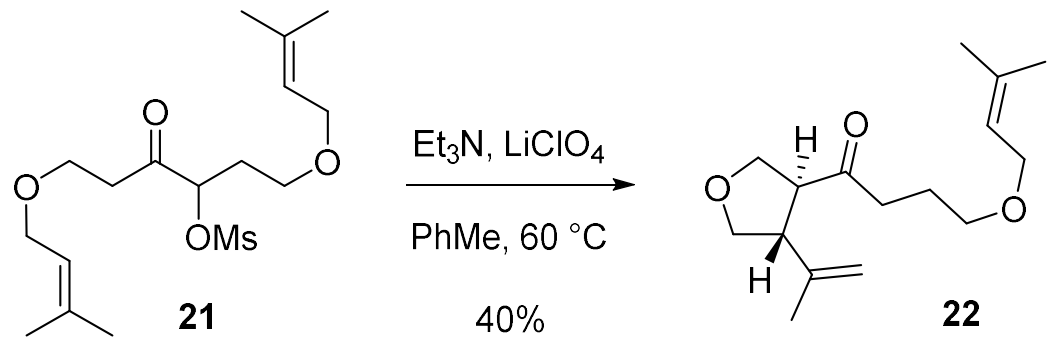

According to the General procedure - Method C. A suspension of mesyloxy ketone 21 (19.5 mg, $0.0538 \mathrm{mmol}, 1 \mathrm{eq})$, triethylamine $(15 \mu \mathrm{L}, 0.107 \mathrm{mmol}, 2 \mathrm{eq})$ and lithium perchlorate $(11.4 \mathrm{mg}$, $0.107 \mathrm{mmol}, 2 \mathrm{eq})$ in toluene $(0.18 \mathrm{~mL})$ was stirred at $60^{\circ} \mathrm{C}$ for $20 \mathrm{~min}$. The reaction mixture was directly purified by column chromatography (petroleum-ether/ethyl acetate $=85 / 15$ ) to afford 5.7 mg $(40 \%)$ of compound 22 , as a colorless oil.

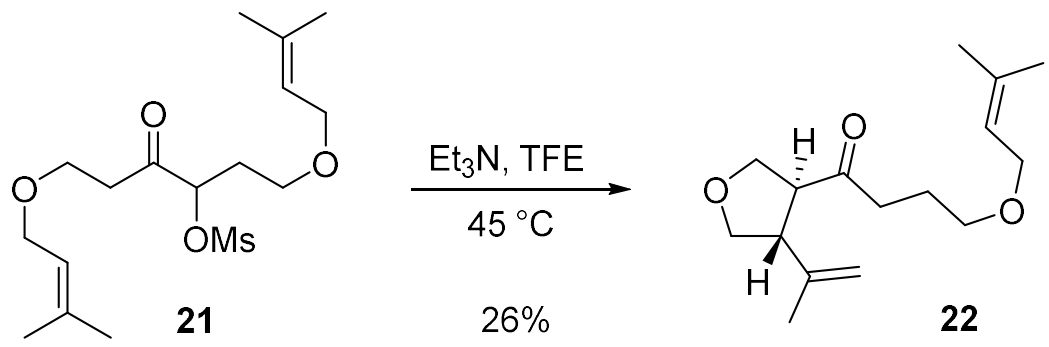

According to the General procedure - Method A. Using 21 (28.1 mg, $0.078 \mathrm{mmol}, 1 \mathrm{eq})$, triethylamine $(17 \mu \mathrm{L}, 0.124 \mathrm{mmol}, 1.6 \mathrm{eq})$ and trifluoroethanol $(0.25 \mathrm{~mL})$ at $45^{\circ} \mathrm{C}$, during $10 \mathrm{~min}$. Purification by column chromatography (petroleum-ether/ ethyl acetate $=85 / 15$ ) afforded $5.4 \mathrm{mg}$ (26\%) of compound 22 , as colorless oil. 
${ }^{1} \mathbf{H}$ NMR $\left(500 \mathrm{MHz}, \mathrm{CDCl}_{3}\right) \delta 5.34-5.28(\mathrm{~m}, 1 \mathrm{H}), 4.85-4.81(\mathrm{~m}, 2 \mathrm{H}), 4.03(\mathrm{t}, J=8.4 \mathrm{~Hz}, 1 \mathrm{H})$, $3.98(\mathrm{t}, J=8.1 \mathrm{~Hz}, 1 \mathrm{H}), 3.95-3.89(\mathrm{~m}, 3 \mathrm{H}), 3.65(\mathrm{dd}, J=7.2 \mathrm{~Hz}, 1 \mathrm{H}), 3.40$ (t, $J=6.4 \mathrm{~Hz}, 2 \mathrm{H})$, $3.17(\mathrm{dd}, J=14.9,7.3 \mathrm{~Hz}, 1 \mathrm{H}), 3.10(\mathrm{dd}, J=14.7,7.4 \mathrm{~Hz}, 1 \mathrm{H}), 2.62-2.48(\mathrm{~m}, 2 \mathrm{H}), 1.90-1.83$ $(\mathrm{m}, 2 \mathrm{H}), 1.76(\mathrm{~s}, 3 \mathrm{H}), 1.74(\mathrm{~s}, 3 \mathrm{H}), 1.66(\mathrm{~s}, 3 \mathrm{H})$.

${ }^{13} \mathrm{C}$ NMR $\left(125 \mathrm{MHz}, \mathrm{CDCl}_{3}\right) \delta$ 209.2, 143.5, 136.9, 121.1, 112.5, 72.5, 70.2, 68.9, 67.2, 55.7, 50.2, 39.4, 25.8, 23.7, 20.0, 18.0 .

IR (ATR) $v_{\text {max }}: 2968,2925,2856,1712,1645,1446,1409,1376$.

HRMS (ESI) calcd. for $\mathrm{C}_{16} \mathrm{H}_{26} \mathrm{O}_{3} \mathrm{Na}^{+}[\mathrm{M}+\mathrm{Na}]^{+}: 289.1774$, found: 289.1774 .

trans-(E)-4-(but-2-en-1-yloxy)-1-(4-vinyltetrahydrofuran-3-yl)butan-1-one (24trans) and cis-(E)-4-(but-2-en-1-yloxy)-1-(4-vinyltetrahydrofuran-3-yl)butan-1-one (24cis)
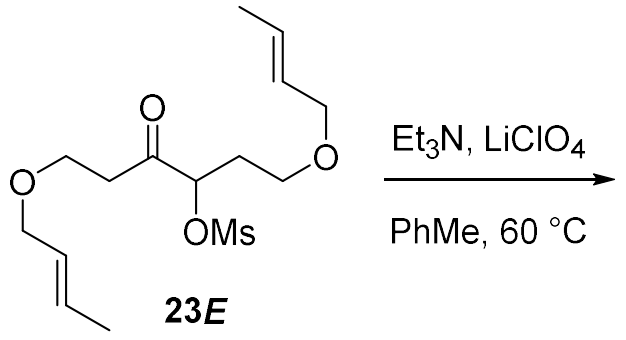

PhMe, $60{ }^{\circ} \mathrm{C}$

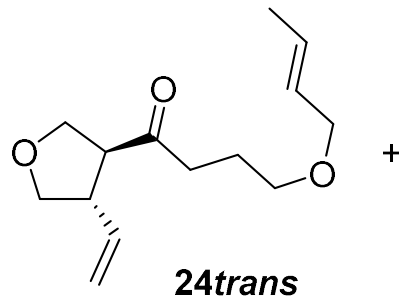

$44 \%$

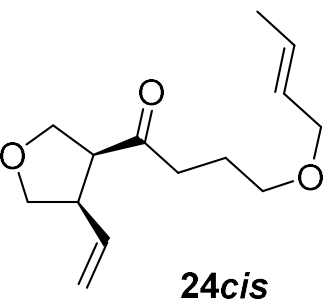

$13 \%$

According to General procedure - Method C. A suspension of 23E (59.4 mg, $0.1776 \mathrm{mmol}, 1 \mathrm{eq})$, triethylamine $(50 \mu \mathrm{L}, 0.36 \mathrm{mmol}, 2 \mathrm{eq})$ and lithium perchlorate $(38 \mathrm{mg}, 0.107 \mathrm{mmol}, 2 \mathrm{eq})$ in toluene $(0.8 \mathrm{~mL})$ was stirred at $60{ }^{\circ} \mathrm{C}$ for 1 hour. The reaction mixture was directly applied to a column and purified by column chromatography (petroleum ether/ ethyl acetate $=7 / 3$ ) to afford

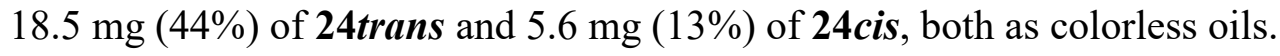

Spectral data for 24trans:

${ }^{1}$ H NMR $\left(500 \mathrm{MHz}, \mathrm{C}_{6} \mathrm{D}_{6}\right) \delta 5.57-5.50(\mathrm{~m}, 2 \mathrm{H}), 5.44\left(\mathrm{ddd}, J^{1}=17.6, J^{2}=9.9, J^{3}=8.8 \mathrm{~Hz}, 1 \mathrm{H}\right)$, $4.90(\mathrm{~d}, J=17.0 \mathrm{~Hz}, 1 \mathrm{H}), 4.85(\mathrm{~d}, J=10.1 \mathrm{~Hz}, 1 \mathrm{H}), 3.98\left(\mathrm{dd}, J^{1}=8.5, J^{2}=7.0 \mathrm{~Hz}, 1 \mathrm{H}\right), 3.87(\mathrm{t}, J$ $=7.9 \mathrm{~Hz}, 1 \mathrm{H}), 3.79-3.70(\mathrm{~m}, 3 \mathrm{H}), 3.33(\mathrm{t}, J=8.0 \mathrm{~Hz}, 1 \mathrm{H}), 3.22(\mathrm{t}, J=6.0 \mathrm{~Hz}, 2 \mathrm{H}), 2.93(\mathrm{p}, J=$ $7.6 \mathrm{~Hz}, 1 \mathrm{H}), 2.56\left(\mathrm{dd}, J^{1}=15.1, J^{2}=7.5,1 \mathrm{H}\right), 2.31-2.22(\mathrm{~m}, 1 \mathrm{H}), 2.22-2.13(\mathrm{~m}, 1 \mathrm{H}), 1.85-$ $1.78(\mathrm{~m}, 2 \mathrm{H}), 1.53\left(\mathrm{dd}, J^{1}=3.4, J^{2}=1.0,3 \mathrm{H}\right)$.

${ }^{13} \mathrm{C}$ NMR (125 MHz, $\left.\mathrm{C}_{6} \mathrm{D}_{6}\right) \delta 207.4,138.2,128.6(2 \times \mathrm{C}), 116.3,73.4,71.6,70.0,68.9,57.7,47.8$, 39.6, 24.2, 17.7 .

IR (ATR) $v_{\max }: 3079,3013,2934,2857,1826,1712,1642,1446,1362,1257,1103$.

HRMS (ESI) calcd. for $\mathrm{C}_{14} \mathrm{H}_{23} \mathrm{O}_{3}{ }^{+}[\mathrm{M}+\mathrm{H}]^{+}: 239.1642$, found: 239.1635 . 
Correlations (NOESY) for 24trans:

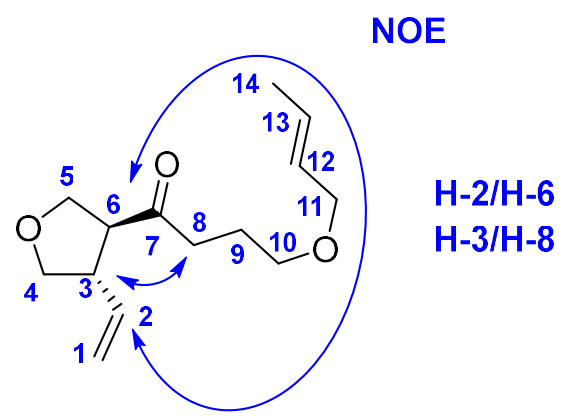

Spectral data for 24cis:

${ }^{1}$ H NMR $\left(500 \mathrm{MHz}, \mathrm{C}_{6} \mathrm{D}_{6}\right) \delta 5.63\left(\mathrm{dt}, J^{1}=17.1, J^{2}=9.9,1 \mathrm{H}\right), 5.58-5.53(\mathrm{~m}, 2 \mathrm{H}), 4.86-4.79$ $(\mathrm{m}, 2 \mathrm{H}), 4.28\left(\mathrm{dd}, J^{l}=17.1, J^{2}=9.9,1 \mathrm{H}\right), 3.79-3.59(\mathrm{~m}, 5 \mathrm{H}), 3.28-3.20(\mathrm{~m}, 2 \mathrm{H}), 2.77\left(\mathrm{dd}, J^{l}\right.$ $\left.=15.5, J^{2}=7.8,1 \mathrm{H}\right), 2.60-2.52(\mathrm{~m}, 1 \mathrm{H}), 2.29\left(\mathrm{dt}, J^{1}=17.5, J^{2}=7.1,1 \mathrm{H}\right), 2.14\left(\mathrm{dt}, J^{1}=17.7, J^{2}\right.$ $=7.0,1 \mathrm{H}), 1.92-1.75(\mathrm{~m}, 2 \mathrm{H}), 1.56-1.51(\mathrm{~m}, 3 \mathrm{H})$.

${ }^{13} \mathrm{C}$ NMR (125 MHz, $\left.\mathrm{C}_{6} \mathrm{D}_{6}\right) \delta 207.4,136.0,128.7,117.0,73.3,71.6,69.1,68.8,55.2,47.6,40.9$, 24.0, 17.7 .

IR (ATR) $v_{\max }: 3080,3012,2933,2858,1711,1674,1639,1447,1362,1255,1179,1103$.

HRMS (ESI) calcd. for $\mathrm{C}_{14} \mathrm{H}_{23} \mathrm{O}_{3}{ }^{+}[\mathrm{M}+\mathrm{H}]^{+}: 239.1642$, found: 239.1632 .

Note: In $\mathrm{CDCl}_{3}$ 24cis isomerizes to 24trans (probably due to acidity of deuterochloroform). Isomerization is slow and incomplete: after ten days at room temperature solution od 24cis in $\mathrm{CDCl}_{3}$ gave final ratio of 24cis:24trans $=1.00: 0.38$.

\section{1,1-Dimethyloctahydroindeno[7,1-bc]furan-2a(1H)-ol (26)}<smiles>COC1C(=O)CCCC1CCC=C(C)C</smiles>

25

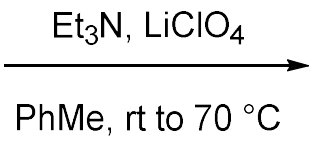

$55 \%$

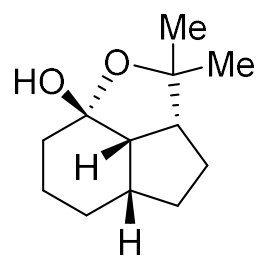

26

According to the General procedure - Method C. A suspension of mesylate 25 (11.5 mg, 0.042 $\mathrm{mmol})$, triethylamine $(12 \mu \mathrm{L}, 0.086 \mathrm{mmol})$ and lithium perchlorate $(8.9 \mathrm{mg}, 0.084 \mathrm{mmol})$ in dry toluene $(0.14 \mathrm{~mL})$ was stirred at room temperature for $2 \mathrm{~h}$, then at $50{ }^{\circ} \mathrm{C}$ for $2 \mathrm{~h}$, and at $70{ }^{\circ} \mathrm{C}$ for $12 \mathrm{~h} . \mathrm{SiO}_{2}(50 \mathrm{mg})$ was added to the reaction mixture, the volatiles were removed under reduced pressure and the residue was purified by column chromatography (petroleum-ether/ethyl acetate = $4 / 1)$ to afford $4.5 \mathrm{mg}(55 \%)$ of compound $\mathbf{2 6}$, as colorless oil. 
${ }^{1} \mathbf{H}$ NMR $\left(500 \mathrm{MHz}, \mathrm{CDCl}_{3}\right) \delta 2.70-2.62(\mathrm{~m}, 2 \mathrm{H}), 2.17-2.09(\mathrm{~m}, 1 \mathrm{H}), 2.02-1.97(\mathrm{~m}, 1 \mathrm{H}), 1.89$ (s, $1 \mathrm{H}), 1.82-1.76(\mathrm{~m}, 1 \mathrm{H}), 1.69-1.65(\mathrm{~m}, 1 \mathrm{H}), 1.55-1.48(\mathrm{~m}, 2 \mathrm{H}), 1.47-1.31(\mathrm{~m}, 5 \mathrm{H}), 1.45$ (s, 3H), $1.19(\mathrm{~s}, 3 \mathrm{H})$.

${ }^{13}$ C NMR $\left(125 \mathrm{MHz}, \mathrm{CDCl}_{3}\right) \delta 104.4,84.4,53.3,52.2,38.51,38.0,33.9,31.6,29.6,26.7,24.5$, 17.9.

IR (ATR) $v_{\max }: 3320,2938,2854,1459,1357,1175,1145,1094,1065,1009,996$.

HRMS (ESI) calcd. for $\mathrm{C}_{12} \mathrm{H}_{19} \mathrm{O}^{+}\left[\mathrm{M}+\mathrm{H}-\mathrm{H}_{2} \mathrm{O}\right]^{+}:$: 179.1430; found: 179.1431 .

\section{(E)-2,3,3a,5,6,7,8,10a-Octahydrocyclopenta[9]annulen-4(1H)-one (28)}

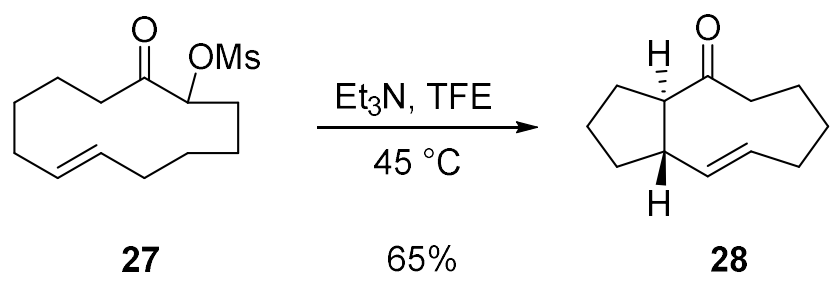

According to the General procedure - Method A. Triethylamine ( $36 \mu \mathrm{L}, 0.258 \mathrm{mmol})$ was added to the solution of mesylate $27(39.6 \mathrm{mg}, 0.145 \mathrm{mmol})$ in trifluoroethanol $(3.8 \mathrm{~mL})$ and the reaction mixture was stirred at $45{ }^{\circ} \mathrm{C}$ for $16 \mathrm{~h}$, under an argon atmosphere. The reaction mixture was concentrated in vacuo and the residue was purified by column chromatography (petroleumether/ethyl acetate $=16 / 1)$ to afford $16.9 \mathrm{mg}(65 \%)$ of product 28 , as relatively volatile colorless liquid with characteristic odor.

The corresponding tosylate was also used as a substrate for this cyclization, and under identical conditions ( $\mathrm{Et}_{3} \mathrm{~N}, \mathrm{TFE}, 45^{\circ} \mathrm{C}, 16 \mathrm{~h}$ ) gave compound $\mathbf{2 8}$ in $50 \%$ yield. Besides, tosylate is more difficult to prepare (tosylation of $\alpha$-hydroxy ketone has to be performed with 4 eq. $\mathrm{TsCl}$ in concentrated pyridine solution over $48 \mathrm{~h}$ ) and purify then mesylate 27 .

${ }^{1} \mathbf{H}$ NMR $(500 \mathrm{MHz}, \mathrm{CDCl} 3) \delta 5.57-5.48(\mathrm{~m}, 1 \mathrm{H}), 5.03\left(\mathrm{dd}, J^{1}=15.6, J^{2}=9.9 \mathrm{~Hz}, 1 \mathrm{H}\right), 3.19(\mathrm{dt}$, $\left.J^{l}=11.3, J^{2}=7.7 \mathrm{~Hz}, 1 \mathrm{H}\right), 3.06\left(\mathrm{dt}, J^{l}=10.8, J^{2}=8.1 \mathrm{~Hz}, 1 \mathrm{H}\right), 2.36-2.19(\mathrm{~m}, 3 \mathrm{H}), 2.07\left(\mathrm{ddt}, J^{l}\right.$ $\left.=13.6, J^{2}=9.7, J^{3}=6.9 \mathrm{~Hz}, 1 \mathrm{H}\right), 1.92-1.73(\mathrm{~m}, 5 \mathrm{H}), 1.64-1.27(\mathrm{~m}, 5 \mathrm{H})$.

${ }^{13} \mathbf{C}$ NMR (126 MHz, $\left.\mathrm{CDCl}_{3}\right) \delta 215.1,133.0,132.8,54.8,46.6,44.4,34.3,33.2,30.7,27.1,27.0$, 23.7.

IR (ATR) $v_{\max }: 2930,2859,1704,1448,1343,1078,980$.

HRMS (ESI) calcd. for $\mathrm{C}_{12} \mathrm{H}_{19} \mathrm{O}^{+}[\mathrm{M}+\mathrm{H}]^{+}: 179.1430$; found: 179.1432 . 
Correlations (NOESY) for $\mathbf{2 8}$ :

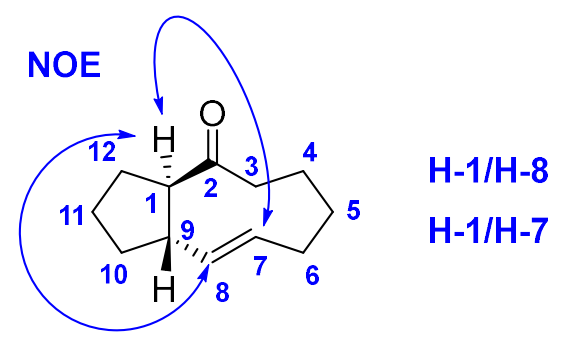

1-(2-Methyl-5-vinylidenecyclopentyl)-2-phenylethanone (30)

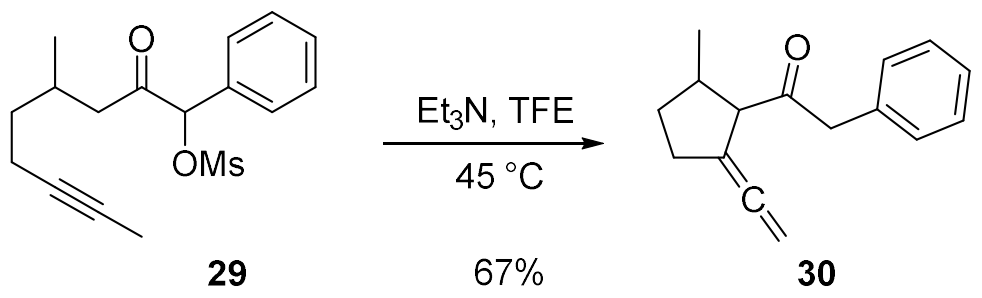

According to the General procedure - Method A. Using mesyloxy ketone 29 (99.3 mg, 0.308 mmol, 1 eq), triethylamine ( $69 \mu \mathrm{L}, 0.49 \mathrm{mmol}, 1.6 \mathrm{eq})$ and trifluoroethanol $(1 \mathrm{~mL})$, during $2 \mathrm{~h}$. Purification by column chromatography (petroleum-ether/diisopropyl ether $=95 / 5$ ) afforded 47.0 $\mathrm{mg}(67 \%)$ of allene $\mathbf{3 0}$, as a colorless oil.

${ }^{1} \mathbf{H}$ NMR $\left(500 \mathrm{MHz}, \mathrm{CDCl}_{3}\right) \delta 7.34-7.29(\mathrm{~m}, 2 \mathrm{H}), 7.27-7.22(\mathrm{~m}, 1 \mathrm{H}), 7.21-7.16(\mathrm{~m}, 2 \mathrm{H})$, $4.86-4.77$ (m, 2H), $3.86(\mathrm{~d}, J=15.6 \mathrm{~Hz}, 1 \mathrm{H}), 3.78(\mathrm{~d}, J=15.6 \mathrm{~Hz}, 1 \mathrm{H}), 3.26-3.20(\mathrm{~m}, 1 \mathrm{H})$, $2.54-2.38(\mathrm{~m}, 3 \mathrm{H}), 1.98-1.90(\mathrm{~m}, 1 \mathrm{H}), 1.35-1.25(\mathrm{~m}, 1 \mathrm{H}), 0.91(\mathrm{~d}, J=6.7 \mathrm{~Hz}, 3 \mathrm{H})$.

${ }^{13} \mathbf{C}$ NMR $\left(125 \mathrm{MHz}, \mathrm{CDCl}_{3}\right) \delta 206.9,202.7,134.2,129.7,128.5,126.8,103.1,77.5,61.8,48.9$, 37.6, 34.1, 30.8, 18.7 .

IR (ATR) $v_{\max }: 3028,2953,2925,2865,1957,1707,1600,1494,1453,1280$.

HRMS (ESI) calcd. for $\mathrm{C}_{16} \mathrm{H}_{19} \mathrm{O}^{+}[\mathrm{M}+\mathrm{H}]^{+}: 227.1430$, found: 227.1422 .

1-(5-Methyl-2-vinylcyclopent-1-enyl)-2-phenylethanone (31)

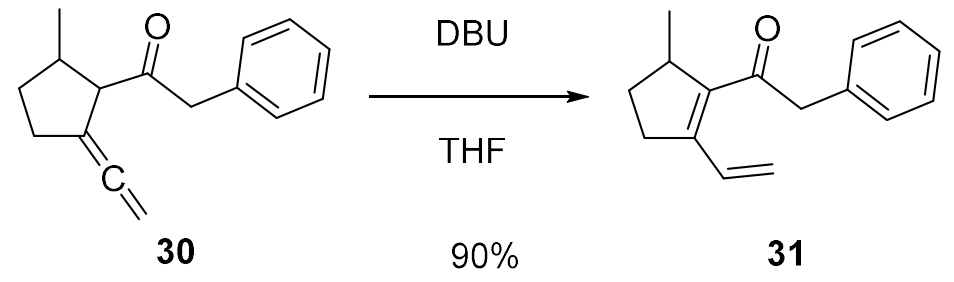


$\operatorname{DBU}(5 \mu \mathrm{L}, 0.034 \mathrm{mmol}, 0.2 \mathrm{eq})$ was added to the solution of allene 30 (38.8 $\mathrm{mg}, 0.17 \mathrm{mmol}, 1$ eq) in THF $(0.5 \mathrm{~mL})$ and the reaction mixture was stirred at room temperature for $2 \mathrm{~h}$. The reaction mixture was quenched with sat. $\mathrm{NH}_{4} \mathrm{Cl}$ solution, extracted with diethyl ether and washed with water. The combined organic extract was dried over anh. $\mathrm{MgSO}_{4}$ prior to concentrating in vacuo. Purification of the residue by column chromatography (petroleum-ether/ethyl acetate $=95 / 5$ ) afforded $35 \mathrm{mg}(90 \%)$ of dienone $\mathbf{3 1}$, as a colorless oil.

${ }^{1} \mathbf{H}$ NMR $\left(500 \mathrm{MHz}, \mathrm{CDCl}_{3}\right) \delta 7.33-7.28(\mathrm{~m}, 2 \mathrm{H}), 7.26-7.21(\mathrm{~m}, 1 \mathrm{H}), 7.20-7.16(\mathrm{~m}, 2 \mathrm{H})$, $7.11(\mathrm{dd}, J=17.5,10.8 \mathrm{~Hz}, 1 \mathrm{H}), 5.41(\mathrm{~d}, J=17.4 \mathrm{~Hz}, 1 \mathrm{H}), 5.35(\mathrm{~d}, J=10.8 \mathrm{~Hz}, 1 \mathrm{H}), 3.90(\mathrm{~d}, J=$ $15.6 \mathrm{~Hz}, 1 \mathrm{H}), 3.83(\mathrm{~d}, J=15.6 \mathrm{~Hz}, 1 \mathrm{H}), 3.26-3.18(\mathrm{~m}, 1 \mathrm{H}), 2.75-2.67(\mathrm{~m}, 1 \mathrm{H}), 2.56-2.48(\mathrm{~m}$, $1 \mathrm{H}), 2.15-2.06(\mathrm{~m}, 1 \mathrm{H}), 1.53-1.46(\mathrm{~m}, 1 \mathrm{H}), 1.10(\mathrm{~d}, J=6.8 \mathrm{~Hz}, 3 \mathrm{H})$.

${ }^{13}$ C NMR (125 MHz, $\left.\mathrm{CDCl}_{3}\right) \delta 199.6,148.2,143.7,134.3,131.8,129.5,128.4,126.7,120.6,48.9$, 41.7, 31.6, 30.8, 20.0.

IR (ATR) $v_{\max }:$ 3088, 3062, 3028, 2955, 2867, 1667, 1607, 1562, 1495, 1454, 1347.

HRMS (ESI) calcd. for $\mathrm{C}_{16} \mathrm{H}_{18} \mathrm{O}\left[\mathrm{M}+\mathrm{Na}^{+}\right]$: 249.1250, found: 249.1242.

\section{2-Phenyl-1-(4-vinyl-2,5-dihydrofuran-3-yl)ethanone (33)}

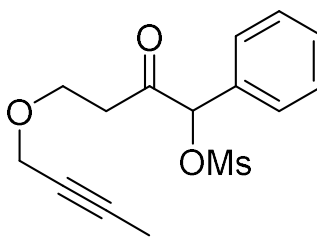

32

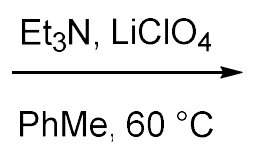

$25 \%$

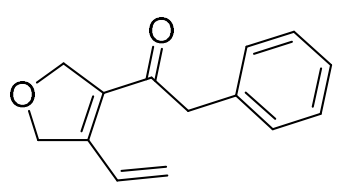

33

According to the General procedure - Method C. Using mesyloxy ketone $32(37.7 \mathrm{mg}, 0.128$ mmol, $1 \mathrm{eq})$, triethylamine $(36 \mu \mathrm{L}, 0.256 \mathrm{mmol}, 2 \mathrm{eq})$, lithium perchlorate $(27 \mathrm{mg}, 0.256 \mathrm{mmol}, 2$ eq), and toluene $(0.5 \mathrm{~mL})$, during $20 \mathrm{~min}$. Purification by dry-flash chromatography (petroleumether/ ethyl acetate $=9 / 1)$ afforded $6.8 \mathrm{mg}(25 \%)$ of dienone 33 , as a colorless oil.

According to the General procedure - Method A, dienone 33 was isolated in only $8 \%$ yield.

${ }^{1} \mathbf{H}$ NMR $\left(500 \mathrm{MHz}, \mathrm{CDCl}_{3}\right) \delta 7.41-7.31(\mathrm{~m}, 3 \mathrm{H}), 7.30-7.25(\mathrm{~m}, 1 \mathrm{H}), 7.20-7.15(\mathrm{~m}, 2 \mathrm{H}), \quad 5.54$ $(\mathrm{d}, J=11.0 \mathrm{~Hz}, 1 \mathrm{H}), 5.35(\mathrm{~d}, J=17.8 \mathrm{~Hz}, 1 \mathrm{H}), 3.98-3.90(\mathrm{~m}, 4 \mathrm{H}), 3.78(\mathrm{~s}, 2 \mathrm{H})$.

${ }^{13} \mathbf{C ~ N M R}\left(125 \mathrm{MHz}, \mathrm{CDCl}_{3}\right) \delta 194.2,146.8,133.2,132.2,129.5,128.9,128.4,127.3,123.6,76.6$, $76.0,49.8$.

IR (ATR) $v_{\max }:$ 3062, 3030, 2923, 2861, 1728, 1682, 1627, 1535, 1496, 1453, 1358.

HRMS (ESI) calcd. for $\mathrm{C}_{16} \mathrm{H}_{18} \mathrm{O}\left(\mathrm{M}+\mathrm{H}^{+}\right): 227.1430$, found: 227.1422 . 


\section{Side reactions}

\section{2,2,2-Trifluoroethyl (E)-3-(2-(hept-2-en-1-yl)phenyl)propanoate (35)}

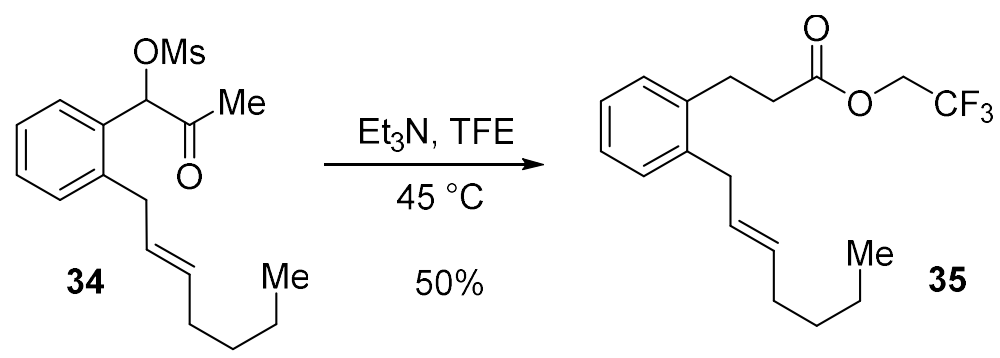

According to the General procedure - Method A. Triethylamine ( $7 \mu \mathrm{L}, 0.050 \mathrm{mmol})$ was added to the solution of mesylate $34(10.0 \mathrm{mg}, 0.031 \mathrm{mmol})$ in trifluoroethanol $(1.0 \mathrm{~mL})$ and the reaction mixture was stirred at $45{ }^{\circ} \mathrm{C}$ for $3 \mathrm{~h}$, under an argon atmosphere. The reaction mixture was concentrated in vacuo and the residue was purified by column chromatography (petroleumether/ethyl acetate $=94 / 6)$ to afford $5.1 \mathrm{mg}(50 \%)$ of trifluoroethyl ester $\mathbf{3 5}$, as a colorless oil with characteristic odor.

${ }^{1}$ H NMR (500 MHz, CDCl $) \delta 7.22-7.09(\mathrm{~m}, 4 \mathrm{H}), 5.57-5.48(\mathrm{~m}, 1 \mathrm{H}), 5.48-5.39(\mathrm{~m}, 1 \mathrm{H})$, $4.47(\mathrm{q}, J=8.5 \mathrm{~Hz}, 2 \mathrm{H}), 3.35\left(\mathrm{dd}, J^{1}=6.2, J^{2}=1.0 \mathrm{~Hz}, 2 \mathrm{H}\right), 2.99\left(\mathrm{dd}, J^{l}=9.8, J^{2}=6.2 \mathrm{~Hz}, 2 \mathrm{H}\right)$, $2.70\left(\mathrm{dd}, J^{1}=9.8, J^{2}=6.2 \mathrm{~Hz}, 2 \mathrm{H}\right), 2.01\left(\mathrm{dd}, J^{l}=13.3, J^{2}=6.6 \mathrm{~Hz}, 2 \mathrm{H}\right), 1.40-1.25(\mathrm{~m}, 4 \mathrm{H}), 0.88$ (t, J $=7.1 \mathrm{~Hz}, 3 \mathrm{H})$.

${ }^{13}$ C NMR (126 MHz, CDCl 3 ) 171.5, 138.9, 138.0, 132.4, 130.0, 129.0, 128.4, 126.9, 126.6, 123.1 $(\mathrm{q}, J=277.1 \mathrm{~Hz}), 60.5(\mathrm{q}, J=36.5 \mathrm{~Hz}), 36.2,34.8,32.4,31.7,27.6,22.4,14.0$.

IR (ATR) $v_{\max }: 3065,3021,2960,2929,2871,1761,1490,1454,1413,1282,1172,1145,974$.

HRMS (APPI) calcd. for $\mathrm{C}_{18} \mathrm{H}_{23} \mathrm{~F}_{3} \mathrm{O}_{2}{ }^{+}[\mathrm{M}]^{+}:$328.1645; found: 328.1637 .

\section{(E)-1-(Hept-2-en-1-yl)-2-vinylbenzene (36)}

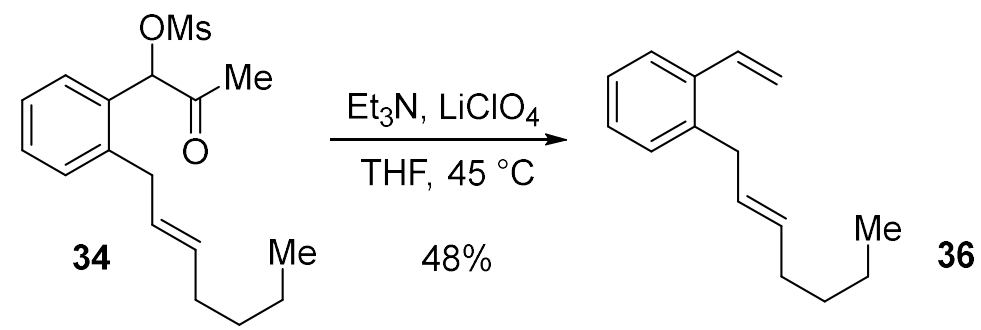

Triethylamine $(17.5 \mu \mathrm{L}, 0.125 \mathrm{mmol})$ and $\mathrm{LiClO}_{4}(9.5 \mathrm{mg}, 0.089 \mathrm{mmol})$ were added to the solution of mesyloxy ketone $34(5.0 \mathrm{mg}, 0.015 \mathrm{mmol})$ in tetrahydrofuran $(0.30 \mathrm{~mL})$, and the reaction 
mixture was stirred at $45{ }^{\circ} \mathrm{C}$ for $24 \mathrm{~h}$, under an argon atmosphere. The reaction mixture was carefully concentrated under reduced pressure and the residue was purified by column chromatography (petroleum-ether/ethyl acetate $=49 / 1$ ) to afford $1.5 \mathrm{mg}(48 \%)$ of styrene $\mathbf{3 6}$, as very volatile colorless oil.

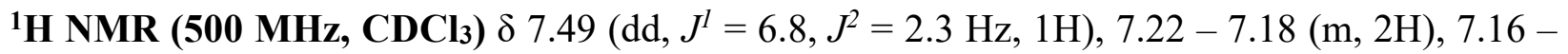
$7.14(\mathrm{~m}, 1 \mathrm{H}), 6.98\left(\mathrm{dd}, J^{1}=17.4, J^{2}=11.0 \mathrm{~Hz}, 1 \mathrm{H}\right), 5.63\left(\mathrm{dd}, J^{l}=17.4, J^{2}=1.4 \mathrm{~Hz}, 1 \mathrm{H}\right), 5.53$ $\left(\right.$ dddd, $\left.J^{1}=7.5, J^{2}=6.2, J^{3}=4.4, J^{4}=3.1 \mathrm{~Hz}, 1 \mathrm{H}\right), 5.41\left(\mathrm{ddd}, J^{1}=15.2, J^{2}=10.1, J^{3}=6.0 \mathrm{~Hz}\right.$, $1 \mathrm{H}), 5.27\left(\mathrm{dd}, J^{1}=11.0, J^{2}=1.4 \mathrm{~Hz}, 1 \mathrm{H}\right), 3.38(\mathrm{~d}, J=5.3 \mathrm{~Hz}, 2 \mathrm{H}), 2.00\left(\mathrm{dd}, J^{1}=13.2, J^{2}=6.8\right.$ $\mathrm{Hz}, 2 \mathrm{H}), 1.35-1.25(\mathrm{~m}, 4 \mathrm{H}), 0.87(\mathrm{t}, J=7.1 \mathrm{~Hz}, 3 \mathrm{H})$.

${ }^{13}$ C NMR (126 MHz, CDCl 3$) \delta 138.3,136.8,134.9,132.2,129.7,128.2,128.0,126.5,125.8$, $115.4,36.5,32.4,31.76,22.4,14.1$.

IR (ATR) $v_{\max }:$ 3086, 3062, 3021, 2957, 2926, 2856, 1626, 1482, 1452, 969, 912, 767.

HRMS (ESI) calcd. for $\mathrm{C}_{15} \mathrm{H}_{19}{ }^{+}[\mathrm{M}-\mathrm{H}]^{+}:$199.1487; found: 199.1484 .

\section{(E)-4-(5-Acetyl-3-((benzyloxy)methyl)tetrahydrofuran-3-yl)but-2-en-1-yl acetate (38)}
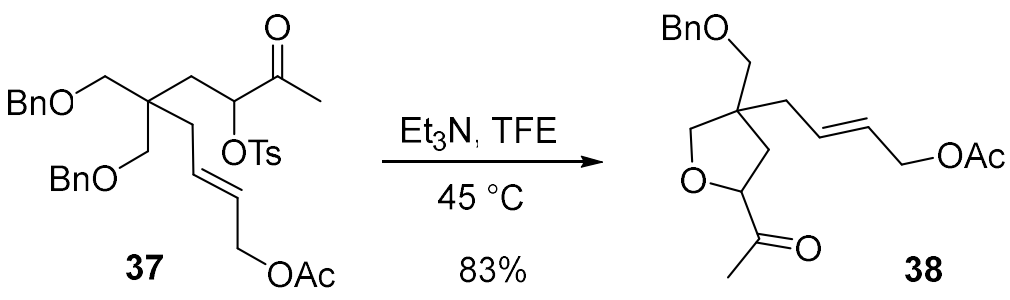

According to the General procedure - Method A. Triethylamine ( $9 \mu \mathrm{L}, 0.065 \mathrm{mmol})$ was added to the solution of tosylate $37(23.0 \mathrm{mg}, 0.038 \mathrm{mmol})$ in trifluoroethanol $(1.5 \mathrm{~mL})$ and the reaction mixture was stirred at $45{ }^{\circ} \mathrm{C}$ for $1 \mathrm{~h}$, under argon atmosphere. The reaction mixture was concentrated in vacuo and the residue was purified by column chromatography (petroleumether/ethyl acetate $=3 / 1)$ to afford $10.9 \mathrm{mg}(83 \%)$ of compound $\mathbf{3 8}$, as a colorless oil. Mixture of cis and trans isomers in a relative ratio 1.86:1, according to ${ }^{1} \mathrm{H}$ NMR spectrum integration of [CCHH-C in the ring] signals at $1.78\left(\mathrm{dd}, J^{1}=13.1, J^{2}=7.8 \mathrm{~Hz}, 1 \mathrm{H}_{\text {minor }}\right)$ and $1.72\left(\mathrm{dd}, J^{l}=13.1, J^{2}\right.$ $=8.2 \mathrm{~Hz}, 1 \mathrm{H}$ major). However, we were not able to assignate which isomer is cis and which one is trans, i. e., we do not know whether the cis-isomer is the major, or the minor one.

Spectral data for the mixture of diastereoisomers $\mathbf{3 8}$ :

${ }^{1} \mathbf{H}$ NMR (500 MHz, CDCl3) $\delta 7.42$ - 7.27 (m, 5H minor+major), 5.73 - 5.53 (m, 2H minor+major), $4.55-4.43$ (m, $4 \mathrm{H}$ minor+major), $4.39-4.27\left(\mathrm{~m}, 1 \mathrm{H}\right.$ minor+major), 3.83 (dd, $J^{1}$ $=13.7, J^{2}=8.9 \mathrm{~Hz}, 1 \mathrm{H}$ minor+major), $3.65(\mathrm{~d}, J=8.8 \mathrm{~Hz}, 1 \mathrm{H}$ minor+major), $3.37-3.21(\mathrm{~m}, 2 \mathrm{H}$ 
minor+major), $2.35-1.97$ (m, 9H minor+major), $1.78\left(\mathrm{dd}, J^{1}=13.1, J^{2}=7.8 \mathrm{~Hz}, 1 \mathrm{H}\right.$ minor), 1.72 (dd, $J^{1}=13.1, J^{2}=8.2 \mathrm{~Hz}, 1 \mathrm{H}$ major).

${ }^{13}$ C NMR $\left(126 \mathrm{MHz}, \mathrm{CDCl}_{3}\right) \delta 210.0,209.9,170.9,138.3,138.2,131.23,131.20,128.6,127.89$, $127.85,127.82,127.8,127.7,83.7,76.2,75.9,73.5,73.4,72.9,64.9,47.7,47.6,37.65,37.60,36.9$, 25.88, 25.83, 21.1.

IR (ATR) $v_{\text {max }}: 3062,3029,2938,2861,1738,1452,1362,1235,1097,1026,974,743$.

HRMS (ESI) calcd. for $\mathrm{C}_{20} \mathrm{H}_{26} \mathrm{NaO}_{5}{ }^{+}[\mathrm{M}+\mathrm{Na}]^{+}: 369.1678$; found: 369.1679 


\section{Copies of NMR spectra}

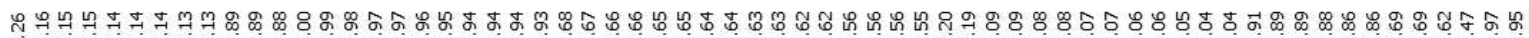

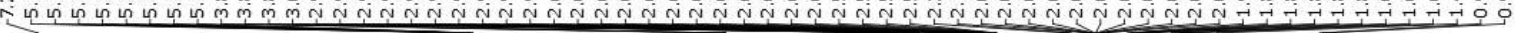
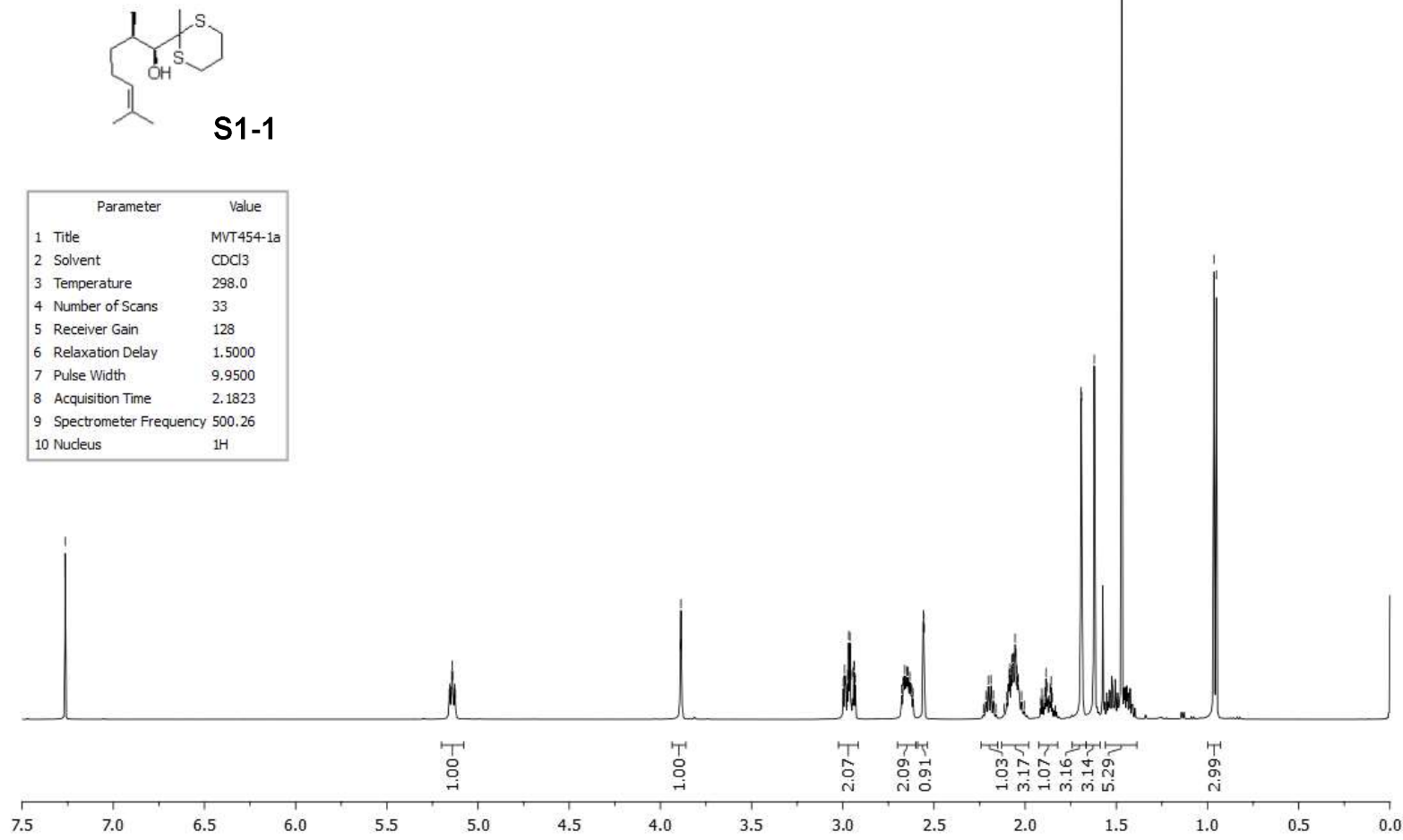

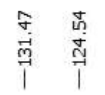

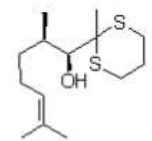

S1-1

\begin{tabular}{|lll|}
\hline & \multicolumn{1}{c}{ Parameter } & \multicolumn{1}{c|}{ Value } \\
1 & Title & MVT 454-1a \\
2 & Solvent & CDCl3 \\
3 & Temperature & 298.2 \\
4 & Number of Scans & 160 \\
5 & Receiver Gain & 2050 \\
6 & Relaxation Delay & 1.5000 \\
7 & Pulse Width & 11.5000 \\
8 & Acquisition Time & 0.5505 \\
9 & Spectrometer Frequency 125.79 \\
10 & Nucleus & $13 \mathrm{C}$ \\
\hline
\end{tabular}

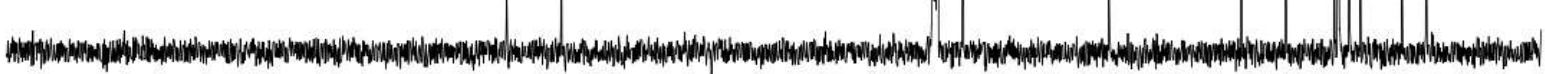

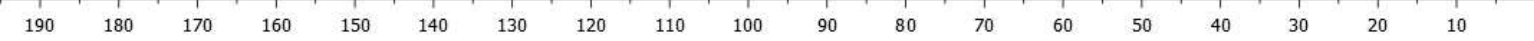



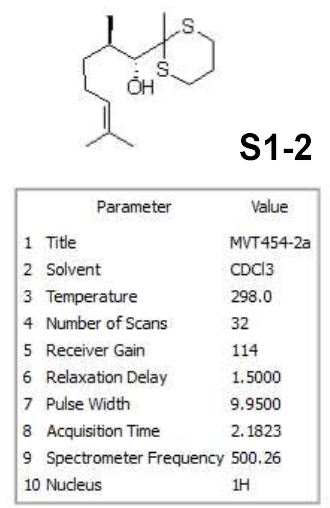

S1-2

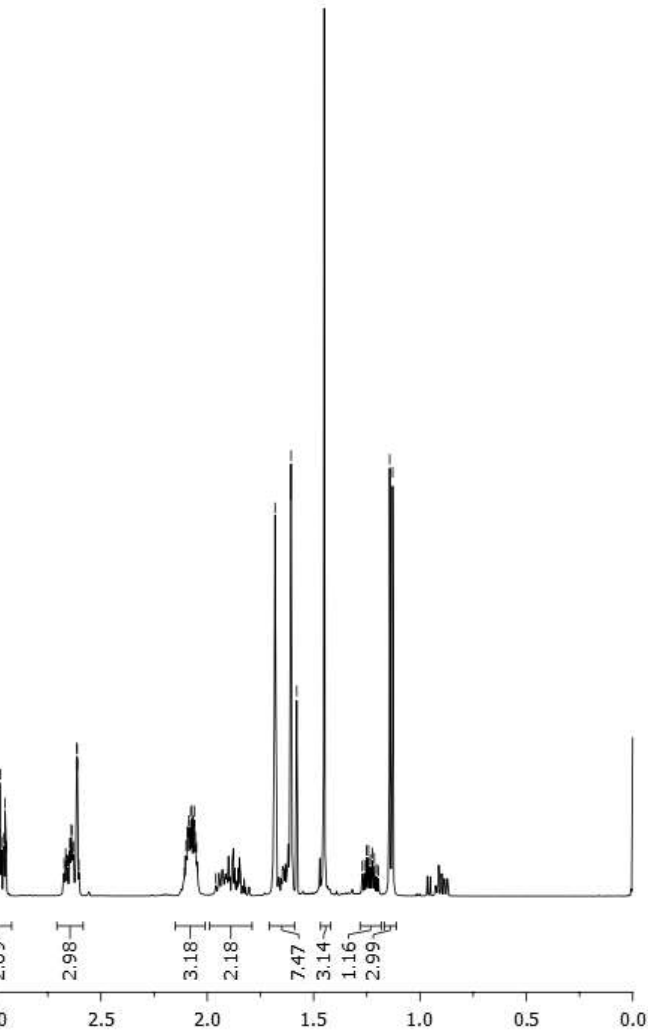

MVT 454-2a

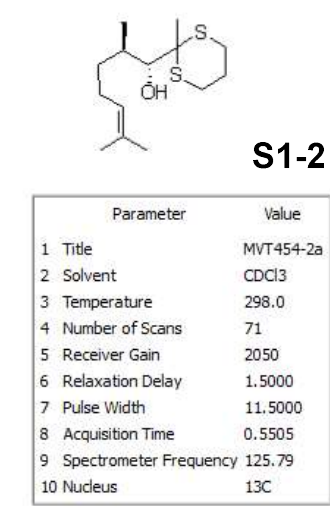

S1-2

\begin{tabular}{|c|c|c|}
\hline & Parameter & Value \\
\hline & Title & MVT 454-2a \\
\hline & Solvent & $\mathrm{CDCl} 3$ \\
\hline & Temperature & 298.0 \\
\hline & Number of Scans & 71 \\
\hline & Receiver Gain & 2050 \\
\hline & Relaxation Delay & 1.5000 \\
\hline & Pulse Width & 11,5000 \\
\hline 8 & Acquisition Time & 0.5505 \\
\hline & Spectrometer Frequency & 125.79 \\
\hline & Nucleus & $13 \mathrm{C}$ \\
\hline
\end{tabular}
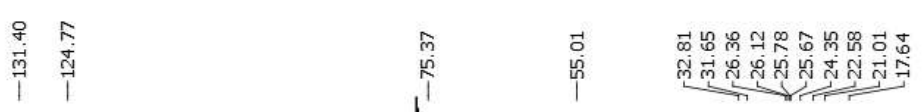

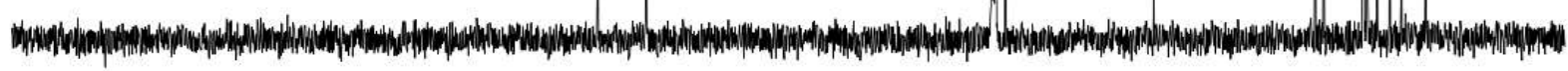

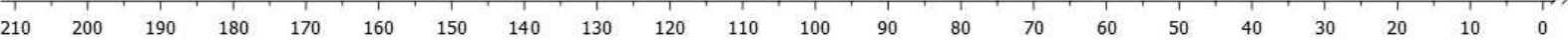



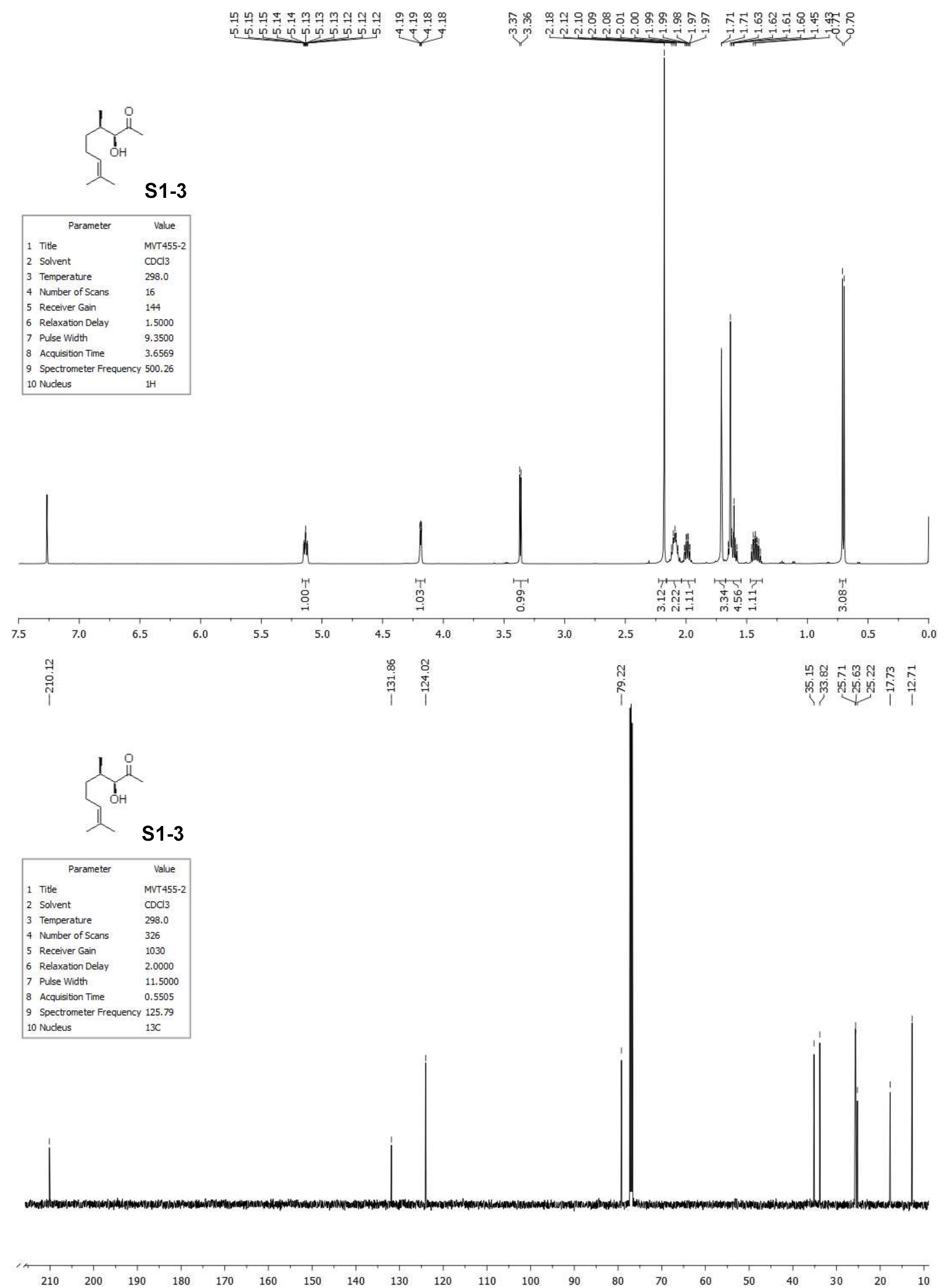

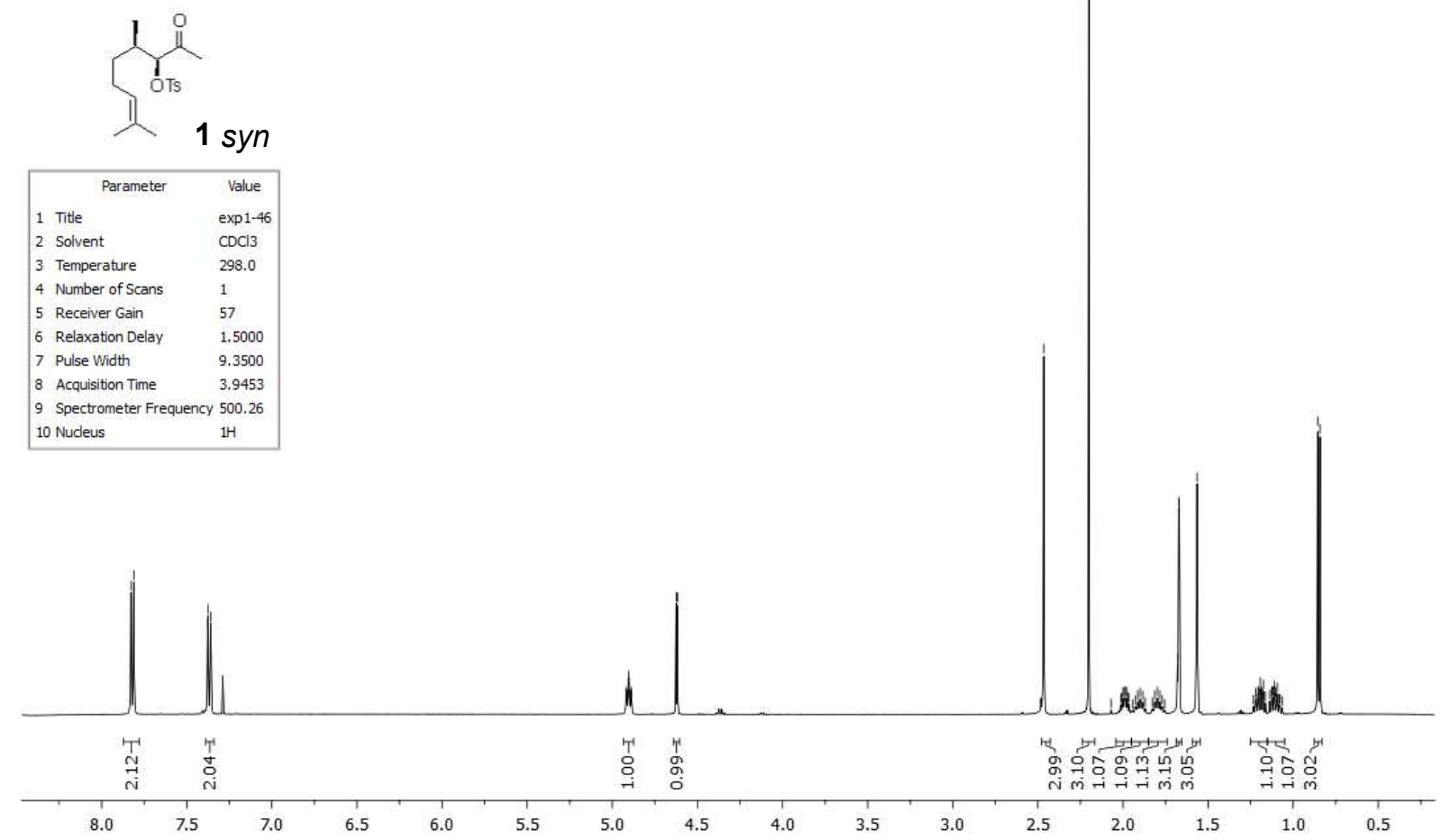

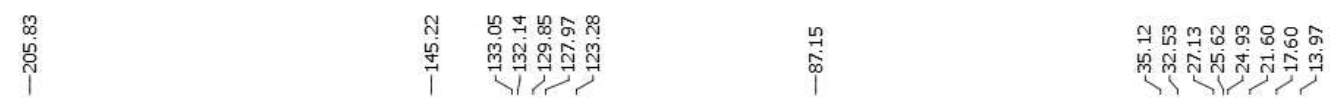
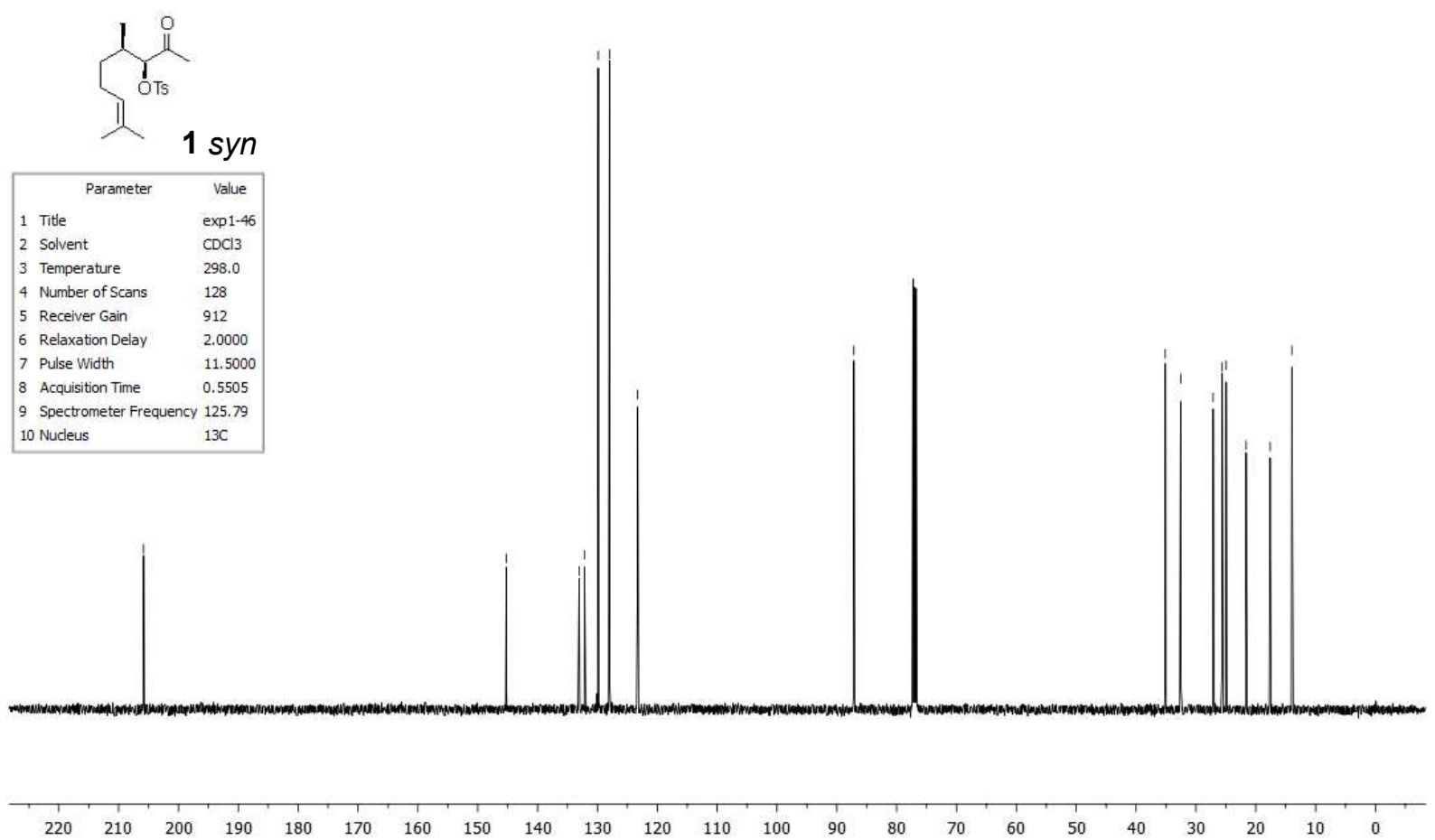

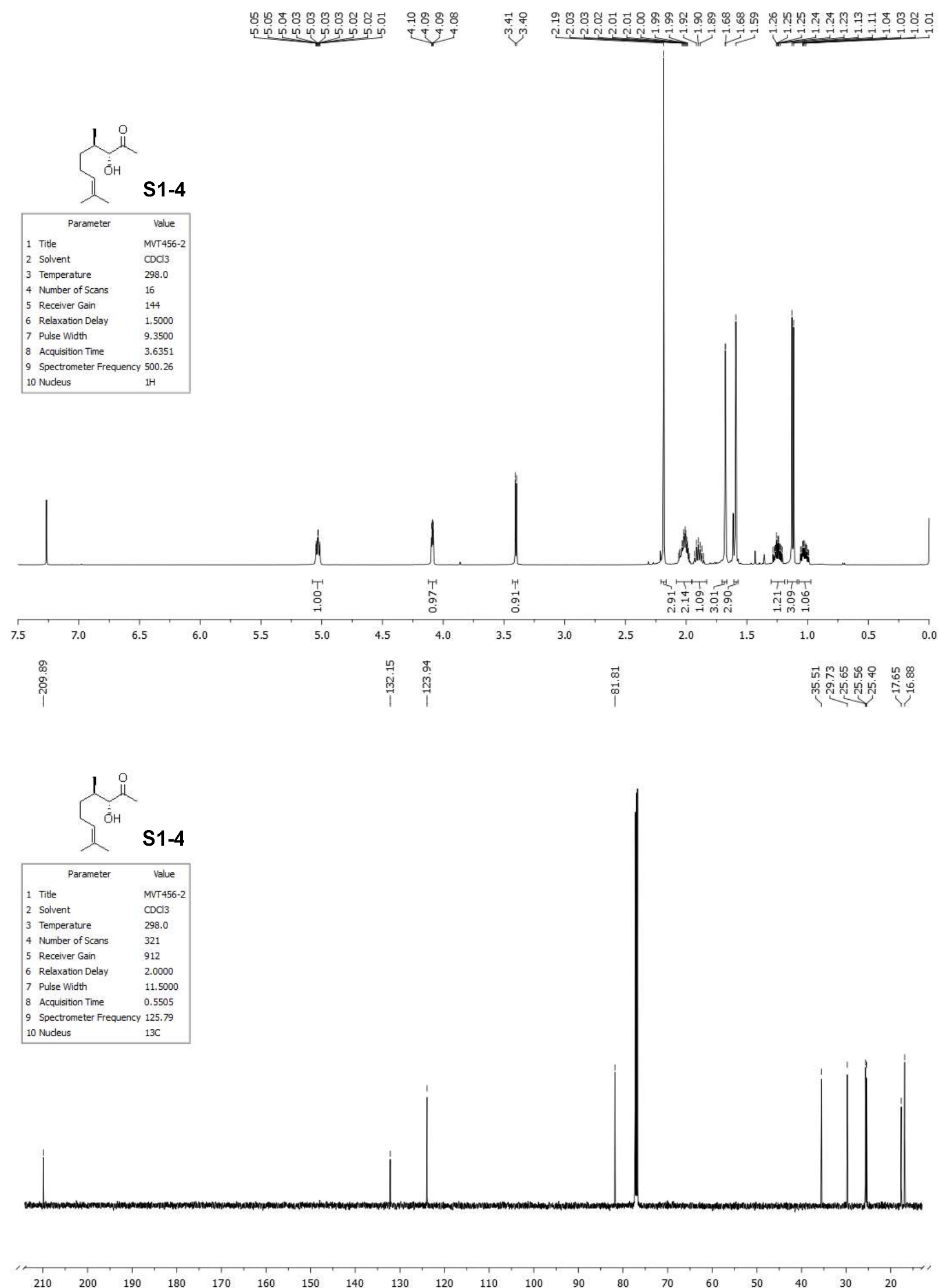


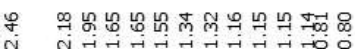
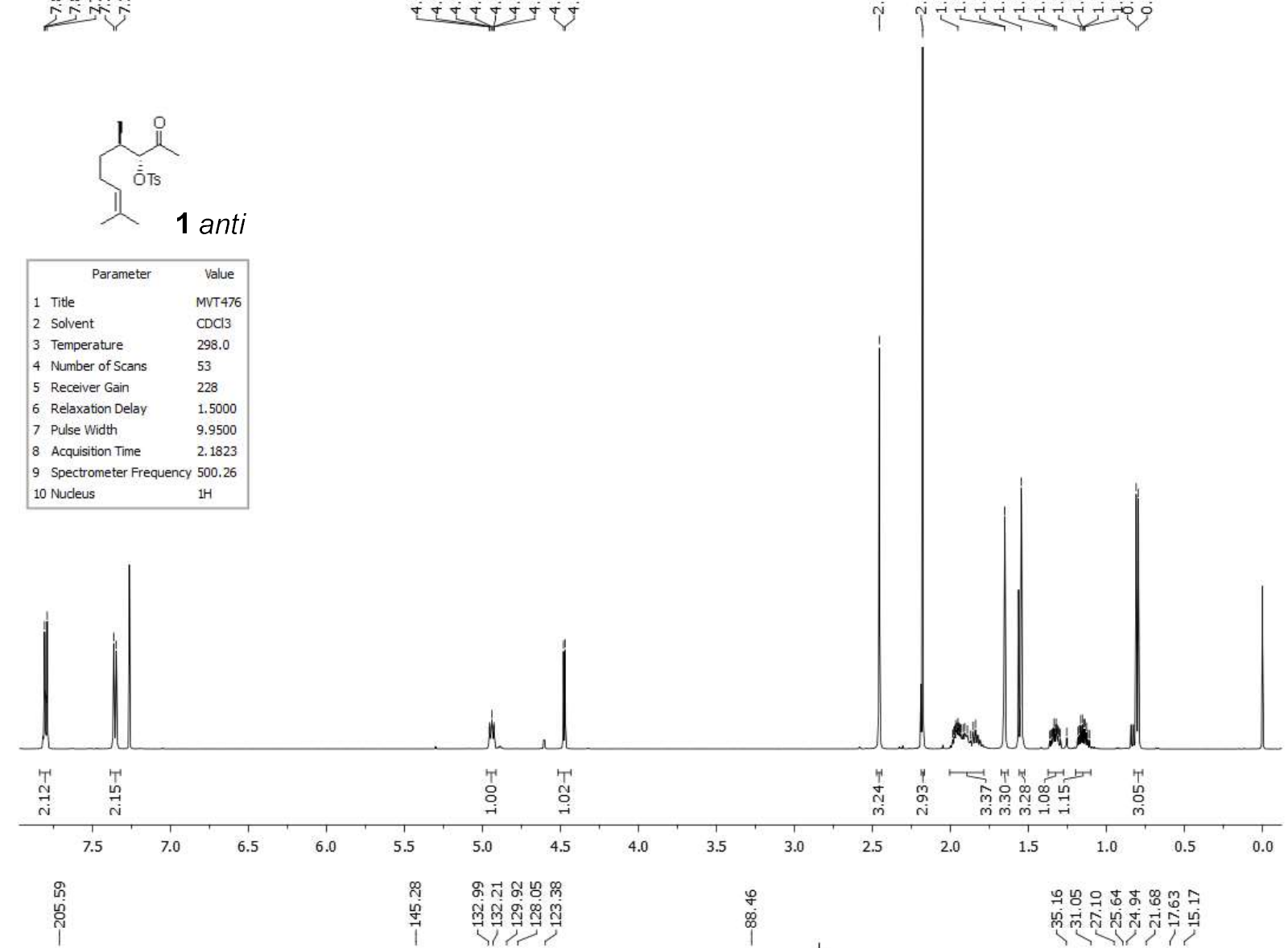

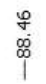

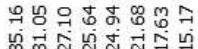

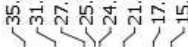
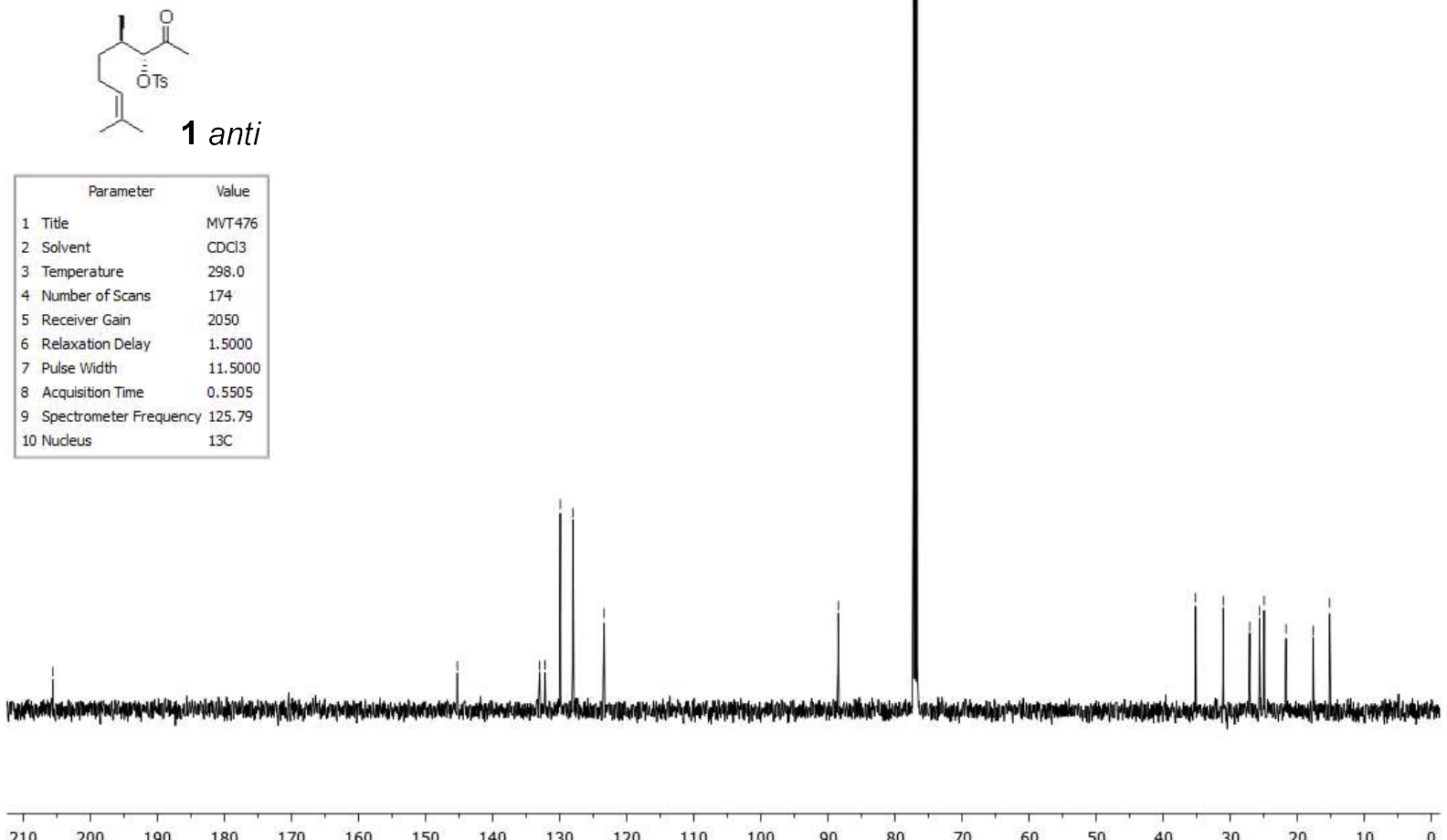
<smiles>CCC(=O)C(C)C(C)CC=C(C)C</smiles>

\section{1 syn+anti}

\begin{tabular}{|lll|}
\hline \multicolumn{1}{|c}{ Parameter } & \multicolumn{1}{c|}{ Value } \\
1 & Title & MVT515 \\
2 & Solvent & CDCl3 \\
3 & Temperature & 298.0 \\
4 & Number of Scans & 16 \\
5 & Receiver Gain & 101 \\
6 & Relaxation Delay & 2.0000 \\
7 & Pulse Width & 9.3500 \\
8 & Acquisition Time & 3.6372 \\
9 & Spectrometer Frequency & 500.26 \\
10 & Nucleus & $1 \mathrm{H}$ \\
\hline
\end{tabular}

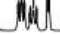

\begin{tabular}{lll|l|l|l|}
\hline 7.0 & 6.5 & 6.0 & 5.5 & 5.0 & 4.5
\end{tabular}

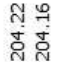

กั

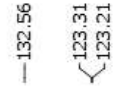

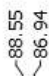

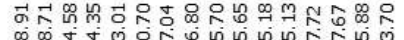

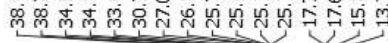

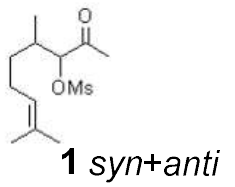

\begin{tabular}{lll}
\hline \multicolumn{1}{c}{ Parameter } & \multicolumn{1}{c}{ Value } \\
1 & Title & MVT515 \\
2 & Solvent & CDCl3 \\
3 & Temperature & 298.0 \\
4 & Number of Scans & 361 \\
5 & Receiver Gain & 912 \\
6 & Relaxation Delay & 2.0000 \\
7 & Pulse Width & 11.5000 \\
8 & Acquisition Time & 0.5505 \\
9 & Spectrometer Frequency & 125.79 \\
10 & Nucleus & $13 \mathrm{C}$ \\
\hline
\end{tabular}
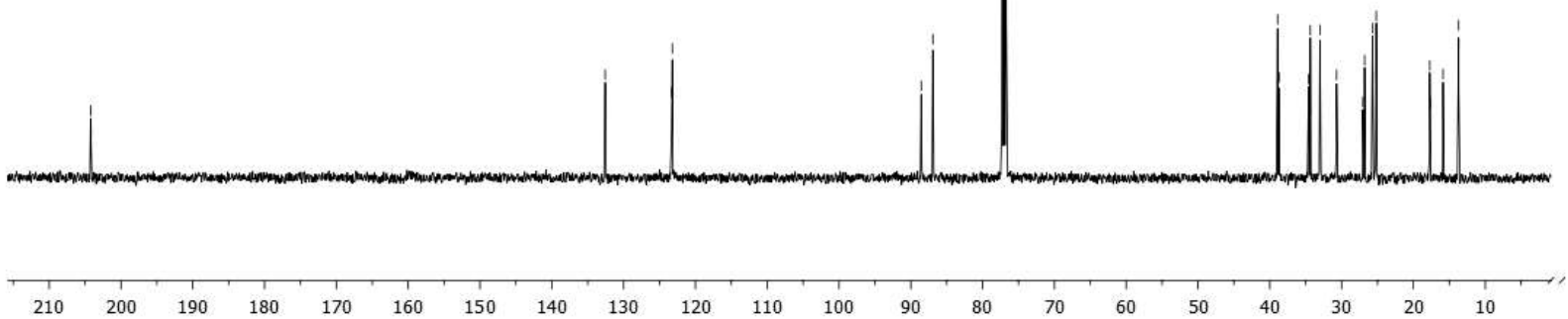

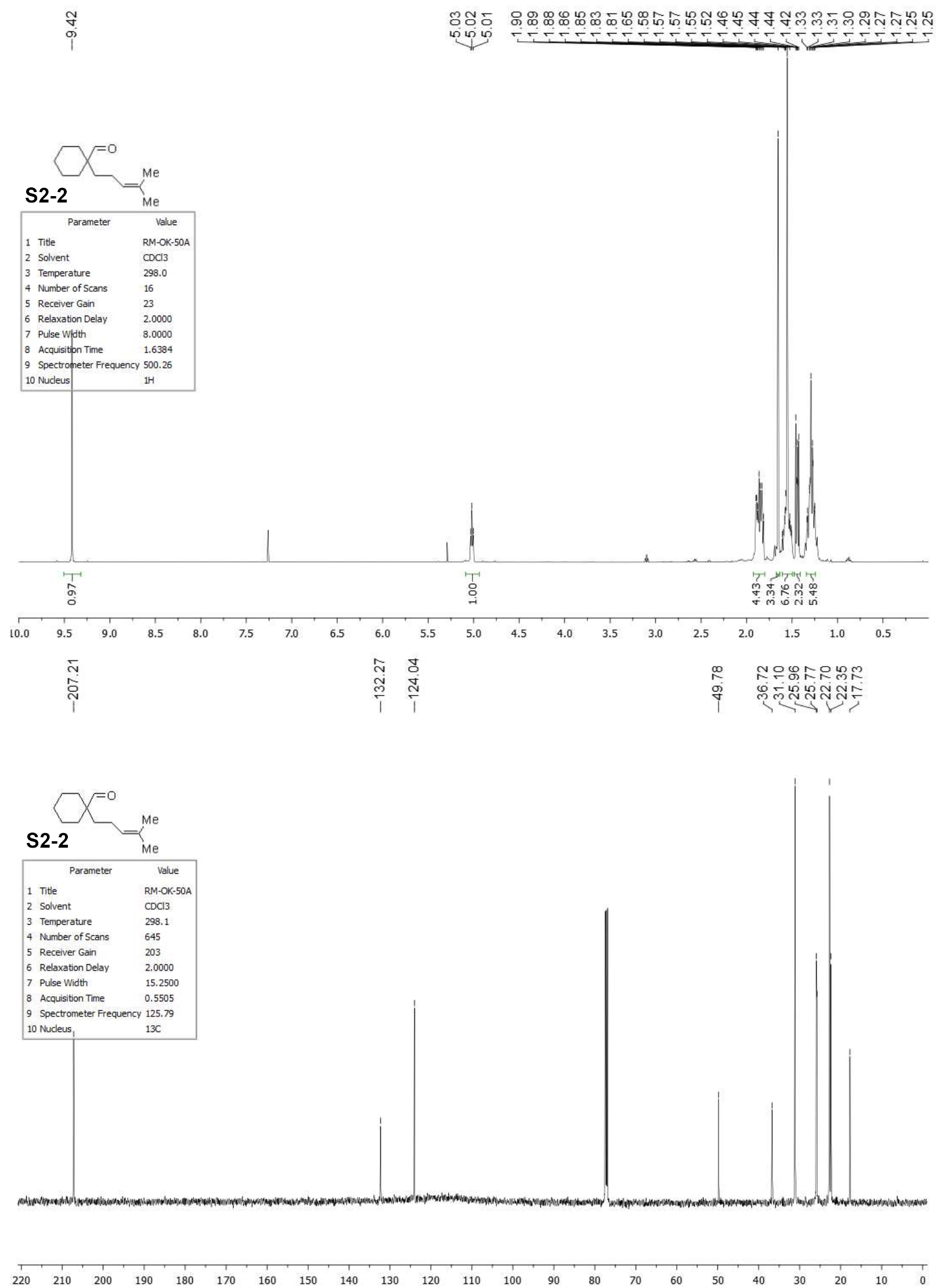

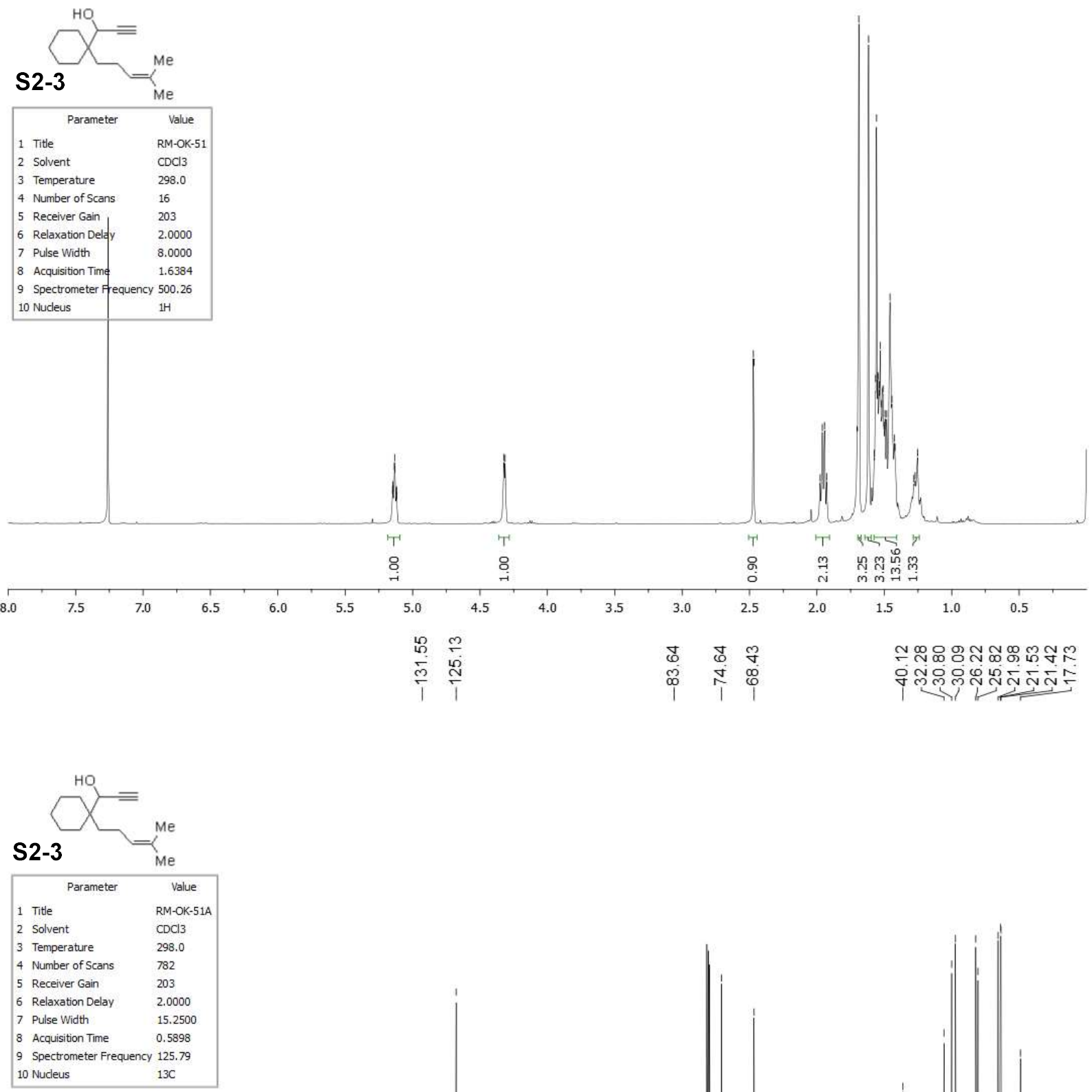


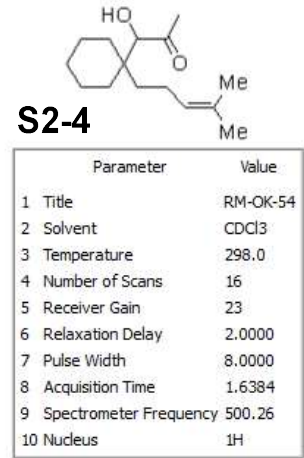

$1 \mathrm{H}$
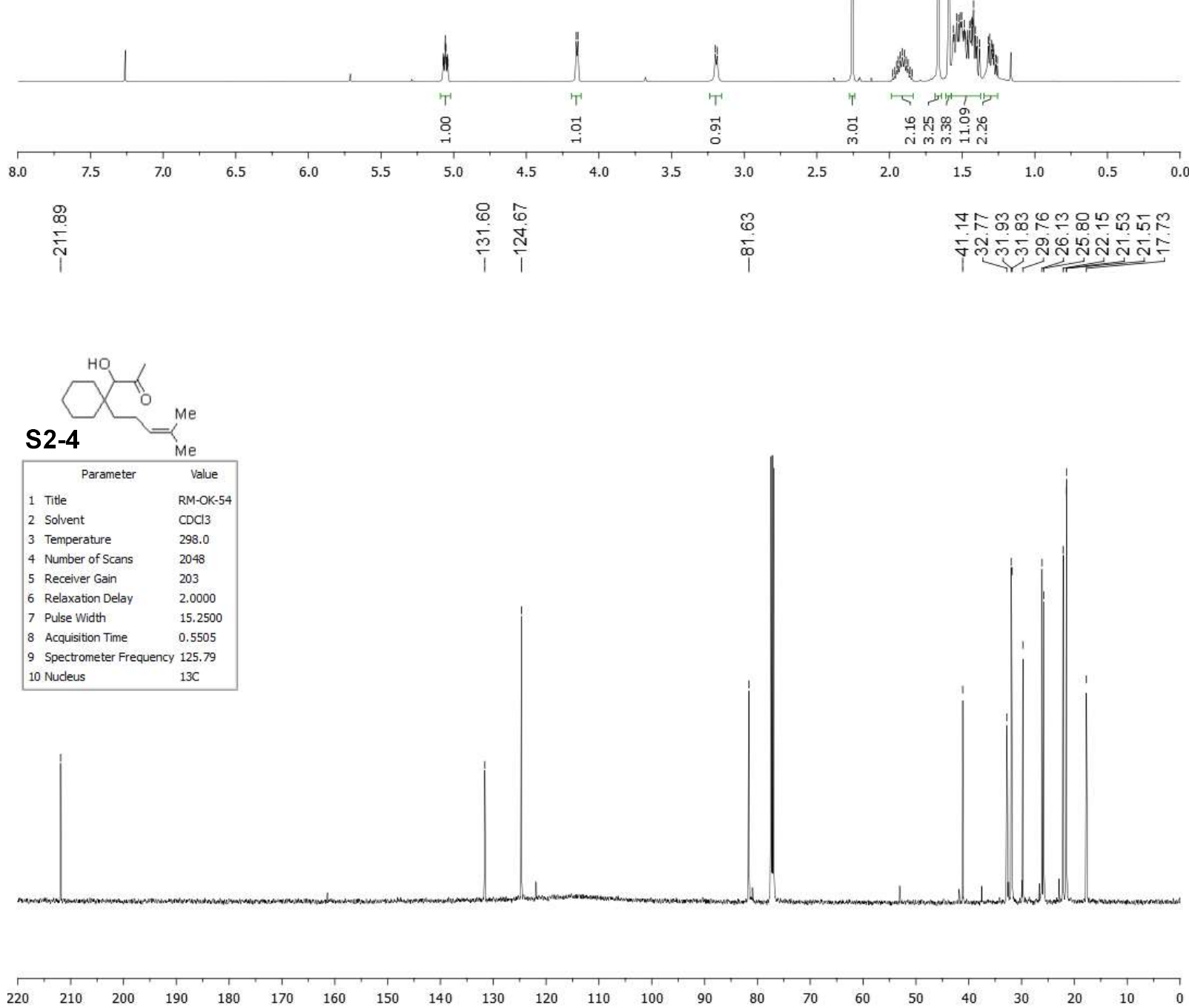

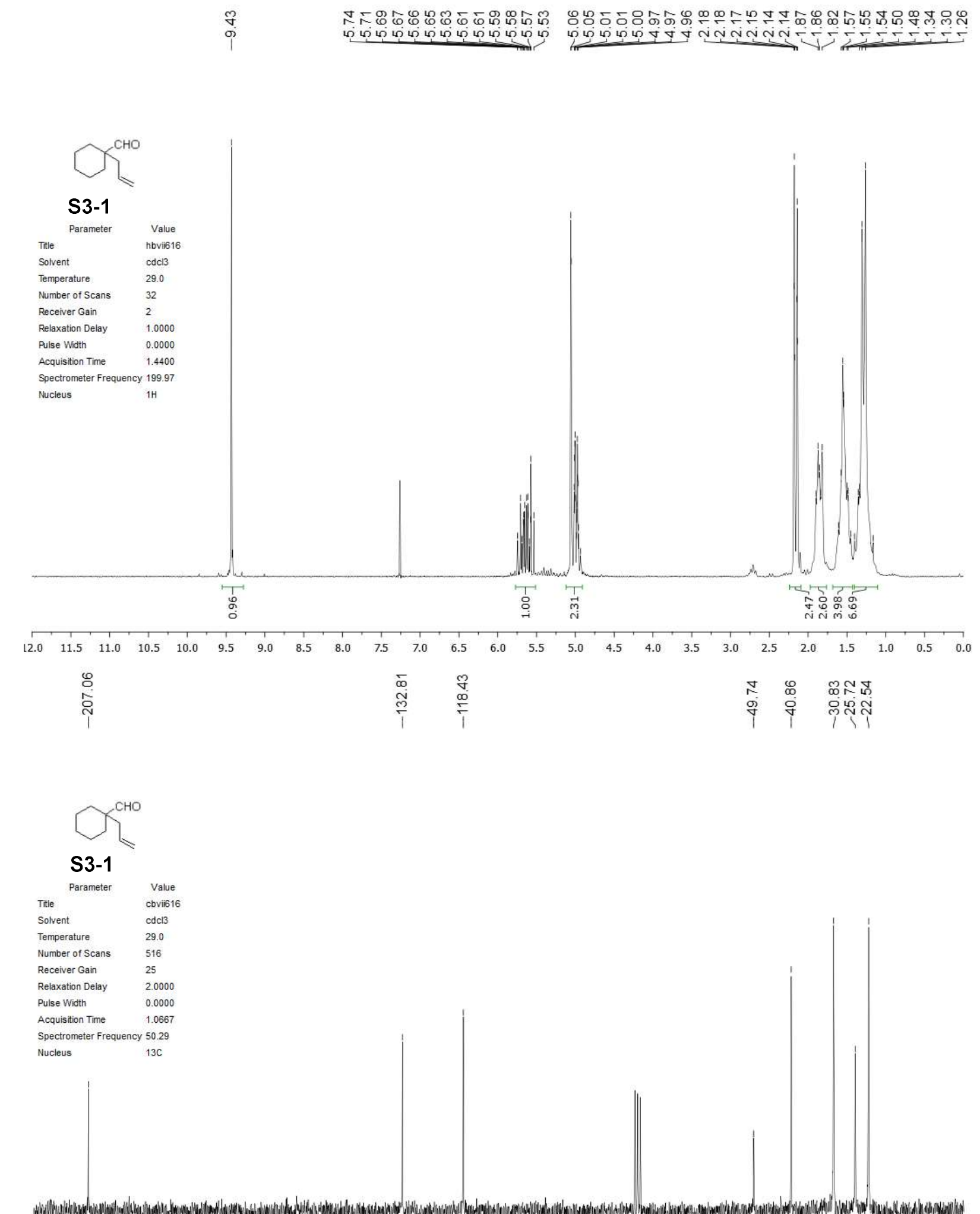

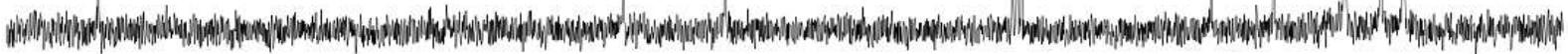

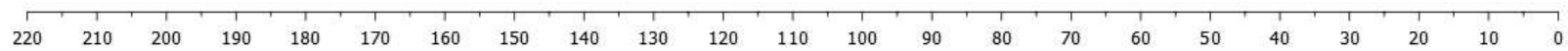



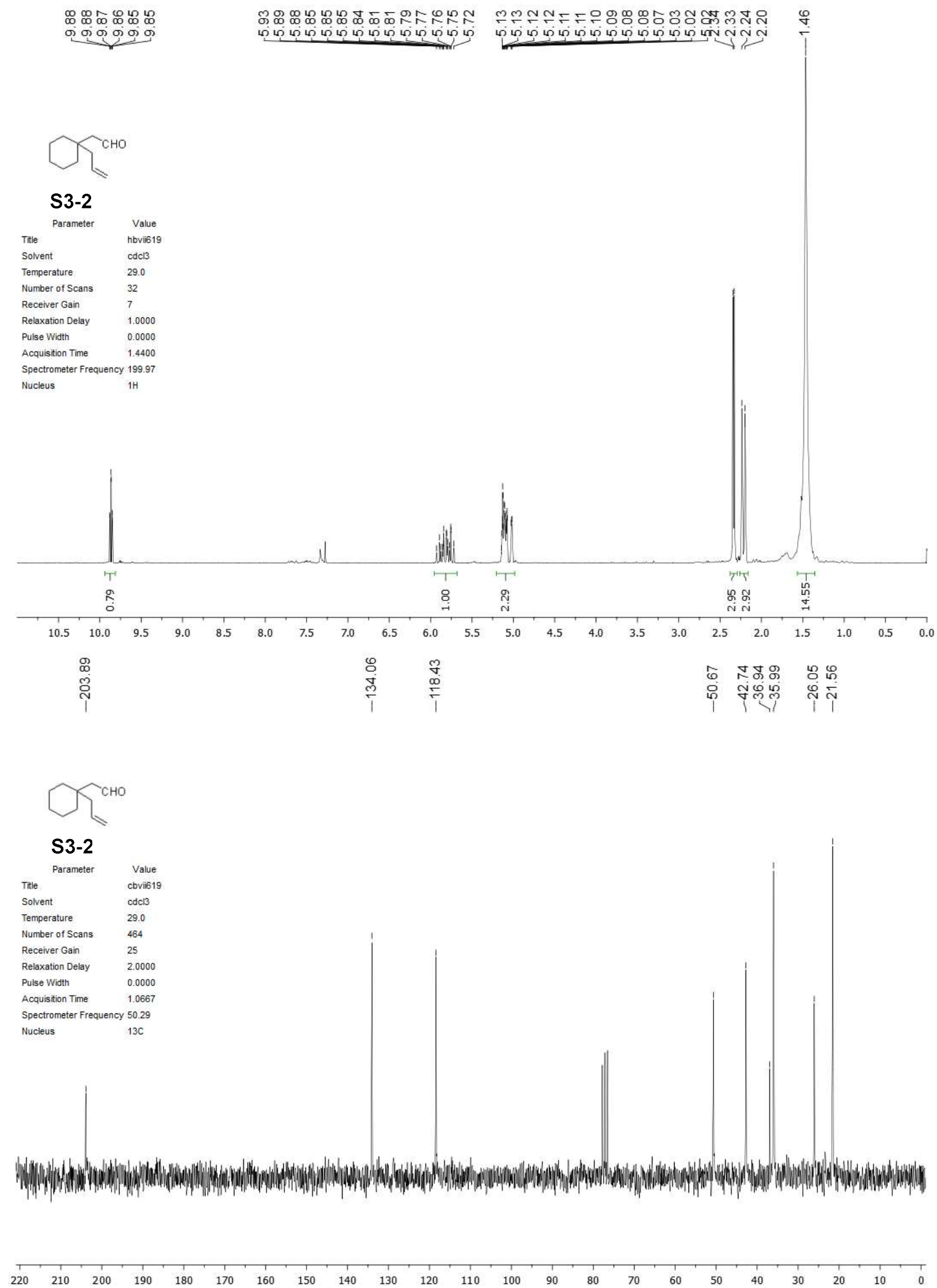

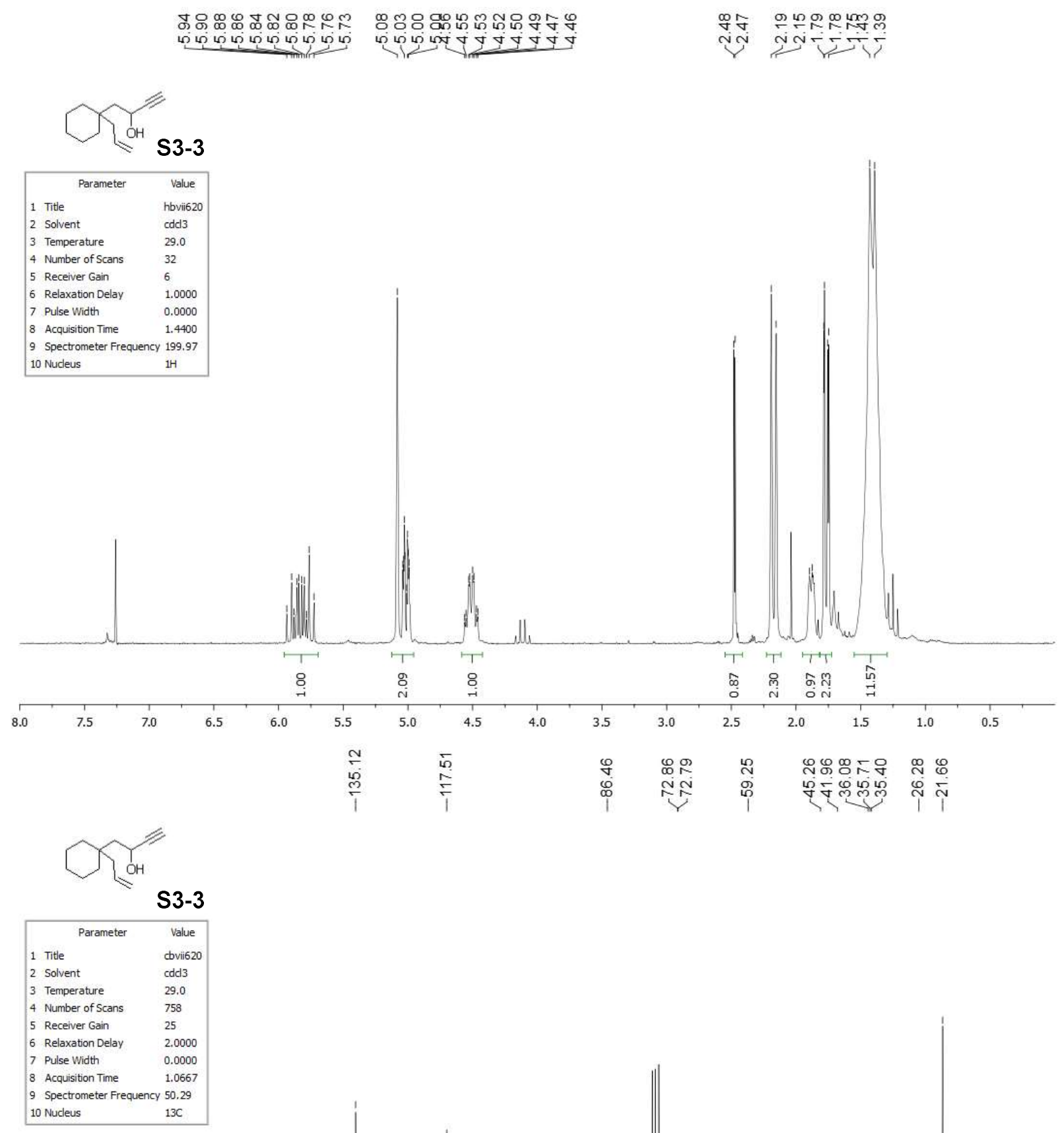

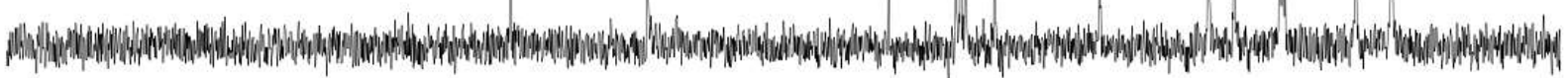

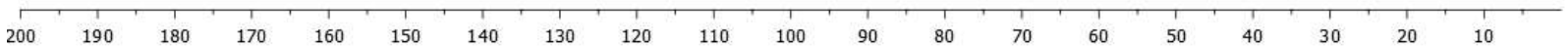



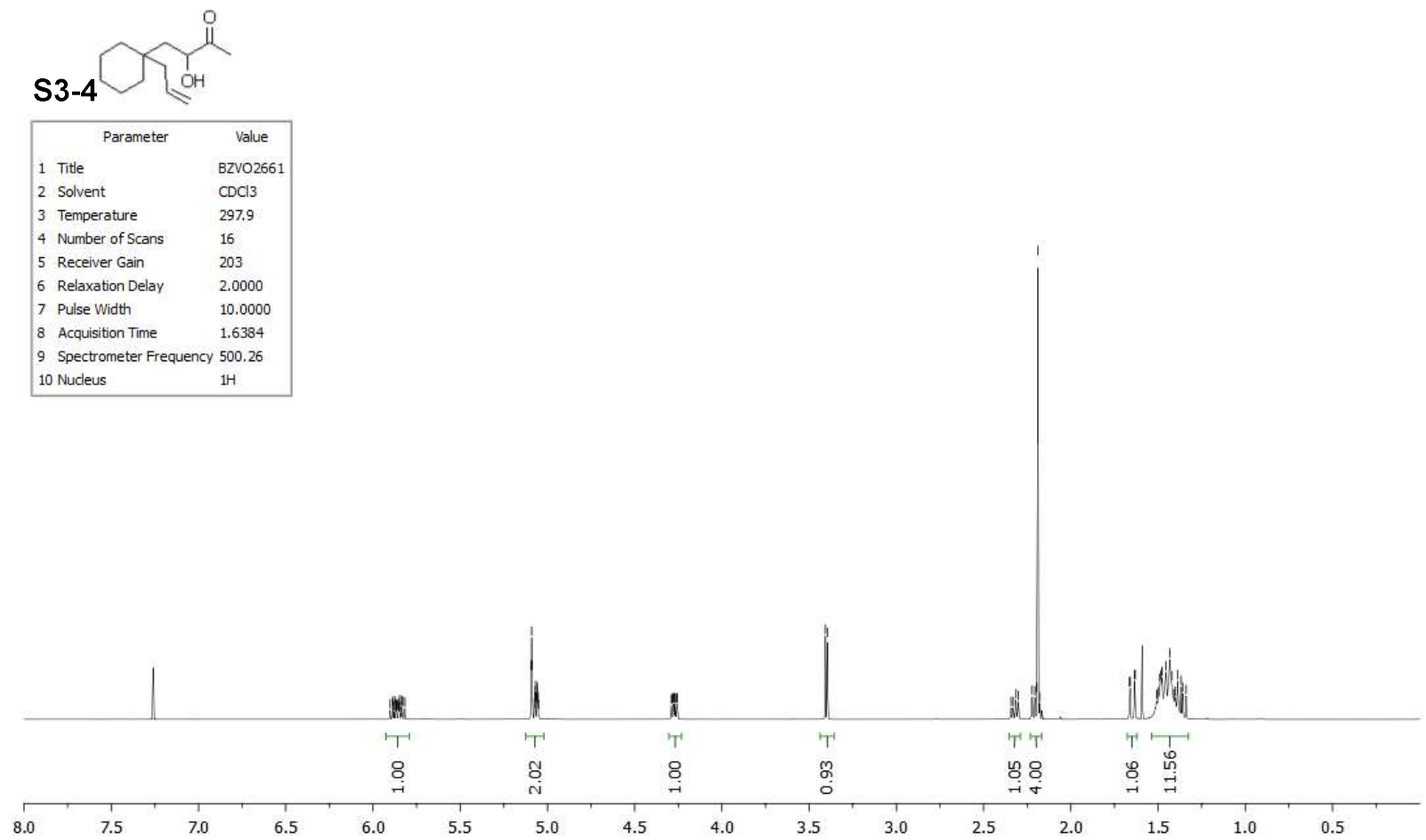

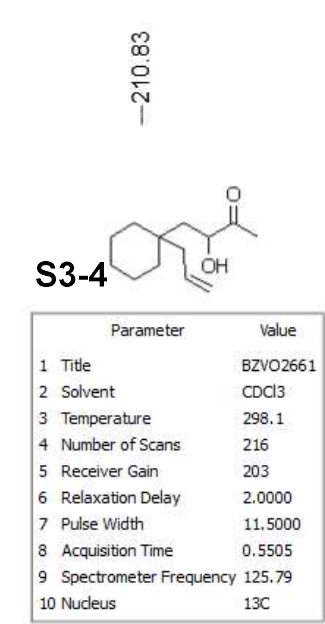
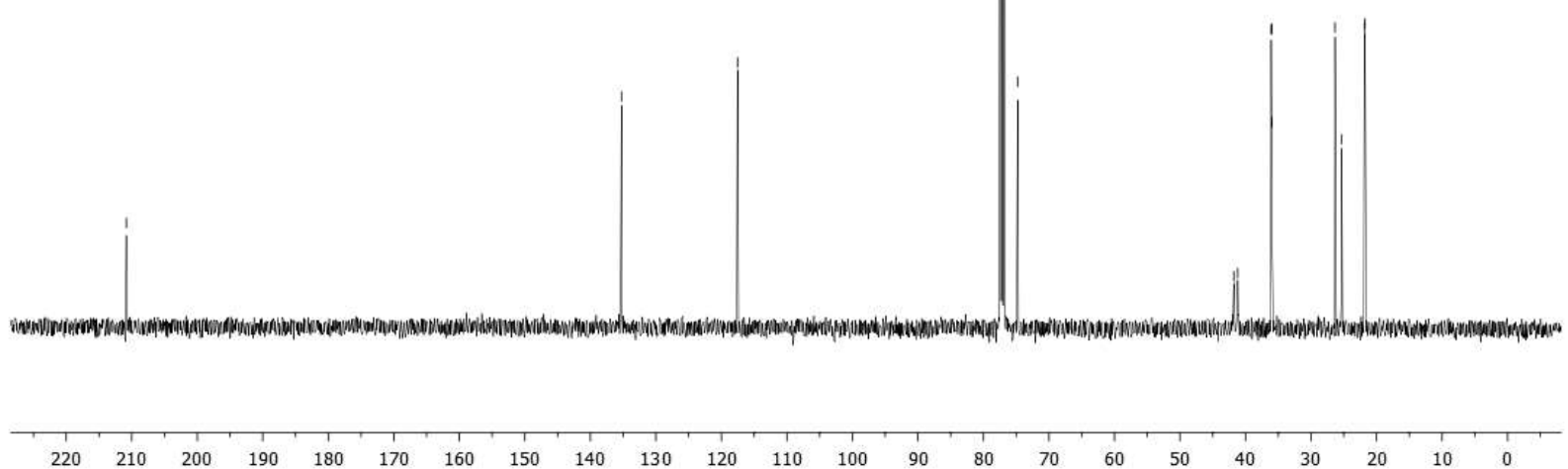

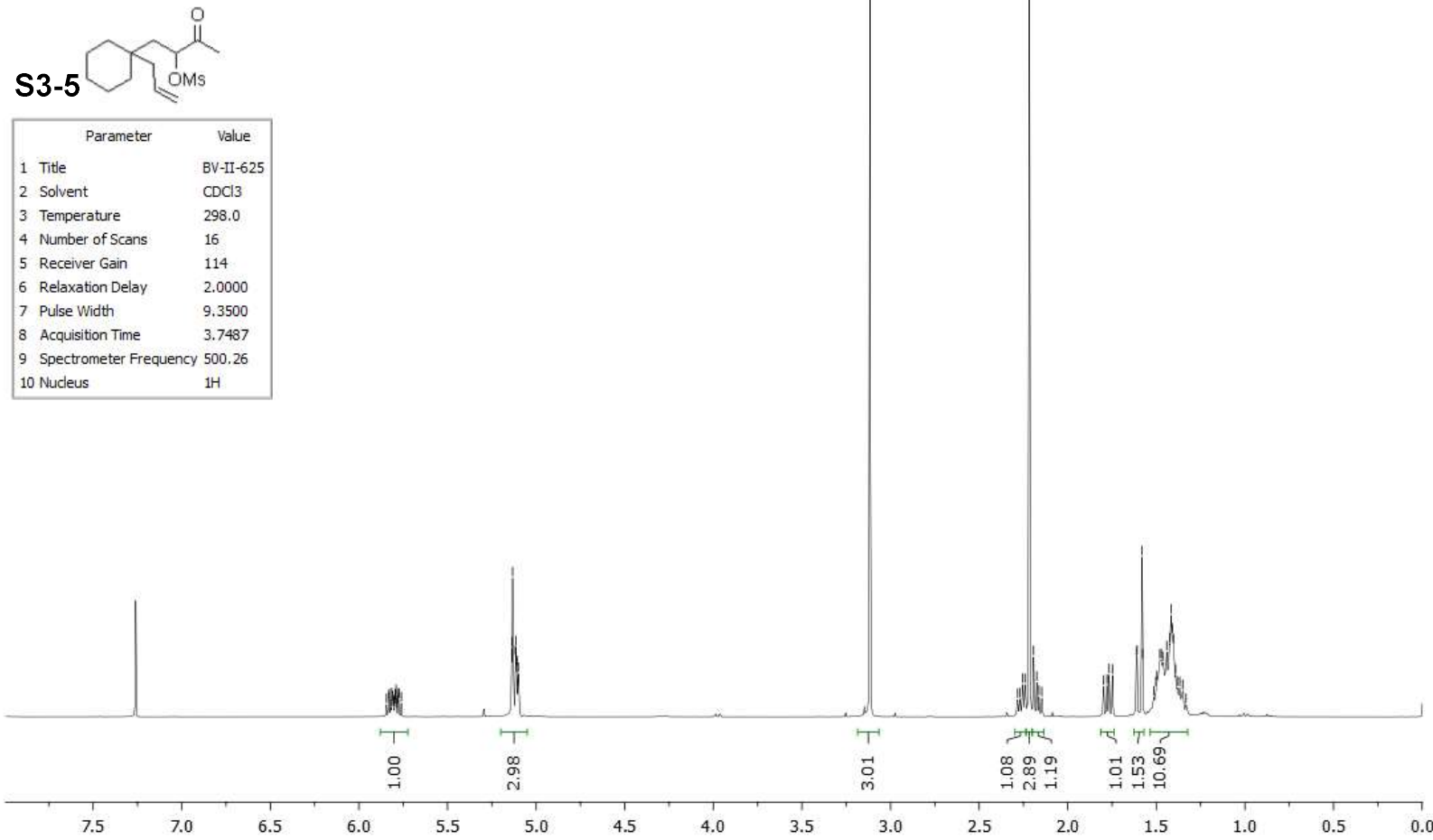

管
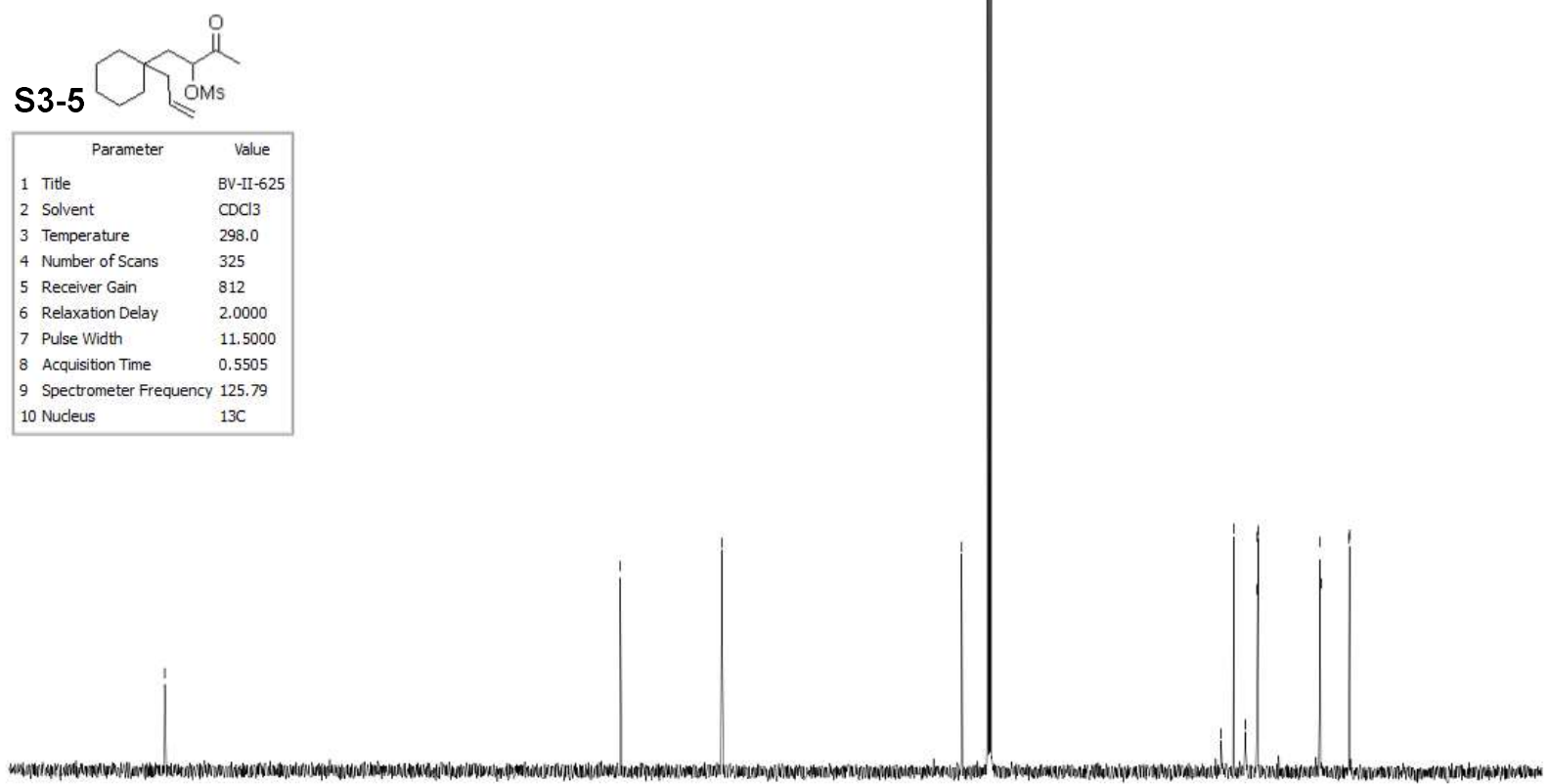

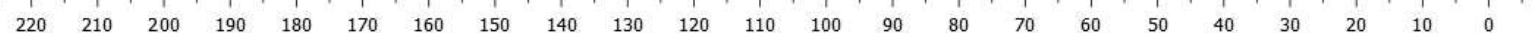




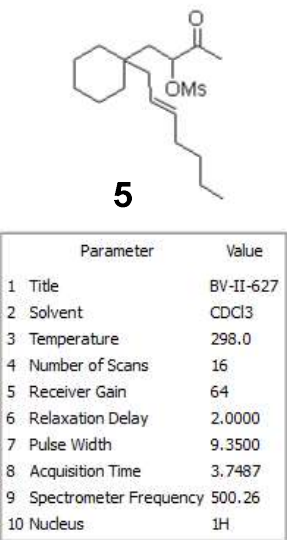$$
1 \mathrm{H}
$$ 

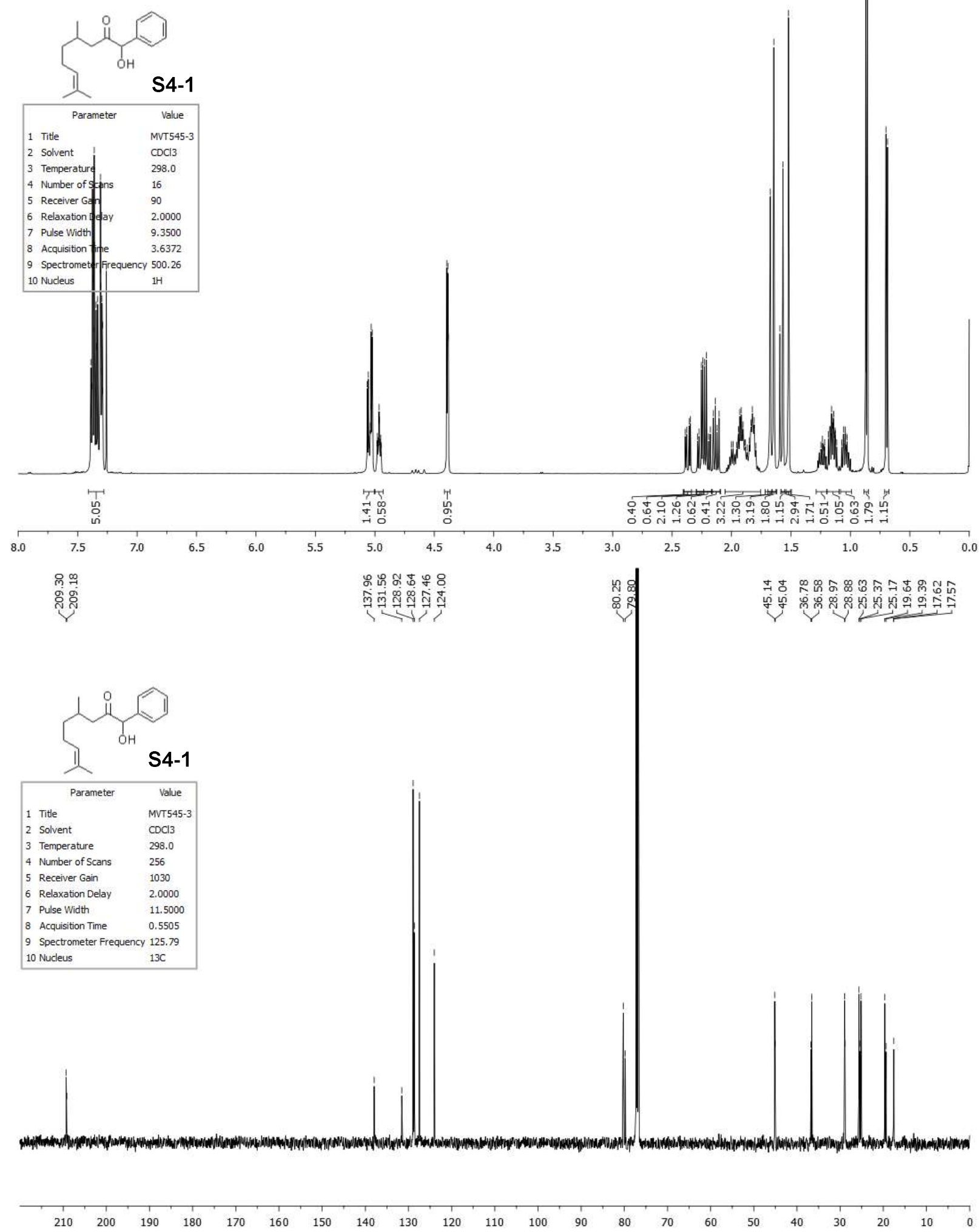

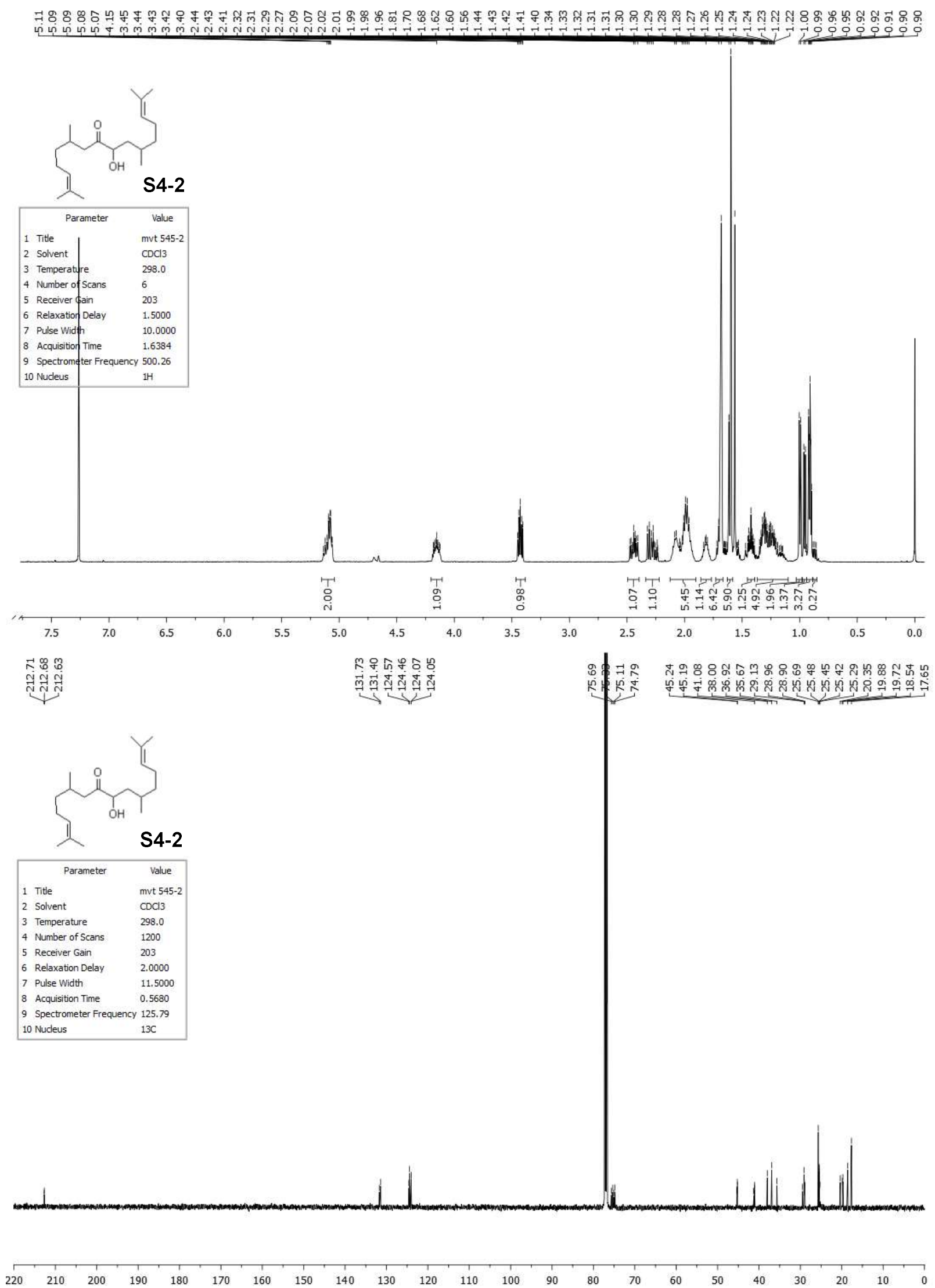

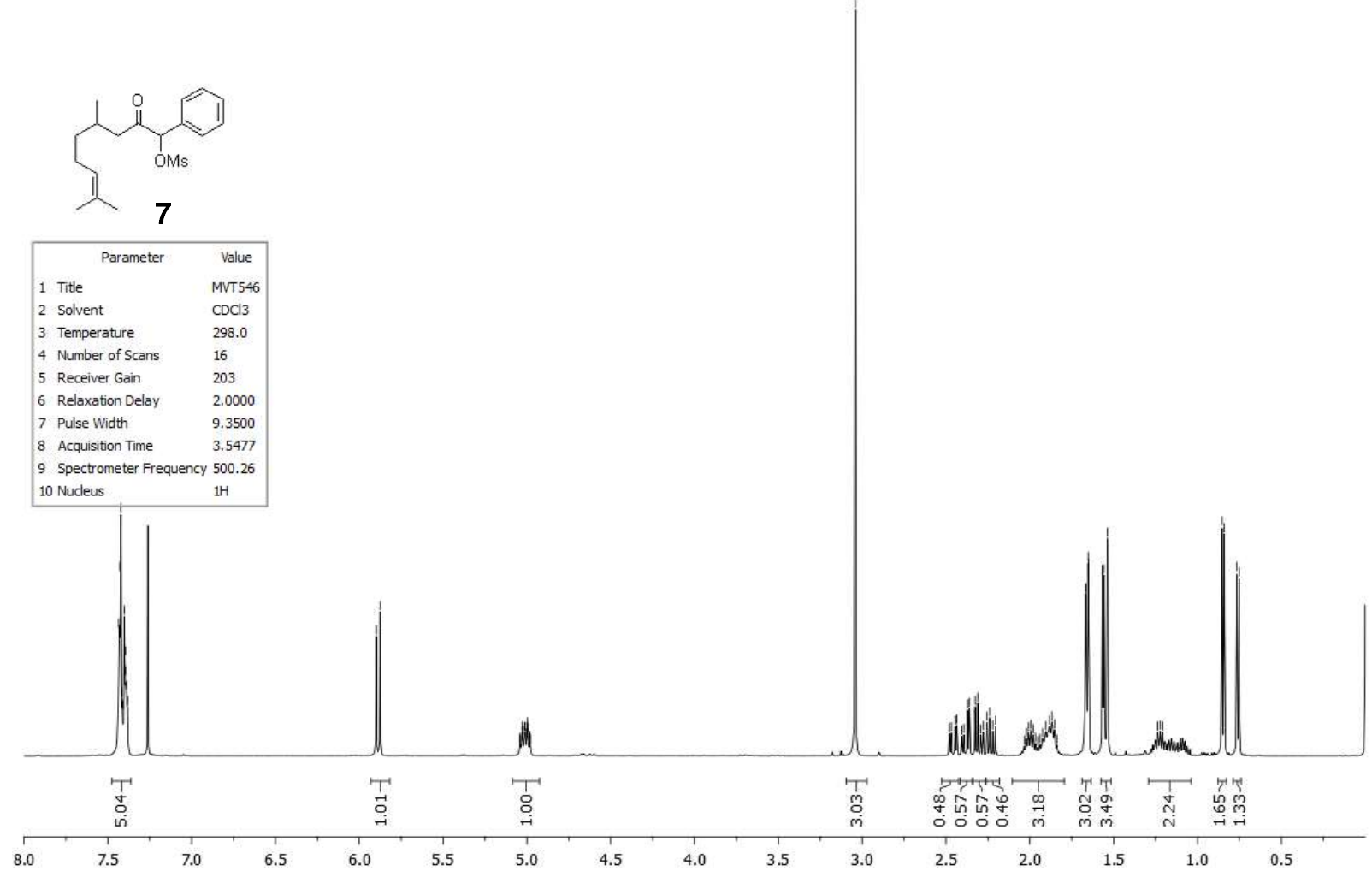

8.0

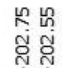

ชู่

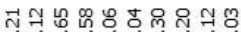

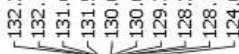

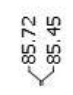

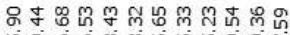

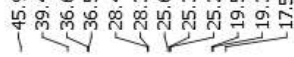

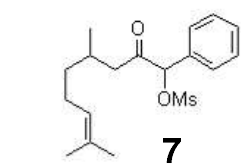

\begin{tabular}{|lll|}
\hline \multicolumn{1}{|c|}{ Parameter } & \multicolumn{1}{c|}{ Value } \\
1 & Title & MVT546 \\
2 & Solvent & $\mathrm{CDCl}$ \\
3 & Temperature & 298.0 \\
4 & Number of Scans & 513 \\
5 & Receiver Gain & 1030 \\
6 & Relaxation Delay & 2.0000 \\
7 & Pulse Width & 11.5000 \\
8 & Acquisition Time & 0.5505 \\
9 & Spectrometer Frequency & 125.79 \\
10 & Nucleus & $13 \mathrm{C}$ \\
\hline \multicolumn{2}{l}{} \\
\hline \multicolumn{2}{l}{} \\
\hline
\end{tabular}
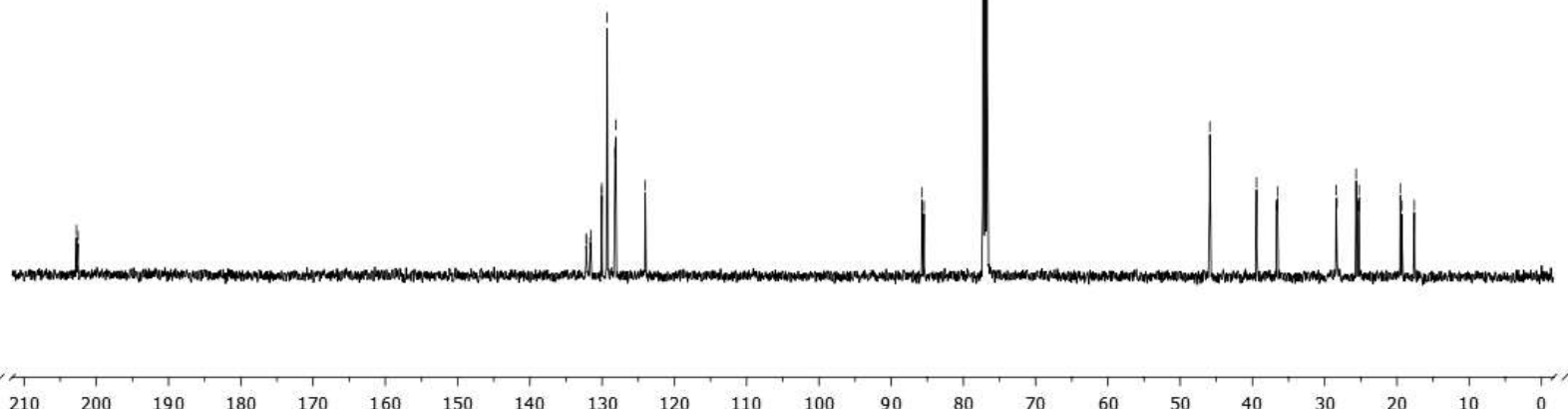


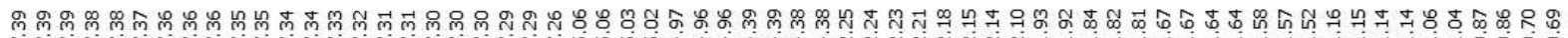

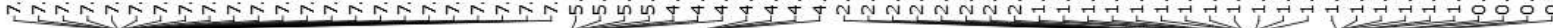
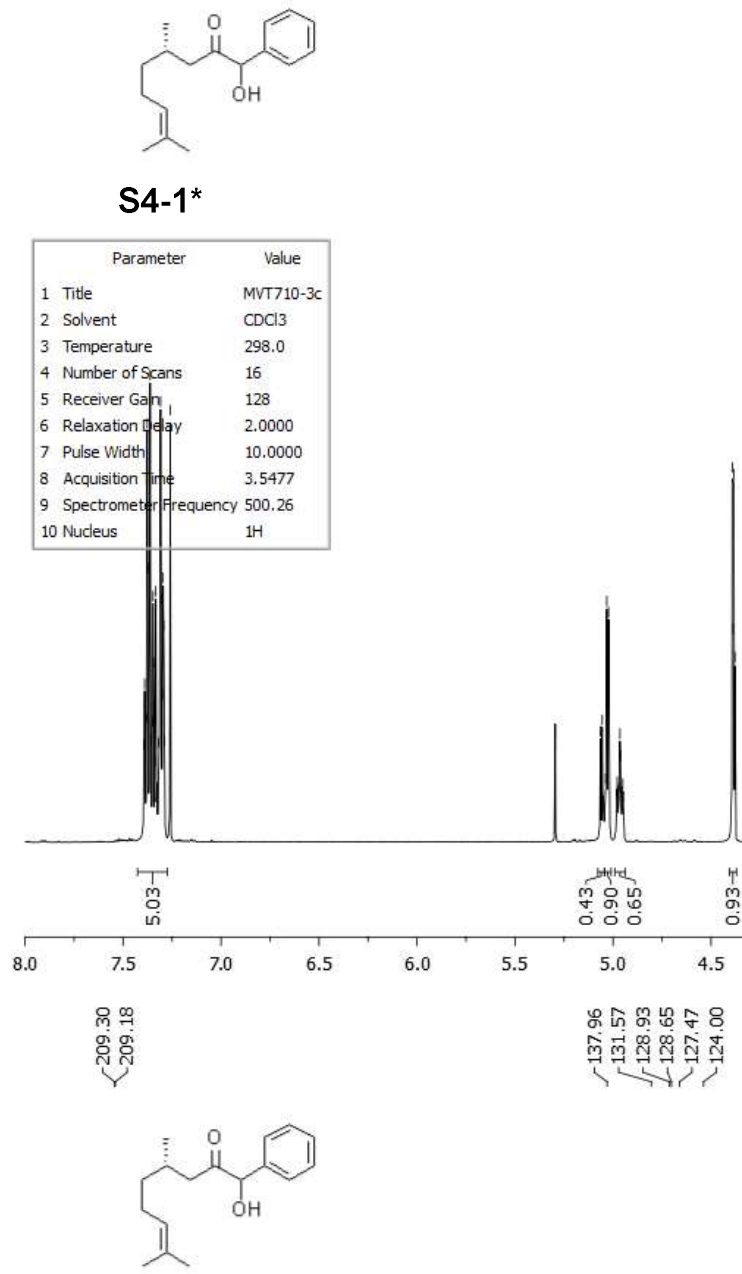

\section{S4-1*}

\begin{tabular}{|lll|}
\hline \multicolumn{1}{|c|}{ Parameter } & \multicolumn{1}{c|}{ Value } \\
1 & Title & MVT 710-3C \\
2 & Solvent & CDCl3 \\
3 & Temperature & 298.0 \\
4 & Number of Scans & 560 \\
5 & Receiver Gain & 203 \\
6 & Relaxation Delay & 2.0000 \\
7 & Pulse Width & 11.5000 \\
8 & Acquisition Time & 0.5505 \\
9 & Spectrometer Frequency 125.79 \\
10 & Nucleus & $13 \mathrm{C}$ \\
\hline
\end{tabular}
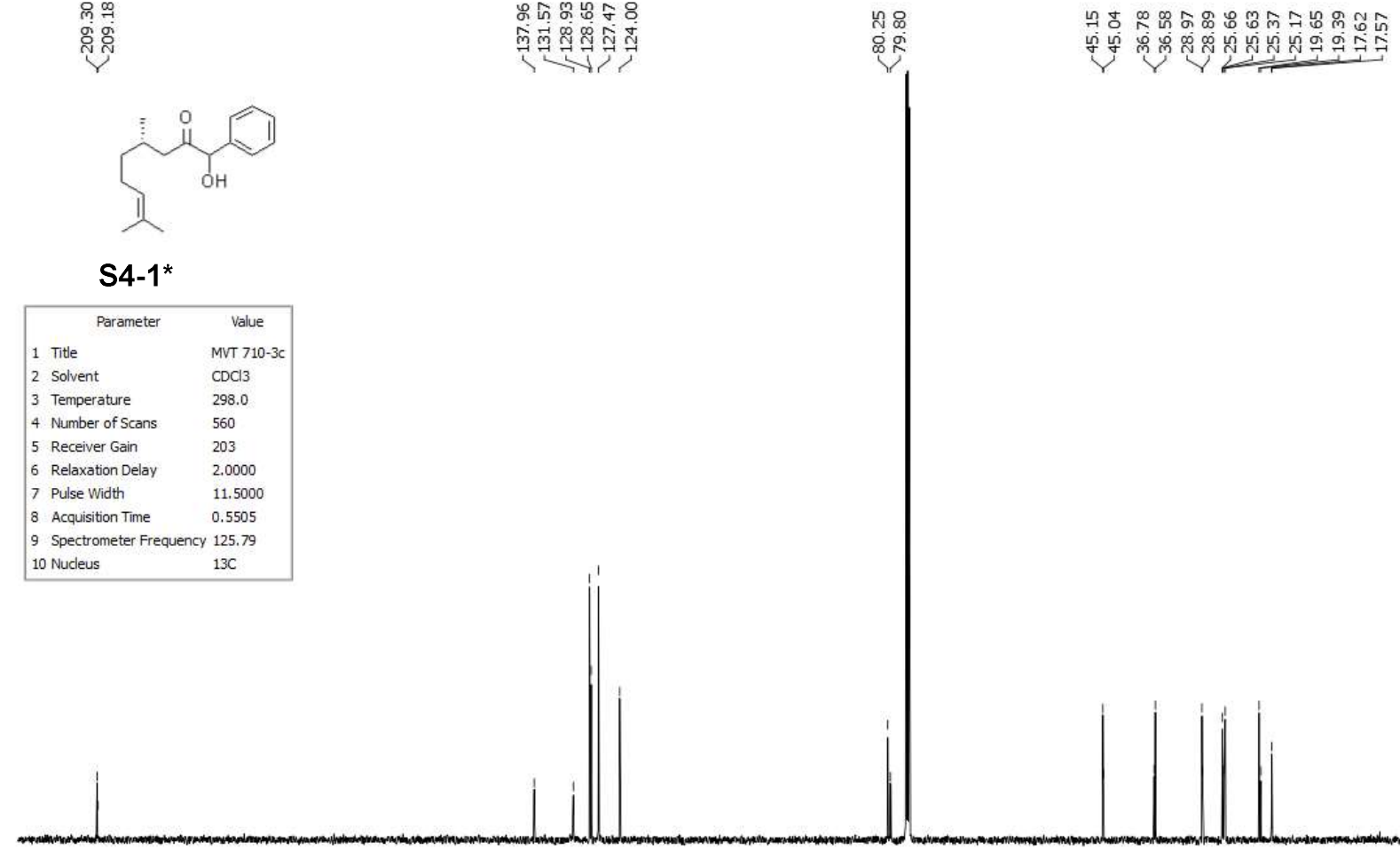

$13 \mathrm{C}$

220
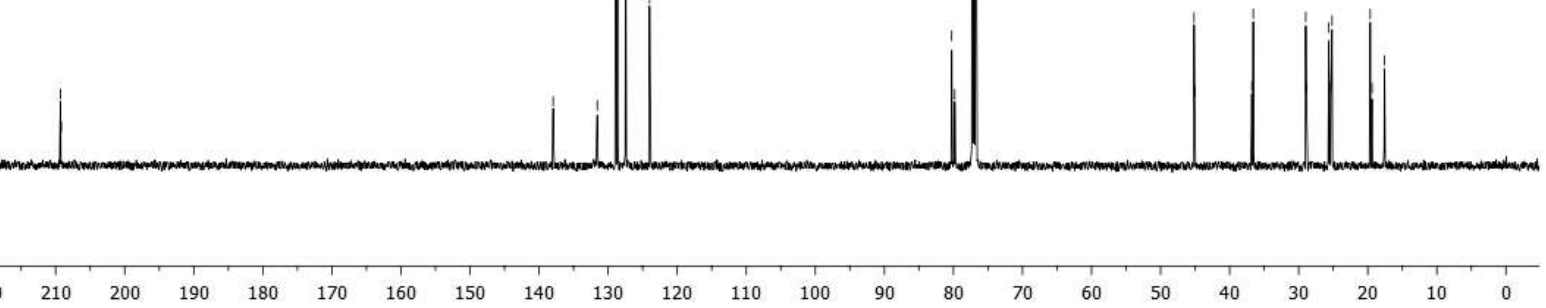

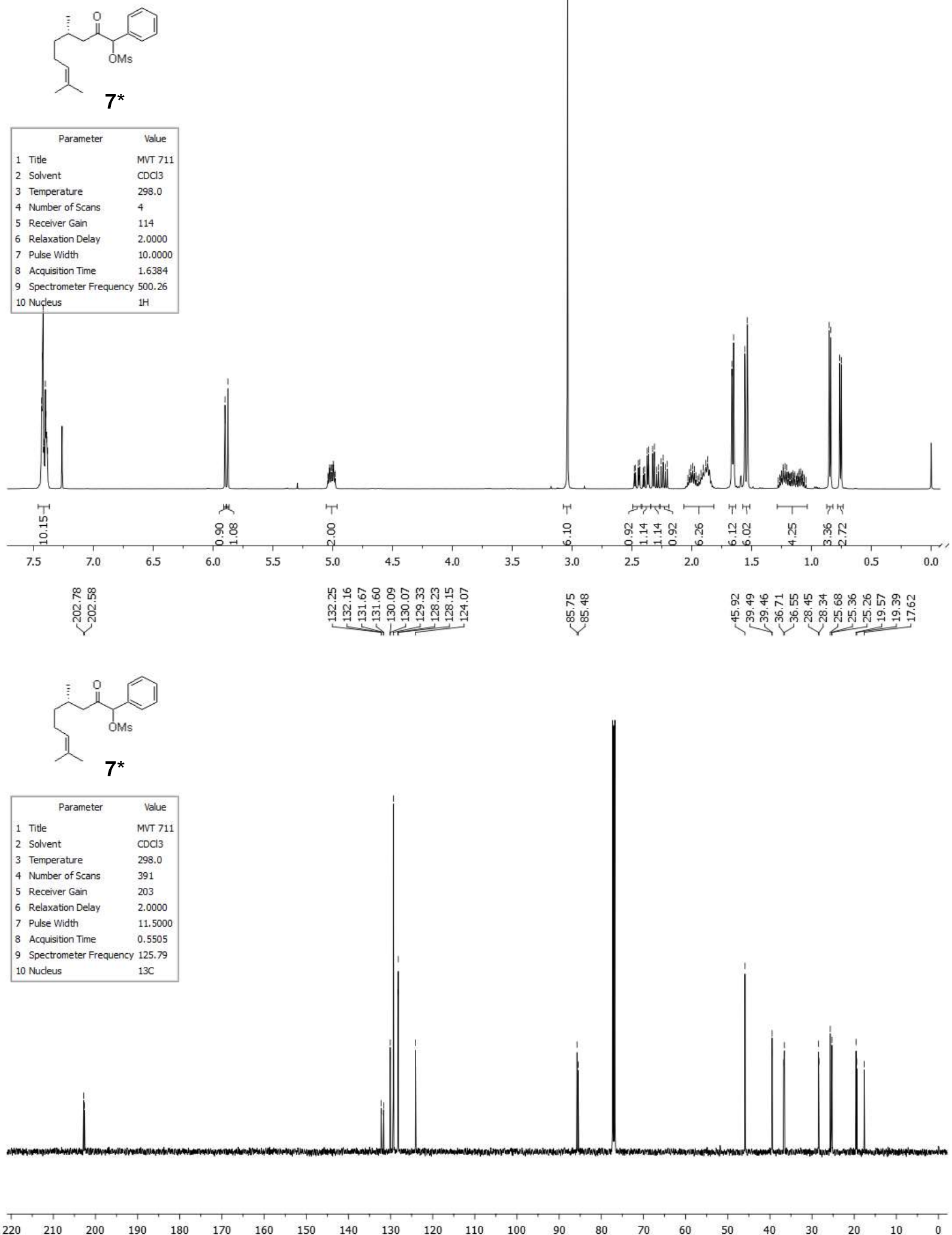

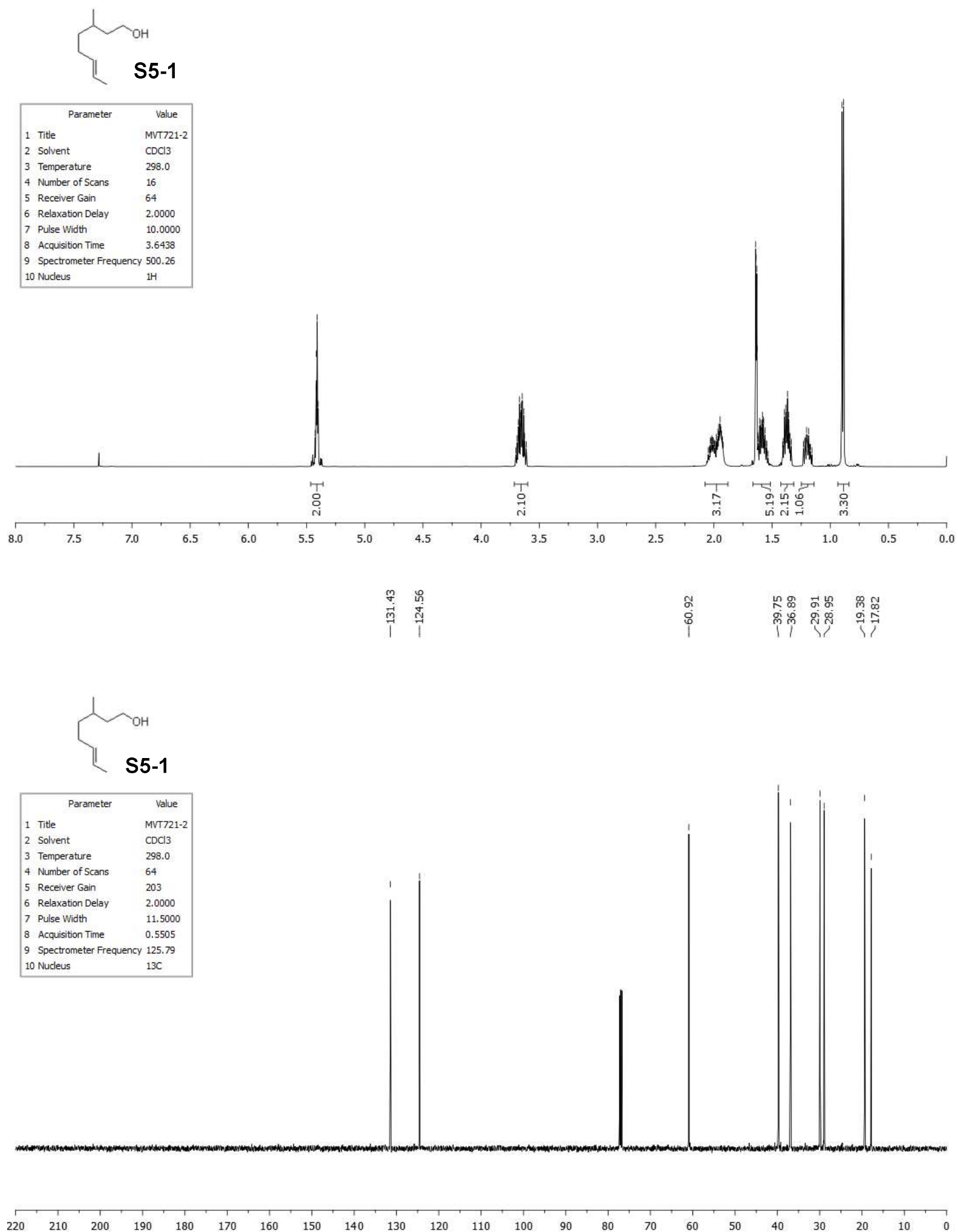


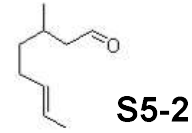

\begin{tabular}{|lll|}
\hline \multicolumn{1}{|c}{ Parameter } & \multicolumn{1}{c|}{ Value } \\
1 & Title & MVT722 \\
2 & Solvent & $\mathrm{CDCl}$ \\
3 & Temperature & 298.0 \\
4 & Number of Scans & 16 \\
5 & Receiver Gain & 64 \\
6 & Relaxation Delay & 2.0000 \\
7 & Pulse Width & 10.0000 \\
8 & Acquisition Time & 2.7918 \\
9 & Spectrometer Frequency & 500.26 \\
10 & Nudeus & $1 \mathrm{H}$ \\
\hline
\end{tabular}

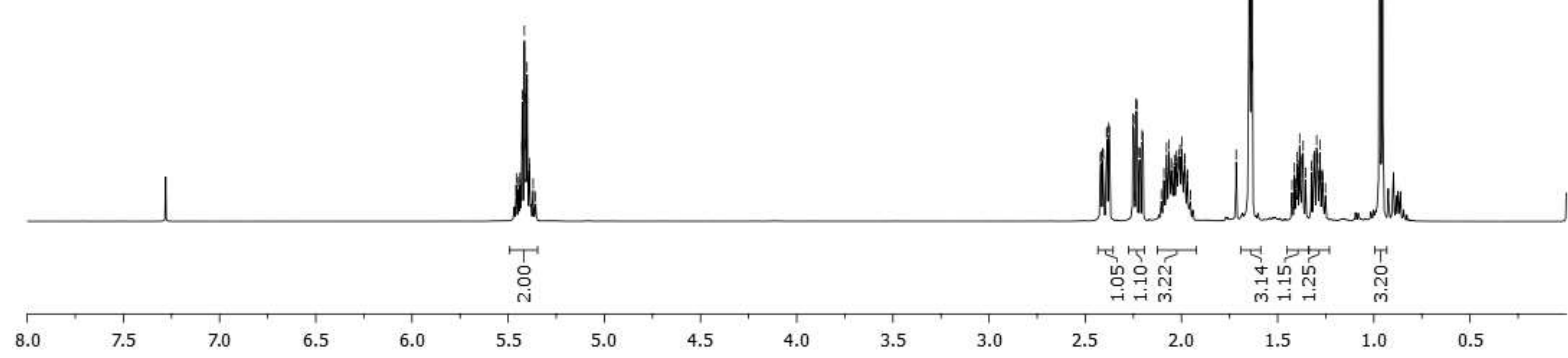

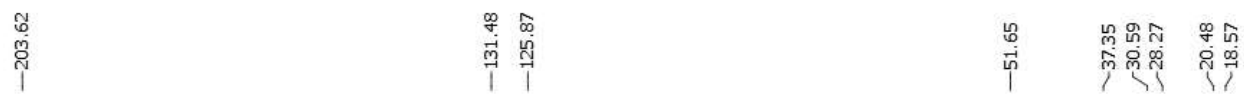
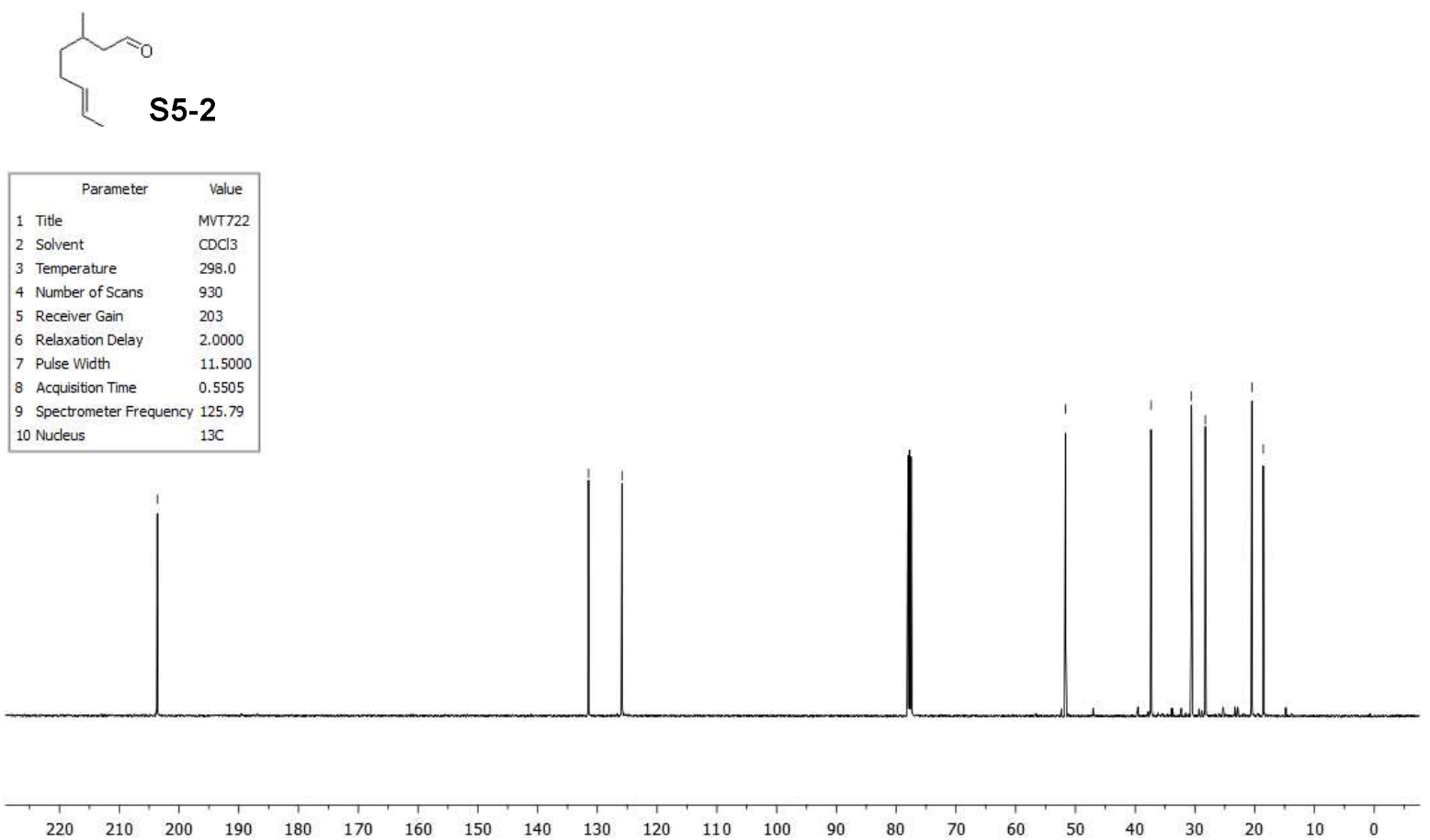

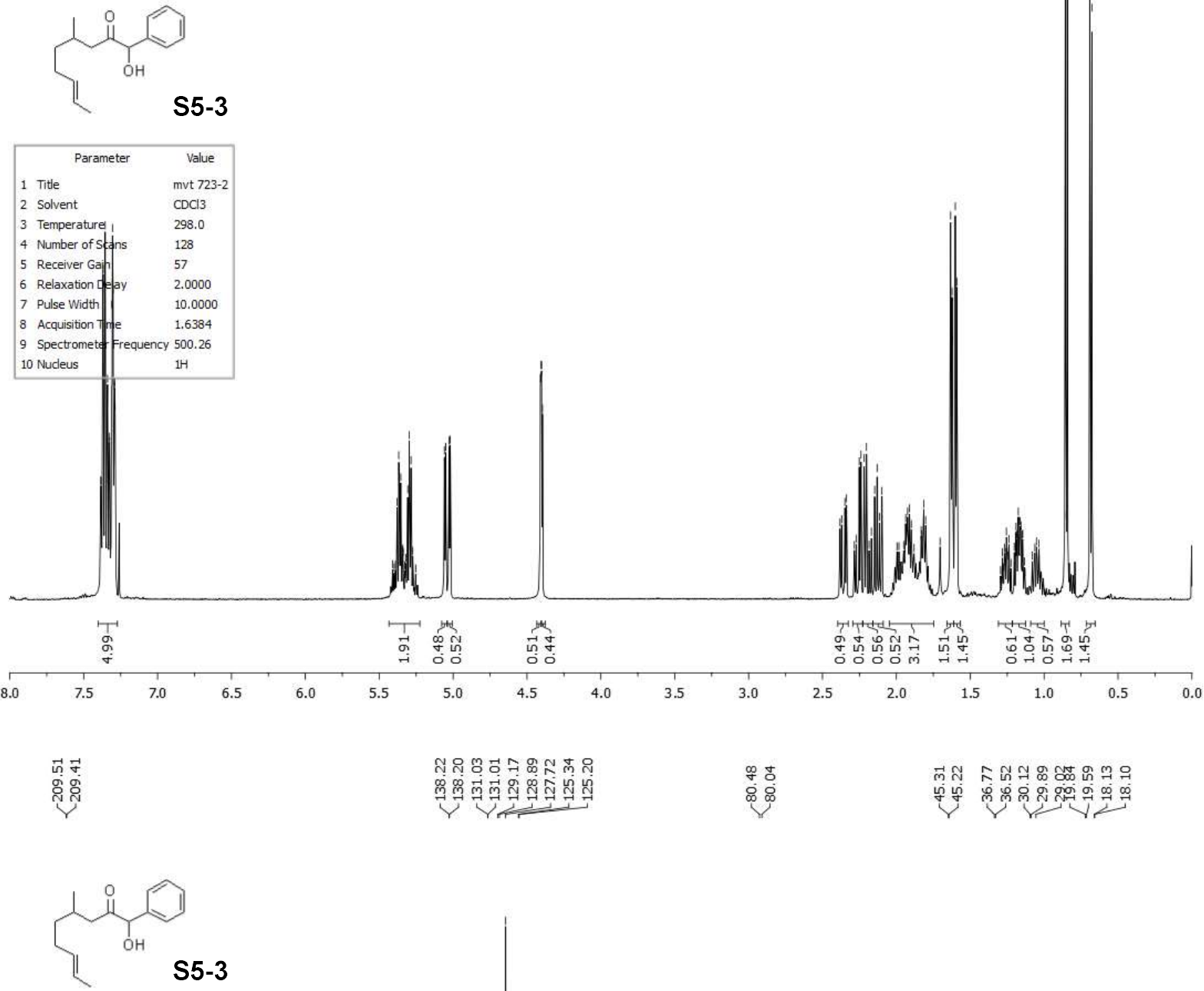

\begin{tabular}{|lll|}
\hline & \multicolumn{1}{c}{ Parameter } & \multicolumn{1}{c|}{ Value } \\
1 & Title & mvt $723-2$ \\
2 & Solvent & $\mathrm{CDCl} 3$ \\
3 & Temperature & 298.0 \\
4 & Number of Scans & 15610 \\
5 & Receiver Gain & 203 \\
6 & Relaxation Delay & 2.0000 \\
7 & Pulse Width & 11.5000 \\
8 & Acquisition Time & 0.5505 \\
9 & Spectrometer Frequency & 125.79 \\
10 & Nucleus & $13 \mathrm{C}$ \\
\hline
\end{tabular}
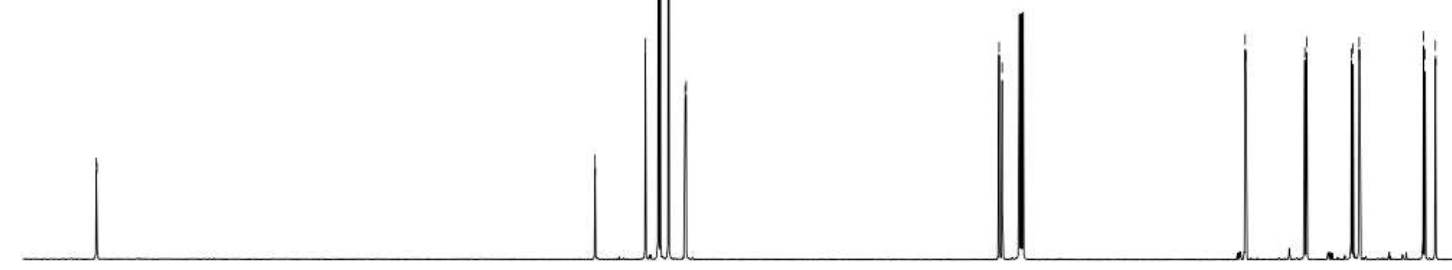

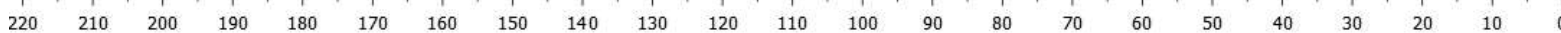




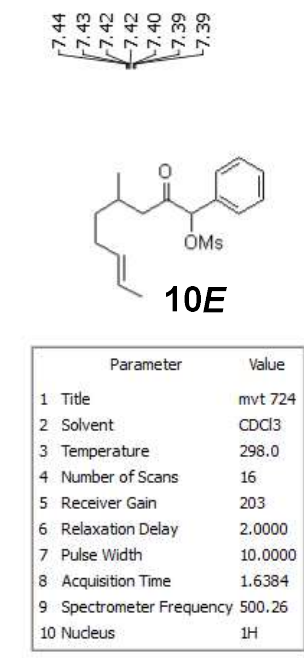

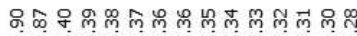

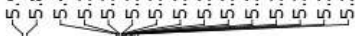

ఫ $\quad$ J

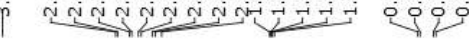
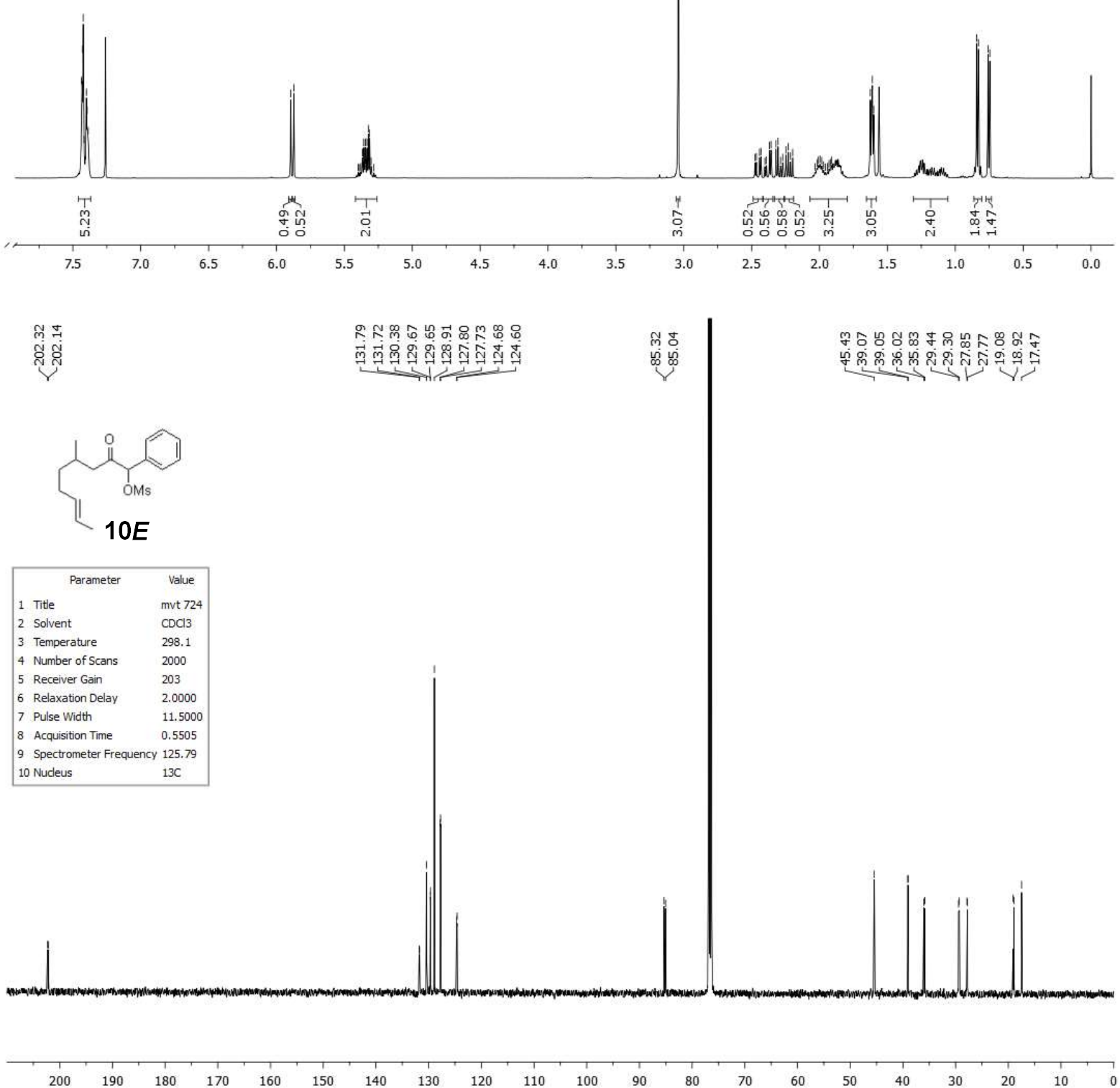


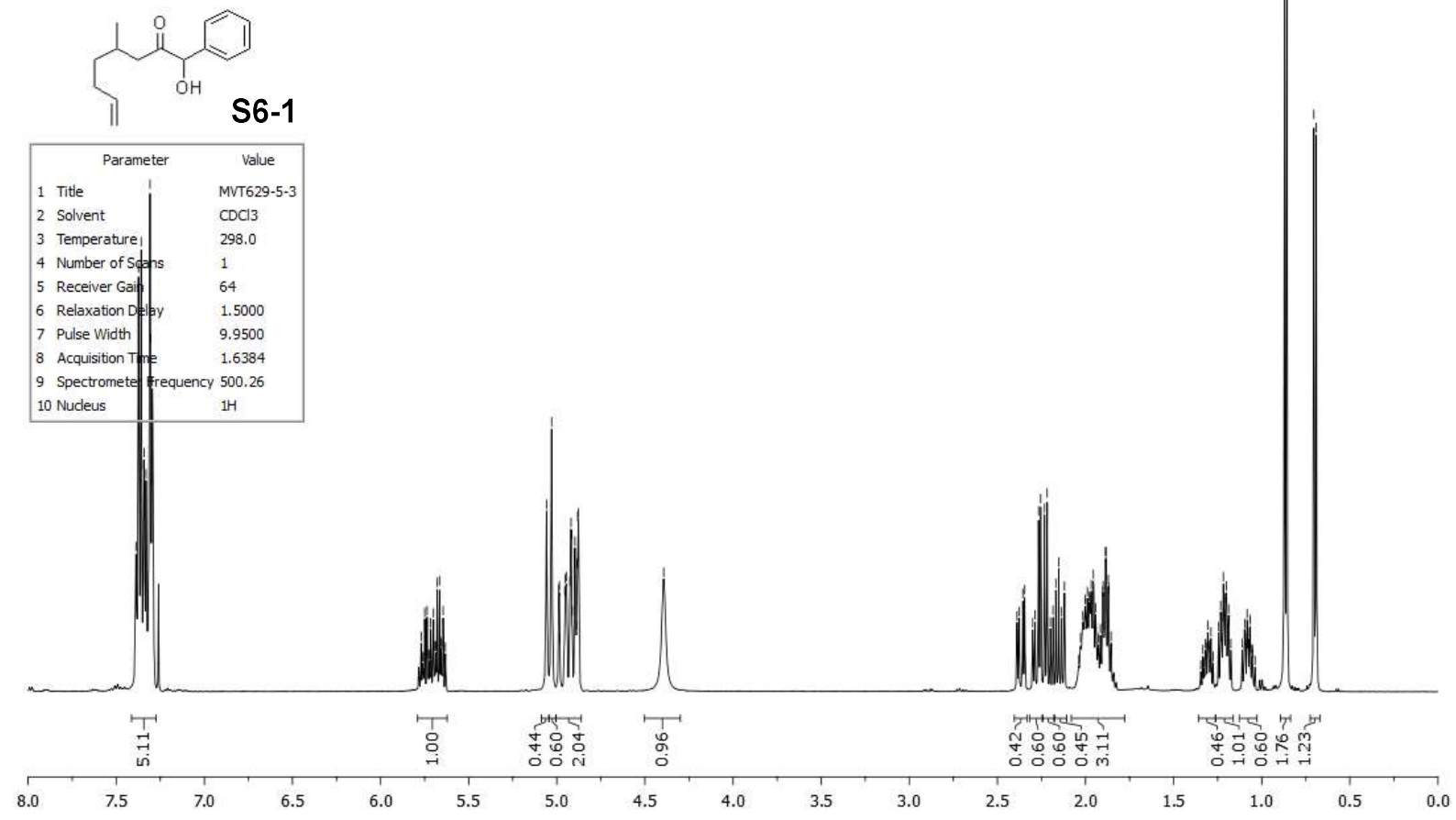

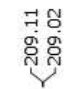

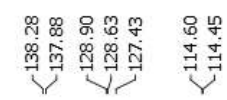

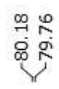

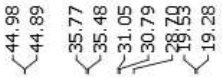
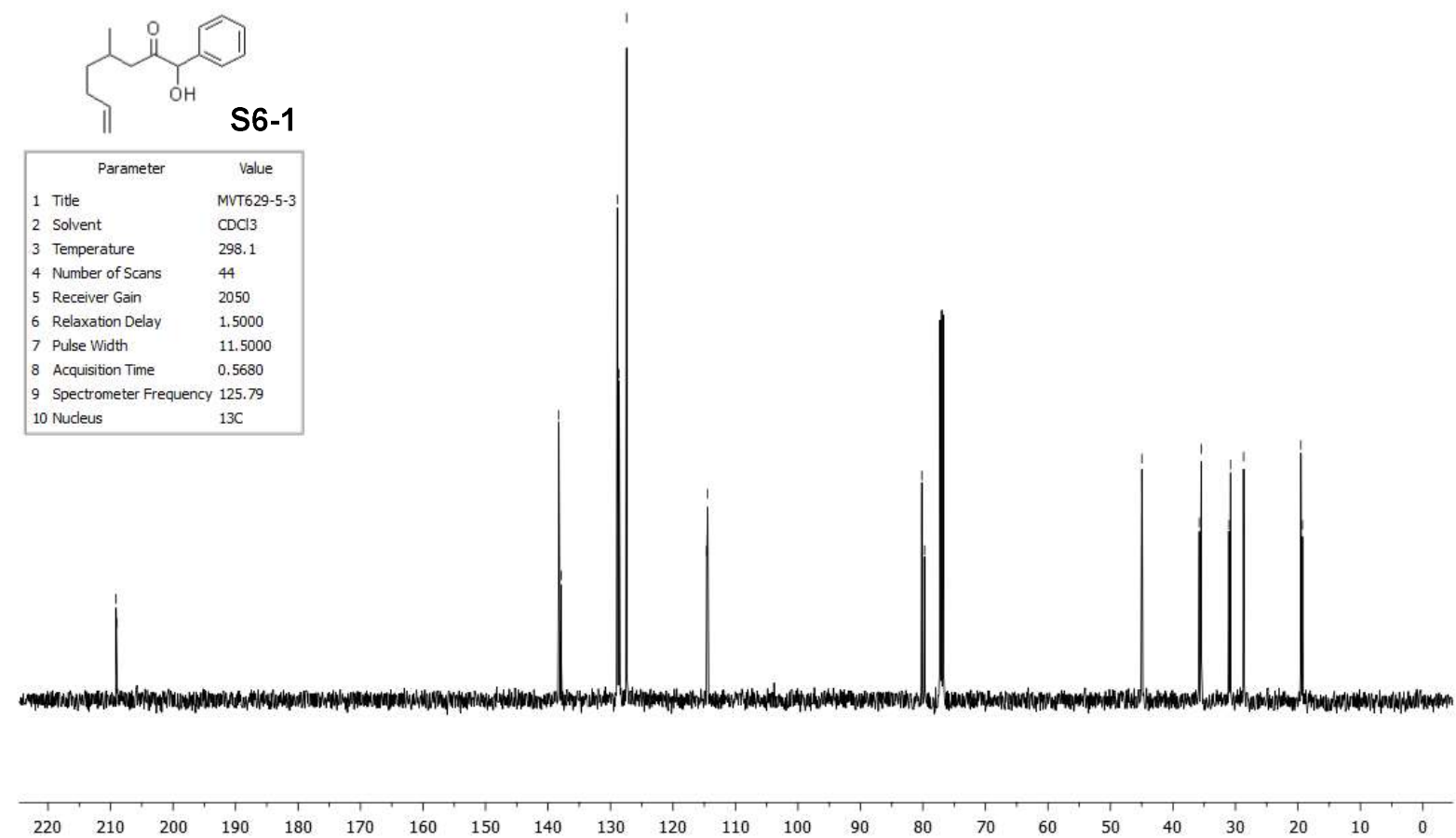

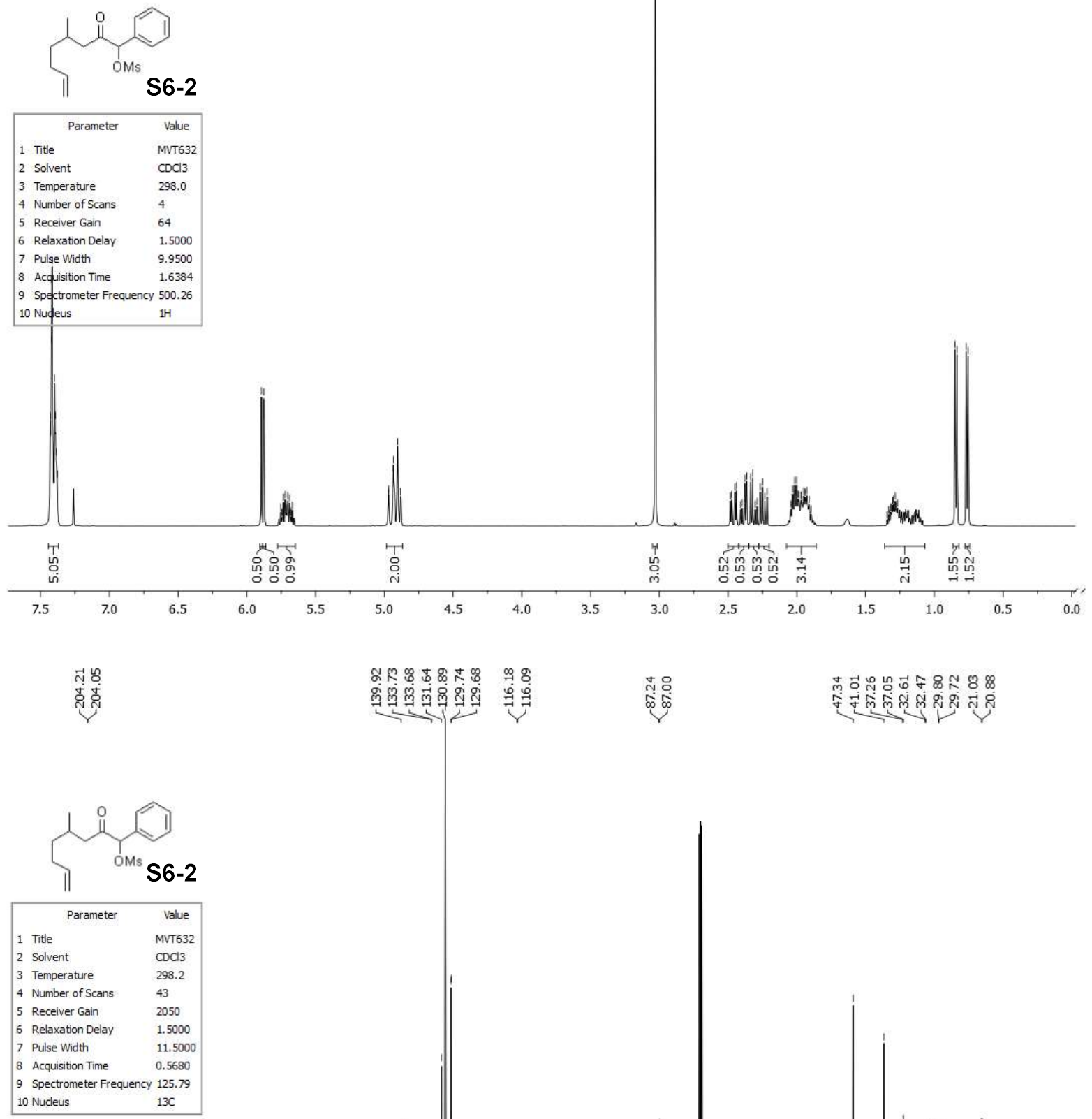

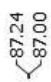

药

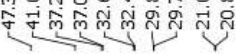

us $\quad 13 \mathrm{C}$ C

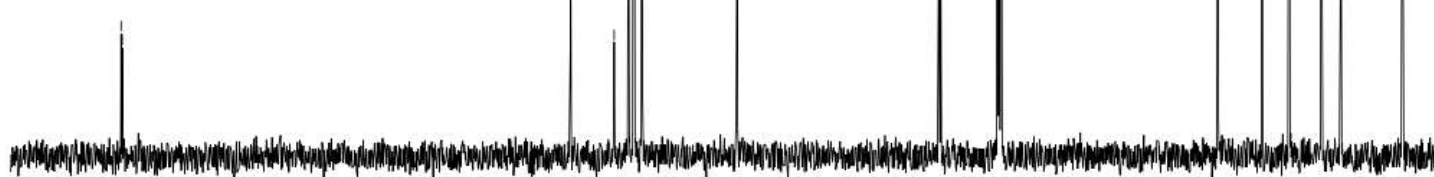

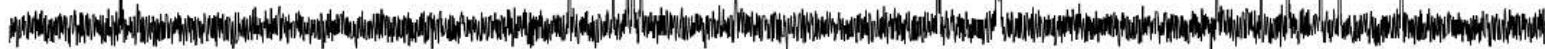

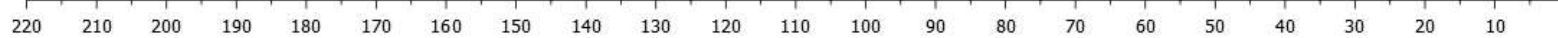



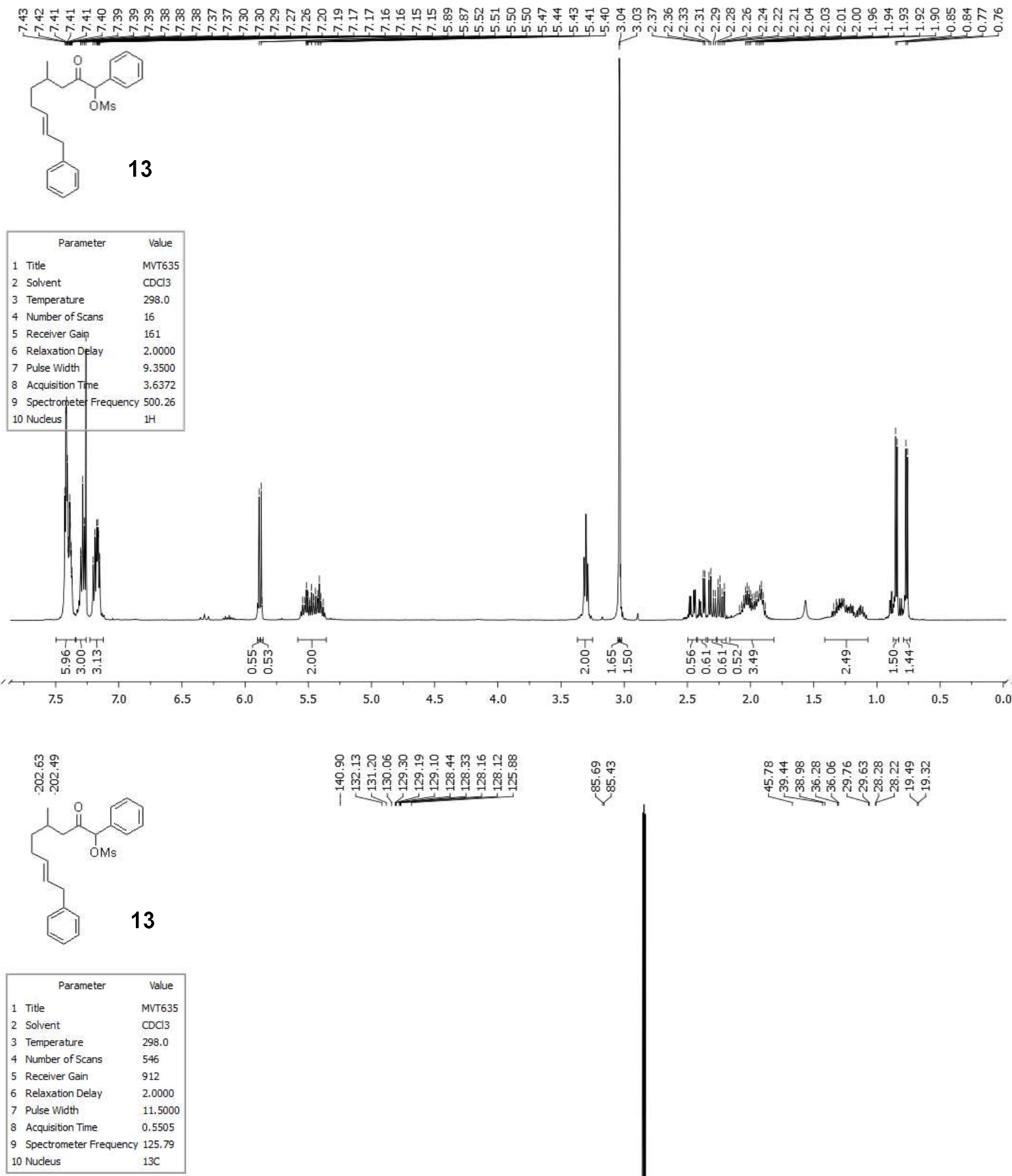

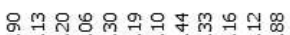

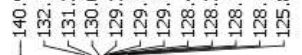

gำ

ஸ்

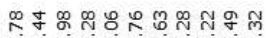

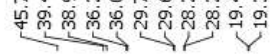

Value

MVT635

298.0

(1)

5000

25.79 $13 \mathrm{C}$
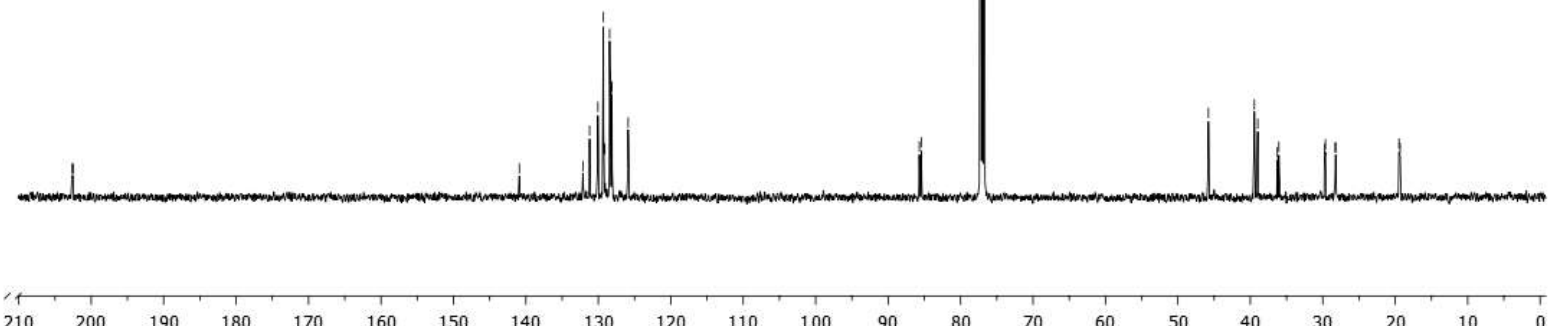

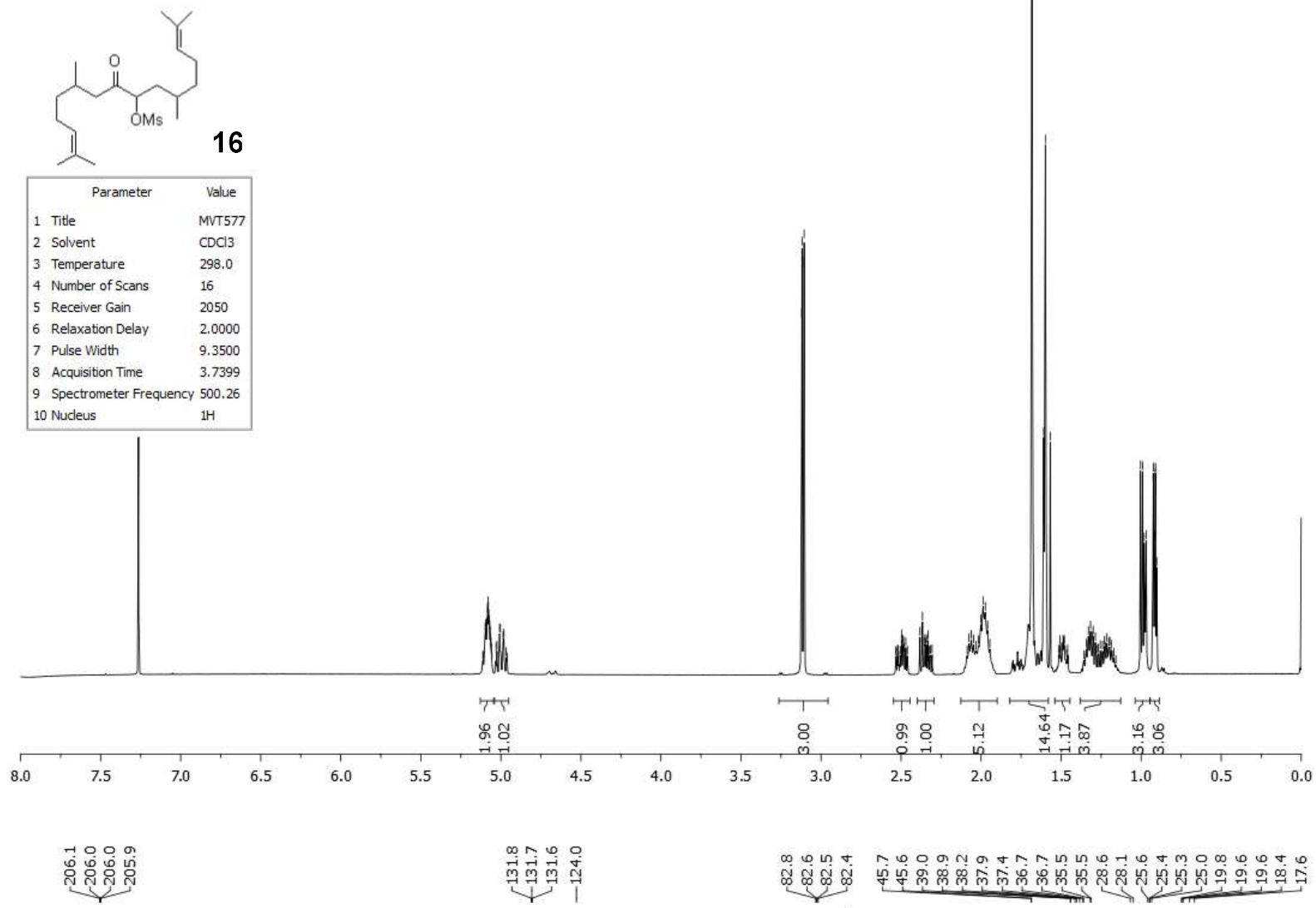

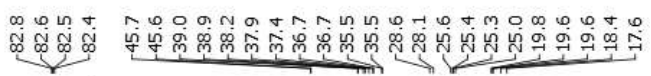

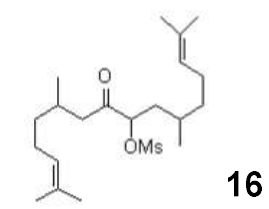

\begin{tabular}{|lll|}
\hline \multicolumn{2}{|c|}{ Parameter } & \multicolumn{1}{c|}{ Value } \\
1 & Title & MVT577 \\
2 & Solvent & $\mathrm{CDCl} 3$ \\
3 & Temperature & 298.0 \\
4 & Number of Scans & 521 \\
5 & Receiver Gain & 2050 \\
6 & Relaxation Delay & 2.0000 \\
7 & Pulse Width & 11.5000 \\
8 & Acquisition Time & 0.5505 \\
9 & Spectrometer Frequency & 125.79 \\
10 & Nucleus & $13 \mathrm{C}$ \\
\hline \multicolumn{2}{|c|}{}
\end{tabular}
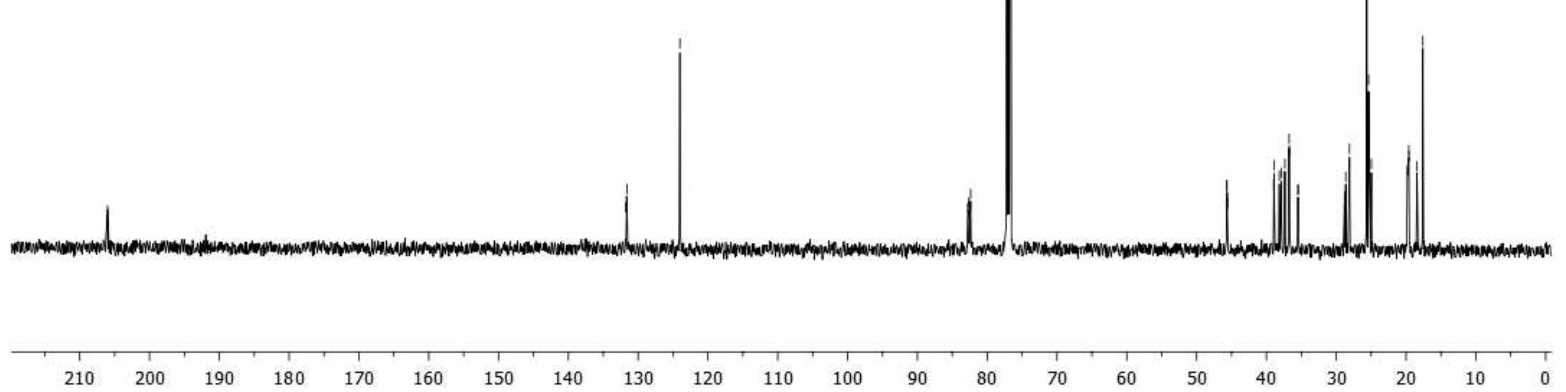

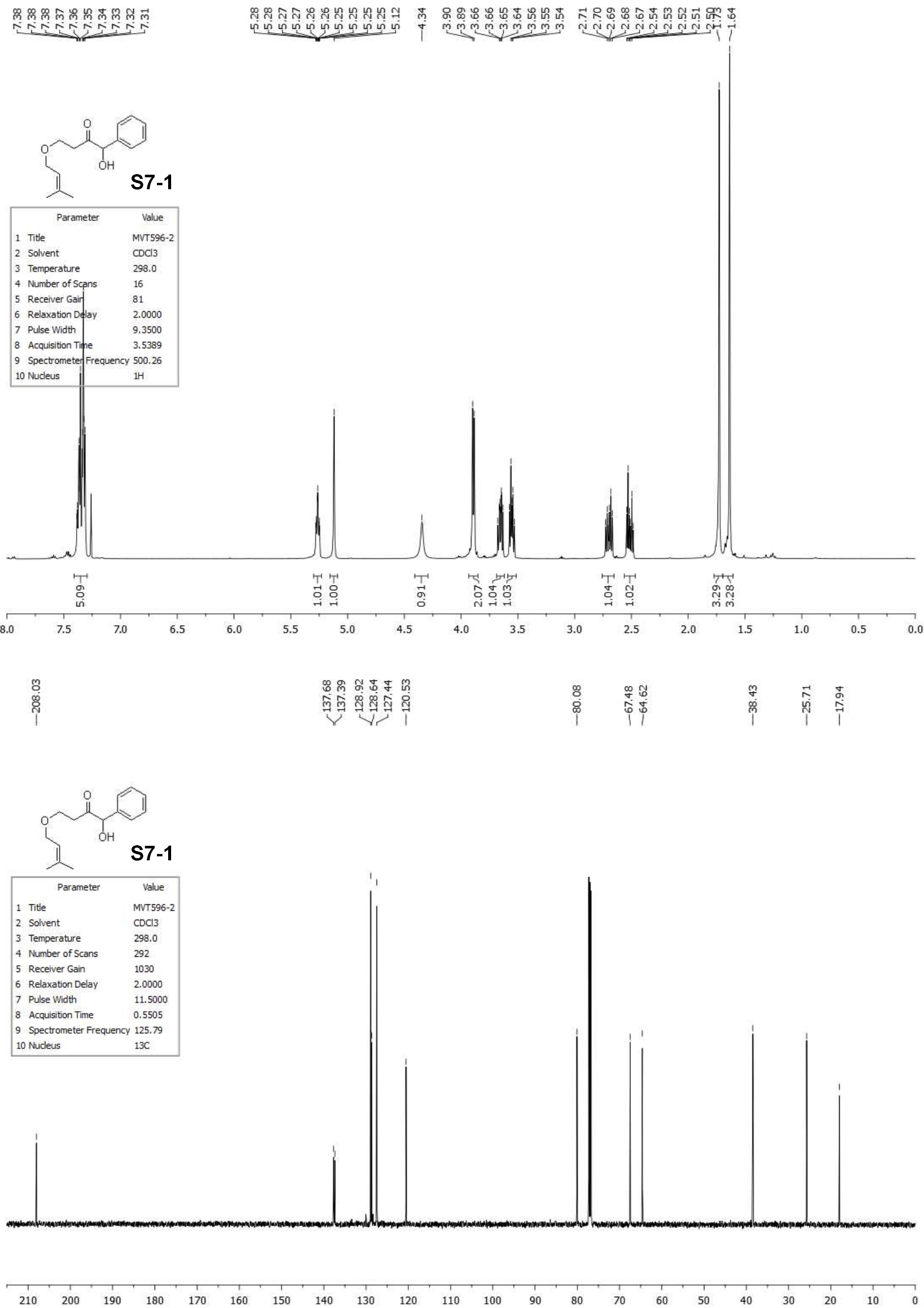

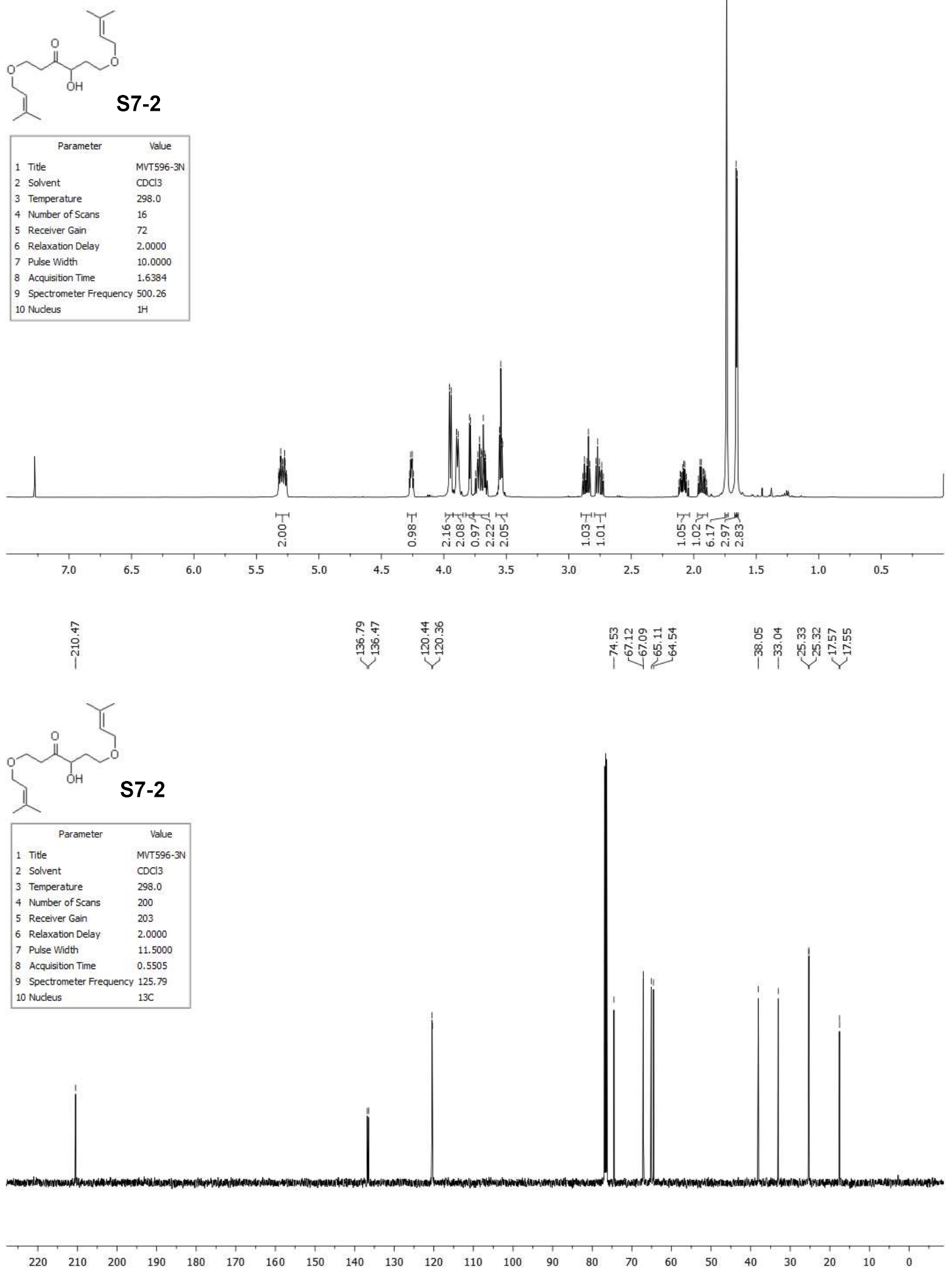

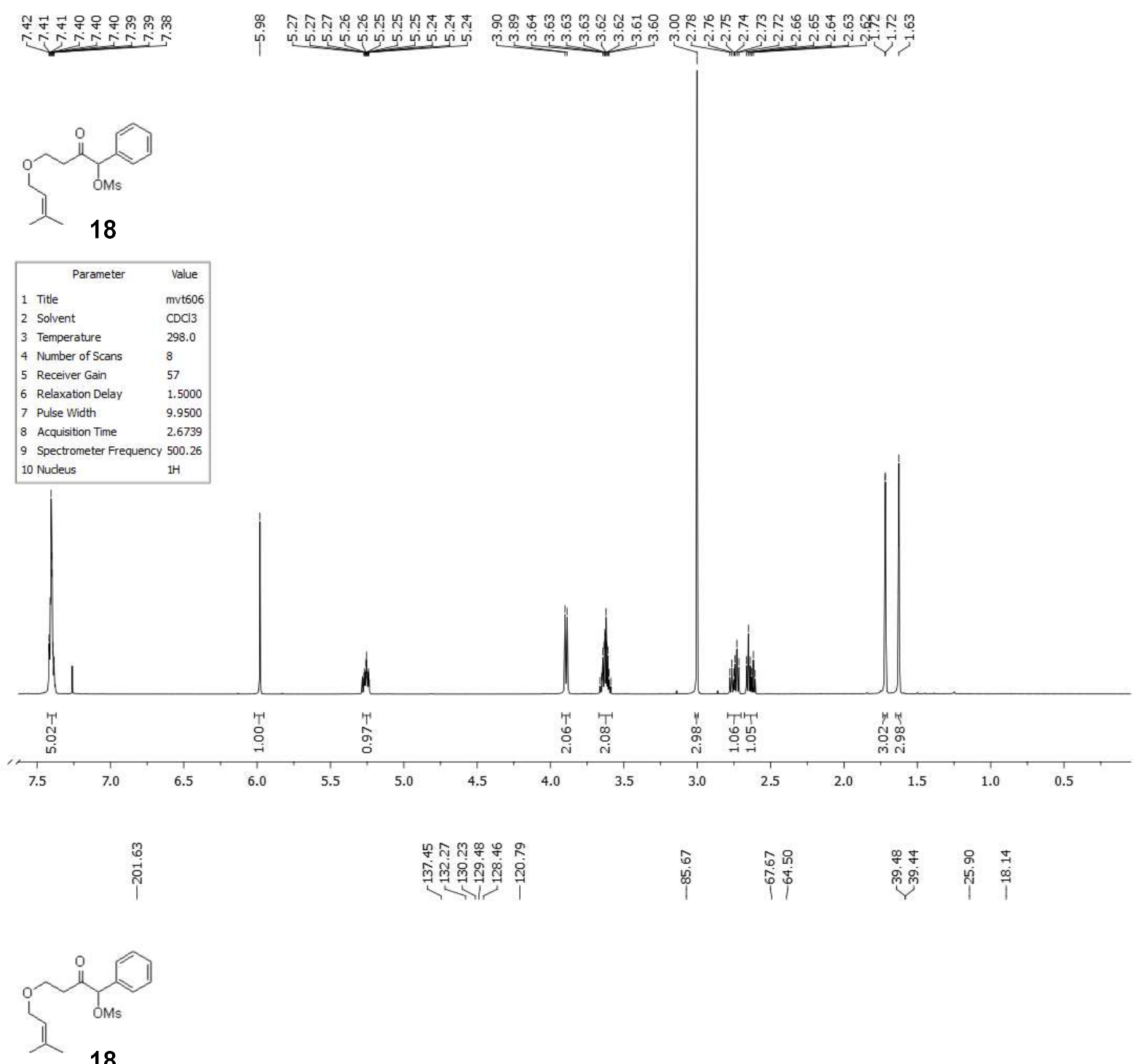

\section{8}

\begin{tabular}{|lll|}
\hline \multicolumn{1}{|c|}{ Parameter } & \multicolumn{1}{c|}{ Value } \\
1 & Title & mvt606 \\
2 & Solvent & $\mathrm{CDCl} 3$ \\
3 & Temperature & 298.0 \\
4 & Number of Scans & 66 \\
5 & Receiver Gain & 2050 \\
6 & Relaxation Delay & 2.0000 \\
7 & Pulse Width & 11.5000 \\
8 & Acquisition Time & 0.5505 \\
9 & Spectrometer Frequency & 125.79 \\
10 & Nudeus & $13 \mathrm{C}$ \\
\hline \multicolumn{2}{|l}{} \\
\hline
\end{tabular}

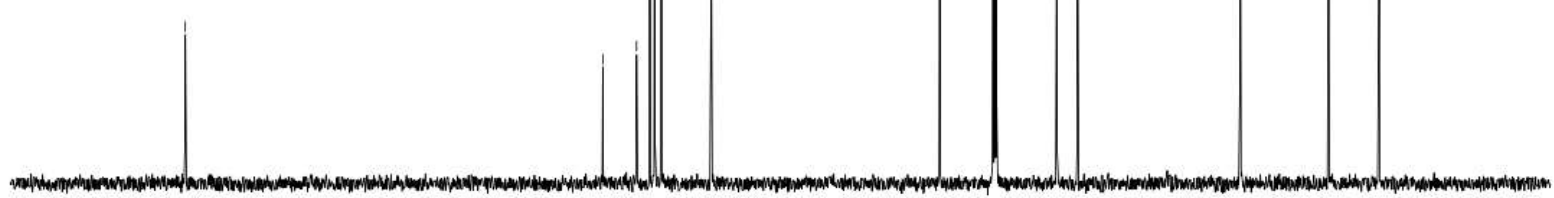

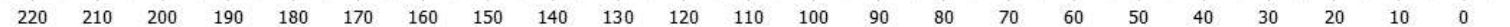



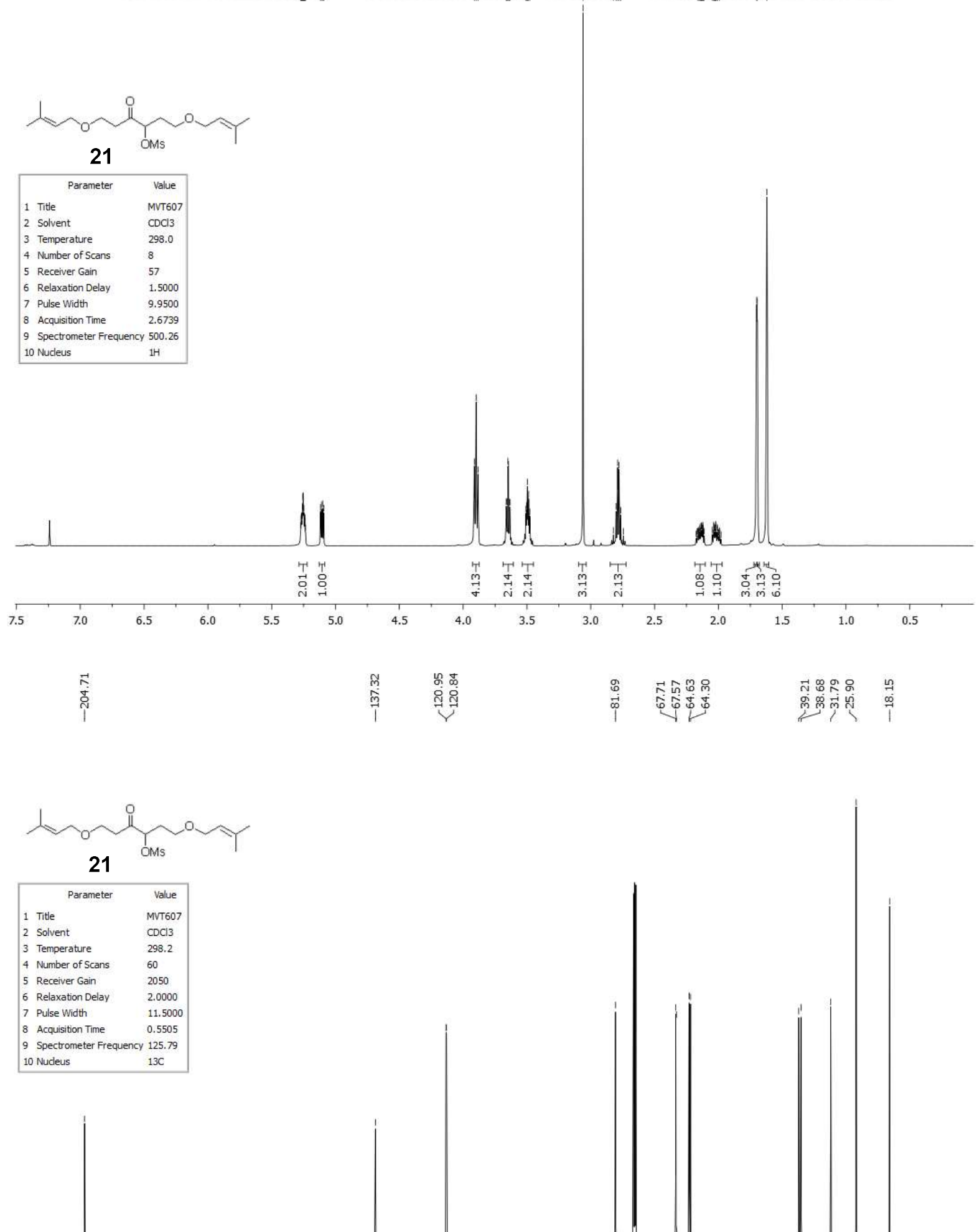

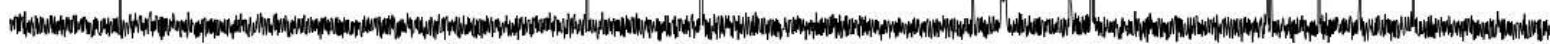

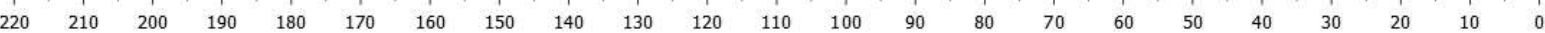



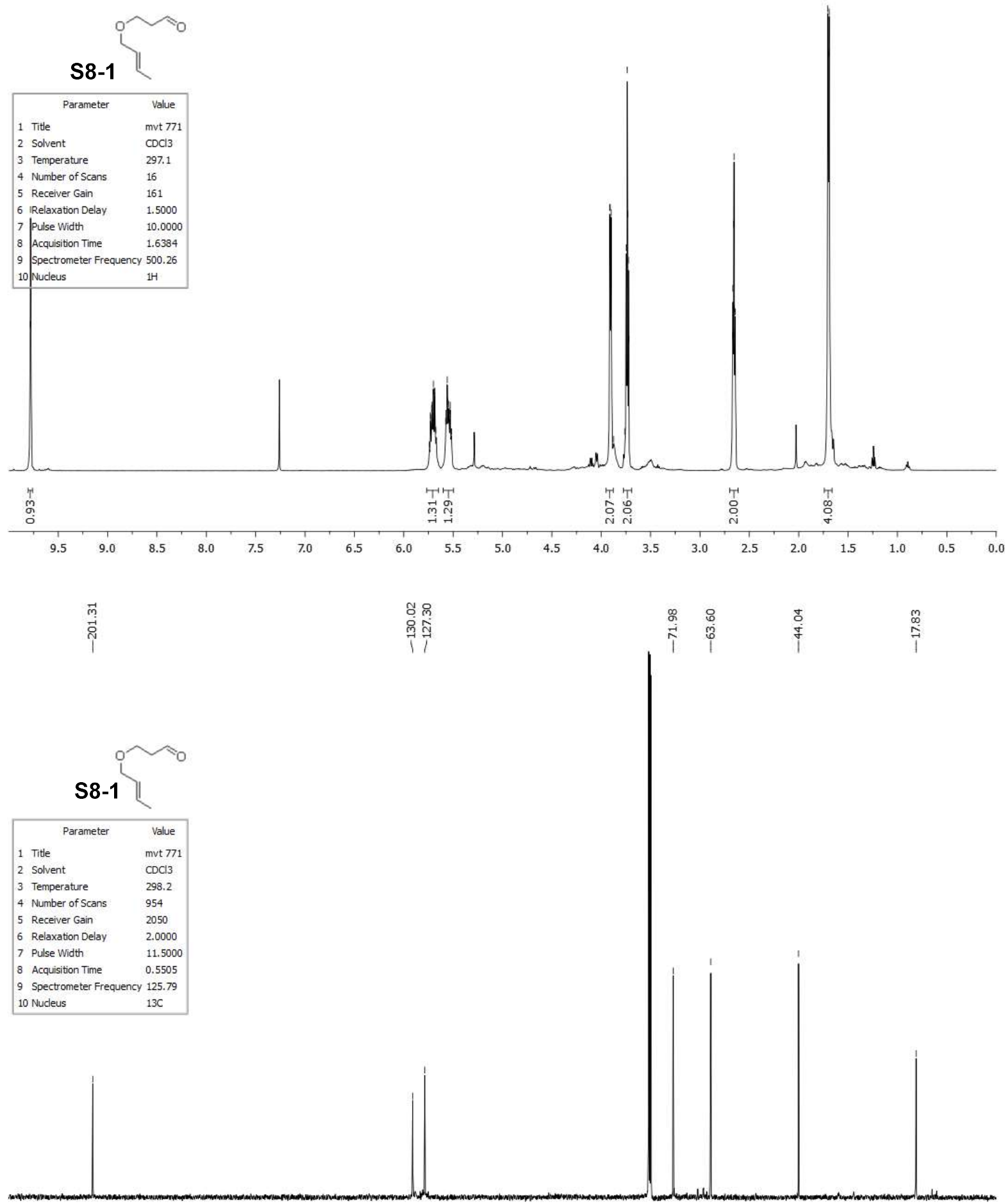

$\begin{array}{lllllllllllllllllll}220 & 210 & 200 & 190 & 180 & 170 & 160 & 150 & 140 & 130 & 120 & 110 & 100 & 90 & 80 & 70 & 60 & 50 & 40\end{array}$ 


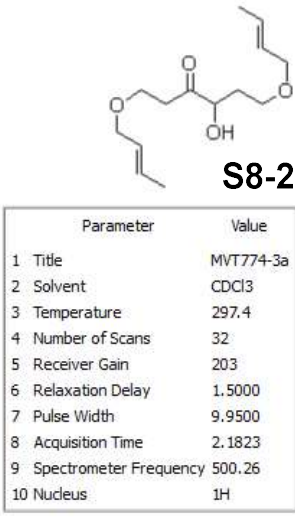

S8-2

\begin{tabular}{|lll|}
\hline & \multicolumn{1}{|c|}{ Parameter } & \multicolumn{1}{c|}{ Value } \\
1 & Title & MVT774-3a \\
2 & Solvent & $\mathrm{CDCl} 3$ \\
3 & Temperature & 297.4 \\
4 & Number of Scans & 32 \\
5 & Receiver Gain & 203 \\
6 & Relaxation Delay & 1.5000 \\
7 & Pulse Width & 9.9500 \\
8 & Acquisition Time & 2.1823 \\
9 & Spectrometer Frequency 500.26 \\
10 & Nucleus & $1 \mathrm{H}$ \\
\hline
\end{tabular}
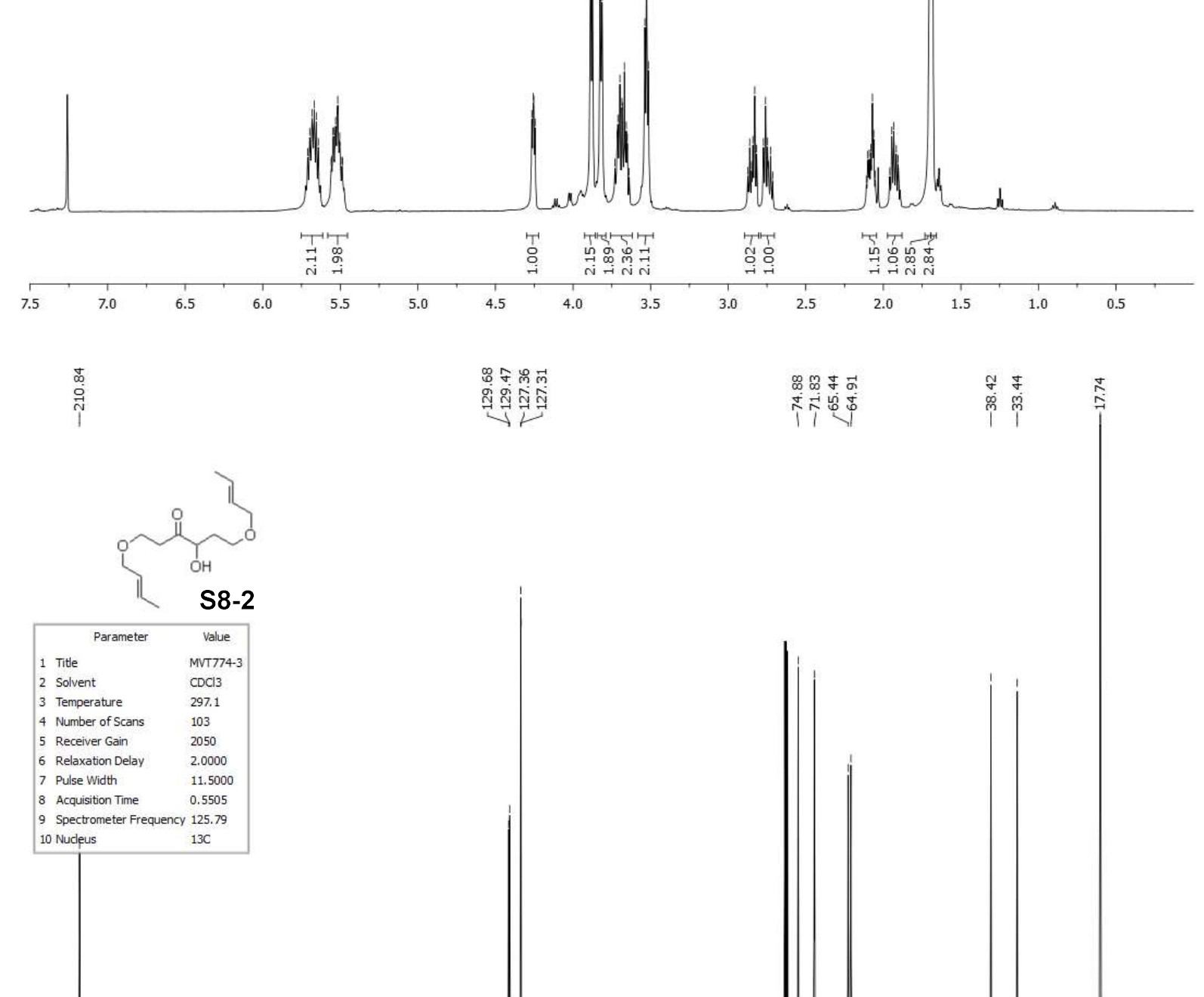

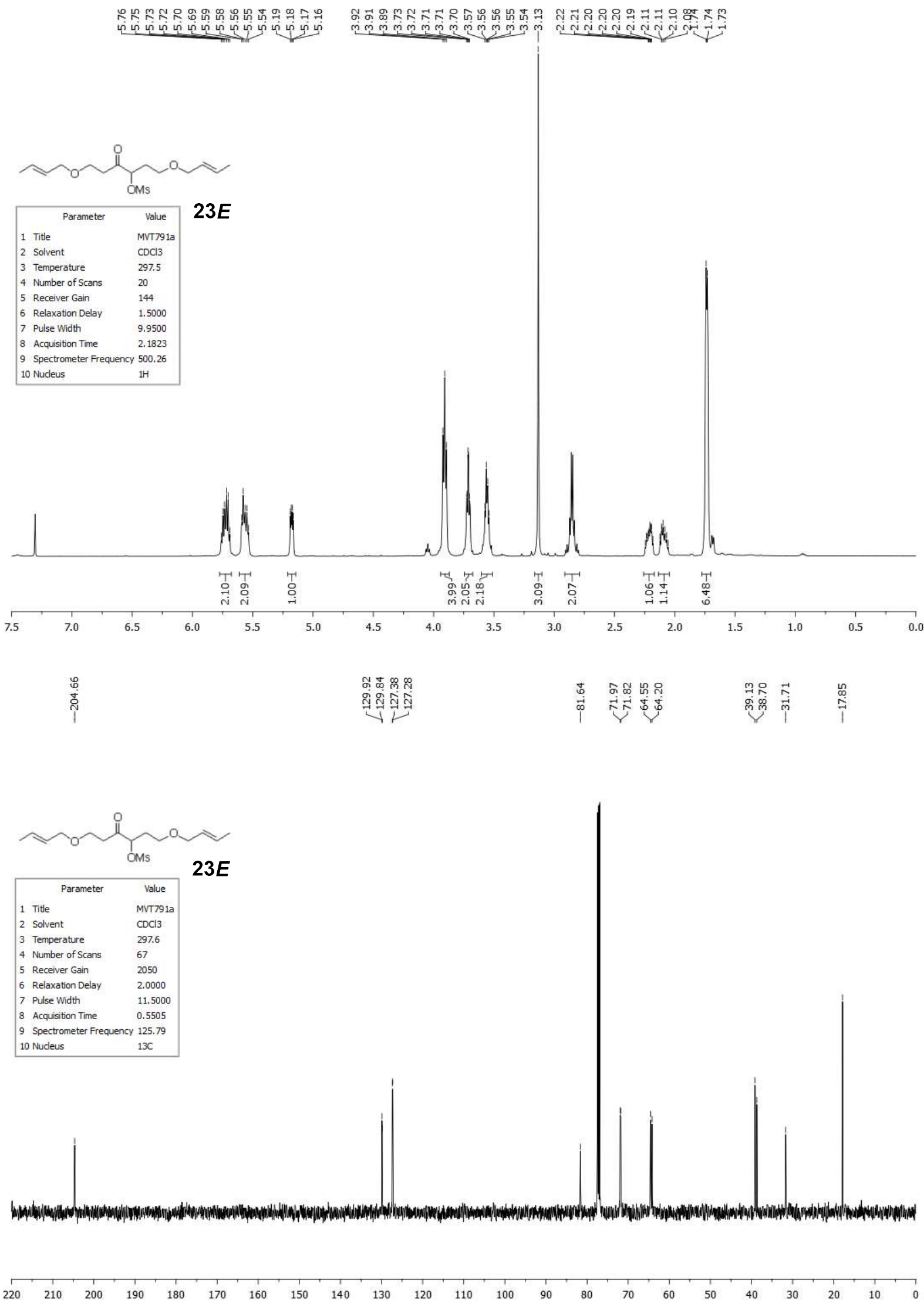


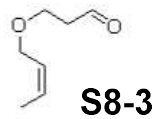

S8-3
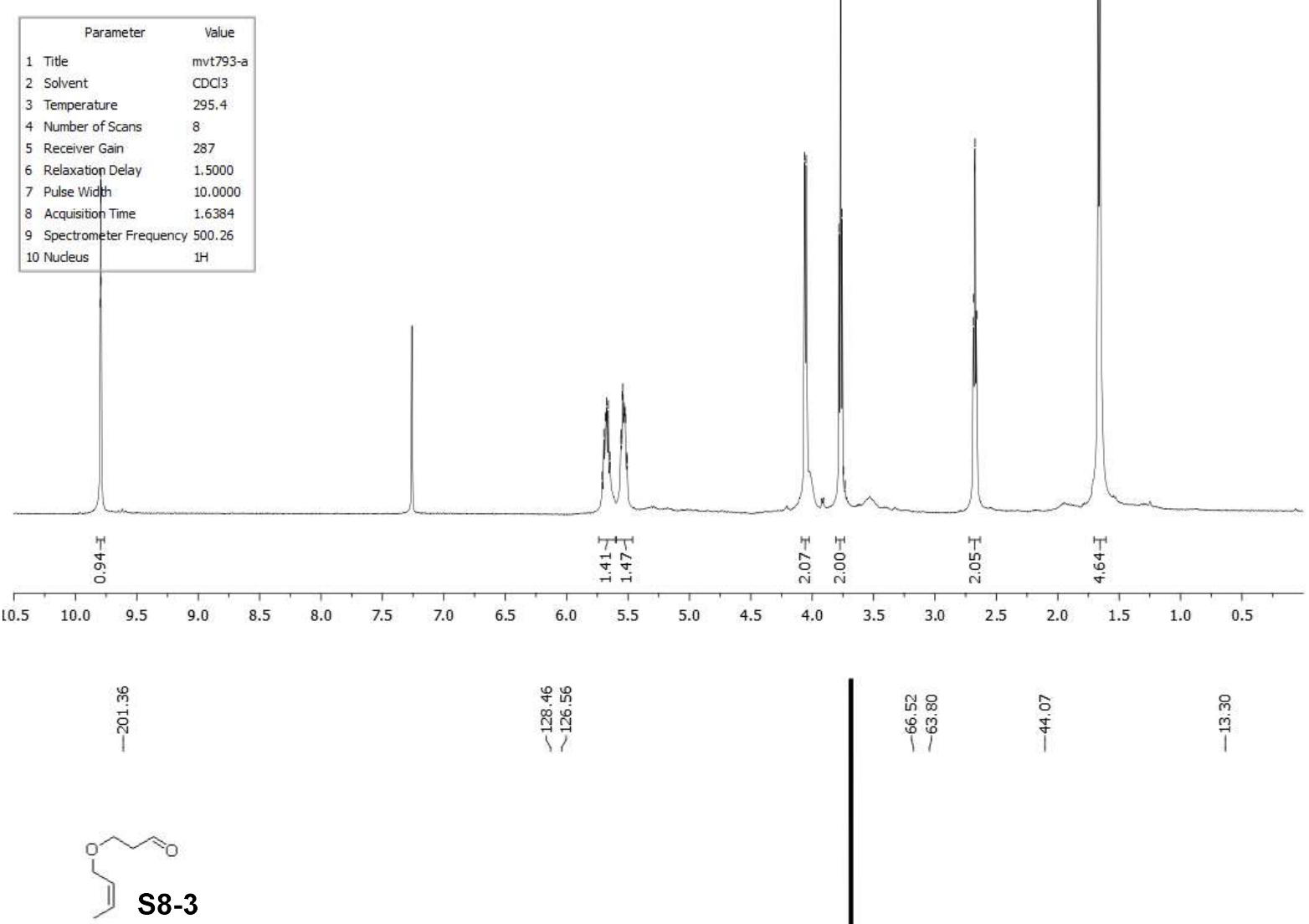

\begin{tabular}{|lll|}
\hline & \multicolumn{1}{|c|}{ Parameter } & \multicolumn{1}{c|}{ Value } \\
1 & Title & mvt793-a \\
2 & Solvent & $\mathrm{CDCl} 3$ \\
3 & Temperature & 296.3 \\
4 & Number of Scans & 147 \\
5 & Receiver Gain & 2050 \\
6 & Relaxation Delay & 2.0000 \\
7 & Pulse Width & 11.5000 \\
8 & Acquisition Time & 0.5505 \\
9 & Spectrometer Frequency & 125.79 \\
10 & Nucleus & $13 \mathrm{C}$ \\
\hline \multicolumn{2}{c}{} \\
\end{tabular}

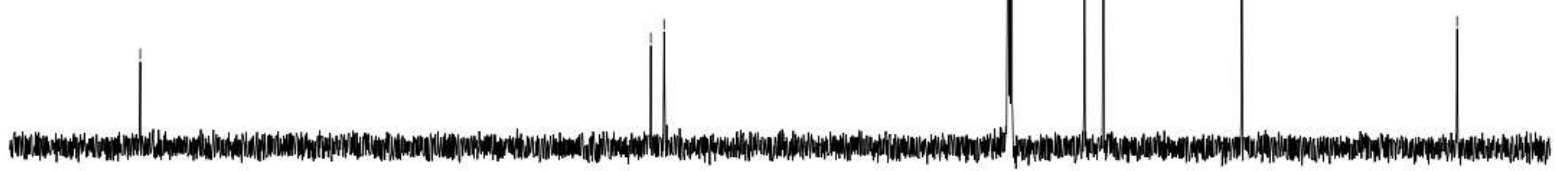

$\begin{array}{lllllllllllllllllllllll}220 & 210 & 200 & 190 & 180 & 170 & 160 & 150 & 140 & 130 & 120 & 110 & 100 & 90 & 80 & 70 & 60 & 50 & 40 & 30 & 20 & 10 & 0\end{array}$ 

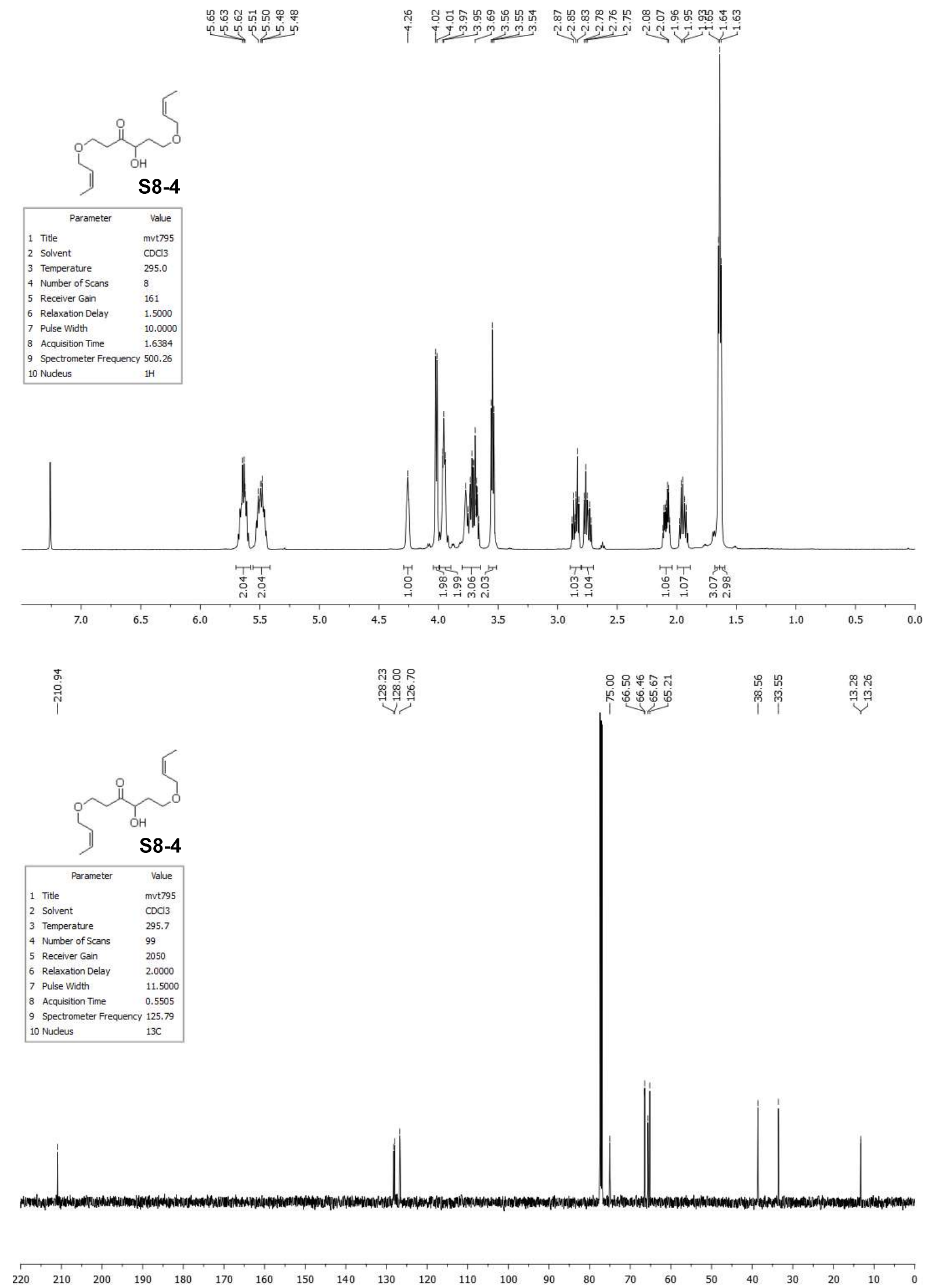


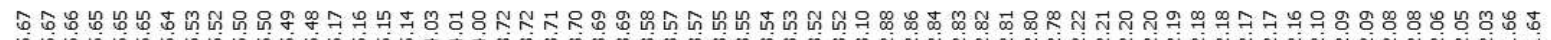

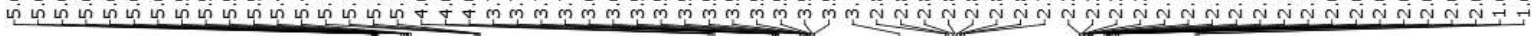
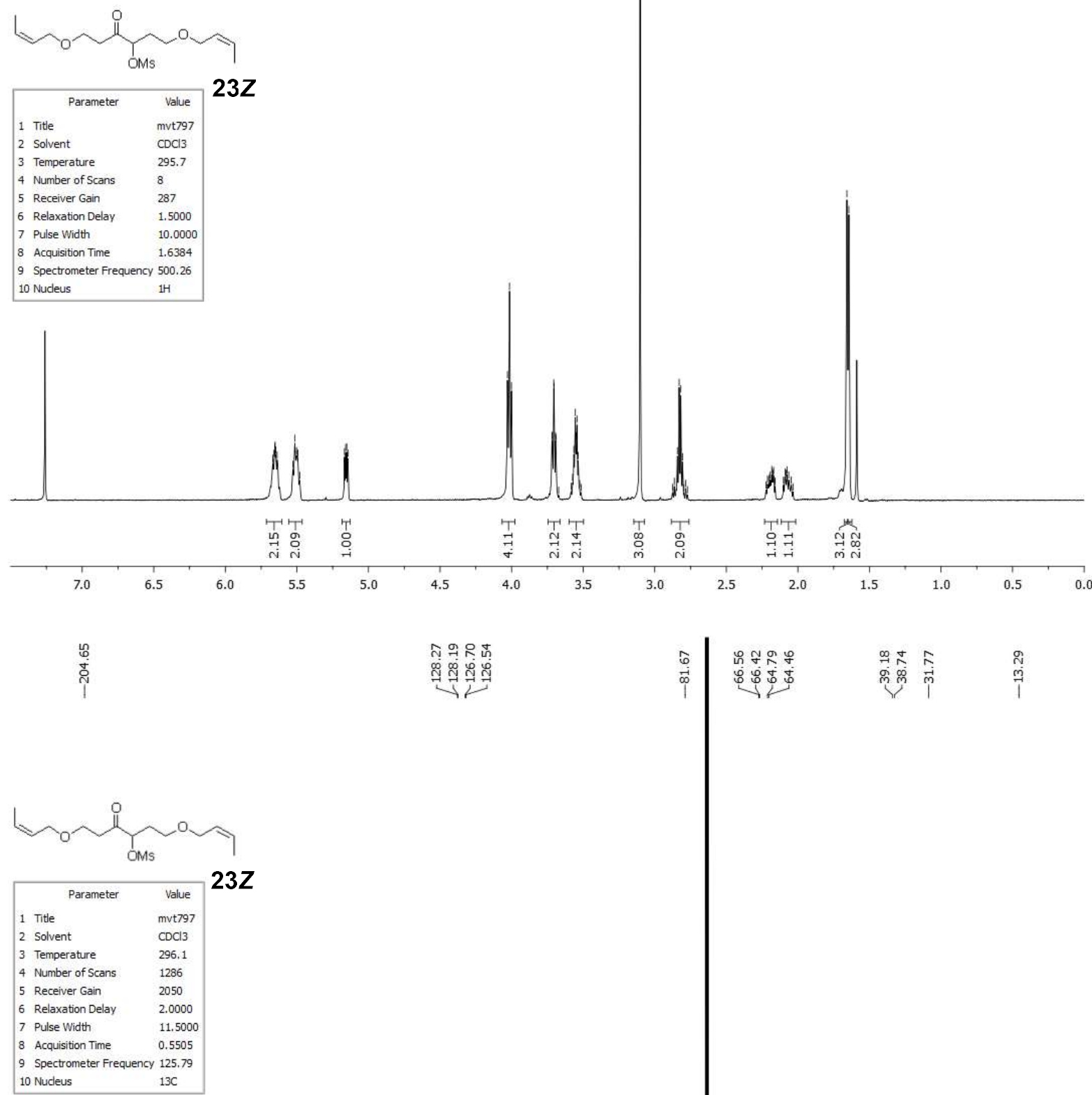

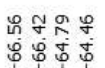

ํㅣ용

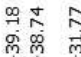

$\prod^{\infty}$

$3 Z$
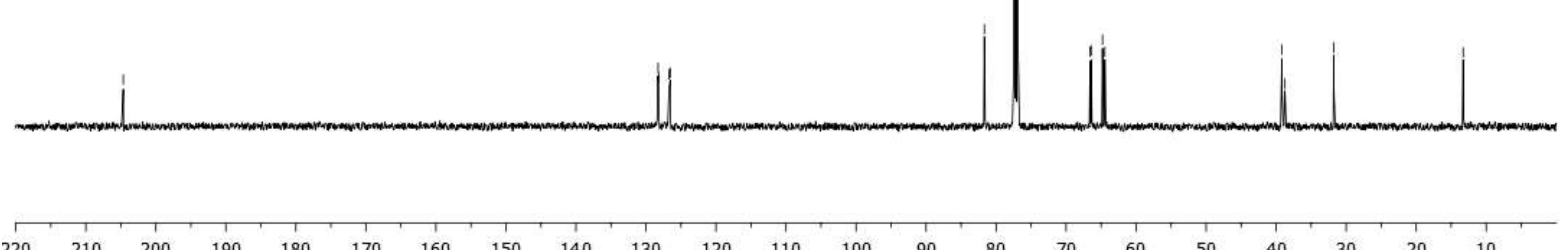


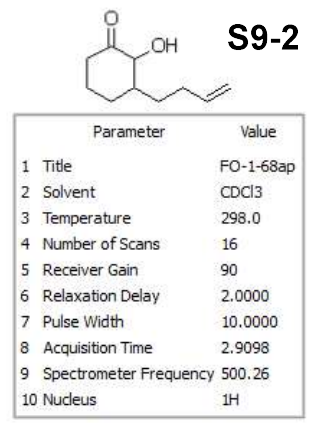

\section{9-2}

Value
$-1-68$ ap
Cl3
.0
000
.0000
098
.26
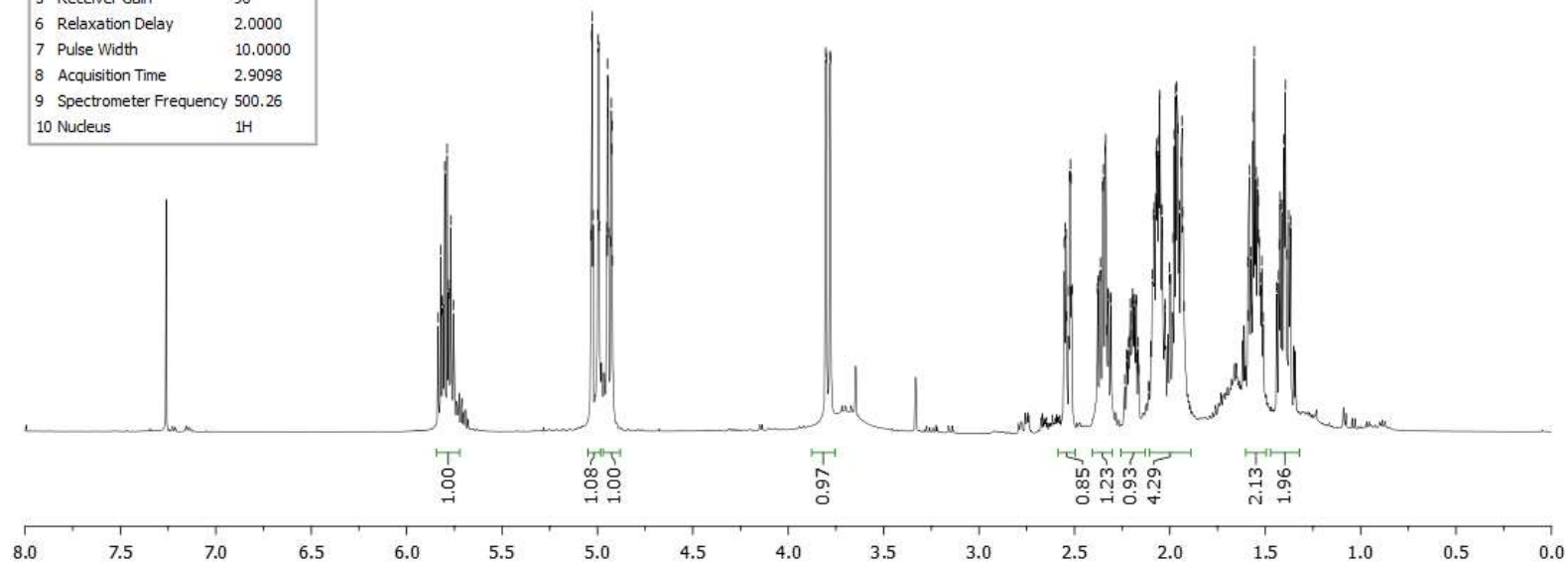

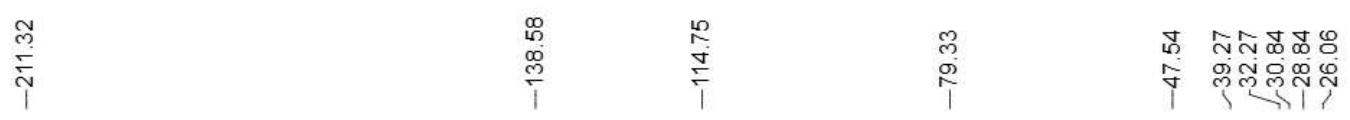

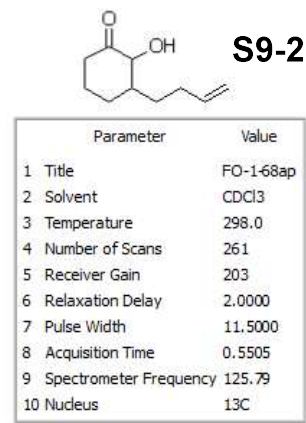

S9-2

Value
0 - 1.68 ap
DCl3
98.0
61
103
0000
1.5000
.5505
125.79
$3 C$
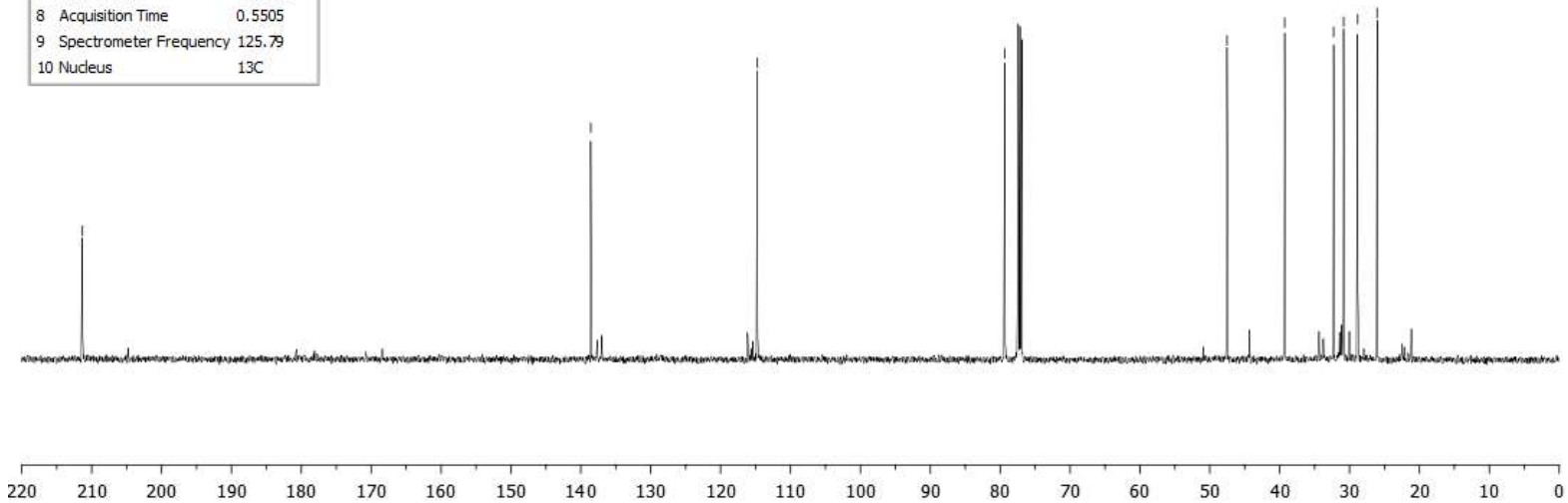


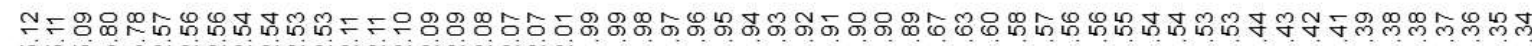

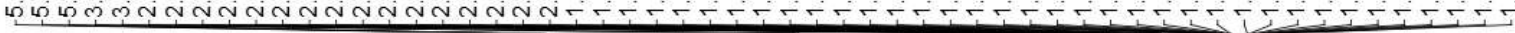

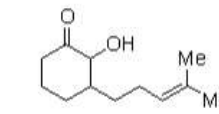

S9-3

\begin{tabular}{|lll|}
\hline \multicolumn{1}{|c|}{ Parameter } & \multicolumn{1}{c|}{ Value } \\
1 & Title & FO- $01-075$ \\
2 & Solvent & $\mathrm{CDCl} 3$ \\
3 & Temperature & 298.0 \\
4 & Number of Scans & 13 \\
5 & Receiver Gain & 101 \\
6 & Relaxation Delay & 2.0000 \\
7 & Pulse Width & 10.0000 \\
8 & Acquisition Time & 1.6384 \\
9 & Spectrometer Frequency & 500.26 \\
10 & Nudeus & $1 \mathrm{H}$ \\
\hline
\end{tabular}

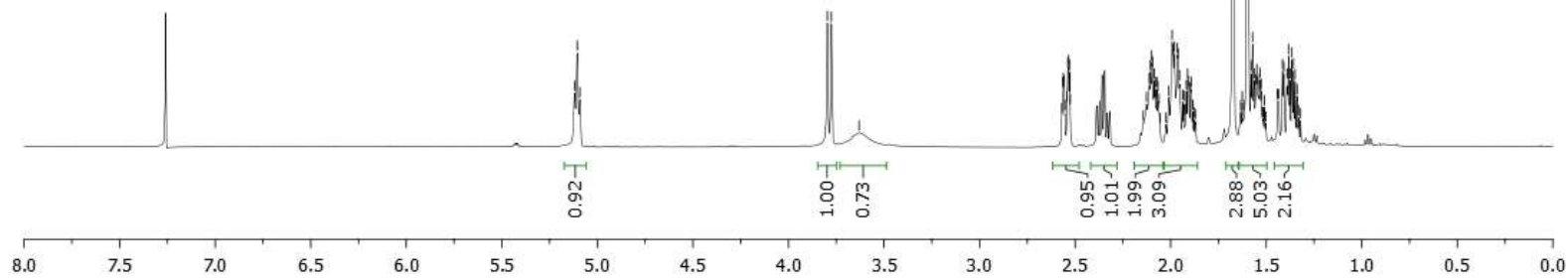

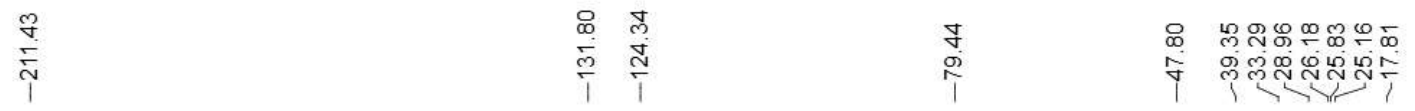
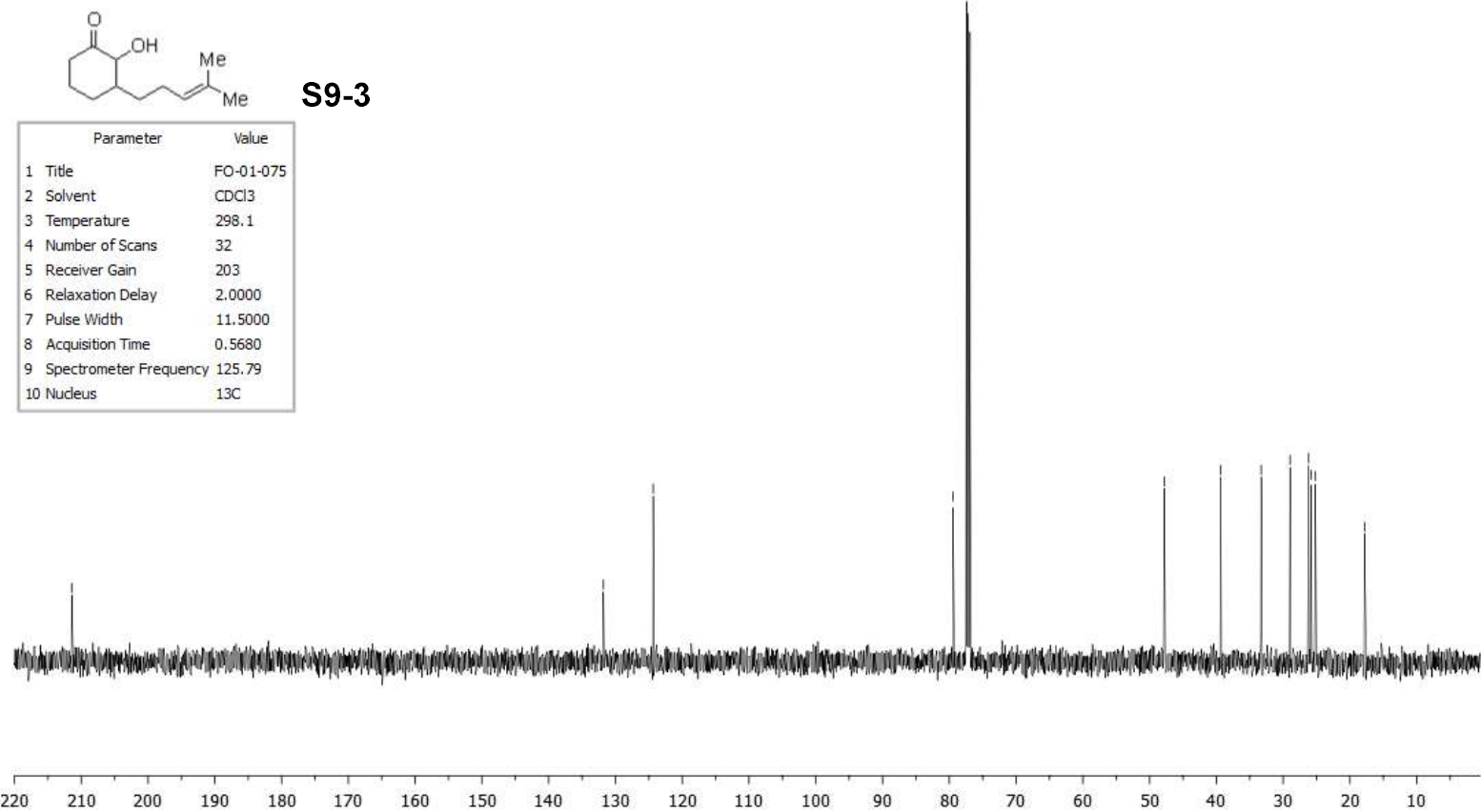


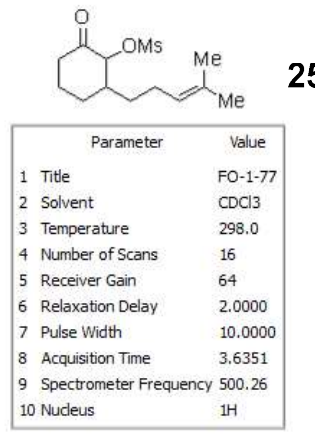

25

$1 \mathrm{H}$

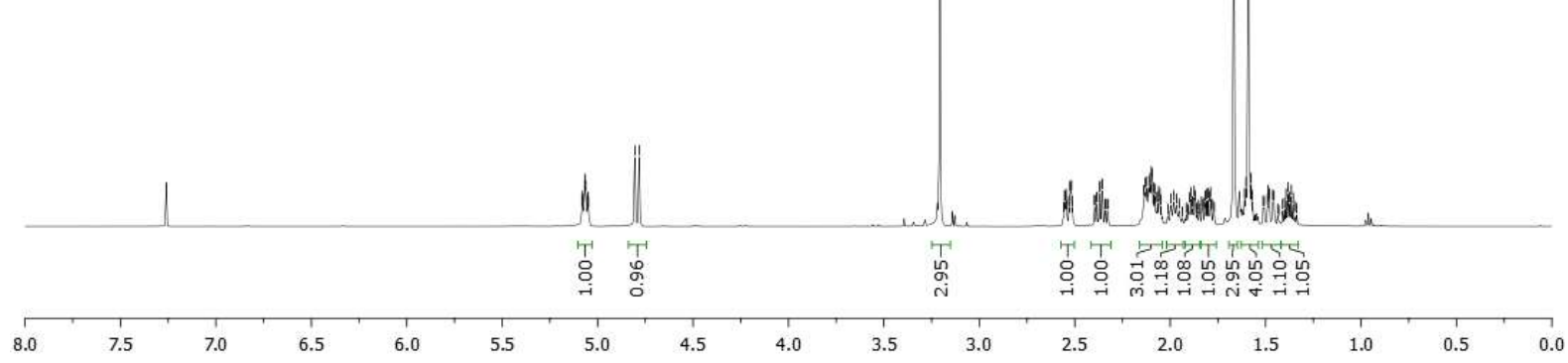

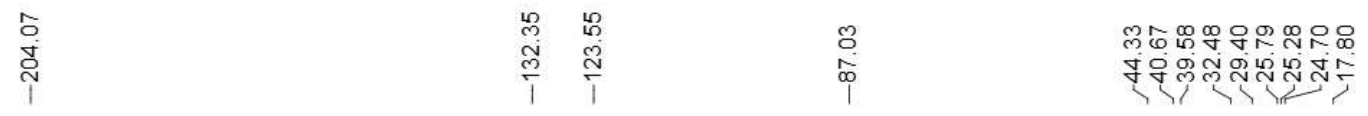

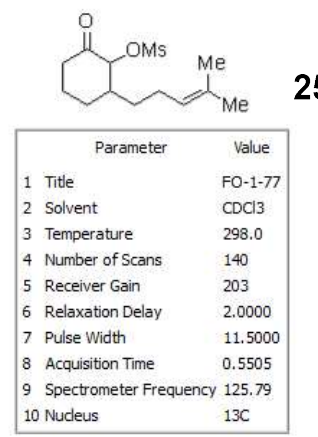

25

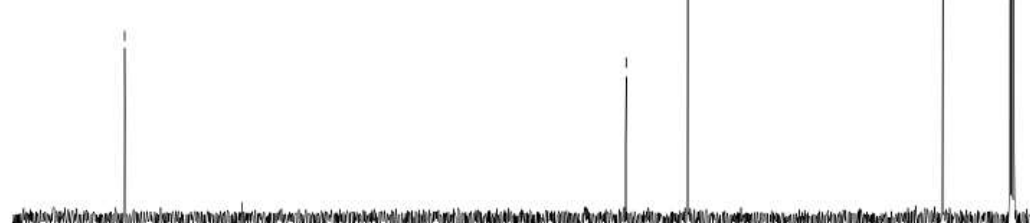

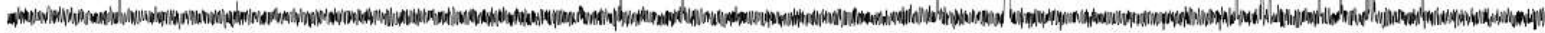

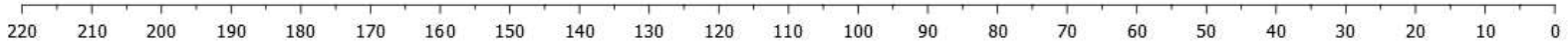




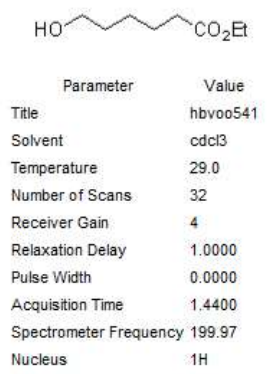

S10-1

Nucleus

$1 \mathrm{H}$

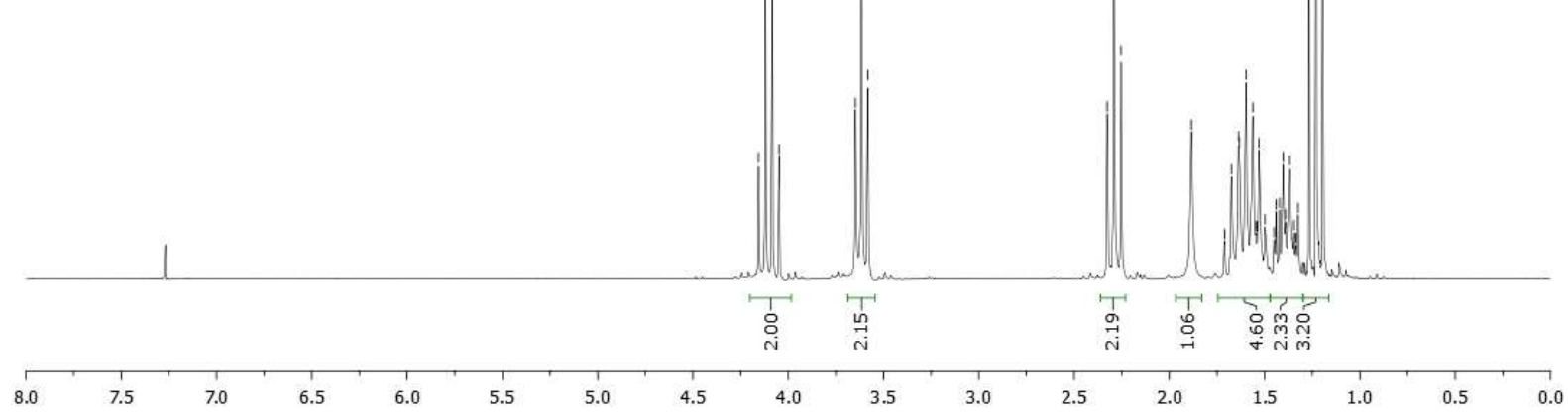

\begin{tabular}{|c|c|}
\hline $\begin{array}{l}\mathscr{\%} \\
\text { ल } \\
\end{array}$ & 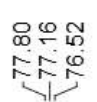 \\
\hline
\end{tabular}
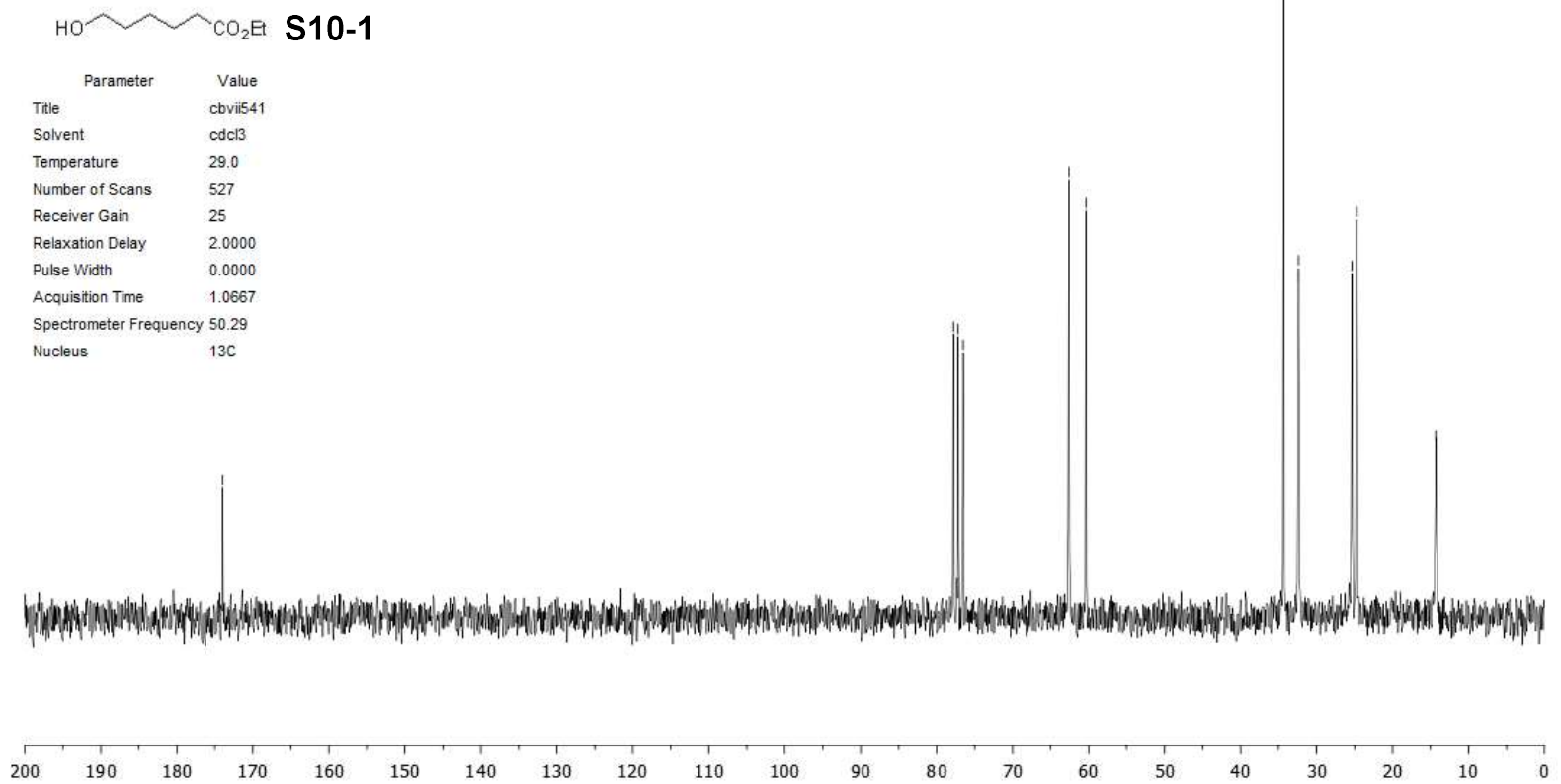

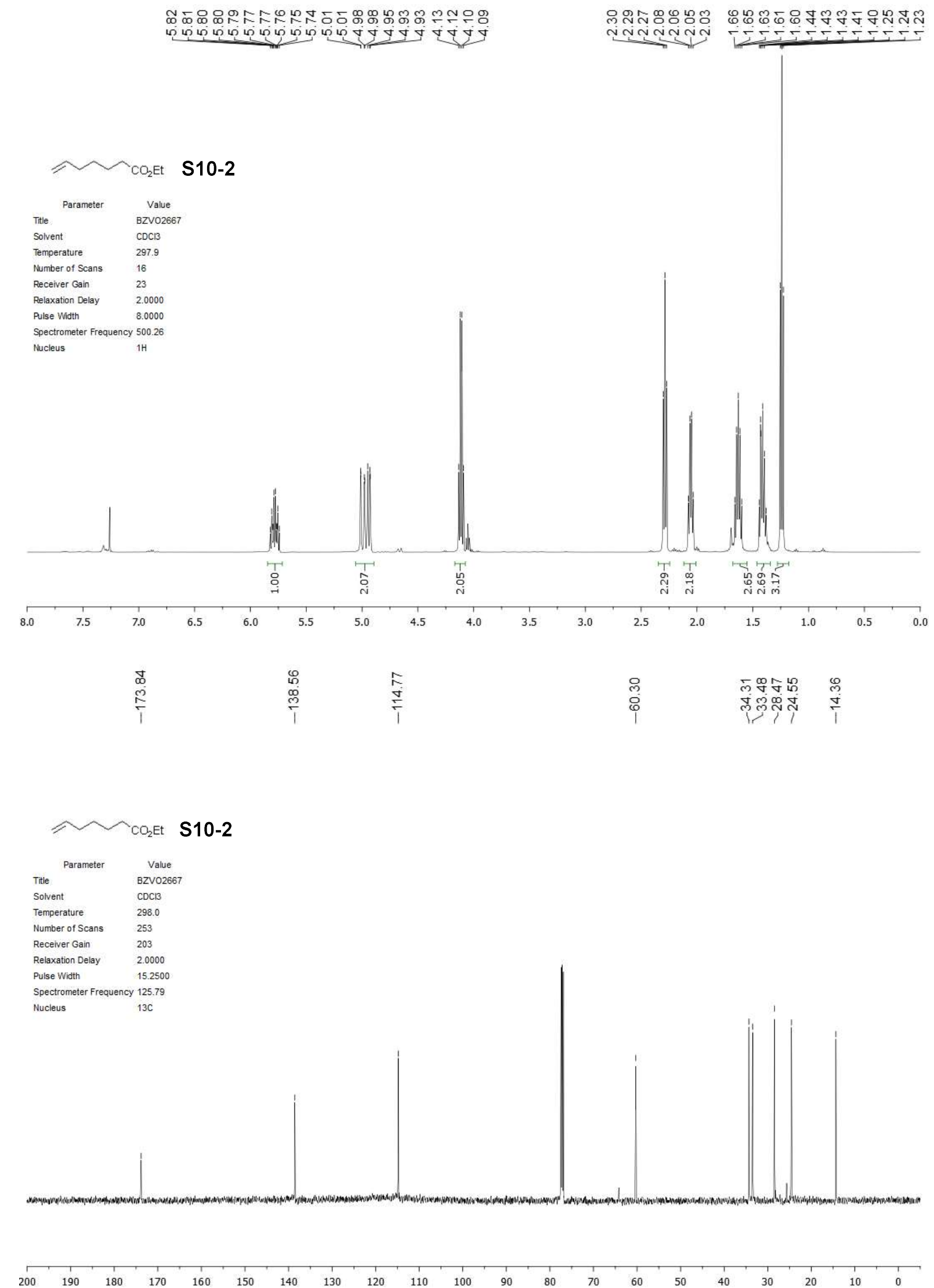
S10-3

$\mathrm{EtO}_{2} \mathrm{C} \sim \mathrm{CO}_{2} \mathrm{Et}$

\begin{tabular}{ll} 
Title & \multicolumn{1}{c}{ Parameter } \\
BZV02668 \\
Solvent & CDC13 \\
Temperature & 298.0 \\
Number of Scans & 8 \\
Receiver Gain & 64 \\
Relaxation Delay & 2.0000 \\
Pulse Width & 8.0000 \\
Spectrometer Frequency & 500.26 \\
Nucleus & $1 \mathrm{H}$
\end{tabular}

Nucleus $1 \mathrm{H}$

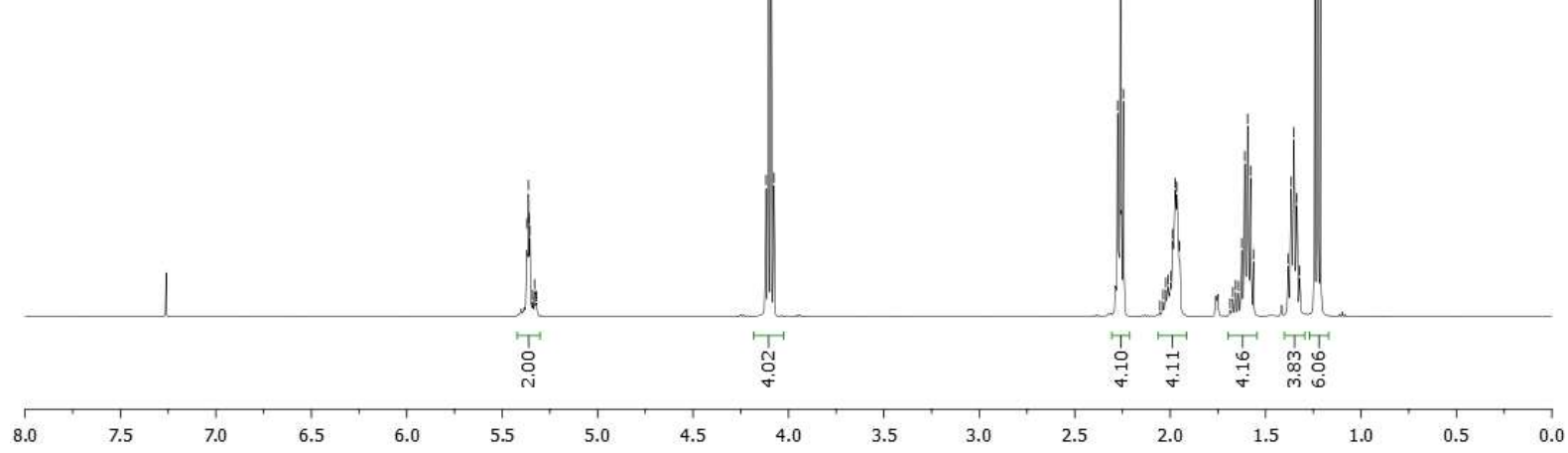

\begin{tabular}{|c|c|c|c|}
\hline 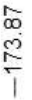 & $\begin{array}{l}\stackrel{6}{N} \\
\stackrel{0}{m} \\
\stackrel{i}{i}\end{array}$ & $\begin{array}{c}\text { n } \\
0 \\
0 \\
1\end{array}$ & 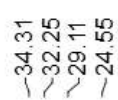 \\
\hline
\end{tabular}

S10-3

$\mathrm{EtO}_{2} \mathrm{C} \leadsto \mathrm{CO}_{2} \mathrm{Et}$

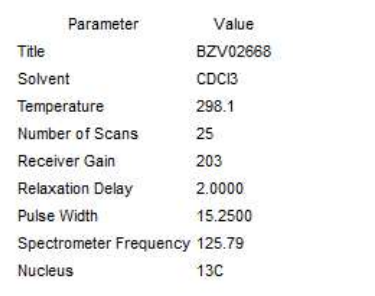

Nucleus $\quad 13 \mathrm{C}$

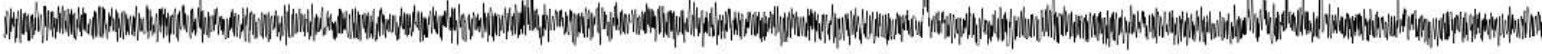

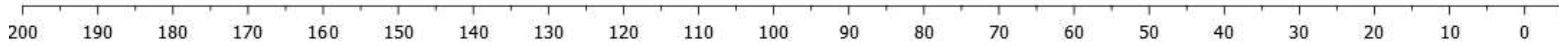




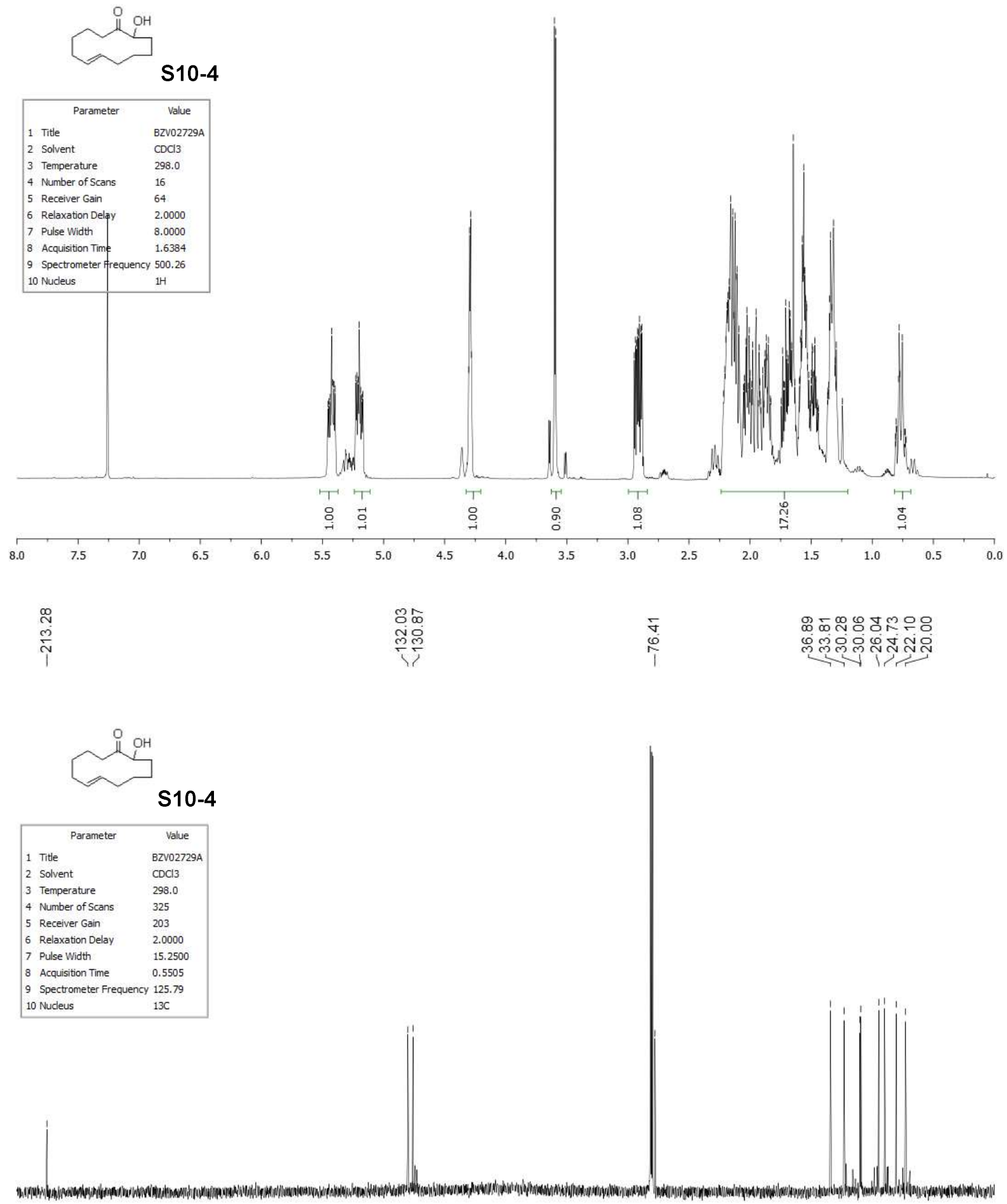

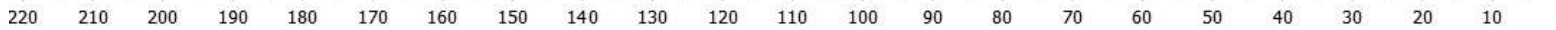


๑

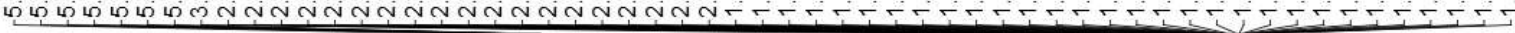

\begin{tabular}{|c|c|}
\hline \multirow[t]{2}{*}{ Parameter } & Value \\
\hline & BZV027398 \\
\hline 2 Solvent & $\mathrm{CDCl} 3$ \\
\hline \multirow{2}{*}{$\begin{array}{l}3 \text { Temperature } \\
4 \text { Number of Scans }\end{array}$} & 298.0 \\
\hline & 32 \\
\hline 5 Receiver Gain & 64 \\
\hline \multirow{2}{*}{$\begin{array}{l}6 \text { Relaxation Delay } \\
7 \text { Pulse Width }\end{array}$} & 2.0000 \\
\hline \multirow{2}{*}{\multicolumn{2}{|c|}{$\begin{array}{l}7 \text { Pulse Width } \\
8 \text { Spectrometer Frequency } 5000.26\end{array}$}} \\
\hline & \\
\hline \multicolumn{2}{|l|}{9 Nudeus } \\
\hline
\end{tabular}

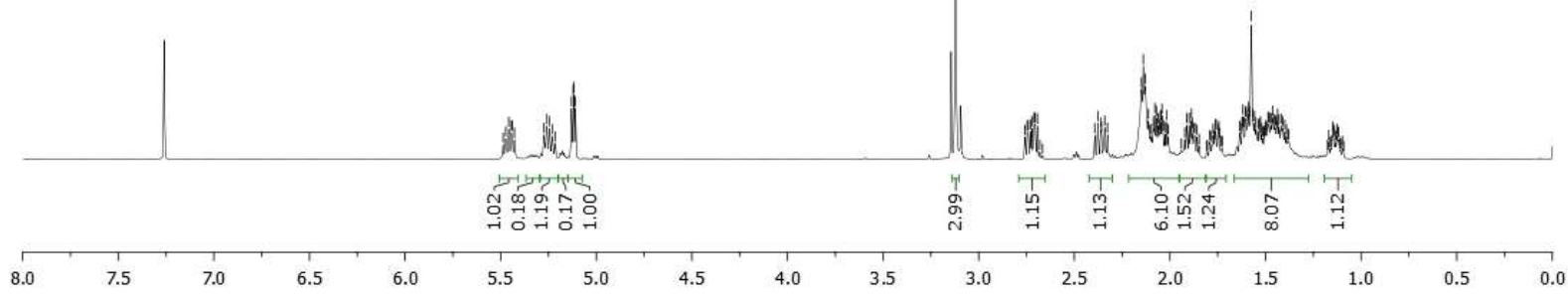

\begin{tabular}{|c|c|c|c|}
\hline $\begin{array}{l}\infty \\
\infty \\
\infty \\
\sim \\
1\end{array}$ & 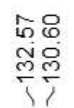 & $\underset{\substack{\infty \\
\infty \\
1}}{\infty}$ & 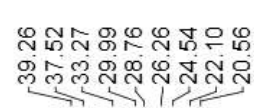 \\
\hline
\end{tabular}
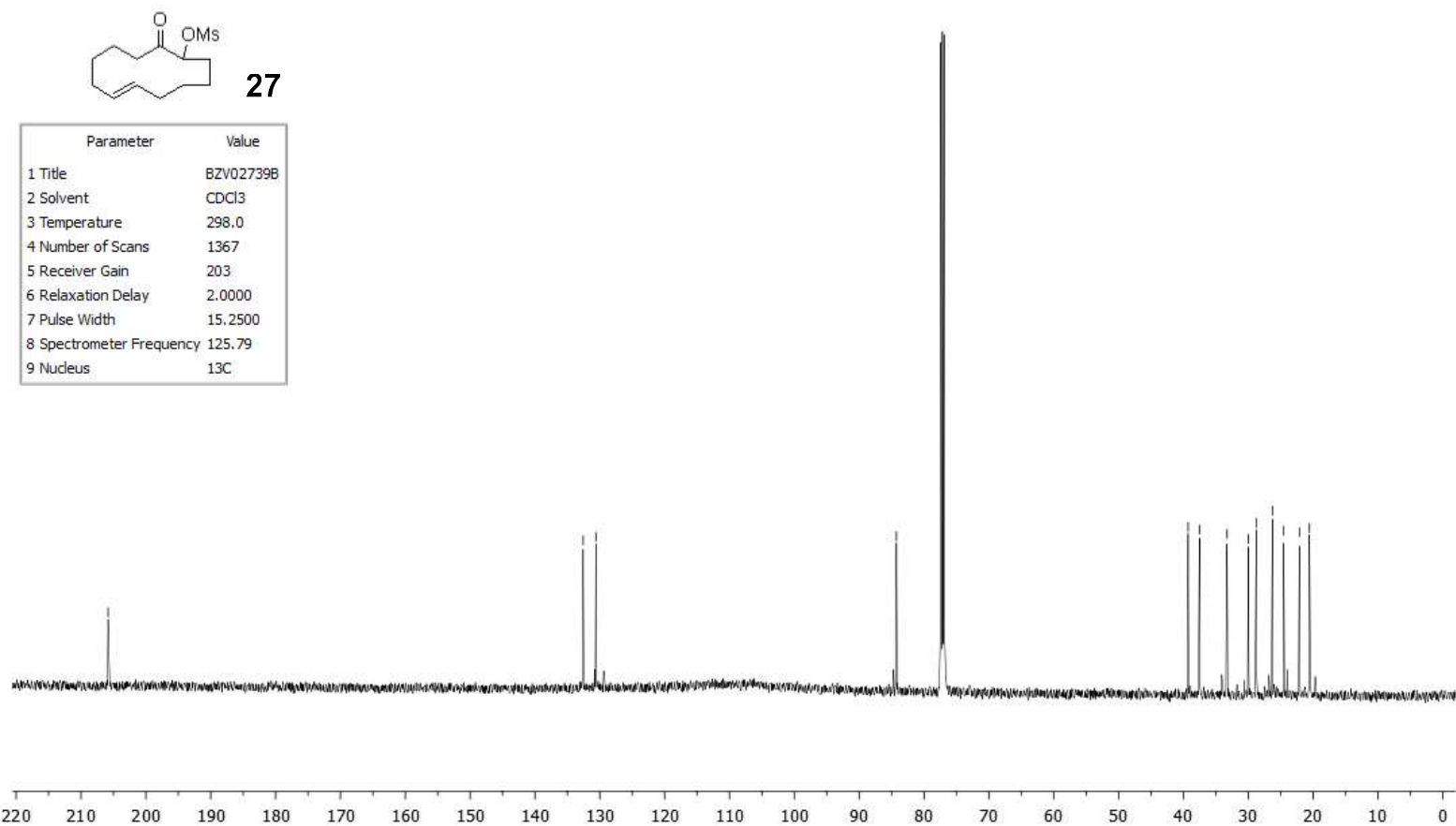

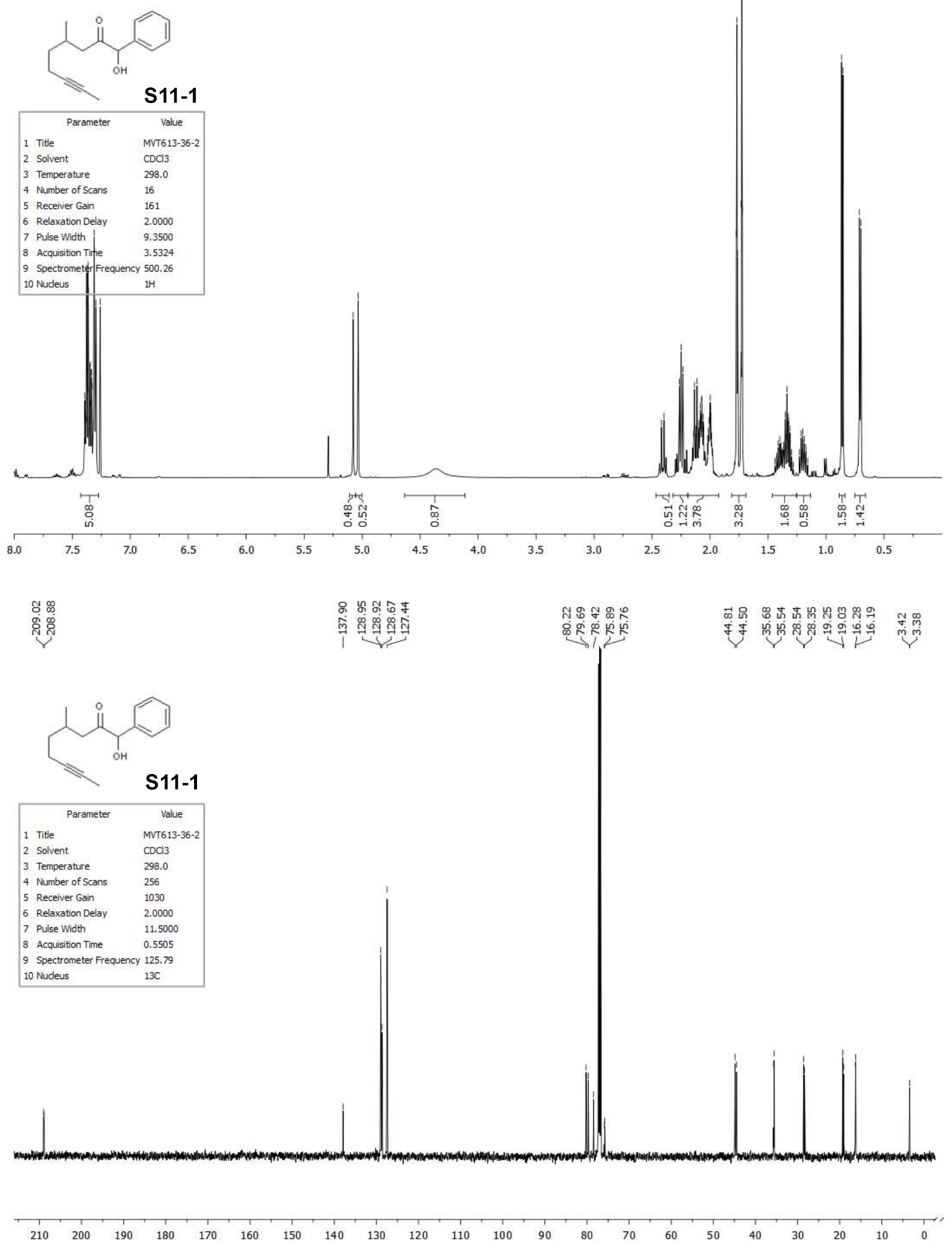


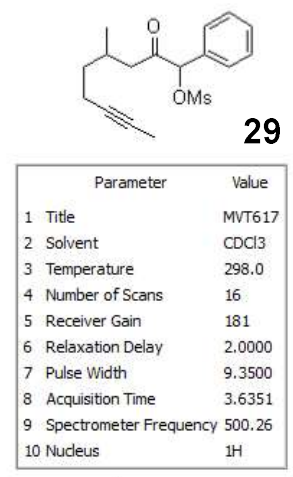

29
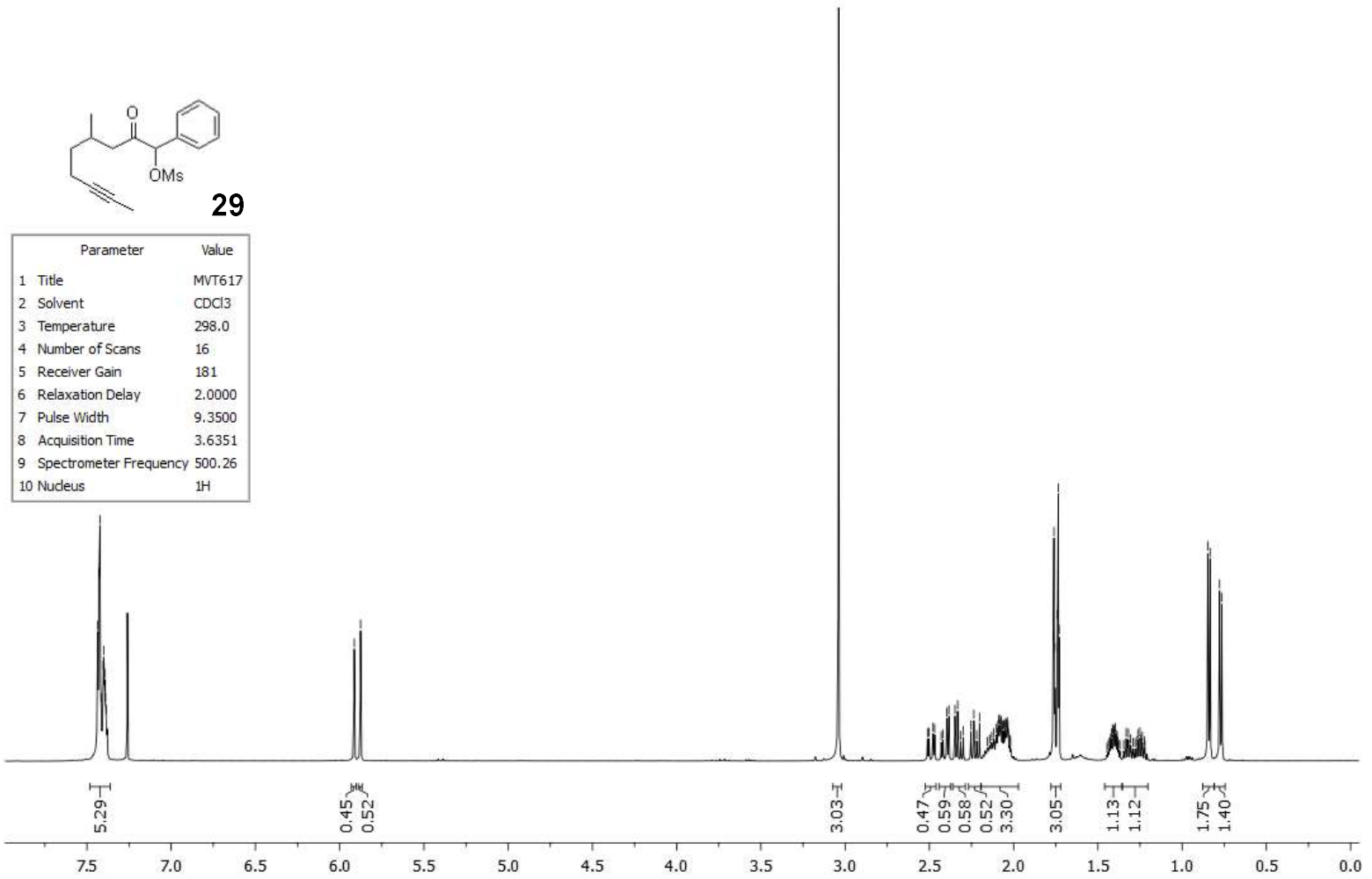

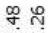

กั่

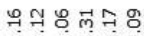

min

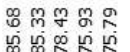

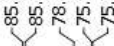

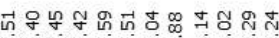

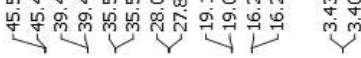

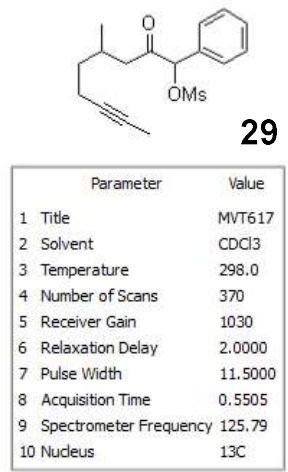

29

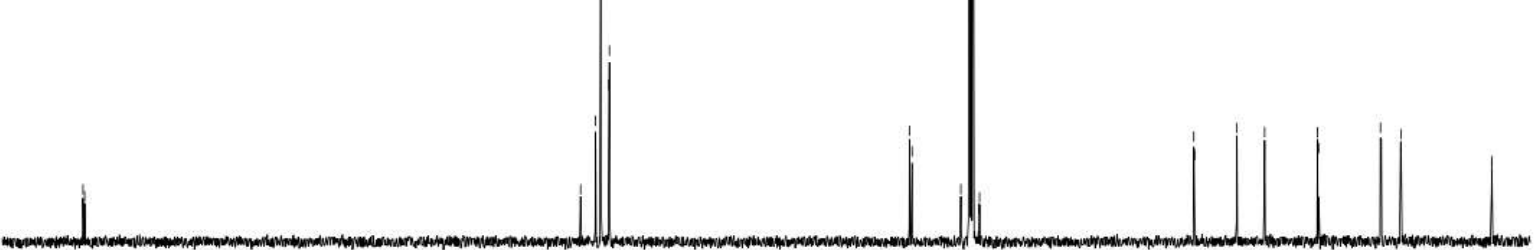

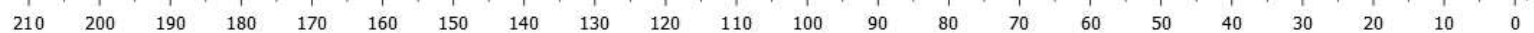



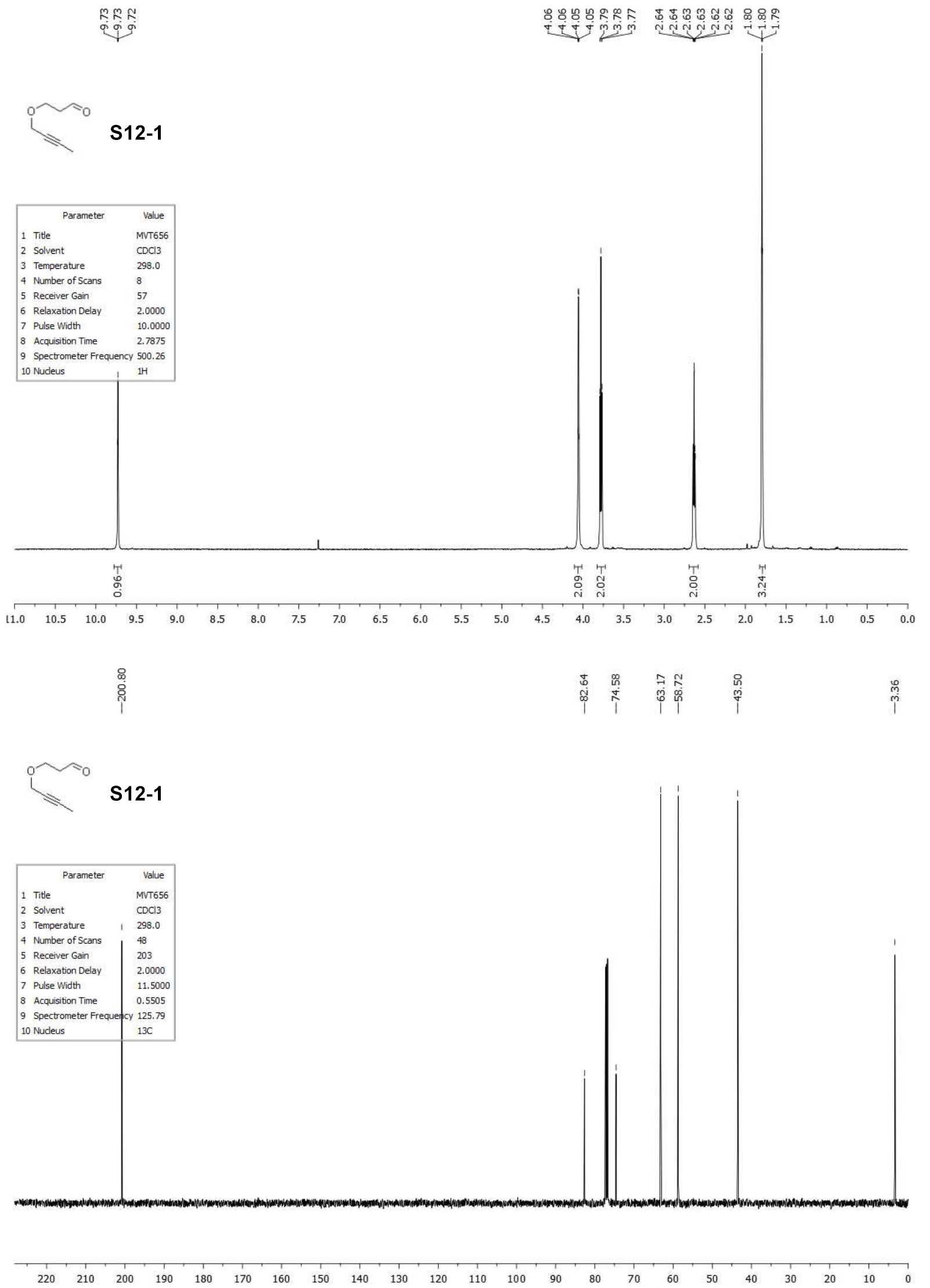

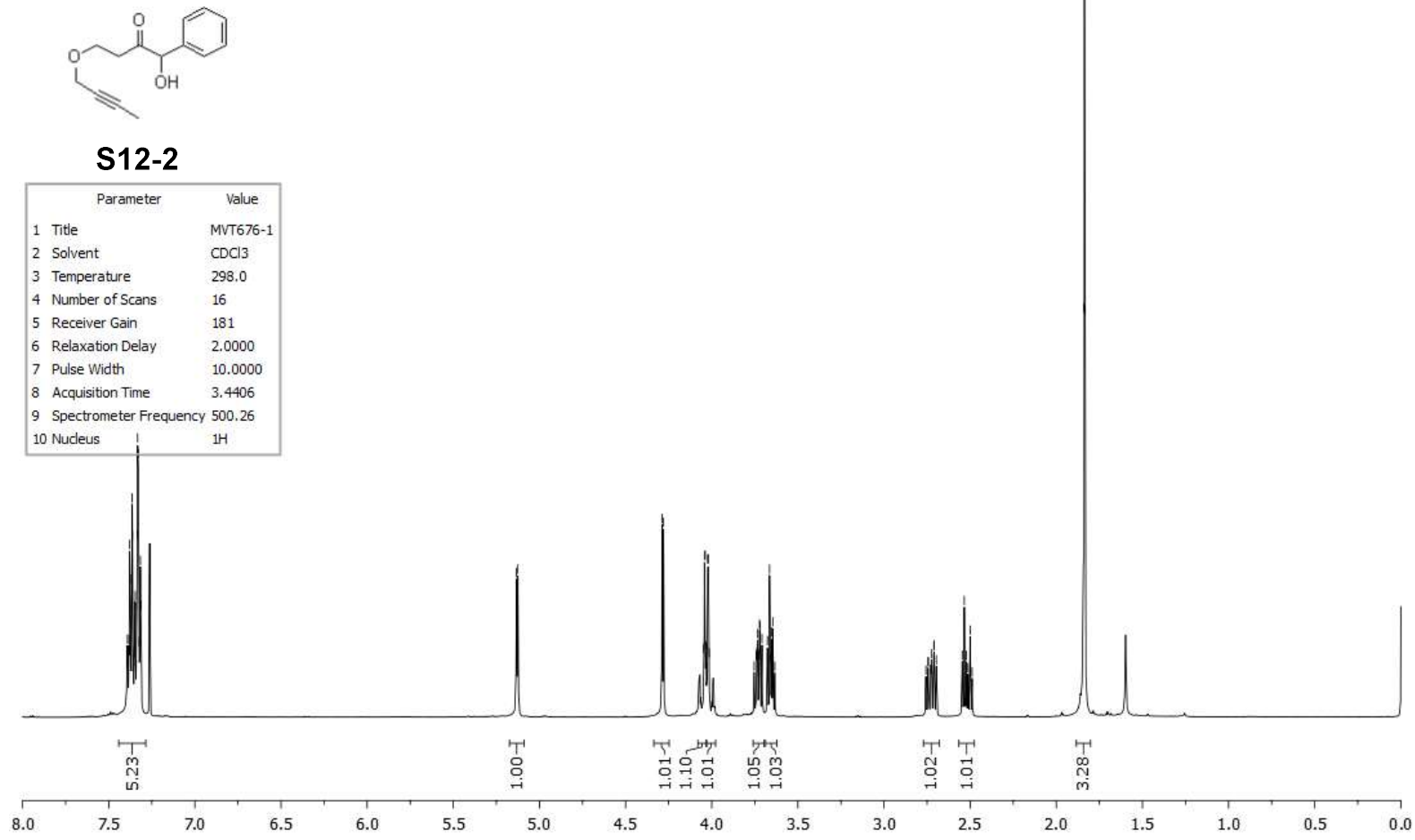

$\stackrel{m}{\stackrel{m}{5}}$
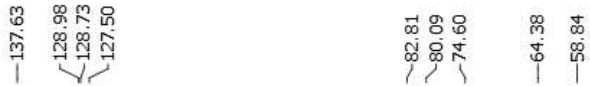

\section{$\underset{\substack{n \\ \infty}}{\substack{0 \\ 0}}$}

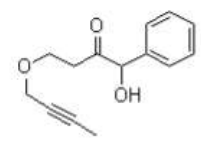

S12-2

\begin{tabular}{|lll|}
\hline \multicolumn{1}{|c|}{ Parameter } & \multicolumn{1}{c|}{ Value } \\
1 & Title & MVT676-1 \\
2 & Solvent & CDCl3 \\
3 & Temperature & 298.0 \\
4 & Number of Scans & 700 \\
5 & Receiver Gain & 203 \\
6 & Relaxation Delay & 2.0000 \\
7 & Pulse Width & 11.5000 \\
8 & Acquisition Time & 0.5505 \\
9 & Spectrometer Frequency & 125.79 \\
10 & Nudeus & $13 \mathrm{C}$ \\
\hline
\end{tabular}
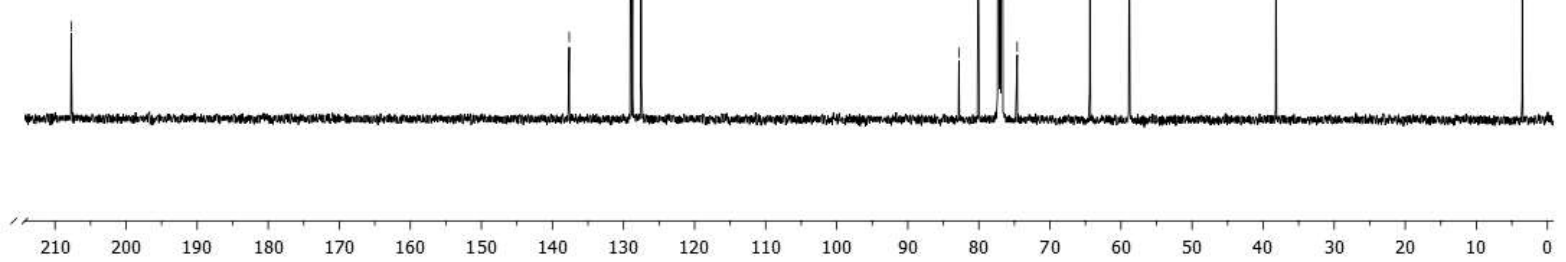

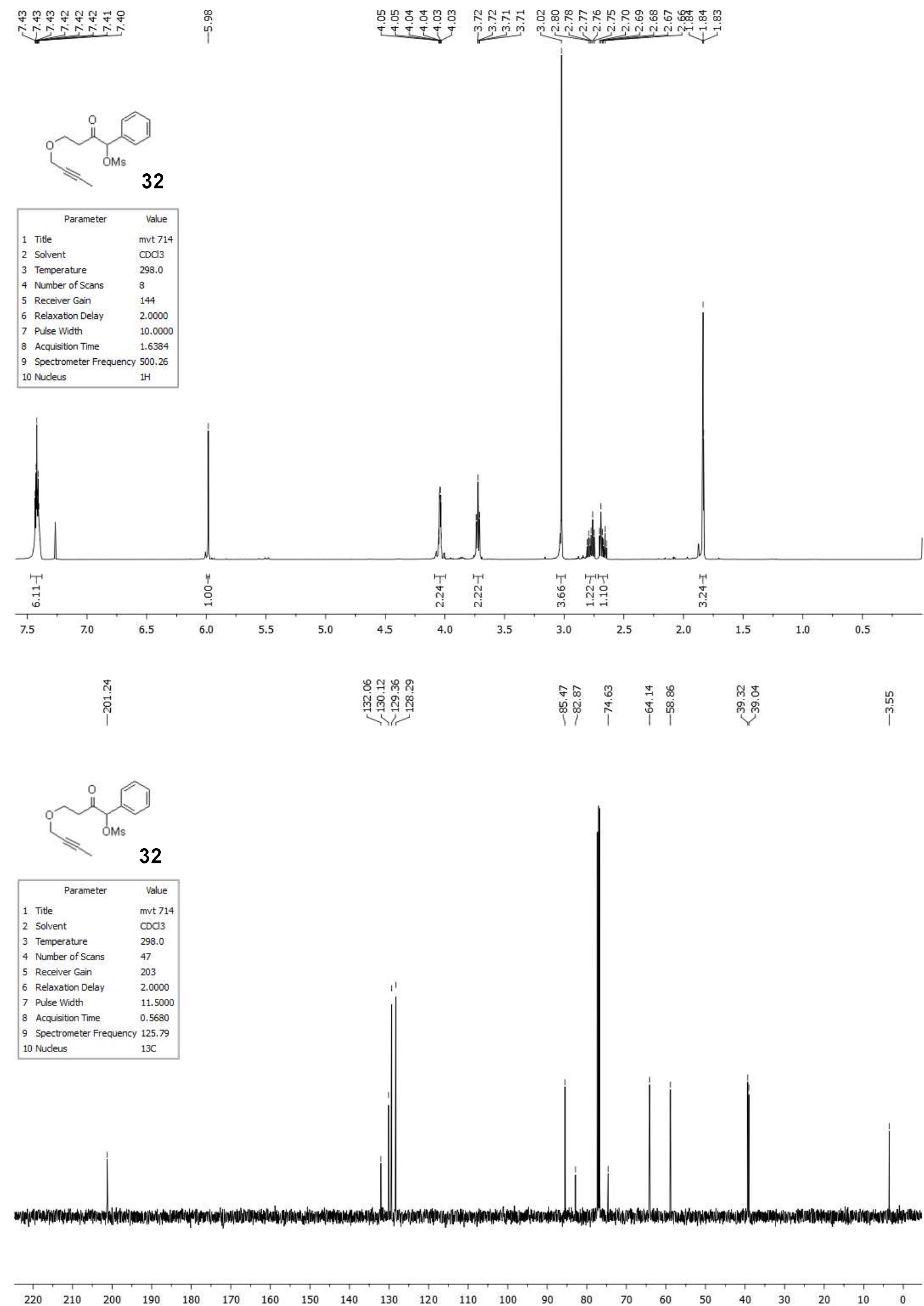


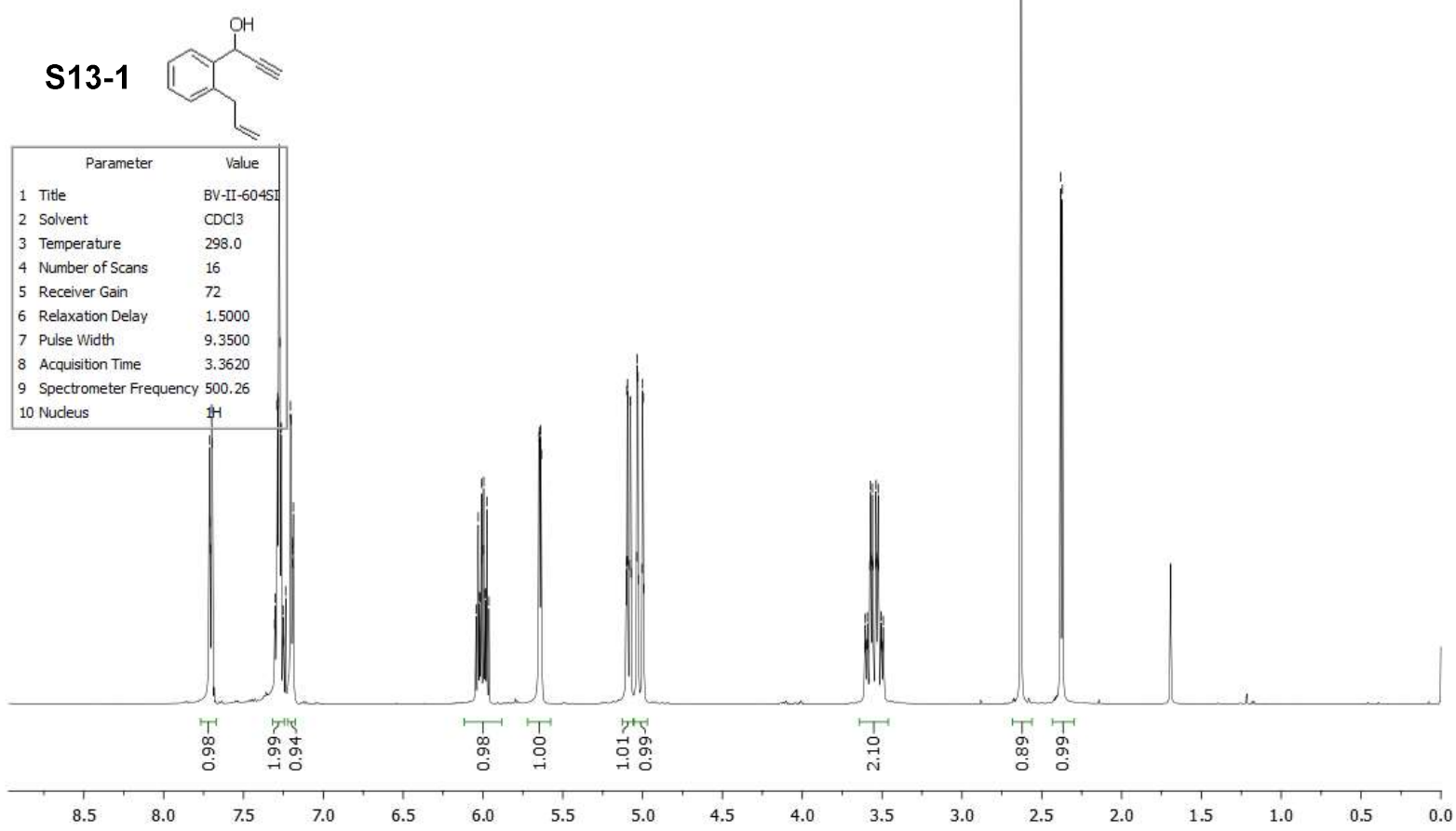

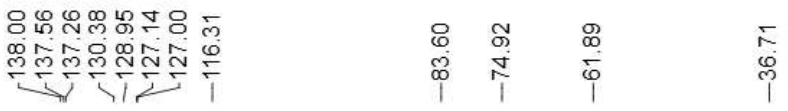
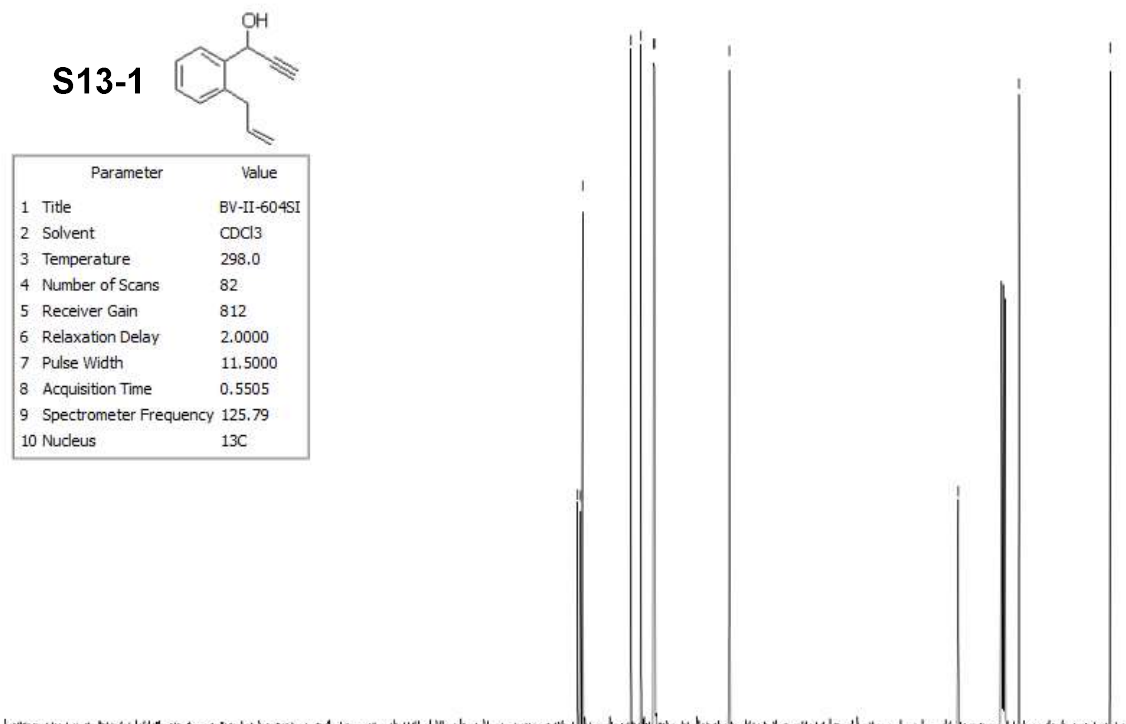

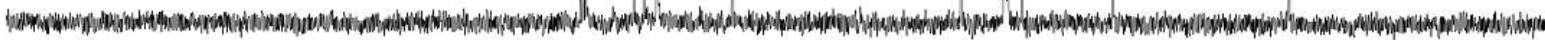

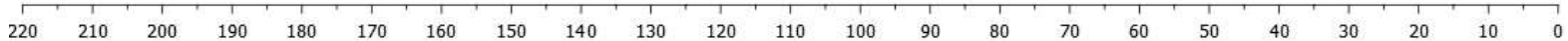



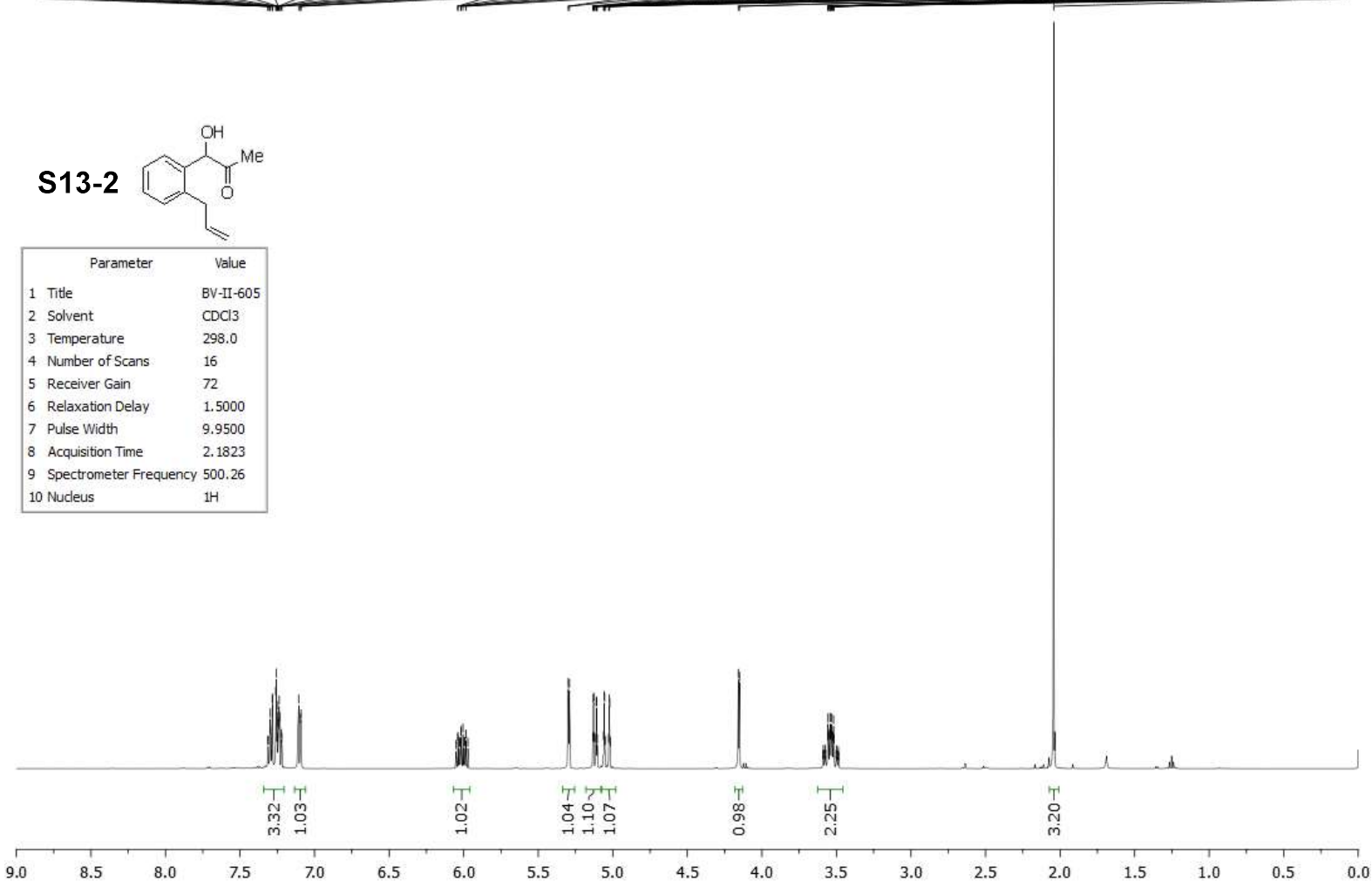

సั.
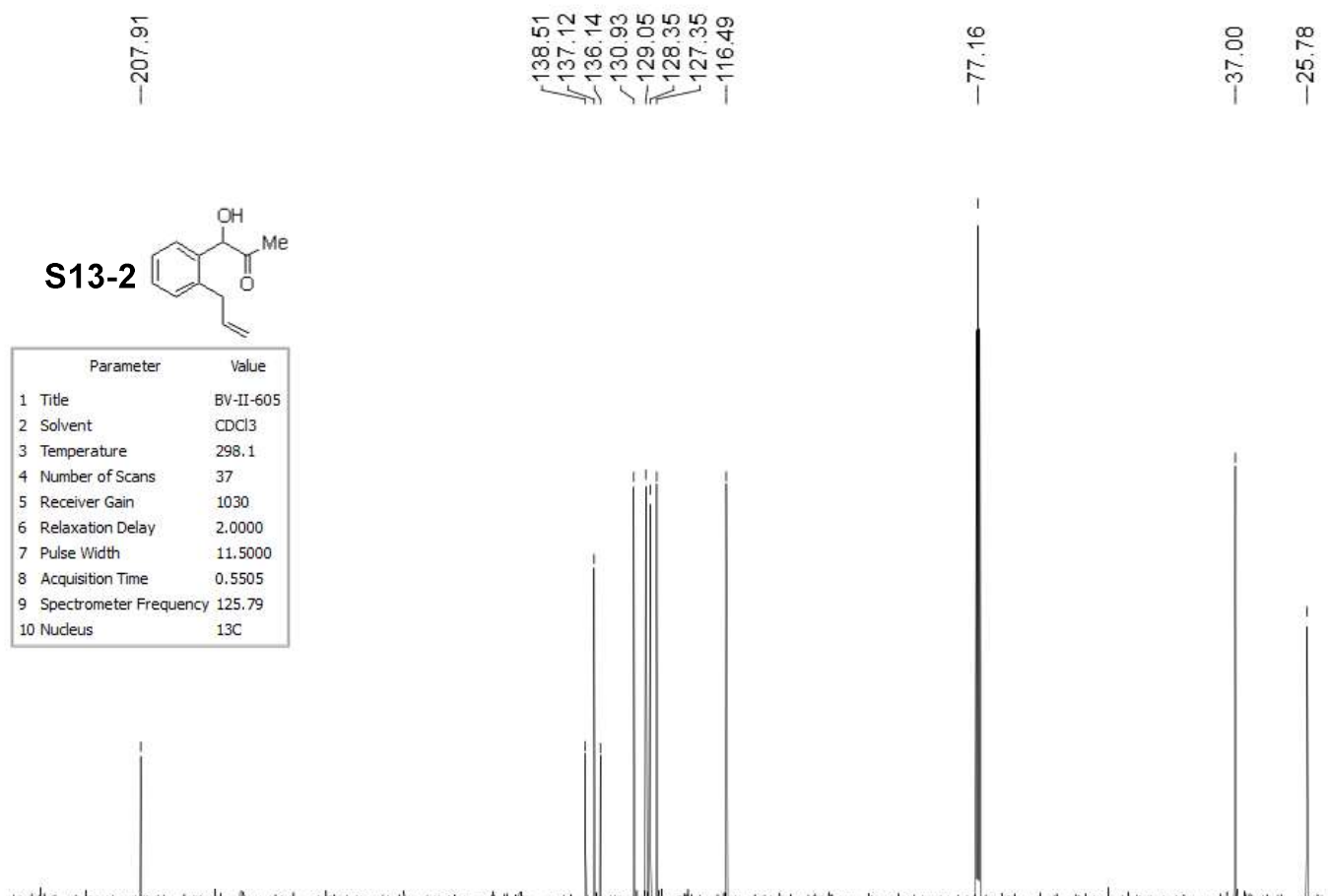

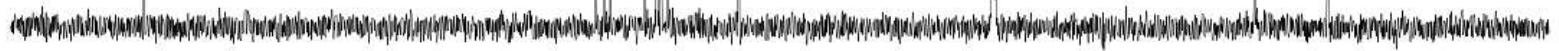

$\begin{array}{lllllllllllllllllllllllllllll}220 & 210 & 200 & 190 & 180 & 170 & 160 & 150 & 140 & 130 & 120 & 110 & 100 & 90 & 80 & 70 & 60 & 50 & 40 & 30 & 20 & 10 & 0 & 1\end{array}$



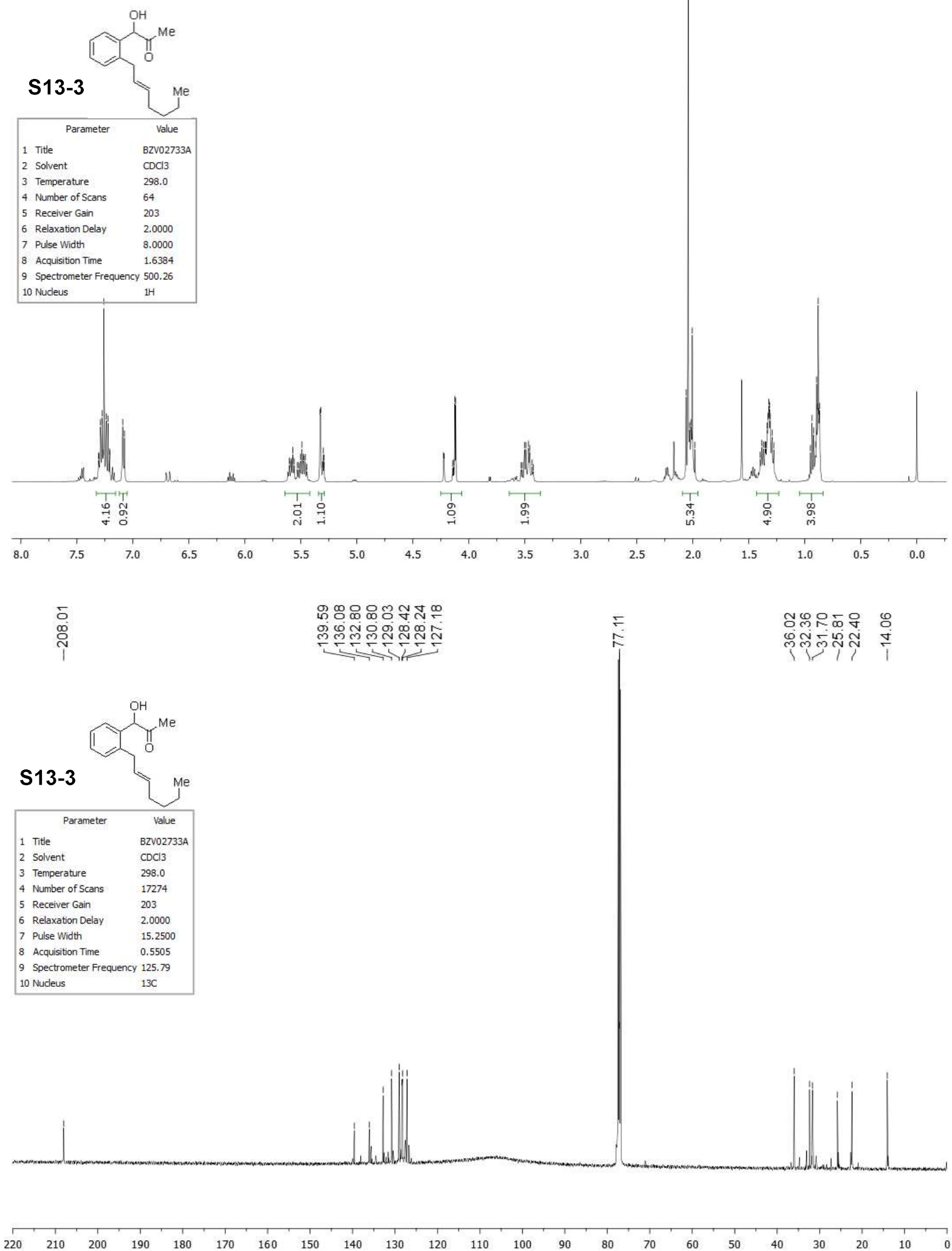

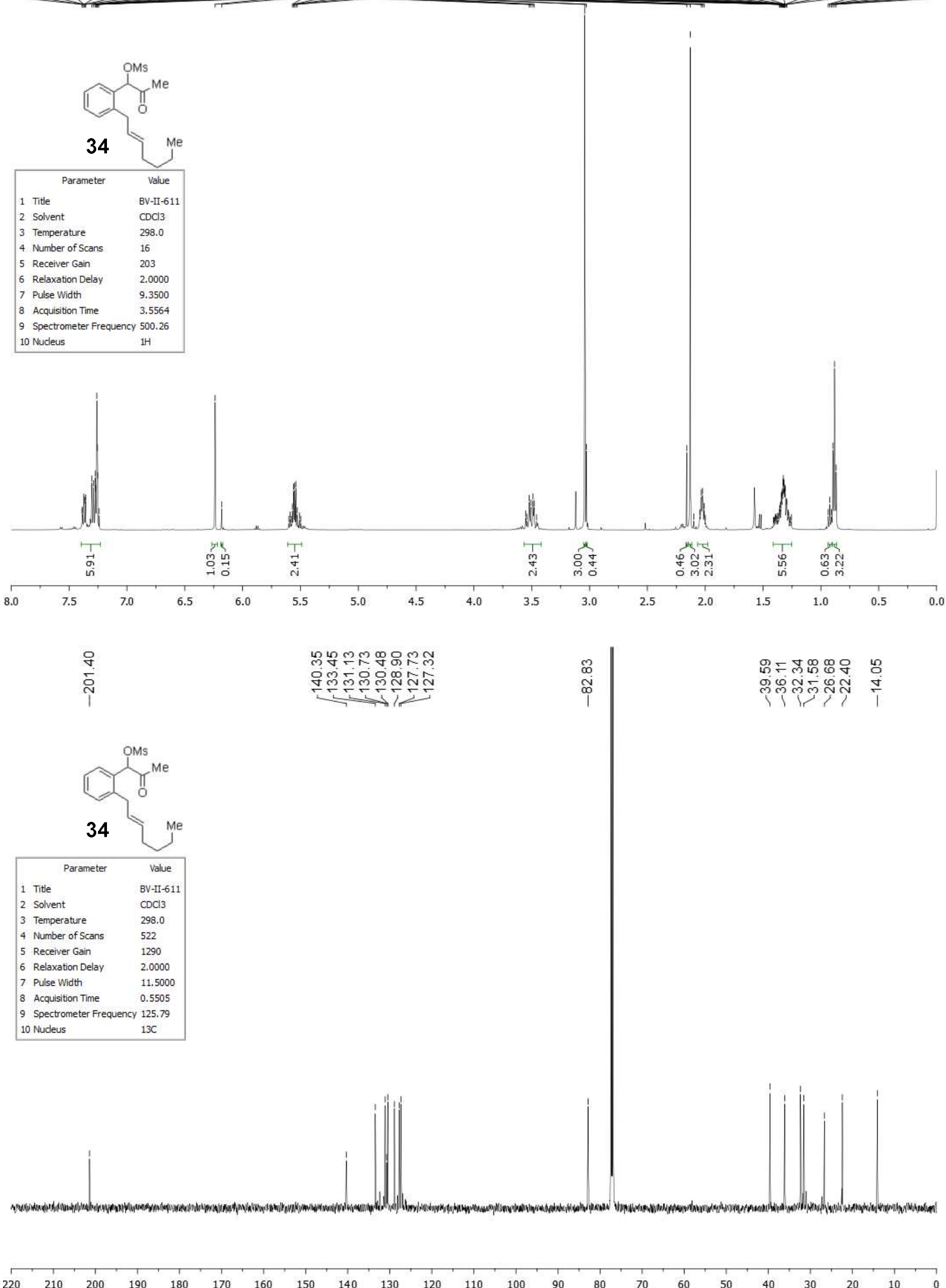

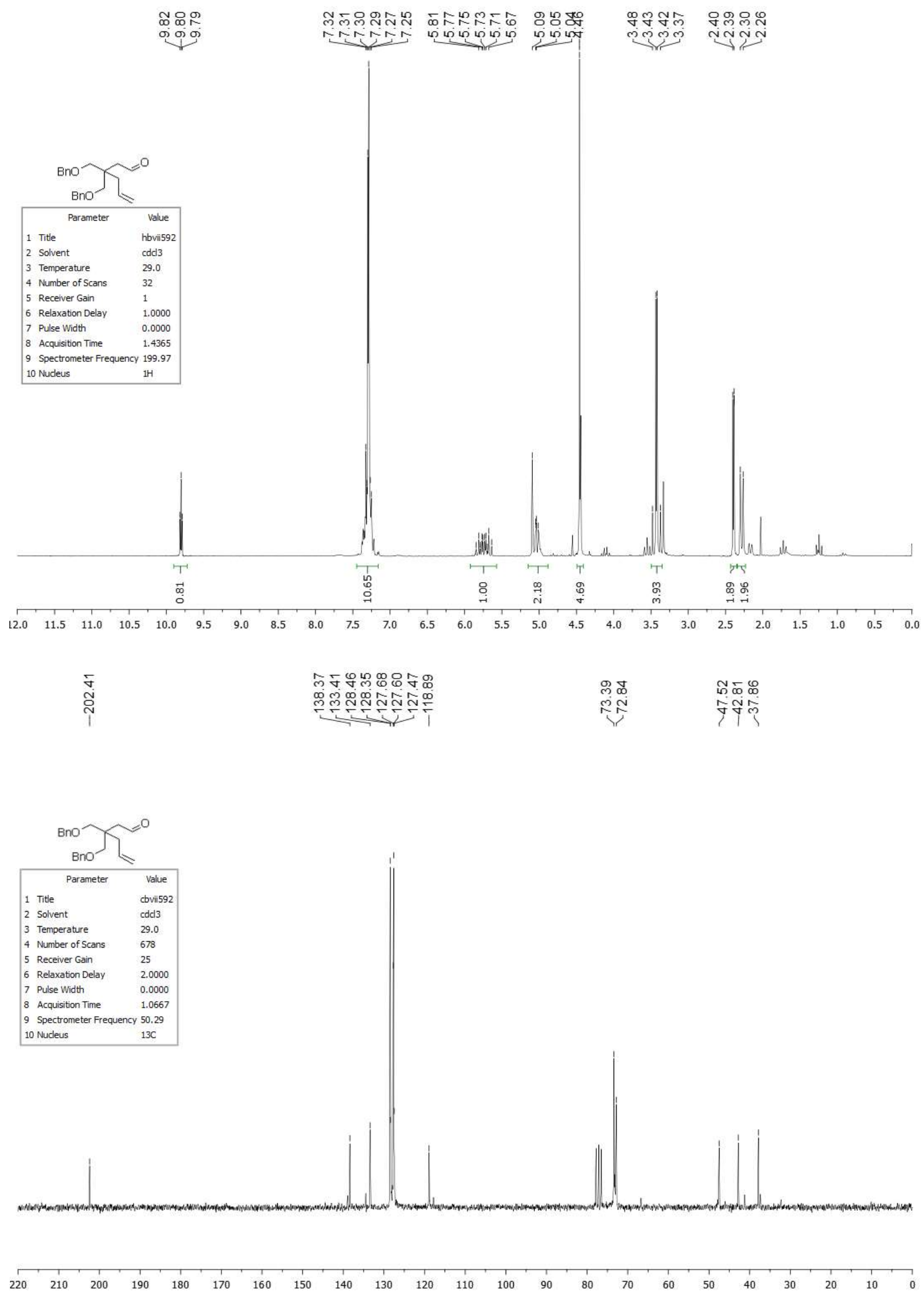


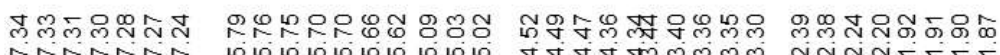

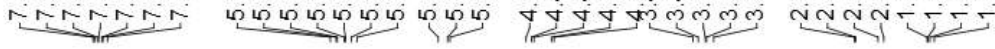
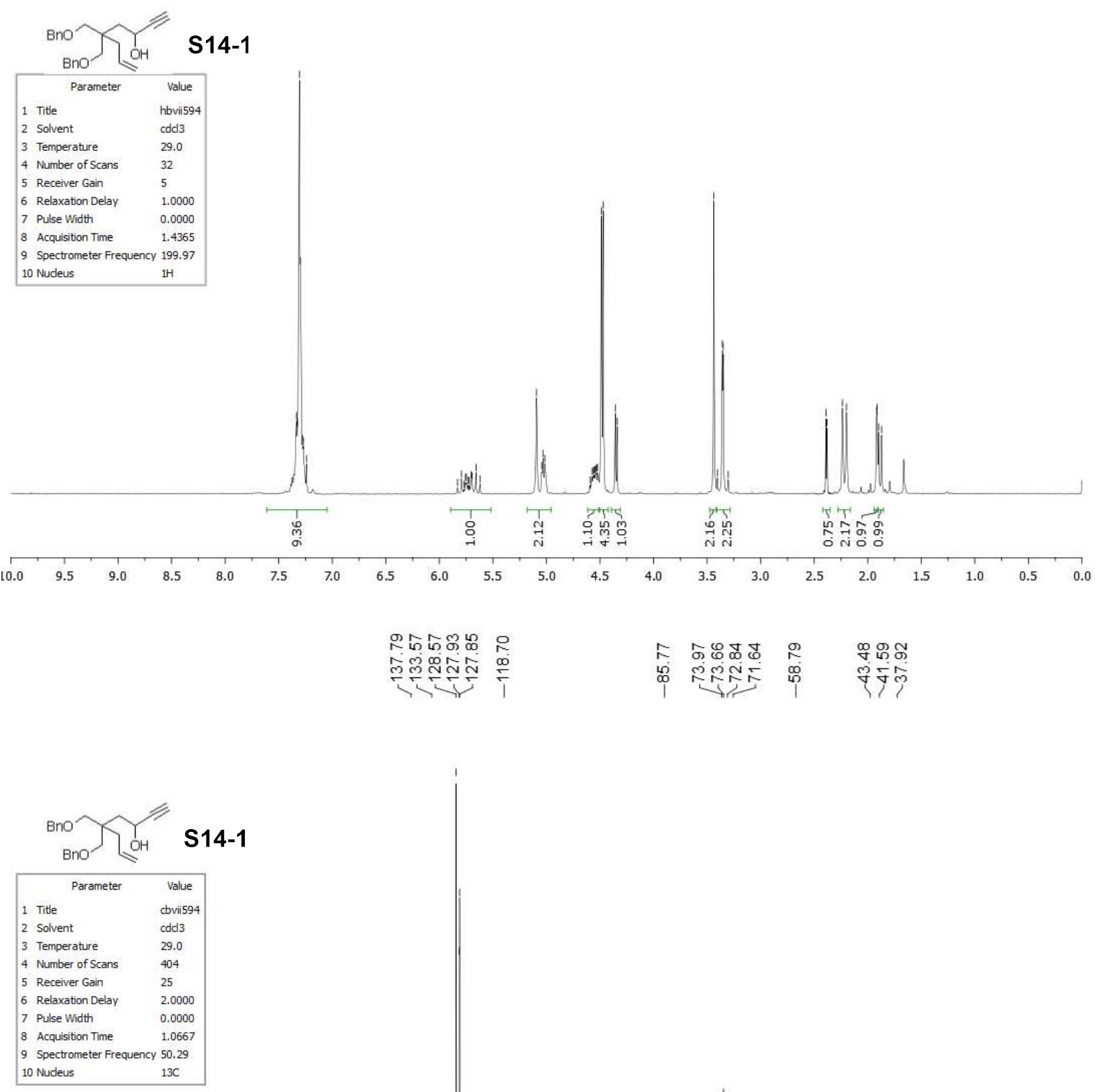

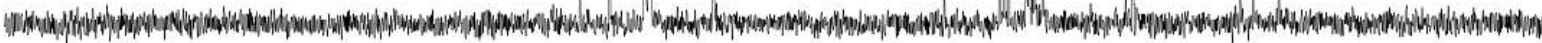

$\begin{array}{llllllllllllllllllllllll}220 & 210 & 200 & 190 & 180 & 170 & 160 & 150 & 140 & 130 & 120 & 110 & 100 & 90 & 80 & 70 & 60 & 50 & 40 & 30 & 20 & 10 & 0\end{array}$ 

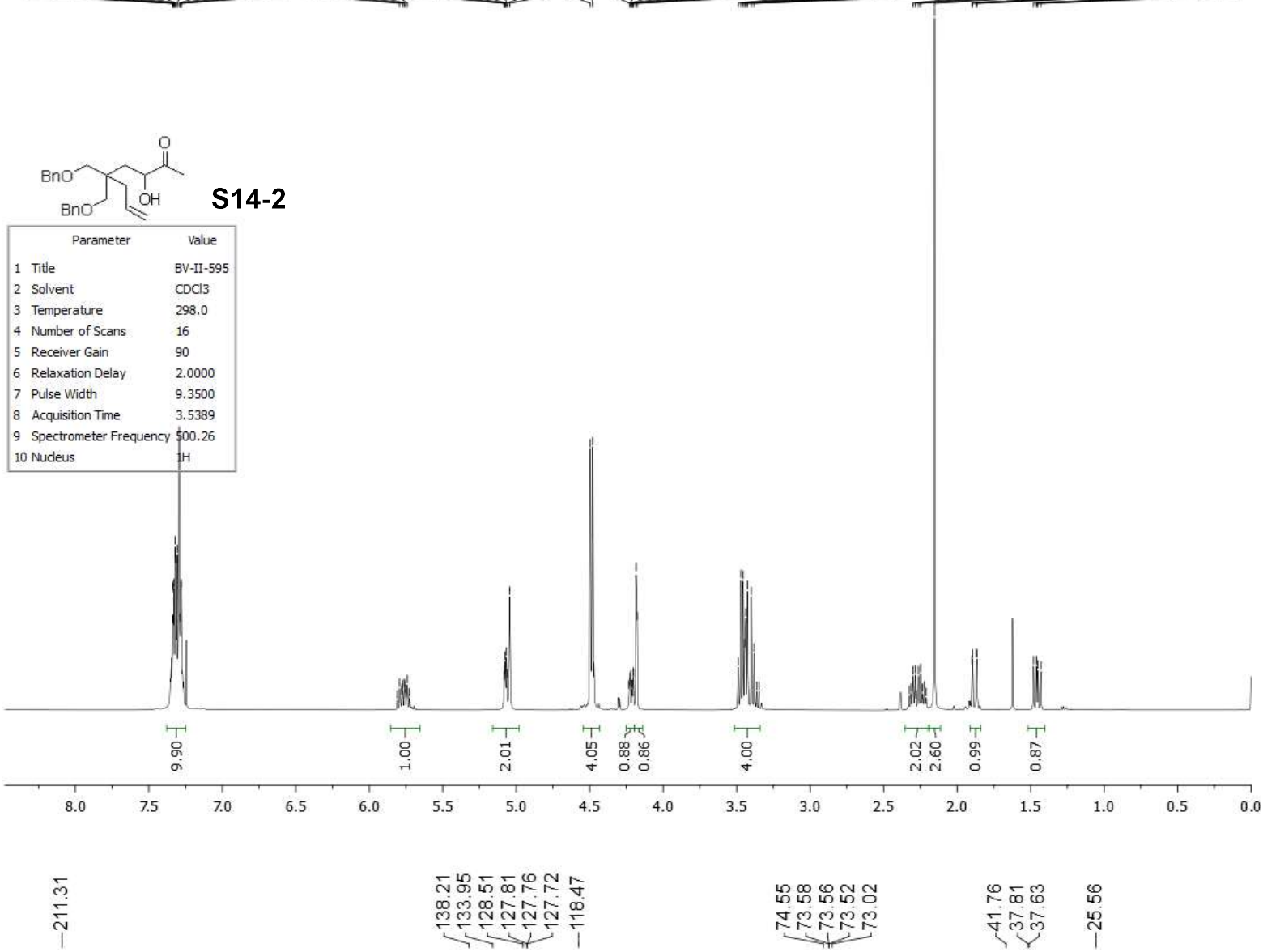

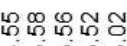

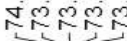

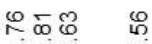

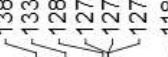

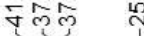
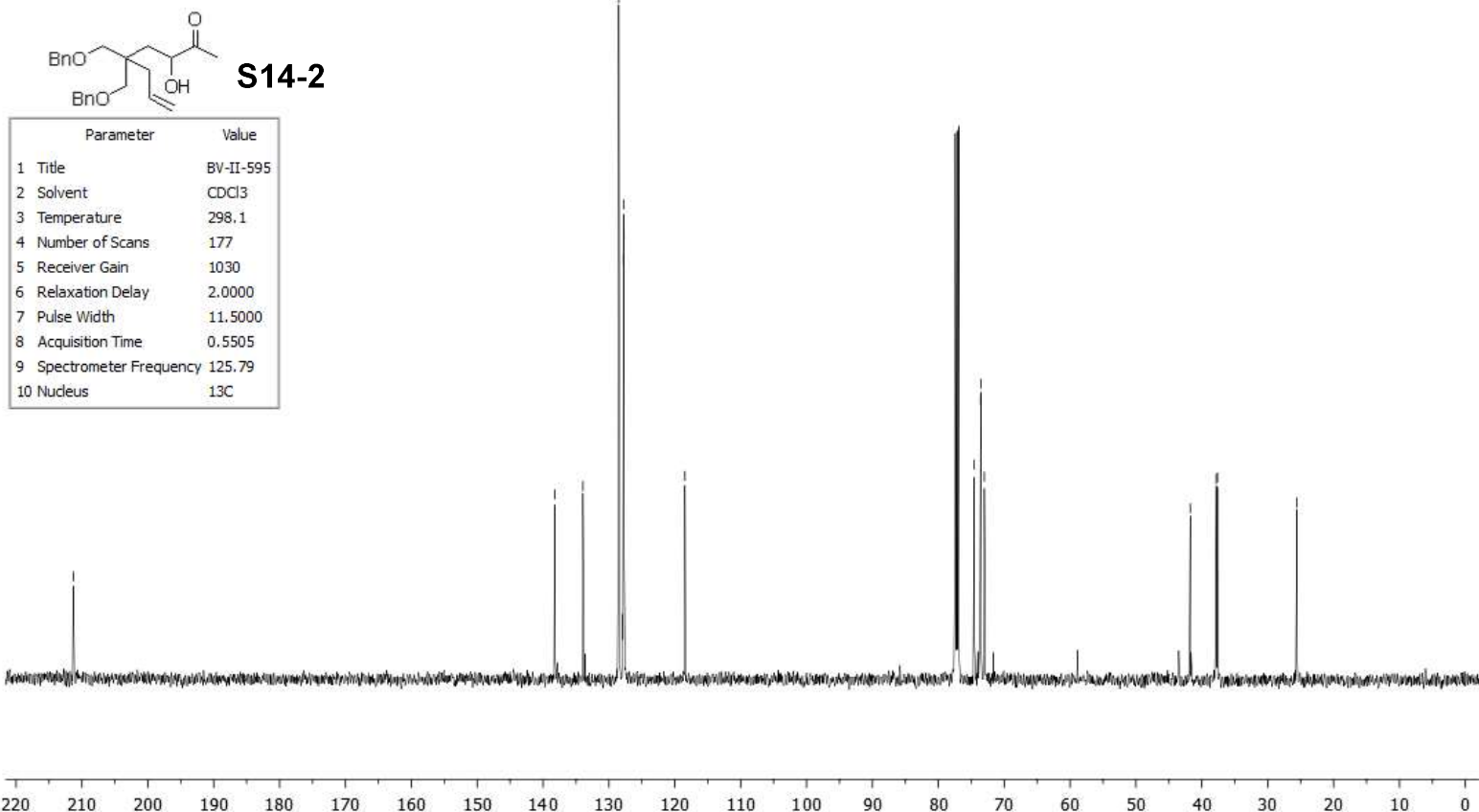


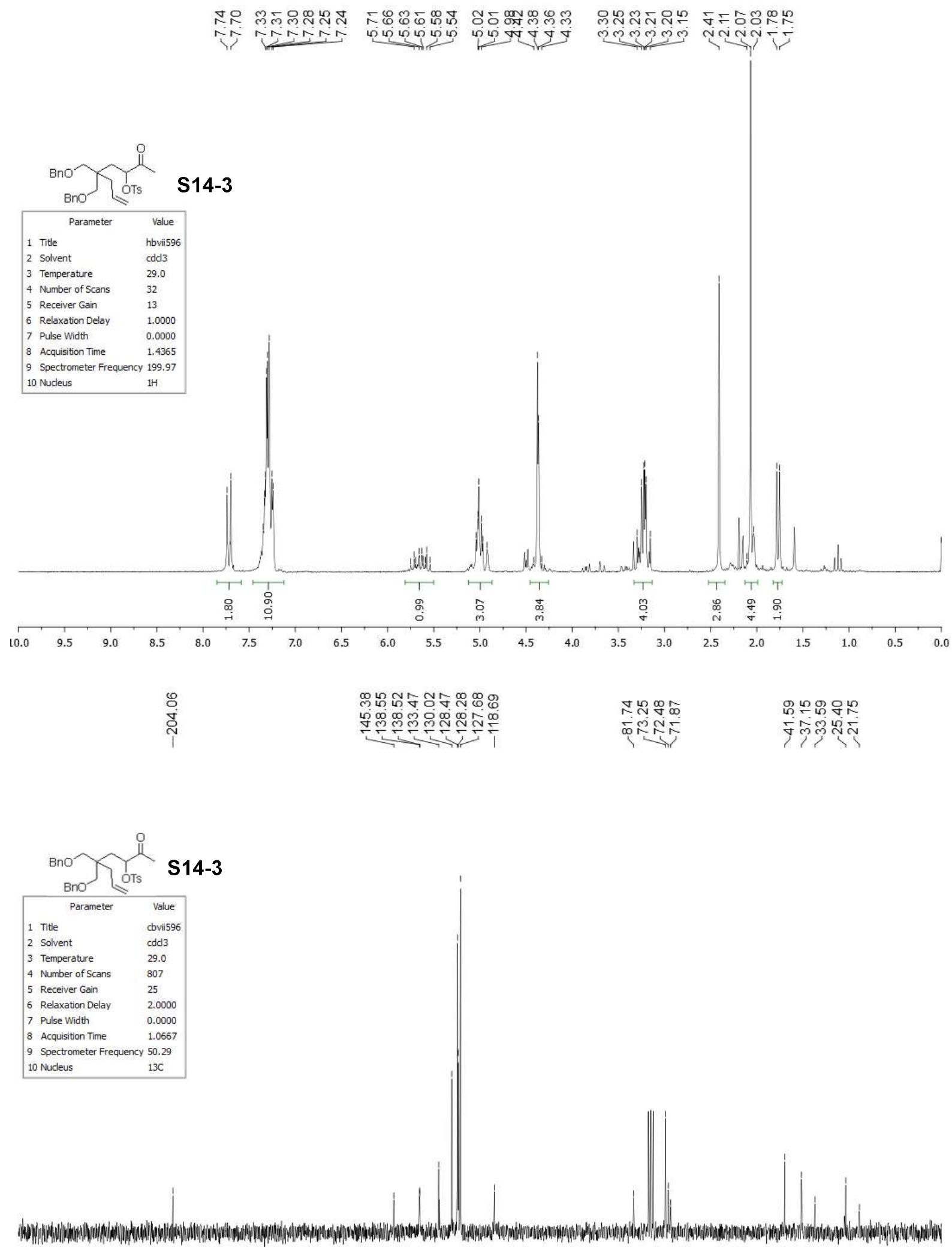

$\begin{array}{lllllllllllllllllllllllll}240 & 230 & 220 & 210 & 200 & 190 & 180 & 170 & 160 & 150 & 140 & 130 & 120 & 110 & 100 & 90 & 80 & 70 & 60 & 50 & 40 & 30 & 20 & 10 & 0\end{array}$ 

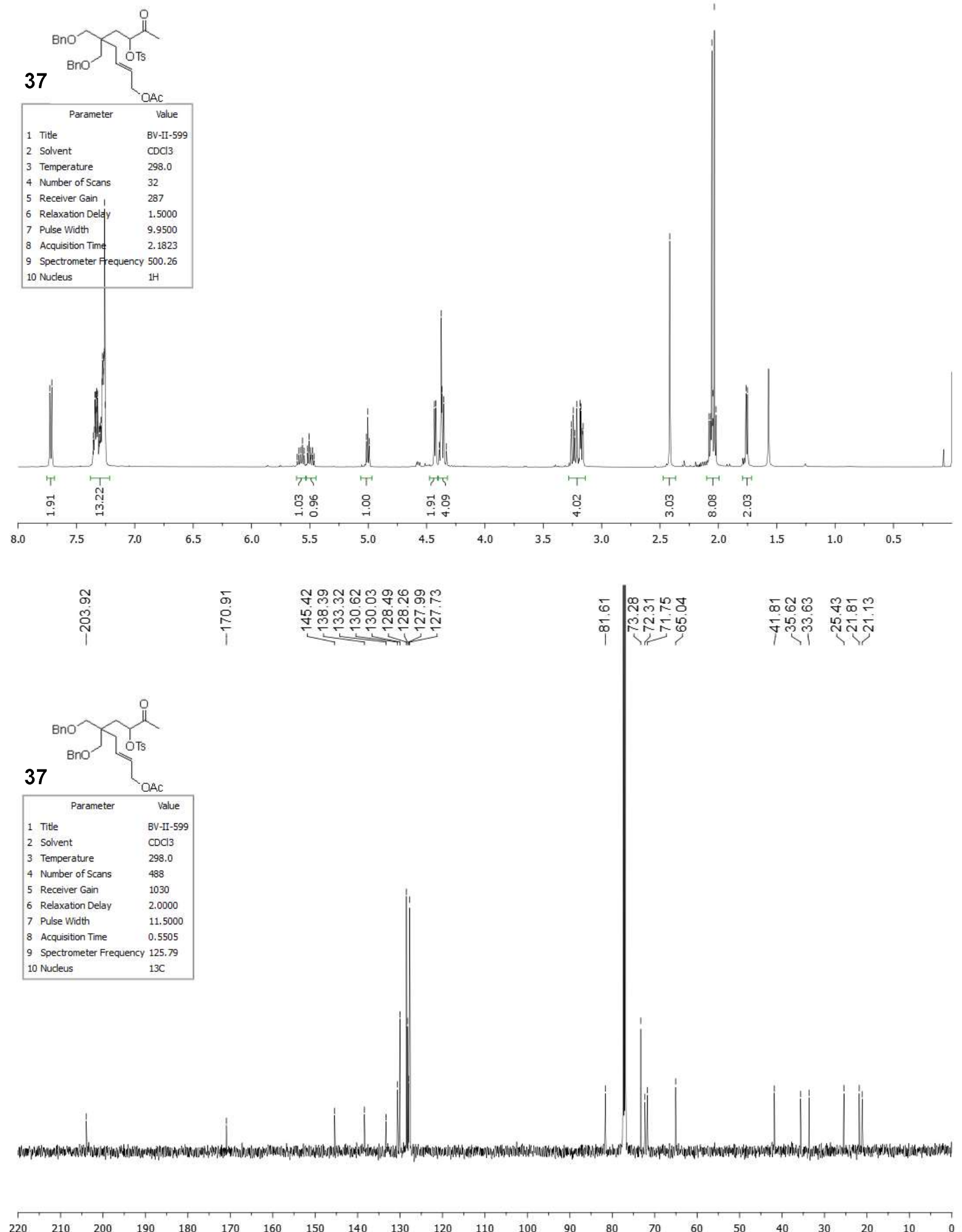
2
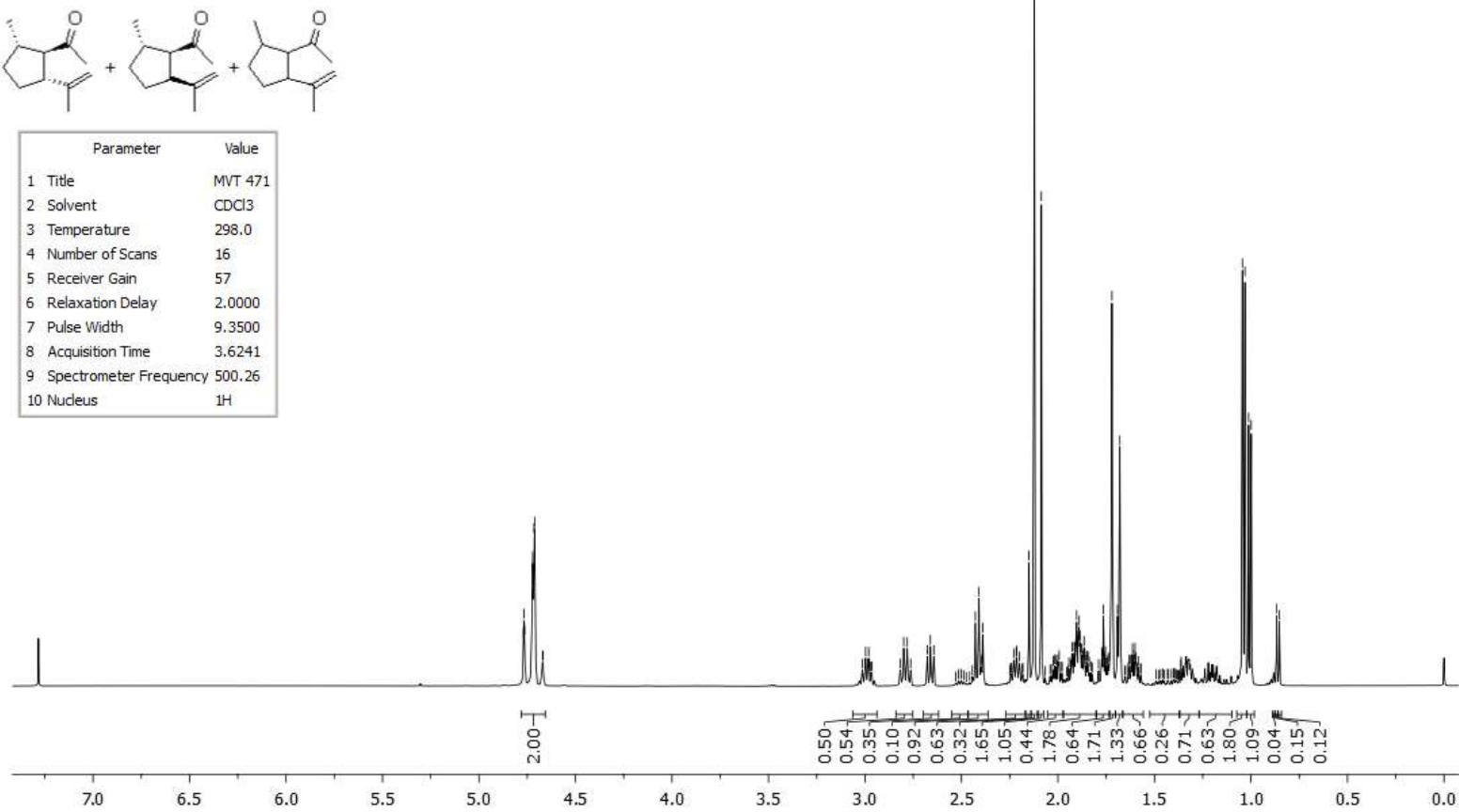

\begin{tabular}{|c|}
\hline 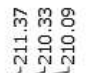 \\
\hline
\end{tabular}

2

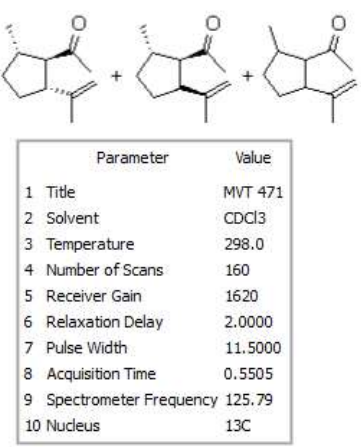

10 Nucleus $-13 \mathrm{C}$
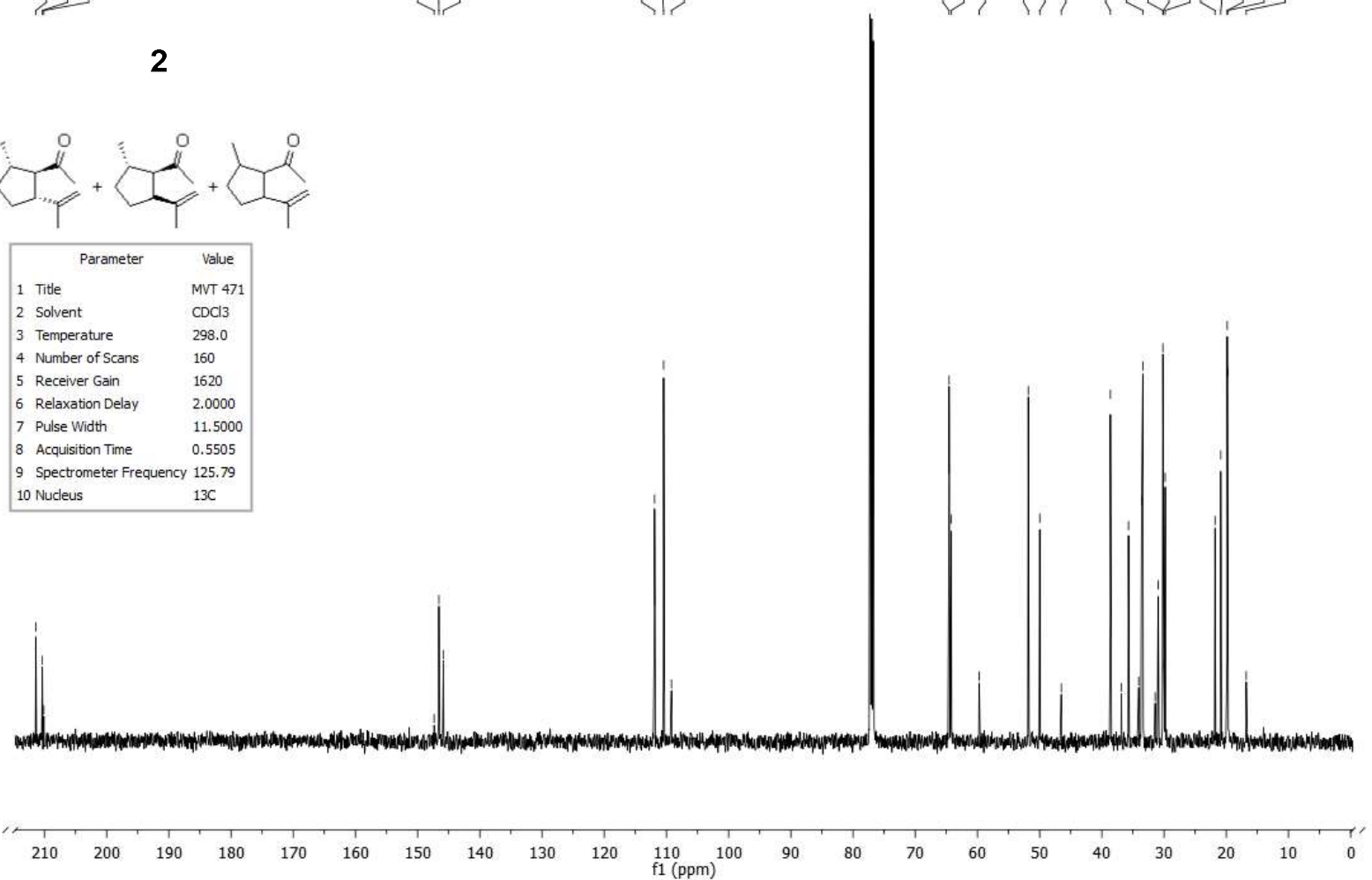
Correlations (NOESY) for major diastereomer 2a:
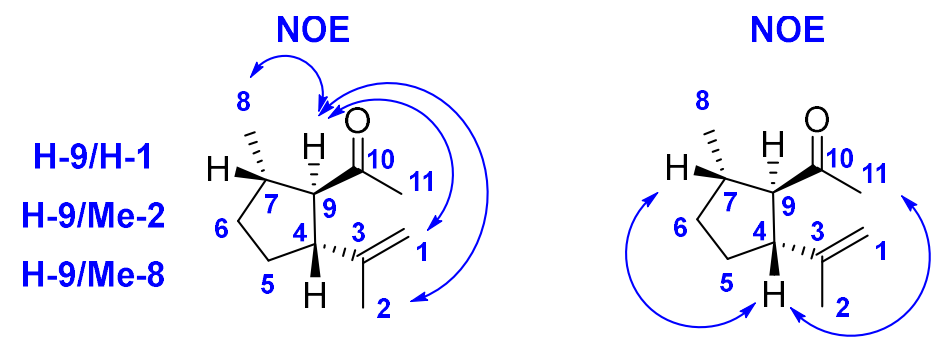

$\mathrm{H}-4 / \mathrm{H}-7$

H-4/Me-11

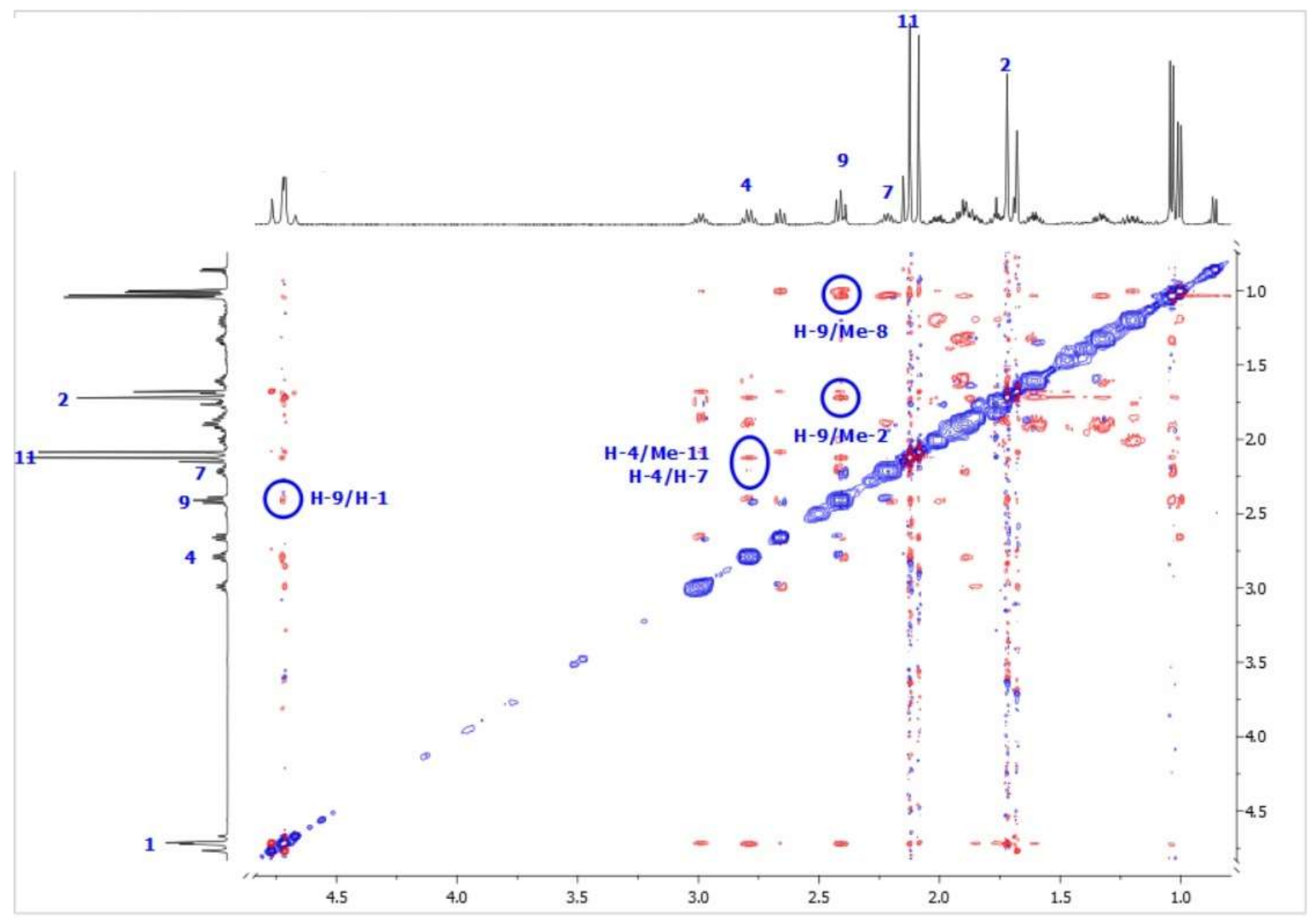



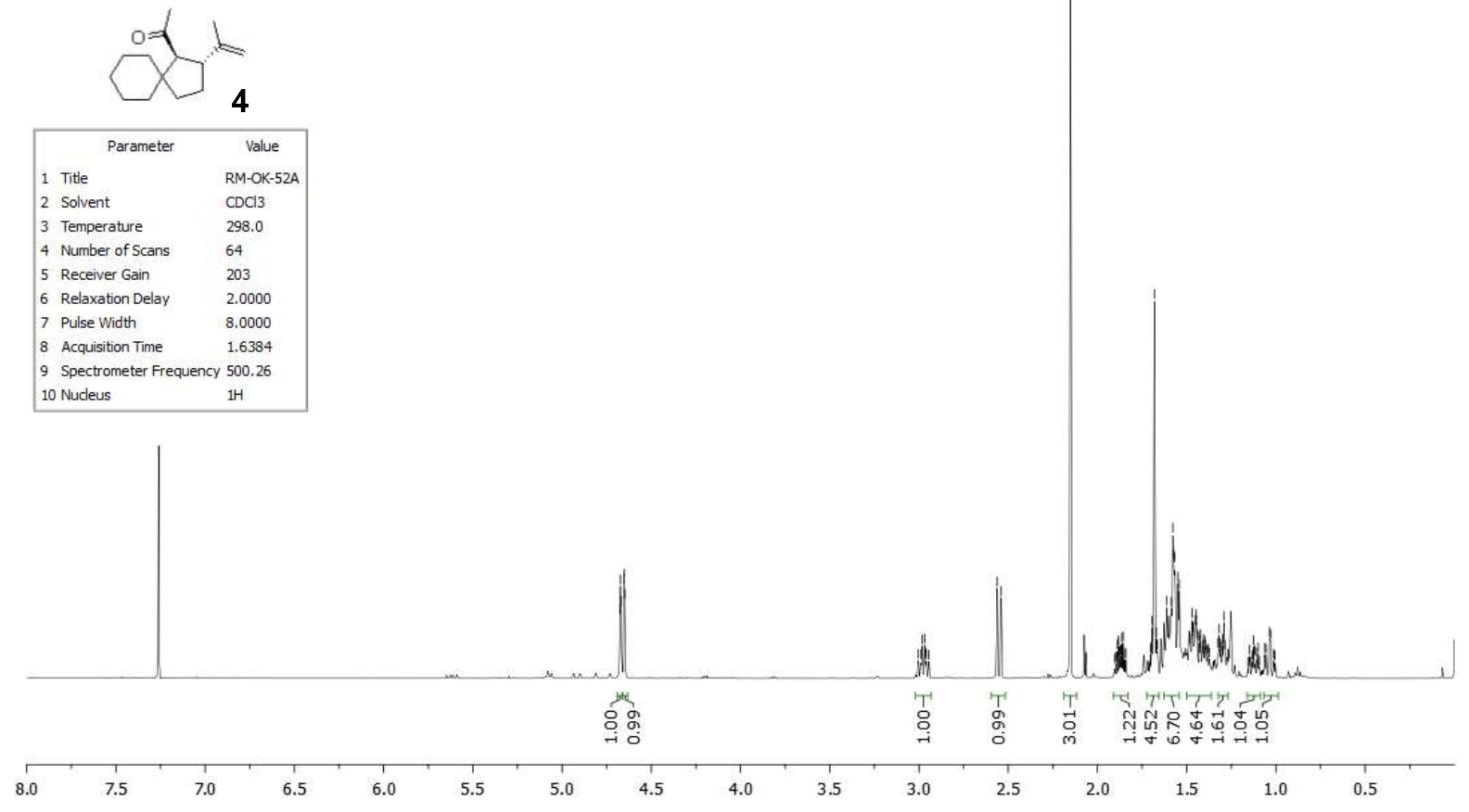

\begin{tabular}{|c|c|c|c|c|}
\hline$\stackrel{\mathscr{O}}{\stackrel{\leftarrow}{\leftarrow}}$ & $\begin{array}{l}\infty \\
\stackrel{0}{\tilde{\sigma}} \\
\end{array}$ & $\frac{9}{2}$ & 占 & 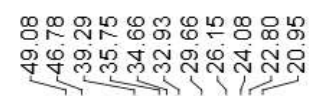 \\
\hline
\end{tabular}
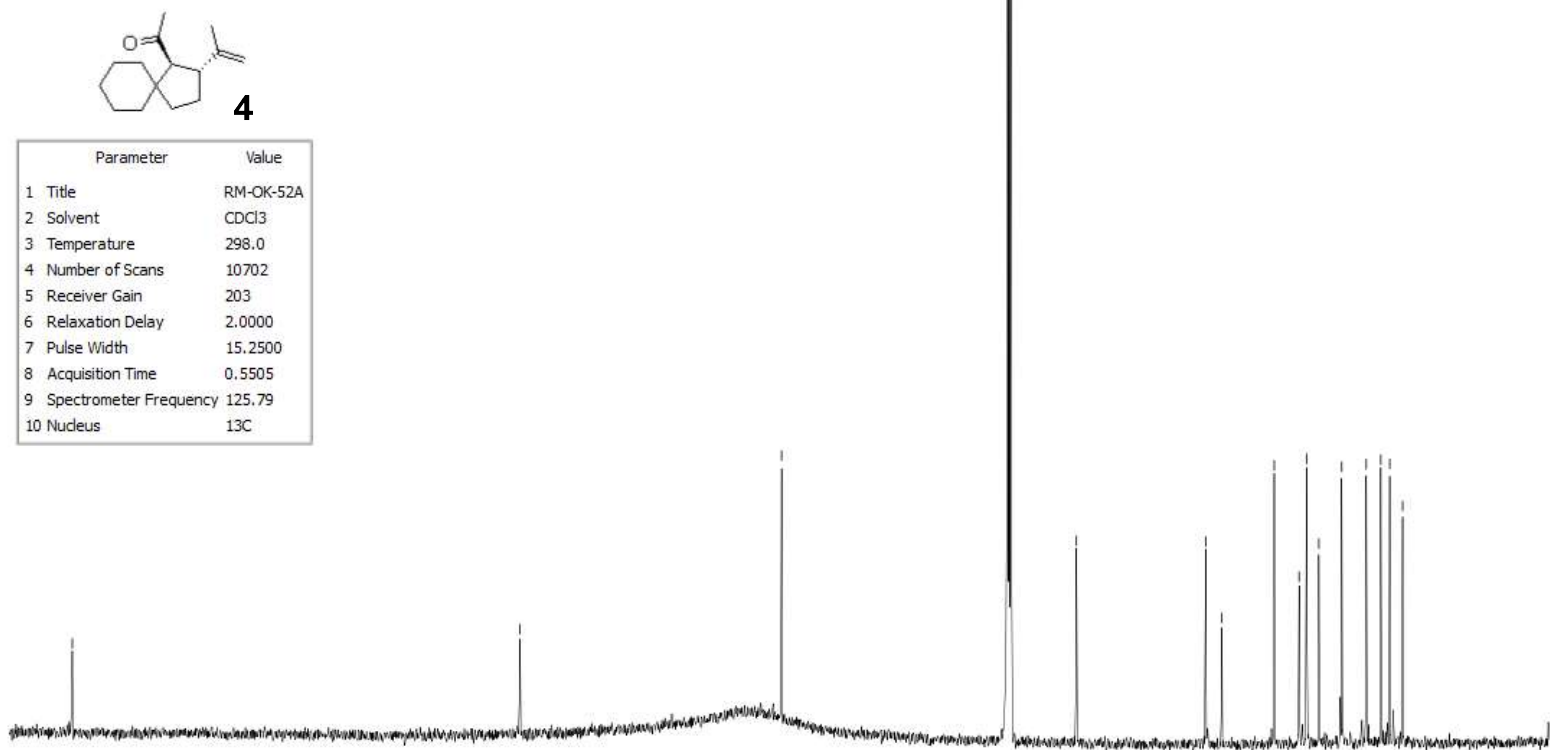

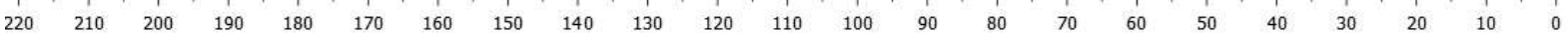


Correlations (NOESY) for compound $\mathbf{4}$ :

\section{4}

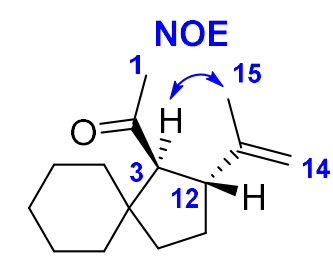

H-3/Me-15

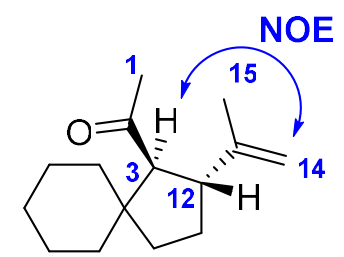

H-3/H-14

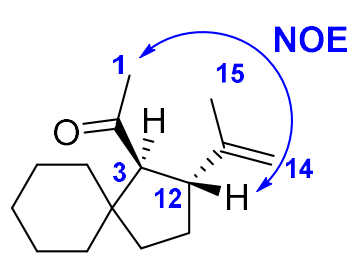

$\mathrm{H}-12 / \mathrm{Me}-1$

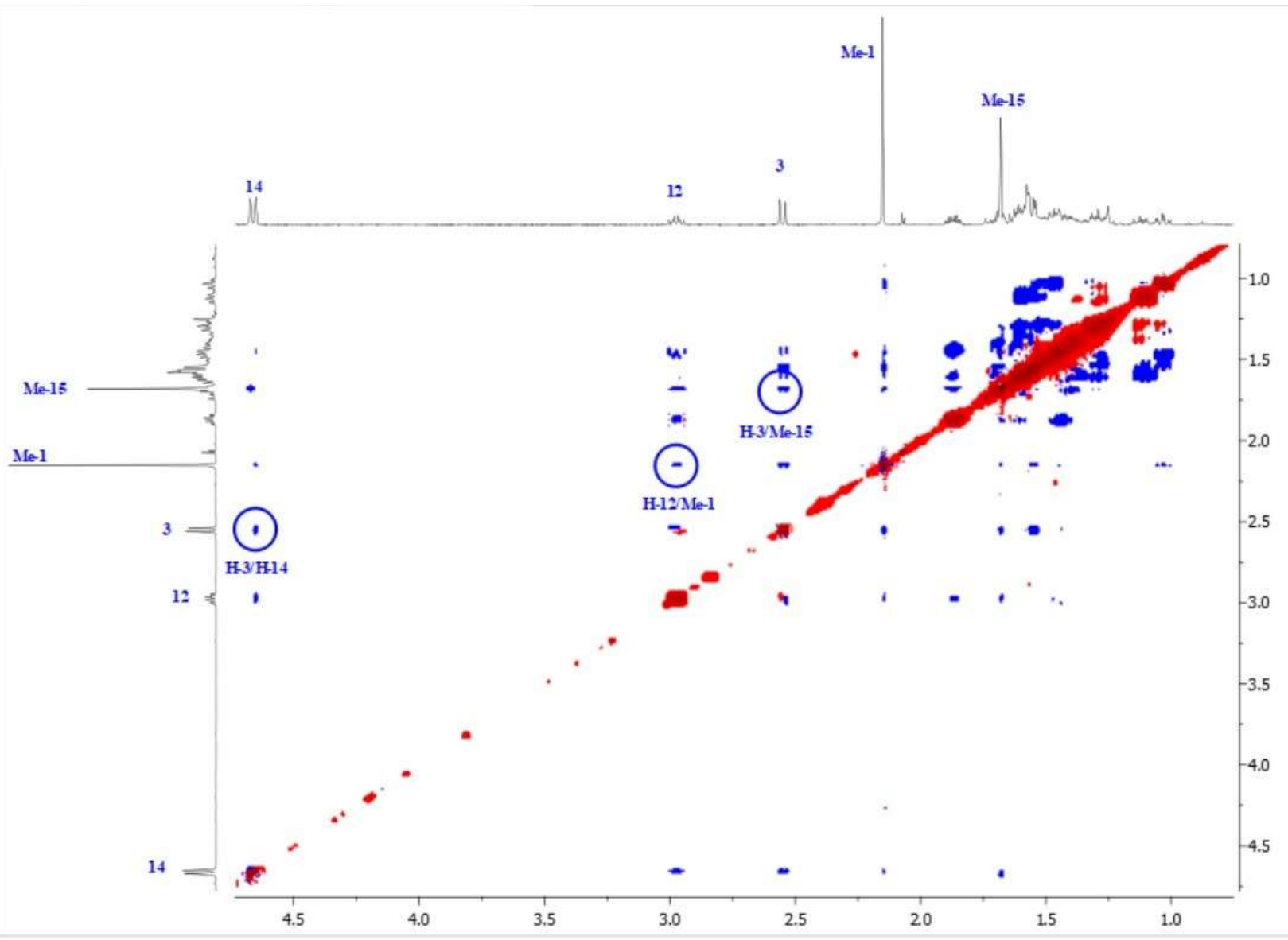




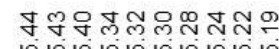

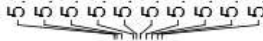

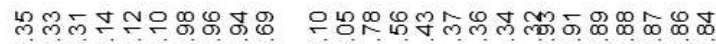

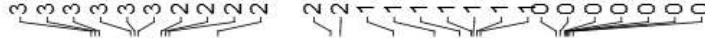
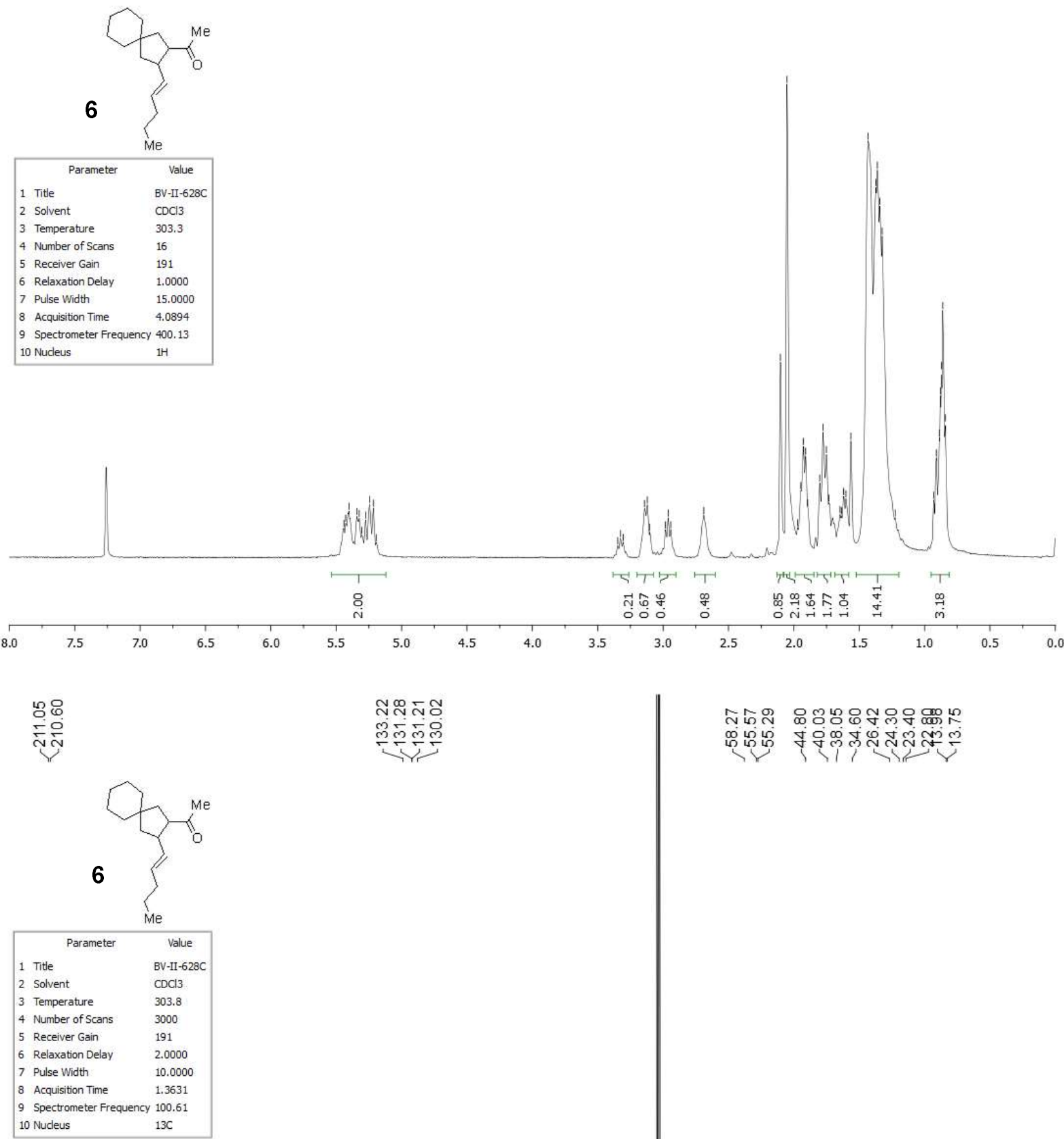

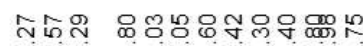

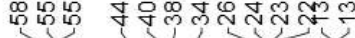

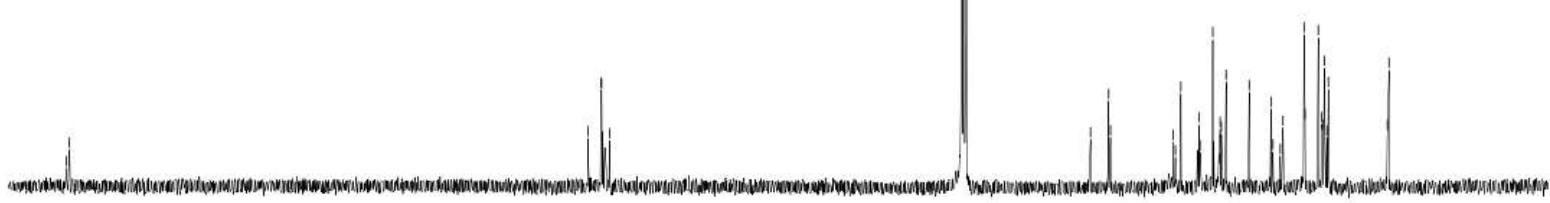

$\begin{array}{llllllllllllllllllllllll}210 & 200 & 190 & 180 & 170 & 160 & 150 & 140 & 130 & 120 & 110 & 100 & 90 & 80 & 70 & 60 & 50 & 40 & 30 & 20 & 10 & 0 & -10\end{array}$ 

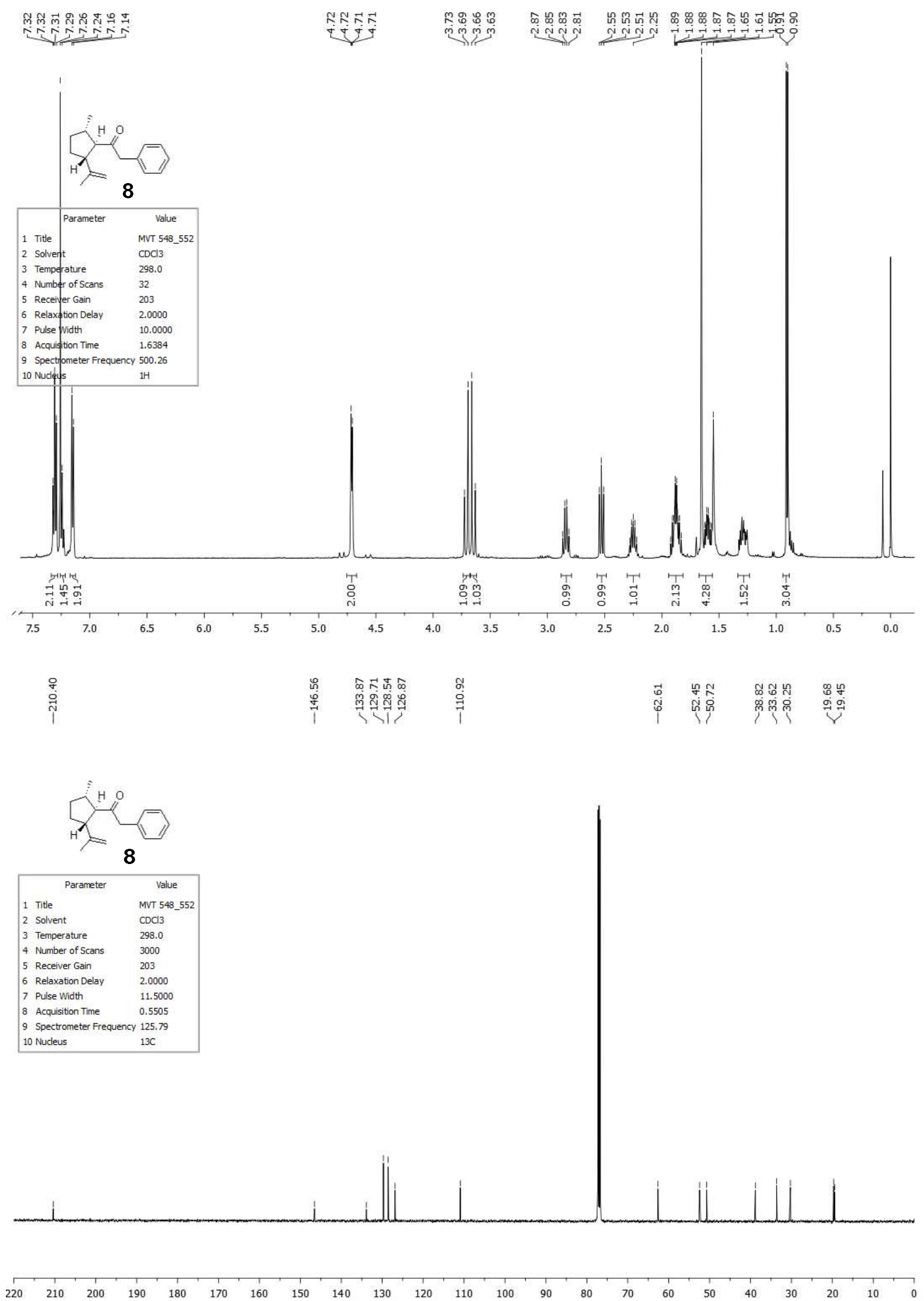


\section{Correlations (NOESY) of $\mathbf{8}$ :}
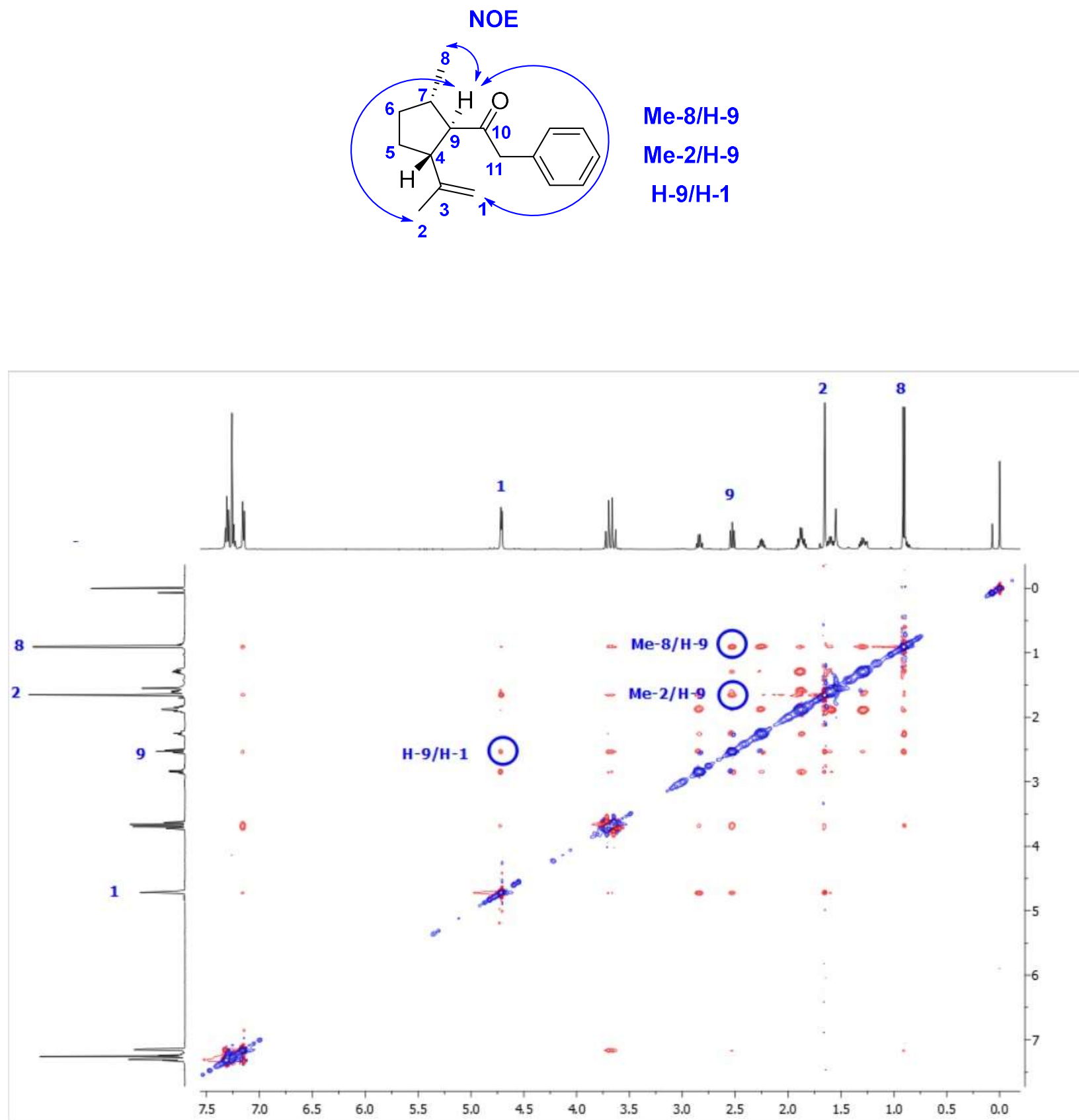


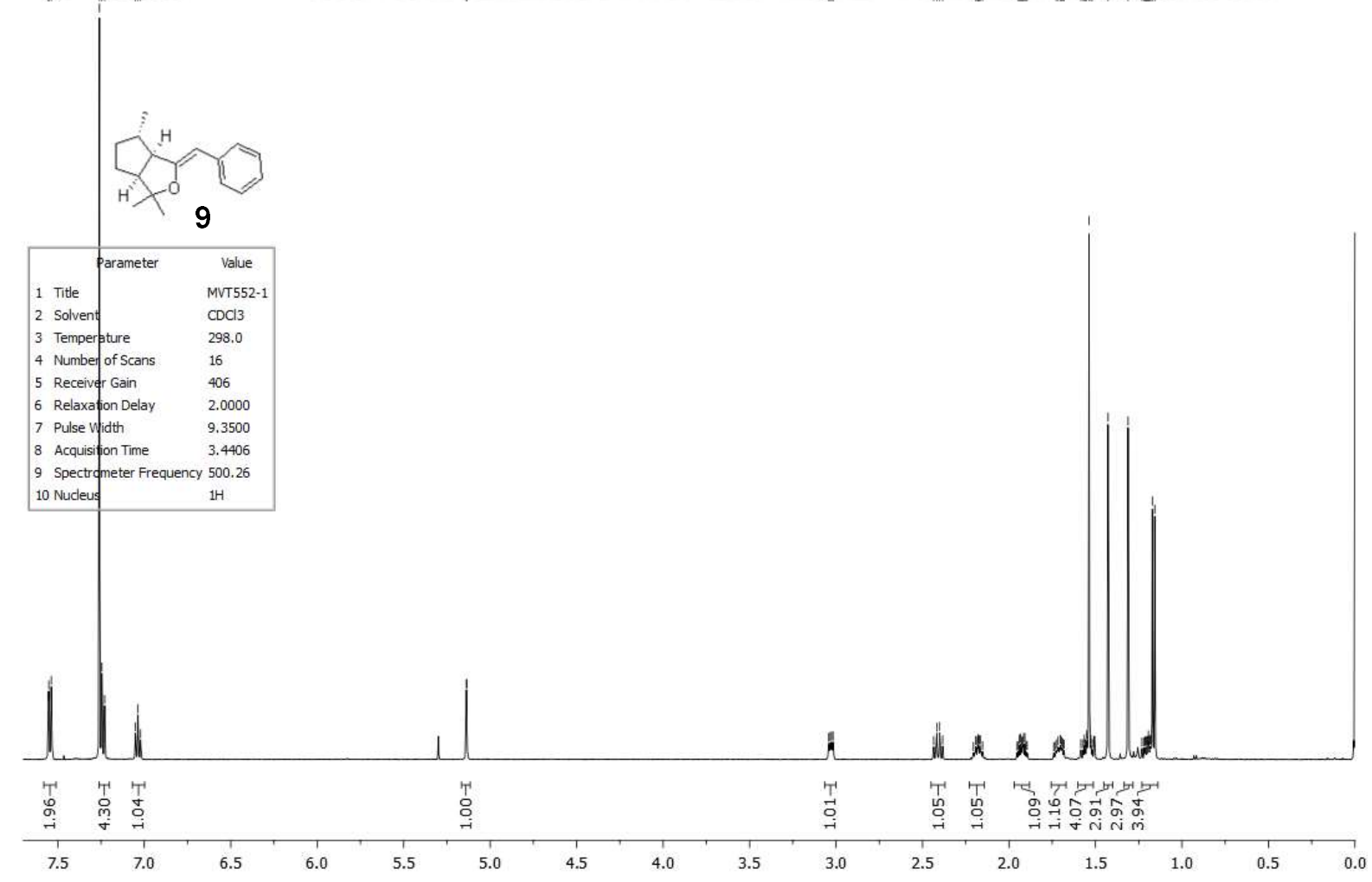

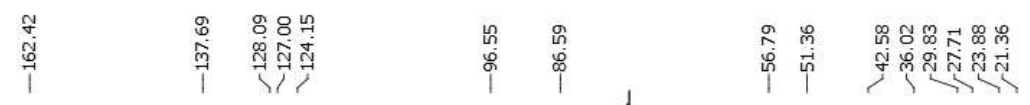
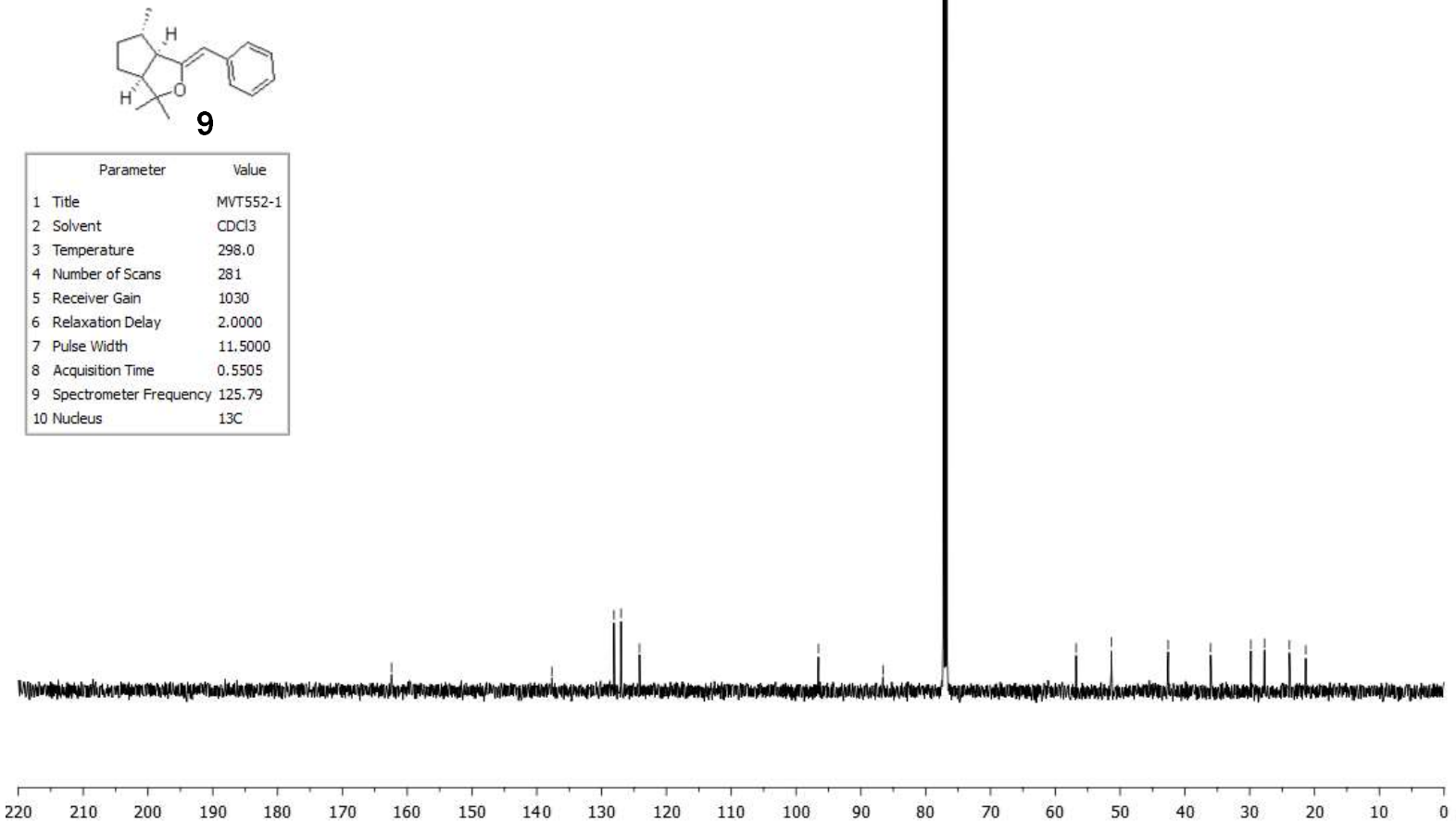

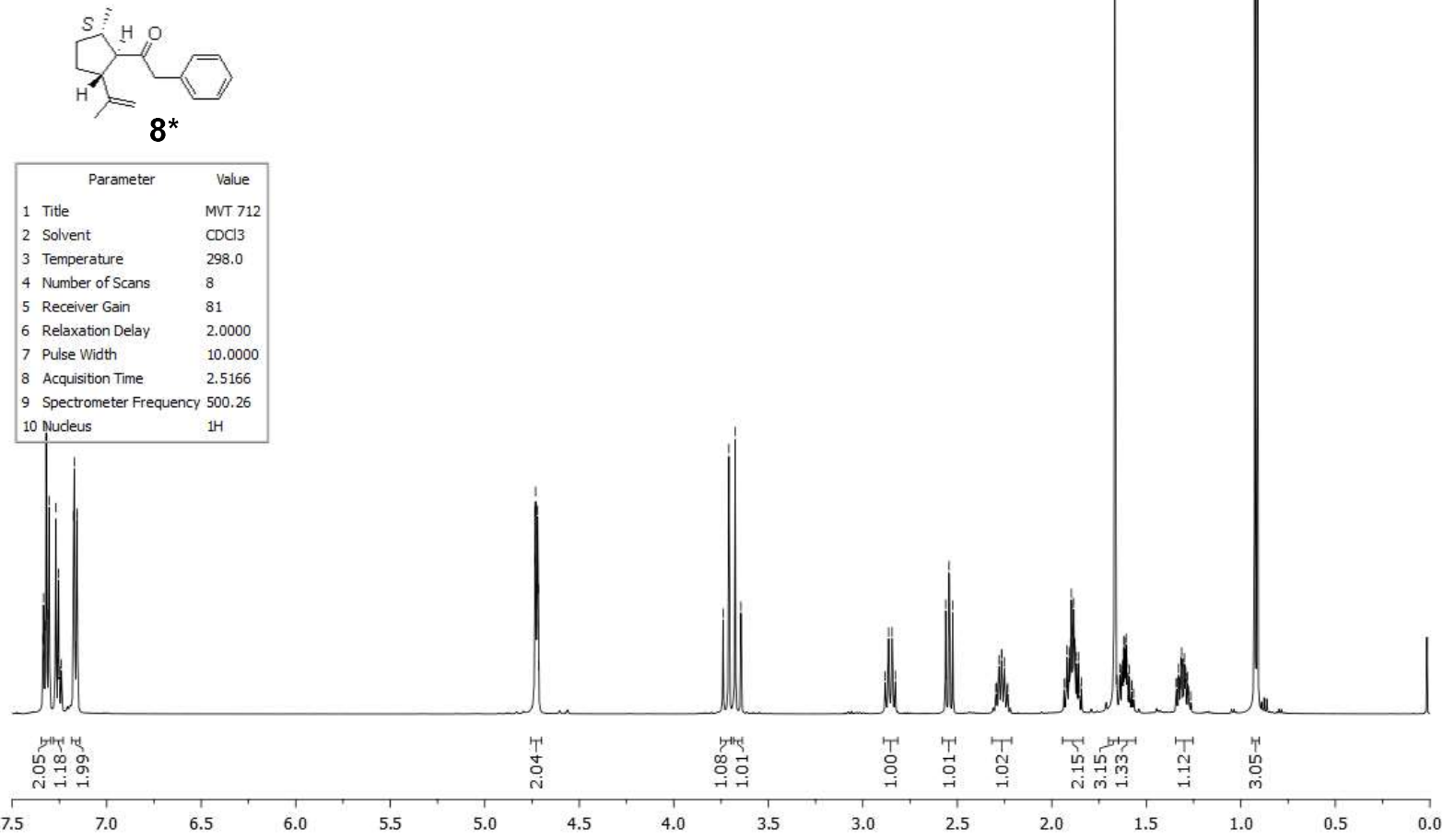

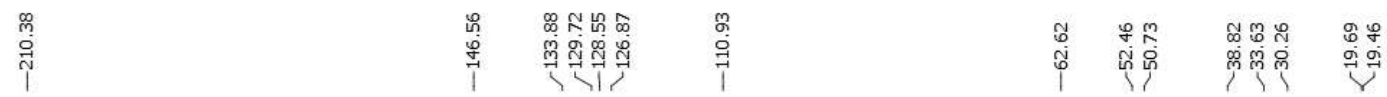

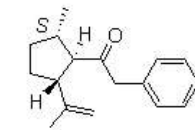

8*

\begin{tabular}{|lll|}
\hline \multicolumn{1}{|c}{ Parameter } & \multicolumn{1}{c|}{ Value } \\
1 & Title & MVT 712 \\
2 & Solvent & CDCl3 \\
3 & Temperature & 298.0 \\
4 & Number of Scans & 95 \\
5 & Receiver Gain & 203 \\
6 & Relaxation Delay & 2.0000 \\
7 & Pulse Width & 11.5000 \\
8 & Acquisition Time & 0.5505 \\
9 & Spectrometer Frequency & 125.79 \\
10 & Nucleus & $13 \mathrm{C}$ \\
\hline
\end{tabular}
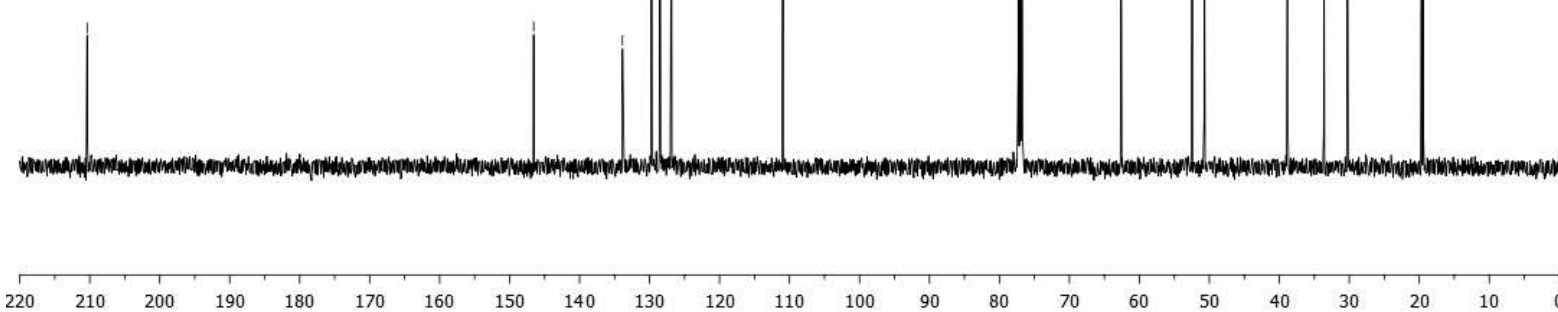
Correlations (NOESY) for $8 *$ :
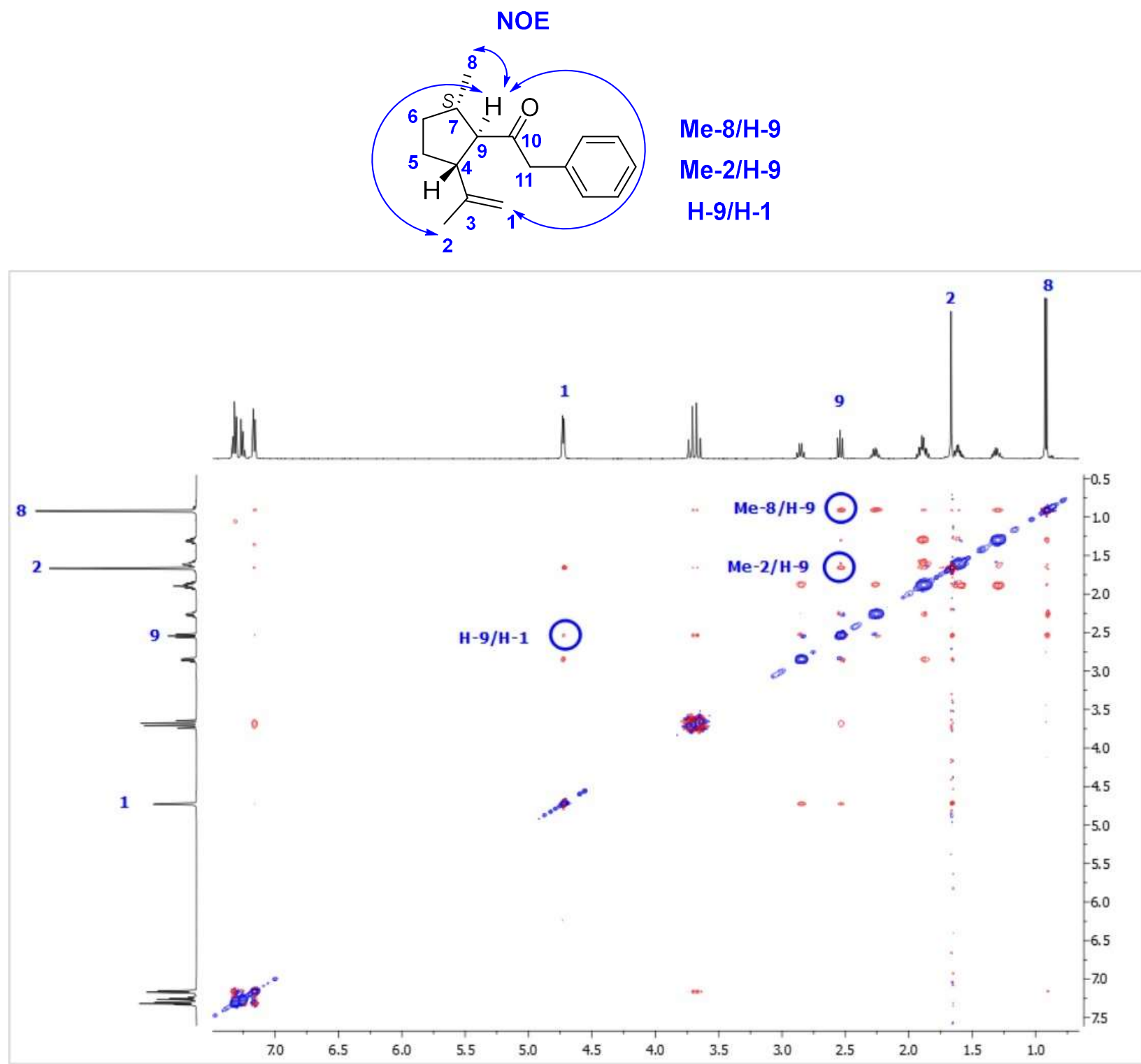

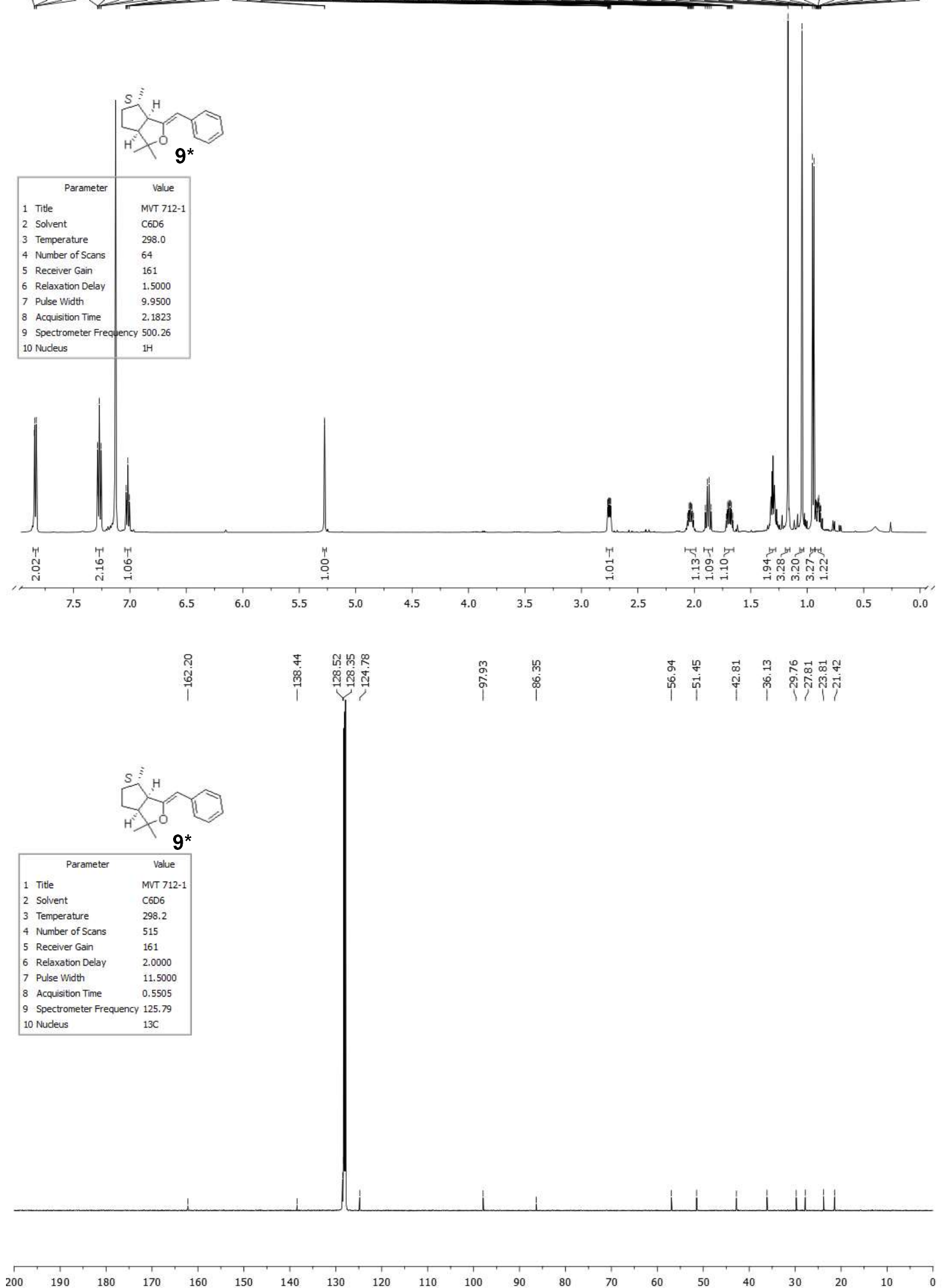

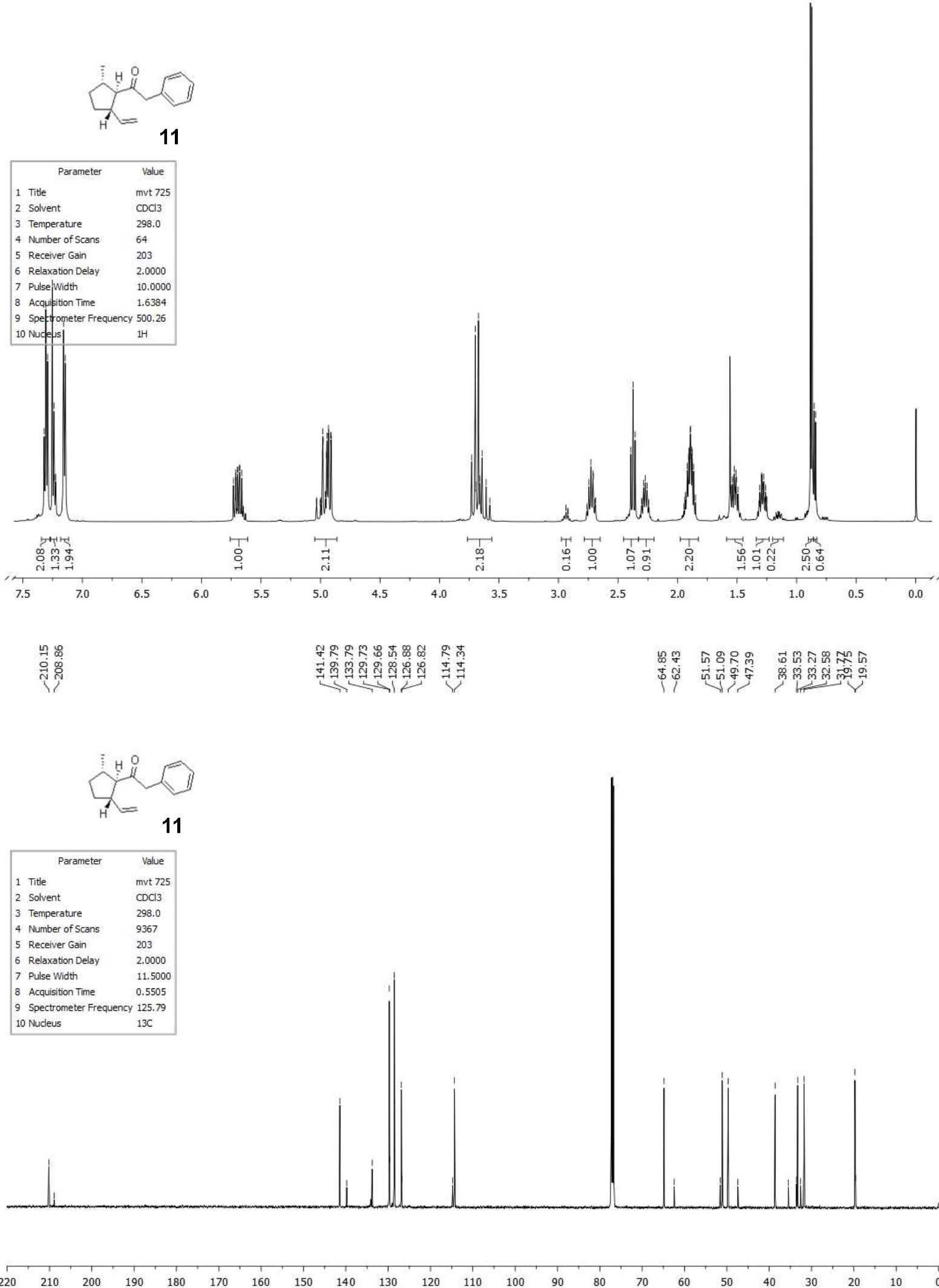


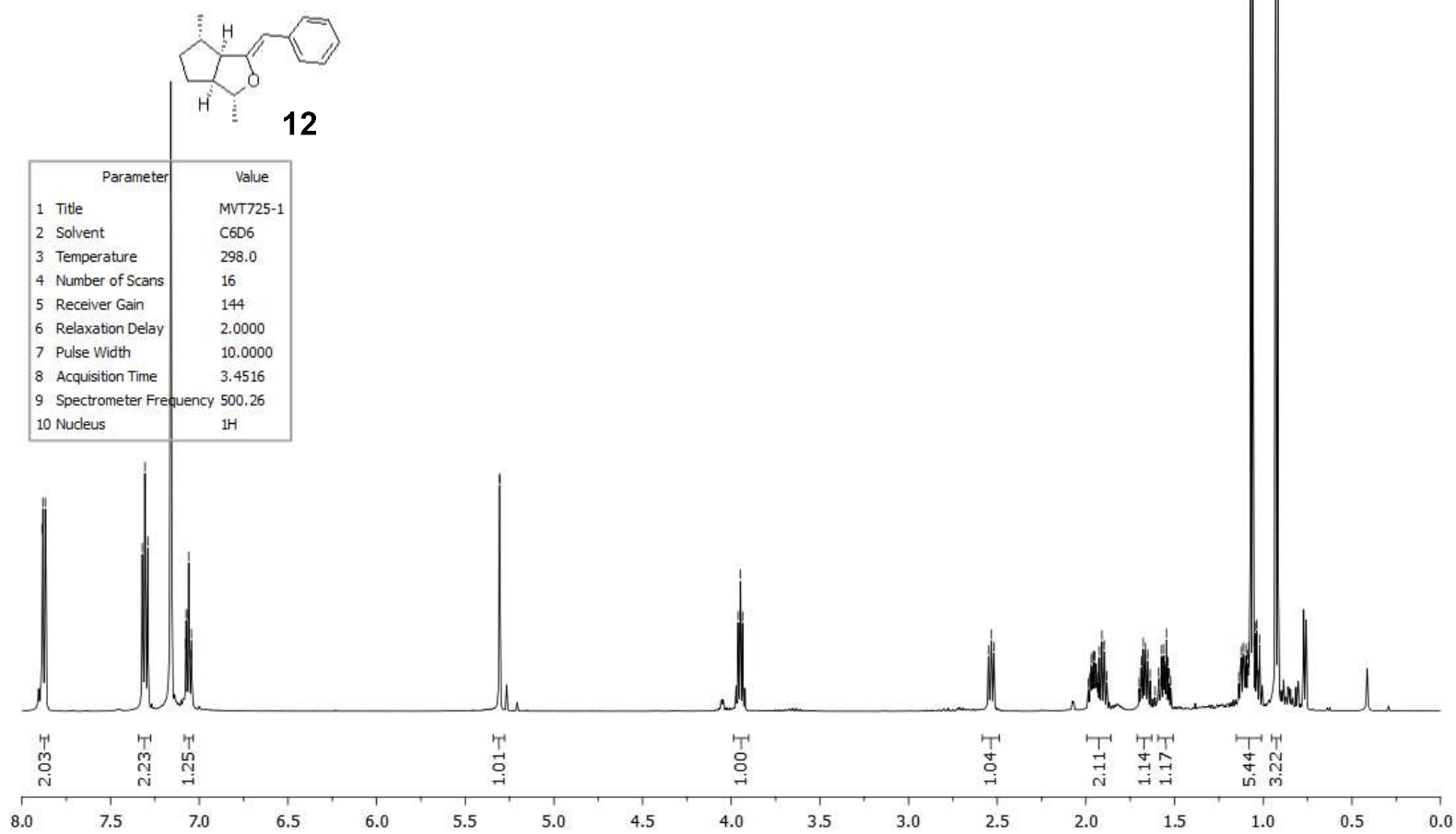

MVT 725-1

Value
MvT725-1
C606
298.0
341
203
2.0000
11.5000
0.5505
125.79
$13 C$

\begin{tabular}{|lll|}
\hline & \multicolumn{1}{c}{ Parameter } & \multicolumn{1}{c|}{ Value } \\
1 & Title & MVT725-1 \\
2 & Solvent & C6D6 \\
3 & Temperature & 298.0 \\
4 & Number of Scans & 341 \\
5 & Receiver Gain & 203 \\
6 & Relaxation Delay & 2.0000 \\
7 & Pulse Width & 11.5000 \\
8 & Acquisition Time & 0.5505 \\
9 & Spectrometer Frequency & 125.79 \\
10 & Nucleus & $13 \mathrm{C}$ \\
\hline & &
\end{tabular}



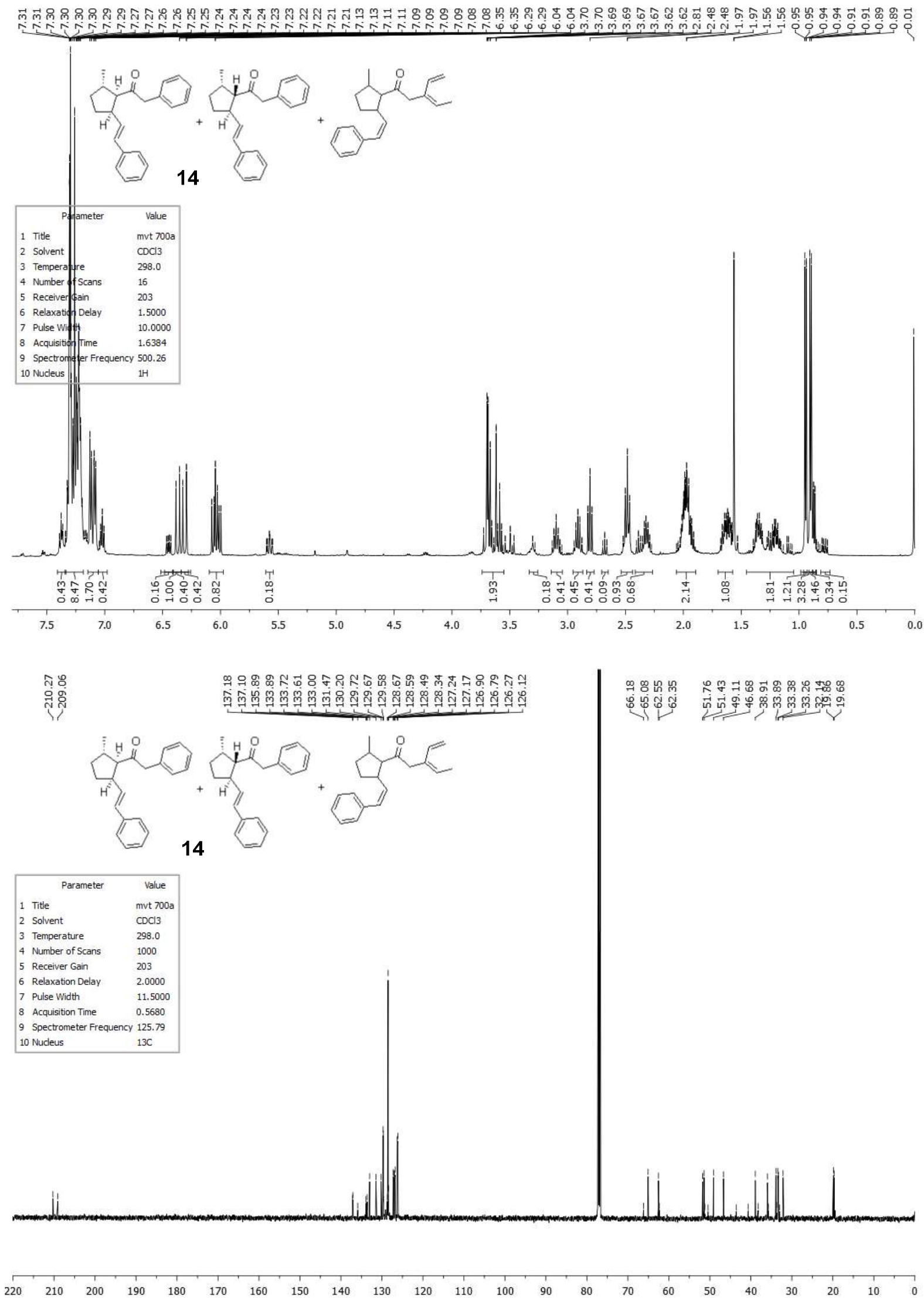

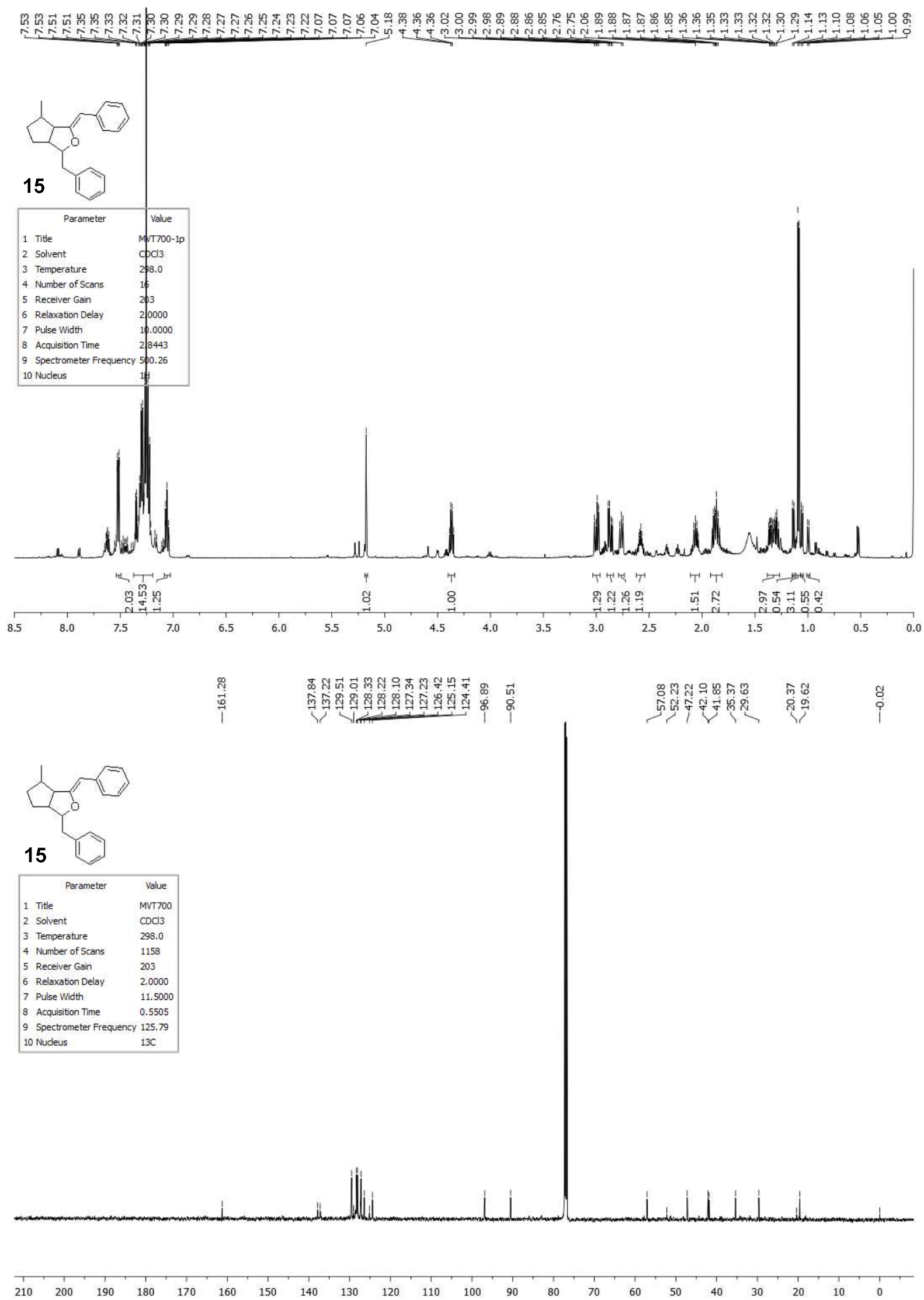

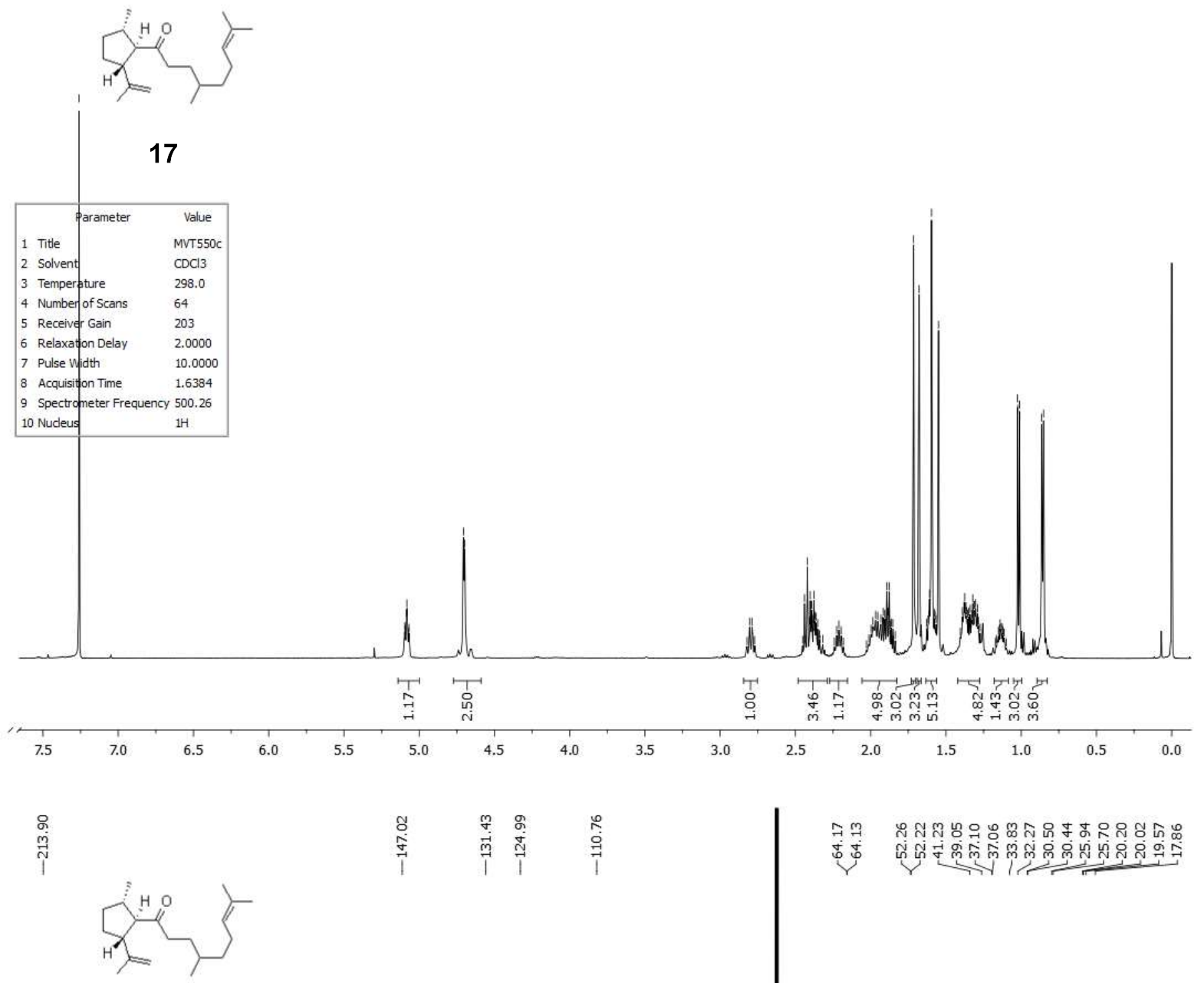

17

\begin{tabular}{|lll|}
\hline & \multicolumn{1}{|c|}{ Parameter } & \multicolumn{1}{c|}{ Value } \\
1 & Title & MVT550C \\
2 & Solvent & $\mathrm{CDCl} 3$ \\
3 & Temperature & 298.0 \\
4 & Number of Scans & 10055 \\
5 & Receiver Gain & 203 \\
6 & Relaxation Delay & 2.0000 \\
7 & Pulse Width & 11.5000 \\
8 & Acquisition Time & 0.5505 \\
9 & Spectrometer Frequency & 125.79 \\
10 & Nucleus & $13 \mathrm{C}$ \\
\hline
\end{tabular}
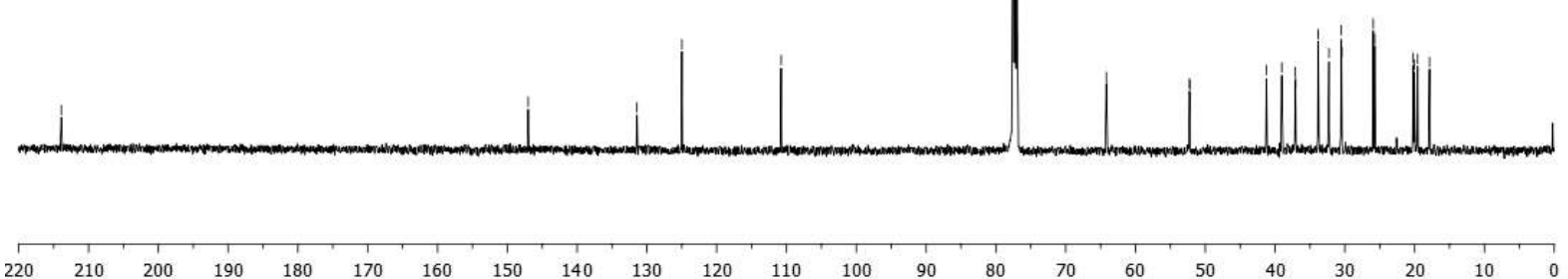

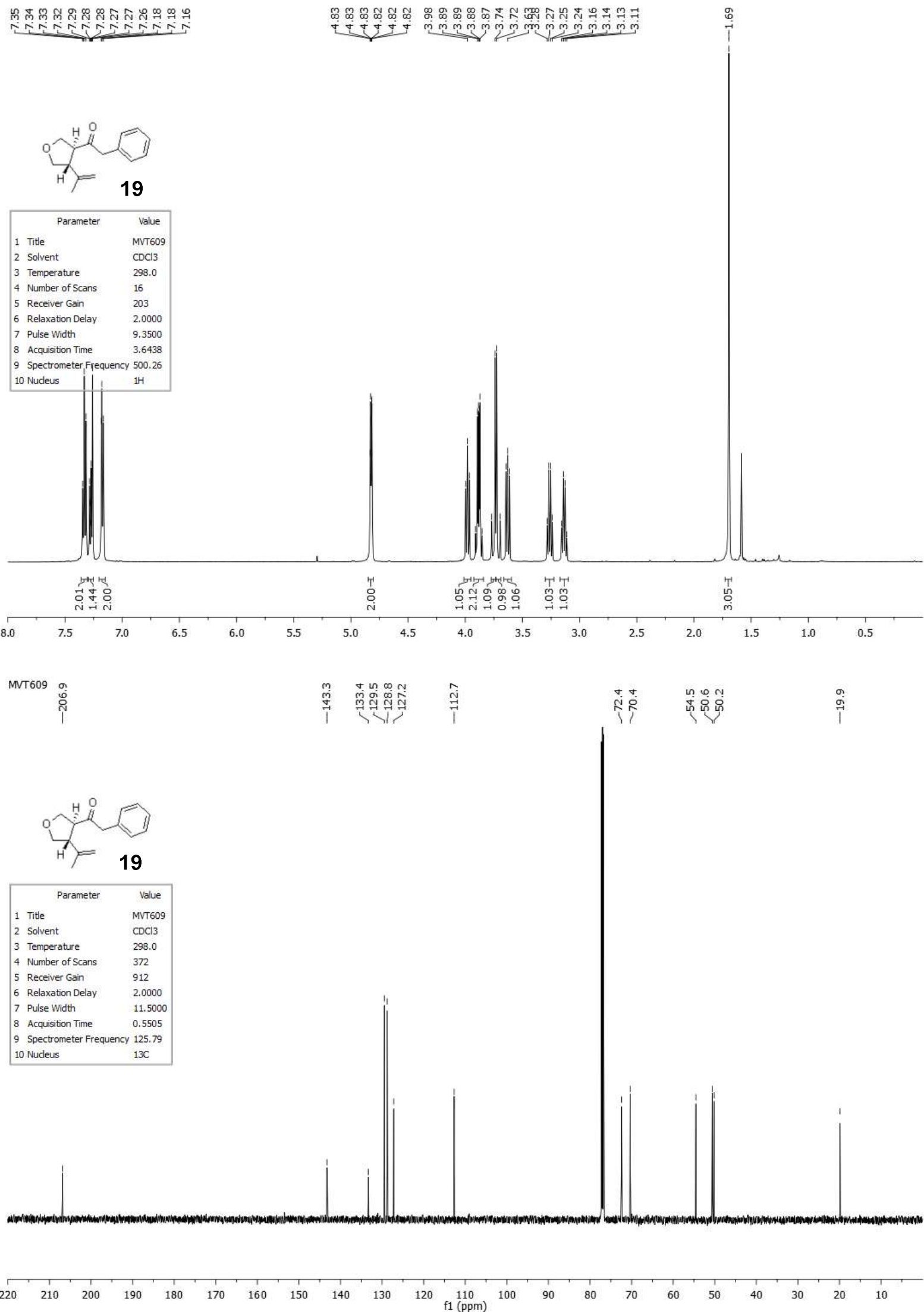


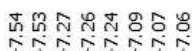

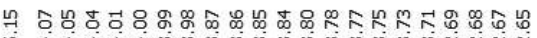

फ

il

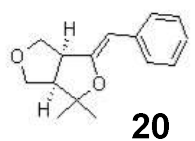

20

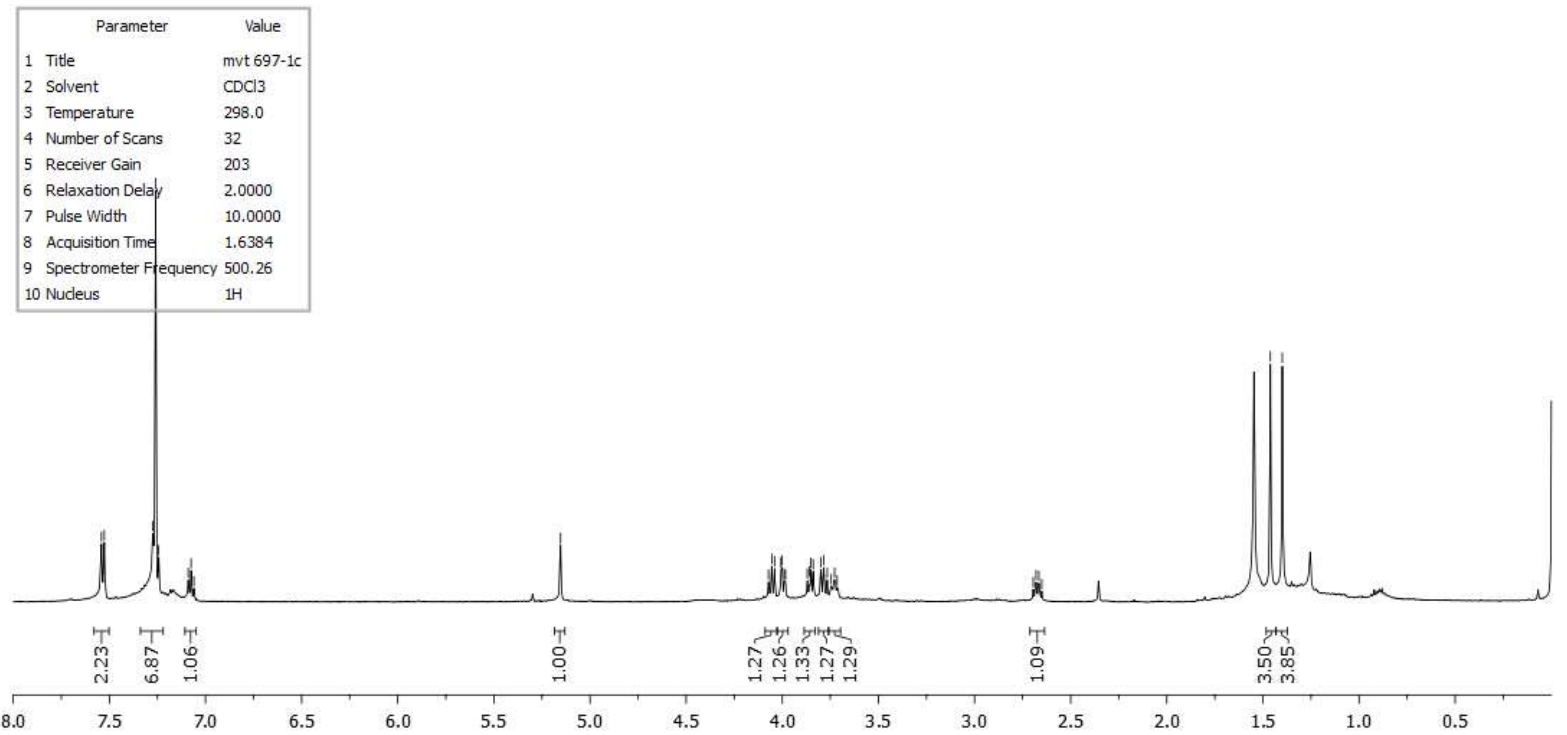

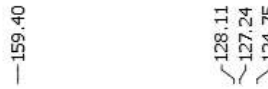

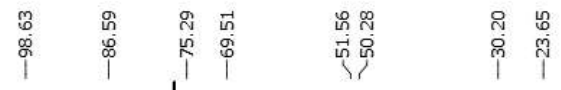

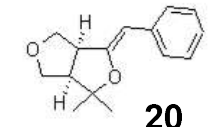

20

\begin{tabular}{|lll|}
\hline \multicolumn{1}{|c|}{ Parameter } & \multicolumn{1}{c|}{ Value } \\
1 & Title & mvt $697-1 \mathrm{C}$ \\
2 & Solvent & $\mathrm{CDCl} 3$ \\
3 & Temperature & 298.0 \\
4 & Number of Scans & 2852 \\
5 & Receiver Gain & 203 \\
6 & Relaxation Delay & 1.5000 \\
7 & Pulse Width & 11.5000 \\
8 & Acquisition Time & 0.5680 \\
9 & Spectrometer Frequency 125.79 \\
10 & Nucleus & $13 \mathrm{C}$ \\
\hline
\end{tabular}
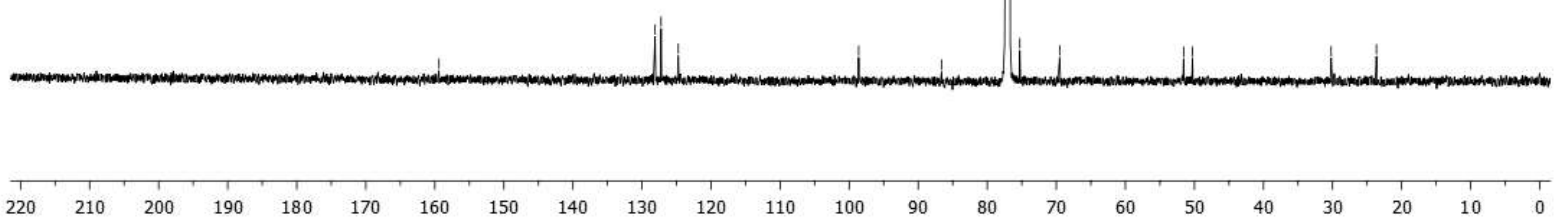


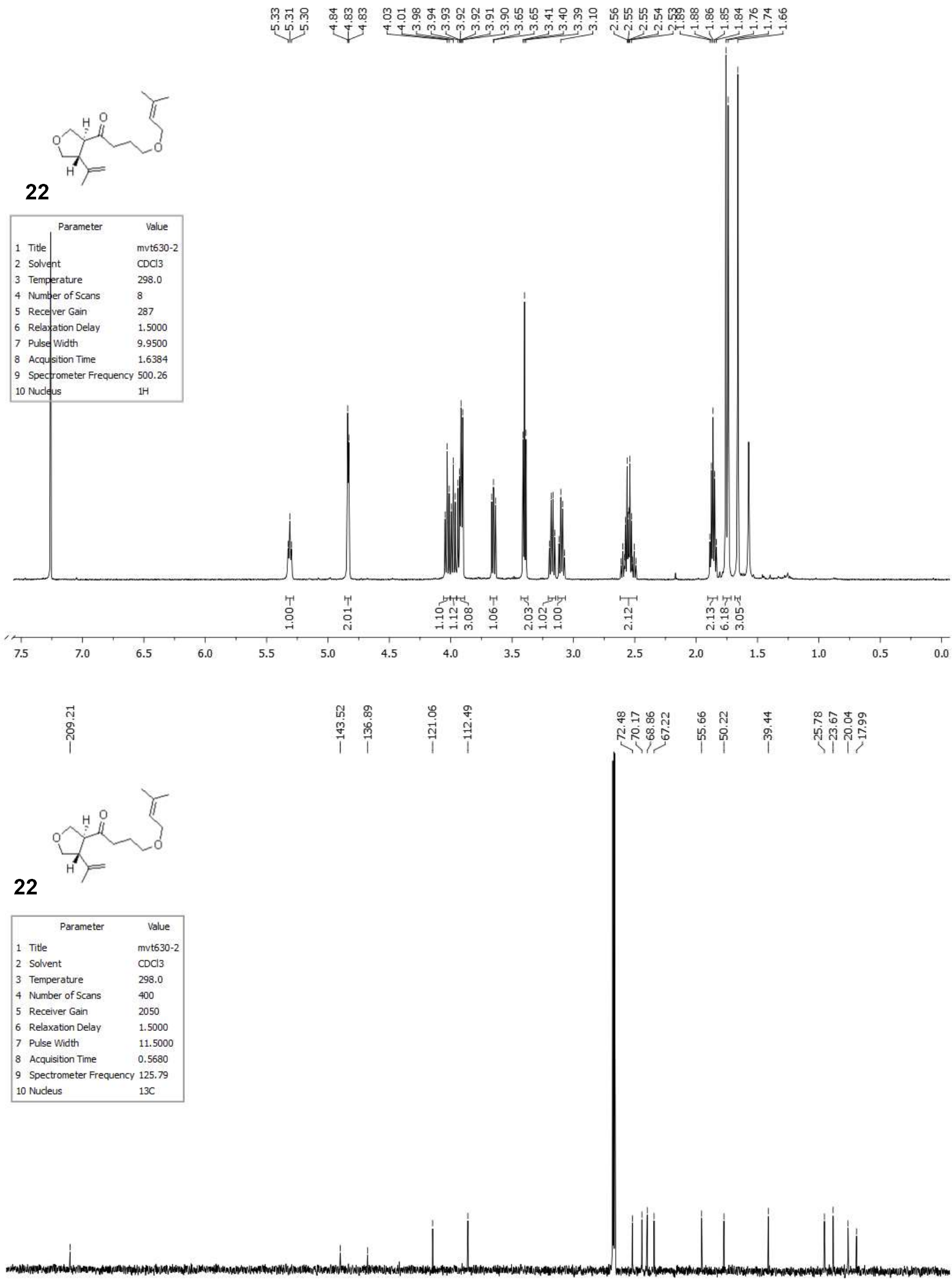

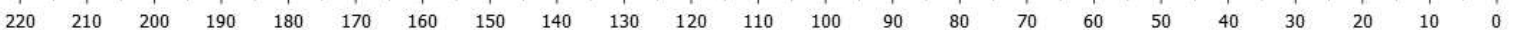



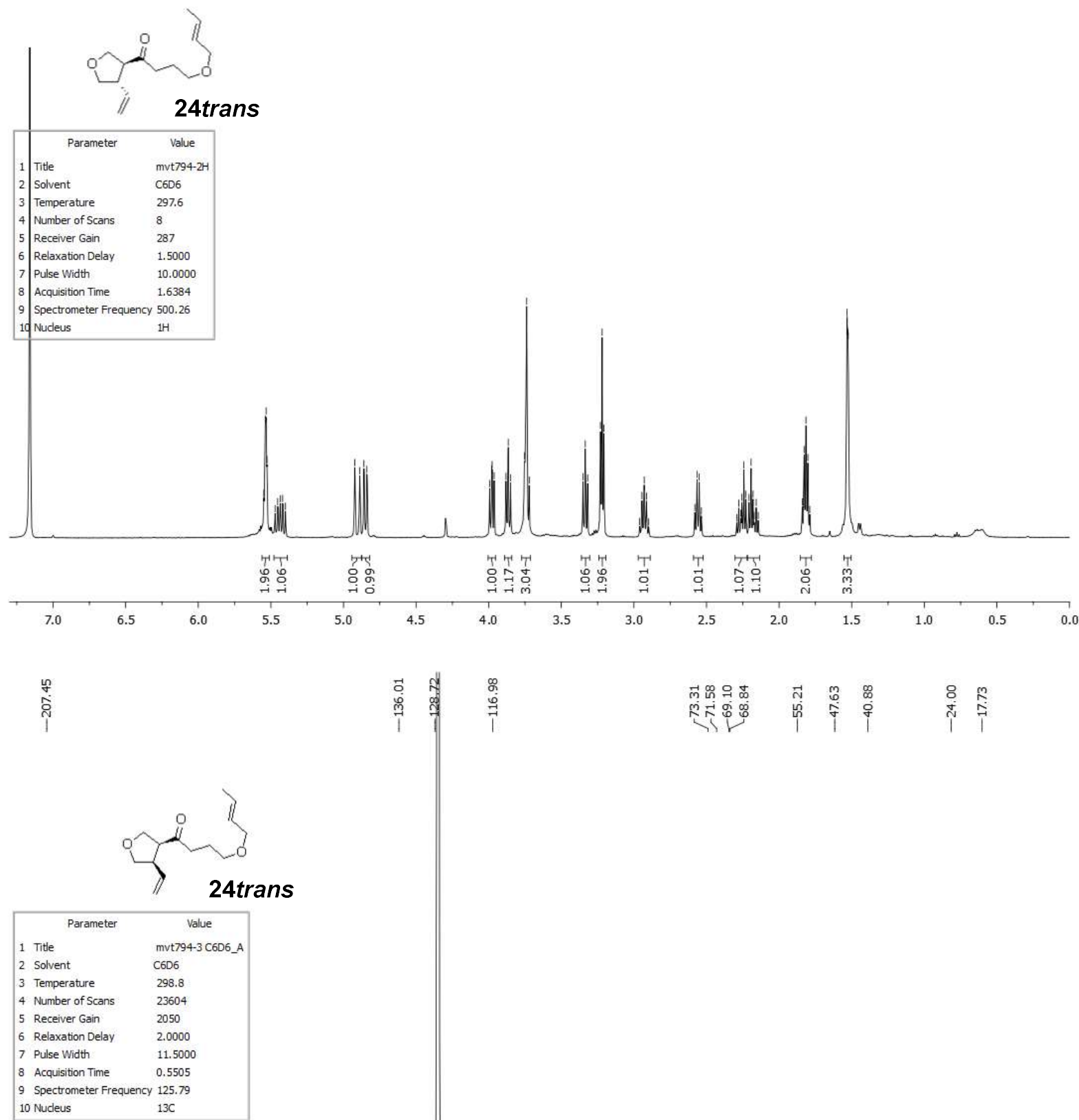

\section{4trans}


Correlations (NOESY) of 24trans:
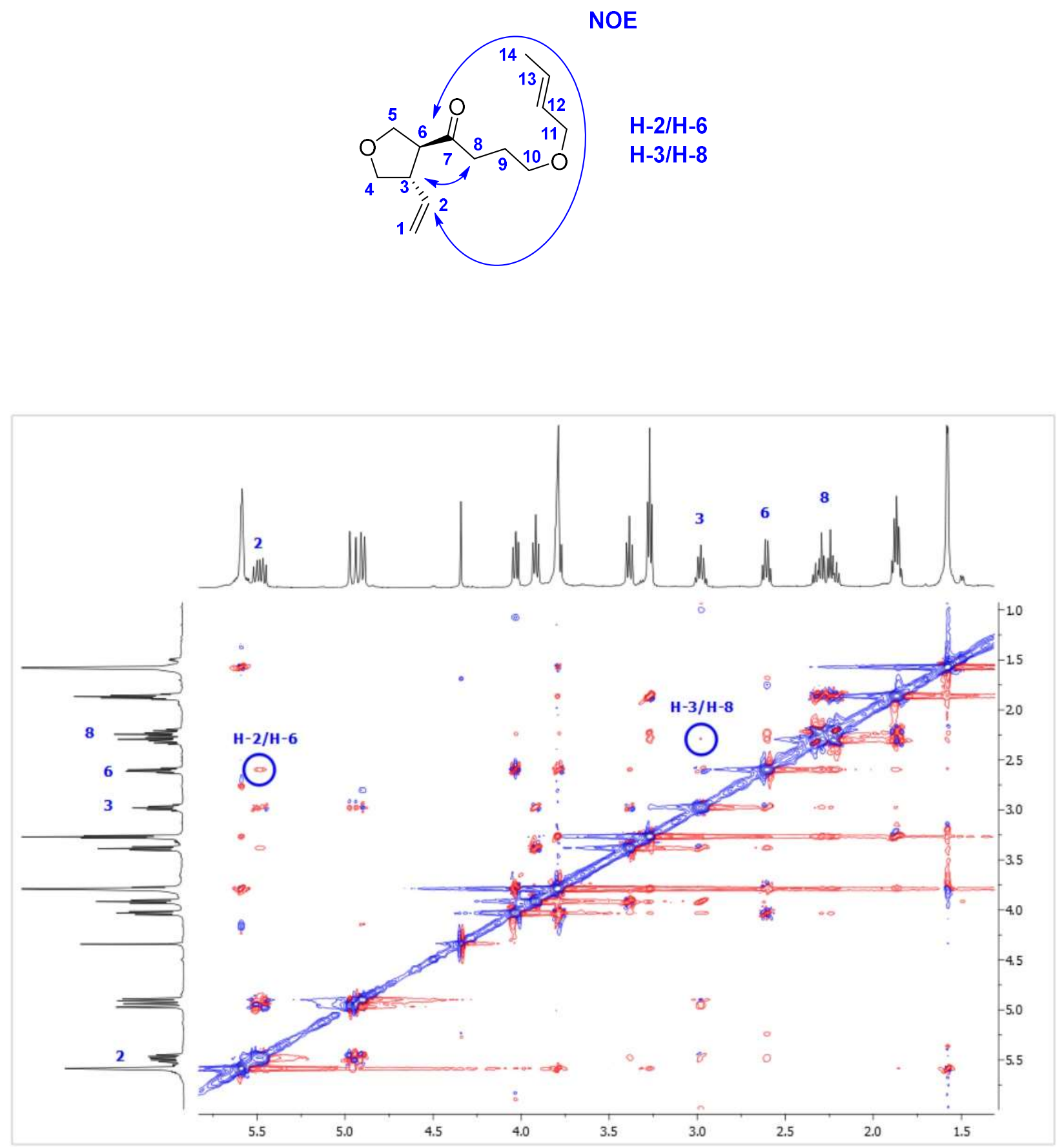

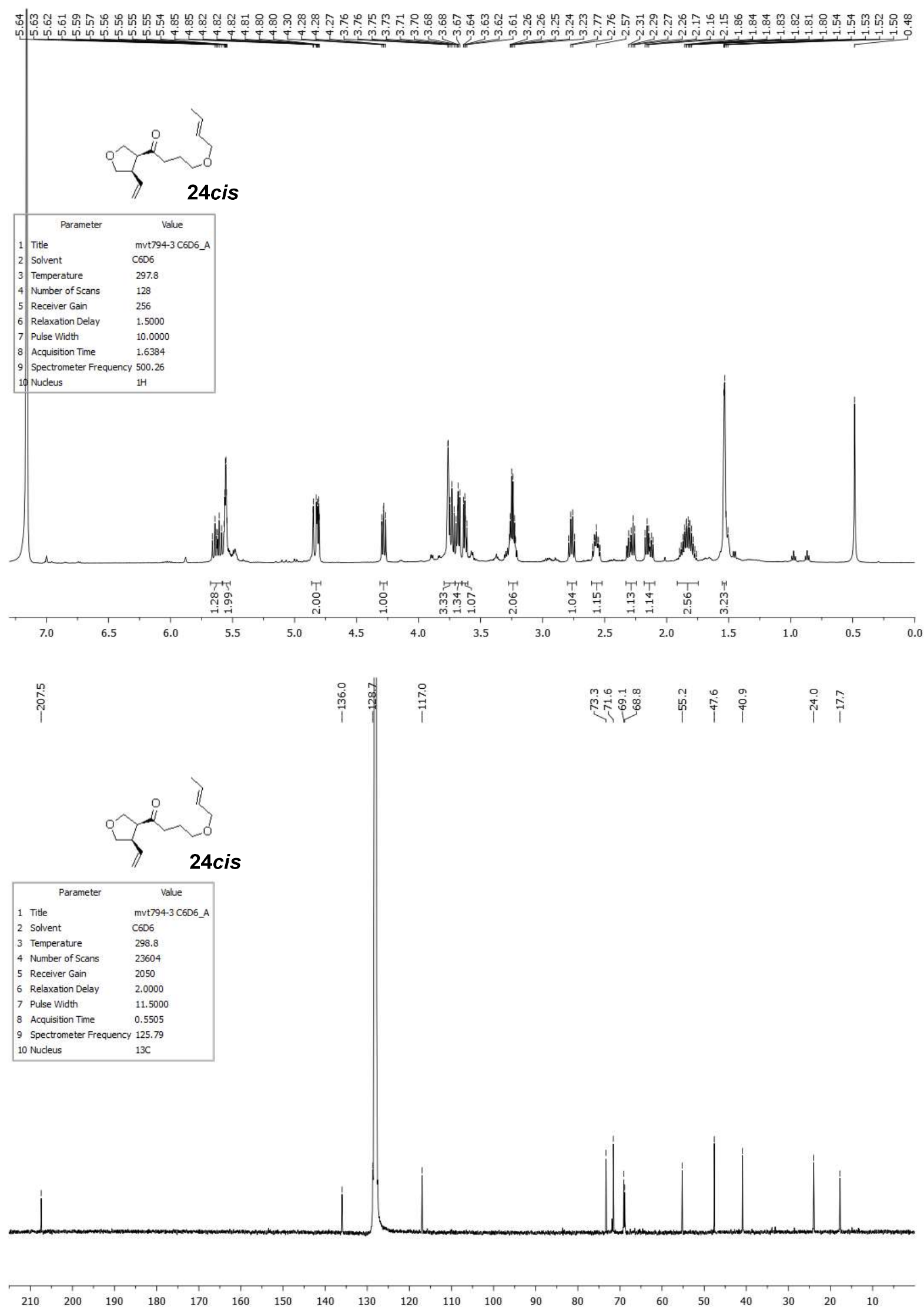
Correlations (NOESY) of 24cis:
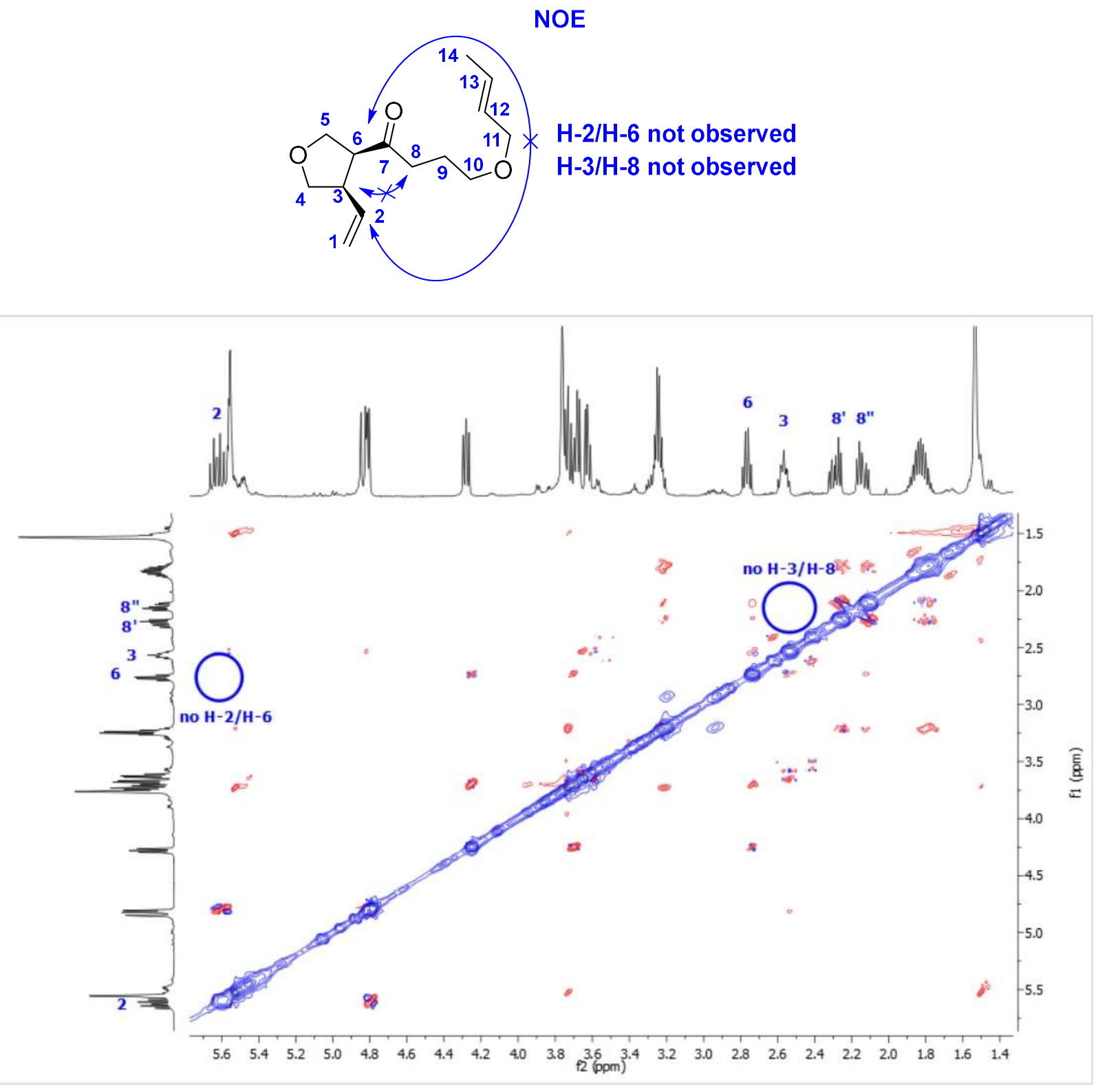


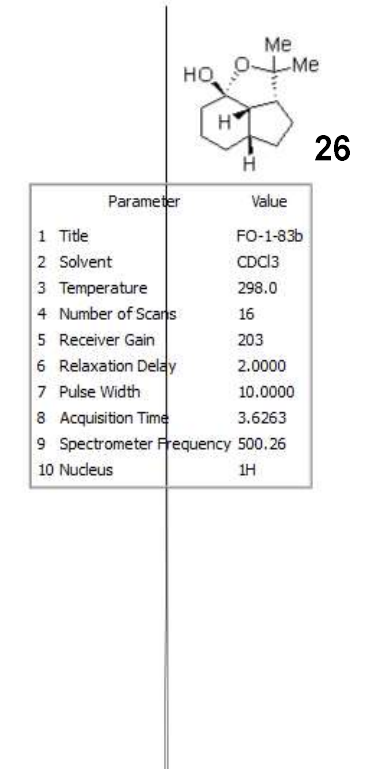

26
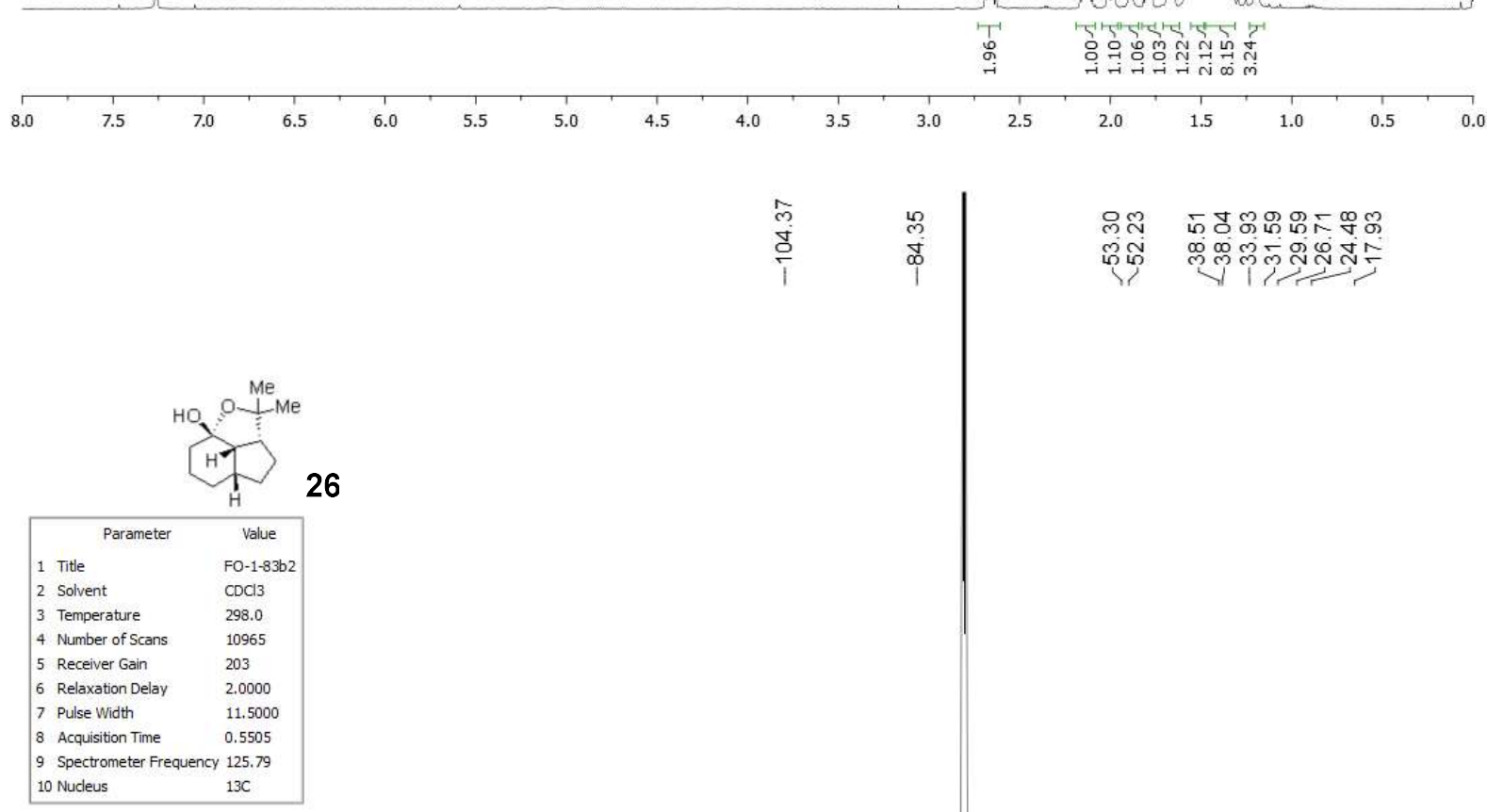

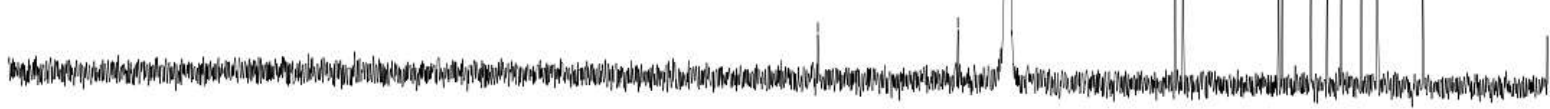

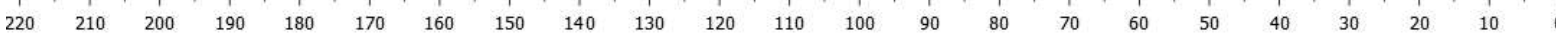




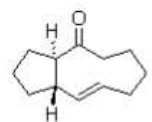

28
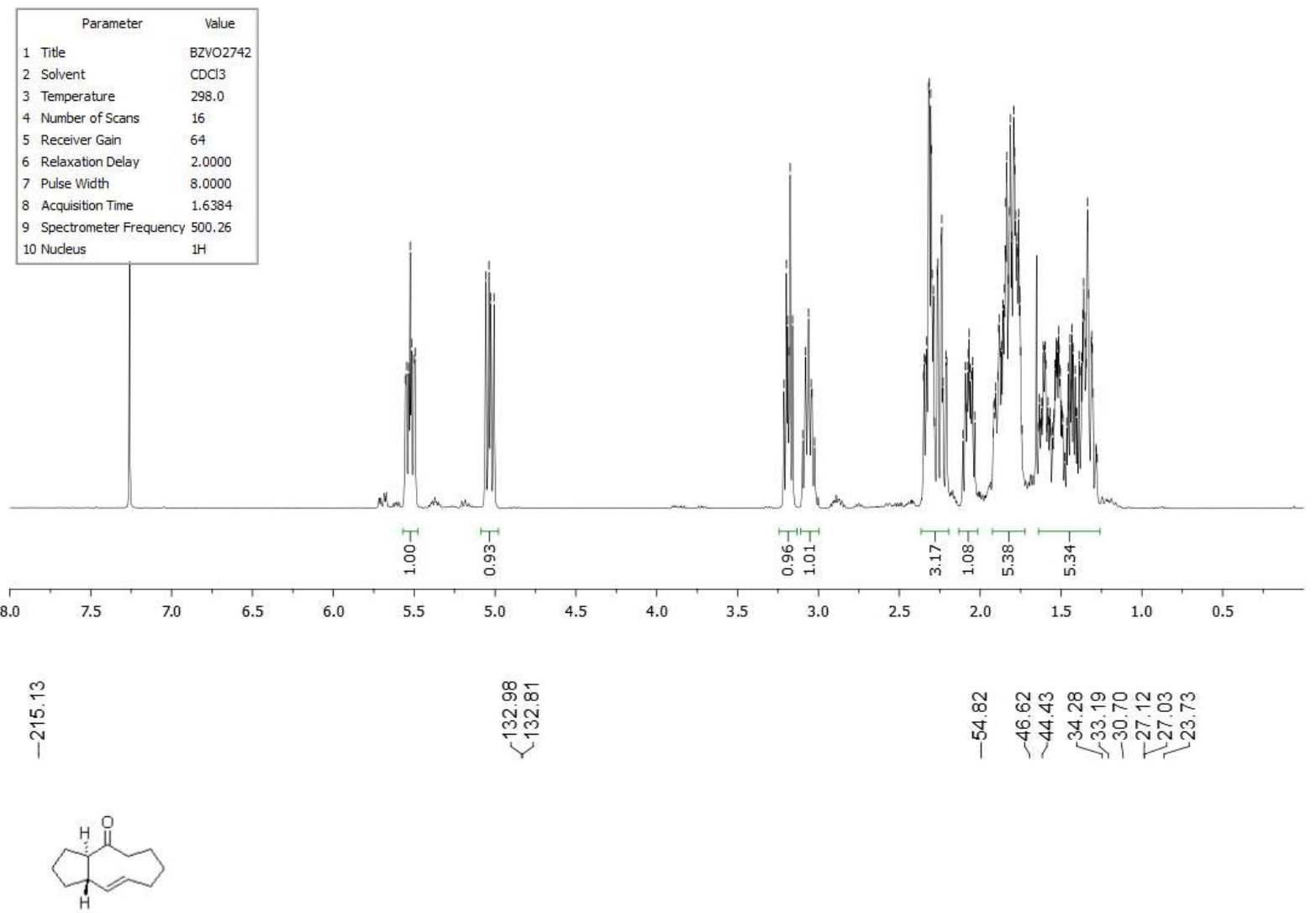

28

\begin{tabular}{|lll|}
\hline \multicolumn{1}{|c|}{ Parameter } & \multicolumn{1}{c|}{ Value } \\
1 & Title & BZvO2742 \\
2 & Solvent & \multicolumn{1}{c|}{$\mathrm{CDCl} 3$} \\
3 & Temperature & 298.0 \\
4 & Number of Scans & 512 \\
5 & Receiver Gain & 203 \\
6 & Relaxation Delay & 2.0000 \\
7 & Pulse Width & 15.2500 \\
8 & Acquisition Time & 0.5505 \\
9 & Spectrometer Frequency & 125.79 \\
10 & Nudeus & $13 \mathrm{C}$ \\
\hline
\end{tabular}

10 Nucleus $13 \mathrm{C}$

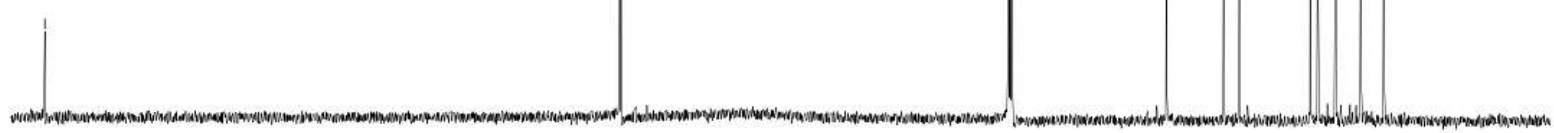

$\begin{array}{lllllllllllllllllllllll}220 & 210 & 200 & 190 & 180 & 170 & 160 & 150 & 140 & 130 & 120 & 110 & 100 & 90 & 80 & 70 & 60 & 50 & 40 & 30 & 20 & 10 & \end{array}$ 
Correlations (NOESY) for $\mathbf{2 8}$ :
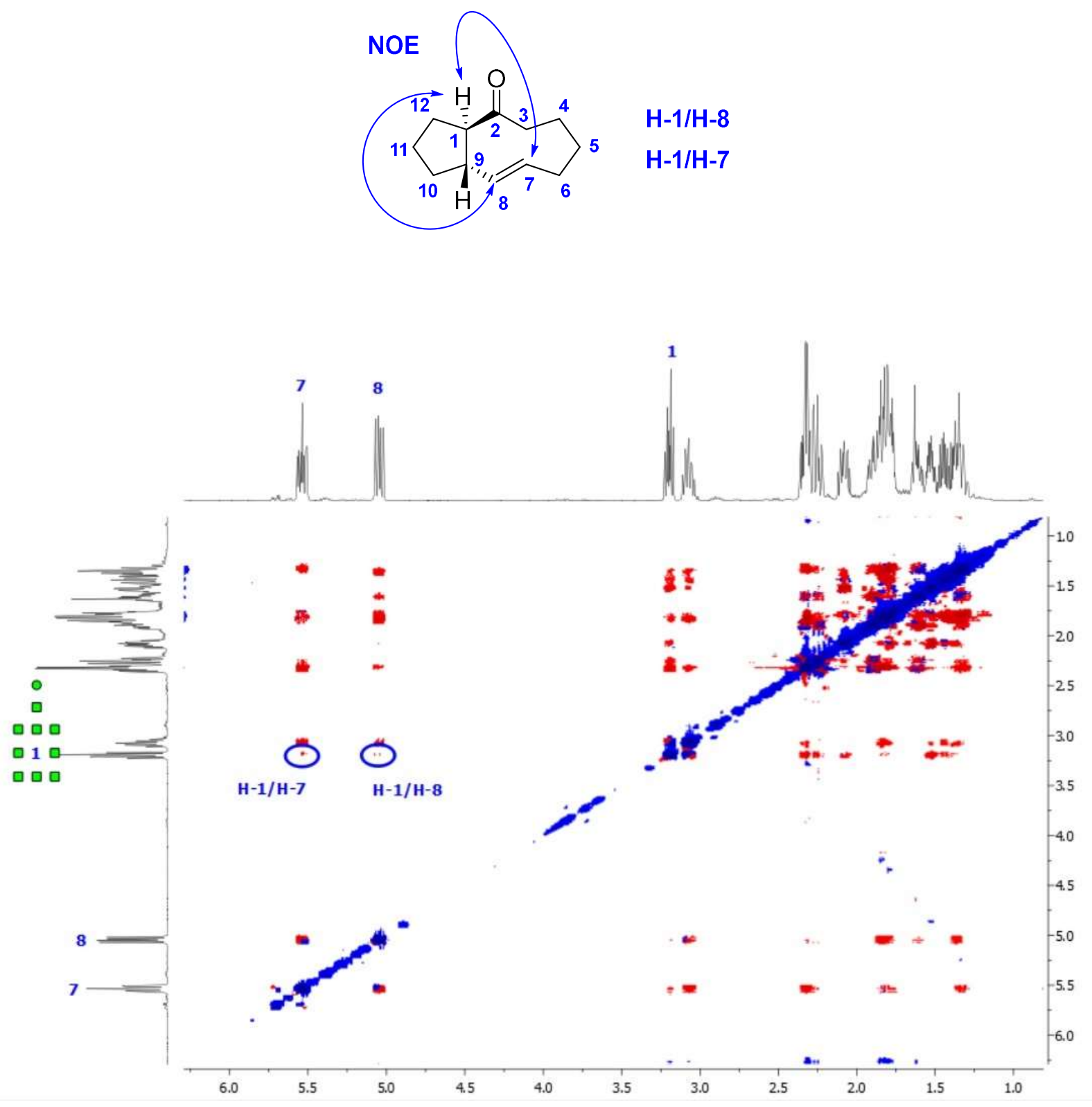

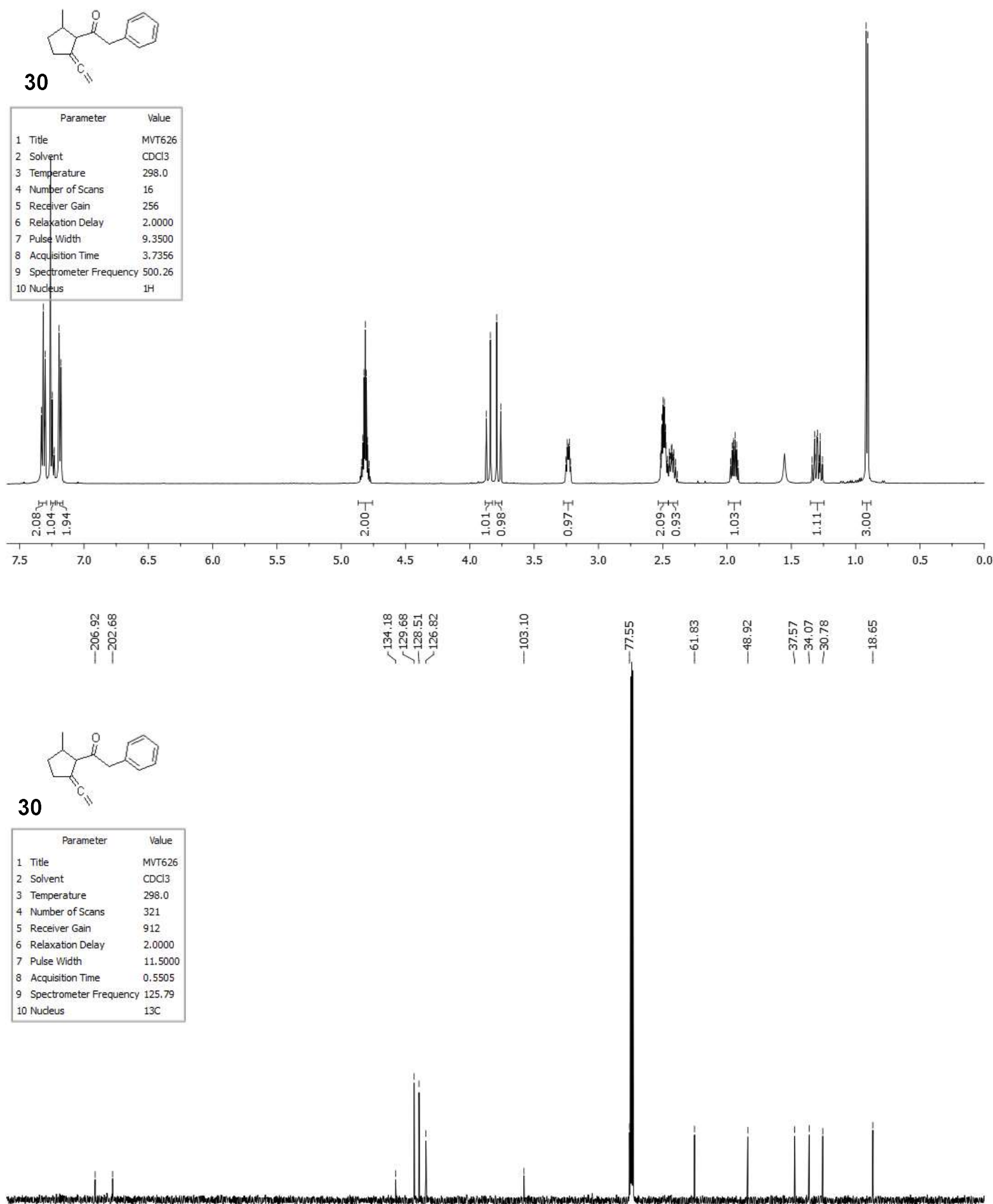

$\begin{array}{lllllllllllllllllllllll}220 & 210 & 200 & 190 & 180 & 170 & 160 & 150 & 140 & 130 & 120 & 110 & 100 & 90 & 80 & 70 & 60 & 50 & 40 & 30 & 20 & 10 & 0\end{array}$ 

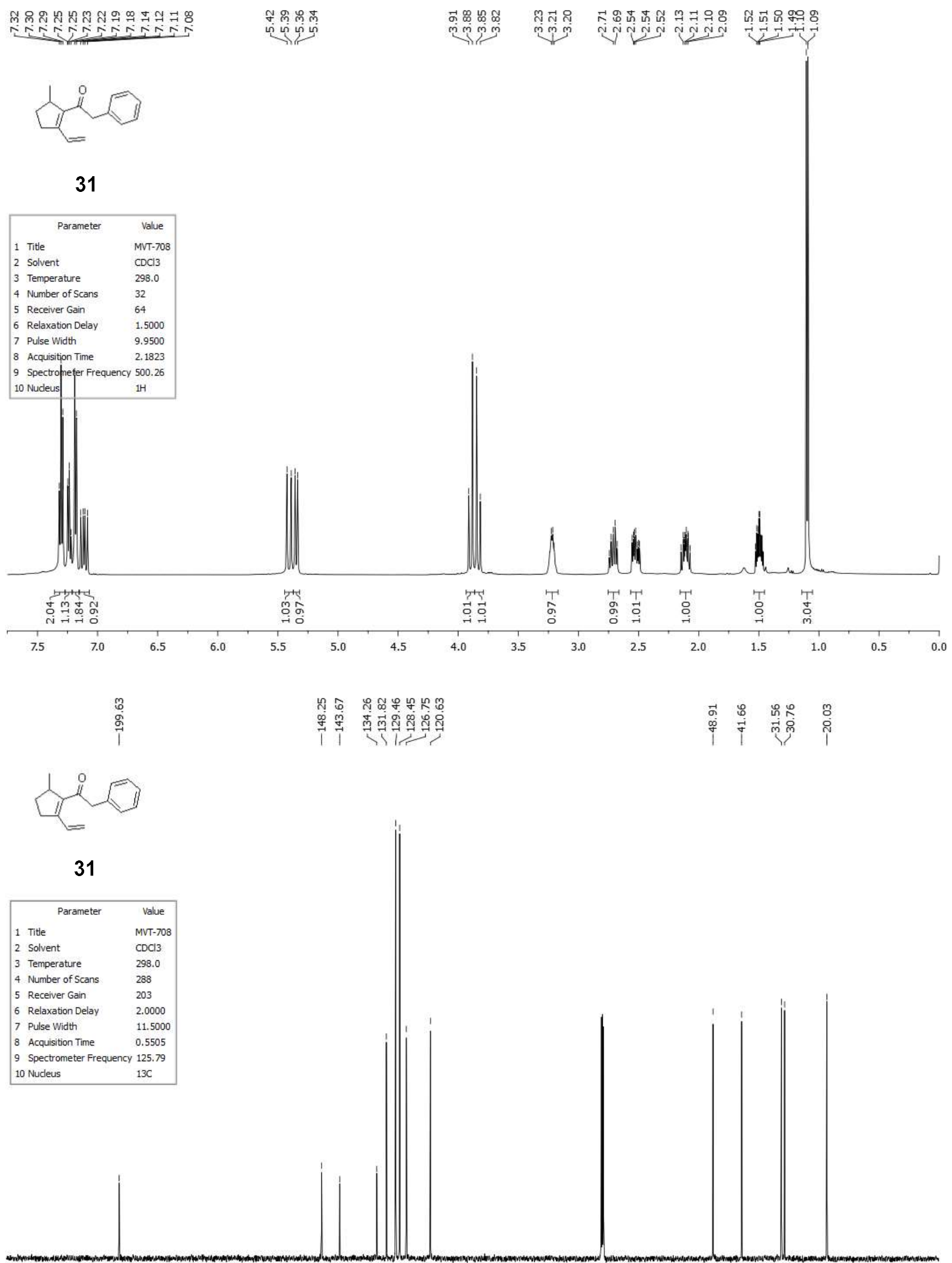

$\begin{array}{llllllllllllllllllllllll}220 & 210 & 200 & 190 & 180 & 170 & 160 & 150 & 140 & 130 & 120 & 110 & 100 & 90 & 80 & 70 & 60 & 50 & 40 & 30 & 20 & 10 & 0 & \end{array}$ 


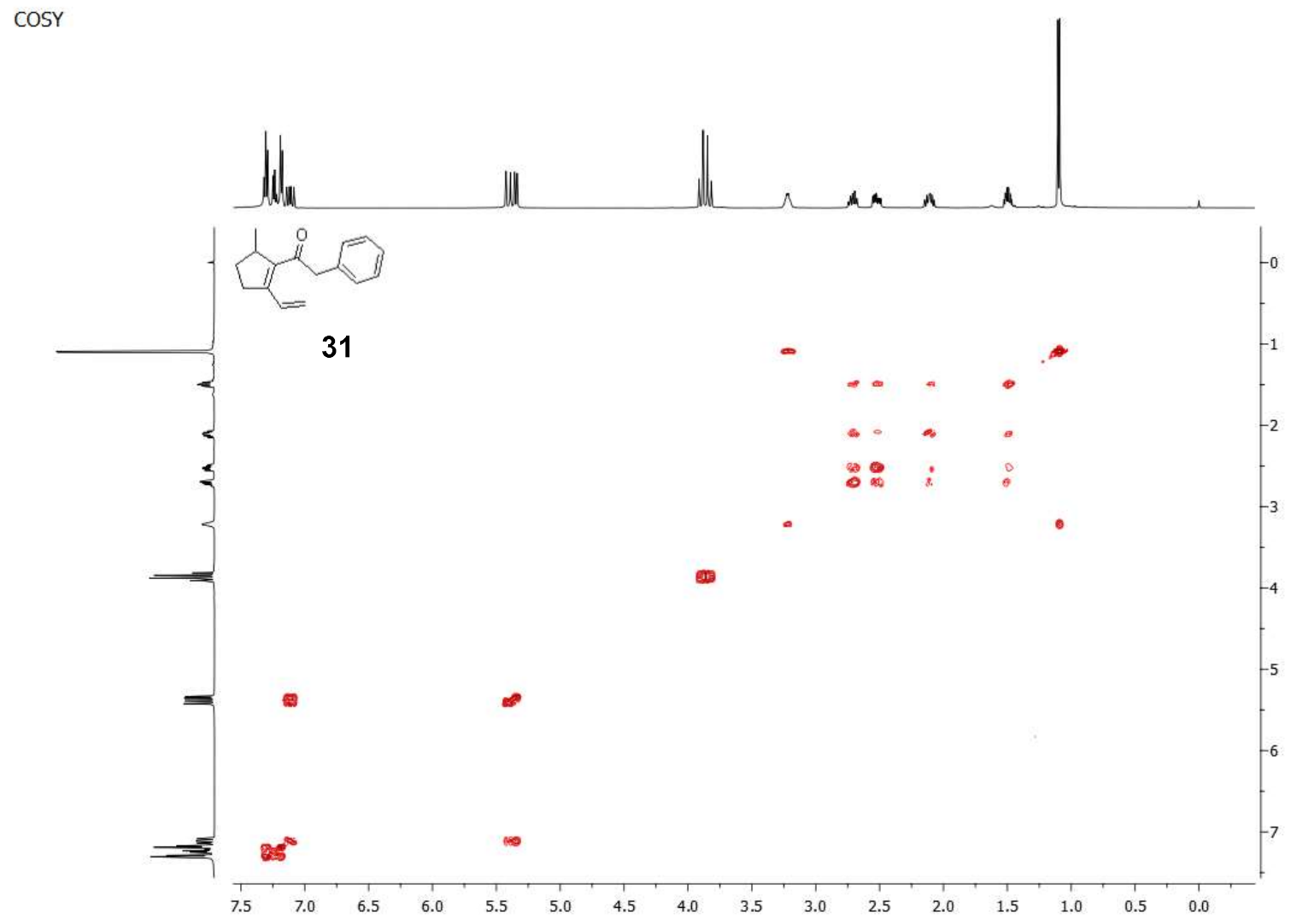

HSQC

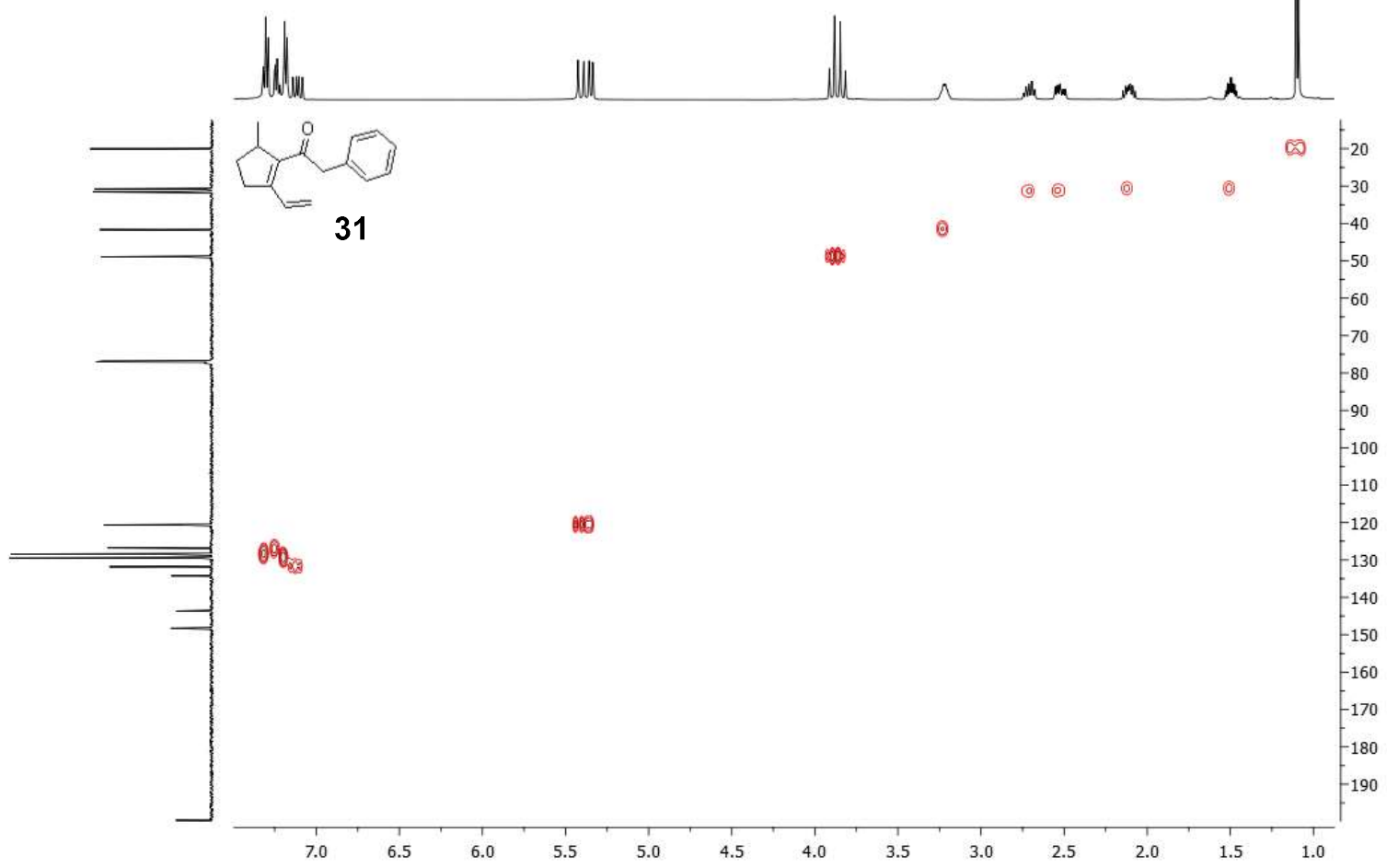



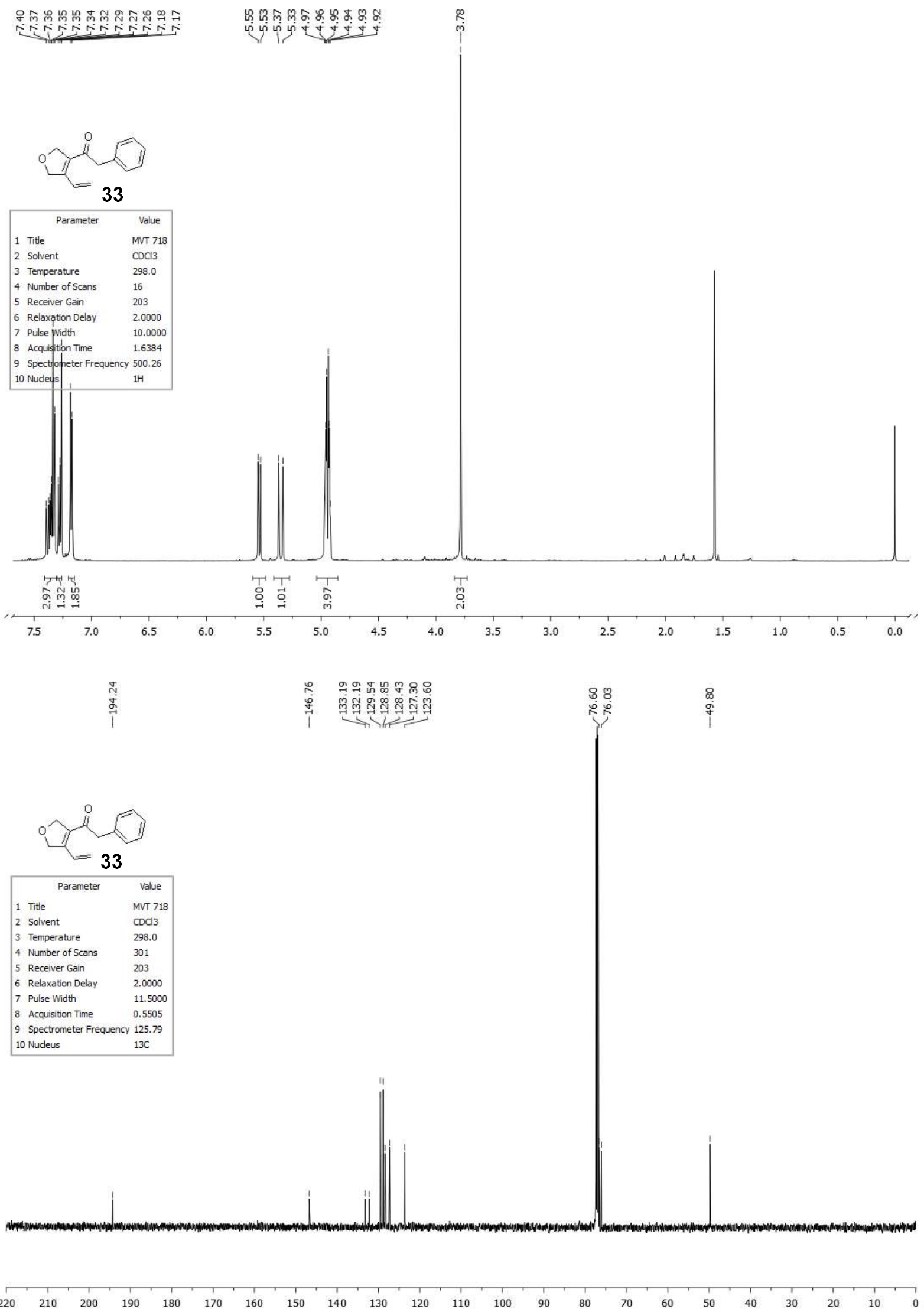


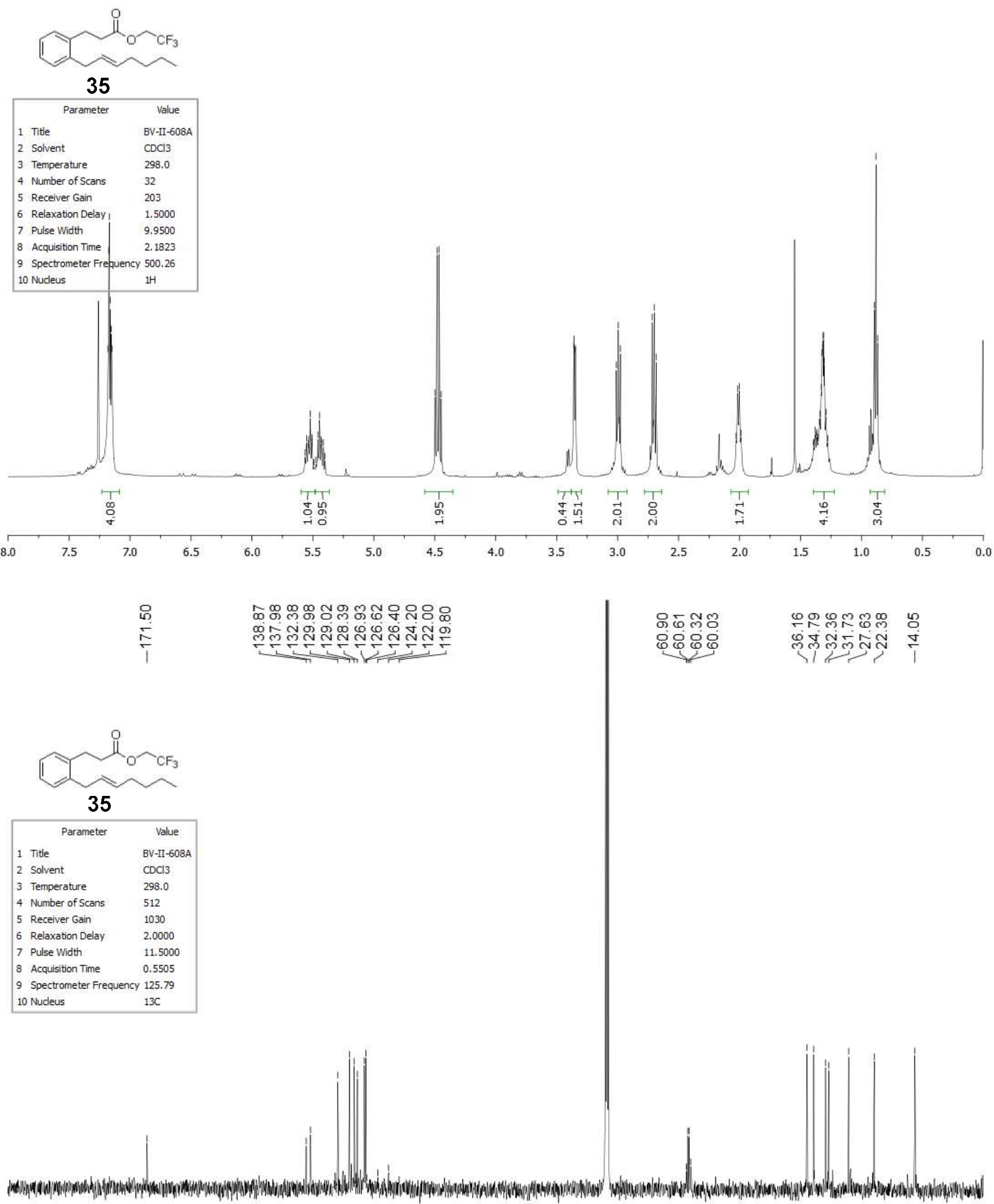

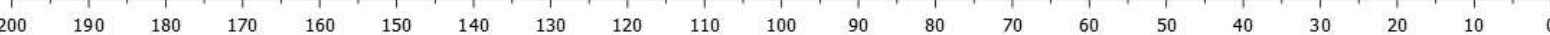



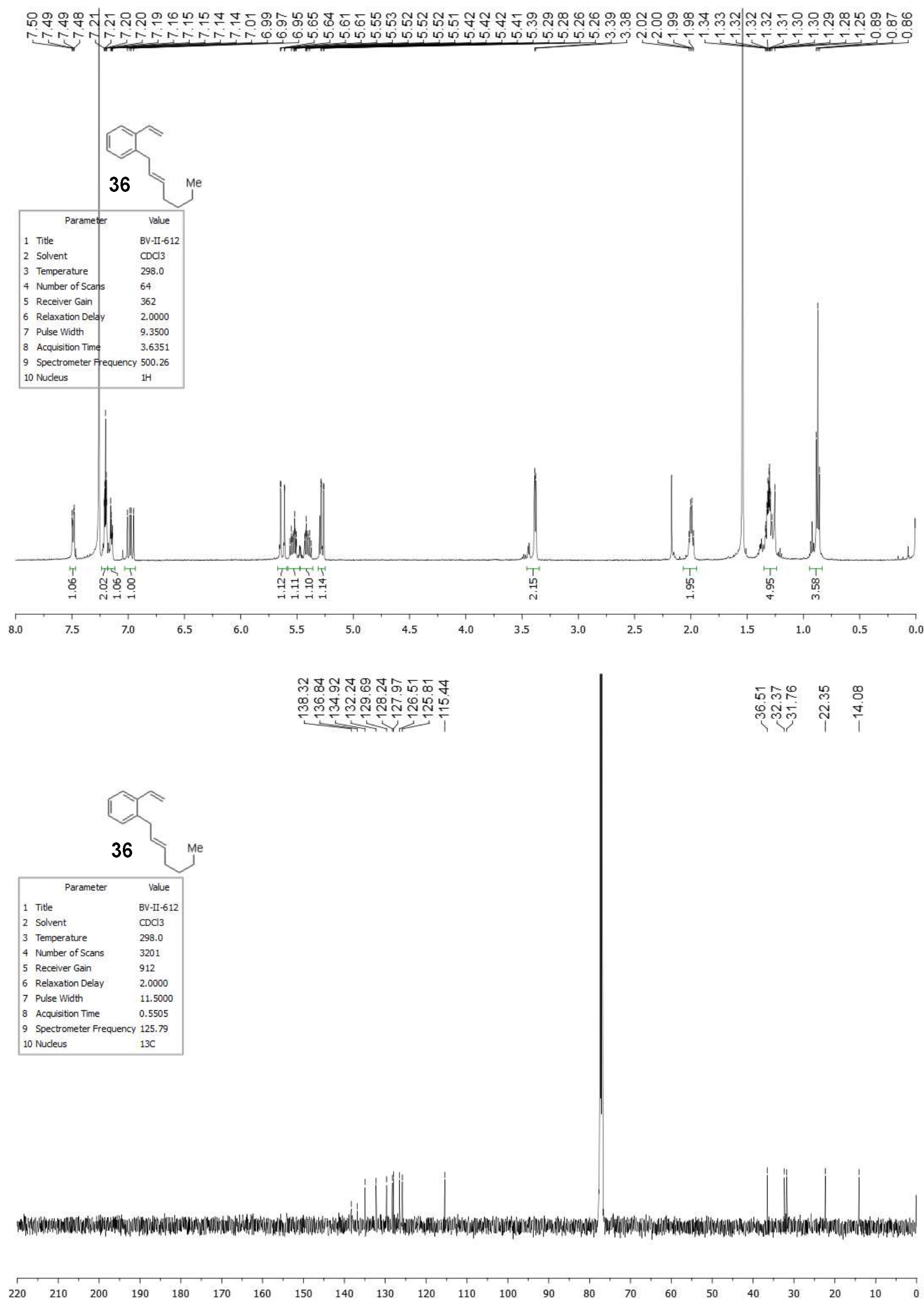

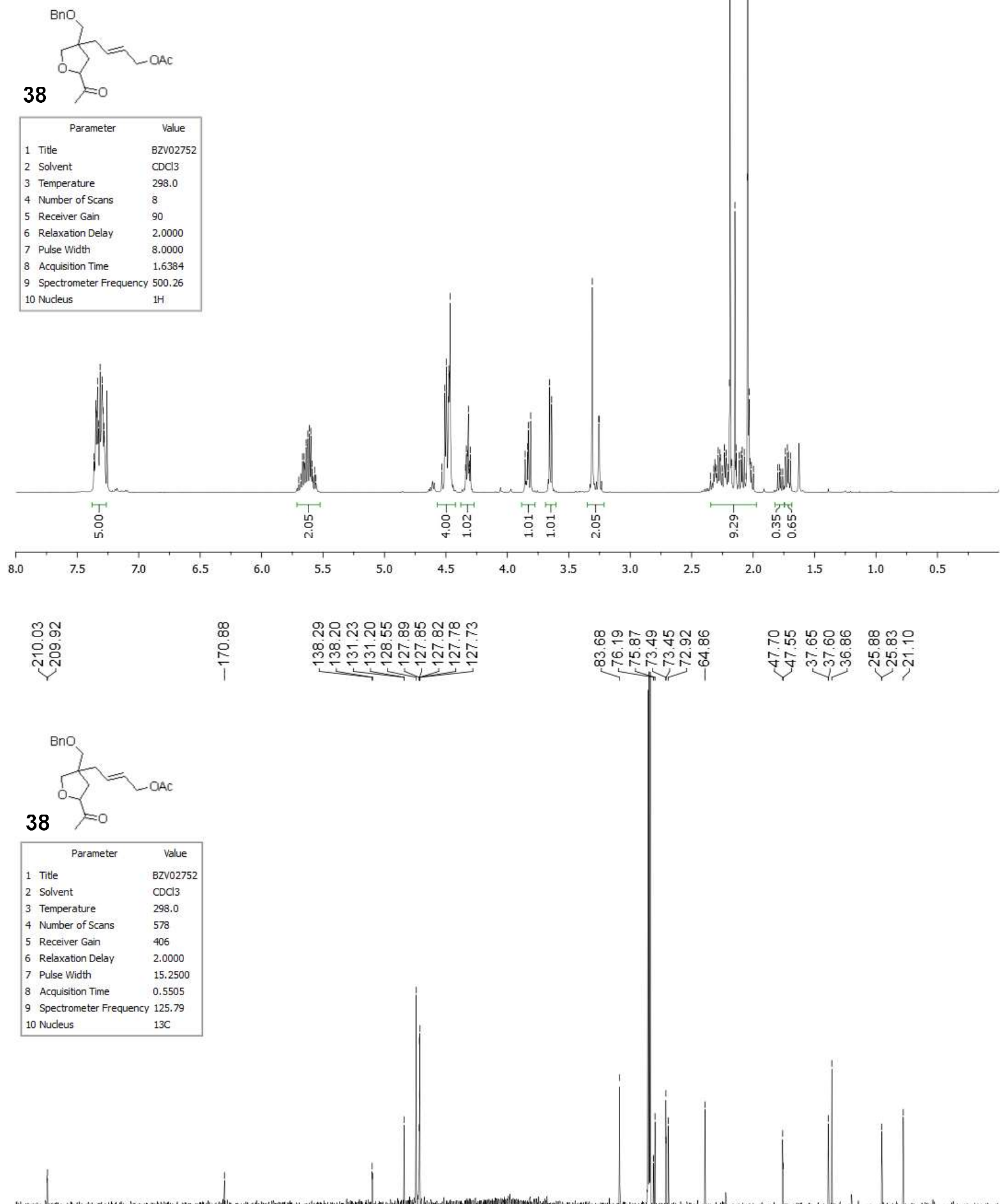

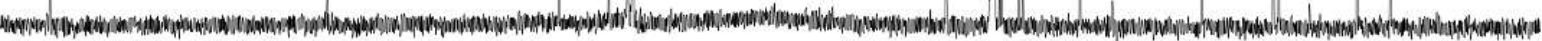

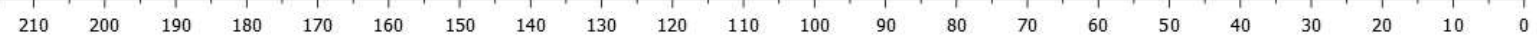

